\title{
Plenary Abstracts
}

\author{
(C) International Osteoporosis Foundation and National Osteoporosis Foundation 2006
}

\section{PL1. UPDATE ON THE MANAGEMENT OF VERTEBRAL FRACTURES: CLINICAL AND ECONOMIC ASPECTS?}

Bengt Jönsson; Centre for Health Economics, Stockholm School of Economics, Sweden

My lecture will cover the following issues:

- What are the consequences of vertebral fractures in terns of costs, quality of life reduction and mortality

- What are the evidence on cost-effectiveness of treating vertebral fractures

- What are the evidence on cost-effectiveness of treatment and prevention of osteoporosis to avoid vertebral fractures

Studies of treatment and prevention of osteoporosis to prevent fractures have focused on hip fractures, due to the well documented consequences for morbidity and mortality. However, it has become more and more apparent that prevention of vertebral fractures is as important due to the frequency, timing, and serious consequences of these fractures.

There is a problem to establish the true incidence of vertebral fractures since not all fractures will be registered in the hospital statistics as is the case with hip fractures. The registration of fractures in ambulatory care is not complete. There is also data showing that silent vertebral fractures also have consequences both in terms of costs and quality of life.

Recent data shows that the quality of life impact of vertebral fractures is greater than previous estimates. For hospitalised patients, the quality of life reduction of vertebral fractures is as great as for hip fractures.

Vertebral fractures are important for the optimal treatment and prevention of osteoporosis since these fractures occur earlier than hip fractures, and are thus key to early intervention for fracture prevention. Effectiveness on vertebral fractures are a major determinant of cost-effective strategies for treatment and prevention of osteoporosis.

\section{PL2. UPDATE ON THE MANAGEMENT OF NON-VERTEBRAL FRAC- TURES: CLINICAL AND ECONOMIC ASPECTS}

Cyrus Cooper; Director and Professor of Rheumatology, MRC Epidemiology Resource Centre, University of Southampton, Southampton General Hospital, Southampton, UK

Osteoporosis constitutes a major public health problem through its association with age related fractures. These fractures typically occur at the hip, spine and distal forearm. It has been estimated from incidence rates derived in North America that the lifetime risk of a hip fracture in caucasian women is $17.5 \%$, with a comparable risk in men of $6 \%$. Hip fractures lead to an overall reduction in survival of around $15 \%$ and the majority of excess deaths occur within the first six months following the fracture. They are also associated with considerable morbidity: they invariably necessitate hospitalization and the average length of hospital stay is around 30 days. Although all vertebral deformities do not come to clinical attention, the lifetime risk of clinically diagnosed vertebral fractures is around $15 \%$ in caucasian women. These fractures tend to be associated with back pain and result in kyphosis. They are also associated with impairment of survival, although this is likely to be due to the clustering of comorbidity which predisposes independently to osteoporosis and premature death. Around a quarter of clinically diagnosed vertebral deformities result in hospitalization.

Hip fractures typically follow a fall from the standing position and their incidence rises exponentially with age. Above the age of 50 years there is a female to male ratio of around $2: 1$. There is marked seasonality in hip fracture incidence, with substantial increases in rates during winter months in temperate countries. Nevertheless, the majority of hip fractures follow falls indoors and are not related to slipping on icy pavements. Age and sexadjusted hip fracture rates are generally higher in caucasian than in Asian populations. Furthermore, the pronounced female preponderance in fracture incidence observed in white populations is not seen amongst blacks or Asians in whom age-adjusted female to male incidence ratios approximate unity. Urbanisation in certain parts of Africa has led to a secular increase in hip fracture incidence rates, but even recently derived African rates are considerably lower than those found in North American or European whites. The incidence of clinically diagnosed vertebral fractures also rises steeply with age and the female to male incidence ratio after age adjustment is also around 2:1.

The ultimate determinants of osteoporotic fractures are bone strength and trauma. Bone strength is related to the quality of bone, its architecture and its mass. These characteristics cannot easily be assessed in vivo, but correlate closely with bone mineral density. There is now convincing longitudinal evidence that a reduction in bone density is an important determinant of fracture risk. The determinants of bone density can be categorised into those influencing the peak which is attainable during growth and consolidation; and the subsequent rate of bone loss. There is a genetic feeling to the peak bone density which can be obtained during the first 25 years of life, which is modified by nutrition, mechanical factors and hormonal status. Important determinants of bone loss include estrogen deficiency in women, low body mass index, cigarette smoking, alcohol consumption, poor dietary calcium intake, physical inactivity, certain drugs such as corticosteroids, and illnesses such as rheumatoid arthritis. Preventive strategies against osteoporotic fracture can be targeted at the general population and at those at the greatest risk. Thus, modification of physical activity and dietary calcium/vitamin D nutrition in the elderly and during mid-life, should complement high risk approaches entailing appropriate measurement of bone mineral density and targeting of antiresorptive and formation stimulating drugs. In addition, the recently developed WHO algorithm for evaluation of 10-year absolute risk of fracture provides a means whereby various antiresorptive and formulation-stimulating therapies can be targeted most cost-effectively to those at the greatest risk. The algorithm incorporates data on clinical risk factors (previous history of fracture, glucocorticoid use, thinness, cigarette smoking and alcohol consumption, and secondary osteoporosis). These risk factors, together with BMD and potentially biochemical indices of bone turnover, can be utilised to derive absolute risks of fracture and cost-utility thresholds at which treatment is justified. These data will provide the basis for translation into coherent public health strategies aiming to prevent osteoporosis both in individuals and in the general population. 


\section{PL3. UPDATE ON FRACTURE RISK ASSESSMENT: CLINICAL AND ECONOMIC ASPECTS}

John A Kanis; WHO Collaborating Centre for Metabolic Bone Diseases, University of Sheffield Medical School, Sheffield, UK

The development of effective interventions for osteoporosis has had a significant impact on our ability to treat the disorder and decrease vertebral and non-vertebral fracture risk. A major problem that needs to be resolved is who best benefits from intervention, particularly in the absence of widespread screening policies with BMD. The resolution of the problem is to optimise fracture risk prediction.

The purpose of predicting fracture risk is to direct interventions to those most at need and to avoid unnecessary treatment. Since the determinants of osteoporotic fracture are multifactorial, risk prediction will always be imperfect.

Hip fracture prediction with BMD alone is at least as good as blood pressure readings to predict stroke. Like blood pressure tests, the test has high specificity, but its sensitivity (detection rate) for fracture outcome is low over most reasonable assumptions. When the WHO thresholds are used, the majority of fractures will occur in those individuals characterised to be at low risk. The predictive value of BMD can be enhanced by the use of other factors such as biochemical indices of bone resorption and clinical risk factors. Validated clinical risk factors that contribute to fracture risk independently of BMD include age, previous fragility fracture, a family history of hip fracture, the prolonged use of oral corticosteroids, rheumatoid arthritis, smoking and excessive consumption of alcohol. The presence of such factors increases fracture risk over and above that which can be explained on the basis of BMD. Therefore, diagnostic thresholds for osteoporosis using BMD differ from intervention thresholds. Intervention thresholds should be based on the absolute risk of fracture. In the absence of validated population screening strategies, a case finding strategy is recommended based on the finding of risk factors and the computation of fracture probability.

\section{PL4. UPDATE ON THE MANAGEMENT OF SECONDARY OSTEOPOROSIS: CLINICAL AND ECONOMIC ASPECTS}

Socrates E Papapoulos; Department of Endocrinology \& Metabolic Diseases, Leiden University Medical Center, The Netherlands

Secondary osteoporosis encompasses a large number of heterogeneous conditions that can increase skeletal fragility. These range from genetic disorders affecting the skeleton (e.g. osteogenesis imperfecta) to use of medications (e.g. glucocorticoids). For some of these conditions a causal relationship with bone fragility has been established while for most a relationship to bone fragility has been derived from association or observation studies. Despite its clinical relevance, the contribution of secondary osteoporosis to the overall burden of the disease is rather small due to its relatively low prevalence in the population. However, identification of underlying causes of osteoporosis in clinical practice helps to define better the risk profile of the individual as such causes increase the risk of fractures above that associated with known risk factors such as, for example, previous fragility fracture, BMD and family history of fracture. In addition, in some patients with secondary osteoporosis treatment of the underlying disease can have a direct impact on bone fragility. Therefore, management that aims to reduce fracture risk has two primary objectives: first, the identification of potentially treatable causes of osteoporosis and treatment with disease-specific therapies, when such therapies are available; second, treatment with bone active agents which is applicable to the majority of patients. Although numerous studies with bone active agents have been reported in patients with secondary osteoporosis, these have mainly examined the efficacy of treatments on surrogate endpoints rather than on fracture risk due mainly to the small number of patients included in such studies. Reliable data on fracture frequency have been obtained only in patients with glucocorticoid-induced osteoporosis and several guidelines are currently available for the management of this condition. Because of the excessive risk of fracture of the individual with secondary osteoporosis, effective treatment with bone active agents will generally be more cost-effective compared to treatment of patients with postmenopausal osteoporosis with a similar overall risk profile and several studies have addressed this specific issue particularly in glucocorticoid-treated patients.

\section{PL5. UPDATE ON THE MANAGEMENT OF OSTEOARTHRITIS: CLINICAL AND ECONOMIC ASPECTS}

Jean-Pierre Pelletier, Unité de Recherche en Arthrose, Centre Hospitalier Notre-Dame

Until recently, the main aim of pharmacological treatments of osteoarthritis has been to control the symptoms of the disease. On the other hand, due to their mechanism of action, specific compounds may not only modify the course of symptoms over longterm treatment periods, but also favourably affect joint structure changes and thus the disease progression. The structure-modifying properties of Chondroitin sulfate (CS) were assessed in a double-blind, placebo-controlled trial that included 119 patients with interphalangeal OA. After 3 years, the group taking $400 \mathrm{mg}$ CS three times daily had significantly fewer patients with new erosive OA finger joints $(8,8 \%)$ compared to the placebo group $(29,4 \%)$. More recently, a dose of $800 \mathrm{mg}$ /day of chondroitin sulfate, given for a period of 2 years to patients with primary knee osteoarthritis, prevented the decrease in joint space width and joint space thickness observed in patients having received the placebo for the same duration. To test the long-term effects of Glucosamine sulfate (GS) on the progression of OA joint structural changes in symptoms of the knee 212 patients with knee OA were randomly assigned in a double-blind fashion to a continuous treatment with GS (1500 mg once/day) or placebo for 3 years. Patients treated with placebo had an average joint space narrowing (JSN) of approximatively 0.08 to $0.1 \mathrm{~mm} /$ year, while no joint space narrowing occured in the group treated with glucosamine sulfate. These results were later confirmed by another double-blind, placebo-controlled study, performed in 202 patients with knee OA. In the glucosamine group, the minimum joint space width of the tibio-femoral joint increased by $0.02 \mathrm{~mm}$ after 3 years while it decreases by $0.19 \mathrm{~mm}$ in the placebo group. In a three-year, randomized, double-blind, placebo-controlled study, 507 patients with severe to moderate hip osteoarthritis were randomized to receive either diacerein, $50 \mathrm{mg}$ twice daily or identical placebo. At the end of the trial, $62.7 \%$ of the placebo and $53.7 \%$ of the diacerein treated patients $(\mathrm{p}=0.037)$ had a radiological progression defined as a reduction in the joint space width of at least $0.5 \mathrm{~mm}$. Within the valid completers population $(n=269)$, the annual joint space narrowing rate was significantly lower in those receiving placebo $(0.018 \mathrm{~mm} /$ year versus $0.023 \mathrm{~mm} /$ year; $\mathrm{p}=0.042$ ). There is now a convergent body of evidence that several substances acting either through a synthesis of matrix constituants or through an inhibition of catabolic mediators and processus can significantly interact with the progression of osteoarthritis.

\section{SESSION VI}

Organized under the auspices of the Austrian Society For Bone And Mineral Research

\section{PL6. POSTTRANSPLANTATION BONE DISEASE: ClinICAL AND ECO- NOMIC ASPECTS}

P. Pietschmann ${ }^{1}$, K. Kerschan-Schindl ${ }^{2}$; ${ }^{1}$ Institute of Pathophysiology, Center of Physiology and Pathophysiology Medical University of Vienna, ${ }^{2}$ Department of Physical Medicine and Rehabilitation, Medical University of Vienna, Austria

Posttransplantation bone disease is observed after heart, lung, liver, kidney or bone marrow transplantation. The pathogenesis of bone disease after transplantation is complex and incompletely 
understood; many patients who are candidates for transplantation are treated with drugs that have adverse effects on bone (such as glucocorticoids, loop diuretics or anticoagulants) or have a preexisting bone disease (e.g.postmenopausal osteoporosis or renal osteodystrophy). Bone loss after organ transplantation appears to be caused primarily by glucocorticoids and other immunosuppressive drugs; vitamin D deficiency or hypogonadism are further contributory factors. The risk of fractures in patients after organ transplantation seems to depend on the type of transplantation. While fracture risk has been reported to be relatively low in patients after bone marrow transplantation, recipients of heart or lung transplants have a fracture prevalence of approximately $40 \%$. Despite its high incidence, data on economic aspects of posttransplantation bone disease (e.g. the costs of screening/diagnosis, prevention and treatment) are very limited.

\section{PL7. TREATMENT OF POST-TRANSPLANTATION BONE DISEASE: CLINICAL AND ECONOMIC ASPECTS}

A. Fahrleitner-Pammer, H. Dobnig; Department of Internal Medicine, Division of Endocrinology and Nuclear Medicine, Medical University Graz, Austria

Depending on which organ has failed patients reveal a high prevalence of bone disease already prior to transplantation (TX). In general, the waiting time for TX is several months and during this period minimization of risk factors as well as provision of calcium and vitamin D supplementation and hormone replacement therapy should be part of the pre-transplantation work-up. Except for patients with chronic kidney- or cholestatic liver disease who may be at high risk for adynamic bone disease there is evidence that bisphosphonate therapy prior to TX attenuates bone loss following surgery.

It is well known that during the first 6 to 12 postoperative months bone loss is exceedingly high primarily due to an uncoupling of bone formation and bone resorption driven by combined immunosuppressive therapy. This results in high fracture incidence rates. Although data on randomized double blind placebo controlled trials are still lacking there is evidence that in addition to calcium and vitamin $\mathrm{D}$ supplementation bisphosphonate treatment is beneficial following kidney-, liver-, lungand heart transplantation. Active vitamin D metabolites in patients with renal damage due to cyclosporine side-effects as well as hormone replacement therapy in hypogonadal men also show promising results in some trials.

Taking into account the overall costs of organ transplantation and given the high fracture incidence it seems justified to treat all patients with bisphosphonates following TX independent of their BMD results, at least during the first year following surgery.

\section{PL8. OSTEOPOROSIS ASSOCIATED WITH CHRONIC LUNG DISEASES:} CLINICAL AND ECONOMIC ASPECTS

H.P. Dimai; Department of Internal Medicine, Division of Endocrinology and Nuclear Medicine, Medical University of Graz, Graz, Austria

Long term respiratory conditions such as chronic obstructive pulmonary disease (COPD), asthma and cystic fibrosis (CF) are leading causes of chronic morbidity and mortality.

The overall prevalence of COPD in adults appears to lie between $4 \%$ to $10 \%$ in countries of the Western World. The prevalence of osteoporosis in patients with COPD has been shown to be as high as $60 \%$, depending on the severity of the disease. Besides the frequent use of corticosteroids as a possible reason of the high prevalence of osteoporosis in these patients, COPD itself has been shown to be associated with decreased bone mass and an increased risk of fracture, even in those who had never received a corticosteroid treatment.

The prevalence of asthma in the adult population has been estimated some $5 \%$, and - depending on the type of corticosteroid used and its cumulative dose - the risk of osteoporotic fractures in these patients has been reported to be increased by roughly $70 \%$ compared to a healthy population. In contrast to COPD, there is no clear evidence available that asthma itself, i.e. without corticosteroid treatment, may be associated with an increased risk of osteoporosis.

In adult patients with cystic fibrosis $(\mathrm{CF})$, reduced bone mineral density has been reported in as many as three fourths of these patients. Furthermore the prevalence of vertebral deformities in the same population has been shown to be $27 \%-32 \%$.

Whereas vertebral fractures in general have been shown to be associated with an increased mortality, patients with chronic lung disease who develop vertebral fractures may also experience a further decrease in respiratory function. In this regard, it has been estimated that each vertebral compression fracture may cause a $10 \%$ decrement of the Forced Expiratory Vital Capacity (FVC), a parameter describing the volume of air that can be forcibly and rapidly exhaled following a maximum inspiration.

Overall, the occurrence of osteoporotic fractures substantially contributes to the disability and mortality of patients with chronic lung diseases and also adds to the economic burden of theses diseases. However, because of increasing life expectancy and access to lung transplantation, the prevalence of osteoporosis in patients with chronic respiratory diseases is likely to increase further, and thus should be a major public health concern.

\section{PL9. OSTEOPOROSIS IN CANCER PATIENTS: CLINICAL AND ECO- NOMICAL ASPECTS}

Heinrich Resch; Medical Department II, KH Barmherzige Schwestern (St. Vincent Hospital), School of Medicine, University Vienna, Austria

Bone health is an increasingly important concern in patients surviving malignant diseases. Beside bone loss and consecutive fracture risk induced by most of chemotherapies and adjuvant therapy strategies, it is anticipated that tumor cells per se interact with the bone marrow microenvironment to drive bone destruction and tumor growth in a symbiotic relationship. Cancer cells secrete PTHrP, which is the primary stimulator of osteoblast production of RANK Ligand and responsible for the down-regulation of the OPG production by osteoblasts, thereby stimulating osteoclastogenesis. Furthermore other factors produced and secreted by tumor cells also increase the expression of RANK Ligand resulting finally in osteoporosis and also osteolytic lesions. Women with breast cancer for example are often treated with adjuvant hormonal therapy using antiestrogens (tamoxifen) to compete with endogenous estrogen for binding sites on estrogen receptors and aromatase inhibitors to inhibit conversion of androgens to estrogens. Literature and also own data show that these females are at more than 1.5- to 2-fold higher risk of experiencing vertebral fracture, as compared to patients receiving tamoxifen alone. In males prostate cancer cell proliferation is regulated and stimulated by binding of the androgen receptor by androgens. Most men with prostate cancer are treated with androgen deprivation therapy (ADT) with gonadotropin-releasing hormone $(\mathrm{GnRH})$ agonists when they develop biochemical progression. However, ADT, which inhibits testosterone production also leads to bone loss, which can lead to an increase in the risk of osteoporosis and osteoporotic fractures.

Since the discovery of the OPG/RANK/RANK Ligand signaling pathway, our understanding of the osteoclast and its role in bone biology has advanced considerably. Estrogen deficiency due to aromatase inhibition, chemotherapy, glucocorticoid exposure, and osteotropic tumors are limiting the RANK Ligand/OPG balance to favor osteoclast activation and ultimately bone loss.

Presently there are several pharmakologic therapies (including different galenic preparations of bisphosphonates and raloxifene) available which inhibit accelerated bone loss and decrease the risk of fracture. Furthermore RANK Ligand inhibition has been shown in several preclinical and clinical models to be an effective way to limit bone loss. Those therapies not only reduce fracture risk but also the number of days of bed disability and limited activity due to back pain. 


\section{Oral Communications}

\section{OC10. DENOSUMAB (AMG 162) INHIBITION OF RANK LIGAND INCREASES BONE MINERAL DENSITY IN POSTMENOPAUSAL WOMEN AFTER TWO YEARS OF TREATMENT}

E.M. Lewiecki ${ }^{1}$, P.D. Miller ${ }^{2}$, M.R. McClung ${ }^{3}$, S.B. Cohen ${ }^{4}$, Y. Liu $^{5}$, A. Wang ${ }^{5}$, L.A. Fitzpatrick ${ }^{5}$ ' ${ }^{N M}$ Clin Res \& Osteoporosis Ctr, Albuquerque, NM, USA, ${ }^{2} \mathrm{CO} \mathrm{Ctr}$ for Bone Res, Lakewood, CO, USA, ${ }^{3}$ Oregon Osteoporosis Ctr, Portland, OR, USA ${ }^{4}$ Radiant Research, Dallas, TX, ${ }^{5}$ Amgen Inc, Thousand Oaks, CA, USA

Objective: Denosumab (AMG 162) is a fully human monoclonal antibody that inhibits RANKL, a key mediator of osteoclast activity. Previous reports showed that denosumab treatment significantly increased BMD and decreased bone turnover markers compared with placebo after 12 months in 412 postmenopausal women with low BMD (T-score $\leq-1.8$ ). We now report a preplanned exploratory analysis of denosumab efficacy and safety after 24 months of treatment in this randomized, placebo-controlled, dose-ranging study.

Materials and Methods: Subjects were randomly assigned to 1 of 9 treatment groups. Eight groups received subcutaneous injections of placebo or denosumab $(6,14$, or $30 \mathrm{mg} \mathrm{3-monthly} \mathrm{or}$ $14,60,100$, or $210 \mathrm{mg} 6$-monthly). One group received open-label oral alendronate (ALN; $70 \mathrm{mg}$ once weekly).

Results: A total of $337(82 \%)$ subjects completed 24 months of study ( 38 placebo, 259 denosumab, 40 ALN). Denosumab treatment significantly reduced markers of bone turnover compared with placebo, and the reductions were sustained for 24 months. Subjects treated with denosumab for 24 months had significantly greater increases from baseline in lumbar spine, total hip, distal $1 / 3$ radius, and total body BMD compared with placebo (all $P<0.001$; Table). BMD changes for the denosumab $60-\mathrm{mg}$, 6 -monthly (60 mg Q6M) group and ALN group are also shown.

Table Mean Percent Change in BMD from Baseline at 24 Months

\begin{tabular}{lllll}
\hline & $\begin{array}{l}\text { Denosumab } \\
\text { (all doses) }\end{array}$ & $\begin{array}{l}\text { Denosumab } \\
60 \text { mg Q6M }^{\mathrm{b}}\end{array}$ & $\begin{array}{l}\text { ALN } 70 \mathrm{mg} \\
\mathrm{QW}^{\mathrm{b}}\end{array}$ & Placebo $^{\mathrm{b}}$ \\
\hline $\begin{array}{c}\text { Lumbar } \\
\text { spine }\end{array}$ & $4.25 \%-8.95 \%$ & $7.37 \% \pm 0.63 \%$ & $6.22 \% \pm 0.63 \%$ & $-1.04 \% \pm 0.68 \%$ \\
$\begin{array}{c}\text { Total hip } \\
\text { Distal } 1 / 3 \\
\text { radius }\end{array}$ & $2.76 \%-5.11 \%$ & $5.11 \% \pm 0.40 \%$ & $3.40 \% \pm 0.40 \%$ & $-1.75 \% \pm 0.43 \%$ \\
$\begin{array}{c}\text { Total body } \\
\text { Toti }\end{array}$ & $0.90 \%-4.46 \%$ & $2.63 \% \pm 0.50 \%$ & $1.51 \% \pm 0.52 \%$ & $-1.62 \% \pm 0.54 \%$ \\
\hline
\end{tabular}

${ }^{\mathrm{a}}$ Range of the percent changes for all doses; ${ }^{\mathrm{b}}$ Mean \pm SE percent change

Denosumab was generally well tolerated in these subjects. Occurrence of adverse events was similar among the denosumab, placebo, and ALN groups, and no new pattern of events was observed in the second year of treatment. Neutralizing antibodies to denosumab were absent throughout 24 months.

Conclusions: Denosumab treatment for 2 years led to sustained reductions in bone resorption and significant increases in BMD. The results of this analysis support the continued investigation of denosumab as a potential treatment for postmenopausal women with low BMD.

OC11. COST-EFFECTIVENESS OF BALLOON KYPHOPLASTY FOR SYMPTOMATIC VERTEBRAL COMPRESSION FRACTURES IN OSTHEOPOROTIC PATIENTS

M. Marchetti ${ }^{1}$, M. Caruggi ${ }^{2}$, R. Taylor ${ }^{3} ;{ }^{1}$ Laboratory of Clinical Epidemiology, IRCCS Policlinico San Matteo, Pavia, Italy, ${ }^{2}$ S.A.V.E. Società Analisi e Valutazioni Economiche, Milan, Italy, ${ }^{3}$ Department of Public Health and Epidemiology, University of Birmingham, UK

Objective: To assess the cost-effectiveness of balloon kyphoplasty (BKP) as compared with either conventional medical management (CMM) or vertebroplasty (VP), in osteoporotic patients who incurred vertebral compression fractures (VCF).

Methods: A Markov model was developed in order to assess the incremental cost per quality-adjusted life year (QALY) ratio from the perspective of the Italian Health Services at a 2-year time horizon. In the model, patients were allowed to incur symptomatic or asymptomatic cement leakage (after BKP or VP), recurrent VCF, death. The rate of recurrent VCF was based on patient's age and sex, VCF number, rate of cement leakage and therapy. Pain and quality-of-life data were obtained from a systematic review of literature. Utilities were imputed from SF-36 values using the algorithm of Nichol et al (MDM, 2001). In-hospital resource consumption was recorded through chart review. Basecase analysis modelled a 70-year old female with primary osteoporosis incurring her first VCF.

Results: In basecase analysis, BKP gained 0.42 QALYs and 109 VAS points more than CMM. BKP also gained 0.09 QALYs and 44 VAS points more than VP. At a cost of $€ 4,300$ for BKP material and of $€ 1,000$ for VP material, the incremental costeffectiveness of BKP vs CMM was $€ 4,917$ per QALY $(€ 22$ per VAS point), and BKP vs VP was $€ 14,762$ per QALY ( $€ 48$ per VAS point). The base-case cost-effectiveness ratio were sensitive to the time horizon of the analysis, the length of stay for CMM patients, the number of baseline VCFs and the disutility of a recurrent VCF. In patients with $2 \mathrm{VCF}$ and 2 vertebral levels treated, the incremental cost-effectiveness of BKP was $€ 4,147$ per QALY, as compared with CMM and VP was dominated (extended dominance).

Conclusions: A novel therapy is conventionally considered cost-effective if its incremental cost-effectiveness ratio is below $€ 40,000$ per QALY (Laupacis et al, CMAJ 1992). Consequently, $\mathrm{BKP}$ is a cost-effective therapy for patients with symptomatic VCF. BKP is particularly cost-effective in patients with two or more VCF

\section{OC12. POSTMENOPAUSAL BONE FRAGILITY - A DISEASE OF FAILED ADAPTATION}

P. Szulc ${ }^{1}$, E. Seeman ${ }^{2}$, F. Duboeuf ${ }^{1}$, E. Sornay-Rendu ${ }^{1}$, F Munoz ${ }^{1}$, P.D. Delmas ${ }^{1}$; INSERM 403 Research Unit and Université Claude Bernard Lyon 1, Lyon, France, ${ }^{2}$ Department of Endocrinology, University of Melbourne, Melbourne, Australia

Postmenopausal bone loss results in decreased bone strength and increased fracture risk. However, morphological basis underlying cortical bone loss was only partly studied. We evaluated age-related changes in bone size and mass at one-third distal radius by yearly repeated DXA measurements (Hologic QDR2000) during a long term follow up (mean - 7.1 years) in 846 women aged 31 to 89 .

In premenopausal women, the outward shift of constant amount of bone mineral (bone mineral content [BMC]) increased estimated bending strength (cross-sectional moment of inertia [CSMI], section modulus) despite cortical thinning. In perimenopausal women, slight slowdown of periosteal apposition and mild acceleration of endocortical resorption resulted in a decrease in BMC and cortical thickness but the bending strength remained stable. After menopause, periosteal apposition was slower and endocortical resorption was faster than before (both $\mathrm{p}<0.05$ ). Consequently, BMC, cortical thickness and estimated bending strength declined $(\mathrm{p}<0.0001)$. The higher the remodelling rate (elevated serum levels of osteocalcin [OC] and b-CTX-I), the greater was the decline in $\mathrm{BMC}$, cortical thickness and estimated bending strength. The rate of periosteal expansion was independent of the bone turnover rate. In postmenopausal women with slow bone turnover (lowest quartiles of OC and b-CTX-I), periosteal apposition offset the loss of strength due to low endocortical resorption and the estimated bending strength remained stable despite the BMC loss. In postmenopausal women treated with hormone replacement therapy (HRT), expansion of the medullary cavity and decline in cortical area were less than in 
untreated women. In 637 postmenopausal women, estimates of bone strength predicted the risk of all fracture occurrence (e.g., CSMI - O.R. $=2.21$ per 1 SD decrease, 95\% C.I.: $1.64-2.98)$.

In summary, accelerated endocortical resorption and decreased periosteal apposition after menopause result in the loss of bone mass and bending strength which is partly inhibited by HRT. Increased bone turnover is associated with faster cortical thinning and greater decline in the estimated bone strength. Low estimated bone strength based on morphological parameters is predictive of the increased fracture risk. We conclude that postmenopausal osteoporosis is characterised - in the cortical compartment - by a failure of periosteal apposition to compensate for endocortical resorption.

OC13. REDUCTION IN THE RISK OF BACK PAIN PERSISTS AT LEAST 30 MONTHS AFTER DISCONTINUATION OF TERIPARATIDE TREATMENT: A META-ANALYSIS

J.-Y. Reginster ${ }^{1}$, M.C. Nevitt' ${ }^{2}$, P. Chen ${ }^{3}$, R.K. Dore ${ }^{4}$, D.P. Kiel ${ }^{5}$, J.R. Zanchetta ${ }^{6}$, E.V. Glass ${ }^{3}$, J.H. Krege ${ }^{3} ;{ }^{1}$ University of Liège, Liège, Belgium, ${ }^{2} \mathrm{UCSF}$, San Francisco, CA, USA, ${ }^{3}$ Eli Lilly and Company, Indianapolis, IN, USA, ${ }^{4}$ UCLA, Anaheim, CA, USA, ${ }^{5}$ Hebrew Rehabilitation Center for Aged and Harvard Medical School, Boston, MA, USA, ${ }^{6}$ Instituto de Investigaciones Metabólicas, Buenos Aires, Argentina

Background: Vertebral fractures are the most frequent outcome in osteoporosis and may result in back pain with functional limitations and diminished quality of life.

Objectives: Evaluate the risk of developing new or worsening back pain during treatment with teriparatide [rhPTH (1-34), TPTD] or control +30 months of additional follow up in patients with osteoporosis.

Methods: All four TPTD trials with posttreatment follow-up were included in our analyses: two placebo controlled trials, (Neer 2001, Orwoll 2003) one alendronate $10 \mathrm{mg}$ daily comparator trial, (Body 2002) and one study of hormone replacement therapy (HRT) versus HRT plus TPTD (Ste-Marie 2001). During followup, patients were allowed to receive other osteoporosis treatments. Back pain was defined as either new or worsening in severity after the initiation of study drug through the end of posttreatment follow-up. The interaction of treatment and study in a multivariate Cox proportional hazards model was used to assess the heterogeneity of results among studies and to estimate the relative risk of back pain after adjusting for other osteoporosis drug use following discontinuation of TPTD.

Results: Findings were not statistically heterogeneous among trials $(\mathrm{P}=0.66)$ and there were no differences between groups administered TPTD20 or $40 \mathrm{mcg} /$ day doses $(\mathrm{P}=0.31)$. Therefore, placebo, HRT, and alendronate groups were pooled as a control group and compared with pooled TPTD groups. Relevant data are listed in table below. Overall, patients in the pooled TPTD

Table

\begin{tabular}{lllllll}
\hline Endpoint & Group & N & Cases & $\begin{array}{l}\text { Patient- } \\
\text { years } \\
\text { at risk }\end{array}$ & $\begin{array}{l}\text { Rate } \\
\text { (per100 } \\
\text { patient-yrs) }\end{array}$ & $\begin{array}{l}\text { RR } \\
\text { (95\% C.I.) }\end{array}$ \\
\hline $\begin{array}{c}\text { Any back } \\
\text { pain }\end{array}$ & Control & 691 & 203 & 2187.2 & 9.28 & \\
TPTD & 1222 & 285 & 4157.2 & 6.86 & $\mathbf{0 . 7 3}$ \\
$\begin{array}{c}\text { Moderate/ } \\
\text { severe } \\
\text { back pain }\end{array}$ & Control & 691 & 144 & 2340.8 & 6.15 & $(0.61-0.87)$ \\
$\begin{array}{c}\text { Severe } \\
\text { back pain }\end{array}$ & Control & 691 & 45 & 4761.8 & 1.73 & $(0.58-0.89)$ \\
& TPTD & 1222 & 33 & 2607.9 & 0.69 & $\mathbf{0 . 3 9}$ \\
& & & & & & $(0.25-0.61)$ \\
\hline
\end{tabular}

group had reduced risk for any back pain, moderate or severe back pain, and severe back pain compared with pooled control from initiation of study drug through the end of follow-up. A sensitivity analysis showed that the results were consistent among trials and robust to the removal of each individual trial from the meta-analysis.

Conclusion: The results demonstrate reduced back pain risk in patients with osteoporosis treated with teriparatide and then followed for 30 additional months in four clinical trials.

\section{OC14. THE DIFFERENTIAL EXPRESSION OF OPG/RANKL IN HUMAN OSTEOARTHRITIC SUBCHONDRAL BONE OSTEOBLASTS IS AN INDI- CATOR OF THE METABOLIC STATE OF THESE DISEASE CELLS}

J. Martel-Pelletier ${ }^{1}$, D. Lajeunesse ${ }^{1}$, F. Mineau ${ }^{1}$, H. Fahmi ${ }^{1}$, M. Lavigne ${ }^{2}$, J-P. Pelletier ${ }^{1}$; ${ }^{1}$ Osteoarthritis Research Unit, University of Montreal Hospital Centre (CHUM), Notre-Dame Hospital, Montreal, Quebec, Canada, ${ }^{2}$ Department of Orthopaedics, Maisonneuve-Rosemont Hospital, Montreal, Quebec, Canada

Objective: Although the ratio of OPG to RANKL is considered a key regulator of bone metabolism, there is paucity of data on their role in human osteoarthritic (OA) subchondral bone. We reported that OA osteoblasts (ob) can be discriminated into two subgroups identified by their levels of endogenous production (low and high) of $\mathrm{PGE}_{2}$. Here, we investigated the OPG and RANKL expression levels in ob of both human OA subgroups and in normal cells, and analysed the effects of bone remodeling factors on OPG and RANKL expression.

Methods: Gene expression was determined using real-time PCR, and gene regulation was monitored following treatment with vitamin $\mathrm{D}_{3}(50 \mathrm{nM})$, TGF-B1 (5 ng/ml), IL-1ß (100 pg/ml), TNF- $\alpha(5 \mathrm{ng} / \mathrm{ml}), \mathrm{PGE}_{2}(500 \mathrm{nM})$, or PTH $(100 \mathrm{nM})$.

Results: Human ob demonstrated much higher levels of OPG expression than RANKL. OA ob expressed less OPG than normal cells; this was more pronounced in the low OA cells $(\mathrm{p}<0.05)$. Compared to normal ob, RANKL levels were increased in the low OA and decreased in the high subgroup. The OPG/RANKL ratio was significantly diminished in low OA ob compared to normal or high OA $(\mathrm{p}<0.02, \mathrm{p}<0.03)$, and markedly increased in high $\mathrm{OA}$ compared to normal. In low $\mathrm{OA}$ cells, both vitamin $\mathrm{D}_{3}$ and TGF- $\beta 1$ significantly reduced the OPG/RANKL ratio, IL-1B demonstrated no effect, and the ratio was significantly increased in response to TNF- $\alpha$ (335-fold), and to a lesser extent by PTH and $\mathrm{PGE}_{2}$ (30- and 7-fold).

Conclusion: OPG and RANKL expression levels, and consequently the OPG/RANKL ratio, differed according to human OA subchondral bone ob classification; OPG/RANKL is decreased in the low and increased in the high OA ob. This suggests that the metabolic state of the low OA ob would promote bone resorption, while that of the high OA ob, which could be reached at another stage in the progression of the disease, would favor reduced resorption. The latter may very well be due to a flare in disease activity in which factors such as TNF- $\alpha$ and $\mathrm{PGE}_{2}$ are up-regulated.

\section{OC15. ARE COSTS OF FRACTURE PROPORTIONAL TO THEIR MOR- BIDITY?}

F. Borgström ${ }^{1,2}$, O. Johnell ${ }^{3}$, B. Jönsson ${ }^{4}$, J.A. Kanis ${ }^{5} ;{ }^{1}$ Medical Management Centre, Karolinska Institutet, Stockholm, Sweden, ${ }^{2}$ Stockholm Health Economics, Stockholm, Sweden, ${ }^{3}$ Department of Orthopaedics, Malmö General Hospital, Malmö, Sweden, ${ }^{4}$ Stockholm School of Economics, Stockholm, Sweden, ${ }^{5}$ Centre for Metabolic Bone Diseases (WHO Collaborating Centre), University of Sheffield Medical School, Sheffield, UK

Background: Intervention thresholds (IT) for osteoporosis can be defined as the 10 year absolute hip fracture risk at which an intervention becomes acceptable. To account for the total burden of osteoporosis the excess morbidity of all fractures compared to hip fracture (a.k.a. hip fracture equivalents) have been calculated and used in intervention threshold estimations (refs). One 
assumption underlying these calculations is that the fracture costs are proportional to fracture disutility.

Objective: To investigate whether the assumption of proportionality between disutility and fracture costs by estimating the excess costs of all osteoporotic fractures compared to hip fracture.

Methods: An approach similar to what was used to assess the excess morbidity of fractures was adapted for costs. A Markov cohort model was built that could measure the downstream of costs related to osteoporotic fracture types for different ages of fracture occurrence. The estimated lifetime related fracture costs were related to age differentiated fracture incidence making it possible to create a hip fracture cost equivalent index. The cost data used in the model was the most recent and appropriate for a Swedish setting.

Results: The estimated hip fracture cost equivalent index varied from 2.33 at the ages $50-54$ to 1.36 for ages $85-89$. This can be compared to the previously calculated hip fracture morbidity equivalents that vary from 6.07 (50-54 year olds) to 1.60 (8589 years olds). This indicate that, with available fracture cost data, hip fractures is related to a higher proportion of the total fracture costs compared to the proportion of disutility related to hip fractures.

Conclusions: The results show that the assumption of proportionality between cost and disutility might not be entirely appropriate. Not taking this into account might have the potential to skew the results in intervention threshold calculations. One solution could be to down adjust the fracture related costs by the ratio between the morbidity and the cost index when estimating the intervention thresholds.

\section{OC16. NEED FOR ADDITIONAL CALCIUM TO REDUCE THE RISK OF HIP OR ANY NONVERTEBRAL FRACTURE WITH VITAMIN D SUPPLEMEN- TATION: EVIDENCE FROM A COMPARATIVE META-ANALYSIS OF RAN- DOMIZED CONTROLLED TRIALS}

S. Boonen ${ }^{1}$, D. Vanderschueren ${ }^{1}$, P. Haentjens ${ }^{2}$, P. Lips ${ }^{3}$; ${ }^{1}$ Leuven University Center for Metabolic Bone Diseases, Leuven, Belgium, ${ }^{2}$ Brussels University Department of Orthopaedics and Traumatology, Brussels, Belgium, ${ }^{3}$ Amsterdam University Department of Endocrinology, Amsterdam, The Netherlands

Objectives: A recent meta-analysis (JAMA. 2005;293:22572264) reported that supplementation with oral vitamin D 700-800 $\mathrm{IU} /$ day reduces the risk of hip or any nonvertebral fracture in elderly individuals by approximately $25 \%$. However, this metaanalysis was unable to define the role of additional calcium supplementation.

The aim of the current study was to assess the need for calcium supplementation in individuals receiving vitamin $\mathrm{D}$ for the prevention of hip and nonvertebral fractures. Methods MEDLINE (search terms: 'vitamin D' AND 'hip fracture'), bibliographies of articles retrieved, and the authors' reference files were used to identify randomized controlled trials (RCTs) of oral vitamin D supplementation with or without calcium supplementation vs placebo/no treatment in postmenopausal women and/or older men (over 50 years) specifically reporting hip fracture risk. Data extraction was independent by 2 authors using predefined criteria, including study quality indicators.

Results: All pooled analyses are based on random-effects models. Based on 4 RCTs (9083 subjects), the pooled relative risk (RR) of hip fracture for vitamin D supplementation alone was 1.10 (95\% confidence intervals [CI] 0.89-1.36). No between-trial heterogeneity was observed. For the 5 RCTs (9227 subjects) of vitamin D supplementation with calcium supplementation, the pooled RR for hip fracture was 0.79 (95\% CI, 0.64-0.97). There was no heterogeneity between trials. The RRs for all nonvertebral fracture were $0.98(0.83-1.16)$ for vitamin $\mathrm{D}$ alone and 0.84 (0.73-0.96) for vitamin D with calcium, with moderate heterogeneity between trials. In an adjusted indirect comparison of the summary RRs from the 2 meta-analyses, the RR for hip fracture for vitamin $\mathrm{D}$ with calcium vs vitamin $\mathrm{D}$ alone was 0.72 (95\% CI, $0.53-0.96)$ and the RR for all nonvertebral fractures was 0.77 (95\% CI, 0.60-0.99).
Conclusions: Oral vitamin D supplementation appears to reduce the risk of hip and any nonvertebral fractures only when calcium supplementation is added. Our findings suggest that to optimize clinical efficacy, vitamin D supplementation should be complemented with calcium supplements.

\section{OC17. INCIDENCE OF HIP FRACTURE OVER A 10-YEAR PERIOD: REVERSAL OF A SECULAR TREND RELATED TO A SPECIFIC DECREASE OF THE AGE-ADJUSTED INCIDENCE IN INSTITUTIONAL-DWELLING ELDERLY WOMEN}

T. Chevalley ${ }^{1}$, E. Guilley ${ }^{2}$, F. Herrmann ${ }^{1}$, P. Hoffmeyer ${ }^{3}$, C.-H. Rapin $^{2}$, R. Rizzoli ${ }^{1}$; ${ }^{1}$ Service of Bone Diseases (World Health Organization Collaborating Centre for Osteoporosis Prevention), Department of Rehabilitation and Geriatrics, Geneva Switzerland, ${ }^{2}$ Centre for Interdisciplinary Gerontology, ${ }^{3}$ Service of Orthopaedic Surgery, University Hospitals of Geneva, Geneva, Switzerland

Hip fracture is the main burden of osteoporosis in terms of mortality, disability, and costs. Many studies have described an increase of age-adjusted hip fracture incidence (secular trend). From 1991 to 2000 , we examined the age distribution of patients with hip fracture, the secular changes in the incidence rates of hip fracture in women and men, and the influence of the residential status (institutional-dwelling or home-dwelling) in a well defined community. We recorded all patients 60 years of age and older discharged with a diagnosis of a hip fracture in the Geneva university hospital, which is receiving $95 \%$ of hip fracture occurring in a well defined area. Over a 10-year period, 3951 hip fractures were recorded among 1409 women and 215 men living in nursing homes and 1776 women and 551 men living in private accommodation. Mean age $( \pm$ SD) of women and men with hip fracture was $86.4 \pm 6.5$ and $84.5 \pm 7.9$ years in institutionaldwelling elderly, and $81.6 \pm 8.5$ and $79.7 \pm 8.9$ years in homedwelling elderly $(p<0.001)$, respectively. From 1991 to 2000 , the population older than 60 years increased by $12.8 \%$. Mean age of hip fractured patients increased each year by +0.13 year $(\mathrm{p}=0.032)$ and by +0.14 year $(\mathrm{p}=0.038)$ in institutional- and home-dwelling elderly women, respectively. In men of the corresponding groups, mean age changes were +0.28 year $(p=0.129)$ and -0.06 year (NS), respectively. The age-adjusted incidence rate of hip fractures, standardized to the 2000 Geneva population, decreased significantly by $1.3 \%$ per year in women $(p=0.039)$ and remained unchanged in men $(+0.5 \%$ per year, $\mathrm{p}=0.686$ ). Among institutional-dwelling elderly women, hip fracture incidence decreased by $1.9 \%$ per year $(\mathrm{p}=0.044)$ whereas it remained stable $(+0.0 \%$ per year, $p=0.978)$ among home-dwelling women. In men, no significant change in hip fracture incidence occurred among institutional-dwelling $(+1.0 \%, \quad p=0.666)$ nor home-dwelling $(+0.8 \%$ per year, $\mathrm{p}=0.565)$.

In conclusion, despite an increase in the population at risk and in the mean age of hip fractured women, the significant decrease in age-adjusted incidence of hip fracture observed in women but not in men was related to a specific decrease in the age-adjusted incidence of hip fracture in institutional-dwelling elderly women.

\section{OC18. ADVANCED ATHEROSCLEROSIS IN THE LUMBAR AORTA POSES AN INCREASED RISK FOR FUTURE CARDIOVASCULAR EVENTS AND HIP FRACTURES IN POSTMENOPAUSAL WOMEN}

L.B. Tankó, Y.Z. Bagger, P. Alexandersen, C. Christiansen; Center for Clinical and Basic Research, Ballerup, Denmark

Objectives: To investigate whether the severity of aorta calcification (AC) - a surrogate marker of atherosclerosis -is an independent predictor of low BMD, accelerated bone loss, and risk of incident fractures in postmenopausal women.

Materials and Methods: Participants were 2662 postmenopausal women $65 \pm 7$ years old, who were followed for 7.5 years. Of them, 847 (31.8\%) had osteoporosis based on BMD T-score 
criteria, $171(6.4 \%)$ had a prevalent vertebral and $20(0.8 \%)$ reported a prevalent hip fracture. Aorta calcification was visualized on lateral lumbar radiographs and graded on a scale from 0 to 24 using a published scoring-system. Outcome variables were the annual rate of bone loss from the lumbar spine and proximal femur (DEXA), incident vertebral fractures (X-ray), hip fractures (self-reported), and acute cardiovascular events. Independent variables included in the multivariate logistic regression analyses were age, years since menopause (YSM), BMI, education level, smoking, daily walking and weekly fitness activities, hypertension, hyperlipidemia, and diabetes. Cut-off value for advanced atherosclerosis was defined by the mean $\pm 1 \mathrm{SD}$ of women with YSM $<10$ years $(1.1 \pm 2.0, \mathrm{n}=694)$.

Results: Women with AC $\geq 3$ had significantly lower BMD at both skeletal sites $(\mathrm{p}<0.001)$. In addition, they had significantly higher annual rate of bone loss from the proximal femur $(-0.38 \pm 0.05 \%$ vs. $-0.25 \pm 0.03 \%, \mathrm{p}=0.02)$, but not from the spine $(0.26 \pm 0.04 \%$ vs. $0.18 \pm 0.03 \%$, respectively). Rate of bone loss from the proximal femur was linearly associated with the parallel rate of atherogenesis $(p=0.01)$. At follow-up, 471 $(16.2 \%)$ vertebral and $36(1.4 \%)$ incident hip fractures was registered. Multivariate logistic regression models including the same set of independent variables indicated that $\mathrm{AC} \geq 3$ is an independent predictor of increased risk for a hip fracture (OR $2.3,95 \%$ CI $1.1-4.8, \mathrm{p}=0.03$ ) or a cardiovascular event (OR $2.4,95 \%$ CI $1.6-3.8, \mathrm{p}<0.001)$. The increase in risk was only suggestive for vertebral fractures (OR 1.2, 95\% CI 1.0-1.5, $\mathrm{p}=0.12$ ).

Conclusions: Peripheral vascular disease seems to contribute to the pathogenesis of osteoporosis, particularly at peripheral skeletal sites. Intervention studies are needed to clarify whether anti-atherogenic medications can improve hip fracture prevention in postmenopausal women.

\section{OC19. EFFECTS OF TIBOLONE AND RALOXIFENE ON BONE: RESULTS OF THE STEP STUDY}

P.D. Delmas ${ }^{1}$, S. Adami ${ }^{2}$, S.R. Davis ${ }^{3}$, S.C. van Os ${ }^{4} ;{ }^{1}$ INSERM Research Unit 403 and Université Claude Bernard Lyon 1, Lyon, France, ${ }^{2}$ Clinical Orthopedic Rehabilitation Center, Verona, Italy, ${ }^{3}$ Monash University, Melbourne, Australia, ${ }^{4}$ Clinical Development Department, N.V Organon, Oss, The Netherlands

Background/Objectives: The Study of Tibolone's Effects on osteoPenia (STEP) was a 2-year, multicenter, double-blind, group comparative trial to determine the effects of tibolone $1.25 \mathrm{mg}(\mathrm{N}=115)$ and raloxifene $60 \mathrm{mg}(\mathrm{N}=120)$ on bone mineral density (BMD) in postmenopausal women with osteopenia.

Methods: 310 otherwise healthy postmenopausal women with osteopenia (lumbar vertebral BMD T score -2.5 to -1.0 ), mean age 66 years, were randomized. Lumbar spine BMD was assessed by dual X-ray absorptiometry (DXA). Adverse events (AEs) were reported.

Results: BMD data are presented as the mean percent change from baseline after 1 and 2 years treatment using the last observation carried forward in the ITT group.

\begin{tabular}{|c|c|c|c|c|}
\hline \multirow[t]{2}{*}{ Treatment group } & \multicolumn{2}{|l|}{ Spine } & \multicolumn{2}{|l|}{ Hip } \\
\hline & Week 52 & Week 104 & Week 52 & Week 104 \\
\hline $\begin{array}{l}\text { Tibolone } \\
\text { Raloxifene }\end{array}$ & $\begin{array}{l}+2.55 \% \\
+1.41 \%\end{array}$ & $\begin{array}{l}+4.38 \% \\
+2.57 \%\end{array}$ & $\begin{array}{l}+0.90 \% \\
+0.55 \%\end{array}$ & $\begin{array}{l}+1.49 \% \\
+0.63 \%\end{array}$ \\
\hline
\end{tabular}

Treatment resulted in a statistical significant difference in favor of the tibolone group for BMD for the lumbar vertebra compared with raloxifene at 1 year $(p=0.0048)$ and 2 years $(p=0.0002)$. For hip BMD, a statistically significant difference in favor of the tibolone group when compared to raloxifene was found after 2 years $(p=0.018)$. Comparison of the frequency of hot flushes presented a higher incidence in the frequency of hot flushes (including menopausal symptoms) in the raloxifene group.

Conclusions: Treatment with tibolone up to 2 years was well tolerated and not only provides a comprehensive treatment for climacteric symptom relief but also is an attractive option for the prevention of postmenopausal bone loss.

\section{OC20. LASOFOXIFENE INCREASES THE SPINE AND HIP BMD AND DECREASES BONE TURNOVER MARKERS IN POSTMENOPAUSAL WOMEN WITH LOW OR NORMAL BMD}

M. McClung ${ }^{1}$, E. Siris ${ }^{2}$, S. Cummings ${ }^{3}$, H. Bone ${ }^{4}$, R. Recker ${ }^{5}$, P. Delmas ${ }^{6}$, J. Zanchetta $^{7}$, M. Lewiecki ${ }^{8}$, C. Zerbini ${ }^{9}$, S. Miller ${ }^{10}$, K. Wolter ${ }^{11}$, J. Proulx ${ }^{11}$, R. Brunell ${ }^{11}$ D. Radecki ${ }^{11}$; ${ }^{1}$ Oregon Osteoporosis Ctr., Portland, OR, USA, ${ }^{2}$ NY Presbyterian Hosp-Columbia Univ, NY, NY, USA, ${ }^{3}$ San Francisco Coordinating Ctr., CA, USA, ${ }^{4}$ Michigan Bone and Mineral Clinic, Detroit, MI, USA, ${ }^{5}$ Creighton Univ, Omaha, NE, USA, ${ }^{6}$ Univ Claude Bernard, Lyon, France, ${ }^{7}$ Instituto de Investigaciones Metabólicas, Buenos Aires, Argentina, ${ }^{8}$ New Mexico Clinical Research \& Osteoporosis Ctr., Albuquerque, NM, USA, ${ }^{9} \mathrm{Hos}-$ pital Helopolis, Sao Paulo, Brazil, ${ }^{10}$ SAM Clinical Research Center, San Antonio, TX, USA, ${ }^{11}$ Pfizer Global Research and Development, Groton, CT, USA

Objective: Increased bone mineral density (BMD) and reduced bone turnover markers have been reported in Phase 2 trials with lasofoxifene, a new SERM. We report the pooled results from two identical Phase 3, double-blind trials.

Method: 1907 postmenopausal women (lumbar spine T-score 0 to -2.5$)$ were randomized to lasofoxifene $0.025 \mathrm{mg} / \mathrm{d}, 0.25 \mathrm{mg} /$ $\mathrm{d}$, or $0.5 \mathrm{mg} / \mathrm{d}$ or placebo (PBO) daily for 2 years. They also received daily calcium and vitamin $\mathrm{D}$. The primary endpoint, change in L1-L4 lumbar spine BMD at 2 years, was evaluated using an a priori fixed sequence multiple comparisons procedure on data pooled from both studies.

Results: At 2 years, patients treated with $0.025 \mathrm{mg} / \mathrm{d}$, $0.25 \mathrm{mg} / \mathrm{d}$, or $0.5 \mathrm{mg} / \mathrm{d}$ lasofoxifene had a significant increase in lumbar spine BMD from baseline: $1.5 \%, 2.3 \%$, and $2.3 \%$, respectively $(\mathrm{p} \leq 0.001)$ compared with a decrease of $0.7 \%$ with placebo. All 3 lasofoxifene doses significantly increased BMD of the total hip and its subregions ( $p \leq 0.001$ vs. PBO). Lasofoxifene significantly increased BMD of the lumbar spine and total hip for all 3 doses at 6 and 12 months. At 6 and 24 months, lasofoxifene groups exhibited significant decreases in bone turnover markers vs. PBO. Median percent changes from baseline at $6 \mathrm{~m}$ for $0.025 \mathrm{mg} / \mathrm{d}, 0.25 \mathrm{mg} / \mathrm{d}$ and $0.5 \mathrm{mg} / \mathrm{d}$ lasofoxifene (underlined) and PBO respectively were osteocalcin: $-9.40 \%,-22.5 \%$, $-20.8 \%, 5.71 \%$; CTX: $-37.5,-49.9 \%,-5 \overline{0.9 \%,}-0.43$; and P1NP: $-30.0 \%,-40.5 \%,-40.5 \%,-4.6 \% \quad(p \leq 0.001)$. Bone biopsies in lasofoxifene-treated subjects showed bone of norma quality. Overall, lasofoxifene was well-tolerated. Adverse events associated with lasofoxifene therapy included hot flushes, leg cramps, and increased vaginal moisture.

Conclusion: In postmenopausal women with low or normal BMD, lasofoxifene treatment significantly increased lumbar spine and hip BMD and decreased bone turnover markers, with beneficial changes observed as early as 6 months. Lasofoxifene may be an appropriate therapy for the prevention of bone loss in postmenopausal women.

\section{OC21. STRONTIUM RANELATE REDUCES THE RISK OF FRACTURE IN ELDERLY WOMEN WITH OSTEOPOROSIS IN THE FIRST YEAR OF TREATMENT}

E. Seeman ${ }^{1}$, B. Vellas ${ }^{2}$, C.L. Benhamou ${ }^{3}$, J.P. Aquino ${ }^{4}$, J. Semler $^{5}$, J.-M. Kaufman ${ }^{6}$, K. Hoszowski ${ }^{7}$, A. Roces Varela ${ }^{8}$, C.E. Fiore ${ }^{9}$, K. Brixen ${ }^{10}$, J.-Y. Reginster ${ }^{11}$, S. Boonen ${ }^{12}$; ${ }^{1}$ Austin Hospital, University of Melbourne, Melbourne, Australia, ${ }^{2} \mathrm{CHU}$ Purpan, Toulouse, France, ${ }^{3}$ Hôpital de la Madeleine, Orléans, France, ${ }^{4}$ Clinique Médicale de la Porte Verte, Versailles, France, ${ }^{5}$ Immanuel Krankenhaus Rheumaklinik, Berlin, Germany, ${ }^{6} \mathrm{U} . Z$. Gent Department of Internal Medicine, Gent, Belgium, ${ }^{7}$ Centrum 
Medyczcne, Warsaw, Poland, ${ }^{8}$ Hospital Universitario N. S. de Candelaria, Dept of Rheumatology, Tenerife, Canary Islands, Spain, ${ }^{9}$ Clinica Medica Ove, University of Catania, Italy, ${ }^{10}$ Odense University Hospital, Odense, Denmark, ${ }^{11}$ University of Liège, Liège, Belgium, ${ }^{12}$ Leuven University Center for Metabolic Bone Diseases and Division of Geriatric Medicine, Leuven, Belgium

Objective: Over 80 year old women are often left untreated because it is believed to be 'too late'. However, women 80 years and over contribute $30 \%$ of the burden of all fragility fractures and $60 \%$ of the burden of all hip fracture because of high prevalence of osteoporosis and high incidence of falls. Despite this, few anti-fractures studies have focussed on the elderly.

Materials and methods: Strontium ranelate is a new antiosteoporotic treatment which reduces bone resorption while allowing continued bone formation. The results of the phase III program including 2 international, double-blind, placebo-controlled studies (SOTI and TROPOS trials), support the efficacy and safety of strontium ranelate $2 \mathrm{~g}$ /day orally in reducing the risk of vertebral and non vertebral fractures including hip fractures in postmenopausal women with osteoporosis.

A pre-planned analysis of the pooled data from these 2 studies was performed to investigate the efficacy of strontium ranelate in women aged 80 years or more $(n=1488)$.

Results: The baseline characteristics of patients were similar in both groups (age 83.5 \pm 3.0 years; lumbar BMD T-score $-2.7 \pm 1.7$; femoral neck BMD T-score $-3.3 \pm 0.7$ ). From the first year of treatment, in the intent-to-treat population, strontium ranelate reduced the risk of vertebral fracture by $59 \%(\mathrm{RR}=0.41 ; 95 \% \mathrm{CI}$ $[0.22 ; 0.75], p=0.002)$, of clinical fractures by $37 \% \quad(R R=0.63$; $95 \%$ CI $[0.44 ; 0.91], \mathrm{p}=0.012)$ and of non-vertebral fracture by $41 \%$ $(\mathrm{RR}=0.59 ; 95 \% \mathrm{CI}[0.37 ; 0.95], \mathrm{p}=0.027)$, compared to placebo. These reductions were confirmed over 3 years: $-32 \%(\mathrm{RR}=0.68$; $95 \%$ CI $[0.50 ; 0.92], p=0.013)$ for vertebral fracture, $-22 \%$ $(\mathrm{RR}=0.78 ; 95 \% \mathrm{CI}[0.61 ; 0.99], \mathrm{p}=0.040)$ for clinical fracture and $-31 \%(\mathrm{RR}=0.69 ; 95 \% \mathrm{CI}[0.52 ; 0.92], \mathrm{p}=0.011)$ for non-vertebral fracture. The drug had a good safety profile, similar to that in younger patients.

Conclusion: Strontium ranelate is the first anti-osteoporotic treatment to demonstrate an early and sustained reduction for vertebral and non-vertebral fractures in patients aged 80 years and more. This early efficacy is of great consequence in elderly.

\section{OC22. MAGNITUDE AND DURATION OF EXCESS MORTALITY FOL- LOWING HIP FRACTURE AMONG POSTMENOPAUSAL WOMEN: A META-ANALYSIS}

P. Haentjens ${ }^{1}$, D. Vanderschueren ${ }^{2}$, K. Venken ${ }^{2}$, S. Boonen ${ }^{2}$; ${ }^{1}$ Department of Orthopaedics and Traumatology, Vrije Universiteit Brussel, Brussels, Belgium, ${ }^{2}$ Leuven University Center for Metabolic Bone Diseases and Division of Geriatric Medicine, Katholieke Universiteit Leuven, Leuven, Belgium

Objectives: To determine the magnitude and duration of excess mortality after hip fracture among postmenopausal women.

Methods: We conducted a systematic review and meta-analysis of the literature to estimate the pooled relative risk of death after hip fracture by time since fracture. We selected only controlled studies that reported data on postmenaupausal women aged 50 years or older, carried out a life-table analysis, and displayed the survival curves of the hip-fracture group and an ageand sex-matched control group. Using random-effects models we calculated the pooled relative risk of death with $95 \%$ confidence intervals $(95 \% \mathrm{CI})$ by time since fracture.

Results: Twenty-three studies contributed to this meta-analysis. The pooled relative risk of dying within three, six, twelve, and twenty-four months following hip fracture was $5.06(95 \% \mathrm{CI}$ : 4.31, 5.93), 3.92 (95\% CI: 3.11, 4.94), 2.71 (95\% CI: 2.33, 3.14), and $2.02(95 \% \mathrm{CI}: 1.83,2.23)$, respectively. Thereafter, excess mortality remained relatively constant. The relative risk of mortality at five years, ten years, and fifteen years post-fracture was
1.44 (95\% CI: $1.29,1.62), 1.40(95 \% \mathrm{CI}: 1.35,1.45)$, and 1.36 (95\% CI, 1.31, 1.41), respectively.

Conclusions: Excess mortality among postmenopausal women having suffered a hip fracture was most apparent immediately after the event, declined steeply during the first years post-fracture, but did not return to that of age- and sex-matched controls, even at the longest duration of follow-up. The impact of a hip fracture on excess mortality among postmenopausal women continued for up to 15 years.

\section{OC23. ASSOCIATIONS BETWEEN INCREASE IN BONE MINERAL DEN- SITY AND DECREASE IN CLINICAL VERTEBRAL FRACTURE INCIDENCE DURING A TREATMENT WITH STRONTIUM RANELATE}

O. Bruyère ${ }^{1}$, C. Roux $^{2}$, J.B. Cannata ${ }^{3}$, R. Rizzoli ${ }^{4}$, K. Brixen ${ }^{5}$, M.L. Brandi ${ }^{6}$, J.-Y. Reginster ${ }^{7} ;{ }^{1,7}$ WHO Collaborating Center for Public Health Aspect of Osteoarticular Disorders, University of Liège, Liège, Belgium, ${ }^{2}$ Hôpital Cochin, Dept of Rheumatology, Paris, France, ${ }^{3}$ Hospital Central de Asturias, Oviedo, Spain, ${ }^{4}$ Dept of Rehabilitation and Geriatrics, Hôpital Cantonal, Geneva, Switzerland, ${ }^{5}$ Odense University Hospital, Odense, Denmark, ${ }^{6}$ Department of Internal Medicine, University of Florence, Florence, Italy

Objective: Strontium ranelate has been shown to increase bone mineral density and to reduce the incidence of vertebral and nonvertebral fractures in postmenopausal osteoporosis. The objective of this study is to analyze the relationship between BMD changes and clinical vertebral fractures incidence during a 3-year treatment with SR.

Material and methods: Patients receiving Strontium ranelate from the Spinal Osteoporosis Therapeutic Intervention study (SOTI) and the Treatment of Peripheral Osteoporosis study (TROPOS) were included in this study. BMD was assessed at baseline and after a follow-up of 3 years at the lumbar spine, the femoral neck and the total hip.

Results: The logistic regression analysis, including age, body mass index and baseline BMD as covariates, showed that 3-year increase in neck, hip, but not lumbar BMD changes was statistically associated with a reduction in the incidence of new clinical vertebral fractures $(p<0.001)$. For each increase of $1 \%$ in neck or hip BMD, the risk to experience a new clinical vertebral fracture after 3 years decreased by $4 \%(95 \%$ CI $2 \%-7 \%$ for neck BMD and $95 \%$ CI $2 \%-6 \%$ for hip BMD). Patients experiencing new fractures gain less BMD $(+3.58 \%$ at the femoral neck and $+4.97 \%$ for the total hip) that patients without new fractures $(+5.69 \%$ and $+7.31 \%$ for the femoral neck and total hip, respectively) $(\mathrm{p}<0.01$ between the two groups). The risk to experience new clinical vertebral fractures in patients in the highest quartile of femoral neck BMD change $(>9.49 \%)$ is reduced by $41 \% \quad(p<0.02)$ compared to patients in the lowest quartiles $(<1.01 \%)$. Patients experiencing an increase in neck BMD $(>0 \%)$ after 3 years of treatment have a $44 \%$ risk reduction $(p=0.001)$ to experience a new clinical vertebral fracture, compared to patients without increase in BMD.

Conclusion: During 3 years of SR treatment, an increase in BMD (femoral neck or total hip) is associated with a reduction of clinical vertebral fracture incidence.

OC24. TERIPARATIDE-MEDIATED CHANGES IN BONE MINERAL DENSITY (BMD) AND FRACTURE RISK REDUCTION IN WOMEN WITH OSTEOPOROSIS

P. Chen ${ }^{1}$, P. Miller ${ }^{2}$, P.D. Delmas ${ }^{3}$, D.A. Misurski ${ }^{1}$, J. Krege ${ }^{1}$; ${ }^{1}$ Eli Lilly and Company, Indianapolis, IN, USA, ${ }^{2}$ Center for Bone Research, University of Colorado, Denver, CO, USA, ${ }^{3}$ INSERM Research Unit 403, Hopital Edouard Herriot, Claude Bernard University of Lyon, Lyon, France

BMD changes account for a small proportion of antiresorptive-mediated fracture risk reduction. The relationship between teriparatide (TPTD)-mediated increases in lumbar spine (LS) BMD and risk of new vertebral fractures was assessed using data 
from the Fracture Prevention Trial. Postmenopausal women with osteoporosis $(n=1637)$ were randomized to placebo or TPTD 20 or $40 \mathrm{mcg} /$ day (median 19 months). LS BMD was assessed at baseline and 18-months. Vertebrae whose fracture status changed were not included. Baseline and endpoint lateral spine $\mathrm{x}$-rays were assessed using a visual semiquantitative technique.

Baseline BMD and BMD change contributed to fracture risk. The mean increase in TPTD-treated patients was $0.09 \mathrm{~g} / \mathrm{cm}^{2}$. This increase was similar across tertiles of baseline BMD. Fracture risk was assessed by logistic regression. Factors included in the model were endpoint BMD, treatment (pooled TPTD versus placebo), and treatment-by-BMD interaction. All were statistically significant $(\mathrm{p}<0.05)$. Compared to placebo, fracture risk was lower with TPTD for all endpoint BMD values. The absolute risk reduction was significantly different across the range of endpoint BMD values (Figure).

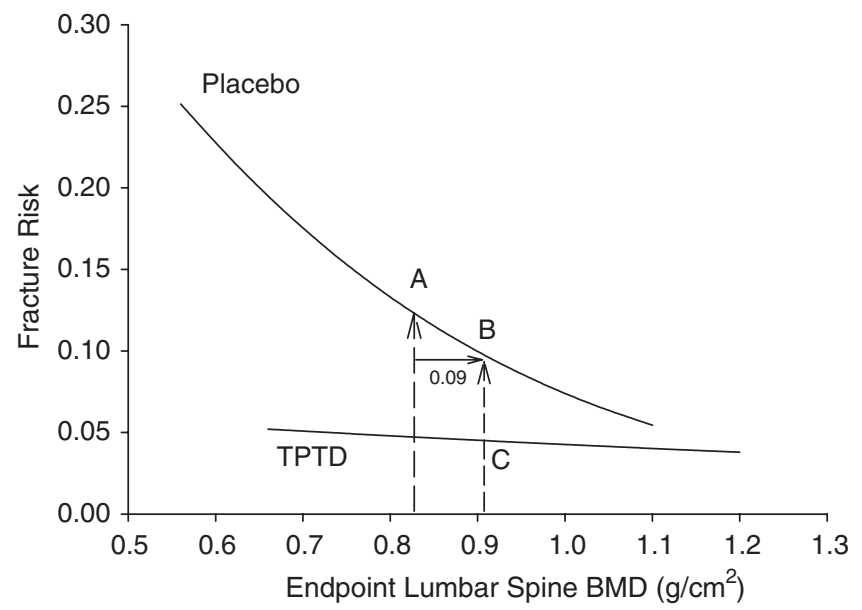

$\mathrm{A}-\mathrm{B}$ and $\mathrm{A}-\mathrm{C}$ represent fracture risk reductions associated with a $0.09 \mathrm{~g} / \mathrm{cm}^{2}$ increase in BMD alone and in response to TPTD, respectively. The proportion of TPTD-mediated fracture risk reduction attributable to the BMD increase can be estimated by $(\mathrm{A}-\mathrm{B}) /(\mathrm{A}-\mathrm{C})$. For " $\mathrm{A}$ " values from 0.7 to $0.9 \mathrm{~g} / \mathrm{cm}^{2}$, the proportion of TPTD-mediated fracture risk reduction attributable to a $0.09 \mathrm{~g} / \mathrm{cm}^{2}$ increase in BMD ranged from 30 to $41 \%$, respectively. The remaining risk reduction with TPTD was due to improvements in other non-BMD determinants of bone strength.

OC25. A COMPARISON OF BONE MINERAL MEASUREMENTS FOR FRACTURE PREDICTION: A META-ANALYSIS

J.A. Kanis, O. Johnell, H. Johansson, A. Oden, P.D. Delmas, J.A. Eisman, S. Fujiwara, C. Gluer, H. Kroger, E.V. McCloskey, D. Melstrom, L.J. Melton III, H.A. Pols, J. Reeve, A. Silman, A. Tenenhouse, WHO Collaborating Centre for Metabolic Bone Diseases, University of Sheffield, UK

The aim of this study was to compare the performance characteristics of bone mineral measurements for fracture prediction. We used the primary data of up to 9 population-based cohorts in which BMD or heel QUS had been measured at base-line. 30,238 men and women were followed for up to 125,600 person-years. The effect of BMD on fracture risk was examined using a Poisson model for each cohort separately, and the results of the different studies were then merged using weighted $\beta$ coefficients. Gradients of risk for fracture prediction ( $\mathrm{RR} / \mathrm{SD}$ decrease in $\mathrm{Z}$-score) were computed for the age of 70 years and are shown in the table below.

There was no significant differences in gradients of risk between men and women. BMD at the femoral neck (FN) provided the highest gradient of risk for hip fracture prediction, which was significantly higher $(p<0.001)$ than BMD at the lumbar spine (LS). For vertebral or all osteoporotic fracture prediction, there was no difference between techniques. Quantitative ultrasound at the heel performed similarly to DXA for hip and all fracture outcomes. We conclude that there is no advantage in measuring lumbar spine over femoral neck or total hip (TH), and that heel ultrasound may perform similarly to central DXA in the assessment of fracture risk.

\begin{tabular}{lllll}
\hline \multicolumn{2}{l}{ Technique/ } & \multicolumn{2}{l}{ Outcome fracture } \\
\cline { 2 - 5 } & & Vertebral & Vertebral & $\begin{array}{l}\text { Any } \\
\text { osteoporotic }\end{array}$ \\
\hline site & Hip & Clinical & morphometric ${ }^{\mathrm{b}}$ & fracture \\
\hline DXA TH & $2.5(1.8-3.5)$ & $1.6(1.4-1.8)$ & $-^{\mathrm{a}}$ & $1.4(1.2-1.5)$ \\
DXA FN & $2.8(2.4-3.2)$ & $1.4(1.4-1.5)$ & $1.7(1.5-1.9)$ & $1.5(1.4-1.6)$ \\
DXA LS & $1.7(1.4-2.1)$ & $1.5(1.4-1.7)$ & $1.6(1.4-1.9)$ & $1.4(1.3-1.5)$ \\
SoS Heel & $2.4(1.6-3.6)$ & $-^{\mathrm{a}}$ & $-^{\mathrm{a}}$ & $1.6(1.3-2.0)$ \\
BUA Heel & $2.6(1.8-3.8)$ & $-^{\mathrm{a}}$ & $-^{\mathrm{a}}$ & $1.4(1.2-1.7)$ \\
\hline
\end{tabular}

a only 1 cohort available

bomputed with logistic regression

OC26. EFFECT OF A ONCE-WEEKLY TABLET CONTAINING ALENDRONATE AND VITAMIN $D_{3}$ FOR THE TREATMENT OF OSTEOPOROSIS

K. Lippuner ${ }^{1}$, N. Binkley ${ }^{2}$, P.D. Delmas ${ }^{3}$, R. Recker ${ }^{4}$, J. Ringe ${ }^{5}$, J. Reed ${ }^{6}$, D. Felsenberg ${ }^{7}$, G. Salzmann ${ }^{8}$, M. Liu ${ }^{8}$, A. Santora ${ }^{8}$; ${ }^{1}$ University Hospital, Berne, Switzerland, ${ }^{2}$ University of Wisconsin, Madison, WI, USA, ${ }^{3}$ Hôpital Edouard Herriot, Lyon, France, ${ }^{4}$ Creighton University Osteoporosis Research Center, Omaha, NE, USA, ${ }^{5}$ University of Cologne, Leverkusen, Germany, ${ }^{6}$ Fallon Clinic, Worcester, MA, USA, ${ }^{7}$ University Hospital Benjamin Franklin, ${ }^{8}$ Merck Research Laboratories, Rahway, NJ, USA

Objective: We conducted a study of the efficacy, safety, and tolerability of a once-weekly tablet containing alendronate $70 \mathrm{mg}$ (ALN) and cholecalciferol 2800 IU (D) in osteoporotic patients. Results of an extension with additional vitamin $\mathrm{D}_{3}$ are described here.

Methods: The base study was a 15-week, randomized, double-blind, multi-center, controlled study in winter/early spring. Postmenopausal women and men with osteoporosis (T $\leq-2.5)$ were randomized to once-weekly ALN with $2800 \mathrm{IU}$ $\mathrm{D}(\mathrm{ALN}+\mathrm{D} ; \mathrm{n}=360)$ or ALN alone $(\mathrm{n}=357)$. Patients with baseline serum 25-hydroxyvitamin D (25OHD) $<9 \mathrm{ng} / \mathrm{mL}$ were excluded. Sunlight and supplements containing vitamin D were to be avoided. The 24-week extension was conducted in summer/fall; all patients received ALN + D plus either additional vitamin $\mathrm{D}_{3} 2800$ IU or matching placebo once weekly. Vitamin D supplements up to $1000 \mathrm{IU} /$ day (in addition to study therapy) were permitted and sunlight was not restricted. The primary focus of the extension was hypercalciuria (24-hour urine calcium $>300 \mathrm{mg}$ in women and $>350 \mathrm{mg}$ in men and $>25 \%$ higher than baseline). Serum 25OHD was the primary endpoint in the base study and secondary in the extension.

Results: 652 patients entered the extension; the percentage of patients with hypercalciuria at the end (week 39 ) was $4.2 \%$ for ALN + D5600 and 2.8\% for ALN + D2800 [RR, $1.48(95 \% \mathrm{CI}$ : $0.64-3.40$ )], similar to the $\sim 4 \%$ at the end of the base study (week 15). There were no reports of hypercalcemia. Serum 25OHD averaged $22 \mathrm{ng} / \mathrm{mL}$ at baseline and declined in the ALN only group, but was $26 \%$ higher in the ALN $+\mathrm{D}$ group at week 15 , compared to ALN alone $(\mathrm{p}<0.001)$. At the end of the extension, the mean 25OHD levels were 27.9 and $25.6 \mathrm{ng} / \mathrm{ml}$ in the ALN + D5600 and ALN + D2800 groups, respectively, and $3.1 \%$ and $5.6 \%$ had serum $25 \mathrm{OHD}<15 \mathrm{ng} / \mathrm{mL}$. Reductions in NTx were similar in both groups at week 15 , and remained stable during the extension.

Conclusions: During the 24-week extension study the safety and tolerability profile was maintained with 5600 IU weekly even when sunlight and additional vitamin $\mathrm{D}$ were permitted. Under these conditions, serum 25OHD further increased, and the proportion of patients with low 25OHD was further reduced. 
OC27. EFFECTS OF GLUCOSAMINE SULFATE ON 6-MONTH CONTROL OF KNEE OSTEOARTHRITIS SYMPTOMS VS PLACEBO AND ACETAMINOPHEN: RESULTS FROM THE GLUCOSAMINE UNUM IN DIE EFFICACY (GUIDE) TRIAL

G. Herrero-Beaumont ${ }^{1}$, J.A. Román ${ }^{2}$, M.C. Trabado ${ }^{3}$, F.J. Blanco $^{4}$, P. Benito ${ }^{5}$, E. Martin-Mola ${ }^{6}$, J. Paulino ${ }^{7}$, J.L. Marenco ${ }^{8}$, A. Porto ${ }^{9}$, A. Laffon ${ }^{10}$, D. Araùjo ${ }^{11}$, M. Figueroa ${ }^{12}$, J. Branco ${ }^{13}$; ${ }^{1}$ Fund. J. Diaz, Madrid, Spain, ${ }^{2} \mathrm{H}$ Peset, Valencia, Spain, ${ }^{3} \mathrm{H}$ Clinic, Barcelona, Spain, ${ }^{4} \mathrm{CH}$ Juan Canalejo, A Coruña, Spain, ${ }^{5} \mathrm{H}$ del Mar, Barcelona, Spain, ${ }^{6} \mathrm{HU}$ La Paz, Madrid, Spain, ${ }^{7}$ CHCR, Ciudad Real, Spain, ${ }^{8}$ H N.S. de Valme, Sevilla, Spain, ${ }^{9} \mathrm{HU}$, Coimbra, Portugal, ${ }^{10} \mathrm{H}$ Princesa, Madrid, Spain, ${ }^{11} \mathrm{H} \mathrm{C}$. Bertiandos, Ponte de Lima, Portugal, ${ }^{12} \mathrm{H}$ Donostia, San Sebastian, Spain, ${ }^{13} \mathrm{H}$ E. Moniz, Lisboa, Portugal

Objective: Two randomised, placebo-controlled, double-blind trials have been lately investigating the efficacy of oral glucosamine in knee osteoarthritis (OA), using a symptomatic medication as reference: GAIT performed by the NIH in the US with nutraceutical glucosamine hydrochloride $500 \mathrm{mg}$ t.i.d. vs celecoxib, and GUIDE performed in Europe with the original prescription glucosamine sulfate $1500 \mathrm{mg}$ once-a-day (u.i.d.) vs acetaminophen, i.e. the preferred symptomatic medication in OA practice guidelines. We report the results of GUIDE.

Materials and Methods: 318 patients (88\% women) with knee OA (ACR criteria) were randomised to double-dummy oral glucosamine sulfate soluble powder $1500 \mathrm{mg}$ u.i.d., or acetaminophen $1000 \mathrm{mg}$ tablets t.i.d. ( $3 \mathrm{~g} /$ day, as recommended in Europe), or placebo, for 6 months. The rescue medication consisted of standardised use of ibuprofen $400 \mathrm{mg}$ tablets (daily diary recording). The primary efficacy parameter was the 6-month ITT change in the Lequesne index, using the LOCF approach for non per-protocol completers ( 34 on placebo, 28 in each acetaminophen and glucosamine sulfate groups, without differences in dropout reasons). Secondary parameters included the changes in the WOMAC index and the OARSI-A responder criteria. Statistical analysis on the indexes was performed by GLM-ANOVA, with Dunnett's pairwise comparisons vs placebo. The proportions of responders and patients using the rescue were compared by the chi-square test.

Results: The groups were comparable for demographics and baseline characteristics. Glucosamine sulfate efficacy vs placebo was significant on all parameters (table). Acetaminophen had more responders than placebo, but failed to reach a significant difference on the Lequesne $(\mathrm{p}=0.18)$ and WOMAC $(\mathrm{p}=0.077)$ indexes.

More patients on placebo used the rescue medication $(\mathrm{p}=0.027$ and $0.045 \mathrm{vs}$ glucosamine sulfate and acetaminophen, respectively). There were no safety differences among groups.

Conclusions: Glucosamine sulfate at the oral once-daily dose of $1500 \mathrm{mg}$ might be the preferred symptomatic medication in knee OA.

Baseline Lequesne and WOMAC, with 6-month ITT changes and \% of OARSI-A responders

\begin{tabular}{|c|c|c|c|c|c|c|}
\hline & \multicolumn{2}{|c|}{$\begin{array}{l}\text { Placebo } \\
(\mathrm{N}=104)\end{array}$} & \multicolumn{2}{|c|}{$\begin{array}{l}\text { Acetaminophen } \\
(\mathrm{N}=108)\end{array}$} & \multicolumn{2}{|c|}{$\begin{array}{l}\text { Glucosamine } \\
\text { sulfate }(N=106)\end{array}$} \\
\hline & Baseline & 6 months & Baseline & 6 months & Baseline & $\begin{array}{l}6 \\
\text { months }\end{array}$ \\
\hline $\begin{array}{l}\text { Lequesne } \\
\text { (points) }^{\mathrm{a}}\end{array}$ & $\begin{array}{l}10.8 \\
(2.6)\end{array}$ & $\begin{array}{l}-1.9(-2.6 \\
\text { to }-1.2)\end{array}$ & $11.1(2.7)$ & $\begin{array}{l}-2.7(-3.3 \\
\text { to }-2.1)\end{array}$ & $\begin{array}{l}11.0 \\
(3.1)\end{array}$ & $\begin{array}{l}-3.1^{*} \\
(-3.8 \\
\text { to }-2.3)\end{array}$ \\
\hline $\begin{array}{l}\text { WOMAC } \\
\text { (points) }^{\mathrm{a}}\end{array}$ & $\begin{array}{l}37.9 \\
(14.3)\end{array}$ & $\begin{array}{l}-8.2 \\
(-11.3 \\
\text { to }-5.1)\end{array}$ & $40.4(14.8)$ & $\begin{array}{l}-12.3 \\
(-14.9 \\
\text { to }-9.7)\end{array}$ & $\begin{array}{l}38.3 \\
(15.2)\end{array}$ & $\begin{array}{l}-12.9^{\dagger} \\
(-15.6 \\
\text { to }-10.1)\end{array}$ \\
\hline $\begin{array}{l}\text { OARSI-A } \\
\text { responders } \\
(\%)\end{array}$ & - & $21.2 \%$ & - & $33.3 \%$ & - & $39.6 \%$ \\
\hline
\end{tabular}

${ }^{\text {a }}$ Mean absolute (SD) at baseline and change $(95 \% \mathrm{CI})$ at 6 months $\mathrm{P}$ vs placebo: $* 0.032$ [difference $=-1.2(-2.3$ to -0.8$)$ ] ${ }^{\dagger} 0.039$ [difference $=-4.7(-9.1$ to -0.2$)$ ]; $\ddagger 0.047 ;{ }^{\S} 0.007$
0C28. EFFECTS OF PARATHYROID HORMONE 1-84 ON CORTICAL AND TRABECULAR BONE AT THE HIP AS ASSESSED BY QCT: RESULTS AT 18 MONTHS FROM THE TOP STUDY

C.E. Bogado ${ }^{1}$, J.R. Zanchetta ${ }^{1}$, A. Mango ${ }^{1}$, A.L. Mathisen ${ }^{2}$, J. Fox $^{2}$; ${ }^{1}$ Intstituto de Investigaziones Metabolicas, Buenos Aires, Argentina, ${ }^{2}$ NPS Pharmaceuticals, Salt Lake City, UT -Nycomed, Roskilde, Denmark

Objective: Treatment with PTH increases trabecular bone mineral density (BMD), but its effects on mixed trabecular and cortical bone sites, are less well established.

Method and material: We used quantitative computed tomography (QCT) to assess the effects of treatment with parathyroid hormone 1-84 (PTH) on volumetric (v) trabecular ( Tb), cortical $(\mathrm{Ct})$ and integral (Int; $\mathrm{Tb}+\mathrm{Ct}$ ) $\mathrm{BMD}$, bone mineral content (BMC) and bone volume (BV) at the total hip and femoral neck of postmenopausal women with osteoporosis. The QCT study consisted of 122 women from the TOP study (BMD T score $\leq-2.5$ at the lumbar spine, total hip or femoral neck) who were randomly assigned to daily injections of PTH $(100 \mu \mathrm{g} ; \mathrm{n}=62)$ or placebo $(n=60)$.

Results: The mean percent change from baseline for placebo and PTH subjects is shown in the Table below.

\begin{tabular}{|c|c|c|c|c|c|c|}
\hline & \multicolumn{3}{|c|}{ Total Hip } & \multicolumn{3}{|c|}{ Femoral Neck } \\
\hline & Placebo & PTH & p-value & Placebo & РTH & p-value \\
\hline vTbBMD & -0.21 & 4.99 & $<0.001$ & -0.42 & 4.11 & 0.006 \\
\hline vTbBMC & -1.06 & 2.91 & 0.003 & -2.56 & 7.80 & 0.003 \\
\hline TbBV & -0.83 & -1.29 & 0.064 & -1.46 & 3.34 & 0.074 \\
\hline vCtBMD & 3.25 & -1.41 & 0.001 & 1.22 & -0.91 & 0.278 \\
\hline vCtBMC & -4.74 & 0.04 & 0.040 & 1.74 & 8.22 & 0.116 \\
\hline CtBV & -7.17 & 1.83 & 0.003 & 0.01 & 11.17 & 0.043 \\
\hline vIntBMD & -0.21 & 4.50 & $<0.001$ & 1.37 & 3.37 & 0.178 \\
\hline vIntBMC & -1.06 & 2.91 & 0.003 & 0.23 & 7.25 & 0.032 \\
\hline IntBV & -0.83 & -2.10 & 0.258 & -1.61 & 4.26 & 0.041 \\
\hline
\end{tabular}

Conclusion: The increase in vTbBMD at total and femoral neck in PTH-treated subjects was due primarily to an increase in vTbBMC; there was no significant change in TbBV at either site. In contrast, $\mathrm{CtBV}$ increased significantly in the PTH group at both hip regions. However, because the increase in CtBMC was smaller than the increase in bone volume, there was a decrease in vCtBMD that was significant at the total hip, but not at the femoral neck. To our knowledge this is the first placebo controlled study of the effects of PTH treatment on vBMD at the hip using QCT. Daily administration of PTH for 18 months increases integral and volumetric trabecular BMD and BMC, and cortical bone volume. We found only a modest decrease in volumetric cortical BMD, which was not significant at the femoral neck region. These results are consistent with changes observed in PTHtreated osteopenic rhesus monkeys in which increases in integral $\mathrm{VBMD}$ and $\mathrm{vBMC}$ at these regions translated into significant increases in bone strength.

OC29. ARTICULAR CARTILAGE VOLUME AND THICKNESS MEASUREMENTS CORRELATE WITH PAIN AND FUNCTION IN INDIVIDUALS WITH MEDIAL TIBIOFEMORAL KNEE OA

K. Beattie ${ }^{1}$, P. Boulos ${ }^{1,2}$, D. Inglis ${ }^{1}$, C. Webber ${ }^{3}$ F. Eckstein ${ }^{4}$, J.D. Adachi ${ }^{1,2}$; ${ }^{1}$ McMaster University, Hamilton, Canada, ${ }^{2}$ St. Joseph's Healthcare, Hamilton, Canada, ${ }^{3}$ Dept. of Nuclear Medicine, Hamilton Health Sciences, Hamilton, Canada, ${ }^{4}$ Dept. of Anatomy, Paracelsus Medizinische Privatuniversität, Salzburg, Austria

Objective: To investigate the relationship between medial tibial cartilage volume and thickness and WOMAC and SF-36 measures of pain and function in osteoarthritic individuals. 
Methods: Men and women $\geq 35$ years old with radiographic evidence of knee OA (K-L grade $\geq 2$ on plain X-ray acquired in fixed-flexion) participated. Volunteers consented to have a $3 \mathrm{D}$ gradient-echo MRI scan (fat saturation) acquired in the sagittal plane (1.5 mm slice thickness) using a 1T OrthOne ${ }^{\mathrm{TM}}$ Peripheral MRI scanner (ONI Medical Systems, Wilmington, MA). One technician analyzed all images for medial tibial cartilage volume and thickness using a proprietary software program (Chondrometrics $\mathrm{GmbH}$, Ainring, Germany). Cartilage segmentation was conducted on a slice-by-slice basis by manually tracing the bonecartilage interface and the cartilage surface of the medial tibial plateau. Participants also completed WOMAC and SF-36 questionnaires.

Results: Of 41 participants, 26 were female and 15 were male. The mean (SD) age, height, weight and BMI for the group were $64.8(10.3)$ years, $167.0(11.4) \mathrm{cm}, 78.9(16.1) \mathrm{kg}$ and 28.3 (5.0) $\mathrm{kg} / \mathrm{m}^{2}$, respectively. Mean (SD) cartilage volume and thickness values were 1495.45 (647.62) $\mu \mathrm{L}$ and $1.19(0.45) \mathrm{mm}$, respectively. All individuals completed the WOMAC and SF-36 questionnaires. Correlation analyses between medial tibial cartilage volume and thickness and subcategories of the WOMAC and SF-36 were performed. Results (Pearson correlation coefficients $=$ CC) are presented in the table $(\mathrm{p}<0.05$ considered significant).

\begin{tabular}{lll}
\hline & $\begin{array}{l}\text { Cartilage Volume } \\
\text { CC }(\mathrm{r})\end{array}$ & $\begin{array}{l}\text { Cartilage Thickness } \\
\mathrm{CC}(\mathrm{r})\end{array}$ \\
\hline WOMAC Pain & $-0.403(\mathrm{p}=0.011)$ & $-0.496(\mathrm{p}=0.001)$ \\
WOMAC Stiffness & $-0.434(\mathrm{p}=0.006)$ & $-0.469(\mathrm{p}=0.003)$ \\
WOMAC Function & $-0.484(\mathrm{p}=0.002)$ & $-0.604(\mathrm{p}=0.001)$ \\
WOMAC Total & $-0.481(\mathrm{p}=0.0021)$ & $-0.592(\mathrm{p}=0.001)$ \\
Physical Function & $0.442(\mathrm{p}=0.007)$ & $0.637(\mathrm{p}=0.001)$ \\
(SF-36) & & \\
\hline
\end{tabular}

Relationships between medial tibial cartilage volume and thickness and OA symptoms

Conclusions: In osteoarthritic individuals, symptoms of pain and physical function correlated well with cartilage volume and thickness measurements yielded through the analysis of sagittal MR images using a cartilage segmentation program. However, correlations were stronger with volume, normalized to bone size, and cartilage thickness. Larger, longitudinal studies should be conducted to determine if changes in cartilage volume and thickness also reflect changes in patient symptoms.

\section{OC30. IBANDRONATE IS COST-EFFECTIVE IN THE TREATMENT OF POSTMENOPAUSAL OSTEOPOROSIS: A COMPARISION OF BIS- PHOSPHONATES}

N.O. Lynch ${ }^{1}$, S.R. Earnshaw ${ }^{2}$, S. Beard ${ }^{3}$, W. Cowell ${ }^{4}$; ${ }^{1}$ GlaxoSmithKline, Middlesex, UK, ${ }^{2}$ RTI Health Solutions, Research Triangle Park, NC, ${ }^{3}$ RTI Health Solutions, Manchester, UK, ${ }^{4}$ Roche Products Ltd, Welwyn, UK

Objectives: We estimated the cost-effectiveness of monthly ibandronate compared to weekly bisphosphonate (BP) treatments for women with established postmenopausal osteoporosis, in the United Kingdom (UK).

Methods \& Material: A Markov model was developed to evaluate the lifetime cost-effectiveness of three BPs: monthly ibandronate and weekly alendronate, and risedronate. Vertebral, hip and wrist fracture efficacy were assigned a bisphosphonate class effect as estimated by the literature. Persistence with weekly therapy was referenced to rates reported for natural populations receiving weekly BPs (43\% at 1 year) and to an absolute $7 \%$ improved persistence (to $50 \%$ at 1 year) for ibandronate. The analysis population was post-menopausal women aged $\geq 50$ years with prevalent radiologic vertebral deformity and hip BMD Tscore $\leq-2.5$. Baseline fracture risk and mortality were obtained from the published literature. Both fracture risk and mortality were allowed to increase as patient's age. Yearly drug cost was referenced to acquisition cost for each BP. Direct health resource costs for fracture states were estimated from published literature and discounted at a $3.5 \%$ yearly rate. All costs were reported in $2004 £ U K$.

Results: More fractures per 1000 patients were avoided (vs. no treatment) with monthly ibandronate (24.4) than with weekly alendronate (21.2) or risedronate (21.2). Under conditions of improved persistence, fracture care costs decrease but drug costs are higher with ibandronate compared to alendronate and risedronate ( $£ 315, £ 300 \& £ 267$ respectively). The incremental cost per fracture avoided (vs. no treatment) was lower with ibandronate $(£ 2,876)$ compared to alendronate $(£ 4,733)$ and risedronate $(£ 3,179)$, demonstrating that ibandronate is costeffective in comparison to other bisphosphonates. Changing assumptions in the model (primarily model horizon to 10 years) to that of previously published cost-effectiveness models produced similar results, providing external validity for the robustness of this model.

Conclusion: Ibandronate is a cost-effective intervention for the treatment of post-menopausal osteoporosis. Incremental persistence with BP therapy thus improves the benefit realised in patient populations. These benefits include fewer fractures for patients without a significant increase in cost to payers. As a result, when compared to other bisphosphonates, ibandronate is at least as cost-effective and potentially more cost-effective, than both alendronate and risedronate.

\section{OC31. STRONTIUM RANELATE: AN ANTI-OSTEOPOROTIC TREATMENT DEMONSTRATED VERTEBRAL AND NONVERTEBRAL ANTI FRACTURE EFFICACY OVER 5 YEARS IN POST MENOPAUSAL OSTEOPOROTIC WOMEN}

J.-Y. Reginster ${ }^{1}$, P.J. Meunier ${ }^{2}$, C. Roux ${ }^{3}$, J. Compston ${ }^{4}$, S. Ortolani ${ }^{5}$; ${ }^{1}$ University of Liège, Liège, Belgium, ${ }^{2}$ Faculty Laennec, Lyon, France, ${ }^{3}$ Hospital Cochin, Paris, France, ${ }^{4}$ Department of Medicine, University of Cambridge, Cambridge, UK, ${ }^{5}$ Istituto Auxologico Italiano, Milano, Italy

Objective: The objective of this work was to describe the long term efficacy of strontium ranelate over 5 years treatment.

Materials and methods: Strontium ranelate is a new antiosteoporotic treatment with an innovative mode of action both increasing bone formation and decreasing bone resorption.

In the phase III program, two double blind placebo controlled studies were performed in Caucasian women with postmenopausal osteoporosis in 75 European and Australian centres: SOTI (Spinal Osteoporosis Therapeutic Intervention) including 1649 patients aged 69.3(7.2) years [mean (SD)] with lumbar spine bone mineral density (BMD) T-score -3.6(1.3) and TROPOS (Treatment Of Peripheral OSteoporosis) in 5091 patients aged 76.7(5) years with femoral neck BMD T-score -3.1(0.6). In SOTI, patients were randomly assigned to receive strontium ranelate $2 \mathrm{~g}$ /day or placebo for 4 years and during the $5^{\text {th }}$ year, half of the strontium ranelate group received placebo while the other half continued their treatment with strontium ranelate. In TROPOS, patients were randomly assigned to receive strontium ranelate $2 \mathrm{~g}$ /day or placebo for 5 years. In both studies, all patients received supplementation with calcium and vitamin $\mathrm{D}$ adapted to their needs. The main statistical analysis was performed after 3 years of treatment and demonstrated efficacy of strontium ranelate at vertebral and nonvertebral sites.

Results: In the SOTI study, the long term efficacy data confirmed a significant reduction in the risk of new vertebral fracture by $33 \%$ in the strontium ranelate group $(n=719)$ as compared to the placebo group $(n=723)$ in the intention to treat population over the four years treatment period $(\mathrm{RR}=0.67$; 95\% CI[0.55;0.81],Cox model: $\mathrm{p}<0.001)$.

The 5 years results of TROPOS established the long term efficacy of strontium ranelate, with a $24 \%$ reduction in vertebral fracture $(\mathrm{RR}=0.76 ; 95 \% \mathrm{CI}[0.65 ; 0.87]$ Cox model: $\mathrm{p}<0.001)$ and a $15 \%$ reduction in nonvertebral fracture $(\mathrm{RR}=0.85 ; 95 \%$ 
CI[0.77;0.99] Cox model: $\mathrm{p}=0.03)$ in the intent-to-treat population $(n=2479$ in strontium ranelate group and 2453 in placebo group).

Conclusion: These results demonstrate, uniquely for anti-osteoporotic treatments, that strontium ranelate provides sustained efficacy over five years against both vertebral and nonvertebral fractures.

\section{OC32. PRONOUNCED INCREASES IN BONE MINERAL DENSITY WITH INTERMITTENT INTRAVENOUS IBANDRONATE INJECTIONS: 2-YEAR RESULTS FROM DIVA}

P.D. Delmas ${ }^{1}$, J.A. Eisman ${ }^{2}$, J. Stepan ${ }^{3}$, C. Hughes ${ }^{4}$, N. Mairon ${ }^{4}$, D. Masanauskaite ${ }^{4}$, D. Felsenberg ${ }^{5}$; ${ }^{1}$ Claude Bernard University and INSERM Research Unit 403, Lyon, France, ${ }^{2}$ Garvan Institute of Medical Research, St Vincent's Campus \& UNSW, Sydney, Australia, ${ }^{3}$ Charles University, Prague, Czech Republic, ${ }^{4}$ F. Hoffmann-La Roche Ltd, Basel, Switzerland, ${ }^{5}$ Charité-University Medicine Berlin, Berlin, Germany

Objectives: Bisphosphonates are widely used in the treatment of postmenopausal osteoporosis. However, oral administration may be unsuitable or contraindicated in some patients. A proven intravenous (i.v.) preparation could be a practical alternative for use in these patients. Ibandronate (Bonviva) is a potent, nitrogen-containing bisphosphonate that can be administered intermittently, either orally or by rapid (1530 seconds) i.v. injection. The DIVA study compared the efficacy on bone mineral density (BMD) of i.v. ibandronate dosing with a daily oral ibandronate regimen that has previously shown antifracture efficacy (3-year vertebral fracture risk reduction: $62 \%{ }^{1}$ ).

Materials and methods: DIVA was a randomised, doubleblind, phase III, non-inferiority study. Women (aged 5580 years and $=5$ years since menopause) with osteoporosis (spinal [L2-L4] BMD T-score $<-2.5$ and $\geq-5$ ) were randomised to receive either $2 \mathrm{mg}$ every 2 months [q2 mo] or $3 \mathrm{mg}$ every 3 months [q3 mo] i.v. ibandronate injections or $2.5 \mathrm{mg}$ daily oral ibandronate. Participants were given daily calcium $(500 \mathrm{mg})$ and vitamin D (400 IU). Changes (\%) from baseline in lumbar spine, total hip, femoral neck and hip trochanter BMD were measured at 2 years.

Results: In total, 1,395 women were randomised. Similar and substantial increases in lumbar spine BMD were observed in the i.v. treatment arms (Table). At 2 years, as at 1 year, both i.v. regimens were proven non-inferior and superior $(\mathrm{p}<0.001)$ to the oral regimen at the spine. At all hip sites, greater increases in BMD were observed in the i.v. groups than the oral group (Table). A greater proportion of patients in the i.v. arms achieved maintenance or increase in lumbar spine and/or total hip BMD above baseline ( $\leq \leq 0.004$ for all comparisons).

Conclusions: Compared with daily oral dosing, intermittent i.v. ibandronate injections provide larger gains in BMD at the lumbar spine and hip. I.v. administration may be advantageous in patients for whom oral bisphosphonates are contraindicated or unsuitable.

1. Chesnut CH, et al. J Bone Miner Res 2004; 19:1241-9.

Table Mean change $(\%)$ from baseline BMD at 2 years

\begin{tabular}{llll}
\hline & $\begin{array}{l}2.5 \mathrm{mg} \text { daily } \\
\text { oral IBN } \\
(\mathrm{n}=330)\end{array}$ & $\begin{array}{l}2 \mathrm{mg} \mathrm{q} 2 \mathrm{mo} \\
\text { i.v. IBN } \\
(\mathrm{n}=316)\end{array}$ & $\begin{array}{l}3 \mathrm{mg} \mathrm{q} 3 \mathrm{mo} \\
\text { i.v. IBN } \\
(\mathrm{n}=333)\end{array}$ \\
\hline Lumbar spine* & 4.8 & $6.4^{\dagger}$ & $6.3^{\dagger}$ \\
Total hip & 2.2 & $3.4^{\dagger}$ & $3.1^{\dagger}$ \\
Femoral neck & 2.2 & 2.7 & 2.8 \\
Trochanter & 3.5 & $5.0^{\dagger}$ & $4.9^{\dagger}$ \\
\hline
\end{tabular}

$*_{\mathrm{n}}=334,320$ and 334 for the daily, $\mathrm{q} 2 \mathrm{mo}$ and $\mathrm{q} 3$ mo groups, respectively ${ }^{\dagger} \mathrm{p}<0.001$ vs daily
OC33. PERSISTENCE WITH RISEDRONATE THERAPY IS ALREADY HIGH AND FURTHER IMPROVED BY A PATIENT EDUCATIONAL PROGRAM

P. Hadji ${ }^{1}$, V. Ziller ${ }^{1}$, M. Hartmann ${ }^{2}$, B. Niggeschulze ${ }^{2}$, S. Kroder ${ }^{2}$, W. Möhrke ${ }^{2}$ ' Universitätsklinikum Marburg, Marburg, Germany, ${ }^{2}$ Procter \& Gamble Pharmaceuticals-Germany GmbH, Weiterstadt, Germany

Objective: There is concern that persistence with therapy for chronic, asymptomatic diseases, i.e. postmenopausal osteoporosis is be suboptimal and this may affect clinical efficacy. Recent analyses, however, show a satisfactory 1-year persistence as high as $68 \%$ for weekly bisphosphonates. To further improve persistence with risedronate therapy a patient educational program was introduced in 2000 in Germany by the Alliance for Better Bone Health. Patients can enrol into the program sending in a reply card. They receive reminder letters together with leaflets informing them about osteoporosis in regular intervals over the first 7 month period. In this study we evaluated whether persistence with risedronate therapy improved in patients enrolled into the program versus a control group.

Methods: Telephone interviews were conducted with postmenopausal women with osteoporosis enrolled into the program $(n=204)$ and a control group of patients that was recruited from physicians in private practice who were not enrolled into the program $(n=204)$. Treatment start was equally distributed between February 2003 and February 2005. Survival analyses were used to determine persistence at 12, 24, and 29 months. Log rank test was used to statistically compare the two groups.

Results: Average age in both groups was similar (69 years). The majority of patients were taking weekly risedronate. Persistence with risedronate therapy in the patient group enrolled into the program was significantly higher than in the control group ( $p<0.03$ ). Persistence at 12 months was $91.6 \%$ for the group enrolled in the program versus $84.7 \%$ in the control group. After 24 months persistence was $84.6 \%$ versus $75.7 \%$, and at 29 months persistence was $79.1 \%$ versus $75.7 \%$.

Conclusion: Persistence with risedronate therapy is high in Germany. These data suggest that enrolling patients in a mailed educational program can further improve persistence.

\section{OC34. TRABECULAR NUMBER AS A RISK FACTOR IN OSTEOPOROSIS AND THE ROLE OF NASAL SPRAY CALCITONIN (NS-CT) IN ITS MODI- FICATION}

M. Olson ${ }^{1}$, L. Mindeholm ${ }^{1}$, E.F. Eriksen ${ }^{1}$, S. Majumdar ${ }^{2}$, M. Azria ${ }^{1}$, C. Chesnut ${ }^{3}$; ${ }^{1}$ Novartis Pharma, Basel, Switzerland, ${ }^{2}$ University of California, San Francisco, CA, USA, ${ }^{3}$ University of Washington, Medical Center, Seattle, WA, USA

Objective: Bone loss associated with increasing age and osteoporosis is characterized by loss of trabecular elements, which can be quantified as trabecular number (Tb.No). In the QUEST study bone loss in the forearm was associated with a decrease in Tb.No and increased trabecular spacing (1). In this post hoc analysis we evaluated baseline trabecular number (Tb.No) assessed by high resolution MRI as predictor for subsequent disease progression at 24 months in placebo and NS-CT $(200 \mathrm{IU} / \mathrm{d})$ treated patients.

Methods: We used the baseline median Tb.No (region 4, distal radius) to categorize patients into risk cohorts. Summary statistics were calculated for the percent change from baseline to month 24 for Tb.No by treatment group. Within and between group changes were assessed using linear contrasts from an analysis of variance model.

Results: Figure 1 summarizes the percent change from baseline to month 24 for Tb.No by baseline Tb.No cohort and treatment. Subjects on placebo with low baseline Tb.No exhibited significant loss in Tb.No $(-12.7 \%, p=0.0003)$, whereas the loss in the cohort with high baseline Tb.No was non-significant $(-1.0 \%$, $\mathrm{p}=0.75$ ). In the NS-CT group Tb.No increased slightly, irrespective of baseline Tb.No. 


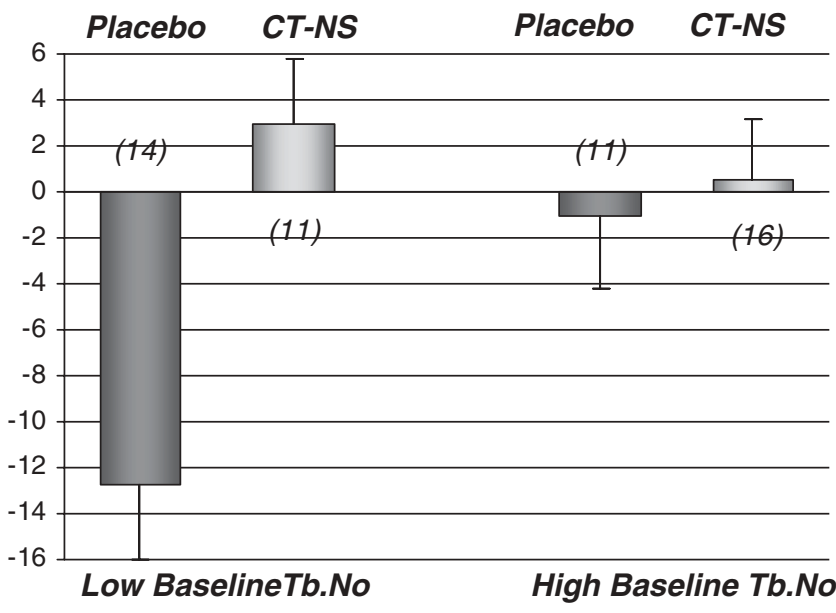

Fig. 1 Percent change from baseline to month 24 for MRI Tb.No (N)

These differences resulted in a significant treatment effect of NS-CT in subjects with low baseline Tb.No (difference $15.7 \%$, $\mathrm{p}=0.0007)$. The treatment difference in the cohort with high baseline Tb.No was $1.5 \%$ (ns).

Conclusion: Baseline Tb.No as assessed by HR-MRI can be used to define patients at risk of excessive trabecular bone loss. In a similar analysis, BMD did not show the same predictive value. Moreover, NS-CT preserved trabecular architecture regardless of trabecular bone structure at baseline.

\section{Reference}

1. Chesnut C et al JBMR, April 2005.

\section{OC35. EFFECT OF GLUCOSAMINE SULFATE ON NEWLY PROPOSED/ RECOMMENDED CLINICAL TRIAL END-POINTS IN PATIENTS WITH KNEE OSTEOARTHRITIS}

O. Bruyer̀e ${ }^{1}$, G. Giacovelli ${ }^{2}$, K. Pavelka ${ }^{3}$, L.C. Rovati ${ }^{2}$, J.-Y. Reginster ${ }^{1}$; ${ }^{1}$ Bone and Cartilage Research Unit, University of Liège, Liège, Belgium, ${ }^{2}$ Clinical Pharmacology Department, Rottapharm, Monza, Italy, ${ }^{3}$ Institute of Rheumatology, Prague, Czech Republic

Objective: To help in interpreting results of clinical trials, 3 recent publications suggest the use of dichotomised end-points for the assessment of the efficacy of drugs in osteoarthritis. Two of them propose assessment based on clinical relevance i.e. the use of the Minimal Clinically Important Improvement (MCII) and $\mathrm{Pa}$ tient Acceptable Symptom State (PASS) (Tubach et al., Ann Rheum Dis 2005) for WOMAC. Including clinical and radiological features, the assessment of "responders" or "failures" is recommended by the GREES (Abadie et al., Osteoarthrtis Cartilage 2004). The objective of this study is to assess the efficacy of glucosamine sulfate on these news end-points, in two recent longterm trials.

Material and methods: Patients completing the 3-year treatment course in the randomised, double-blind, placebo-controlled trials of Reginster 2001 and Pavelka 2002, were pooled in a single database. The MCII and PASS for WOMAC pain and function scores were calculated according to Tubach 2005. The rate of "GREES responders" were calculated according to Abadie 2004 (a joint space narrowing less than $0.5 \mathrm{~mm}$ and an improvement in total WOMAC of at least $20 \%$ ). The proportion of patients achieving such thresholds was compared between groups by the chi-square test.
Results: Out of the 259 completers (126 with placebo and 133 with glucosamine sulfate), over $50 \%$ were in PASS at the end of the studies regardless of treatment. However, they were $68.4 \%$ for WOMAC pain with glucosamine sulfate vs. $55.6 \%$ with placebo $(\mathrm{p}=0.033)$, and $63.9 \%$ vs. $50.0 \%$ on WOMAC function $(\mathrm{p}=0.024)$. Patients reporting MCII on WOMAC pain were $39.8 \%$ vs. $27.8 \%$, respectively $(p=0.040)$. In the glucosamine sulfate group, 71 patients $(53 \%)$ were considered responders (JSN $<0.5 \mathrm{~mm}$ and at least $20 \%$ improvement in total WOMAC) compared to 41 patients $(32 \%)$ in the placebo group $(\mathrm{p}=0.0007)$.

Conclusions: Glucosamine sulfate induces a statistically significant benefit compared to placebo independently of the responders criteria used.

OC36. LONG TERM EVALUATION OF DISEASE PROGRESSION THROUGH THE QUANTITATIVE MAGNETIC RESONANCE IMAGING OF SYMPTOMATIC KNEE OSTEOARTHRITIS PATIENTS: CORRELATION WITH CLINICAL SYMPTOMS AND RADIOGRAPHIC CHANGES

J-P. Raynauld ${ }^{1}$, J. Martel-Pelletier ${ }^{1}$, M-J. Berthiaume ${ }^{1}$, G. Beaudoin $^{1}$, D. Choquette ${ }^{1}$, B. Haraoui ${ }^{1}$, H. Tannenbaum ${ }^{2}$, J.M. Meyer ${ }^{3}$, J.F. Beary ${ }^{3}$, G.A. Cline ${ }^{3}$, and J-P. Pelletier ${ }^{1}$; ${ }^{1}$ Osteoarthritis Research Unit, University of Montreal Hospital Centre (CHUM), Notre-Dame Hospital, Montreal, Quebec, Canada, ${ }^{2}$ McGill University Health Centre (MUHC), Montreal General Hospital, Montreal, Quebec, Canada, ${ }^{3}$ Procter \& Gamble Pharmaceuticals, Mason, Ohio, USA

Objective: The objective of this study was to further explore the cartilage volume changes in knee OA over time using quantitative MRI. These were correlated to demographic, clinical, and radiological data to better identify the disease risk features.

Methods: 107 patients were selected from a large trial $(\mathrm{n}=$ 1232) evaluating the effect of a bisphosphonate on OA knees. The MRI acquisitions of the knee were done at baseline, 12, and 24 months. Cartilage volume from the global, medial, and lateral compartments was quantified. The changes were contrasted with clinical data and other MRI anatomic features.

Results: Knee OA cartilage volume losses were statistically significant compared to baseline values: $-3.7 \pm 3.0 \%$ for global cartilage and $-5.5 \pm 4.3 \%$ for the medial compartment at 12 months, and $-5.7 \pm 4.4 \%$ and $-8.3 \pm 6.5 \%$ at 24 months, respectively. Three different populations were identified according to cartilage volume loss: fast $(\mathrm{n}=11) ;-13.2 \%$, intermediate ( $=48 ;-7.2 \%)$, and slow $(n=48 ;-2.3 \%)$ progressors. The predictors of fast progressors were the presence of severe meniscal extrusion $(\mathrm{p}=0.001)$, severe medial tear $(\mathrm{p}=0.005)$, medial and or lateral bone edema $(\mathrm{p}=0.03)$, high body mass index $(\mathrm{p}<$ 0.05 , fast vs. slow), weight ( $\mathrm{p}<0.05$, fast vs. slow) and age ( $\mathrm{p}<$ 0.05 fast vs. slow). The loss of cartilage volume was also slightly associated with less knee pain. No association was found with other WOMAC scores, joint space width, or urine biomarker levels.

Conclusion: Meniscal damage and bone edema are closely associated with more cartilage volume loss. These data confirm the significant advantage of quantitative MRI for reliably measuring knee structural changes at as early as 12 months, and for identifying risk factors associated with OA progression.

\section{OC37. RADIOLOGIC EVOLUTION OF THE KNEE JOINT: A FOUR-YEAR PROSPECTIVE STUDY}

D. Gensburger, M. Arlot, E. Sornay-Rendu, F. Munoz, P.D. Delmas; INSERM Research Unit 403 and Université Claude Bernard Lyon 1, France

Background: Osteoarthritis (OA) of the knee is a growing public health problem, especially because of ageing of the population and secular increase in body weight. Its natural history remains poorly documented as most epidemiologic studies con- 
cern elderly OA populations, without standardized radiographic protocol. Consequently, the existence of a "physiological" agerelated joint space narrowing is controversial.

Objective: To determine if there is a reduction of joint space width in the tibio-femoral compartment, even in the absence of signs of osteoarthritis, in a four year prospective study of healthy women from a population based cohort.

Patients and methods: 415 women from the OFELY cohort, 39 to 87 years old, had radiographs of both knees in semiflexion with a standardized fluoroscopically assisted protocol at baseline and four years later. Assessment of radiographs was quantitative, using a data-processing software measuring the joint space width after digitalisation, and qualitative, according to a modified Altman score. Both at baseline and at follow-up, clinical risk factors (age, BMI, height, hormonal statute and WOMAC), biological data (biochemical markers of bone and cartilage metabolism) and BMD (hip and lombar spine) were recorded.

Results: Reproducibility of measures was satisfactory. Quantitative assessment showed a significant reduction of tibio-femoral joint space width at the four compartments, even in women without OA. This joint space narrowing appears more significant in the medial compartment and increases with the initial joint space width. There was a significant correlation between osteophytes and urinary CTX-II, a specific marker of cartilage degradation.

Conclusion: We showed, in this longitudinal study, a joint space loss with age in the tibio-femoral compartment, even in women without clinical or radiological features of osteoarthritis.

\section{OC38. SCLEROSTIN INHIBITION IN OSTEOPOROTIC RATS COM- PLETELY REVERSES ONE YEAR OF OVARIECTOMY-INDUCED SYSTEMIC BONE LOSS}

K. Warmington ${ }^{1}$, M. Ominsky ${ }^{1}$, B. Bolon ${ }^{2}$, R. Cattley ${ }^{1}$, P. Stephens $^{3}$, A. Lawson ${ }^{3}$, D. Lightwood ${ }^{3}$, V. Perkins ${ }^{3}$, H. Kirby ${ }^{3}$, A. Moore ${ }^{3}$, M. Robinson ${ }^{3}$, P.J. Kostenuik ${ }^{1}$, W.S. Simonet ${ }^{1}$, D.L. Lacey ${ }^{1}$, C. Paszty ${ }^{1}$; ${ }^{1}$ Amgen Inc., Thousand Oaks, CA, USA, ${ }^{2}$ GEMpath Inc., Cedar City, UT, USA, ${ }^{3}$ Celltech R and D Ltd., Slough, United Kingdom

Objective: Sclerostin is a secreted protein that has been shown to play a key role in controlling bone mass throughout life, apparently by negatively regulating the anabolic output of the osteoblast lineage. Thus, in both humans and mice, the absence of sclerostin results in increased bone formation and high bone mass. We used a monoclonal antibody (MAb) approach to explore the therapeutic potential of inhibiting sclerostin for the treatment of postmenopausal osteoporosis (PMO).

Materials and Methods: Six month-old female rats were ovariectomized (OVX), allowed to lose bone for 1 year, and then treated for 5 weeks with a sclerostin-neutralizing MAb or PTH 134. Following treatment, bone mineral density (BMD) was determined in vivo by DXA and ex vivo by Micro-CT. Serum TRAP-5b, an osteoclast-specific bone resorption marker, was also measured.

Results: Bone loss, at 1 year post-OVX, was similar at various skeletal sites with a $\sim 12 \%$ decrease in BMD vs agematched, sham-operated controls. Sclerostin inhibition led to statistically significant increases in BMD in lumbar vertebrae $(26 \%)$, whole leg $(16 \%)$, femoral metaphysis $(28 \%)$, and femoral diaphysis $(9 \%)$ vs vehicle-treated controls. These increases in BMD were similar to those seen in the PTH-treated group. Micro-CT analysis of the femur showed that sclerostin inhibition led to improved trabecular architecture (increased BV/TV [118\%], BMD [64\%], trabecular number [50\%], trabecular thickness [57\%]) and cortical geometry [increased cortical thickness $(26 \%)$ and decreased endosteal circumference $(14 \%)]$. Serum TRAP-5b was statistically significantly decreased in the Mab-treated rats (43\% vs vehicle), suggesting that the sclerostin pathway, in addition to controlling osteoblasts, may also affect osteoclasts, perhaps by modulating osteoblast-expressed osteoprotegerin and RANK ligand levels. Gross observation, serum clinical chemistry, and hematological and histopathological analyses revealed no abnormalities associated with pharmacological inhibition of sclerostin.

Conclusion: In summary, very impressive increases in BMD were achieved in a rat model of PMO after 5 weeks of treatment with a sclerostin-neutralizing Mab. These data suggest that inhibition of sclerostin may be effective in building bone in the clinical setting of PMO, even in cases where osteoporosis has progressed to the point where significant bone loss has already occurred.

OC39. SB-462795 (RELACATIB), A CATHEPSIN INHIBITOR, PREVENTS THE LOSS OF BONE MASS AT SEVERAL SITES IN OVARIECTOMIZED CYNOMOLGUS MONKEYS

S. Kumar ${ }^{1}$, C. Jerome ${ }^{2}$, G. Stroup ${ }^{1}$; ${ }^{1}$ GlaxoSmithKline Pharmaceuticals, Collegeville, PA, USA, ${ }^{2}$ MDS Pharma, Bothell, WA, USA

Objective: Cathepsin $\mathrm{K}$ has been shown to play an important role in bone matrix degradation during osteoclastic bone resorption. We studied the effects of SB-462795, a novel, potent inhibitor of cathepsins $\mathrm{K}, \mathrm{L}$ and $\mathrm{V}$ on bone turnover in aged ovariectomized (Ovx) female monkeys.

Methods: Adult female cynomolgus monkeys were randomized to 6 groups of 20 each, of which one was sham-Ovx (Sham) and the remainder Ovx. Ovx animals were treated with vehicle, SB-462795 at 1,3 , or $10 \mathrm{mg} / \mathrm{kg} / \mathrm{d}$ by oral gavage, or alendronate at $0.05 \mathrm{mg} / \mathrm{kg}$ by IV injection once every 2 weeks for 9 months. All statistical comparisons are with Ovx, vehicletreated animals.

Results \& Conclusions: SB-462795 dose-dependently decreased urinary excretion of NTx with a significant effect observed earlier for SB-462795 versus vehicle than alendronate. Unlike alendronate which decreased bone formation markers, treatment with SB-462795 at $10 \mathrm{mg} / \mathrm{kg} / \mathrm{d}$ decreased bone formation markers to a lesser extent and significantly increased serum osteocalcin. Animals treated with SB-462795 had dosedependent improvement in aBMD at the lumbar spine and proximal and distal femur. At the distal femur SB-462795 prevented $73 \%$ of the loss in aBMD while alendronate showed a non-significant $23 \%$ protection. At the proximal femur the level of protection by the two agents were comparable. At the lumbar spine, SB-462795 protected $42 \%$ while alendronate protected $100 \%$ of the loss in aBMD. Femoral neck analysis using pQCT, showed a similar level of protection of total vBMD by SB-462795 $(58 \%, \mathrm{p}<0.05)$ and alendronate $(51 \%$, $\mathrm{p}>0.1)$. Both agents had a similar, positive effect on trabecular vBMD and BMC. Treatment with SB-462795 at the high dose significantly increased cortical area and cortical BMC compared to vehicle. This statistical significance was not achieved with alendronate. Histomorphometry data revealed a dose-dependent reduction in both bone resorption and formation parameters at cancellous sites with both agents. However, a stimulatory effect on femur periosteal bone (Ps.BFR/BV) was observed with SB462795 but not with alendronate treatment. These findings suggest that SB-462795 significantly differs from alendronate in that it may have classic anti-resorptive effects in cancellous bone, and a novel, beneficial, stimulatory effect on cortical bone.

OC40. ZOLEDRONIC ACID SAFETY AND EFFICACY OVER 5 YEARS IN POST-MENOPAUSAL OSTEOPOROSIS

J.P. Devogelaer ${ }^{1}$, P. Burckhardt ${ }^{2}$, P. Meunier ${ }^{3}$, J.M. Kaufman ${ }^{4}$, K. Lippuner ${ }^{5}$, J.J. Body ${ }^{6}$, G. Samsioe 7 , D. Felsenberg ${ }^{8}$, J. Brown $^{9}$, P. Garnero ${ }^{3}$, L. Sanna ${ }^{10}$, C.E. Ortmann ${ }^{10}$, J. Krasnow ${ }^{11}$, E.F. Eriksen ${ }^{10}$, T. Fashola ${ }^{10}$, U. Trechsel ${ }^{10}$; ${ }^{1}$ Cliniques Universitaires St Luc, Brussels, Belgium, ${ }^{2}$ Centre Hospitalier Universitaire Vaudois, Lausanne, Switzerland, ${ }^{3}$ Hôpital Edouard Herriot,Inserm, Lyon, France, ${ }^{4}$ University Hospital of Ghent, Ghent, Belgium, ${ }^{5}$ Leiter Einheit fur Osteoporose, Inselspital, Bern, Switzerland, ${ }^{6}$ Institut Jules Border, Brussels, Belgium, 
${ }^{7}$ Kvinnokliniken, Universitets Sjukhuset, Lund, Sweden, ${ }_{9}^{8}$ Universitätsklinikum Benjamin Franklin, Berlin, Germany, ${ }^{9}$ Centre Hospitalier Universitaire de Québec, Sainte-Foy, Québec, Canada, ${ }^{10}$ Novartis Pharma AG, Basel, Switzerland, ${ }^{11}$ Novartis Pharmaceuticals Inc, US

Objective(s): Zoledronic acid (ZOL) administered as a single intravenous dose has been shown to increase BMD and decrease biochemical markers of bone turnover similar in magnitude to that observed with oral bisphosphonates in post-menopausal osteoporosis $(\mathrm{PMO})^{(1)}$. To explore the long term safety and efficacy of ZOL, the results from an open label extension to the study of Reid et al ${ }^{(1)}$ with total annual dose of $4 \mathrm{mg}$ up to 5 years are presented.

Materials and Methods: ZOL doses administered in the core and extension phases are:

Treatment groups

\begin{tabular}{lll}
\hline Group & Group 2: 3 years & Group 3: 5 years \\
1: 2 years & of ZOL treatment & of ZOL treatment \\
of ZOL & $\mathrm{N}=78$ & $\mathrm{~N}=22$ \\
treatment & & \\
$\mathrm{N}=19$ & &
\end{tabular}

\begin{tabular}{|c|c|c|c|c|c|}
\hline $\begin{array}{l}\text { Core study } \\
\text { (1 year } \\
\text { treatment } \\
\text { or placebo)* }\end{array}$ & Placebo & $4 \times 0.25 \mathrm{mg}$ & $4 \times 0.5 \mathrm{mg} 4 \times 1 \mathrm{mg}$ & $2 \times 2 \mathrm{mg}$ & $1 \times 4 \mathrm{mg}$ \\
\hline $\begin{array}{l}\text { E1 study } \\
\text { (2-year } \\
\text { treatment for } \\
\text { all patients)* }\end{array}$ & $4 \times 1 \mathrm{mg}$ & $4 \times 1 \mathrm{mg}$ & $4 \times 1 \mathrm{mg} \quad 4 \times 1 \mathrm{mg}$ & $4 \times 1 \mathrm{mg}$ & $4 \times 1 \mathrm{mg}$ \\
\hline $\begin{array}{l}\text { E2 study } \\
\text { (2-year } \\
\text { treatment or } \\
\text { calcium only)* }\end{array}$ & Calcium & Calcium & Calcium Calcium & $1 \times 4 \mathrm{mg}$ & $1 \times 4 \mathrm{mg}$ \\
\hline
\end{tabular}

* all patients received $1 \mathrm{~g}$ calcium daily during the 5 years

Results: After 5 years, the lumbar spine BMD increased $8.5 \pm 5.80 \%$ (mean \pm SD) in group $1,9.0 \pm 4.97 \%$ in group 2 and $6.3 \pm 6.60 \%$ in group 3, while total hip BMD increased $5.5 \pm 3.70 \%, 4.9 \pm 4.04 \%$ and $5.2 \pm 4.53 \%$ respectively over baseline. Bone turnover markers (CTX and BSAP) following the first year of treatment were within the premenopausal range in all 3 groups. Repeated dosing of ZOL did not result in further suppression of bone turnover and no patient revealed sustained suppression below the premenopausal range over the 5 years. During the 2 year offset period in group 1, bone markers revealed an upward trend within the premenopausal range. No clinically significant renal safety issues were identified and no cases of osteonecrosis of the jaw was reported.

Conclusion(s): In the limited number of PMO patients, the use of iv ZOL $4 \mathrm{mg}$ for up to 5 years was generally safe and well tolerated Continuing treatment resulted in maintenance of bone turnover within the premenopausal range. After discontinuation of treatment, bone turnover increased over time, showing preservation of remodeling capacity. The anti-fracture efficacy of annual i.v ZOL $5 \mathrm{mg}$, is currently being investigated in large Phase III trials in Osteoporosis.

(1) Reid I et al. NEJM Feb 2002, 346:653-661

OC41. INCIDENT FRACTURES IN PATIENTS WITH INADEQUATE RESPONSE TO ANTIRESORPTIVE OSTEOPOROSIS THERAPY: ONE-YEAR RESULTS FROM THE OSSO STUDY

C. Cooper ${ }^{1}$, F. Jakob ${ }^{2}$, C. Chinn ${ }^{3}$, E. Martín-Mola ${ }^{4}$, P. Fardellone, S. Adami ${ }^{5}$, N.C. Thalassinos, J. Melo-Gomes ${ }^{8}$, D. Torgerson ${ }^{9}$, T. Nicholson ${ }^{3}$, F. Marín ${ }^{3}$; ${ }^{1}$ MRC Environmental Epidemiology Unit, Southampton General Hospital, Southampton, UK, ${ }^{2}$ Dept. Orthopaedics, Julius-Maximilians-Universität, Wurzburg, Germany, ${ }^{3}$ Lilly Research Center, Windlesham, UK, ${ }^{4}$ S. Reumatología, Hospital La Paz, Madrid, Spain, ${ }^{5}$ S. Rhumatologie, Hopital Nord, Amiens, France, ${ }^{6}$ Centro
Ospedaliero Clinicizzatodi Valeggio sul Mincio, Verona, Italy, ${ }^{7}$ Dept. Endocrinology, Evangelismos General Hospital, Athens, Greece, ${ }^{8}$ Instituto Portugues de Reumatologia, Lisbon, Portugal, ${ }^{9}$ Department Health Sciences, University of York, York, UK

Objective: Currently available osteoporosis treatments reduce the risk of fractures, but up to $50 \%$ of patients have an inadequate clinical outcome to therapy. We describe the incidence of osteoporotic fractures and the associated risk factors in patients included in OSSO (Observational Study on Severe Osteoporosis) after one year of follow-up.

Patients and Methods: OSSO is an observational study conducted in six European countries, that included 2,322 postmenopausal women [mean age (SD): 70.6(9.0) years)] with established osteoporosis and an inadequate clinical response to osteoporosis drug treatment. At baseline, patients were allocated to either the index fracture (IF) cohort (those who had experienced a fragility fracture despite osteoporosis drug therapy for at least one year; $\mathrm{n}=1,313)$, or the compliance/side effect $(\mathrm{C} \backslash \mathrm{SE})$ cohort (those who discontinued osteoporosis drug therapy due to compliance problems and/or side effects; $n=1,009$ ), and prospectively followed-up for one year.

Results: $86.7 \%$ of patients were receiving at least one osteoporosis drug at baseline.

Fracture outcomes were available in 1,885 patients during the follow-up period $(81.2 \%) .79 .5 \%$ of the women completed the scheduled 1-year visit. After a median of 365 days of follow-up [mean (SD): 351 (88) days], 166 women (8.8\%) (IF: 100 $(10.1 \%)$; C $\backslash$ SE: $66(7.3 \%)$ ) sustained 209 fractures, including 98 spinal, 24 forearm/wrist, 17 hip, 19 sternum/rib, 13 humerus, and 13 pelvic fractures. 126 patients sustained a single incident fracture $(6.7 \%)$, and $37(2 \%)$, and $3(0.2 \%)$ sustained 2 and 3 fractures respectively. Women in the incident fracture group had similar age, demographics and lifestyle characteristics, and reproductive history to women in the non-incident fracture group. However, they reported more falls in the previous year, history of fragility fractures after age 40, hearing problems, current use of glucocorticoids and antidepressants, and lower bone mineral density. By the end of follow-up, women with incident fractures had more comorbidities $(41.9 \%$ vs $28.1 \%)$, and $6.5 \%$ of them were not taking any osteoporosis drug therapy.

Conclusion: The frequency of a new fragility fracture in patients with inadequate clinical response to osteoporosis therapy is high $(8.8 \%)$ within only one year of follow-up. Early recognition and intervention in this population of patients should be implemented to decrease the risk of fractures.

\section{OC42. HOW CAN WE IDENTIFY AND PLAN INTERVENTION AGAINST HIP FRACTURES IN THE POPULATION?}

O. Johnell ${ }^{1}$, H. Lundgren ${ }^{2}$, D. Noreen ${ }^{2}$, H. Weibull ${ }^{2}$, T. Lithman ${ }^{2}$ ${ }^{1}$ Dept of Orthopaedics UMAS, Malmo, Sweden, ${ }^{2}$ Region Scania, Lund, Sweden

The true risk factors for hip fractures are hard to establish since most cohort studies have only included mentally healthy individuals. We identified all hip fractures 145 years of age occurring January to September 2005 in the Scania (southern) Region of Sweden $(n=1.572)$. All hospital and primary care visits both outpatients and inpatients were analysed one year before the hip fracture. Within the year before the hip fracture, $10.5 \%$ (165 years) had visited the health care system with a diagnosis of dementia compared to $1.2 \%$ in the general population standardized to the age and sex distribution of the hip fractures. $10.9 \%$ of patients had other psychiatric diagnoses compared to $1.5 \%$. Anaemia had $3.1 \%$ compared to $0.6 \%$, dizziness and fainting $7.3 \%$ to $1.4 \%$, diabetes $9.1 \%$ to $2.4 \%$, hypertension $18.2 \%$ to $6.1 \%$, other injuries $26 \%$ to $3.2 \%$, stroke $13.3 \%$ to $1.8 \%$ in the general population. To find out the risk of hip fracture we then identified in 2004/2005 all with these different diagnoses and identified those who had a hip fracture prospectively January to September 2005 in the 
time span of one year. $3 \%$ of those with dementia had a hip fracture within 1 year and the RR (Relative Risk) for men and women compared to the general population was $13.1(1,8-94,0)$ at the age of 45-64 and $2.1(1,7-2,7)$ at the age of 185 . For those with other psychiatric diagnoses the corresponding figures were RR $4.5(2,8-7,3)$ and $1.7(1,3-2,3)$, anaemia $8.1(2,0-32,9)$ and 0.9 (NS), dizziness and fainting 1.6 and 1.5, diabetes 2.5 and $0.8(\mathrm{NS})$, hypertension 2.5 and $1.1(\mathrm{NS})$, other injuries 6.5 and 2.3, stroke 17.2 and 1.5 and for alcohol abuse 11.2 and 2.6
(75-84 years). Thus individuals with dementia, other psychiatric diagnosis ( incl. depression) and alcohol have a high increased risk of having a hip fracture within the next year, high enough to warrant an intervention. For many diagnoses, such as hypertension, diabetes etc., a similar pattern was also observed for having a myocardial infarction, stroke etc. Therefore, also strategies can be implemented for several diseases. The main exception was dementia. 


\section{Poster Presentations}

\section{P100. THE MANAGEMENT OF PATIENTS WITH SECONDARY OSTEO- POROSIS AFTER TRAUMA OF EXTREMITIES}

E. Popova Ramova, M. Schurbevski, C. Schumanova; Department of Physical Therapy and Rehabilitation, Military Hospital, Skopje, FYRO Macedonia

The patients with circulatory insufficiency and trauma of extremities had a local post traumatic osteoporosis. The circulatory insufficiency and osteoporosis are slowing the rehabilitation and work condition of patients. The patients with circulatory insufficiency are on anti trombosis medical therapy. The aim of our study is to represent our management of treatment of this local osteoporosis.

Material and method: We had treated 220 patients, 98 female and 122 male, with over age of 52.7 years, in period of 6 months. The managements is consisting of medical treatment of circulatory insufficiency and local osteoporosis (calcitonin 200 IE pro day nasal spray, Ca and Vit.D3) and physical therapy (interferential current, impulse magnetic field, iontoforesis with vasoactive material) and exercises with muscles pump effect. The medical support was 2 months and all rehabilitation program 6 months. The follow clinical parameters were: pain, swelling, skin color, joint mobility, fibrosis and radiological picture of treated bone. The doses of physical therapy applications and medical support were exactly determinate.

Results: The total points before treatment were $12 \%$, and after 6 months $89.9 \%$ by score. Two patients had complication of circulation, and the physical therapy was exclude one month. The medical support with calcitonin was good accepted by patients and the radiological pictures were with regression of osteoporosis after two months.

Conclusion: We try to help the patients with circulatory insufficiency, because the bone condition was by all of them with local osteoporosis. The good therapy effect was proved with good clinical picture, good radiological picture and economic effect of faster good work condition.

\section{P101. THE MANAGEMENT AND ROLE OD ORDINARY PRACTICE DOC- TOR IN PREVENTION AND DIAGNOSIS OF OSTEOPOROSIS}

C. Schumanova, E. Popova Ramova; Ordinary Practice Department, Military Hospital, Skopje, FYRO Macedonia

The prevention and early diagnosis of osteoporosis is one of way to low the complication of this disaster. The ordinary practice doctor is knowing the common health condition of his patients. He is in situation to detect the risk factors, and to sent the patients for diagnostic methods, but also to follow the patient by treatment. The aim of our study is to represent our experience in management of early detection of risk group patients and his treatment.

Material and method: There were 45 patient included in our study with risk factors group. There were $90 \%$ of women, and with over age of 68.3 years. The early detection is consisting of: 1. risk group by age, sex, and other disaster, 2. clinical examination with subjective pain in bones and muscles, 3. labor: osteocalcin, calcitonin, PTH, and pyrilinx-d (bone marker). 4. examination by specialist for osteoporosis. The medical treatment was including 6 months, and after that control labor.

Results: The two patients had osteomalacija, one after suckling, and the other on extreme diet both 31 years old, 5 of them after gynecology surgery treatment, 12 with diabetes, and 26 by age. The treatment was with calcitonin one month, and the rest with biphosponate, diet, exercises and vitamin D3 and Ca. The 2 patients (31 year old) had only one month treatment with antiresorptive bone drugs. The control labor and subjective pain and self-condition were goodness after 6 months. Discussion: We try to detect the osteoporosis with risk factors groups and bone markers, the DEHA and other procedures are too expensive, also we try to adapted the medical treatment with social status of patients.

Conclusion: Our management was good proved with good socioeconomic status and patient with low of pain.

\section{P102. HYSTERECTOMY, BODY MASS INDEX AND CALCANEAL BMD IN LEBANESE POPULATION}

Y. Yaghi ${ }^{1}$, A. Kanaan ${ }^{2}$, R. Halabi ${ }^{1}$; Hammoud Hospital, University Medical Center, Saida, Lebanon, ${ }^{2}$ Caritas, Health Care Center, Saida, Lebanon

Introduction: Several studies have demonstrated that low body mass index (BMI) and hysterectomy are predictors of osteoporosis. We examined this relationship between calcaneal BMD, surgical menopause and BMI in Lebanese population.

Materials and Methods: The study was conducted at one center (Caritas-Health care center, SAIDA-LEBANON. Enrollment was targeted for 186 Lebanese post-hysterectomy Women (surgical menopause). Age of subjects ranged from 37 to 81 years. They were subdivided into 3 groups according to the date of surgery, each of 10 years length (table 1). The ratio weight/height $2\left(\mathrm{wt} / \mathrm{ht}^{2}\right)$ was calculated as body mass index (BMI), which is a common and reliable rating of body fat. A patient was considered over-weight when BMI exceeds $25 \mathrm{~kg} /$ $\mathrm{m}^{2}$. Analysis of groups was done according to patients BMI. BMD was measured using Hologic Sahara QUS machine at calcaneal bones for all women included in this study. During the length of the study one technician did all the measurements.

Results: Our study shows that a decrease in BMI is always associated with an increased bone turnover and significant decrease of the women level of calcaneal BMD. Women with high BMI who underwent hysterectomy maintained a higher calcaneal BMD levels than those with low BMI (table 2).

Conclusion: Based on our results we conclude that BMI is an important risk factor for developing osteoporosis.

Table 1

\begin{tabular}{ll}
\hline Group & Years of Hysterectomy \\
\hline I & $0-10$ \\
II & $11-20$ \\
III & 20 and more
\end{tabular}


Table 2

\begin{tabular}{|c|c|c|c|c|c|c|}
\hline \multirow[t]{2}{*}{ GROUP } & \multicolumn{3}{|c|}{ BMI less than 25} & \multicolumn{3}{|c|}{ BMI more than 25} \\
\hline & $\#$ & $\begin{array}{l}\text { Calcaneal } \\
\text { BMD }\end{array}$ & $\begin{array}{l}\text { Mean } \\
\text { Age }\end{array}$ & $\#$ & $\begin{array}{l}\text { Calcaneal } \\
\text { BMD }\end{array}$ & $\begin{array}{l}\text { Mean } \\
\text { Age }\end{array}$ \\
\hline I & 26 & 0.432 & 51.6 & 30 & 0.463 & 53.96 \\
\hline II & 42 & 0.325 & 61.66 & 62 & 0.400 & 63.12 \\
\hline III & 12 & 0.296 & 71 & 14 & 0.397 & 65.8 \\
\hline
\end{tabular}

P103. COMPARISON OF CALCANEAL BMD BETWEEN RIGHT, LEFT, DOMINANT AND NON-DOMINANT SIDES IN LEBANESE POPULATION

Y. Yaghi, R. Halabi, A. Elbaba, D. Makki; Hammoud Hospital, University Medical Center, Saida, Lebanon

Introduction: Several studies demonstrated higher bone mineral content (BMC) and bone mineral density (BMD) values in the proximal femur, forearm and phalangeal bones in the dominant side. We studied differences in calcaneal BMD between right, left, dominant and non-dominant sides in Lebanese population.

Materials and Methods: A group of 784 women were included in this study. Age of subjects ranged from $22-84$ years. They were divided into groups according to age, each of 10 years length (table1) 702 were right handed and 82 were left handed. Measurements of calcaneal bones were performed on all subjects. Patients with history of fractures were excluded from this study. BMD was measured by QUS machine (SAHARA). During the length of the study one machine was used and one technician did all the measurements.

Results: BMD values showed a significant correlation between right and left sides, but when compared to the non-dominant side, the dominant side was $4.5 \%$ higher in the younger age group and this difference became considerably greater with older age groups (table 2).

Conclusions: Significant differences in calcaneal BMD occur between dominant and non-dominant sides. These differences should be considered when interpreting results.

Table 1

\begin{tabular}{lll}
\hline & AGE: years & Number of subjects \\
\hline GROUPI & Less than 39 & 56 \\
GROUPII & $40-49$ & 188 \\
GOUP III & $50-59$ & 256 \\
GROUPIV & $60-69$ & 196 \\
GROUPV & $70-$ & 88 \\
\hline
\end{tabular}

Table 2

\begin{tabular}{lllll}
\hline \multicolumn{5}{c}{ CALCANEAL } \\
\cline { 2 - 5 } & LEFT & RIGHT & DOMINANT & $\begin{array}{l}\text { NON- } \\
\text { DOMINANT }\end{array}$ \\
\hline I & 0.475 & 0.493 & 0.498 & 0.471 \\
II & 0.487 & 0.491 & 0.504 & 0.476 \\
III & 0.448 & 0.452 & 0.465 & 0.435 \\
IV & 0.423 & 0.419 & 0.434 & 0.408 \\
V & 0.372 & 0.369 & 0.380 & 0.358 \\
\hline
\end{tabular}

P104. THE PREDICTIVE ROLE OF BIOCHEMICAL MARKERS IN BONE MINERAL DENSITY CHANGES IN MEN

O.S. Donescu ${ }^{1}$, M.C. Battie ${ }^{2}$, T. Videman ${ }^{3} ;{ }^{1}$ Faculty of Rehabilitation Medicine, University Alberta, Edmonton, AB, Canada, ${ }^{2}$ Department of Physical Therapy, University Alberta, Edmon- ton, AB, Canada, ${ }^{3}$ Faculty of Rehabilitation Medicine, University Alberta, Edmonton, AB, Canada

Objectives: The use of biochemical markers as indicators of overall bone metabolism has been suggested as a potentially valuable clinical method in osteoporosis screening, diagnosis and monitoring the effects of different interventions. They reflect small changes in bone turnover in a short timeframe. The aims of this study were to determine whether bone formation (PINP and PICP), and bone resorption (ICTP) markers are predictive of changes in BMD over a 5-year period, and also the ability of bone resorption marker NTx to explain the variance in BMD change over the prior 5 years.

Material and Methods: Both prospective and retrospective cohort study designs were used. Subjects were selected from the population-based Finnish Twin Cohort. The sample was composed of 203 monozygotic male twins 35-69 years old (mean 49.7, SD 8.4). PINP, PICP and ICTP markers were determined from serum by radioimmunoassay (Orion Diagnostica, Finland); NTx was measured in urine using an ELISA resorption assay (Osteomark ${ }^{\circledR}$; Ostex International) $(\mathrm{CV}<10.0 \%)$. BMD was measured with DEXA (Lunar DPX, Madison, WI), at the L1-L4 vertebrae (CV 0.9\%) and femoral neck (CV 1.5\%). Data analysis. Pearson coefficients assessed the correlation between BMD change and baseline marker values in the whole group, and in the group of subjects older than 60 years. The ability of markers to explain change in BMD, with age, fat free weight, height and baseline BMD as possible confounding factors was examined using multiple linear regression.

Results: Among the markers analyzed in the whole group, only NTx correlated with change in femoral neck BMD $(\mathrm{r}=$ $-0.21, p=0.006$ ) and explained $3.8 \%$ of the variance. In the group of subjects older than 60 years $(n=14)$, NTx and PICP significantly correlated with change in spine BMD $(r=0.6$ and $\mathrm{r}=0.5, \mathrm{p}<0.05)$

Conclusions: Among markers, only NTx explained a statistically significant yet quite limited portion of the variance in change BMD in the whole group of men, but only at the femoral neck. The correlations of markers NTx and PICP with the change in spine BMD were notably higher in the group of subjects older than 60 years than in the whole group.

P105. STEROID-RELATED KNEE OSTEONECROSIS TREATED BY PERCUTANEOUS CORE DECOMPRESSION

J.-K. Wu, C.-T. Yu, C.-P. Hsieh, S.-J. Chen, C.-C. Wu, C.-C. Chang, I.-L Chang, C.-Y. Lin, P.-W. Ko, P.-K. Lee; Orthopaedic Department, Changhua Christian Hospital, Changhua, Taiwan

Objectives: To describe the clinical, demographic, and radiographic patterns of patients with steroid-related osteonecrosis of the knee, and present the outcome of percutaneous core decompression.

Material and Methods: Between May 1, 1999 to April 30, 2005, a retrospective chart review of four consecutive patients $(6$ knees) with steroid-related knee osteonecrosis confirmed by magnetic resonance imaging (MRI) and bone scintigraphy. Demographic and radiographic features were characterized. Staging of all films was performed according to the Ficat and Arlet staging system of the hip adapted for the knee. All patients undertook percutaneous core decompression and evaluated by knee society score.

Results: There were two female patients and two male patients; the mean age at diagnosis was forty-seven years (range, 43 to 53 years) and follow up from 18 months to 6 years. All patients had history of using prednisolone (one patient had ALL with bilateral ON knee and unilateral ON hip, one patient had severe aplastic anemia with bilateral ON knee, and two patients had SLE with unilateral ON). All 6 knees had not orthopaedic complications from the percutaneous core depression. Radiographically, there were two knees in stage I) three knees in stage II) and one knee in stage III. Two knees (one stage II and one stage III) undertook twice operation due to recurrent discomfort after index 
surgery. The mean knee society score improved from 46 points preoperative to 91 points at the time of the latest follow-up.

Conclusions: In contrast to the spontaneous osteonecrosis of the knee (SPONK), steroid-related ON occurs younger and often bilateral with multiple joints involved. In our series, all patients achieved excellent and good results, which showed the method is a simple and effective technique with excellent pain relief and function outcome.

\section{P106. MALE STRADDLE-TYPED STRESS FRACTURE}

J.-K. Wu, C.-T. Yu, C.-Y. Lin, C.-C. Chang, P.-K. Lee, I.-L. Chang; Orthopedic Department, Changhua Christian Hospital, Changhua City, Taiwan

Pelvic stress fractures are not common, and most stress fractures occur in the lower extremities, with frequent sites being the tibia $(49.1 \%)$, tarsals $(25.3 \%)$, metatarsals $(8.8 \%)$, femur $(7.2 \%)$, fibula $(6.6 \%)$, sesamoids $(0.9 \%)$, pelvis $(1.6 \%)$, and spine $(0.6 \%)$ Pelvic stress fractures are mechanical disruptions of the bones of the pelvis, resulting from excessive repetitive muscular activity. Most occur in the inferior pubic rami and less commonly in the superior pubic rami.

The etiology of stress fractures is unclear. Intrinsic risk factors include mechanical factors, such as bone density, skeletal alignment, and bone size and composition; physiologic factors, such as bone turnover rate, flexibility, and muscular strength and endurance; and hormonal and nutritional factors. Extrinsic risk factors include mechanical factors such as surface, footwear, and external loading, as well as physical training parameters.

Symptomatic treatment is effective for pelvic stress fractures. Clinicians should suspect pelvic stress fractures in military recruits, joggers, and amateur runners, who present with pain in the inguinal area.

\section{P107. KINETIC ANALYSIS OF POSTERIOR LIGAMENT SUBSITUTE TO- TAL KNEE ARTHROPLASTY}

C.-T. Yu, C.-C. Chang, P.-K. Lee; Orthopedic Department, Changhua Christian Hospital, Changhua, Taiwan

Objective: The purpose of this study was to investigate kinematic and kinetic results of High-Flex ${ }^{\circledR}$ TKA during different motion patterns.

Material and Methods: Twelve patients with bilateral; OA knees were enrolled for study. All operations were performed on the more severe site by the same surgeon. Post-operative results were evaluated by Hospital for Special Surgery (HSS) score and comprehensive motion analysis.

Result: High-Flex ${ }^{\circledR}$ TKA provided excellent improvement in HSS score (88vs. 62). Post-operative evaluation showed an advantage in range of motion (ROM) of different motions. No significant differences were found with regard to gait pattern and ground reaction force. The knee stiffness also increased after surgery.

Conclusion: The results provide better understanding about the kinematics and kinetics of the High-Flex ${ }^{\circledR}$ TKA in Taiwanese and knee stiffness was first used for evaluating arthroplasty result.

\section{P108. INTRAARTICULAR CORTICOSTEROID INJECTIONS. PAIN RELIEF AND FUNCTIONAL IMPROVEMENT IN OSTEOARTHRITIS OF HIP?}

M. Changulani, K. O D'wyer; Department of Orthopaedics, Worcester Royal Hospital, Worcester, UK

Introduction: The use of corticosteroids for intraarticular injections is a common practice in orthopaedics. Though they are known to provide significant benefit in knee OA. Less is known of their effect in Osteoarthritis of hip. The purpose of this study was to evaluate the results of intraarticular corticosteroid hip injections in patients with osteoarthritis of hip.

Methods: A Questionnaire study was performed on 19 hips in 17 patients (mean age 63yrs.) with Osteoarthritis hip between Feb- Dec 2004. Criteria for inclusion was 1. Early Osteoarthritis.
2. Advanced arthritis awaiting joint replacement. 3. Patients with Osteoarthritis unfit for surgery. All patients were injected with $80 \mathrm{mg}$ methylprednisolone $+7 \mathrm{ml} .0 .5 \%$ Marcaine using Image intensifier. Omnipaque was used to confirm intraarticular positioning of the needle. Assessment of patients was done at 3 weeks preinjection and at 6 weeks post injection. At each of these visits, pain and functional ability were assessed using Harris hip score.

Results: Out of 19 hips injected there were no complications noted in any of the patients. $11(58 \%)$ recorded an increase in hip score after the injection (symptoms improved). $2(10.5 \%)$ had the same score (no change). In $6(31.5 \%)$ the score decreased (worsening of symptoms). The group in which score increased the maximum benefit was in pain relief and probably this led to improved functional ability. It was also noticed that in patients with no change in hip score there was an initial improvement in symptoms for 3 weeks but it faded away by 6 weeks.

Conclusion: This study suggests that intraarticular corticosteroids can be useful tool in patients with Osteoarthritis hip although their role still remains equivocal. Though they have been known to provide pain relief and improve function but the effect is very shortlived.

\section{P109. NARGHILE SMOKING AND BMD}

N. Omess, Y. Yaghi, R. Halabi, F. Honeineh; Hammoud Hospital, University Medical Center, Saida, Lebanon

Objectives: The aim of this study was to determine the influence of smoking "Narghile" on bone mineral density (BMD) in a population of Lebanese women.

Patients and Methods: The study was realized in women in the southern region of Lebanon-Saida Caza. Three hundred and twenty women were enrolled in this study. The BMD of spine and hip were measured by means of DEXA machine (Hologic QDR 4000). None of them received any medication known to affect bone metabolism. 180 of them were current smokers of "Narghile" 1-3/day and 140 of them were non-smokers (table 1).

Results: The patients mean age was 61 years (32-82 years). Our results show a statistically significant decrease of BMD values both in the lumber spine and total hip in the Narghile smoking group against the non smokers, this difference became considerably greater with older age groups (table 2).

Conclusion: Narghile (sheesha-hubble bubble-gouza...) has been the standard smoking for countries in the Middle East and Turkey for over 500 years ago. The smoking experience is rapidly growing in popularity all over the world. Awareness campaigns should include the Narghile among other risk factors that lead to osteoporosis.

Table 1

\begin{tabular}{lcc}
\hline & Narghile smokers & Non-smokers \\
\hline Number of patients & 180 & 140 \\
Age & 61 & 60 \\
BMI & 32.4 & 32.1 \\
\hline
\end{tabular}

Table 2

\begin{tabular}{|c|c|c|c|c|}
\hline \multirow[t]{3}{*}{ Age } & \multicolumn{4}{|l|}{$\mathrm{BMD}(\mathrm{g} / \mathrm{cm} 2)$} \\
\hline & \multicolumn{2}{|l|}{ SMOKER } & \multicolumn{2}{|c|}{ NON-SMOKER } \\
\hline & Spine (L1-L4) & Hip (total) & Spine(L1-L4) & Hip (total) \\
\hline $31-40$ years & 1.178 & 0.878 & 1.166 & 0.950 \\
\hline $41-50$ years & 0.855 & 0.840 & 1.030 & 0.926 \\
\hline $51-60$ years & 0.831 & 0.840 & 0.960 & 0.866 \\
\hline $61-70$ years & 0.785 & 0.770 & 0.878 & 0.801 \\
\hline $71-80$ years & 0.770 & 0.717 & 0.860 & 0.737 \\
\hline
\end{tabular}


P110. LIFE AFTER HIP FRACTURE: REHABILITATION AND SOCIAL OUTCOME

K.H. $\operatorname{Lin}^{1}$, Y.W. Lim ${ }^{1}$, Y.J. Wu ${ }^{2}$, K.S. Lam ${ }^{1}$; ${ }^{1}$ Department of Orthopaedic, Changi General Hospital, Singapore, Singapore, ${ }^{2}$ Clinical Trials and Epidemiology Research Unit, Singapore, Singapore

Aims: To prospectively study the functional and social outcome following proximal hip fractures

Materials and Methods: Prospectively collected data from sixty-eight consecutive patients who had been admitted to a regional hospital from May 2001 to September 2001 were reviewed. Patients were followed prospectively to determine the functional and social status associated with hip fracture over a 2-year follow-up period. Seventeen patients passed away during the follow up period, leaving fifty-one patients for review at end of 2 years follow up. A basic questionnaire and Barthel index of activities of daily living were used to assess the morbidity and social status of the patients.

Results: There is significant changes in total Barthel index over time $(p<0.0001)$. The mean total Barthel score at baseline is significantly higher than at 6 months, 1 year, 2 years $(p<0.0001)$. The mean total Barthel score at 6 month, 1 year and 2 years is significantly higher than in hospital $(p<0.0001)$. There is no significant difference between the mean total Barthel score of 6 months, 1 year and 2 years. The mean total Barthel score of the patient who survived showed a persistent decrease compared with their mean total Barthel score before the hip fracture. After fracture, improvement reached a plateau at 6 months.

At the end of 2 years, only 35.3 percent $(18 / 51)$ of the patients were back to their initial ambulatory status and only 25.5 percent $(13 / 51)$ of the patients felt they could walk equally well when compared to their pre-fracture status. 72.6 percent $(37 / 51)$ of the patients stayed in their own home before the fracture and 51 percent $(26 / 51)$ of patients return to their own home upon discharge. The numbers of patients requiring nursing home care at 2 years $(14 / 51)$ were not significantly higher than the prefracture number $(13 / 51)$. Only one family had to stop working to take care of the patient.

Conclusions: There is an increase in the requirement for social support amongst survivors, but the overall burden on long term institutional care (nursing home) at end of 2 years follow-up or families having to stop working were not significantly changed by proximal hip fracture in our study.

P111. SHORT-TERM RESULTS WITH THE INVERSE DELTA-III PROTH ESIS IN OMARTHROSIS AND MULTIPLE FRAGMENT FRACTURES OF THE PROXIMAL HUMERUS

M. Klein, B. Hinkenjann, B. Scherger, P.A.W. Ostermann; Department of Trauma-, Reconstructive- and Orthopaedic Surgery, St.-Agnes-Hospital Bocholt, Bocholt, Germany

Introduction: How are the clinical and radiological and shortterm results after implanting the inverse Delta-III prothesis in patients with arthritis and multiple fragment fractures of the proximal humerus.

Methods: Between July 1st 2002 and October 30th 200446 Delta-III protheses were implanted in patients with omarthrosis and multiple fragment fractures of the proximal humerus. 44 patients (30f, $14 \mathrm{~m})$ with 46 implanted protheses and a mean age at the time of operation of 73 years (range 53-88) could be followed up after a mean of 13 months (range 6-30). Patients at the time of follow-up were examined and an X-ray was done a.p. and axial. The postoperative functional results were measured by the Constant Score.

Results: A high Constant Score with 80,82 points was evaluated. Compared with the results of other type of shoulder protheses this outcome is high. 6 patients had complications. In one patient a loosening of the prothesisshaft was found. Here an exchange was done. In 3 patients a dislocation happened. In 1 patient the inlay was changed, in another patient the epiphysial component was enlarged to $9 \mathrm{~mm}$ and 1 patient only used a reduction without operation. 3 patients had a soft tissue infect (2 early-, 1 late infection).

Conclusion: Because of the excellent functional results after implanting a Delta-III prothesis we conclude that the indication for implanting this type of prothesis can be enlarged.

P112. SUPERIORITY OF A COMBINED TREATMENT OF ALFACALCIDOL AND ALENDRONATE COMPARED TO THE MONOTHERAPIES IN POSTMENOPAUSAL OSTEOPOROSIS

K. Ones ${ }^{1}$, E. Schacht ${ }^{2} ;{ }^{1}$ Physical Medicine and Rehabilitation, Istanbul Physical Medicine and Rehabilitation Training and Research Hospital, Istanbul, Turkey, ${ }^{2}$ Rheumatology and Rehabilitation, University Clinic Balgrist, Zurich, Switzerland

A prospective, randomized, observer-blind controlled study was performed on 197 postmenopausal osteoporotic women over the course of 24 months.

The objectives of the Study were to compare the efficacy and safety of $0,5 \mu \mathrm{g}$ alfacalcidol plus $10 \mathrm{mg}$ alendronate (Group A), $0,5 \mu \mathrm{g}$ alfacalcidol (Group B), $10 \mathrm{mg}$ alendronate (Group C), and $500 \mathrm{mg}$ calcium as the control group (Group D) once daily per os. All patients in Groups A, B and C received $500 \mathrm{mg}$ of calcium daily in addition to their Study medication. The 4 groups were well matched in terms of mean age, height, weight, time since the onset of menopause, biochemical, calcium in serum and urine and BMD values. 188 patients completed the Study. In all patients BMD was measured at the lumbar spine and the femur neck by DXA (LUNAR DPX) at the beginning and after 12 and 24 months. During the 2-year study we observed a medium percentage significant increase of BMD at the lumbar spine of $6.4 \%$ $(\mathrm{p}<0.01)$ in Group A according to the baseline values and of $1.7 \%$ (NS) in Group B and of 4.8\% (p < 0.01) in Group C, and a decrease of $-1.4 \%$ (NS) in Group D. We also observed a significant medium increase of BMD at the femur neck of $5.0 \%$ $(\mathrm{p}<0.01)$ in Group A vis-à-vis baseline data, of $1.2 \%(\mathrm{NS})$ in Group $\mathrm{B}$, of $3.6 \%(\mathrm{p}<0.05)$ in group $\mathrm{C}$, and a significant decrease of $-6.9 \%(\mathrm{p}<0.05)$ in Group D. There were significant differences $(p<0.05)$ between alfacalcidol and controls for both bone compartments. The differences between the combined therapy were significant in comparison to alfacalcidol and to alendronate therapy at the lumbar spine and the femoral neck $(p<0.05)$. No case of hypercalcuria or hypercalcemia was recorded. Gastrointestinal side effects occurred in 4 patients in Group A, and in 5 patients in Group C, which resulted in the termination of treatment.

In conclusion, the combined treatment of alfacalcidol and alendronate was found to be superior in significantly increasing lumbar and femoral BMD, as compared to the monotherapies.

\section{P113. THE ETHICS OF GENERICS-MEDICAL AND ECONOMIC ADVAN- TAGES OF A GENERIC ALENDRONATE IN TREATING OSTEOPOROSIS PATIENTS}

\section{Halperin; Teva Pharmaceutical Industries, Netanya, Israel}

Since Hippocrates (460-377 B.C.) first formulated the basic ethical precept "First, Do no harm", there have been on going additions to the ethical imperatives. In 2001, the American Medical Association adopted a 9 points Code of Ethics, containing the following imperatives: a physician shall, while caring for a patient, regard responsibility to the patient as paramount. A physician shall support access to medical care for all people. (AMA's House of Delegates June 17, 2001.) Cost containment in the Health System is a high priority for Governments all over the world. In Israel, it is implemented by a strict policy of reimbursement for medications. This puts limitations on the use of Alendronate, which is reimbursed, according to the Government Health Basket, for patients with a T Score of less than 2.5 Standard deviations or an existing fracture. A generic Alendronate has been approved by the Israeli Ministry of Health, based on Bioequivalence Studies. Since 2001, hundreds of thousands of packs have been marketed, with an excellent 
efficacy and safety profile. The cost reduction of the generic product compared to the innovative drug is about $60 \%$. We postulate that a wide adoption of the generic Alendronate by Doctors and patients will lessen the need for cost containment in the Osteoporosis segment and lead to the widening of the reimbursement indications, thus affording access to medical care to all Osteoporosis patients, according to the Ethical imperative of the medical profession.

\section{P114. PYRMONT ASSESSMENT OF COMPLAINTS AND QUALITY OF} LIFE IN PATIENTS WITH SPINAL OSTEOPOROSIS (PACO)

H.W. Minne ${ }^{1}$, C. Hinz ${ }^{1}$, M. Pfeifer ${ }^{1}$, S. Schmitt ${ }^{2}$, D. Lazarescu ${ }^{1}$; ${ }^{1}$ Institute of Clinical Osteology Gustav Pommer and Clinic "DER FÜRSTENHOF", Bad Pyrmont, Germany, ${ }^{2}$ Procter \& Gamble Pharmaceuticals, Weiterstadt, Germany

Objectives: So far, several questionnaires to assess quality of life in patients with osteoporosis are available. These include generic instruments such as the Nottigham Health Profile (NHP) or the Short Form 36 (SF-36) as well as disease specific instruments such as QUALEFFO-41 and OPTOQol. Clinical experience, however, indicate that the degree of disease specificity is not high enough to reveal the whole picture of osteoporosis and to reflect the burden of disease in its complexity and various appearances in different patients.

Materials and Methods: In the first step, 104 questions were selected out of clinical experience of the investigators. In its first and crude version, this new instrument entered a validation phase to test for within-subject reproducibility, internal consistency and construct validity. Finally, 24 items with a significant Odds Ratio $>10$ were identified in a case-control-study comparing patients with back pain due to spinal degenerative disease and patients with back pain due to osteoporotic vertebral fractures. These 24 items were included into the final version of PACO. The items of PACO will now be randomly mixed with QUALEFFO-41 and SF-36 in order to compare reproducibility, consistency, and validity.

Results: Especially, items such as "to carry home shoppings" $(\mathrm{OR}=19.7 ; 95 \%$ C.I.: $5.4-71.4)$, "to move a vacuum cleaner" $(\mathrm{OR}=21.3 ; 95 \%$ C.I.: $2.6-170.6)$, "to drive a car" $(\mathrm{OR}=13.1$; 95\%C.I.: $3.4-51.1)$, "to go to a movie theater" $(\mathrm{OR}=19.8$; $95 \%$ C.I.: $2.5-158.9)$, "to retreat from circle of acquaintances" $(\mathrm{OR}=48.4$; 95\%C.I.: $5.9-293.3)$, or "gardening" $(\mathrm{OR}=11.5$; 95\% C.I.: 2.4 - 54.7) were highly specific for osteoporosis. In a ROC-analysis PACO-24 showed a sensitivity of 0.88 with a specificity of 0.70 .

Conclusion: The newly developed PACO-24 discriminates between cases and controls and differentiates between pain caused by osteoporotic vertebral fractures and pain caused by spinal degenerative disease. Furthermore, PACO-24 demonstrates a good sensitivity and specificity and reveals severe impairments of activities in daily living in patients suffering from postmenopausal osteoporosis resulting in vertebral fractures.

\section{P115. THE ORTHOSES SPINOMED AND SPINOMED ACTIVE IMPROVES POSTURE, TRUNK MUSCLE STRENGTH AND QUALITY OF LIFE IN POSTMENOPAUSAL WOMEN WITH VERTEBRAL FRACTURES: A CON- TROLLED, RANDOMIZED, AND PROSPECTIVE CLINICAL TRIAL}

H.W. Minne, M. Pfeifer; Institute of Clinical Osteology Gustav Pommer and Clinic "DER FÜRSTENHOF", Bad Pyrmont, Germany

Spinal orthoses may play an important role in the treatment of spinal fractures due to osteoporosis. So far, however, clinical trials addressing efficacy according to evidence-based medicine are rare. In a first pivotal study, an improvement in posture, trunk muscle strength, and quality of life after wearing the orthosis Spinomed has been demonstrated (Pfeifer M et al. 2004).

In this study, 110 patients suffering from vertebral fractures and an angle of kyphosis of $60^{\circ}$ and above were recruited in this randomized, prospective clinical trial with the angle of kyphosis being primary endpoint. Secondary endpoints include body height, trunk muscle strength, body sway, pain and limitations of daily living using standardized questionnaires.

Table 1 presents results after six months of treatment (*: $\mathrm{p}<0.01 ; \#: \mathrm{p}<0.05)$ :

\begin{tabular}{lccr}
\hline & $\begin{array}{l}\text { Spinomed active } \\
\text { ("Body"; } \mathrm{N}=48)\end{array}$ & $\begin{array}{l}\text { Spinomed } \\
(\mathrm{N}=31)\end{array}$ & \multicolumn{1}{c}{$\begin{array}{l}\text { Controls } \\
(\mathrm{N}=31)\end{array}$} \\
\hline Age (Years) & $68,5 \pm 10,6$ & $2,8 \pm 7,1$ & $72,3 \pm 6,7$ \\
Vertebral fractures (Number) & $1,5 \pm 2,4$ & $2,0 \pm 2,7$ & $2,1 \pm 2,8$ \\
Angle of kyphosis $\left(\Delta^{\circ}\right)$ & $-6,2 \pm 5,3^{*}$ & $-7,9 \pm 4,9^{*}$ & $-1,6 \pm 5,5$ \\
Body height $(\Delta$ in $\mathrm{mm})$ & $+4,8 \pm 5,7^{*}$ & $+5,3 \pm 6,3^{*}$ & $-0,4 \pm 4,7$ \\
Back ext. strength $(\Delta$ in $\mathrm{N})$ & $+178 \pm 135^{*}$ & $+189 \pm 152^{*}$ & $+7 \pm 55$ \\
Abd. flex. strength $(\Delta$ in $\mathrm{N})$ & $+131 \pm 117^{*}$ & $+94 \pm 71^{*}$ & $+23 \pm 46$ \\
Body sway $(\Delta$ in mm) & $-16,2 \pm 31,2^{*}$ & $-20,4 \pm 40,2^{*}$ & $-1,7 \pm 35,6$ \\
Vital capacity $(\Delta$ in \%) & $+5,6 \pm 18,9 \#$ & $+6,1 \pm 20,5 \#$ & $-9,9 \pm 16,1$ \\
Pain $(\Delta$ Score-Points) & $-1,3 \pm 1,0^{*}$ & $-1,5 \pm 1,2^{*}$ & $+0,1 \pm 0,9$ \\
ADL $(\Delta$ Score-Points) & $-1,4 \pm 1,5^{*}$ & $-2,1 \pm 1,6^{*}$ & $+0,2 \pm 0,8$ \\
\hline
\end{tabular}

Both orthoses Spinomed and Spinomed active led to an improvement in posture, trunk muscle strength, and quality of life in patients suffering from osteoporotic vertebral fractures. Especially Spinomed active, which is completely invisible below normal clothes is characterized by a very high compliance and acceptance among patients and thus comes very close to an ideal orthosis for the treatment of osteoporosis.

P116. EFFECTS OF VITAMIN D AND CALCIUM SUPPLEMENTATION ON FALLS AND PARAMETERS OF MUSCLE FUNCTION - A PROSPECTIVE, RANDOMIZED, DOUBLE-BLIND MULTI-CENTER STUDY

H.W. Minne ${ }^{1}$, H. Dobnig ${ }^{2}$, M. Pfeifer ${ }^{1}$, K. Suppan ${ }^{2},{ }^{1}$ Institute of Clinical Osteology "Gustav Pommer", Bad Pyrmont, Germany, ${ }^{2}$ Dept.of Endocrinology, University of Graz, Austria

The effects of calcium and vitamin D supplements on falls, falls-related fractures and functional measures are well-known (Pfeifer et al. 2000, Bischoff et al. 2003, Dukas et al. 2004, Larsen et al. 2004). This prospective study was undertaken to test the influence of latitude, seasonal variations, possible threshold effects and duration of vitamin D efficacy after cessation of therapy. 242 healthy male and female subjects over 70 years of age and a 25 $\mathrm{OH}-\mathrm{D} 3$ serum level below $78 \mathrm{nmol} / 1$ were recruited in Bad Pyrmont $\left(52^{\circ} \mathrm{N}\right)$ and $\operatorname{Graz}\left(48^{\circ} \mathrm{N}\right)$ and were randomly assigned to two treatment groups: one receiving $1000 \mathrm{mg} \mathrm{Calcium} /$ day $(\mathrm{Ca})$ and the other $1000 \mathrm{mg}$ Calcium and 800 I.U. Vitamin D (Ca + D) over 12 months. This double blind intervention phase was followed by a treatment free observation phase over another 8 months. Falls as the primary efficacy parameter were recorded by diaries and phone calls every two months. Parameters of muscle function such as quadriceps strength, body sway and "timed up and go test" were measured every four months. Statistical evaluation was carried out using the statistics software of IDV, Gauting (Test + Estimation, Version 5.2, "CRO" Dr. Heinz, Vienna, Austria). Baseline parameters did not differ between groups.

Despite a relatively high inclusion criterion for vitamin D (78 $\mathrm{nmol} / \mathrm{l}$ ) and independent of latitude, we observed a significant reduction of falls and parameters of muscle function after supplementation with vitamin D and calcium (MaxiKalzD3). So far, we did not find any evidence for a threshold phenomenon or an effect of latitude.

Table 1 shows intention-to-treat analysis after 12 months of treatment and 8 months of follow-up:

\begin{tabular}{llcll}
\hline & (Ca) & (Ca + D) & \\
\hline Mean number of falls per group & 0.69 & 0.45 & $\mathrm{P}<0.01$ \\
Number of falls per subject & 1.61 & 0.95 & $\mathrm{p}<0.01$ \\
Body sway frontal (mm) & $12.9 \pm 9.1$ & $9.2 \pm 7.9$ & $\mathrm{p}<0.01$ \\
Quadriceps strength left leg (Newton) & $175 \pm 65$ & $210 \pm 65$ & $\mathrm{p}<0.01$ \\
"Timed up and go Test" (Seconds) & $8.1 \pm 3.4$ & $7.3 \pm 3.8$ & $\mathrm{p}<0.01$ \\
25-OH-Vitamin D after follow-up (nmol/l) & $38 \pm 13$ & $48 \pm 16$ & $\mathrm{p}<0.01$ \\
\hline
\end{tabular}


P117. VITAMIN D STATUS IS NOT ASSOCIATED WITH BONE MINERAL DENSITY, BONE TURNOVER, BONE LOSS AND FRACTURE RISK IN POSTMENOPAUSAL WOMEN. THE OFELY STUDY

P. Garnero, F. Munoz, E. Sornay-Rendu, P.D. Delmas; INSERM unit 403, Lyon, France

Vitamin D status is considered as an important determinant of bone health. This belief is mainly derived from cross-sectional studies. Supplementation trials with vitamin D3 using fracture incidence as an outcome have yielded conflicting results.

Aim: To investigate the relationships between serum levels of $25 \mathrm{OHD}$ and intact PTH, bone turnover, BMD and incidence of fracture in postmenopausal women.

Methods: In the 671 postmenopausal women (mean age: 62.2 yr; range: 50-89 yr) belonging to the population-based OFELY cohort, we measured at baseline serum $25 \mathrm{OH}$ D, intact PTH, bone turnover markers (BTM) and BMD by DXA at the total hip and radius. Women were followed prospectively for a median (IQ) of $11.2 \mathrm{yr}(11-12.3)$. BMD at the radius was repeated annually and all incident fragility fractures, which occurred in 134 women were confirmed by radiographs.

Results: At baseline, serum $25 \mathrm{OH}$ D correlated modestly with intact PTH with no suggestion of a plateau effect $\left(r^{2}=0.023\right.$, $\mathrm{p}<0.0001)$, but not with BTM or BMD after adjustment for age. When serum levels of 25 OHD or PTH were considered as continuous variables or in quartiles, there was no significant association between the levels of these two hormones and radius BMD loss or fracture risk. For example, the relative risk $(95 \% \mathrm{CI})$ of fracture for women with levels in the lowest quartile of $25 \mathrm{OHD}$ was $1.11(0.64-2.03)$ compared to women with levels in the highest quartile. We also separated women using a cut-off of 75 $\mathrm{nmol} / \mathrm{L}$ of serum $25 \mathrm{OH} \mathrm{D}$, which has recently been recommended by a panel of experts as the optimal threshold for fracture prevention (Dawson-Hughes et al; OI, 2005). After adjustment for age, there was no significant difference between the 254 women $(38 \%)$ with $250 \mathrm{HD}$ below $75 \mathrm{nmol} / 1$ and the other women for grip strength, physical activity, $\%$ of fallers in the previous year, serum PTH, BTM, BMD, bone loss and the $\%$ of women with incident fracture $(23 \%$ vs $18 \%, \mathrm{p}=0.80)$. Further adjustment for seasons did not modify these associations.

Conclusion: Vitamin D status may not be an important determinant of bone health in a healthy population of postmenopausal women.

\section{P118. EVALUATION OF BONE MINERAL DENSITY IN TERMS OF VEIL- ING, SOCIOECONOMICAL STATUS AND EDUCATIONAL LEVEL IN TURKISH WOMEN OVER 40 YEARS. VEILING MAY BE A RISK FACTOR FOR OSTEOPOROSIS IN MUSLIM POPULATIONS}

M. Bahceci ${ }^{1}$, M. Ertem ${ }^{2}$, G. Saka ${ }^{2}$, D. Gokalp ${ }^{1}$, Z. Karacomak ${ }^{2}$, N. Akdeniz ${ }^{3}$; ${ }^{1}$ Departments of Endocrinology, Dicle University School of Medicine, Diyarbakir, Turkey, ${ }^{2}$ Public Health, Dicle University School of Medicine, Diyarbakir, Turkey, ${ }^{3}$ Gynecology, Dicle University School of Medicine, Diyarbakir, Turkey

Background and aims: Low BMI, low dietary calcium intake, a short fertile period, and a short period of education are associated with increased risk of hip fracture in Turkish population. Headscarf is a kind of headcover, worn for religious intentions. Lots of veiled women start to wear a headscarf with the onset of puberty in Islamic Peoples. We aimed to evaluate effect of various factors such as parity, menopause period, veiling, education level and antropometrical parameters on BMD in women over 40 years.

Subjects and methods: Four hundred thirty nine women living in different region (suburb and house provided to workers) of city center of Diyarbakir-Turkey, aged over 40 years (with mean age $48,9+11,3$ years) were included to study. The predicted factors influencing BMD was investigated by using a questionnaire. Body weights and heights were measured without shoes and in light clothing, BMI was expressed as weight (kilograms) per height (meters) squared. Body fat percent and fat mass were determined by bioelectric impedance. Bone Mineral Density (BMD) was determined with MetriScan 500-1210-00. Mean BMD of women were analyzed by using $t$ test and ANOVA. PostHoc Turkey analysis was used. Informed and written consent was obtained from all workers and control subjects.

Results: Living in suburban region $(p=0.000)$, family history of osteoporosis $(p<0.002)$, low education level $(p=0.000)$, insufficient calcium intake $(\mathrm{p}<0.001)$, parity over $4(\mathrm{p}=0.000)$, low body height $(\mathrm{p}=0.000)$ and body weight $(\mathrm{p}<0.002)$ and long menopausal period $(\mathrm{p}=0.000)$ were the factors with negative effects on BMD. Additional to those well-known factors veiling was also found to be an important risk factor for low BMD $(p=0.000)$. All results related to osteoporosis were shown in Table.

Conclusions: In addition to well-known factors such as parity, insufficient nutrition, and long menopausal period, veiling may also be an important risk factor for low BMD and therefore, osteoporosis in especially Muslim populations.

\begin{tabular}{|c|c|c|c|}
\hline Parameters & & $\mathrm{BMD} \pm \mathrm{SD}$ & $\mathrm{p}$ value \\
\hline \multirow[t]{2}{*}{ Home type } & $\begin{array}{l}\text { House provided } \\
\text { to workers }\end{array}$ & $56,5 \pm 6,9$ & 0.000 \\
\hline & Squatter's house & $53,1 \pm 8,0$ & \\
\hline \multirow[t]{2}{*}{ Wearing } & Non-veiling & $58,1 \pm 5,8$ & 0,000 \\
\hline & Veiling & $52,6 \pm 8,0$ & \\
\hline \multirow[t]{2}{*}{ BMI $\left(\mathrm{kg} / \mathrm{m}^{2}\right)$} & $<25$ & $54,0 \pm 8,0$ & 0,95 \\
\hline & $\geq 25$ & $54,5 \pm 7,8$ & \\
\hline \multirow[t]{2}{*}{ Smoking } & - & $54,0 \pm 7,9$ & 0,012 \\
\hline & + & $56,4 \pm 7,0$ & \\
\hline \multirow[t]{2}{*}{ Family hist. osteoporosis } & + & $55,9 \pm 7,1$ & 0,002 \\
\hline & - & $53,5 \pm 8,0$ & \\
\hline \multirow[t]{2}{*}{ Sunning } & Frequently & $53,7 \pm 8,7$ & 0,23 \\
\hline & Sometime & $54,8 \pm 7,6$ & \\
\hline \multirow[t]{3}{*}{ Educationl level } & $\begin{array}{l}\text { High School and } \\
\text { above }\end{array}$ & $58,1 \pm 5,7$ & 0,000 \\
\hline & Primary School & $57,8 \pm 6,9$ & 0,000 \\
\hline & Illiterate & $51,7 \pm 7,7$ & 0,000 \\
\hline \multirow[t]{2}{*}{ Nutritional status } & Sufficient & $54,9 \pm 7,6$ & 0.001 \\
\hline & Insufficient & $52,1 \pm 8,4$ & \\
\hline \multirow[t]{2}{*}{ Parity number } & $0-3$ & $58,2 \pm 6,3$ & 0,000 \\
\hline & $4+$ & $53,0 \pm 7,8$ & \\
\hline \multirow[t]{2}{*}{ Body height $(\mathrm{cm})$} & $<150$ & $49,7 \pm 9,6$ & 0,000 \\
\hline & $>151$ & $55,4 \pm 7,0$ & \\
\hline \multirow[t]{2}{*}{ Body Weight (kg) } & $>51$ & $54,8 \pm 7,6$ & 0,002 \\
\hline & $<50$ & $50,9 \pm 8,3$ & \\
\hline \multirow[t]{2}{*}{ Menopause duration (year) } & $0-3$ & $57,4 \pm 6,4$ & 0,000 \\
\hline & $4+$ & $49,5 \pm 7,4$ & \\
\hline
\end{tabular}

P119. EARLY AND LATE RESPONSE OF BMD AT LUMBAR SPINE AND TOTAL HIP TO TERIPARATIDE IN POSTMENOPAUSAL OSTEOPOROSIS

B. Höne ${ }^{1}$, H.-J. Heberling ${ }^{1}$, C. Mehnert-Solzer ${ }^{2}$, T. Nickelsen ${ }^{2}$; ${ }^{1}$ Medizinische Klinik West, Leipzig, Germany, ${ }^{2}$ Lilly Deutschland $\mathrm{GmbH}$, Bad Homburg, Germany

The aim of our analysis was to evaluate the early (6 month) and late response $(12,18,24$ months) of teriparatide treatment in postmenopausal women pretreated with antiresorptive agents at lumbar spine and total hip BMD.

Patients enrolled were mostly participants in EUROFORSStudy $(\mathrm{n}=12)$. A small group were from our outpatient department $(n=3)$.

All patients received treatment with $20 \mu \mathrm{g}$ teriparatide s.c./day and a supplementation with Calcium $(500 \mathrm{mg})$ and Vitamin D (400 E). All patients included had inadequate outcome to longlasting prior antiresorptive therapy (new fractures and or further diminishing of lumbar and/or total hip BMD).

Regular measurements of BMD were performed $(6,12,18$ and 24 months) using a DEXA-machine (Lunar-Prodigy). A steady increase at the lumbar spine could be demonstrated up to 24 months of treatment. The increase of total hip BMD was delayed but could be registered after 24 months teriparatide.

These findings give evidence that treatment with teriparatide is effective even in antiresorptive pretreated patients, at least in this small group. Relevant adverse effects were not observed. No patients withdrew from the above mentioned therapy up to the official 
planned ending. Under treatment, back pain diminished. 15 patients were treated for 12 months, and 10 patients for 24 months.

\section{P120. SELECTIVE ESTROGEN RECEPTOR MODULATOR CIMICIFUGA RACIMOSA IN TREATMENT OF OSTEOPOROSIS IN POSTMENOPAUSAL CHRONIC RENAL DISEASE WOMEN}

L.P. Martynyuk, M.I. Shved, S.M. Butvyn; Ternopol State Medical University, Department of Inner Medicine, Ternopil, Ukraine

Objective: The aim of this study was to evaluate the effects of a combined low-dose therapy of alphacalcidol, calcium carbonate and phytoselective estrogen receptor modulator (phytoSERM), particularly, Cimicifuga racimosa extract BNO 1055 in postmenopausal chronic renal disease women.

Material and methods: The study included 13 patients in the SERM group and 12 in the control group. All 25 patients were diagnosed I-II stages of CRD (glomelular filtration rate 75,4 +2,3 $\mathrm{ml} / \mathrm{min}$ ). Bone mineral density (BMD) was measured at 2 siteslumbar spine (L1-L4) and femoral neck using dual energy X ray absorptiometry at baseline and after 12 months of treatment. After a written informed consent, patients were randomized into two groups: is first group (13 women) were administered $1000 \mathrm{mg}$ of calcium, alfacalcidol at a daily dose of 0,25 microg and Cimicifuga racimosa extract 1 casp twice a day. In second group (12 women) calcium carbonate at daily dose 1,0 gram of elemental calcium was administered. Serum concentration of calcium, phosphorus and alkaline phosphatase (AP) were studied before starting treatment and after 12 months.

Results: In phytoSERM's group the mean serum calcium, increased significantly and alkaline phosphatase (AP) declined significantly after 12 months of treatment. In the control group the levels of calcium, phosphorus and AP were not significantly different at baseline and after 12 months. In the control group, the mean BMD at lumbar spine decreased in $-4,41 \pm 0,70 \%$ from at baseline. In comparison in phytoSERM's group it increased in $1,98 \pm 0,82 \%$ from baseline $(\mathrm{p}<0,01)$. No patients had side effects of the therapy.

Conclusions: The study supports the idea that phytoselective estrogen receptor modulator Cimicifuga racimosa extract and alphacalcidol treatment can be used safely and is recommended for prevention of postmenopausal osteoporosis in CRD women. The possible long-term effects phytoSERM's remain to be determined.

P121 COMPARISON OF THE TREATMENT EFFECT OF OSSEINHYDROXYAPATITE COMPOUND AND CALCIUM CARBONATE WITH VITAMIN D3 ON BONE TISSUE HISTOMORPHOMETRIC PARAMETERS IN THE OVARIECTOMIZED (OVX) RATS

L.B. Nugmanova, A.Y. Kandilyotu, K.Y. Agababyan, V.M.Vorojeikin; Institute of Endocrinology, Uzbekistan Public Health Ministry, Tashkent, Uzbekistan

Aim: to study effect of ossein-hydroxyapatite compound on the ovariectomized rat bone tissue and to compare the effect with calcium carbonate with vitamin D3. 3-month old white rats-virgins with body mass of $120-150 \mathrm{~g}$ were divided into 5 groups. The 1st control group $(n=10)$-intact animals, the 2 nd one - sham OVX rats $(n=10)$, the 3 rd one - OVX untreated rats $(n=20), 4$ th - 10 OVX rats receiving ossein-hydroxyapatite compound (per os $32 \mathrm{mg}$ ) and 5th $-10 \mathrm{OVX}$ rats receiving calcium carbonate with vitamin D3 (per os 3,5mg and 0,85 IU per day respectively). The 3 rd group was subdivided into two subgroups, 10 rats decapitated a month after castration and 10 rats- two months after castration, respectively. Thighbones were prepared; their proximal metaphyses underwent histomorphometry. Histomorphometry of sham OVX rats was similar to the one in the control structure. The $3 \mathrm{rd}$ group rat bone histomorphometry "a month after castration" showed marked signs of osteoporosis. Thighbone mass reduction in female rats by $24 \%$ is the leading sign. The process is caused by both cortical layer and trabeculae thickness reduction. In the 3rd group rat bones " 2 months after castration" revealed reduction in mass of non-calcified thighbone by $32 \%$ of the initial one, decrease of cortical layer area by $30 \%$, the highest $(35 \%)$ reduction being observed in trabecular area. The trabeculae are thin and "trabecular nodes", their connection zones, are practically absent. Histomorphometry of the 4th group rat bones showed: the bone resorption intensity decrease. Though thighbone mass is $6,1 \%$ lower than the one in the condition "a month after castration", it is $4,8 \%$ higher than that in the condition "two months after castration". The increase of cortical layer area by $2,8 \%$ in the average and the one of trabecular apparatus by $5,7 \%$, are the evidence for the fact. In the 5th group rats: highbone mass reduction by $6,8 \%$ as compared with the one under condition "a month after castration" and its increase by $4,1 \%$ as compared with the one under condition "two months after castration". Cortical layer area increased by $2,2 \%$ in the average, the one of trabecular apparatus by $4.8 \%$.

These results demonstrate that both the treatment with ossein-hydroxyapatite compound and the treatment with calcium carbonate with vitamin D3 stimulated osteogenesis. However, effect of ossein-hydroxyapatite compound was higher, probably, because it consists insulin-like growth factors I, II and osteocalcin.

\section{P122. HEALTH-RELATED QUALITY OF LIFE (HRQOL) ASSESSMENT IN WOMEN WITH ESTABLISHED OSTEOPOROSIS TREATED WITH TERI- PARATIDE (PTH 1-34)}

A. Anastasilakis ${ }^{1}$, G.D. Giaglis ${ }^{1}$, Z. Efstathiadou ${ }^{1}$, M. Kita ${ }^{1}$, G. Koukoulis $^{2}$, A. Abramidis ${ }^{1}$; ${ }^{1}$ Department of Endocrinology, Hippocration General Hospital, Thessaloniki, Greece, ${ }^{2}$ Department of Endocrinology, Clinic of Internal Medicine, Medical School of Thessalia, Larisa, Greece

Objective: Osteoporosis may result in pain, decreased physical function, immobility, social isolation and depression, especially when complicated by fracture. All these factors together may diminish health-related quality of life (HRQoL). Teriparatide (PTH 1-34) is the only anabolic agent available for treatment of osteoporotic patients. The aim of this study was to evaluate the effects of teriparatide on the HRQoL of women with established osteoporosis.

Material and Methods: 10 women with established osteoporosis (age $68.7 \pm 6.9$ years - menopause age $45.4 \pm 4.2$ years) participated in this prospective study. HRQoL was measured usin SF-36, a generic HRQoL questionnaire, which provides two major scales: the physical component summary (PCS) and the mental component summary (MCS), and 8 subscales. The questionnaire was completed by the patients before and six months after teriparatide initiation, and was scored with the American norm-based scoring algorithm.

Results: Patients' scores initially were below national average, thus showing the burden of the disease. After teriparatide initiation, both PCS and MCS revealed a slight but not statistically significant improvement $(44.4 \pm 11.1$ vs $45.8 \pm 9.5, p=N S$ and $37.4 \pm 13.3$ vs $39.5 \pm 8.4, p=N S$ accordingly). Assessment of the 8 subscales showed a similar trend but not statistical significance: Physical Functioning (PF) $41.9 \pm 10.1$ vs $42.9 \pm 9.6$, Role Physical (RP) $43.2 \pm 13.3$ vs $44.9 \pm 12.3$, Bodily Pain (BP) 41.5 \pm 17.5 vs $42.4 \pm 11.3$, General Health $(\mathrm{GH}) 38.4 \pm 11.8$ vs 43.3 \pm 7.8 , Vitality (VT) $44.8 \pm 11.3$ vs $45.8 \pm 6.0$, Social Functioning (SF) $42.4 \pm 15.8$ vs $41.8 \pm 9.2$, Role Emotional (RE) 41.1 \pm 15.2 vs $41.2 \pm 11.4$, Mental Health $(\mathrm{MH}) 31.1 \pm 12.9$ vs 36.5 \pm 5.5 .

Conclusions: Our findings in this preliminary study with a small number of patients suggest that teriparatide treatment causes a slight but not statistically significant improvement in both physical and mental component of health related quality of life. As expected, even for a potentially effective treatment a larger time interval is needed, before patients experience a significant improvement of their quality of life. 
P123. COSTING FRACTURES FOR USE IN HEALTH ECONOMICS MODELLING

F. Borgström ${ }^{1,2}$, O. Johnell ${ }^{3}$, B. Jönsson ${ }^{4}$, J.A. Kanis ${ }^{5} ;{ }^{1}$ Medical Management Centre, Karolinska Institutet, Stockholm, Sweden, ${ }^{2}$ Stockholm Health Economics, Stockholm, Sweden, ${ }^{3}$ Department of Orthopaedics, Malmö General Hospital, Malmö, Sweden, ${ }^{4}$ Stockholm School of Economics, Stockholm, Sweden, ${ }^{5}$ Centre for Metabolic Bone Diseases (WHO Collaborating Centre), University of Sheffield Medical School, Sheffield, UK

Background: Intervention thresholds for osteoporosis can be defined as the 10-year absolute hip fracture risk at which an intervention becomes acceptable. To account for the total burden of osteoporosis an index of the excess morbidity of all fractures compared to hip fracture (a.k.a. hip fracture equivalents) have been calculated. One assumption underlying these calculations is that the fracture costs are proportional to fracture disutility.

Objective: Investigate how accurately the cost-effectiveness is estimated when using hip fracture equivalents compared to a model including all fractures explicitly and to examine whether a down adjustment of fracture costs lead to a better fit.

Methods: A Markov cohort model as built to be able to estimate the cost per QALY gained based on fracture specific health states explicitly and by the use of hip fracture equivalents and thereby implicitly taking all fractures into account. All fracture types that were used in the calculation of excess morbidity of hip fracture were included. The model was populated with Swedish data. The cost-effectiveness was estimated for a 5-year long intervention that was assumed to reduce the fracture risk by $35 \%$.

Results: The cost per QALY gained was estimated for women at population risk of fracture with increasing starting age of intervention. On average, the cost-effectiveness ratio was $\$ 16180$ lower when using hip fracture equivalents compared to all fractures explicitly. The incremental QALY was found to be very similar between the two approaches (average difference of 0.002 units or $6 \%$ ) but the incremental cost differed somewhat more (on average $\$ 279$ or $30 \%$ ). When down adjusting the fracture related costs by the excess fracture cost of all osteoporotic fractures compared to hip fracture the average difference in cost per QALY gained decreased to $\$ 2629$.

Conclusions: The results shows that the estimated QALYs using hip fracture equivalents compare well with an all fracture model but less so in terms of fracture costs. This might indicate that the assumption of proportionality between costs and utility loss needs some modification. When down adjusting the fracture costs a better congruence was achieved between the hip fracture equivalent and the all fracture approach.

\section{P124. THE POTENTIAL COST-EFFECTIVENESS OF COMPLIANT PA- TIENTS TREATED WITH STRONTIUM RANELATE. AN ANALYSIS BASED ON THE PPS-POPULATION}

F. Borgström ${ }^{1,2}$, O. Ström ${ }^{1}$, B. Jönsson ${ }^{3}$; ${ }^{1}$ Stockholm Health Economics, Stockholm, Sweden, ${ }^{2}$ Medical Management Centre, Karolinska Institutet, Stockholm, Sweden, ${ }^{3}$ Stockholm School of Economics, Stockholm, Sweden

Objectives: Strontium ranelate, a new therapy for the treatment of osteoporosis, has been shown to reduce the risk of vertebral, non vertebral and hip fractures in post-menopausal women in two phase III clinical trials: the Spinal Osteoporosis Therapeutic Intervention study (SOTI) and the TReatment Of Peripheral OSteoporosis study (TROPOS). By measuring blood strontium levels among patients in the TROPOS study it was possible to derive a sample of patients with adequate strontiemia during the first 18 months of the study period the study period. The objective of this study was to estimate the cost-effectiveness of this sub sample of patients called the Per Protocol Set (PPS) population among women in a Swedish setting.

Material and Methods: The cost-effectiveness, from a societal perspective, of strontium ranelate compared to no treatment was estimated in a Markov cohort model populated with Swedish specific cost, utility and epidemiological data. In the base case, the cost-effectiveness was estimated for patient groups similar in characteristics to the patients in the PPS population, i.e. 77- year old women with low BMD (mean T-score -3.1). In line with results in the clinical trial it was assumed that treatment reduced the risk of any osteoporotic fractures fractures. The intervention was set to three years. The cost effectiveness was estimated without and with costs in added life years.

Results: Intervention with strontium ranelate compared to no treatment based on the PPS population in Sweden was found to be cost saving when excluding cost in added life years and at $€ 484$ per QALY gained when they were included.

Conclusion: The results indicate that, based on the PPS population, strontium ranelate is highly cost-effective in a Swedish setting. The results based on the PPS-population could be interpreted as the cost-effectiveness for a good compliant patient group.

\section{P125. MEDIAL TIBIOFEMORAL MINIMUM JOINT SPACE WIDTH MEA- SUREMENTS CORRELATE WITH PAIN AND FUNCTION IN INDIVIDUALS WITH KNEE OA}

K. Beattie ${ }^{1}$, P. Boulos ${ }^{1,2}$, J. Duryea ${ }^{3}$, C. Webber ${ }^{4}$, J.D. Adachi ${ }^{2}$; ${ }^{1}$ McMaster University, Hamilton, Canada, ${ }^{2}$ St. Joseph's Healthcare, Hamilton, Canada, ${ }^{3}$ Dept. of Radiology, Brigham and Womens Hospital, Boston, United States, ${ }^{4}$ Dept. of Nuclear Medicine, Hamilton Health Sciences, Hamilton, Canada

Objective: To investigate the relationship between medial tibiofemoral minimum joint space width (mJSW) in the knee and WOMAC and SF-36 scores in those with OA.

Methods: Females and males $\geq 35$ years of age who had been clinically diagnosed with knee OA participated. All volunteers completed the WOMAC and SF-36 questionnaires and underwent a single, fixed-flexion radiograph of their most severely affected knee. Radiographs were graded according to the KellgrenLawrence (K-L) scale. Films were digitized and analyzed for medial mJSW using an automated computer algorithm.

Results: Of 41 participants, 26 were female and 15 were male. The mean (SD) age, height, weight and BMI for the females were $61.1(10.9)$ years, $160.5(7.4) \mathrm{cm}, 73.3(13.0) \mathrm{kg}$ and $28.6(5.6) \mathrm{kg}$ $\mathrm{m}^{2}$, respectively and, for the male group, were 63.1 (9.7) years, $178.9(7.7) \mathrm{cm}, 88.8(14.5) \mathrm{kg}$ and $27.8(3.7) \mathrm{kg} / \mathrm{m}^{2}$, respectively. The distribution of K-L scores for the females was grade $0: 2$, grade $1: 8$, grade $2: 6$, grade $3: 5$, grade $4: 5$, and for the males was grade $0: 1$, grade 1: 2, grade $2: 3$, grade $3: 3$, grade $4: 6$. All participants completed WOMAC and SF-36 questionnaires with scores for each subcategory presented in the table. Correlation analyses were conducted to determine if WOMAC and SF-36 subcategories were significantly related to mJSW in females and males. Pearson correlation coefficients (CC) and their 95\% confidence intervals $(\mathrm{CI})$ are presented in the table.

Relationships between mJSW and pain, stiffness and function (WOMAC questionnaire)

$\mathrm{ns}=$ not significant, $\mathrm{N} / \mathrm{A}=$ not applicable

\begin{tabular}{|c|c|c|c|c|c|c|}
\hline & \multicolumn{3}{|l|}{ Females } & \multicolumn{3}{|l|}{ Males } \\
\hline & $\begin{array}{l}\text { Mean } \\
\text { (SD) } \\
\text { Score }\end{array}$ & $\mathrm{CC}(\mathrm{r})$ & $\begin{array}{l}95 \% \mathrm{CI} \\
\text { of } \mathrm{r}\end{array}$ & $\begin{array}{l}\text { Mean } \\
(\mathrm{SD}) \\
\text { Score }\end{array}$ & $\mathrm{CC}(\mathrm{r})$ & $\begin{array}{l}95 \% \mathrm{CI} \\
\text { of } \mathrm{r}\end{array}$ \\
\hline $\begin{array}{l}\text { WOMAC } \\
\text { Pain }\end{array}$ & $7.2(4.9)$ & -0.500 & $\begin{array}{l}-0.743 \\
-0.14\end{array}$ & $5.3(4.1)$ & $-0.474 \mathrm{~ns}$ & $\mathrm{~N} / \mathrm{A}$ \\
\hline $\begin{array}{l}\text { WOMAC } \\
\text { Stiffness }\end{array}$ & $3.7(1.9)$ & $-0.377 \mathrm{~ns}$ & $\mathrm{~N} / \mathrm{A}$ & $3.1(2.3)$ & $-0.411 \mathrm{~ns}$ & $\mathrm{~N} / \mathrm{A}$ \\
\hline $\begin{array}{l}\text { WOMAC } \\
\text { Function }\end{array}$ & $25.5(16.3)$ & -0.530 & $\begin{array}{l}-0.761 \\
-0.180\end{array}$ & $20.5(12.2)$ & -0.705 & $\begin{array}{l}-0.894 \\
-0.302\end{array}$ \\
\hline $\begin{array}{l}\text { Total } \\
\text { WOMAC }\end{array}$ & $28.7(25.1)$ & -0.528 & $\begin{array}{l}-0.759 \\
-0.177\end{array}$ & $28.8(17.3)$ & -0.662 & $\begin{array}{l}-0.876 \\
-0.227\end{array}$ \\
\hline $\begin{array}{l}\text { Physical } \\
\text { Function } \\
\text { (SF-36) }\end{array}$ & $36.4(22.7)$ & 0.591 & $\begin{array}{l}0.265 \\
0.796\end{array}$ & $66.7(50.0)$ & 0.629 & $\begin{array}{l}0.173 \\
0.863\end{array}$ \\
\hline
\end{tabular}


Conclusions: Radiographs acquired in the fixed flexion position and analyzed for mJSW using an automated algorithm demonstrated significant correlations with WOMAC and SF-36 outcomes. Previously published cross-sectional studies have shown no such correlations. Larger longitudinal studies should be performed to further investigate these relationships.

\section{P126. TERIPARATIDE PERFORMANCE IN OSTEOPOROSIS OF WOMAN}

A. Bazarra-Fernandez, M.A. Bazarra-Castro; Department of ObGyn Juan Canalejo, University Hospital Medicine, Santiago University, La Coruna, Spain

Background: Elderly women with osteoporosis can significantly and safely improve their bone mass with a combination therapy of hormone replacement and bisphosphonates. After three years at the lumbar spine the mean increases in BMD were 10.4 percent with combination of therapy of hormone replacement plus alendronate. Bisphosphonates can yeild side effects in the long term and conjugated estrogen with or without medroxyprogesterone are not beta-estradiol and progesterone. So its effects and risks can not be extrapolated like it was done.

Aim: Looking into new possibilities as treatment to osteoporosis.

Material and method: 16 women who were 45 to 80 years old have been recruited. They were assigned to two groups according age, 45-65, 66-80. Bone density was determined by DEXA. They were given subcutaneous teriparatide injection of $20 \mathrm{microg} /$ day for three months. Fasting blood and urine samples were collected at baseline, at three and six months. Biochemistry: Serum calcium, PTH, 25-hydroxyvitamin D, osteocalcin, alkaline phosphatase, bone-specific alkaline phosphatase. 24-hour urine collection: PYD, calcium without and with calcium intake. Calcium supplement was $1500 \mathrm{mg}$ per day with $0.266 \mathrm{mg}$ vitamin D twice a week.

Results: In 45-65, 66-80 group the mean BMD was T-score 1.8 and -3.20 . At base line fasting blood and urine sample mean: Serum calcium $9.1 ; 8.9 \mathrm{mg} / \mathrm{dl}, 25$-hydroxyvitamin D $16 ; 12 \mathrm{ng} / \mathrm{ml}$, alkaline phosphatase 173; $162 \mathrm{U} / \mathrm{L}$ bone-specific alkaline phosphatase $26.3 \% ; 23 \%$. PID 9;15 nM PYD/mM creatinine, urinary calcium 64; 240. At three months: Serum calcium 9.9; $9.3 \mathrm{mg} / \mathrm{dl}$, 25-hydroxyvitamin D 68;88 ng/ml, alkaline phosphatase $193 ; 180$ $\mathrm{U} / \mathrm{L}$ bone-specific alkaline phosphatase $45.3 \% ; 32 \%$. PID $4.8 ; 8.7$ $\mathrm{nM}$ PYD/mM creatinine, urinary calcium $189 ; 309$. At six months with seven and six cases the results are similar in relation to both groups.

Conclusions: Increasing of bone formation is given but is different in both groups. The bone development is not continuous but in cycle basis because of we think one cycle basis approach is needed to be found with teriparatide and inhibitors of osteoclastmediated bone resorption of short action without deposit for long in bones. So, proposal of study is made with estradiol and progesterone plus teriparatide.

\section{P127. OBSERVER PERFORMANCE IN IDENTIFYING POSTMENOPAUSAL WOMEN WITH OSTEOPOROSIS BY DENTAL PANORAMIC RADIO- GRAPHS: RESULTS FROM THE OSTEOPOROSIS SCREENING PROJECT IN DENTISTRY (OSPD)}

A. Taguchi ${ }^{1}$, A. Asano ${ }^{2}$, M. Ohtsuka ${ }^{3}$, T. Nakamoto $^{3}$, Y. Suei ${ }^{1}$, M. Tsuda ${ }^{4}$, Y. Kudo ${ }^{4}, \mathrm{~K}$. Inagaki ${ }^{5}, \mathrm{~T}$. Noguchi ${ }^{5}, \mathrm{~K}$. Tanimoto ${ }^{3}$, E. Klemetti ${ }^{6}$, S.C. White ${ }^{7}$, K. Horner ${ }^{8}$; ${ }^{1}$ Department of Oral and Maxillofacial Radiology, Hiroshima University Hospital, Hiroshima, Japan, ${ }^{2}$ Division of Mathematical and Information Sciences, Faculty of Integrated Arts and Sciences, Hiroshima University, Higashi-Hiroshima, Japan, ${ }^{3}$ Department of Oral and Maxillofacial Radiology, Division of Medical Intelligence and Informatics, Graduate School of Biomedical Sciences, Hiroshima University, Hiroshima, Japan, ${ }^{4}$ Department of Obstetrics and Gynecology, Division of Clinical Medical Science, Graduate School of Biomedical Sciences, Hiroshima University, Hiroshima,
Japan, ${ }^{5}$ Department of Periodontology, School of Dentistry, Aichi-Gakuin University, Nagoya, Japan, ${ }^{6}$ Faculty of Dentistry, Kuwait University, Kuwait, Section of Oral and Maxillofacial Radiology, UCLA School of Dentistry, Los Angeles, USA, ${ }^{8}$ Unit of Oral Radiology, University Dental Hospital of Manchester, Manchester, UK

Objectives: Mandibular cortical erosion detected on dental panoramic radiographs may be useful for identifying women with osteoporosis. There is little known about the variation in diagnostic efficacy of observers worldwide.

The purpose of this study was to measure the accuracy of diagnosis of osteoporosis in a worldwide group of observers using dental panoramic radiographs.

Materials and Methods: We constructed a website that included background information about osteoporosis screening and instruction regarding interpretation of mandibular cortical erosion. Dental panoramic radiographs of 100 Japanese postmenopausal women aged 50 years or more who had completed skeletal bone mineral measurements by dual energy x-ray absorptiometry were digitized at $300 \mathrm{dpi}$. These were displayed on the Osteoporosis Screening Project in Dentistry (OSPD) website and used for the evaluation of diagnostic efficacy. Sixty-one observers aged 25 to 66 years recruited from 16 countries participated in this study. Sixty classified cortical erosion (none, mild to moderate, and severe) according to Klemetti's classification on the website via the Internet twice with approximately a two-week interval. One individual completed only the first observation. The diagnostic efficacy of the Osteoporosis Self-assessment Tool (OST), a questionnaire based screening tool, was also calculated and compared with that of cortical erosion.

Results: Intra-observer agreement was sufficient (weighted kappa values $>0.6$ ) in 36 observers. This was significantly increased in observers who specialized in oral radiology $(\mathrm{P}<0.05)$. In these 36 observers, mean sensitivity, specificity, positive predictive value (PPV) and negative predictive value (NPV) in identifying women with osteoporosis by any cortical erosion were $83.5 \%, 48.7 \%, 48.3 \%$ and $85.7 \%$. Those by OST were $85.7 \%$, $35.4 \%, 41.7 \%$ and $82.1 \%$. Mean PPV and NPV were significantly higher in 36 observers with sufficient intra-observer agreement than in 24 observers with insufficient intra-observer agreement. In 5 observers with weighted kappa values $>0.8$, mean sensitivity, specificity, PPV and NPV were $92.0 \%, 55.4 \%, 53.1 \%$ and $92.6 \%$.

Conclusions: This data reconfirms the efficacy of the Klemetti's classification for identifying women with osteoporosis among observers worldwide with sufficient intra-observers agreement.

Acknowledgements: We thank the 61 observers who volunteered to participate in the OSPD international collaborative group.

\section{P128. CONTRIBUTION OF BODY FAT AND LEAN MASS TO THE VARI- ABILITY IN BONE MINERAL DENSITY OF THE LUMBAR SPINE}

M. Boyanov, P. Popivanov; Endocrinology Clinic, Alexandrovska Hospital, Medical University of Sofia, Sofia, Bulgaria

Objective: To determine the relative contributions of body fat and lean mass to the variability in BMD of the lumbar spine in Bulgarian women.

Materials and Methods: 180 women aged from 21 through 76 years were examined (mean age $50.84 \pm 9.69$ years). 130 of them were postmenopausal. After taking anthropometric measurements lumbar spine bone mineral density (BMD) was measured by dual-energy X-ray absorptiometry (DXA) followed by a whole-body scan for body composition examination. We applied DXA on a Hologic QDR 4500 A device.

Results: $18.1 \%$ of the variability in lumbar spine BMD are attributable to body fat mass; whereas $16.0 \%$ - to lean body mass. Total body weight was responsible for $23.4 \%$ of BMD variability. The combined contribution of fat and lean body mass to lumbar spine variability was $20.5 \%$. The relative impact of fat mass on L1-L4 BMD is, however, higher than that of lean mass 
(standardized regression coefficient 0.291 versus 0.199 for lean mass).

Conclusion: This study is the first of this kind in our country. It shows that total body weight is well suitable for osteoporosis risk screening and no further assessment of the type of obesity or overweight are needed.

P129. SERUM 25-HYDROXYVITAMIN D LEVEL DISTRIBUTION IN MEN AND POSTMENOPAUSAL WOMEN WITH OSTEOPOROSIS (THE FACTOR STUDY)

F. Chouha ${ }^{1}$, J. Sampalis ${ }^{2,3}$, E. Psaradellis ${ }^{2}$, A.C. Karaplis ${ }^{4}$; ${ }^{1}$ Clinical Research, Merck Frosst Canada Ltd, Kirkland, Québec, Canada, ${ }^{2}$ Department of Epidemiology and Biostatistics, McGill University, Montreal, Canada, ${ }^{3}$ JSS Medical Research Inc., Montreal, Canada, ${ }^{4}$ Department of Medicine, McGill University, Montréal, Canada

Objective: To describe the distribution of serum 25-hydroxyvitamin D [25-(OH) D] levels in Canadian men and postmenopausal women with osteoporosis.

Methods: This was a multicenter, observational study. Subjects with osteoporosis were recruited from physicians' private practices across eight Canadian provinces. Eligible women were postmenopausal for at least 6 months. Osteoporosis was defined as bone mineral density $\geq 2.5$ standard deviations (SD) below normal mean bone mass at any of the following sites: PA lumbar spine (L1-L4), the hip trochanter, total hip or femoral neck.

Results: Of the 449 subjects, $379(84.4 \%)$ were female and 325 $(72.4 \%)$ were Caucasian. Mean (SD) age was 67.4 (10.3) years and nearly $25.0 \%$ of patients were receiving vitamin D supplementation at baseline. Osteoporosis risk factors reported were inadequate physical activity $(\mathrm{n}=130,29.0 \%)$, low calcium intake $(\mathrm{n}=125,27.8 \%)$ and advanced age $\geq 75$ years old $(\mathrm{n}=107$, $23.8 \%)$. The mean (SD) of 25-(OH) D level was $66.3(24.7)(\mathrm{nmol} /$ $\mathrm{L})$. The cumulative distribution of $25-(\mathrm{OH}) \mathrm{D}$ concentrations were as follows: $8(1.8 \%)$ with $25-(\mathrm{OH}) \mathrm{D}$ levels $<22.5 \mathrm{nmol} / \mathrm{L}$, $57(12.7 \%)$ with $<37.5 \mathrm{nmol} / \mathrm{L}, 123(27.4 \%)$ with $<50 \mathrm{nmol} / \mathrm{L}$, $202(45.0 \%)$ with $<62.5 \mathrm{nmol} / \mathrm{L}$ and $286(63.7 \%)$ with $<75$ $\mathrm{nmol} / \mathrm{L}$. No significant differences between genders were observed. The table describes the prevalence of low $(<75 \mathrm{nmol} / \mathrm{L})$ $25-(\mathrm{OH}) \mathrm{D}$ levels by age categories.

Conclusion: The results of this interim report shows that a significant proportion of individuals with osteoporosis have vitamin $\mathrm{D}$ inadequacy as defined by a low $(<75 \mathrm{nmol} / \mathrm{L})$ serum concentration of $25-(\mathrm{OH}) \mathrm{D}$, regardless of age.

\begin{tabular}{|c|c|c|c|c|}
\hline \multirow[t]{2}{*}{ Age (years) } & \multicolumn{4}{|c|}{$\begin{array}{l}\text { Distribution of } 25-(\mathrm{OH}) \text { D Serum Concentrations } \\
(\mathrm{nmol} / \mathrm{L}) \mathrm{N}(\%)\end{array}$} \\
\hline & $<75$ & $\geq 75$ & Total & P-value* \\
\hline$=65$ & $125(66.5 \%)$ & $63(33.5 \%)$ & 188 & 0.286 \\
\hline $66-75$ & $83(58.5 \%)$ & $59(41.5 \%)$ & 142 & \\
\hline$>75$ & $78(65.5 \%)$ & $41(34.5 \%)$ & 119 & \\
\hline Total & $286(63.7 \%)$ & $163(36.3 \%)$ & 449 & \\
\hline
\end{tabular}

*P-value based on chi square test

P130. A COMPARISON OF THE EFFECT OF ALENDRONATE AND RISEDRONATE ON BONE MINERAL DENSITY IN POSTMENOPAUSAL WOMEN WITH OSTEOPOROSIS OVER 24 MONTHS IFOSAMAX ${ }^{\circledR}$ ACTONEL ${ }^{\circledR}$ COMPARISON TRIALS - INTERNATIONAL: FACTS - INTERNATIONAL)

D.M. Reid ${ }^{1}$, D. Hosking ${ }^{2}$, D. Kendler ${ }^{3}$, M.L. Brandi ${ }^{4}$, J.D. Wark $^{5}$, G. Weryha ${ }^{6}$, J.F. Marques-Neto ${ }^{7}$, N. Verbruggen ${ }^{8}$, C.M. Hustad $^{8}$, M.E. Melton ${ }^{8}$; ${ }^{1}$ Department of Medicine \& Therapeutics, University of Aberdeen, Aberdeen, UK, ${ }^{2}$ Dept. of Bone and Mineral Metabolism, Nottingham City Hospital, Nottingham, UK, ${ }^{3}$ Providence Health Care, Vancouver, Canada, ${ }^{4}$ Department of Medicine, University of Florence, Florence, Italy, ${ }^{5}$ Department of Medicine, Royal Melbourne Hospital, University of
Melbourne, Parkville, Victoria, Australia, ${ }^{6}$ Service d'Endocrinologie, Hôpital de Brabois, Nancy, France, ${ }^{7}$ Department of Rheumatology, State University (Unicamp), Campinas, São Paulo, Brazil, ${ }^{8}$ Merck \& Co., Inc., Whitehouse Station, New Jersey, USA

Objectives: To compare alendronate $70 \mathrm{mg}$ once weekly to risedronate $35 \mathrm{mg}$ once weekly with respect to change in bone mineral density (BMD), biochemical markers, and upper gastrointestinal tolerability over 24 months.

Methods: The study was a 12-month extension to the randomized, double blind, 12-month FACTs-international study. Postmenopausal women with osteoporosis were randomly assigned to treatment with either alendronate $70 \mathrm{mg}$ once weekly or risedronate $35 \mathrm{mg}$ once weekly for 12 months in a 1:1 ratio for the base study. All patients who completed the base study and met all extension entry criteria were enrolled. Patients continued the same double blind study medication during the extension study. Efficacy measurements were BMD at the hip trochanter, lumbar spine, total hip, and femoral neck at 24 months. Other assessments included levels of four bone turnover markers at 24 months. Tolerability was assessed by adverse experience reporting. The primary hypothesis was that treatment with alendronate would produce a greater mean percent increase from baseline in hip trochanter BMD at 24 months than that observed with risedronate.

Results: Of 936 patients randomized into the base study, 854 patients completed the 12-month visit, and 798 patients entered the extension (403 alendronate and 395 risedronate patients). Trochanter BMD increased significantly from baseline to Month 24 in both treatment groups, with a significantly larger increase with alendronate: adjusted mean treatment difference of $1.50 \%$ (95\% CI: $0.74 \%, 2.26 \% ; p<0.001)$. This between-group difference was numerically larger than that seen at Month 12. Similar results were seen at all other BMD sites. Significant geometric mean percent decreases $(p<0.001)$ from baseline were seen for all four markers of bone turnover in both the alendronate and risedronate groups, with a significantly larger decreases $(p<0.001)$ with alendronate: adjusted mean treatment differences ranged from $8.9 \%$ to $25.3 \%$. No significant differences were seen between groups in the incidence of upper gastrointestinal adverse events.

Conclusions: Patients taking alendronate $70 \mathrm{mg}$ once weekly had significantly greater gains in BMD and larger decreases in levels of bone turnover markers than those taking risedronate 35 mg once weekly over 24 months of treatment, with no difference in upper gastrointestinal tolerability.

\section{P131. ALENDRONATE IMPROVES SCREW FIXATION IN OSTEOPOROTIC BONE: A CLINICAL STUDY OF PERTROCHANTERIC FRACTURES}

A. Hoang-Kim, C. Faldini, F. Pegreffi, A. Moroni; Department of Orthopaedic Surgery and Trauma, Rizzoli Orthopaedic Institute, Bologna, Italy

Objective: Recently, animal studies have demonstrated the efficacy of using antiresorptive agents such as alendronate as a fixation augmentation technique. In this study, we used the systemic administration of bisphosphonates to investigate osteointegrative properties of hydroxyapatite-coated implants in osteoporotic patients treated with external fixation.

Methods: Sixteen consecutive patients with pertrochanteric fractures were selected. Inclusion criteria were: female, age $\geq 65$ years, BMD T-score $<-2.5 \mathrm{SD}$. Exclusion criteria included pin insertion torque $\leq 1000 \mathrm{~N} / \mathrm{mm}$ and bisphosphonate treatment during the two-year period prior to fracture. Fractures were fixed with a pertrochanteric fixator and $4 \mathrm{HA}$-coated pins. Two pins were implanted in the femoral head (pin positions 1 and 2) and two in the femoral diaphysis (pin positions 3 and 4). Patients were randomized to therapy with either ALN (Group A) or control (Group B) for a three-month post-operative period. Group A patients received an oral dose of $70 \mathrm{mg}$ of ALN per week. Fixators were removed at 3 months post-op in all patients. 
Results: All the fractures healed. No pin loosening or infection occurred in either group and no differences between femoral neck-shaft angles at 6 months versus post-op were observed. There was no significant difference in pin insertion torque between the two groups. The combined mean extraction torque of the pins implanted at positions 1 and 2 (cancellous bone of the femoral head) was $3181 \pm 1385 \mathrm{~N} / \mathrm{mm}$ in Group A and $1890 \pm 813 \mathrm{~N} / \mathrm{mm}$ in Group B $(\mathrm{p}<0.001)$. The combined mean extraction torque of the pins implanted at positions 3 and 4 (cortical bone of the femoral diaphysis) was $4327 \pm 1720 \mathrm{~N} /$ $\mathrm{mm}$ in Group A and $3785 \pm 1181 \mathrm{~N} / \mathrm{mm}$ in Group B (ns).

Conclusions: These data show that weekly systemic administration of ALN improves fixation in elderly patients with osteoporosis. We observed a two-fold fixation increase in the pins implanted in cancellous bone. With cortical bone, the difference in pin fixation was less marked. We believe that the effect of ALN on implant fixation could be even more pronounced with standard metal screws, which have a lower osteointegrative ability than HA-coated screws.

\section{P132. BONE MINERAL DENSITY IN WOMEN WITH BREAST CANCER}

Z. Kocaaga ${ }^{1}$, Y. Turan ${ }^{1}$, A. Duransoy ${ }^{2}$, A. Gurgan ${ }^{1}$; ${ }^{1}$ Ataturk Research and Education Hospital, Department of Physical Medicine and Rehabilitation, Izmir, Turkey, ${ }^{2}$ Ataturk Research and Education Hospital, Department of Radiation Oncology, Izmir, Turkey

Objective: The aim of this study was to investigate osteopenia and osteoporosis in patients having the diagnosis of breast cancer.

Material and methods: The study group $(n=20)$ consisted of patients with breast cancer and the control group $(n=20)$ consisted of healthy individuals. Patients' previous history of chemotherapy protocols, radiotherapy and surgery were noted. Bone mineral density (BMD) of the patients was measured by DEXA. The diagnosis of osteopenia and osteoporosis were based on the WHO criteria. Antero-posterior and lateral radiographs of the thoracic spine and the lumbar spine were graded according to Kleerekoper Vertebral Deformity Score. Short Form- 36 (SF- 36) questionnaire was used to assess the quality of life.

Results: The mean age of the study group was $49.5 \pm 13.6$ years, while that of the control group was $52.4 \pm 8.5$ years $(\mathrm{p}=$ $0,425)$. The distribution of patients according to menopausal state was $55 \%$ premenopausal, $20 \%$ perimenopausal, $25 \%$ postmenopausal while all of the individuals in the control group was in the postmenopausal state. Duration of menopause in the study group was $33.6 \pm 73.8$ months compared to $108.6 \pm 84.4$ months in the control group $(p=0.005)$. The patients in the study group received 6 cycles of chemotherapy consisting of cyclophosphamide, epirubicin and fluorouracil and radiotherapy was applied to $60 \%$ of the patients. There was statistically significant difference between patients with breast cancer and the control group in terms of lumbar BMD $(p=0.05)$, femoral neck BMD $(p=0.006)$. According to Kleerekoper Score $75 \%$ of patients had grade 1, 25 $\%$ had grade 2 deformities in the thoracal region while $80 \%$ had grade $1,5 \%$ had grade 2 deformities in the lumbar region. The most affected subgroup of SF-36 was pain sensation in the study group $(88.10 \pm 17.37)$.

Conclusions: Aggressive chemotherapy procedure may cause side effects on the bone tissue and may induce premature menopause. We observed that even the menopause duration was obviously short in the study group with higher percentages of osteopenia and osteoporosis. BMD evaluation of patients with breast cancer who have had chemotherapy and/or radiotherapy and treatment of bone mineral loss in these patients is very important in increasing the quality of life.

\section{P133. WHO AND WHEN SHOULD WE TREAT FOR OSTEOPOROSIS?}

O. Ström ${ }^{1}$, F. Borgström ${ }^{1,2}$; ${ }^{1}$ Stockholm Health Economics, Stockholm, Sweden, ${ }^{2}$ Medical Management Centre, Karolinska Institutet, Stockholm, Sweden
Objective: In health economic evaluations of therapies for osteoporosis the results are often highly dependant on what population in terms of age, t-score and prevalence of prior fractures is targeted. The choice of when to initiate therapy will affect all these factors. The aim of this study was to, in a societal perspective, estimate the cost-effectiveness of initiating osteoporosis therapy in unfractured female patients with low BMD compared to waiting and initiate therapy after a fracture occurs.

Material and methods: In an individual state transition model we examined the effects of an intervention costing $€ 600 /$ year given for 5 years that decreased the risk of osteoporotic fractures (hip, vertebral, wrist) by $35 \%$ followed by an offset time of 5 years. In the base case, patients started simulations 70 years old and with a t-score of -2.5 . Since prior fracture is an important risk factor for subsequent fractures, elevated risks were programmed to take effect directly after a fracture event. In one arm all patients were treated from the start and could not receive any additional treatment effect after the first $5+5$ years. In the other arm, treatment was only initiated when and if a fracture occurred. The model was populated with data relevant for a Swedish setting.

Results: The cost per QALY gained of treating a base casepatient without knowing if they were to sustain any fractures was $€ 32000$ while for treating a low risk patient (age $=60$ and $\mathrm{t}$-score -2 ) it was $€ 117000$ compared to waiting for a fracture to occur. It was found cost saving to directly treat a high-risk patient (age $=80$ and $t$-score -3 ). If a screening cost of $€ 300$ was added for the directly treated arm the cost per QALY increased to $€ 40000$.

Conclusion: These results indicate that high-risk patients in Sweden should receive treatment regardless of prevalent fracture status while the decision regarding treatment of patients with lower fracture risks and without prior fracture is dependant on the societal willingness to pay for a QALY gained. P134. BELGIAN BONE SPECIALISTS ARE EAGERLY LOOKING TO THE
AVAILABILITY OF NEW MEDICATIONS IN OSTEOPOROSIS

S. Goemaere ${ }^{1}$, P. Bergmann ${ }^{2}$, J.J. Body ${ }^{3}$, S. Boonen ${ }^{4}$, Y. Boutsen $^{5}$, J.-P. Devogelaer ${ }^{6}$, J.-M. Kaufman ${ }^{7}$, S. Rozenberg ${ }^{8}$, J.-Y. Reginster ${ }^{9}$; ${ }^{1}$ Unit for Osteoporosis and Metabolic Bone Diseases, Ghent University Hospital, Ghent, Belgium, ${ }^{2}$ Department of Nuclear Medicine, CHU Brugmann, Free University of Brussels, Brussels, Belgium, ${ }^{3}$ Department of Internal Medicine, Free University of Brussels, Brussels, Belgium, ${ }^{4}$ Center for Metabolic Bone Diseases, Katholieke University Leuven, Leuven, Belgium, ${ }^{5}$ Rheumatology, Saint-Luc University Hospital, MontGodinne, Belgium, ${ }^{6}$ Rheumatology Unit, Saint-Luc University Hospital, Katholieke University of Leuven, Brussels, Belgium, ${ }^{7}$ Unit for Osteoporosis and Metabolic Bone Diseases, Ghent University Hospital, Ghent, Belgium, ${ }^{8}$ Department of Gynaecology-Obsetrics, Free University of Brussels, Brussels, Belgium, ${ }^{9}$ Department of Public Health, Epidemiology and Health Economics, University of Liège, Liège, Belgium

Several medications are currently available, in Belgium, for the management of osteoporosis, and reimbursed based either on the presence of low BMD (DXA) or at least one moderate vertebral fracture. In order to assess whether the needs of the practitioners are fulfilled with these treatment options, the Belgian Bone Club organised a survey within the clinician members of the society $(n=144)$. 61 questionnaires have been returned, which means a $43.0 \%$ participation rate. $64.5 \%$ and $96.8 \%$ of the clinician responders feel that the drugs currently available, in females and males, respectively, are insufficient or largely insufficient. When dealing with severe osteoporosis, these figures are $63.3 \%$ and $89.7 \%$ respectively. $70.1 \%$ of the responders were unsatisfied with the drugs available (event if not reimbursed) for treating osteopenia. Considering the interest of new drugs potentially becoming available in females, parathyroid hormone is considered as bringing a moderate to high benefit in osteoporosis or severe osteoporosis for $61.3 \%$ and $96.8 \%$ of the responders. In the same population, the respective figures for 
strontium ranelate are $84 \%$ and $87.1 \%$. If these drugs are considered for males with osteoporosis or severe osteoporosis, PTH would bring a significant benefit for $70 \%$ and $93.6 \%$ of the responders. Although not investigated for men, the figures for strontium ranelate would be $45 \%$ and $53.3 \%$ respectively. New SERMs with no effect on DVT and new bisphosphonates with less than weekly (monthly or yearly) regimens are also considered as providing a moderate to high benefit for the management of osteoporosis, for the vast majority of the responders. The figures are particularly striking for parathyroid hormone in female patients with severe osteoporosis, for bisphosphonates and strontium ranelate in women with low bone density without prevalent fractures and for new bisphosphonates with less than weekly regimen for male osteoporosis.

In conclusion, the clinicians, members of the Belgian Bone Club, feel that the choice of drugs currently available in Belgium for the overall management of osteoporosis in males and females, is either insufficient or largely insufficient. New therapeutic options are eagerly requested.

\section{P135. BONE LOSS AND DECLINE OF BONE STRENGTH ACCELERATE IN MEN AFTER 70 YEARS OF AGE. A LONG TERM FOLLOW UP OF THE MINOS COHORT}

P. Szulc, P.D. Delmas; INSERM 403 Research Unit and Université Claude Bernard Lyon 1, Lyon, France

The apparent bone loss (decrease in areal bone mineral density [aBMD]) is determined by the endosteal bone loss and the periosteal expansion. The net bone loss (decrease in bone mineral content $[\mathrm{BMC}]$ ) better reflects the negative net balance of bone mineral. We assessed the apparent and net bone loss in a cohort of 714 men aged 50-85 years followed up prospectively for up to 7.5 years (median -7.5 years) by using repeated measurements by DXA (Hologic 1000W and Osteometer DTX 100). Projected surface increased at the lumbar spine, total hip, femoral neck and distal forearm. BMC and aBMD increased at the lumbar spine and decreased at total hip, whole body, distal forearm and ultradistal radius. At the distal radius and ulna, cortical thickness and area as well as bending strength estimated by using crosssectional moment of inertia and section modulus decreased with time. At the femoral neck, aBMD and cortical thickness decreased whereas buckling ratio increased resulting in a loss of bone stability, despite the increase in BMC and estimated bending strength. Apparent and net bone loss accelerated with age at the total hip and distal forearm whereas the rate of periosteal expansion did not vary with age. This resulted in a parallel acceleration of the loss of bending strength at the distal radius and ulna. In men aged more than 70, loss of bone mineral and bending strength was 2 to 7 times faster than in men aged less than 60 at baseline.

Conclusion: In elderly men, apparent bone loss (aBMD) prospectively assessed was due to a significant net bone loss (BMC) and not due to the outward shift of bone mineral (except at femoral neck). The loss of bone mineral and bending strength accelerates with age despite a stable rate of periosteal expansion. This acceleration can be due the increasing endosteal bone loss, consistent with the age-related increase in bone resorption. Our data contribute to a better understanding of the morphological basis underlying the ageing-related increase in fracture incidence in men.

\section{P136. RISK FACTORS FOR OSTEOPOROSIS IN PREMENOPAUSAL- WOMEN}

N. Pilipovic, S. Brankovic, N. Vujasinovic-Stupar, D. PalicObradovic; Institute of Rheumatology, Belgrade, Serbia and Montenegro

The aim of study was to examine risk factors for osteoporosis (OP) in premenopausal (PMP) women.

Methods: We examined 566 women aged 20-79 $(48,70)$, from urban part of Belgrade randomly selected from population register. $292(51,59 \% \%)$ were premenopausal and $45(15,41 \%)$ of them had low bone mineral density (BMD). BMD of spine was measured by DEXA, using a Lunar DPX-L device. We investigated risc factors for OP in 45 PMP women with low BMD $(1,017$ $\mathrm{gr} / \mathrm{cm}^{2}$ ), meanage 41,11 and in 247 PMP women with normal $\operatorname{BMD}(1,255 \mathrm{gr} / \mathrm{cm} 2)$ meanage 39,62.

Conclusion: Premenopausal women with low BMD had not any risc factor for osteoporosis except low BMI.

Results are on the table

\begin{tabular}{lcc}
\hline Risc factors & $\begin{array}{l}\text { Women with low } \\
\text { BMD (45) }\end{array}$ & $\begin{array}{l}\text { Women with normal } \\
\text { BMD }(264)\end{array}$ \\
\hline $\begin{array}{l}\text { Fracture } \\
\text { Sedentary type of life }\end{array}$ & $5((11,11 \%)$ & $23(9,31 \%)$ \\
Cigarette smoking & $19(42,89 \%)$ & $154(62,35 \%)$ \\
Alcohol abuse & $1(2,22 \%)$ & $119(48,18 \%)$ \\
Coffein abuse & $29(64,44 \%)$ & $6(2,43 \%)$ \\
Low Ca intake & $22(48,89 \%)$ & $149(60,329 \%)$ \\
Low Ca intake in child- & $5(11,11 \%)$ & $132(53,44 \%)$ \\
hood & & $28(11,34 \%)$ \\
Low sun exposure & $18(40,00 \%)$ & $119(48,18 \%)$ \\
Concomitant diseases re- & $8(17,78 \%)$ & $43(17,42 \%)$ \\
levant for OP & & \\
Family his.of OP or frac. & $6(13,33 \%)$ & $51(20,65 \%)$ \\
Late menarcha & $15(33,33 \%)$ & $50(20,24 \%)$ \\
Irregular cycles & $8(17,78 \%)$ & $28(11,34 \%)$ \\
Long breast feeding & $12(26,67 \%)$ & $67(31,58 \%)$ \\
Loss of height & $18(40,00 \%)$ & $97(39,27 \%)$ \\
Low BMI & $10(22,22 \%) *$ & $24(9,72 \%)^{*}$ \\
\hline P & &
\end{tabular}

${ }^{*} \mathrm{P}<0.05$

P137. BONE IN BONE IMAGE AFTER TREATMENT WITH INTRAVENOUS INFUSIONS OF DISODIUM PAMIDRONATE IN TWIN CHILDREN SUF. FERING FROM OSTEOGENESIS IMPERFECTA

J.-P. Devogelaer ${ }^{1}$, F. Lecouvet ${ }^{2}$, G. Depresseux ${ }^{1}$; ${ }^{1}$ Rheumatology and Radiology ${ }^{2}$ Units, St-Luc University Hospital, Brussels, Belgium

Two twin boys aged 12 suffering from osteogenesis imperfecta, complicated by several peripheral and thoracic vertebral fractures were treated by intravenous infusions of disodium pamidronate (1 mg/kg per infusion), 3 consecutive days every 3 months for 2 years. Their BMD measured by DXA increased from 0.416 to $0.600 \mathrm{~g} / \mathrm{cm}^{2}$ at the (L1-L4) lumbar spine and from 0.590 to $0.804 \mathrm{~g} / \mathrm{cm}^{2}$ at the total hip after 4 years. In the same time, they grew $16 \mathrm{~cm}$. The views of the postero-anterior DXA scan of the lumbar spine obtained 2 years after the last course of therapy demonstrated the presence of "arrest lines" close to the plateaus of the vertebral bodies. X-ray films confirmed that this aspect was attributable to a "bone in bone image" due to bisphosphonate therapy, a well-known radiological aspect in growing children treated by cyclical intermittent bisphosphonates (1). This DXA aspect corresponding to the radiological image should be recognized, because part of the bone gain can be explained by it.

(1) Devogelaer J-P. New uses of bisphosphonates: osteogenesis imperfecta. Curr Opin Pharmacol 2002; 2: 748-753.

P138. 18F-FLUORIDE POSITRON EMISSION TOMOGRAPHY: A NEW TOOL TO ASSESS MONOSTOTIC PAGET'S DISEASE OF BONE

A. Nzeusseu Toukap ${ }^{1}$ G. Depresseux ${ }^{1}$, J. Installe ${ }^{2}$, M. Lonneux ${ }^{2}$, J.-P. Devogelaer ${ }^{1}$; ${ }^{1}$ Department of Rheumatology, St-Luc University Hospital, Brussels, Belgium, ${ }^{2}$ St-Luc University Hospital, Department of Nuclear Medicine, Brussels, Belgium

Introduction: The activity of monostotic Paget's disease of bone (PDB) may be difficult to assess by biochemical parameters. $18 \mathrm{~F}$-fluoride positron emission tomography (PET) is able to quantify local skeletal metabolic activity. Therefore, we planned to use $18 \mathrm{~F}$-fluoride PET to assess PDB activity and to measure the response of PDB to bisphosphonate (BP) therapy, particularly 
in patients with monostotic disease, in which biochemical parameters frequently remain in the normal range.

Patients and Methods: 9 patients with monostotic PDB underwent a one-hour dynamic 18F-fluoride PET scan before one month, six months and 12 months after BP therapy. Six patients with the polyostotic form served as controls. Serum total alkaline phosphatase (TAP), bone-specific (B) AP, serum CTX and fasting urinary NTX over creatinine were also measured at the same times. Changes in bone metabolism as measured by PET scans were assessed by a semi-quantitative index such as standardized uptake values (SUVmax), and by plasma clearance of $18 \mathrm{~F}$-fluoride to bone mineral (Ki). Wilcoxon signed rank test and simple regression analysis were used for statistics.

Results: SUV and Ki correlated significantly with each other both at baseline and during the follow-up $(\mathrm{p}<0.0001$ at all times) in all patients. In the nine patients with monostotic PDB, the mean 18 F-fluoride uptake decreased significantly up to 70.4 and 72.7 percent of initial values after the first month of BP therapy, as measured by both SUVmax $(\mathrm{p}<0.05)$ and Ki measurements $(\mathrm{p}<0.01)$, respectively. Six months later, further reductions of the mean $18 \mathrm{~F}$-fluoride uptake were observed up to respectively $56.0 \%$ by SUVmax and $60.3 \%$ by Ki measurements ( $\mathrm{p}<0.01$ for both values). After 12 months, these values, measured in 8 patients amounted to $56.9 \%$ and $54.5 \%$, respectively. The mean serum TAP simultaneously decreased borderline significantly to $86.4 \%, 79.3 \%$ and $76.2 \%$ of initial values after 1,6 and 12 months, respectively.

Conclusions: $18 \mathrm{~F}$-fluoride PET is able to demonstrate metabolic bone changes in localized PDB early after BP therapy. This could be particularly interesting for patients with focal pagetic bone. If it becomes more readily available, 18F-fluoride PET could be helpful to monitor the therapeutic response in patients with monostotic PDB.

\section{P139. CONCURRENT OCCURRENCE OF PAGET'S DISEASE OF BONE} AND HYPERPARATHYROIDISM

C. Leens, J.-P. Devogelaer; Department of Rheumatology, St-Luc University Hospital, Brussels, Belgium

Simultaneous occurrence of Paget's disease (PD) and hyperparathyroidism (HPT) has been observed relatively frequently. An elevation of iPTH has been documented in around $18 \%$ of cases. At least thirty-three cases of Paget's disease complicated by adenomata and 5 cases with hyperplasia of the PTH glands have been reported. We have looked in our files what was the frequency of both conditions occurring simultaneously in our hospital. 256 cases of PD were reviewed. Measurements of iPTH (at least at once) was retrieved in $26.5 \%$ of cases $(\mathrm{n}=68)$. An elevated iPTH was observed in $37.9 \%$, and a simultaneous elevation of iPTH and sCa in $10.7 \%$. In $19 \%$, $25 \mathrm{OHVitamin} \mathrm{D}$ was low, out off whom $56.5 \%$ had concurrent elevated iPTH. In $15 \%, 25 \mathrm{OHVitamin} \mathrm{D}$ was normal (i.e. superior to $20 \mathrm{ng} / \mathrm{ml}$ ), but still $43.5 \%$ of these patients had elevated iPTH. Elevated iPTH was not seen more frequently in resistant PD than in good responders. There was a trend to have increased iPTH, more elevated the number of courses of therapy with antipagetic drugs. The effect of age and age-related creatinine increase was the main explanation for that, however. What is the nature of the association PD and PHPT? It could be fortuitously coincidental. However, the prevalence of HPT in PD is significantly increased as compared to the general population. Some cases could be explained by hypovitaminosis D, which could lead to secondary HPT. A stress on extracellular fluid calcium of variable duration and degree when lytic lesions become denser might be capable to induce secondary HPT. Repetitive episodes of hypocalcemia provoked by several courses of antiresorptive drugs could also provoke secondary hypersecretion of the parathyroid glands, potentially leading to their hypertrophy.
P140. ESTIMATION FROM EARLY CHANGES OF SERUM PROCOLLAGEN TYPE I AMINOTERMINAL PROPEPTIDE OF THE LUMBAR BMD GAIN AFTER 2 YEARS IN CHILDREN SUFFERING FROM SEVERE OSTEOGENESIS IMPERFECTA

J.-P. Devogelaer, G. Depresseux; Rheumatology Unit, St-Luc University Hospital, Brussels, Belgium

Three-day intravenous (IV) pamidronate every 3 months has become a classical therapy of severe osteogenesis imperfecta (OI) in children. However, this therapy is rather heavy, and a rapid determination of its future efficacy should be welcome. We have therefore determined whether the measurement of changes of serum procollagen type I aminoterminal propeptide (PINP), a biological marker of bone formation, between day 0 and day +3 could help to foresee the BMD response after 2 years. We have treated 11 patients [M, 9; F, 2; aged $10.4(3.7 \mathrm{SD})]$ suffering from severe OI with IV pamidronate. sPINP was measured on day 0 and +3 , by radioimmunoassay (Orion Corporation Espoo, Finland). The results were expressed as a percentage value of day 0 and day 3. Lumbar-BMD (L1-L4-BMD) was measured by DXA (QDR 4500 Elite, Hologic Inc, Bedford MA) at time 0 and at time +24 months. On average, after 3 days of therapy, PINP value decreased from 398 (251) to 194 (193) ?g/1 (- $52 \%$, $\mathrm{p}<0.001)$ and L1-L4-BMD increased from $0.454(0.134)$ at time 0 to $0.648(0.155) \mathrm{g} / \mathrm{cm}^{2}(+43 \%, \mathrm{p}<0.001)$ after 24 months. A significant inverse correlation was observed between PINP changes $(0-3$ days $)$ and L1-L4-BMD $(0-24$ months $)[r=-0.60$ $(\mathrm{p}=0.05)]$.

In conclusion, a significant decrease of PINP between day 0 and day 3 during a 3 -day IV pamidronate therapy is accompanied by a significant increase of L-BMD after two years. The PINP measurements could constitute a promising biological measurement leading to a better compliance to therapy.

\section{P141. INULIN AND FRUCTO-OLIGOSACCHARIDES DIFFER IN THEIR ABILITY TO ENHANCE BOTH THE BONE MINERAL DENSITY AND THE MUCOSAL LEVELS OF 9-KDA AND 28-KDA CALCIUM BINDING PRO- TEINS IN RATS}

D.H. Manicourt, A. Nzeusseu Toukap, J.-P. Devogelaer; Rheumatology Unit, St-Luc University Hospital, Brussels, Belgium

The increase in BMD observed in growing rats fed with chicory fructans can be related, at least partly, to an increase in calcium absorption in the colon. It is generally believed that products of the colonic fermentation of fructans improve calcium absorption by increasing the solubility of calcium salts by lowering the luminal $\mathrm{pH}$ and by inducing hypertrophy of cecal walls, but direct effects such as an increase in the levels of calcium binding proteins (CaBPs) in the mucosal cells of the large bowel cannot be excluded. Therefore, for a 3 -month time period, 3 groups of 13 six-week-old male rats each received either the standard AO4 diet (CTL group), the standard diet containing 5\% inulin (INU group) or the standard containing 5\% chicory oligofructose (OLF group). At sacrifice, rats of the 2 fructan groups both had a similar median cecal wall weight $(1.5 \mathrm{~g})$ that was significantly higher than that of the CTL group $(0.9 \mathrm{~g}$; $\mathrm{p}<0.001)$. Mucosal proteins were subjected to Western blots and semi-quantification of blots probed with anti- 9-kDa-Calbindin antibodies showed that the levels of this CaBP was increased by a factor of 2 in the OLF group and by a factor of 3 in the INU group. Surprisingly, a monoclonal anti-28-kDa-Calbindin antibody detected a signal at the expected molecular weight in all specimens; further, when compared to the CTL group, the signal intensity was increased by a factor of 3 in the OLF group and by a factor of 5 in the INU group. Tibia and the third lumbar vertebra (L-3) stripped off adherent tissues were analyzed by using pQCT (Research SA+, Stratec, Germany). In both L3 and proximal tibia, the BMD of cancellous bone was significantly increased by INU $\left(0.27\right.$ versus 0.23 and 0.33 versus $0.22 \mathrm{~g} / \mathrm{cm}^{3}$, respectively; $\mathrm{p}<0.001)$ and to a lower extent by OLF $(0.26$ and $\left.0.28 \mathrm{~g} / \mathrm{cm}^{3} ; \mathrm{p}<0.05\right)$. Further, inulin but not OLF, enhanced the 
BMD of cortical bone in both L-3 $\left(0.98\right.$ versus $0.93 \mathrm{~g} / \mathrm{cm}^{3}$; $\mathrm{p}<0.01)$ and tibia $\left(0.88\right.$ versus $\left.0.83 \mathrm{~g} / \mathrm{cm}^{3} ; \mathrm{p}<0.01\right)$.

In conclusion, although inulin and OLF both have a positive effect of the BMD of the axial and peripheral skeleton of growing rats, the stronger effect of inulin cannot be related to an increase in the exchange surface area of the gut, as assessed by the cecal wall weight, but is likely to be related, at least partly, to a strongest increase in the mucosal levels of the $9 \mathrm{kDa}$ and $28 \mathrm{kDa}$ calcium binding proteins which both contribute to intestinal calcium absorption.

\section{P142. THE DIFFERENTIAL REGULATION OF THE BONE MORPHOGENIC PROTEIN ANTAGONIST CHORDIN IN HUMAN NORMAL AND OSTEOAR- THRITIC CHONDROCYTES}

G. Tardif ${ }^{1}$, J-P. Pelletier ${ }^{1}$, D. Hum ${ }^{1}$, C. Boileau ${ }^{1}$, N. Duval ${ }^{2}$, J. Martel-Pelletier ${ }^{1}$; ${ }^{1}$ Osteoarthritis Research Unit, University of Montreal Hospital Centre, Notre-Dame Hospital, Montreal, Quebec, Canada, ${ }^{2}$ Pavillon des Charmilles, Vimont, Quebec, Canada

Objectives: Bone morphogenic proteins (BMPs) are factors that play essential roles during adult bone remodeling and repair as well as during cartilage formation and maintenance. BMP activities are modulated by proteins known as BMP antagonists that prevent BMPs from binding to their specific receptors. In this study, we investigated the distribution and level of the BMP antagonist, chordin, in normal and osteoarthritic (OA) cartilage and synovial membrane, and the regulation of its expression in chondrocytes and synovial fibroblasts following treatment with inflammatory (IL-1B, TNF-a, IFN-g) and growth (BMP-2, TGFB1, Activin A, EGF, bFGF, and PDGF-BB) factors.

Methods: Localization of chordin in cartilage and synovial membrane as well as protein levels was performed by immunohistomorphometric analysis on cartilage explants. Gene expression was determined using real-time PCR on human chondrocytes and synovial fibroblasts.

Results: In normal cartilage, chordin was found at low levels ( $7.6 \pm 1.3 \%$ of positive cells) mainly at the very superficial layers. In OA, chordin was also found at the superficial layers $(8.9 \pm 1.1 \%)$ but at a significantly higher level $(24.7 \pm 1.5 \%, \mathrm{p}<0.0001)$ in the middle and deep layers of the cartilage. Interestingly, chordin also demonstrated a differential regulation between normal and OA chondrocytes. In contrast to normal cells, chordin was markedly $(p<0.01)$ down-regulated in OA chondrocytes by all the growth factors tested. IFN- $\gamma$ significantly $(\mathrm{p}<0.0002)$ stimulated its expression in normal, but did not affect chordin in OA chondrocytes. However, both cell types were not affected by IL-1ß and TNF-a. Protein levels showed patterns similar to those of the mRNAs. The distribution and regulation of chordin was similar in normal and OA synovial membranes/fibroblasts.

Conclusion: The increased level of chordin in OA cartilage as well as its location and its differential regulation between normal and OA chondrocytes suggest a role of this BMP antagonist in the OA process. Chordin may reduce the capacity of BMPs to induce OA cartilage repair, therefore favoring this tissue catabolism. The lack of effect by the inflammatory factors IL-1 1 and TNF- $\alpha$ indicates that inflammatory processes do not trigger chordin expression.

\section{P143. UPREGULATION OF PROTEINASE-ACTIVATED RECEPTOR (PAR)- 2 IN HUMAN OSTEOARTHRITIC TISSUES: A NEW PATHWAY FOR THE MEDIATION OF JOINT DESTRUCTION}

J-P. Pelletier, C. Boileau, F. Mineau, C. Geng, M. Boily, J. Martel-Pelletier; Osteoarthritis Research Unit, University of Montreal Hospital Centre (CHUM), Notre-Dame Hospital, Montreal, Quebec, Canada

Objective: Proteinase-activated receptors (PARs) represent a family of transmembrane domain $\mathrm{G}$ protein coupled receptors. They are activated by a proteolytic cleavage generating a tethered activating ligand at the N-terminus. Four members of this family have been identified, and named PAR-1, $-2,-3$ and -4 . PAR-2 plays a role in both neurogenic inflammation and hyperalgesia by promoting the release of substance P. PAR-2 was also recently suggested to be involved in inflammation and to induce the synthesis of catabolic and inflammatory factors. Little has been reported on the involvement of this factor in human osteoarthritic articular tissues. Here, we investigated PAR-2 levels and modulation in human normal and osteoarthritic cartilage and synovial membrane as well as its role in the in situ synthesis of catabolic factors, including metalloprotease (MMP)-1 and cyclooxygenase (COX)-2 production.

Methods: PAR-2 levels were determined using immunohistochemistry. The protein modulation was investigated on osteoarthritic tissue explants treated for 48 hours as follows: for cartilage and synovial membrane with a PAR-2 activating peptide (400 $\mu \mathrm{M})$, IL-1B $(100 \mathrm{pg} / \mathrm{ml})$ or TNF- $\alpha(5 \mathrm{ng} / \mathrm{ml})$; and for cartilage with a specific inhibitor of MEK1/2, PD $98059(10 \mu \mathrm{M})$, P38, SB $202190(10 \mu \mathrm{M})$, NF-кB, SN50 $(50 \mu \mathrm{M})$, or COX-2, NS-398 (10 $\mu \mathrm{M})$. MMP-1 and COX-2 production were also evaluated following stimulation using the PAR-2 activating peptide.

Results: PAR-2 is produced in both human cartilage and synovial membrane; its expression is significantly increased in osteoarthritic tissues compared to normal $(\mathrm{p}<0.03)$. PAR-2 production is significantly upregulated by IL-1B, TNF- $\alpha$, and PAR-2 activating peptide in both articular tissues. The PAR-2 activating peptide also markedly induces MMP-1 and COX-2 production. Interestingly, only the specific p38 inhibitor, SB 202190, was able to down-regulate PAR-2.

Conclusions: This study documents that PAR-2 is present at increased levels in human osteoarthritic cartilage and synovial membrane, and identifies the major signaling pathway regulating its synthesis. We also demonstrated, for the first time, that the specific PAR-2 stimulation can activate major pathophysiological pathways of the disease such as the synthesis of MMP and COX2. These results suggest PAR-2 as a potential new therapeutic target for the treatment of inflammation in osteoarthritis.

\section{P144. OSTEOPROTEGERIN LEVELS IN SMOKERS}

A. Supervía ${ }^{1,2}$, A. Enjuanes ${ }^{2}$, L. Mellibovsky ${ }^{1,2}$, S. Ruíz-Gaspà ${ }^{2}$, J. Blanch ${ }^{2}$, R. Carreras ${ }^{2}$, X. Nogués ${ }^{1,2}$, A.Díez-Pérez ${ }^{1,2}$; ${ }^{1}$ Department of Internal Medicine, Hospital del Mar, Autonomous University of Barcelona, Barcelona, Spain, ${ }^{2}$ URFOA, IMIM, Barcelona, Spain

Objective: Osteoprotegerin (OPG) is a cytokine that regulates osteoclast recruitment and activity. Serum levels of OPG increase with age in men and healthy postmenopausal women, and are higher in osteoporotic postmenopausal women. A correlation between serum levels of OPG and biochemical markers of bone turnover (type osteocalcin and type I collagen crosslink telopeptides) has been described. Finally, serum OPG is correlated with cardiovascular risks factors. Tobacco smoking is a well-recognised risk factor for osteoporosis. Several studies have evaluated the variations in serum levels of OPG in smokers with conflicting results. The aim of this study was to evaluate the influence of smoking on the serum levels of OPG, and to assess the effects of smoking cessation.

Material and methods: All the study participants were healthy volunteers without cardiovascular risk factors. Twenty active smokers ( 9 men and 11 women) were included. Control group was formed by 49 healthy never smoker volunteers ( 22 men and 27 women). A subgroup of 15 smokers ( 7 men and 8 women) followed a period of abstinence of one month. Serum levels of calcium, parathyroid hormone, and OPG were analysed by ELISA. In the abstinent subgroup, blood analysis was repeat one month later. Statistical analysis was performed with SPSS software.

Results: Both groups were comparable in sex, serum levels of calcium and parathyroid hormone. Smokers were older than never smokers were $(36.2$ (8.6) vs 30.9 (5.6) year old; $p=0.018)$. No differences in OPG serum levels were found between both groups even after adjusting by age differences. OPG serum levels in smokers before and after smoking abstinence were also not different. 
Conclusions: Tobacco smoking does not affect serum levels of OPG in healthy young volunteers. We have found no variations in OPG serum levels after smoking cessation.

\section{P145. DETERMINATION OF T-SCORE CONFIDENCE LIMITS FOR DIAG- NOSING OSTEOPOROSIS}

K.G. Faulkner, C. Verboven, L.S. Weynand, W.K. Wacker, H.S. Barden; GE Healthcare, Madison, WI, USA

Objectives: Effective use of serial DXA measurements for monitoring $\mathrm{BMD}$ change requires the minimization of precision error. However, even a single BMD result is subject to both offset (accuracy) errors and variability (precision) errors, which will influence the T-score. We determined the influence of precision error on the T-score for the diagnosis of osteoporosis.

Materials and Methods: Precision depends on operator experience, type of DXA system used, and the skeletal site. Using the Lunar Prodigy, expert precision error $\left(\mathrm{g} / \mathrm{cm}^{2}\right)$ in women (mean age $63 \pm 9$ years) was 0.010 at the L1-L4 spine, 0.013 at the femoral neck, and 0.008 for the total hip region (1). At clinical centers using the Lunar Prodigy, precision error in women (mean age $61 \pm 10$ years) was reported as 0.014 at the L1-L4 spine, 0.025 at the femoral neck, and 0.012 at the total hip (2). Assuming BMD is normally distributed, the $95 \%$ confidence interval (CI) for a single Tscore measurement can be estimated using the following equation:

95\% CI $=$ T-score \pm 1.96 (Precision/Population SD).

Results: Population SD from the reference database for the Lunar Prodigy is $0.12 \mathrm{~g} / \mathrm{cm}^{2}$ at the spine, femoral neck, and total hip. Using this model, the $95 \%$ CI for T-scores at each skeletal site are shown in Table 1.

Conclusions: We conclude that clinical DXA T-scores have $95 \%$ confidence limits of \pm 0.2 at the spine and total hip and \pm 0.4 at the femoral neck. T-scores variations of \pm 0.2 or less at the spine or total hip and \pm 0.4 at the femoral neck should not be considered significant when diagnosing osteoporosis. Minimizing precision error can increase confidence in T-scores by reducing the confidence limits to less than \pm 0.2 at the spine and total femur and to \pm 0.2 at the femoral neck.

\section{References}

1. Shepherd et al. WCO 2004

2. Weynand et al. ASBMR 2004

Table 1 95\% CI for T-scores based on Expert and Clinical Precision Errors

\begin{tabular}{llll}
\hline & Spine & Neck & Total Hip \\
\hline Expert & \pm 0.16 & \pm 0.21 & \pm 0.13 \\
Clinical & \pm 0.23 & \pm 0.41 & \pm 0.20 \\
\hline
\end{tabular}

\section{P146. A SINGLE DOSE OF ZOLEDRONIC ACID 5mg ACHIEVES MORE SUSTAINED BIOCHEMICAL REMISSION VERSUS DAILY $30 \mathrm{mg}$ RISEDRONATE IN PATIENTS WITH PAGET'S DISEASE}

I. Reid ${ }^{1}$, P. Miller ${ }^{2}$, J. Brown ${ }^{3}$, W. Fraser ${ }^{4}$, D. Hosking ${ }^{5}$, J.P.

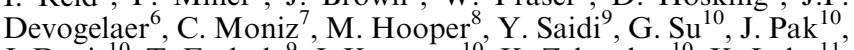
J. Davis ${ }^{10}$, T. Fashola ${ }^{9}$, J. Krasnow ${ }^{10}$, K. Zelenakas ${ }^{10}$, K. Lyles ${ }^{11}$; ${ }^{1}$ University of Auckland, Auckland, New Zealand, ${ }^{2}$ Colorado Center for Bone Research, Lakewood, CO, USA, ${ }^{3}$ Le Centre Hospitalier Universitaire de Quebec, Sainte-Foy, Quebec, Canada, ${ }^{4}$ Royal Liverpool University Hospital, Liverpool, UK, ${ }^{5}$ Nottingham City Hospital, Nottingham, UK, ${ }^{6}$ Cliniques Universitaires St Luc, Brussels, Belgium, ${ }^{7}$ Kings College Hospital, London, UK, ${ }^{8}$ Repatriation General Hospital, Concord, Australia, ${ }^{9}$ Novartis Pharma AG, Basel, Switzerland, ${ }^{10}$ Novartis Pharmaceuticals Corporation, East Hanover, NJ, USA, ${ }^{11}$ Duke University and VA Medical Centers, Durham, NC, USA

Objective(s): Zoledronic acid (ZOL) has been shown to have superior efficacy and faster time to onset than risedronate (RIS) in Paget's disease from pooled data of the 6-month core period of two identical, randomized controlled trials comparing a single ZOL $5 \mathrm{mg} 15$-minute infusion with oral RIS $30 \mathrm{mg} /$ day for 60 days (1), suggesting the potential for longer and more sustained biochemical remission by ZOL. To assess this, the results from the extended observation period (EOP) are presented.

Materials and Methods: The primary efficacy endpoint of the core study was therapeutic response at 6 months, defined as a $\geq 75 \%$ reduction in serum alkaline phosphatase (ALP) excess or its normalization. Of the $169 \mathrm{ZOL}$ and 125 RIS patients eligible for entry, 152 ZOL and 115 RIS responders entered the EOP and had ALP tested every 6 months. Time to first loss of therapeutic response, time to first partial disease relapse (defined as a $\geq 50 \%$ increase from the ALP measurement at month 6 and at least 1.25 times the upper normal limit) and complete disease relapse (defined as ALP level returned within $20 \%$ of baseline ALP value) were evaluated.

Results: As of October 21, 2005, 57/115 RIS patients have lost therapeutic response compared to only $3 / 152$ ZOL patients $(\mathrm{P}<.0001)$. Furthermore, 49/115 and 9/115 RIS patients experienced partial and complete disease relapse compared to only $3 /$ 152 and $0 / 152$ ZOL patients, respectively $(\mathrm{P}<.0001$ and $\mathrm{P}<.001$, respectively). Within the first 3 days of drug administration, ZOL patients experienced about 2-fold higher rate of post-dose symptoms than RIS patients. The symptoms were mild to moderate in severity and mostly resolved after 4 days of onset. After 3 days of drug administration, side effects rate was similar between treatment groups.

Conclusion(s): A single ZOL 5mg infusion shows longer duration of effect relative to the reduction in ALP than RIS Treatment efficacy was sustained in the majority of ZOL patients compared to the substantial number of RIS patients who either lost their therapeutic response or experienced partial or complete disease relapse. Hence, a single ZOL $5 \mathrm{mg}$ dose offers the potential for multi-year biochemical remission in patients with Paget's disease.

(1) Reid et al. N Engl Jmed 2005; 353:898-90

\section{P147. WHY PATIENTS WITH OSTEOPOROSIS IN BULGARIA ARE NOT TREATED}

A.-M. Borissova, R. Kovatcheva, A. Shinkov; University Hospital of Endocrinology, Sofia, Bulgaria

According to the IOF information bulletin many patients discontinue their treatment before the first year after its start and thus the fracture risks returns to initial levels. The reasons for stopping therapy are as follows: $23 \%$ does not want to stay upright for half an hour after the pill, 20\% experience adverse events, $17 \%$ do not agree to fast before and after the pill intake' $13 \%$ forget to take the pills. What is the situation in Bulgaria? Between August and September 2005 the Bulgarian League for the Prevention of Osteoporosis conducted a questionnaire among 150 physicians (endocrinologists, rheumatologists, gynecologists and orthopedists), 50 from the capital Sofia and 100 from the country, on their attitudes and approach with osteoporosis. The preferred medication was as follows: bisphosphonates $-48 \%$, calcitonins $-30 \%$, HRT $-12 \%$, raloxifene $-6 \%$ and phytoestrogens $-4 \%$. One year after commencing therapy $42 \%$ in the capital and $7 \%$ in the country were still continuing treatment. The primary cause of drug discontinuation cited by $96 \%$ of specialists in Sofia and $88 \%$ of those in the country, respectively, was the high cost of the medication. Adverse effects were cited as a cause for premature discontinuation of therapy by only 265 and $10 \%$ of physicians, respectively. The questionnaire among patients (100 in the capital and 200 in the country) showed that $23 \%$ of the subjects never started the prescribed drug due to financial reasons. Only $20 \%$ were still on the medication more than one year after its commencement. The patients needs for information on osteoporosis were rated in the following order: $55 \%$ of subjects needed more information on 
the available therapy, $50 \%$ wanted to know more about the disorder and $42 \%$ needed information about exercise and diet in osteoporosis. The current trends in the work the Bulgaria are towards: 1.) Improved communication between patients and physicians, 2.) Improved communication with the health authorities for better reimbursement of antiosteoporotic medication. The latter will allow access to effective treatment for a larger proportion of patients with osteoporosis at the current economic state in the country.

\section{P148. ACCUMULATED BREAST-FEEDING OF 6 OR MORE MONTHS IS ASSOCIATED WITH OSTEOPOROTIC FRACTURES AND LOW BONE MASS IN A COHORT OF POSTMENOPAUSAL WOMEN}

X. Nogués, M.J. Peña, M.A. Checa, I. Aymar, L. Mellibovsky, A. Supervia, A. Garrido, J. Carbonell, L. Perez-Edo, J.C. Monllau, E. Caceres, R. Carreras, A. Díez-Pérez; URFOA-IMIM Hospital del Mar Barcelona, Autonomous University of Barcelona, Barcelona, Spain

Objectives: Breast-feeding is related with hormonal alterations and increased calcium expenditure in the mother to provide sufficient calcium to the neonate. Therefore, some bone loss occurs is this period although their relationship with of osteoporosis in postmenopausal women is controversial. We analyze this subject in a cohort of Spanish postmenopausal women.

Patients and methods: Seventeen hundred and thirty eight consecutive unselected Spanish postmenopausal women with at least one pregnancy, aged $53.1+7.4$ years (mean \pm SD) from a Menopausal Unit were recruited in a prospective observational study. Medical record, including gynecological data, history of bone fracture and risk factors for osteoporosis, was collected. Bone mineral density (BMD) was measured in lumbar spine (LBMD) and femoral neck (Fn-BMD) using dual-energy X-ray absorptiometry (Hologic QDR 4500 SL). Women with secondary osteoporosis were excluded. Data were analyzed using SPSS 12.01. T-test and ANCOVA were performed.

Results: Age at menopause was 47.8 + 4.9, menarche $12.8+$ 1.6 and accumulates breast-feeding months (ABF) $11.9+13.7$. In a $69.3 \%$ of women ABF were six or more. L-BMD was $0.901+$ $0.14 \mathrm{gr} / \mathrm{cm}^{2}$ in women with less than 6 month ABF vs. $0.862+$ 0,14 in those with 6 or more $(p=0.004)$. Fn-BMD was also significantly different $(0.720+0.11$ vs. $0.709+0.11, \mathrm{p}=0.018$ respectively). Previous osteoporotic fracture was present in $7.8 \%$ of women with $\mathrm{ABF}<6$ months vs. 17.5 in those with 6 or more (RR 1.98; 95\% CI 1.42-2.76; $p<0.0001$ ). ABF in women without fractures were $7.19+10,2$ and in women with fractures were 12.8 $+22.8 ; \mathrm{p}=0.0001$

Conclusion: ABF is relevant for the development of osteoporosis after menopause. Six or more months of ABF are associated with decreased lumbar spine and femoral neck BMD as well as with increased prevalent fractures in a cohort of Spanish postmenopausal women.

\section{P149. MENARCHE OLDER THAN 12 IS ASSOCIATED WITH OSTEOPO- ROTIC FRACTURES AND LOW BONE MASS IN A COHORT OF POST- MENOPAUSAL WOMEN}

X. Nogués, M.J. Peña, M.A. Checa, I. Aymar, L. Mellibovsky, A. Garrido, J. Blanch, P. Benito, J.C. Monllau, E. Caceres, R. Carreras, A. Díez-Pérez; URFOA-IMIM Hospital del Mar Barcelona, Autonomous University of Barcelona, Barcelona, Spain

Objective: Estrogens are related with postmenopausal osteoporosis. Years since menopause and fertile years are strong predictors of low bone mass. However studies on age at menarche by itself are controversial. We study this variable in a cohort of Spanish postmenopausal women.

Patients and methods: Seventeen hundred and twenty seven consecutive unselected Spanish postmenopausal women aged 53.5 +7.6 years (mean \pm SD) from a Menopausal Unit. Medical record, including gynecological data, history of fractures and risk factors for osteoporosis, was collected. Bone mineral density (BMD) was measured in lumbar spine (L-BMD) and femoral neck (Fn-BMD) using dual-energy X-ray absorptiometry (Hologic QDR $4500 \mathrm{SL}$ ). Women with surgical menopause and secondary osteoporosis were excluded. Data were analyzed using SPSS 12.01 and t-test, ANCOVA and multiple regression analysis were performed.

Results: Age of menopause was $48.6+4.3$ and menarche 12.8 +1.6 . A $55.1 \%$ of women had menarche $>12$. L-BMD in women with menarche $<12$ was $0.902+0.14 \mathrm{gr} / \mathrm{cm}^{2}$ and in women with menarche at 13 or older $0.867+0,14(\mathrm{p}<0.0001$, weight-adjusted). Fn- BMD were also significantly different 0.724 +0.11 vs. $0.694+0.11$ respectively ( $\mathrm{p}<0.000$, weight-adjusted). Prevalent osteoporotic fracture was present in $11 \%$ of women with menarche $<12$ vs. $15.6 \%$ in those $\geq 12$ (RR $1.43 ;$ IC $95 \%$ $1.02-2.01 ; \mathrm{p}=0.03)$. Age of menarche (mean $+\mathrm{SD})$ in women without fractures was $12.8+1.6$ and in women with fractures was13.4 $+1.8 ; \mathrm{p}=0.001$. By multiple regression analysis age of menarche and body weight remained significantly associated with L-BMD and femoral neck BMD.

Conclusion: Age of menarche is a strong predictor of low bone mass and fractures in a cohort of Spanish postmenopausal women. Preventive measures before menopause could be recommended in women with menarche older than 12 in Spanish population.

\section{P150. CONSTRUCT VALIDATION OF THE „PATIONNAIRE“TM, A QUES- TIONAIRE TO ASSESS SYMPTOMS AND DISABILITIES OF THE MUSC- ULOSCELETAL SYSTEM}

U. Zuberbuehler ${ }^{1}$, J.F. Huber ${ }^{1}$, M.D. Zumstein ${ }^{1}$, J. Huesler ${ }^{2}$, G.B. Ruflin'; ${ }^{1}$ Orthopaedic Clinic, Kantonsspital AG, Aarau, Switzerland, ${ }^{2}$ Institut for Mathematical Statistics, University of Berne, Bern, Switzerland

Introduction: The 'pationnaire' is a questionaire for assessment of any disorder of the musculosceletal system. The questionaire consists out of four domains, with one page each to report: symptoms, disabilities, treatments and main complaint, socio-ethno-demography, morphometry and self-perception. For ease of understanding it was designed with colors and pictograms. To improve simple utilisation and quick analysis the VCS (visual circle scale, Poster ECCEO 2005) was used ( $r>0.85$ to VAS, Likert Scale).

The aim of this study was to test the construct validity (agreement), and the ability and time to fill it out without help.

Persons/patients and methods: The persons/patients were randomly selected. The time to completion was measured. After that they were personally interviewed to assess the correlation and uncover any sources of misunderstanding or misinterpretation. At the end every person/patient was asked about their understanding, formulations, difficulties, missing questions and general impression.

Results: 78 persons/patients (50 women, 28 men) were included, average age was 46.3 years. $97 \%$ (76) could fill out the ,pationnaire' without help, 2 needed help. Average time for completion was 9.9 min (range 3 - 45 mins). Complete agreement between the questionaire and the perceived symptoms/disabilities was found in $94 \%(n=73)$, it was partial in $3.8 \%(n=3)$, and, no agreement" occurred in $2.2 \% \quad(n=2$, persons, both of whom needed support). The understanding was rated very good in $98 \%$ and difficult in $2 \%$ (elderly persons $>80$ years). The formulation 'my symptoms are' was preferred by everybody. The overall rating was good or very good for all persons, although older people with co-morbidities needed help.

Conclusion: The "pationnaire" can be used to assess symptoms, disabilities, therapies, main complaint, sociodemography, morphometry and self-perception. Most people ( $>95 \%)$ can fill it out within 15 minutes. The agreement between individual perception and answer on the pationnaire is high. 
P151. COMPARISON OF PERIPHERAL QUANTITATIVE COMPUTED TOMOGRAFIE (PQCT) AND DIGITAL X-RAY RADIOGRAMMETRY AS A NEW METHOD FOR QUANTIFICATION OF JUXTA-ARTICULAR DEMINERALIZATION IN RHEUMATOID ARTHRITIS

J. Böttcher ${ }^{1}$, A. Pfeil ${ }^{1}$, B. Heinrich ${ }^{1}$, M.L. Schäfer ${ }^{1}$, G. Lehmann ${ }^{2}$, A. Petrovitch ${ }^{1}$, A. Malich ${ }^{3}$, G. Hein ${ }^{2}$, G. Wolf ${ }^{2}$, W.A. Kaiser'; ${ }^{1}$ Institute of Diagnostic and Interventional Radiology, FriedrichSchiller-University Jena, Germany, ${ }^{2}$ Clinic of Internal Medicine III, Department for Rheumatology and Osteology, FriedrichSchiller-University Jena, Germany, ${ }^{3}$ Sued-Harz Klinikum, Department of Radiology, Nordhausen, Germany

Aims: As a generalized inflammatory disease rheumatoid arthritis involves several joints as well as synovial sheaths of tendons and bursae. Both, rheumatoid arthritis and its extended treatment (i.e. cortison therapy) cause a significant systemic bone loss in a high number of patients and additionally a periarticular disease-related osteoporosis, especially at the hands and feet in an early stage of the disease. To evaluate changes of bone mineral density (BMD) using a radiogrammetrically based bone densitometric technology on patients suffering from rheumatoid arthritis with and without corticoid therapy. To compare this method with peripheral Quantitative Computed Tomography.

Patients and Methods: 90 patients underwent a prospective analysis of BMD via DXR and pQCT. Radiographs have been subjected to DXR for estimating BMD from a plain radiograph of the non dominant hand using Pronosco X-Posure System (Sectra, Sweden), which digitizes a radiograph with a scanner and subsequently derives a BMD of the three middle metacarpals; pQCT calculated BMD (total, trabecular, cortical) regarding distal radius. All patients were divided into a subgroup $(n=52)$ with (5 $\mathrm{mg}$ cortison /d for 6 months) and without corticoid therapy $(\mathrm{n}=38)$.

Results: The mean value of DXR-BMD decreased from 0.57 $\mathrm{g} / \mathrm{cm}^{2} \pm 0.08$ (Larsen Score 1) to $0.45 \mathrm{~g} / \mathrm{cm}^{2} \pm 0.11$ (Larsen Score 5). The relative decrease of BMD measured by DXR between the highest and lowest score was $20 \%(\mathrm{p}<0.05)$. The relative decrease of BMD (pQCT) from Larsen-Score 1 to Score 5 showed a significant result regarding pQCT-BMD (trabecular, as most metabolic active bone tissue) with $16 \%$ $(\mathrm{p}<0.05)$. No significant demineralization confirmed for $\mathrm{pQCT}$ BMD (total) with $12 \%$ and pQCT-BMD (cortical) with $2 \%$. Correlation between DXR-BMD and pQCT-BMD (total and trabecular) demonstrated a significant result $(\mathrm{R}=0,53$ versus $0,55 ; p<0,01)$ for the cortison-subgroup. Correlation of DXRBMD with pQCT-BMD (cortical) was lower $(\mathrm{R}=0,37$, $\mathrm{p}<0,05)$. Equal results were verified for patients without corticoid therapy.

Conclusion: The digital radiogrammetry can exactly measure cortical differences of bone mineralization on patients suffering from rheumatoid arthritis and seems to be able to quantify disease-related periarticular loss of bone mineral density depend on severity and independent from corticoid influence.

P152. THE IMPACT OF BODY FAT ON METACARPAL BONE MINERAL DENSITY MEASURED BY DIGITAL X-RAY RADIOGRAMMETRY (DXR)

J. Böttcher ${ }^{1}$, A. Pfeil ${ }^{1}$, F. Teufl ${ }^{3}$, A. Petrovitch ${ }^{1}$, G. Lehmann ${ }^{2}$, M.L. Schäfer ${ }^{1}$, A. Malich ${ }^{4}$, H.-J. Mentzel ${ }^{1}$, G. Wolf ${ }^{2}$, G. Hein ${ }^{2}$, W.A. Kaiser ${ }^{1}$; ${ }^{1}$ Institute of Diagnostic and Interventional Radiology, Friedrich-Schiller-University Jena, Germany, ${ }^{2}$ Clinic of Internal Medicine III, Department for Rheumatology and Osteology, Friedrich- Schiller-University Jena, Germany, ${ }^{3}$ Clinic of Diagnostic Radiology, Stollberg, Germany, ${ }^{4}$ Sued-Harz Klinikum, Department of Radiology, Nordhausen, Germany

Objective: In addition to many established osteodensitometric methods, Digital X-ray Radiogrammetry is considered to be a reliable and precise method for the measurement of cortical bone mineral density (BMD) on metacarpals. To investigate the association between different body constitution characteristics and osteodensitometric parameters measured by DXR in healthy adults.

Material and Methods: In a prospective study 246 healthy individuals without bone tissue-affecting diseases in their clinical history underwent analysis by DXR. Radiographs of the non dominant hand have been used for the measurements of BMD, Porosity Index (PI) and Metacarpal Index (MCI) by the Pronosco X-Posure System (Version 2, Sectra, Sweden), which digitized images with a scanner and derived radio-geometrical parameters from the three middle metacarpals. For each person height, weight and body mass index (BMI) were recorded. All individuals are divided into subgroups as follows: Under-weight (BMI < 25), norm-weight (BMI $20-25)$ and over-weight (BMI $>25)$.

Results: For all individuals and for all BMI subgroups there was a close association between both height $(0.55<20)$ our study documents an increase of PI which characterizes cortical porosity at the metacarpals. Therefore DXR, which provides a precise and widely available technique without influence of soft tissue at the measurement site, seems to be a promising tool for the quantification of marginal alterations in cortical BMD.

\section{P153. EVALUATION OF PERIPHERAL AND CENTRAL BONE MINERAL DENSITY IN RHEUMATOID ARTHRITIS}

J. Böttcher ${ }^{1}$, A. Pfeil ${ }^{1}$, M.L. Schäfer ${ }^{1}$, G. Lehmann ${ }^{2}$, A. Petrovitch $^{1}$, A. Malich ${ }^{1}$, G. Hein ${ }^{2}$, G. Wolf ${ }^{2}$, W. A. Kaiser ${ }^{1}$; ${ }^{1}$ Institute of Diagnostic and Interventional Radiology, Friedrich-SchillerUniversity Jena, Germany, ${ }^{2}$ Clinic of Internal Medicine III, Department for Rheumatology and Osteology, Friedrich- Schiller-University Jena, Germany

Objective: To evaluate the ability to measure variations of bone mineral density (BMD) using Digital X-ray Radiogrammetry (DXR) on patients with rheumatoid arthritis (RA) and to differentiate systemic from disease-related demineralization. To compare this method with Dual Energy X-ray Absorptiometry (DXA) including comparison between patients with and without corticoid therapy.

Material and Methods: 152 patients with verified RA underwent analysis of BMD by DXR, which calculated BMD and Metacarpal Index (MCI) from a plain radiograph of the non dominant hand using Pronosco X-Posure System TM (Sectra, Sweden). This technique digitized a radiograph with a scanner and subsequently calculated cortical thickness of the three middle metacarpals. Based on the mean bone volume per area and the estimated porosity of the cortical bone, DXR-BMD was computed. DXA (Hologic QDR-4500) measured BMD regarding total femur and lumbar spine.

Results: Correlation of BMD-DXR versus BMD-DXA was significant (femur: $R=0.59, \mathrm{p}<0.01$; lumbar spine: $\mathrm{R}=0.47$, $\mathrm{p}<0.01)$. The mean value of BMD decreased from $0.55 \mathrm{~g} /$ $\mathrm{cm}^{2} \pm 0.08$ (Larsen Score 1) to $0.44 \mathrm{~g} / \mathrm{cm}^{2} \pm 0.11$ (Larsen Score 5). Equal results were verified for the Steinbroker Stage and MCI. The relative decrease of BMD measured by DXR between the highest and lowest score was $20 \%$ for both scores $(p<0.05)$, whereas DXA showed no significant results depend on severity. Correlation regarding BMD-DXR versus BMD-DXA (femur) was $\mathrm{R}=0.60(\mathrm{p}<0.01)$ in patients with corticoid therapy and $\mathrm{R}=0.34$ (n.s.) without corticoid influence.

Conclusion: Calculated by DXR a significant reduction of BMD depending on severity of RA has been verified, whereas DXA-BMD has been demonstrated no significant results. These results indicate that the progress of RA itself allows a quantification of the sum of disease-related periarticular cortical demineralization via DXR. Furthermore the correlation between DXR-BMD and DXA-BMD (total femur) shows only a closed and significant association in patients with steroid therapy. This result points at the ability of DXA and DXR for exact determination of systemic bone mineral density loss, but also this fact may be shows the lack of DXA in the detection of periarticular disease-related demineralization. Possible application of DXR should be the additional BMD- 
calculation on routinely performed follow-up radiographs for quantification of demineralization.

P154. PERIARTICULAR DEMINERALIZATION AND JOINT SPACE NARROWING IN RHEUMATOID ARTHRITIS DETECTED BY DIGITAL X-RAY RADIOGRAMMETRY (DXR) AND RADIOGRAMMETRY KIT (RK)

J. Böttcher ${ }^{1}$, A. Pfeil ${ }^{1}$, A. Petrovitch ${ }^{1}$, A. Kramer ${ }^{1}$, G. Lehmann ${ }^{2}$, M.L. Schäfer ${ }^{1}$, A. Malich ${ }^{3}$, G. Hein ${ }^{2}$, G. Wolf ${ }^{2}$, W.A. Kaiser', ${ }^{1}$ Institute of Diagnostic and Interventional Radiology, FriedrichSchiller-University Jena, Germany, ${ }^{2}$ Clinic of Internal Medicine III, Department for Rheumatology and Osteology, FriedrichSchiller-University Jena, Germany, ${ }^{3}$ Sued-Harz Klinikum, Department of Radiology, Nordhausen, Germany

Objective: This study evaluates Digital X-ray Radiogrammetry (DXR) and Radiogrammetry Kit (RK) as new diagnostic tools for quantification of disease-related periarticular osteoporosis and joint space narrowing dependent on the course of 6 years in patient suffering form rheumatoid arthritis (RA).

Material and Methods: 258 patients with verified RA (ACRCriteria) underwent computerized calculations of Bone Mineral Density (BMD) and Metacarpal Index (MCI) by DXR. BMD and MCI were estimated on the three middle metacarpals from radiographs of hand using the Pronosco X-Posure System (Version 2, Sectra, Sweden). The RK estimated the Joint Space Distance of the metacarpal-phalangeal articulation II -V (JSDMCP). This RK-technique performed a joint space analysis of the metacarpal-phalangeal articulation II $-\mathrm{V}$. The distance between the joint edges were given in $\mathrm{cm}$. A digital performed radiograph of the non-dominant hand for DXR- and RK measurements was annually acquired for each patient.

Results: All RK- and DXR-parameters significantly decreased depending on the course of RA. The relative joint space narrowing ranged from $-18.8 \%$ (JSD-MCP V) to $-31.6 \%$ (JSDMCP II). The JSD-MCP (mean) showed a relative reduction of $-23.5 \%$. On an average our study verified an annual narrowing of the JSD-MCP (mean) with $-2.0 \%$. In the first year of RA manifestation, thus technique showed a narrowing of $-11.8 \%$ (JSD-MCP mean). After a disease duration of more than one year a more flattened decline of JSD-MCP (mean) was observed $(-11.7 \%)$. Over a period of six years a relative decrease of DXRBMD $(-32.1 \%)$ and DXR-MCI $(-33.3 \%)$ was observed, which were pronounced in early RA (disease duration: $<1$ year).

Conclusion: In this longitudinal study significant marked reduction of JSD-MCP as well as DXR-BMD could be verified accentuated in the early and prolonged course of RA. Therefore the operator-independent RK- and DXR-technology could be an important diagnostic tool in RA by which the clinician can detect those patients who require more aggressive management of their RA in order to prevent joint destruction, which will inevitably lead to major disability.

\section{P155. MUSCLE MASS AND FUNCTIONAL OUTCOME AFTER HIP} FRACTURE IN 200 ELDERLY WOMEN

M. Di Monaco ${ }^{1}$, F. Vallero ${ }^{1}$, R. Di Monaco ${ }^{2}$, R. Tappero ${ }^{1}$, A. Cavanna ${ }^{1}$; ${ }^{1}$ Osteoporosis Research Center, Presidio Sanitario San Camillo, Torino, Italy, ${ }^{2}$ Institute of Social and Economic Research SRF, Società Ricerca e Formazione, Torino, Italy

Objective: The loss of muscle mass with aging (sarcopenia) has been suggested to affect the ability to function in the elderly, imposing a relevant economic burden on healthcare services. Disability after hip fracture is common: only $40 \%$ of the patients fully regain their pre-fracture level of independence, and up to $25 \%$ may require long-term nursing home care. Sarcopenia may play a pivotal role in causing disability after hip fracture, but this hypothesis is not supported by the literature. Our aim was to investigate the association between muscle mass and functional outcome in a sample of hip-fracture women.

Methods: 200 of 230 hip-fracture women admitted consecutively to a rehabilitation hospital were investigated in this survey study. Lean mass (LM) was assessed by dual-energy x-ray absorptiometry, $23.1 \pm 7.9$ (mean \pm SD) days after fracture occurrence. Appendicular LM (aLM) was calculated as the sum of LM in arms and legs. Because metal implants (prostheses and nails) affect the regional assessment of body composition, aLM was corrected by substituting LM in unfractured leg for LM in fractured leg: corrected aLM $=(\mathrm{LM}$ in unfractured leg $\mathrm{x} 2)+$ LM in arms. We used two approaches to adjust corrected aLM for body size: corrected aLM divided by height squared (aLM/ $\mathrm{ht}^{2}$ ), and corrected aLM adjusted for height and fat mass (residuals). Functional outcome was assessed by using Barthel index scores

Results: After adjustment for body size, corrected aLM was neither significantly correlated with Barthel index scores nor with the change in Barthel index scores following rehabilitation. The results did not change after further adjustment for age, fat mass, Barthel index scores before rehabilitation, and time spent between fracture occurrence and DXA assessment. Also, after stratification for quintiles of aLM/ht ${ }^{2}$ and residuals, no significant differences in functional recovery were found among the five groups.

Conclusions: Because LM assessed after hip fracture was not associated with the functional outcome in women, LM assessment should not be recommended for predicting the functional recovery. Furthermore, caution is needed in using LM as a surrogate endpoint in clinical trials aimed at improving the ability to function in the elderly.

P156. 25-HYDROXYVITAMIN D, PARATHYROID HORMONE, AND FUNCTIONAL OUTCOME AFTER HIP FRACTURE

M. Di Monaco'1, F. Vallero ${ }^{1}$, R. Di Monaco ${ }^{2}$, R. Tappero ${ }^{1}$, A. Cavanna ${ }^{1}$; ${ }^{1}$ Osteoporosis Research Center, Presidio Sanitario San Camillo, Torino, Italy, ${ }^{2}$ Institute of Social and Economic Research SRF, Società Ricerca e Formazione, Torino, Italy

Objectives: There is increasing interest in the effects of vitamin $\mathrm{D}$ and parathyroid hormone (PTH) on extraskeletal tissues, including the muscle. These effects may explain impairment in functional ability found in vitamin D deficient subjects. Our aim was to investigate the role both of vitamin D and of PTH in affecting the ability to perform activities of daily living after hip fracture.

Methods: We evaluated 521 hip-fracture patients who had been admitted consecutively to our Physical Medicine and Rehabilitation service. Eighteen of the 521 patients were excluded because their hip fracture was caused by either major trauma or cancer affecting the bone. Twenty-six patients were excluded because they died or were transferred to other hospitals. Twenty-one of the remaining 477 patients were excluded because they had abnormally high serum calcium. The final study sample included 456 patients. A blood sample was collected $22.3 \pm 7.1$ days (mean \pm SD) after fracture occurrence, while patients were fasting. PTH was assessed by two-site chemiluminescent enzyme-labelled immunometric assay; 25-hydroxyvitamin D by an immunoenzymatic assay. Functional outcome was assessed after acute inpatient rehabilitation by using the Barthel index score.

Results: The functional scores were significantly correlated with serum levels of 25 -hydroxyvitamin $\mathrm{D}(\mathrm{r}=0.190 ; \mathrm{p}<0.001)$, PTH $(\mathrm{r}=-0.164 ; \mathrm{p}<0.001)$, and 25-hydroxyvitamin D/PTH ratio $(r=0.261 ; p<0.001)$. At multiple regressions, 25 -hydroxyvitamin D and PTH levels were independently associated with Barthel index scores. The correlation between 25 -hydroxyvitamin $\mathrm{D} / \mathrm{PTH}$ ratio and Barthel index scores was significantly stronger than the one between 25-hydroxyvitamin D and Barthel index scores (difference between the two correlation coefficients $=0.071$; $95 \% \mathrm{CI}=0.009-0.133 ; \mathrm{p}=0.011)$. The significant association between 25-hydroxyvitamin D/PTH ratio and Barthel index scores persisted after adjustment for twelve prognostic factors $(\mathrm{p}=$ 0.012 ). On the whole, the panel of prognostic factors we studied predicted $50.1 \%$ of the variance of the functional score.

Conclusions: Data shows that both PTH and 25-hydroxyvitamin $\mathrm{D}$ were significantly associated with ability to function after hip fracture, and suggests that the two hormones act through 
independent mechanisms. The 25 -hydroxyvitamin $\mathrm{D} / \mathrm{PTH}$ ratio significantly contributed to a predictive model of functional outcome.

\section{P157. THE INFLUENCE OF BODY MASS INDEX AND TEXTILE MATERIAL} ON REPRODUCIBILITY OF DUAL X-RAY AND LASER (DXL)

A. Pfeil ${ }^{1}$, J. Böttcher ${ }^{1}$, A. Kramer ${ }^{1}$, A. Petrovitch ${ }^{1}$, G. Lehmann ${ }^{2}$, M. Schmidt ${ }^{1}$, M.L. Schäfer ${ }^{1}$, A. Malich ${ }^{3}$, G. Wolf ${ }^{2}$, G. Hein ${ }^{2}$, W.A. Kaiser ${ }^{1}$; ${ }^{1}$ Institute of Diagnostic and Interventional Radiology, Friedrich-Schiller-University Jena, Germany, ${ }^{2}$ Clinic of Internal Medicine III, Department for Rheumatology and Osteology, Friedrich- Schiller-University Jena, Germany, ${ }^{3}$ Sued-Harz Klinikum, Department of Radiology, Nordhausen, Germany

Objective: Dual Energy X-ray Absorptiometry (DXA) is a widely available technique for the assessment of osteoporosis. The usual DXA has an accuracy between $2 \%$ and $20 \%$. But a high precision is necessary for diagnosis and monitoring of minor changes in the course of osteoporosis. Dual X-Ray and Laser (DXL) is a new technique based on DXA technology combined with a Laser for measurements peripheral Bone Mineral Density (BMD) at the calcaneus. The purpose of this study is to evaluate reproducibility of DXL dependent on body fat and textile material in the measurement area.

Material and Methods: Reproducibility of DXL was measured on five healthy volunteers. The DXL (Demetech AB, Solna, Sweden) technique uses two X-ray energies in combination with laser measurement of the object thickness in order to determine all three tissue components (bone mineral, lean soft tissues, and fatty tissues). For each subjects measurements of DXL-BMD on the right and left calcaneus was performed. Data of height, weight and BMI (Body Mass Index) were acquired and the study group was subdivided into individuals with underweight $(\mathrm{BMI}<25)$, normal-weight (BMI 20-25) and overweight (BMI $>25)$. The influence of textile material was evaluated by five measurements with a textile sock (material: $100 \%$ cotton, material thickness: 1,3 $\mathrm{mm})$.

Results: Reproducibility of the DXL-BMD was CV $=1.75 \%$ (left) and $\mathrm{CV}=1.05 \%$ (right) for the underweight group. The over-weight group showed higher coefficients of variation (left: $\mathrm{CV}=2.51 \%$, right: $2.23 \%$ ) compared to the norm-weight group (left: $\mathrm{CV}=3.08 \%$, right $1.10 \%$ ). The data showed the highest coefficient of variation for under-weight individuals $\mathrm{CV}=3.30 \%$ regarding reproducibility influenced by textile material. Improved results were observed for the norm-weighted group $(\mathrm{CV}=2.97 \%)$ as well as over-weight $(\mathrm{CV}=3.04 \%)$.

Conclusion: The DXL shows a remarkable high reproducibility. However our data showed an influence by body mass and body fat. Based on this results a accurate and precise measurement of BMD by this new technique is available. In addition DXL seems to be a promising diagnostic tool in the assessment of osteoporosis at peripheral measurements.

\section{P158. PERIARTICULAR DEMINERALIZATION IN EARLY RHEUMATOID} ARTHRITIS DETECTED BY DIGITAL X-RAY RADIOGRAMMETRY (DXR)

A. Pfeil ${ }^{1}$, J. Böttcher ${ }^{1}$, A. Petrovitch ${ }^{1}$, G. Lehmann ${ }^{2}$, M.L. Schäfer ${ }^{1}$, A. Malich ${ }^{3}$, G. Hein ${ }^{2}$, G. Wolf ${ }^{2}$, W. A. Kaiser ${ }^{1}$; ${ }^{1}$ Institute of Diagnostic and Interventional Radiology, FriedrichSchiller-University Jena, Germany, ${ }^{2}$ Clinic of Internal Medicine III, Department for Rheumatology and Osteology, FriedrichSchiller-University Jena, Germany, ${ }^{3}$ Sued-Harz Klinikum, Department of Radiology, Nordhausen, Germany

Objective: Rheumatoid arthritis (RA) is a systemic inflammatory disease, which prematurely affected the small joints of the hand leading to destruction of periarticular tissue, including juxta-articular bone. Osteoporosis is a major clinical complication in RA and occurs in two forms: periarticular osteoporosis in near proximity to inflamed joints, which is a typical phenomenon in early RA, and generalised osteoporosis affecting the axial and appendicular bones occurring during the course of rheumatoid disease. Recently receptor activator of nuclear $\mathrm{kB}$ ligand (RANKL) and osteoprotegerin (OPG) have been identified as central regulators of osteoclast recruitment and activation and appear to systemically influence bone resorption in the juxtaarticular bone. Our study evaluates the ability of DXR to measure inflammation-related variations of cortical bone partition in patients with early rheumatoid arthritis compared with Dual X-ray absorptiometry (DXA).

Material and Methods: 77 patients with early rheumatoid arthritis (disease duration: $<1$ year) underwent a prospective analysis of Bone Mineral Density (BMD) and Metacarpal Index (MCI) by DXR. BMD and MCI were estimated from plain radiographs of the non-dominant hand using the Pronosco $\mathrm{X}$ Posure System (Version 2.0, Sectra, Sweden), which digitized images with a scanner and derived radio-geometrical parameters from the three middle metacarpals. DXA (Hologic QDR 4500, Waltham, USA) calculated BMD on lumbar spine and proximal femur. C-reactive Protein (CRP) and Erythrocyte Sedimentation Rate (ESR) were recorded. The patients were devided into two groups: group 1: CRP $>20$ and ESR $>15 \mathrm{~mm} /$ first hour $(\mathrm{n}=36)$, group 2: CRP $<20$ and ESR $<15 \mathrm{~mm} /$ first hour $(\mathrm{n}=41)$.

Results: The relative decrease of DXR-BMD between group 1 and 2 was $-15.1 \%(\mathrm{p}<0.01)$. For group 1 versus group 2 DXRMCI showed a significant reduction of $-17.5 \%$. The reduction of DXA-BMD revealed only significant results regarding measurements at the lumbar spine with $-7.7 \%$.

Conclusion: DXR can quantify inflammation-related cortical BMD-loss in patients suffering from early rheumatoid arthritis, whereas Dual X-ray absorptiometry only documents minor alterations of bone tissue at the axial measurement site.

P159. EXPERIMENTAL STUDY TO DETECT THE INFLUENCE OF IMAGE CAPTURING PARAMETERAS ON DIGITAL X-RAY RADIOGRAMMETRY (DXR)

A. Pfeil ${ }^{1}$, J. Böttcher ${ }^{1}$, M.L. Schäfer ${ }^{1}$, A. Petrovitch ${ }^{1}$, G. Lehmann $^{2}$, B. Heinrich ${ }^{1}$, W. Lin ${ }^{3}$, A. Malich ${ }^{4}$, G. Wolf ${ }^{2}$, G. Hein $^{2}$, W.A. Kaiser ${ }^{1}$; ${ }^{1}$ Institute of Diagnostic and Interventional Radiology, Friedrich-Schiller-University Jena, Germany, ${ }^{2}$ Clinic of Internal Medicine III, Department for Rheumatology and Osteology, Friedrich- Schiller-University Jena, Germany, ${ }^{3}$ Institute of Anatomy I, Friedrich-Schiller-University Jena, ${ }^{4}$ Sued-Harz Klinikum, Department of Radiology, Nordhausen, Germany

Objective: To evaluate the importance of different image capturing conditions, which may influence the characteristics of radiographs and consequently may impact calculation of bone mineral density (BMD) using Digital X-ray Radiogrammetry (DXR). The investigated parameters include film-focus distance (FFD), film quality (sensitivity of 200 versus 400), film brand (Kodak T-MAT-Plus, Agfa Curix), exposure level (mAs) and tube voltage $(\mathrm{kV})$. Furthermore the impact of the imaging technology used in terms of conventional radiographs versus digital $\mathrm{x}$ ray images is considered (Siemens Polydoros SX 80, Diagnost Philips Optimus), either in the original digital format or through digitization of print-outs. The reproducibility of DXR-BMD and Metacarpal Index (MCI) are compared across these different image modalities.

Material and Methods: Radiographs of the left hands of deceased males were acquired three times using systematically varied parameters: 4-8 mAs, 40-52kV, FFD: $90-130 \mathrm{~cm}$, film sensitivity: 200/400 and image modality/source (conventional versus original digital radiographs as well as digital print-outs). All BMD and MCI measurements were obtained with the DXR-technology (Pronosco-Xposure system, Sectra Pronosco A/S, Denmark), which is originally calibrated for analysis of conventional radiographs.

Results: BMD-calculation was not noticeably affected by changes of FFD (conventional: $\mathrm{CV}=0.98 \%$ ), exposure level (conventional: $\mathrm{CV}=0.49 \%$; digital: $\mathrm{CV}=0.35 \%$ ), film sensitivity/ film brand (conventional: $\mathrm{CV}=0.33 \% / 0.49 \%$ ), but was influenced by tube voltage (conventional: $\mathrm{CV}=1.32 \%$ for Agfa and 
$\mathrm{CV}=0.99 \%$ for Kodak; digital: $\mathrm{CV}=0.57 \%$ for Philips printouts and $\mathrm{CV}=0.50 \%$ with original digital images versus $\mathrm{CV}=2.05 \%$ for Siemens print-outs and $1.35 \%$ with original digital images). No significant differences in the sensitivity towards image capturing parameters were observed between BMD and MCI.

Conclusion: Digital X-ray Radiogrammetry provides measurements of MCI and BMD with high precision. The measurements are unaffected by all tested image capturing conditions with the exception of tube voltage. Regarding conventional and digital radiographs, especially digital printouts, our data revealed a significant influence of varied tube voltage on DXR-BMD and MCI measurements. In addition an influence of the applied image modality is detected between the original digital images and their corresponding printed versions.

P160. DISEASE ACTIVITY DEPENDENT PERIARTICULAR BONE LOSS IN RHEUMATOID ARTHRITIS MEASURED BY MULTI-SITE QUANTITATIVE ULTRASOUND AND DIGITAL X-RAY RADIOGRAMMETRY (DXR)

A. Pfeil ${ }^{1}$, J. Böttcher ${ }^{1}$, H.-J. Mentzel ${ }^{1}$, A. Petrovitch ${ }^{1}$, G. Lehmann $^{2}$, M.L. Schäfer ${ }^{1}$, A. Malich ${ }^{3}$, G. Wolf ${ }^{2}$, G. Hein ${ }^{2}$, W. A. Kaiser ${ }^{1}$; ${ }^{1}$ Institute of Diagnostic and Interventional Radiology, Friedrich-Schiller-University Jena, Germany, ${ }^{2}$ Clinic of Internal Medicine III, Department for Rheumatology and Osteology, Friedrich- Schiller-University Jena, Germany, ${ }^{3}$ Sued-Harz Klinikum, Department of Radiology, Nordhausen, Germany

Objective: To differentiate the influence of inflammation and soft tissue swelling on the measurements of peripheral bone status in patients with rheumatoid arthritis (RA) using multi-site Quantitative Ultrasound (QUS) compared to Digital X-ray Radiogrammetry (DXR).

Material and Methods: 53 patients with verified RA underwent measurements of QUS (Sunlight multi-site Omnisense 7000, Sunlight Medical Ltd., Tel Aviv, Israel) with estimation of the Speed of Sound (QUS-SOS) at the distal radius and at the phalanx III. Also Bone Mineral Density (DXR-BMD) and Metacarpal Index (DXR-MCI) were estimated on the metacarpals II-IV using DXR-technology (Pronosco X-Posure System ${ }^{\mathrm{TM}}$ Version 2.0, Pronosco/Sectra ASS, Denmark). Disease activity of RA was estimated by Erythrocyte Sedimentation Rate (ESR; in $\mathrm{mm} / 1$ st hour).

Results: For the group with minor disease activity $(\mathrm{ESR} \leq 20$ $\mathrm{mm} / 1$ st hour) QUS-SOS (phalanx) was significantly associated to DXR-BMD $(\mathrm{R}=0.67, \mathrm{p}<0.01)$ and DXR-MCI $(\mathrm{R}=0.54$, $\mathrm{p}<0.05)$. Lower correlation coefficients were revealed between QUS-SOS (radius) and DXR-parameters (DXR-BMD: $\mathrm{R}=0.39$, $\mathrm{p}<0.05$; DXR-MCI: $0.49, \mathrm{p}<0.05$ ). In the case of high disease activity (ESR $>20 \mathrm{~mm} / 1 \mathrm{st}$ hour) QUS-SOS of the radius showed a closed correlation to DXR-BMD $(\mathrm{R}=0.74 ; \mathrm{p}<0.01)$ and DXRMCI $(R=0.89 ; p<0.01)$, which surpassed the correlation coefficients compared to patients with minor disease activity of RA. For QUS-SOS (phalanx) no significant correlation to DXRparameters could be observed in patients with high disease activity.

Conclusion: Multi-site QUS and in a significant extent DXR can also distinguish between patients with minor disease activity and patients with active RA which demonstrates accelerated bone loss characterized by decreased values of DXR- and QUSSOS parameters. In this study the multi-site QUS-device is surpassed by DXR. In patients with accentuated disease activity no significant association among the QUS-parameters themselves and between QUS-SOS (phalanx) and the DXR-parameters exists. These data seem to indicate a significant influence of soft tissue swelling caused by active RA on QUS estimates at the phalanx as measurement site. In patients with increased disease activity QUS-SOS measurements at the radius should be consistently prefered.
P161. SEVERITY DEPENDENT PERIARTICULAR DEMINERALIZATION EVALUATED BY DIGITAL X-RAY RADIOGRAMMETRY AND PERIPHERAL MULTI-SITE QUANTITIATIVE ULTRASOUND IN PATIENT SUFFERING ON RHEUMATOID ARTHRITIS

A. Pfeil ${ }^{1}$, J. Böttcher ${ }^{1}$, H.-J. Mentzel ${ }^{1}$, A. Petrovitch ${ }^{1}$, G. Lehmann $^{2}$, M.L. Schäfer ${ }^{1}$, A. Malich ${ }^{3}$, G. Hein ${ }^{2}$, G. Wolf ${ }^{2}$, W.A. Kaiser ${ }^{1}$; ${ }^{1}$ Institute of Diagnostic and Interventional Radiology, Friedrich-Schiller-University Jena, Germany, ${ }^{2}$ Clinic of Internal Medicine III, Department for Rheumatology and Osteology, Friedrich- Schiller-University Jena, Germany, ${ }^{3}$ Sued-Harz Klinikum, Department of Radiology, Nordhausen, Germany

Objective: The development of secondary osteoporosis in rheumatoid arthritis (RA) is recently well recognized, showing demineralization at axial and in particular at periarticular peripheral bone sites. The purpose of our study was to evaluate the ability of multi-site Quantitative Ultrasound (QUS) compared to Digital X-ray Radiogrammetry (DXR) for the quantification of cortical bone loss dependent on the severity of rheumatoid arthritis.

Material and Methods: 53 patients with verified RA underwent measurements of QUS (Sunlight multi-site Omnisense 7000, Sunlight Medical Ltd., Tel Aviv, Israel) with estimation of the Speed of Sound (QUS-SOS) at the distal radius and at the phalanx III. Also Bone Mineral Density (DXR-BMD) and Metacarpal Index (DXR-MCI) were estimated on the metacarpals II-IV using DXR-technology (Pronosco X-Posure System ${ }^{\mathrm{TM}}$ Version 2.0, Pronosco/Sectra ASS, Denmark). Additionally Larsen-Score and Steinbroker Stage were assessed by digital radiographs.

Results: Regarding the Larsen-Score the DXR-BMD showed a significant decline from $-25.9 \%$ and DXR-MCI also revealed a reduction upto $-38.6 \%$. QUS-SOS (radius) decreased from score 1 to score 5 with $-2.6 \%(\mathrm{p}=$ n.s.). Regarding QUS-SOS (phalanx) a reduction of $-3.9 \%(\mathrm{p}=$ n.s. $)$ was observed. For Steinbroker Stage DXR-BMD significantly decreased with $-27.1 \%$ from stage 1 to stage 4 . In this context the relative reduction of DXR-MCI was $-38.6 \%(\mathrm{p}<0.001)$. QUS-SOS (radius) showed a decline of $-2.7 \%(\mathrm{p}=\mathrm{n} . \mathrm{s}$.), whereas QUS-SOS (phalanx) documented a relative reduction with $-4.0 \%(\mathrm{p}=$ n.s. $)$.

Conclusion: Digital X-ray Radiogrammetry reveal a significant reduction of DXR-BMD as well as DXR-MCI dependent on the severity of RA. Regarding multi-site QUS (radius and phalanx) no significant severity dependent demineralization was verified. Consequently Digital X-ray Radiogrammetry is a promising tool for the diagnosis of periarticular bone loss in RA.

P162. INTER-AND INTRA-RADIOGRAPH REPRODUCIBILITY OF DIGITAL X-RAY RADIOGRAMMETRY (DXR)

A. Pfeil ${ }^{1}$, J. Böttcher ${ }^{1}$, A. Malich ${ }^{4}$, M.L. Schäfer ${ }^{1}$, G. Lehmann ${ }^{2}$, A. Petrovitch ${ }^{1}$, W. Lin $\beta^{3}$, G. Wolf ${ }^{2}$, G. Hein ${ }^{2}$, W. A. Kaiser ${ }^{1}$; ${ }^{1}$ Institute of Diagnostic and Interventional Radiology, FriedrichSchiller-University Jena, Germany, ${ }^{2}$ Clinic of Internal Medicine III, Department for Rheumatology and Osteology, FriedrichSchiller-University Jena, Germany, ${ }^{3}$ Institute of Anatomy I, Friedrich-Schiller-University Jena, ${ }^{4}$ Sued-Harz Klinikum, Department of Radiology, Nordhausen, Germany

Objective: The potential of Digital X-ray Radiogrammetry for estimating cortical bone loss seems to be promising, in particular because of the high precision. To evaluate the inter- and intraradiograph reproducibility of Digital X-ray Radiogrammetry (DXR) under consideration of both conventional and digital performed radiographs.

Material and Methods: Radiographs of the non-dominant hand from two deceased males were performed according to a predefined study protocol. For conventional radiographs (Philips Super $80 \mathrm{CP}$ ) our study considered the following standardized parameters: $42 \mathrm{kV}, 6 \mathrm{mAs}$, Kodak T-MAT-Plus 200 or Agfa Curix 200 film, FFD $100 \mathrm{~cm}$. For digital print-outs (Diagnost Philips 
Optimus and Siemens Polydoros SX 80) the constant image capturing conditions were as follows: $42 \mathrm{kV}, 4 \mathrm{mAs}$, FFD $100 \mathrm{~cm}$. Inter-radiograph-reproducibility of Bone mineral Density (BMD) and Metaxarpal Index (MCI) were evaluated by acquiring ten repeated radiographs with re-positioning under standard settings. To verify intra-radiograph reproducibility, a single conventional image and a single printout underwent 10 repeated DXR analyses $(42 \mathrm{kV}, 6 \mathrm{mAs}$, FFD $100 \mathrm{~cm})$ with the Pronosco-X posure System (Version 2, Sectra, Sweden).

Results: The inter-radiograph reproducibility of DXR-BMD using conventional images under standardised conditions was calculated to be $\mathrm{CV}=0.49 \%$ for Agfa Curix film and $\mathrm{CV}=0.33 \%$ for Kodak T-MAT-Plus film, whereas reproducibility error using digital images ranged from $\mathrm{CV}=0.57 \%$ (Philips, digital printouts) to $\mathrm{CV}=1.18 \%$ (Siemens, digital print-outs). The intraradiograph reproducibility error was observed to be $\mathrm{CV}=0.13 \%$ and $\mathrm{CV}=0.26 \%$ (conventional, Kodak and Agfa film) versus $\mathrm{CV}=0.27 \%$ and $\mathrm{CV}=0.05 \%$ (digital print-outs, Philips and Siemens). No significant differences in the sensitivity towards image capturing parameters were observed between the BMD and MCI.

Conclusion: DXR shows a high inter- and intra - radiograph reproducibility for conventional as well as digital images and seems to be a reliable osteodensitometric technique for evaluation of the peripheral cortical bone which approve the quantification of minor disease-related alterations of the cortical bone partition.

\section{P163. REPRODUCIBILITY OF DUAL ENERGY X -RAY ABSORPTIOMETRY} MEASURED ON RADIUS AND CALCANEUS

A. Pfeil ${ }^{1}$, J. Böttcher ${ }^{1}$, M. Schmidt ${ }^{1}$, G. Lehmann ${ }^{2}$, A. Kramer ${ }^{1}$, A. Petrovitch ${ }^{1}$, M.L. Schäfer ${ }^{1}$, A. Malich ${ }^{3}$, Wolf $^{2}$, G. Hein ${ }^{2}$, G. W. A. Kaiser ${ }^{1}$; ${ }^{1}$ Institute of Diagnostic and Interventional Radiology, Friedrich-Schiller-University Jena, Germany, ${ }^{2}$ Clinic of Internal Medicine III, Department for Rheumatology and Osteology, Friedrich- Schiller-University Jena, Germany, ${ }^{3}$ SuedHarz Klinikum, Department of Radiology, Nordhausen, Germany

Objective: The Dual Energy X-ray Absorptiometry (DXA) is worldwide used in diagnosis and monitoring of osteoporosis. In the following study reproducibility of a new peripheral DXASystem EXA-3000 was verified dependent on Body Mass Index (BMI) and reposition.

Materials and Methods: DXA-Reproducibility was measured on healthy volunteers. For each subject a measurement of peripheral DXA-BMD (EXA-3000, OsteoSys Co Ltd, Seoul, Korea) on the left radius and calcaneus was performed. Data of height, weight and BMI (Body Mass Index) were acquired and the study group was subdivided into individuals with underweight $(\mathrm{BMI}<25)$, normal-weight (BMI 20-25) and overweight $(B M I>25)$. To verify the impact of reposition on DXA-measurements five measurements under reposition were performed. The influence of textile material was evaluated by five measurements with a textile sock at the left foot (material: 100\% cotton, material thickness: $1,3 \mathrm{~mm}$ ).

Results: For healthy controls the reproducibility for the BMD (radius) was $\mathrm{CV}=1.20 \%$ (under-weight), $\mathrm{CV}=2.21 \%$ (normweight) and $\mathrm{CV}=2.29 \%$ (over-weight). Regarding reposition our data showed $\mathrm{CV}=1.12 \%$ (over-weight individuals) and $\mathrm{CV}=1.29 \%$ (under- and norm-weight group). Improved results were observed for the calcaneus: $\mathrm{CV}=1.60 \%$ (under-weight group), $\mathrm{CV}=1.00 \%$ (norm-weight group) as well as $\mathrm{CV}=1.58 \%$ (over-weight group). Reproducibility presented major CV-values for the calcaneus under reposition: $\mathrm{CV}=1.52 \%$ (under-weight group), $\quad \mathrm{CV}=2.89 \% \quad$ (norm-weight group) and $\mathrm{CV}=2.06 \%$ (over-weight group). Regarding textile sock at the foot no increased coefficients of variation with $\mathrm{CV}=1.93 \%$ (under-weight group), $\mathrm{CV}=1.92 \%$ (norm-weight group) and $\mathrm{CV}=1.61 \%$ (overweight group) were observed.

Conclusion: The DXA shows a high reproducibility in comparison to a usual DXA-System. In this study an impact of body fat, reposition and textile material on BMD measurements by EXA-3000 could not be clarified. EXA-3000 seems to be promising technique for quantification peripheral bone mineral density.

\section{P164. SHORT-PRECISION OF MULTI-SITE QUANTITATIVE ULTRA- SOUND (QUS)}

A. Pfeil ${ }^{1}$, J. Böttcher ${ }^{1}$, H.-J. Mentzel ${ }^{1}$, M. L. Schäfer ${ }^{1}$, A Kramer $^{1}$, A. Petrovitch ${ }^{1}$, G. Lehmann ${ }^{2}$, A. Malich ${ }^{3}$, G. Wolf ${ }^{2}, G$ Hein $^{2}$, W.A. Kaiser ${ }^{1}$; Institute of Diagnostic and Interventional Radiology, Friedrich-Schiller-University Jena, Germany, ${ }^{2}$ Clinic of Internal Medicine III, Department for Rheumatology and Osteology, Friedrich- Schiller-University Jena, Germany, ${ }^{3}$ SuedHarz Klinikum, Department of Radiology, Nordhausen, Germany

Objective: To evaluate inter- and intraobserver-reproducibility of multi-site QUS considering the effects of Body Mass Index (BMI) for healthy individuals and quantifying the impact of disease activity in patients suffering from rheumatoid arthritis (RA).

Material and Methods: For the measurement of the SOS on phalanx III and radius a multi-site QUS device (Sunlight multisite Omnisense 7000, Sunlight Medical Ltd., Tel Aviv, Israel) was used. Inter-observer and intra-observer reproducibility were measured including three healthy volunteers from each BMIgroup (BMI£25, mean: 21.7) and over-weight controls (BMI $>25$, mean: 32.6 ), and also six RA patients with advanced disease activity $(\mathrm{n}=3, \mathrm{CRP}>25 \mathrm{mg} / \mathrm{l})$ as well as low disease activity $(\mathrm{n}=3, \mathrm{CRP} 25 \mathrm{mg} / \mathrm{l})$. Inter-observer reproducibility of QUS-SOS was evaluated by three trained observers with ten repeated measurements at the distal radius and third phalanx of patients with RA versus healthy controls for each subgroup at the same day. To verify intra-observer reproducibility a single observer performed 10 repeated QUS-SOS estimates for all 12 individuals.

Results: For healthy controls the intra-observer reproducibility for the QUS-SOS ranged between CV $=0.90 \%$ (normweight, radius) and $\mathrm{CV}=2.55 \%$ (over-weight, phalanx). The over-weight group showed higher CV-values (phalanx $\mathrm{CV}=2.55 \%$, radius $1.43 \%$ ) compared to the norm-weight group (phalanx $\mathrm{CV}=1.05 \%$, radius $0.90 \%$ ). The intra-observer reproducibility of measurements taken from patients with advanced RA were limited (phalanx $\mathrm{CV}=1.88 \%$, radius $1.03 \%$ ). Inter-observer reproducibility for healthy individuals showed the highest coefficient of variation for over-weight individuals regarding QUS-SOS at the distal radius $(\mathrm{CV}=1.79 \%)$ and QUS-SOS at the phalanx $(\mathrm{CV}=2.17 \%)$. Improved results were observed for the BMI group $<25$ (radius: $C V=1.66 \%$, phalanx: $C V=1.40 \%$ ). Inter-observer reproducibility showed minor $\mathrm{CV}$-values for the advanced disease activity group $(\mathrm{CV}=0.94 \%)$ compared to patients with low RA activity $(\mathrm{CV}=0.65 \%)$ measured by QUS-SOS (radius). Regarding QUS-SOS (phalanx), increased coefficients of variation with $\mathrm{CV}=1.81 \%$ (low disease activity) versus $\mathrm{CV}=1.89 \%$ (advanced disease activity) were observed.

Conclusion: QUS-SOS is characterized by a reduced shortterm precision, which is significantly more noticeable in the healthy individuals with pronounced BMI and patients with advanced RA. It may be concluded that the multi-site QUS is of limited priority for patients with active inflammation of the soft tissue in RA and with an elevated BMI, using measurements at the phalanx.

\section{P165. MEASUREMENT OF JOINT SPACE NARROWING BY RADIOG- RAMMETRY KIT (RK) IN RHEUMATOID ARTHRITIS}

A. Pfeil ${ }^{1}$, J. Böttcher ${ }^{1}$, A. Petrovitch ${ }^{1}$, B. E. Seidl ${ }^{1}$, G. Lehmann ${ }^{2}$, A. Kramer ${ }^{1}$, A. Malich ${ }^{3}$, G. Hein ${ }^{2}$, G. Wolf ${ }^{2}$, W. A. Kaiser'; ${ }^{1}$ Institute of Diagnostic and Interventional Radiology, FriedrichSchiller-University Jena, Germany, ${ }^{2}$ Clinic of Internal Medicine III, Department for Rheumatology and Osteology, FriedrichSchiller-University Jena, Germany, ${ }^{3}$ Sued-Harz Klinikum, Department of Radiology, Nordhausen, Germany 
Objective: Inflammatory-related joint alteration in rheumatoid arthritis is associated by cartilage destruction and a consecutive joint space narrowing. This cross sectional study presents the Radiogrammetry Kit (RK) as a new diagnostic tool for quantification of disease-related joint space narrowing dependent on the severity of rheumatoid arthritis (RA).

Material and Methods: Two-hundred fifty-eight Caucasian patients were enrolled without preselection regarding the grade of RA based on ACR-Criteria. For every patient a radiograph of the non-dominant hand was performed. The RK (Version 1.3.5; Sectra Pronosco A/S; Denmark) estimated the Joint Space Distance of the metacarpal-phalangeal articulation II -V (JSDMCP). This RK-technique performed a joint space analysis of a finger joint by detection of the joint edges within a rectangular Region of Interest (ROI). The software based on an edge filtering of the ROI and automatically determined the distance between the two edges. The distance between the bone edges were given in $\mathrm{cm}$ for the metacarpal-phalangeal articulation II $-\mathrm{V}$. Severity of RA were assessed using Larsen Score, Steinbroker Stage and Sharp Score.

Results: For Steinbroker Stage JSD-MCP (mean) shows a significant narrowing of $-52.9 \%$. Regarding the various joints a relative decrease between $-40.0 \%$ (JSD-MCP V) and $-63.2 \%$ (JSD-MCP II) was observed. Regarding the Larsen-Score the Joint space narrowing varied from $-25.0 \%$ (JSD-MCP IV) to $-44.4 \%$ (JSD-MCP II). A reduction of $-35.3 \%$ for JSD-MCP (mean) was observed. In both Sharp Scores JSD-MCP (mean) showed an narrowing of $-41.2 \%$ for the Joint Space Narrowing segment of the score, whereas the Sharp Erosion Score revealed a lower decline of JSD-MCP (mean) with $-29.6 \%$. For the other joints there was a relative reduction between $-16.5 \%$ (JSD-MCP IV, Sharp Erosion part) and $-52.6 \%$ (JSD-MCP II, Sharp Joint Space Narrowing part).

Conclusion: The Radiogrammetry Kit could be a promising, widely available diagnostic tool to supplement the different scoring methods of RA with quantitative data; allowing an earlier and improved diagnosis of RA and more precision in determining disease progression.

\section{P166. DENOSUMAB PHARMACOKINETICS (PK) AND PHARMACODY- NAMICS (PD) AFTER VARIOUS MULTIPLE SUBCUTANEOUS (SC) DOS- ING REGIMENS IN POSTMENOPAUSAL WOMEN WITH LOW BONE MASS}

M.C. Peterson ${ }^{1}$, B.J. Stouch ${ }^{1}$, S.W. Martin ${ }^{1}$, P.D. Miller ${ }^{2}$, M.R. McClung $^{3}$, L. Fitzpatrick ${ }^{1}$; ${ }^{1}$ Amgen Inc., Thousand Oaks, CA, USA, ${ }^{2}$ Colorado Center for Bone Research, Lakewood, CO, USA, ${ }^{3}$ Oregon Osteoporosis Center, Portland, OR, USA

Objective: Receptor activator of NF-кB ligand (RANKL) is essential for the differentiation, function, and survival of osteoclasts. The PK and PD of denosumab (AMG 162), a fully human monoclonal $\mathrm{IgG}_{2}$ antibody to RANKL, were evaluated in a randomized, double-blind, placebo-controlled, multidose study in postmenopausal women with low bone mass (average T-score -2.2 ).

Materials and Methods: Nine cohorts ( $\sim 40$ women per cohort) were randomly assigned to receive the following dosing regimens: placebo, denosumab $6 \mathrm{mg}, 14 \mathrm{mg}$, or $30 \mathrm{mg}$ 3-monthly; denosumab $14 \mathrm{mg}, 60 \mathrm{mg}, 100 \mathrm{mg}$, or $210 \mathrm{mg}$ 6-monthly, or openlabel alendronate $70 \mathrm{mg}$ once weekly. A validated ELISA method was used to quantify serum levels of denosumab. PK parameters were calculated by noncompartmental methods. PD was assessed by measuring change from baseline in the bone resorption marker, serum C-telopeptide (sCTx). Results after one year of treatment are reported.

Results: Denosumab absorption was rapid and prolonged with serum levels ( $\sim 70 \%$ to $80 \%$ of maximum) detectable within 72 hours of dosing. Maximum serum concentrations occurred between 3 and 29 days post-dose and generally increased linearly with dose and dosing frequencies. Following peak concentrations, serum levels generally declined linearly until reaching a concentration threshold below which concentrations fell more rapidly, producing a biphasic concentration-time curve. Denosumab exhibited nonlinear clearance across the dose range investigated, increasing 1.4fold as dose decreased from 30 to $6 \mathrm{mg} \mathrm{3-monthly} \mathrm{and} \mathrm{increasing}$ 2.6 -fold as dose decreased from 210 to $14 \mathrm{mg}$ 6-monthly. Over one year, denosumab did not accumulate appreciably at any dose or dosing frequency investigated, and the PK did not change substantially following multiple dosing. Consistent with PK, multiple dosing of denosumab caused dose-dependent and reproducible reductions in the levels of sCTX, which were rapid (within 72 hours), substantial ( $>70 \%$ reduction), sustained (up to 6 months), and reversible. Cohorts receiving denosumab did not exhibit a significantly greater number of adverse events compared with those receiving placebo or alendronate.

Conclusion: The PK, multiple dose disposition, and PD of denosumab were well characterized in postmenopausal women with low bone mass. These results support infrequent dosing of denosumab in future studies.

\section{P167. ECONOMIC EVALUATION OF ACLASTATM (ZOLEDRONIC ACID 5 MG) VERSUS ACTONELTM (RISEDRONATE) IN TREATMENT OF PAGET'S DISEASE}

N. Mittmann ${ }^{1,2}$, P.K. Isogai ${ }^{2}$, J.D. Adachi ${ }^{3}$, C.M. Kindundu ${ }^{4}$, M. Barbeau ${ }^{4}$; ${ }^{1} \mathrm{HOPE}$ Research Centre, Division of Clinical Pharmacology, Department of Medicine, Sunnybrook and Women's College Health Sciences Centre, Toronto, Ontario, Canada, ${ }^{2}$ Department of Pharmacology, University of Toronto, Toronto, Ontario, Canada, ${ }^{3}$ St. Joseph's Healthcare, McMaster University, Hamilton, Ontario, Canada, ${ }^{4}$ Novartis Pharmaceuticals Canada Inc., Dorval, Quebec, Canada

Objective: Aclasta ${ }^{\mathrm{TM}}$ (zoledronic acid $5 \mathrm{mg}$ ) is a new agent available for the treatment of Paget's disease of the bone (PDB). The objective of this evaluation is to conduct an economic evaluation of Aclasta ${ }^{\mathrm{TM}}$ and Actonel ${ }^{\mathrm{TM}}$ (risedronate) in a PDB population. Actonel ${ }^{\mathrm{TM}}$ is the standard of care in Canada.

Material and methods: Perspective of the study was from the publicly funded Canadian provincial health care system. Target population was PDB patients with serum alkaline phosphatase levels at least twice the upper limit normal, symptomatic, or at risk of PDB complications. Treatment cost (2005 CAN\$) was determined using efficacy and safety data obtained from the pooled analysis of two comparative trials recently published. Direct medical costs included drug, physician, remission, laboratory, diagnostic and adverse event costs. Frequency of physician visits; laboratory and diagnostic tests were based on published treatment guidelines. No infusion costs for AclastaTM were included since Novartis will fund the infusions. A 5\% discount rate was used after one-year. The acquisition cost of Aclasta $^{\mathrm{TM}}$ was equivalent to Actonel $^{\mathrm{TM}}$.

Results: A single 5-mg IV dose of AclastaTM was shown clinically to have superior efficacy, faster onset and longer-lasting effect, compared to $30 \mathrm{mg}$ of daily Actonel ${ }^{\mathrm{TM}}$ for 60 days. Overall PDB treatment costs were \$2,051.69 for AclastaTM and $\$ 2,498.96$ for ActonelTM $^{\mathrm{TM}}$. Based on remission rates, drug costs

Table 1 Cost breakdown of PDB treatment with Aclasta ${ }^{\mathrm{TM}}$ and Actonel ${ }^{\mathrm{TM}}$ over 2-years

\begin{tabular}{lll}
\hline & Aclasta $^{\mathrm{TM}}$ & Actonel $^{\mathrm{TM}}$ \\
\hline Initial drug cost & $\$ 716.04$ & $\$ 716.04$ \\
$\begin{array}{l}\text { Drug cost due to relapse } \\
\text { Additional drug cost }\end{array}$ & $\$ 151.76$ & $\$ 560.98$ \\
$\quad$ (antipyretic and opiate agonist) & $\$ 679.42$ & $\$ 679.42$ \\
Infusion cost & Supported by Novartis & None \\
Physician cost & $\$ 404.80$ & $\$ 404.80$ \\
Laboratory cost & $\$ 77.94$ & $\$ 107.49$ \\
$\begin{array}{l}\text { Diagnostic cost } \\
\text { Adverse event cost } \\
\text { (flu like symptoms) }\end{array}$ & $\$ 19.76$ & $\$ 29.08$ \\
Total treatment cost & $\$ 1.97$ & $\$ 1.15$ \\
\hline
\end{tabular}


over two-years was $\$ 867.80$ for AclastaTM and $\$ 1,277.02$ for ActonelTM. A breakdown of costs is shown in Table 1 . No incremental cost-effectiveness ratio was calculated as treatment with AclastaTM was clinically more effective and cost less than ActonelTM.

Conclusions: Aclasta ${ }^{\mathrm{TM}}$ for PDB treatment was clinically and economically superior over a two-year time period compared to Actonel ${ }^{\mathrm{TM}}$.

\section{P168. THE EFFICACY OF COMBINED CONDROPROTECTIVE TREAT- MENT IN THE OSTEOARTHRITIS OF THE KNEE}

R.I. Yatsyshyn, Y.M. Neyko, N.G. Yatsyshyn; Department of Internal Diseases, State Medical University, Ivano-Frankivsk, Ukraine

Objectives: The aim of the study was the evaluation of the combined condroprotective treatment in patients with osteoarthritis of the knee.

Methods: The study included 75 patients with osteoarthritis of the knee (ACR criteria) with the mean age $63 \pm 1,25$ years, disease duration 7,1 $\pm 0,82$ years, radiological stage I-III (Kellgren-Lawrence). All patients were randomly divided in 3 groups: 1st group 25 patients received Synvisc 2,0 ml intra-articular weekly, Nr. 3. Second group - 25 patients received "Artron Complex", Unifarm, USA - 2 tablets per os daily, 6 weeks. Third group - 25 patients received a combined treatment: Synvisc $2,0 \mathrm{ml}$ intra-articular, weekly, Nr. 3 + Artron Complex - 2 tablets per os, daily - 6 weeks. The treatment duration was 1 year, with courses repeated every 3 months. As evaluation criteria - Lequesne, WOMAC indexes and health assessment questionnaire were used. Biochemical and radiological investigation were performed before the beginning of treatment.

Results: The clearest results were observed in the 3rd group: the functional index Lequesne decreased with $82 \%(64 \%$ in the 1 st group, $56 \%$ in the 2 nd group), WOMAC index (pain) decreased with $91 \% \quad 69 \%$ in the 1st group, $63 \%$ in the 2 nd group). The quality of life improved with $90 \%$ in the 3 rd group, with $71 \%$ in the group 2 and with $65 \%$ in the group 1 . None of the patients from the 3 rd group presented radiological progression of the process (comparing to $15 \%$ in the 1 st group and $23 \%$ in group 2 ).

Conclusion: The combined condroprotective treatment of osteoarthritis of knee was effective, well tolerated and represents a promising trend in the management of osteoarthritis.

\section{P169. ESTIMATE OF THE PREVALENCE JOINT PAIN AND SWELLING IN POPULATION OF IRKUTSK-CITY (EAST SIBERIA, RUSSIA)}

L.V. Menshikova ${ }^{1}$, T.N. Petrachkova ${ }^{2}$; ${ }^{1}$ Institute for Medical Advanced Studies, ${ }^{2}$ Regional Diagnostic Center, Irkutsk, Russia

Purpose: To estimate prevalence of joint complains in the population.

Materials and methods: The cross-sectional epidemiological investigation of the population of Irkutsk-city, older than 18 years old, has been conducted by the method of forming representative selection with the use of screening questionnaire. The investigation was conducted within multicentral study under the guidance of the Institute of Rheumatology of RAMS (coordinator Sh.Erdes).

Results: 4070 people older than 18 years old have been screened $(32,4 \%$ men and $67,6 \%$ women). The response amounted to $81,4 \%$. The mean age of the people under investigation was $44,7 \pm 16,5$ years old. Prevalence arthralgia, first of all in knee and coxofemoral joints, reaches $45,8 \%$ (in people older than 80 years $-91,8 \%$ ). Complains of joint swelling during life or at the moment of question reaches $30,2 \%$. The average age of people, who had complains of joint pains, amounted to $50,8 \pm 15,8$ years old. Joint swelling was prevalent in women more often than in men: $64,8 \%$ and $19,9 \%$ accordingly, $p<0,001$. The average age of the people, who were questioned and complained of swelling in the region of at least one joint in the history, is higher, than in the people, who did not complain of swelling $(53,1 \pm 14,5$ and $38,5 \pm 15,1$ years accordingly; $p<0,001)$. The complains of joint pain and swelling in one person were diagnosed togerther more often then separately $(\mathrm{p}<0,001)$. Only in small part of population $(12,1 \%)$ painful syndrome in joints was arrested since course of time.

Conclusion: The prevalence and frequency of joint pain and swelling are associated with age and sex.

\section{P170. THE PREVALENCE OF OSTEOARTHRITIS AND OTHER DISEASES OF JOINTS IN POPULATION OF IRKUTSK-CITY (EAST SIBERIA, RUSSIA)}

L.V. Menshikova ${ }^{1}$, T.N. Petrachkova ${ }^{2}$; ${ }^{1}$ Institute for Medical Advanced Studies, ${ }^{2}$ Regional Diagnostic Center, Irkutsk, Russia

Purpose: To study the prevalence of osteoarthritis (OA) and other joint diseases in population of Irkutsk-city.

Materials and Methods: The epidemiological study among the population of Irkutsk-city has been conducted by the method of forming representative selection in the limits of multicentral investigation under the guidance of the Institute of Rheumatology RAMS (coordinator Sh.Erdes). Screening with the help of questionnaire for revealing arthritis and arthralgia has been conducted on the first stage of investigation (the questionnaires have been filled for every adult person of 18 years old and older). Later the diagnostic stage to verify pathology has been conducted.

Results: The screening was conducted in 4070 people $(32,4 \%$ men and $67,6 \%$ women) that amounted to $81,4 \%$. The average age of the people, who participated in investigation, was $44,7 \pm$ 16,5 years old. The results of the analysis of screening stage demonstrated scales of the problem of joint pathology; prevalence of arthralgia amounted to $58,1 \%$ and increased with age. 1095 $(88,6 \%)$ people were examined during diagnostic stage of investigation, who showed the presence of swelling and joint pains. In 295 people (255 women and 40 men) OA was diagnosed (Altman, 1991). The prevalence OA in population of people aged 18 years and older amounted to $7,2 \%$. RA (ARA, 1987) was prevalent in 18 people $(0,4 \%)$ (17 women and 1 men), gout was prevalent in $(0,34 \%)$ of people. The average age of patients with OA amounted to $64,1 \pm 10,3$ years and reliably older, than in patients with RA $-50,8 \pm 8,6$ years $(\mathrm{p}<0,001)$.

Conclusion: Analysing the results of screening, one may estimate preliminary the high spreading joint complains in population of Irkutsk, especially in eldery age groups. Frequency of manifesting OA in people older than 18 years amounted to $7,2 \%$, RA $-0,4 \%$, gout $-0,34 \%$. One should agree that for estimating diseases, which are characterized with pain and functional restriction of joints, individual examination (diagnostic stage) is the only best source of information.

\section{P171. TEMPOPARY DISABILITY IN PEOPLE WITH PAINS IN JOINTS AND BACK IN IRKUTSK-CITY (EAST SIBERIA, RUSSIA)}

L.V. Menshikova ${ }^{1}$, T.N. Petrachkova ${ }^{2}$, A.V. Doroshov ${ }^{1} ;{ }^{1}$ Institute for Medical Advanced Studies, ${ }^{2}$ Diagnostic Center, Irkutsk, Russia. back.

Purpose: To define disability in people with pains in joints and

Materials and methods: Questionnairing representative selection of the population of Irkutsk-city older than 18 years old -4070 people: 1318 men $(32,4 \%)$, average age amounted to $42,1 \pm 16,1$ years old and 2752 women $(67,6 \%)$, average age $-45,9 \pm 16,6$ years old. The questionnaire included the question: Did you take sick-list on the occasion of pain in joints and/or in back?

Results: 1360 people $(33,4 \%)$ answered the question positively. Disability on the occasion of back pain takes place more often, than on the occasion of joint diseases ( 772 people against 586), and is the main factor of working losses in working population aged to 60 years old. The average age of disability on back pain amounted to $52,2 \pm 13,0$ years and on joint pains $53,9 \pm 12,9$ years. Sick-lists were given by physicians of various 
profiles: rheumatologists, therapeutists, neurologists, surgeons, orthopedists.

Conclusion: The diseases of osteomuscular system influence negatively upon economic and working potential of the society.

\section{P172. GLUCOCORTICOIDS-INDUCED OSTEOPOROSIS IN POSTMENO-} PAUSAL WOMEN WITH BRONCHIAL ASTMA

M.L. Menshikov, B.A. Tcherniak, L.V. Menshikova; Regional Diagnostic Center, Irkutsk, Russia

Objective: to determine the prevalence of secondary causes of osteoporosis (OP) in postmenopausal women with bronchial asthma.

Material and methods: 66 postmenopausal women with mean age $59,7(7,2)$ y.o. (range $47-74)$ with asthma using continuous oral corticosteroids (CS), mean dose consist of $9 \mathrm{mg} /$ day, and 33 patients without CS (mean age 57,2 y.o.). Control group consisted of 326 females, matched with respect to age, body weight and body mass index, menopausal status. BMD was measured by DEXA (dual-energy X-ray absorptiometry DPX-IQ, Lunar) at lumbar spine (L2-L4) and hip in the all group. Biochemical markers of bone turnover (serum osteocalcin, beta-crosslaps, deoxypiridinoline) were measured.

Results: according to the DEXA parameters among patients without CS osteopenia and OP were observed in $24,2 \%$ and $18,2 \%$ at the lumbar spine, and in $45,4 \%$ and $9 \%$ at the femoral neck respectively. The patients with CS had consistently the lowest BMD (Bone mineral density). OP was prevalent at the lumbar spine $51,5 \%$ than of femoral neck $-31,8 \%$, osteopenia had $34,8 \%$ and $48,5 \%$. T-score $=-2,6 \mathrm{SD}$ and $-1,8 \mathrm{SD}$; mean $\mathrm{BMD}$ was $0,819(0,164) \mathrm{g} / \mathrm{cm}^{2}$ and $0,756(0,130) \mathrm{g} / \mathrm{cm}^{2}$ respectively. Fractures in minimal trauma had 38(57,6\%) women using CS. Mean age menopause was 46,6 y.o. and less than in the control group and in the women without CS. We find negative correlation with postmenopause period, cumulative prednisolone dose, low body mass index. There were at the same time the increased markers of bone resorption, decreased markers of bone formation.

Conclusions: The results of this study can suggest that, prolonged oral CS therapy increases the risk of secondary osteooposis as well as osteopenia and fractures.

\section{P173. RELATIONSHIP BETWEEN DELAYED MENARCHE AND BONE MINERALIZATION IN PATIENTS AFFECTED BY JUVENILE IDIOPATHIC ARTHRITIS (JIA)}

A. Lurati, R. Cimaz, M. Gattinara, V. Gerloni, B. Teruzzi, I. Pontikaki, A. Salmaso, G. De Marco, F. Fantini; Gaetano Pini Institute, Pediatric Rheumatology Unit, Milan, Italy

Background: Puberty is an essential step in bone mass accrual. Growth failure and impairment of sexual maturation are frequent manifestations of chronic illnesses in pediatric population, and chronic rheumatologic disorders such as juvenile idiopathic arthritis (JIA) are no exception to this.

Methods: The aim of our study was to prospectively evaluate bone density in adolescents' females with JIA, and to correlate results with clinical variables, in particular with age at menarche. Lumbar spine (L2-L4) bone mineral density (BMD) (assessed by Dual X-ray Absorbiometry, DXA) was monitored every 6-12 months in a group of 38 girls with JIA. The evaluated bone mass accrual during the peripubertal time as well as absolute and relative ( $\mathrm{Z}$ score) BMD in relationship with age at menarche, mother's age at menarche, JIA type, disease activity (as evaluated by ESR and $\mathrm{Hgb}$ ), body mass index (BMI) and corticosteroid treatment (mean pro kg daily dose, cumulative dose) was assessed.

Results: Patients were divided into two groups:

- Group I included girls with menarche age within normal limits for Italian standards;

- Group II included girls with delayed menarche.

The BMD values and $\mathrm{Z}$ scores in Group I were not significantly different to normal population. The BMD values and $\mathrm{Z}$ scores in Group II were significantly decreased when compared to the normal population $(\mathrm{p}<0.001)$. With a multivariate analysis only age at menarche seemed independently related to peripubertal mineralization $(p=0.025, r$ between -0.65 and -0.75$)$.

With a binary logistic analysis only disease activity (ESR and $\mathrm{Hgb}$ values) seems indipendently related to a menarche delay (OR 1.16 for each $\mathrm{mm} / \mathrm{h}$ ).

Conclusion: Our data show a critical role for disease activity in determination of a regular pubertal onset, and a normal age at menarche in determination of optimal bone mineralization.

P174. FRACTURES, BONE MINERAL DENSITY AND BONE TURNOVER IN A DUTCH SARCOIDOSIS POPULATION

A.C. Heijckmann ${ }^{1,2}$, M.S.P. Huijberts ${ }^{1}$, J. De Vries ${ }^{3,5}$, B.H.R. Wolffenbuttel $^{4}$, P. Geusens ${ }^{1,5,6}$, M. Drent ${ }^{5}$; ${ }^{1}$ Intern Med., University Hospital Maastricht, ${ }^{2}$ Intern Med, Hosp Bernhoven Veghel/Oss, ${ }^{3}$ Med Psych, Tilburg, ${ }^{4}$ Endocrinology, Univ Med Ctr Groningen, The Netherlands, ${ }^{5}$ Sarcoidosis management center, ${ }^{6}$ Biomedical Research Center, University Hasselt, Belgium

Background: Sarcoidosis is a chronic T-cell-driven disease in which patients can have disturbed calcium homeostasis and are often treated with glucocorticoids (GC). However, little is known about the risk of osteoporosis.

Objectives: To evaluate bone mineral density (BMD), bone turnover, and the prevalence of fractures in patients with sarcoidosis and to identify their determinants.

Methods: BMD of the hip and morphometry of the vertebrae were evaluated in 138 patients with sarcoidosis using dual X-ray absorptiometry. Markers of bone resorption (serum C-terminal telopeptide of type I collagen, ICTP) and formation (serum procollagen type I N-telopeptide, PINP) were also measured. Determinants of BMD and bone markers were assessed using multivariate analysis.

Results: In the total group, Z-scores for BMD at the femoral neck and trochanter were normal. Z-scores were negatively related to $1,25(\mathrm{OH})_{2} \mathrm{D}_{3}$ and at the trochanter to $\mathrm{GC}$ use. Z-scores at the trochanter were significantly increased in GC-naïve patients [0.5 (CI: $0.1,0.7), \mathrm{p}<0.005]$ and this was paralleled by increased Z-scores for PINP [0.3 (CI: 0.0,0.6)] (figure). In the total group, Z-scores for ICTP were increased [1.0 (CI: 0.6,1.3), $\mathrm{p}<0.05]$ and related to inflammatory markers (IL2R, ACE) especially in patients using GC ( $\mathrm{r}=0.534$ and 0.414 , respectively). The prevalence of fractures was low and was associated with longer disease duration and more GC use.

Conclusions: Evaluation of this large cohort of 138 patients with sarcoidosis shows that the overall effects of this inflammatory T-cell-driven disease on BMD are limited. BMD appeared to be normal in patients with sarcoidosis and even increased in GC-

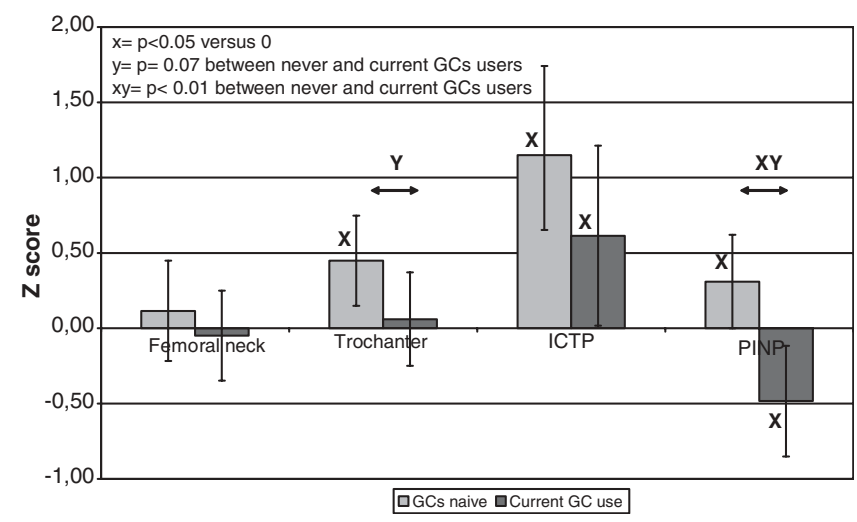

Fig. 1. Z-scores in the GC-na group $(\mathrm{n}=52)$ versus current $\mathrm{GC}$ users $(n=52)$ 
naïve patients, who also demonstrated increased bone formation. Bone resorption was increased and correlated with inflammation. Fracture risk does not seem to be increased in this population.

\section{P175. NON-ISOMERISED C-TELOPEPTIDES OF TYPE I COLLAGEN} ( $a$ CTX) AS A SENSITIVE INDICATOR OF METASTATIC BONE DISEASE

D.J. Leeming ${ }^{1}$, M. Koizumi ${ }^{2}$, G. Delling ${ }^{3}$, K. Henriksen ${ }^{1}$, M. Karsdal $^{1}$, P. Qvist ${ }^{1}$, L.B. Tanko ${ }^{4}$, C. Christiansen ${ }^{4}$, I. Byrjalsen ${ }^{1}$; ${ }^{1}$ Nordic Bioscience, Herlev, Denmark, ${ }^{2}$ Cancer Institute, Hospital, Tokyo, Japan, ${ }^{3}$ Institute of Bone Pathology Universityhospital Hamburg-Eppendorf, Hamburg, Germany, ${ }^{4} \mathrm{CCBR}$, Ballerup, Denmark

Objective: To assess the utility of measuring urinary excretion of the non-isomerized $(\alpha)$ form of the $\mathrm{C}$ - telopeptide of collagen type I ( $\alpha \mathrm{CTX})$ for detection of metastatic bone disease. This was addressed by combining investigations of 1) the tumor loaddependency of the urinary excretion of $\alpha \alpha \mathrm{CTX}$, and the presence of the $\alpha \mathrm{CTX}$ epitope in the proximity of a bone metastasis (BM).

Methods: Participants were 161 cancer patients; 90 breast cancer (45 with BM), 29-lung cancer (15 with BM), and 42 prostate cancer (17 with BM). Presence of BM was determined by Tc99m scintigraphy and the skeletal involvement was graded according to the Soloway score as follows: $1:<6$ metastases, 2: 620 metastases, $3:>20$ metastases but no super-scan, $4:$ superscan (i.e. $>75 \%$ of ribs, vertebrae and pelvic being affected). Urinary $\alpha \alpha C T X$ was measured by a specific sandwich-ELISA. Histological sections from breast cancer patients were stained for the presence of tumor cells (anti-cytokeratin antibody), osteoclasts (TRAcP activity) and $\alpha$ CTX (anti- $\alpha$ CTX antibody).

Results: The urinary concentration of $\alpha \alpha \mathrm{CTX}$ appeared to increase with the severity of skeletal tumor-load in cancer patients (Fig. 1). Immunohistochemistry indicated accumulation of TRAcP + osteoclasts and intense staining for $\alpha$ CTX epitopes in the proximity of cytokeratin + BM. Areas of $\alpha$ CTX-staining showed unstructured bone tissue under polarized light indicating high local bone remodeling. In breast cancer patients with BM, there was a significant linear association between the number of $\mathrm{BM}$ and the relative increases in urinary $\alpha \alpha \mathrm{CTX}$, independent of age and BMI $(r=0.56, p<0.001)$. The estimated relative increases in $\alpha \alpha$ CTX associated with the presence of 1,2 , or 3 metastases were $38 \%, 57 \%$, and $81 \%$, respectively.

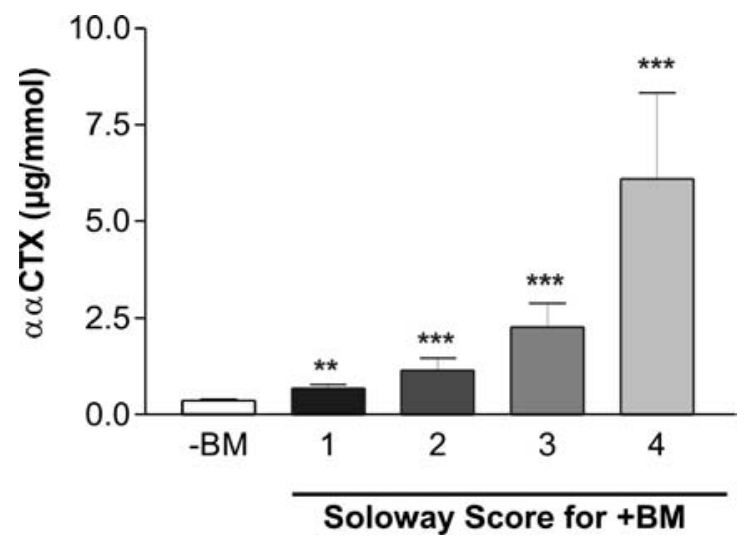

Conclusions: $\alpha \alpha \mathrm{CTX}$ could be a sensitive non-invasive biochemical indicator of the presence and extent of metastatic bone involvement in cancer patients.

P176. OBSERVATIONAL STUDY ON THE PREVALENCE OF INDIVIDUAL AND ENVIRONMENTAL RISK FACTORS FOR HIP FRACTURE IN ELDERLY POPULATION

M. Rossini, G. James, L. Masiero, L. Benedetti, O. Viapiana, D. Gatti, M. Ensunza, C. Detogni, S. Adami; Centro di Riferimento per l'Osteoporosi Regione Veneto, ULSS20-Azienda OspedalieraUniversità di Verona, Verone, Italy

Objective: If on one hand, individual and environmental risk factors for hip fracture are rather well known, the prevalence and the impact of each of these single factors on the local reality are unknown and their knowledge is of utmost importance in order to undertake useful strategies for prevention among the population, above all non-pharmacological strategies.

Methods: elderly patients, of both sexes, consecutively admitted to a hospital for a first femoral fracture of non-cancerous nature. Specific and validated questionnaires are distributed among the patients by specifically trained operators. Furthermore, data regarding personal identification, anthropometric data, medical history and living conditions were gathered. Simultaneously, the same questionnaire is being distributed among a control group.

Preliminary results: a first analysis of 100 elderly patients (76 female and 24 male) with hip fracture are reported. The fracture is not due to an efficient trauma and the patients are in a sufficient neurological state to fill in the questionnaire. The average age was $81 \pm 8$ years. Before the fracture, more than a $50 \%$ of the patients walked less than 30 minutes a day or walked less than $1 \mathrm{~km}$ a day or stood upright less than 4 hours a day; $20 \%$ of them needed walking assistance and $10 \%$ was not able to stand up from a chair. Among the risk factors for fracture present in their medical history, only $3 \%$ reported a hip fracture in the family (mother) and only $5 \%$ smoked or used to smoke. In $96 \%$ of the cases, the fracture was associated to a fall, in $74 \%$ of the cases the fall was from standing height. In $73 \%$ of the cases, the accident occurred at home, mostly in the kitchen or in the bedroom and in $23 \%$ of the cases an object contributed to the fall. $40 \%$ of the patients had already fallen at least once during the last year and $25 \%$ of them more than once. In $34 \%$ of the cases, the patient had already suffered from a fracture after the age of 50 ; only $15 \%$ of the patients had done a densitometry, $12 \%$ had taken therapy for osteoporosis during the last year and only $8 \%$ had taken vitamin D.

These preliminary results already point out in which direction we should move in order to prevent femoral fractures among the elderly.

\section{P177. PREVALENCE OF OSTEOPOROSIS AND RISK FACTORS MET IN POSTMENOPAUSAL WOMEN LIVING IN SEASIDE}

E.Circo $^{1}$, S.Circo ${ }^{2}$, I.Chirca ${ }^{1}$, D.Shavazov ${ }^{1}$; ${ }^{1}$ Department of Endocrinology, Ovidius University of Constanta, Constanta, Romania, ${ }^{2}$ Carol Davila University of Bucharest, Bucharest, Romania

Aims: In Dobrugea lives approximately 1.000 .000 people $35 \%$ of which are women at risk for osteoporosis. Evaluating the prevalence of osteoporosis and pointing out the risk factors that influence it in postmenopausal women living in the seaside.

Methods: We surveyed 1032 postmenopausal womenby using dual-energy X-ray absorptiometry in accordance with $\mathrm{T}$ and $\mathrm{Z}$ scores (by WHO criteria). It included: Age, age when menopause was installed, particularities of menstrual life, number of births. Life style habits included: consumption of milk products, calcium preparates, physical exercise, alcohol intake, coffe, smoking. Women originating from low socio-economic class were asked about the quality of physical activity, the access they had to information. Secondary causes of osteoporosis were considered as well.

Results: Osteoporosis was diagnosed in 503 or $47.7 \%$ of women. Age range was $61.5+/-8.2$ yrs, age at menopause $44.1+/-$ $1.8 \mathrm{yrs}$, early menopause was seen in $9.1 \%$ of osteoporotic women mostly deu to ovarectomy $8.6 \%$. Menstrual disturbances were noted on $12 \%$, nulliparous were only $13.4 \%$. Life-style habits: milk products were taken regularly by $14 \% ; 5.4 \%$ took calcium preparates regularly, $6.8 \%$ intermitently. Women originating from low socio-economic class mainly belonging to rural area claiming hard working conditions. Consumption of alcohol, coffe, smoking was $0.8 \%, 19.6 \%, 25 \%$ respectively. Exposure to sun was poor only $29 \%$ claiming regular summer holidays 
Conclusions: The prevalence of osteoporosis is high and probably underestimated in dobrugea region. Mean age of menopause installation is lower compared to other EU countries, differences existing between urban and rural areas. An earlier installation of menopause could be explained in terms of existing risk factors being sustained by specific economic conditions, insufficient access to information and decreased exposure to sun.

\section{P178. NONVERTEBRAL FRACTURES IN PATIENTS WITH SEVERE ASTHMA ON ORAL STEROIDS}

O. Lesnyak, M. Lezhnina; Department of Family Medicine, Ural State Medical Academy, Yekaterinburg, Russia

Objectives: Inhaled steroids are the treatment of choice in severe asthma. Nevertheless, systemic steroids are still common. The aim of the study was to assess the frequency of nonvertebral fractures in asthma patients on oral steroids depending on age and gender.

Material and methods: The study was performed in tertiary pulmonary center. 96 consequent asthma patients on oral prednisolone more than 6 months were stratified into four sex-age specific groups: females younger than $50(\mathrm{n}=27)$, females 50 and over $(\mathrm{n}=26)$, males younger than $50(\mathrm{n}=20)$ and males over 50 $(n=23)$, min 23 , max 74 years old. The disease duration and the duration of prednisolone treatment did not differ significantly in these groups. The history of factures as well as doses and duration of systemic steroids were studied.

Results: Being on systemic oral steroids, 21 patients $(21,9 \%)$ developed low-trauma nonvertabral fractures without difference in study groups $(p=0,825)$. The most common localizations were ribs $(46,8 \%)$ and wrist $(25,0 \%)$. The overall number of fractures occurred on prednisolone treatment was 32 , which comprised of $44,4 \%$ of all fractures during life period. In contrast, in group of females of 50 years and older $70,6 \%$ of all fractures developed on prednisolone $(p=0,095)$. In the whole group at the time of first fracture the duration of asthma was $12,2 \pm 7,9$ years, the duration of oral steroids treatment was $7,9 \pm 4,7$ years, the cumulative prednisolone dose $-37480 \pm 25322 \mathrm{mg}$, the average daily dose $-12,9 \pm 5,5 \mathrm{mg} /$ day. In male patients of 50 and over the first fracture occurred earlier after the start of steroids than in other groups $(3,3 \pm 2,1$ years, $p=0,001)$, and on lower cumulative and daily doses of prednisolone $(9400 \pm 4537 \mathrm{mg}$ and $9,21 \pm 3,84 \mathrm{mg} /$ day correspondingly, both $\mathrm{p}=0,001)$.

Conclusions: Nonvertebral low-trauma fractures in male patients with severe asthma on oral steroids are as common as in female patients. In older male patients this kind of fractures developed even earlier in the course of treatment and at the lower doses of steroids as compared to women and younger man.

\section{P179. SHORT-TERM TREATMENT OF OSTEOARTHRITIS OF SMALL JOINTS WITH INTRA-ARTICULAR SODIUM HYALURONATE IGO-ON MINI): A RETROSPECTIVE EVALUATION}

K.K. Förster ${ }^{1}$, W. von Zezschwitz ${ }^{2}$, K. Dittmar ${ }^{3}$; ${ }^{1}$ Medical Dept./ Clin. Research, Opfermann Arzneimittel GmbH, Wiehl, Germany, ${ }^{2}$ Practice for Orthopedics and Sports Medicine, Berlin, Germany, ${ }^{3}$ Orthopedic Practice, Berlin, Germany

Introduction: In general, treatment of osteoarthritis $(\mathrm{OA})$ is a combination management including drug treatment to decrease pain, ameliorate limitation of motion, and to control the evolution of the disease. Intra-articular sodium hyaluronate (Na-HA) is a very effective and safe symptomatic drug treatment in OA.

Objectives: material and methods: Efficacy and safety of Na$\mathrm{HA}$ (GO-ON mini) was retrospectively assessed in a multi-centre study in patients with symptomatic AO of small joints. Patients received 3 intra-articular injections, one per week. 1-2 weeks after the last injection, joint pain, inflammation, and reduced joint mobility as well as patientś global therapy assessment were evaluated.
Results: 33 centres reported on 137 patients ( $71 \%$ women) with OA of the carpometacarpal joint of the thumb $(58.4 \%)$ or other small joints $(41.6 \%)$. Concerning pain, at the individually last visit $89.1 \%$ of the patients reported an "improvement" or even "considerable improvement". Concerning reduced joint mobility, $75.2 \%$ of the patients reported an "improvement" or even "considerable improvement". Joint inflammation occurred in too less patients to assess any change. Concerning patientś global therapy assessment, $19.0 \%$ of the patients reported an "excellent", $51.1 \%$ a "good", and $23.4 \%$ a "satisfactory" therapeutic result. Of all 137 patients (397 injections) there was one patient with local pain, twice after one injection, and one with an hematoma. This is an incidence of local reactions of less than 1 percent $(0.76 \%)$.

Conclusion: The results of this retrospective evaluation show that intra-articular GO-ON mini is a very safe and effective symptomatic treatment of OA of smaller joints, e.g., the carpometacarpal joint of the thumb or other small synovial joints. Therefore, Na-HA is an alternative treatment option, not only in $\mathrm{OA}$ of larger joints (GO-ON) but also - as GO-ON mini - in OA of smaller synovial joints.

P180. EXTRA ARTICULAR MALUNION OF THE DISTAL RADIUS TREATED BY CORRECTIVE OSTEOTOMIE AND INJECTABLE CEMENT

L. Obert, S. Rochet, D. Lepage, Y. Tropet, P. Garbuio; University Hospital Jean Minjoz, Orthopedic and Plastic Surgery unit, Besancon, France

Objective: The purpose of the present study was to report on the author's experience using Injectable cement as a bony substitute in distal radius corrective osteotomies. The interest of such a bone substitute is the real capacity to adapt itself to the bone defect because in avoiding the challenge, which consists in performing a trapezoidal cortico spongious graft which can fill very precisely the void and not more.

Material and Methods: Three patients with an average age of 41 yo (24-48) had a corrective osteotomy for a malunited distal radius fracture using Injectable bone substitute (Eurobone, Jectos, Kasios Inc) as an alternative to an autogenous bone graft. Internal fixation of the osteotomy was achieved by using one plate without postoperative immobilisation. Two patients were stiff at preoperative time.

Results: At an average follow-up evaluation of 26 months (14 $37 \mathrm{mo}$ ) all the osteotomies united. Wrist flexion-extension motion improved from $56^{\circ}$ to $110^{\circ}$, forearm rotation increased from $112^{\circ}$ to $142^{\circ}$, and grip strength had an average increase of $120 \%$ at the time of the final follow-up evaluation. All patients were satisfied but there one report of persistent pain. Radiographic evaluation showed an average volar tilt improvement from a preoperative dorsal angulation shifting into a neutral position in the sagittal plane; Radiographically the injectable cement showed evidence of progressive re-absorption over time but with no complete disappearance.

Conclusions: On the basis of this preliminary experience it is reasonable to consider injectable cement as a viable alternative to bone grafting in conjunction with surgical correction and internal fixation of extra articular distal radius malunion.

\section{P181. POST TRAUMATIC ARTHRITIS SECONDARY TO INTRA ARTICU- LAR MAL UNION OF DISTAL RADIUS TREATED BY CHONDRO COSTAL GRAFT}

D. Lepage, J. Pauchot, Y. Tropet, P. Garbuio; University Hospital Jean Minjoz, Orthopedic and Plastic Surgery unit, Besancon, France

Objective: 4 cases of postraumatic arthritis of the distal radius treated by chondro costal graft are reported with a minimum follow up of 2 years.

Material and methods: One 22 years old patient with a dislocated radiocarpal joint 6 months after an injury, 2 patients (48 
and 53 years old) respectively 1,5 and 2 years after a intraarticular fracture of distal radius and a patient ( 74 years old) with radio lunate arthritis reported two mains complaints: pain and stiffness. Location of the loss of cartilage was central in two cases and palmar in the others. A dorsal approach in one cases, a palmar approach in the three others allowed reduction and reconstruction of the destroyed radial part of joint. A chondro costal graft harvested on the eight's rib was inserted and fixed by plate in place of the articular impaction. Plaster cast of 3 months in the first case and 1 month in the three others cases followed the articular reconstruction

Results: No complication have been pointed. Union was achieved in all 4 cases. Integration and viability of the graft were evaluated with RMI. At the highest follow up functionnal result are excellent in the first case. Motion and grasp are similar than the controlateral side. For the three others patients motion in flexion - extension reached respectively $74 \%, 69 \%, 54 \%$ of controlateral side, and grasp reached respectively $62 \%, 73 \%$ and $68 \%$ of controlateral side.

Conclusion: Reconstruction of a partially destroyed articular surface by a costal graft is reliable and allows filling and resurfacing an articular cartilage void. If chondro costal graft is currently used in maxillo facial surgery it is the first report in post traumatic arthritis secondary to intra articular mal union.

P182. IS QUANTITATIVE ULTRASOUND OF THE CALCANEUS AN USEFULL PREDICTIVE TOOL FOR FRACTURE RISK? 3 YEARS FINAL RESULTS FROM THE ECOSAP PROSPECTIVE STUDY

J. González-Macías ${ }^{1}$, A. Díez-Pérez ${ }^{2}$, J. Vila ${ }^{3}$, M. Puchades ${ }^{4}$, M.A. Martín ${ }^{5}$, F. Aganzo ${ }^{6}$, M.A. Cabrera ${ }^{7}$, J. Aliaga $^{8}$, B. Galán ${ }^{9}$, C. Cancelo ${ }^{10}$, F. Marín ${ }^{11}$; ${ }^{1}$ Dept. Internal Medicine, H. Valdecilla, Santander, Spain, ${ }^{2}$ Dept. Internal Medicine, H. del Mar, Barcelona, Spain, ${ }^{3}$ IMIM, Barcelona, Spain, ${ }^{4}$ C.S. AlgemesíAlfafar, Valencia, Spain, ${ }^{5}$ C.S. Cerro del Aguila, Sevilla, Spain, ${ }^{6}$ C.S. La Almunia, Zaragoza, Spain, ${ }^{7}$ C.S. El Pla Hospital, Alicante, Spain, ${ }^{8}$ C.S. Abarán, Murcia, Spain, ${ }^{9}$ C.S. Fuente Palmera, Córdoba, Spain, ${ }^{10}$ C.S. Brihuega, Guadalajara, Spain, ${ }^{11}$ Medical Research, Eli Lilly and Company, Madrid, Spain

Objective: ECOSAP is a 3-year prospective study to evaluate the ability of calcaneus QUS and several clinical risk factors of osteoporosis and fractures to predict the risk for low-energy nonspinal fractures in women aged 65 years or older from Spain.

Patient and Methods: 5201 women [mean age (SD): 72.3 (3.5) years] were selected using non-probabilistic sampling of consecutive cases regardless of the reason for consultation in 58 Primary Care Centers. They completed an osteoporosis and fracture risk factors questionnaire and were assessed with a Sahara ${ }^{\circledR}$ equipment. A Cox proportional hazard regression analysis with nonspinal fractures as the dependent variable was performed. Independent variables included were all those with a $p$-value $<0.15$. We report here the final study results.

Results: At baseline, 1042 women $(20.1 \%)$ presented a history of fragility fracture in adulthood ( $>35$ years). 4453 women $(87.3 \%)$ completed the 36-month final visit, with a median of 3.01 years of follow-up, representing 14,999 women/years. 311 women $(6.0 \%)$ reported a total of 363 non-spinal fragility fractures. The final model consisted of age, history of falls, prevalent fractures, family history of fractures, and calcium intake from dairy products. The adjusted hazard ratios (and 95\% CI) per SD decrease of each QUS parameter are shown in the Table.
Conclusion: The 3-year final results of the ECOSAP study show that QUS of the heel is a predictor of overall non-spinal, low-trauma in a cohort of elderly Spanish women included regardless their bone mass status. Prediction algorithms using QUS and clinical variables can predict absolute risk in elderly women.

P183. A SINGLE ZOLEDRONIC ACID 5mg INFUSION IS PREFERRED OVER WEEKLY 70mg ORAL ALENDRONATE IN A CLINICAL TRIAL OF POSTMENOPAUSAL WOMEN WITH OSTEOPOROSIS/OSTEOPENIA

R. Lindsay ${ }^{1}$, K. Saag ${ }^{2}$, A. Kriegman ${ }^{3}$, J. Davis ${ }^{3}$, E. Beamer ${ }^{3}$, W. Zhou ${ }^{3}$; Clinical Research Center, Helen Hayes Hospital, West Haverstraw, NY, USA, ${ }^{2}$ Division of Rheumatology, University of Alabama at Birmingham, Birmingham, AL, USA, ${ }^{3}$ Novartis Pharmaceuticals Corporation, East Hanover, NJ, USA

Objective(s): To assess patient preference for annual IV infusion therapy compared to weekly oral therapy in a clinical trial.

Material and Methods: This multi-center, randomized, double-blind, double-dummy trial assessed the onset of action of a single 15-minute infusion of zoledronic acid [ZOL] $5 \mathrm{mg}(\mathrm{N}=69)$ compared to oral weekly alendronate $[\mathrm{ALN}] 70 \mathrm{mg}(\mathrm{N}=59)$ over a 24-week period. At the end of the study, patients were asked to respond to four questions to determine their preference for the different treatment modalities. While still blinded to therapy, patients were asked which treatment was 1) more convenient, 2) more satisfying, 3 ) they would be more willing to take for a long period of time, and 4) was preferred. Safety assessments were evaluated over the duration of the 24 -week study.

Results: Overall, $66.4 \%$ of the patients who completed the questionnaire $(\mathrm{N}=122)$ expressed a preference for a once-a-year IV infusion, compared to $19.7 \%$ who preferred a once-weekly pill; $13.9 \%$ indicated that both treatment modalities were equal.

The percentage of patients who reported an adverse event was similar in the ZOL (91\%) and ALN (86\%) groups. However, more patients in the ZOL treatment group reported transient post-infusion adverse experiences (AE), mostly "flu-like" symptoms $(\mathrm{n}=13,18.8 \%)$, nausea $(\mathrm{n}=8,11.6 \%)$, and/or myalgia $(\mathrm{n}$ $=8,11.6 \%$ ), during the first 3 days after infusion compared to the ALN group. After 3 days, there were no significant differences between groups. Even among patients who reported an AE within the first 3 days and completed the questionnaire $(n=61), 73.8 \%$ expressed an overall preference for once-a-year IV therapy.

Conclusion(s): A once-a-year IV infusion was preferred over a once-weekly pill by $66.4 \%$ of postmenopausal women with

\begin{tabular}{llll}
\hline \multicolumn{3}{l}{ Patient Preference Questionnaire (\% of responders) } & \\
\hline & Once-a-Year IV & Once-a-Week Pill & Both Are Equal \\
\hline $\begin{array}{l}\text { More convenient } \\
(\mathrm{N}=122)\end{array}$ & 66.4 & 15.6 & 18.0 \\
$\begin{array}{l}\text { More satisfying } \\
(\mathrm{N}=121)\end{array}$ & 59.8 & 18.9 & 20.5 \\
$\begin{array}{l}\text { More willing } \\
\text { to take }\end{array}$ & 68.0 & 15.6 & 16.4 \\
$\begin{array}{l}\text { a long time } \\
(\mathrm{N}=122)\end{array}$ & & \\
$\begin{array}{l}\text { Overall preference } \\
(\mathrm{N}=122)\end{array}$ & 66.4 & 19.7 & 13.9 \\
\hline
\end{tabular}

\begin{tabular}{llll}
\hline $\begin{array}{l}\text { Type of fracture } \square \\
\text { (number) }\end{array}$ & $\begin{array}{l}\text { All non-vertebral } \square \\
(\mathrm{n}=311)\end{array}$ & $\begin{array}{l}\text { Major non-vertebral* } \\
(\mathrm{n}=253)\end{array}$ & $\begin{array}{l}\text { Wrist/Forearm } \\
\square(\mathrm{n}=104)\end{array}$ \\
\hline BUA (db/MHz) & $1.33(1.17 ; 1.51)$ & $1.38(1.20 ; 1.59)$ & $1.35(1.08 ; 1.69)$ \\
SOS (m/s) & $1.20(1.08 ; 1.34)$ & $1.24(1.12 ; 1.39)$ & $1.30(1.12 ; 1.51)$ \\
QUI (\%) & $1.31(1.15 ; 1.49)$ & $1.40(1.21 ; 1.62)$ & $1.50(1.19 ; 1.89)$ \\
Estimated heel BMD (g/cm $\left.{ }^{2}\right)$ & $1.32(1.16 ; 1.50)$ & $1.41(1.22 ; 1.63)$ & $1.50(1.19 ; 1.89)$ \\
Estimated heel BMD (T-score) & $1.29(1.14 ; 1.45)$ & $1.39(1.22 ; 1.63)$ & $1.40(1.01 ; 1.95)$ \\
\hline
\end{tabular}


osteoporosis/osteopenia participating in ZOL vs ALN comparative trial.

P184. ZOLEDRONIC ACID $5 \mathrm{mg}$ IV PRODUCES MORE RAPID REDUCTION IN BONE RESORPTION MARKERS COMPARED TO WEEKLY $70 \mathrm{mg}$ ORAL ALENDRONATE IN POST-MENOPAUSAL WOMEN WITH OSTEOPOROSIS/OSTEOPENIA

K. Saag ${ }^{1}$, R. Lindsay ${ }^{2}$, A. Kriegman ${ }^{3}$, J. Davis ${ }^{3}$, W. Zhou ${ }^{3}$; ${ }^{1}$ Division of Rheumatology, University of Alabama at Birmingham, Birmingham, AL, USA, ${ }^{2}$ Clinical Research Center, Helen Hayes Hospital, West Haverstraw, NY, USA, ${ }^{3}$ Novartis Pharmaceuticals Corporation, East Hanover, NJ, USA

Objectives(s): To assess the rapidity of zoledronic acid (ZOL) $5 \mathrm{mg}$ IV versus oral alendronate (ALN) $70 \mathrm{mg}$ weekly in reducing bone resorption markers in post-menopausal women with osteoporosis/osteopenia.

Material and methods: Multi-center, randomized, doubleblind, active-controlled trial to assess rapidity of onset of action of a single infusion of ZOL $5 \mathrm{mg}$ (=69) versus oral weekly ALN $70 \mathrm{mg}(\mathrm{N}=59)$ over 24 weeks. The primary efficacy variable was relative change from baseline of urine $\mathrm{N}$-telopeptide of type 1 collagen (NTx) at 1 week. Secondary variables included relative change from baseline of NTx at weeks 2, 4, 8, 12, and 24 and bone-specific alkaline phosphatase (BSAP) at baseline and 4, 12, and 24 weeks. Safety assessments were evaluated over 24 weeks.

Results: ZOL demonstrated significantly greater reduction in NTx at week 1 versus ALN $(P<.0001)$. Compared to ALN, ZOL significantly reduced NTx levels at all timepoints $(P<.05$ for weeks $2,4,8,12,24)$. Consistent with IV bisphosphonates, reduction in NTx with ZOL was greatest at week 1 post-infusion, then levels gradually increased.

BSAP, which was elevated at baseline, slowly decreased for both ZOL and ALN, and achieved levels within the normal premenopausal reference range from week 12 onward. There were no significant BSAP differences between groups at week 24. As expected, during the first 3 days post-dose, more patients reported adverse experiences with ZOL versus ALN, including flu-like symptoms ( 18.8 vs $5.1 \%)$, myalgia $(11.6 \%$ vs $1.7 \%)$, and/or nausea $(11.6 \%$ vs $5.1 \%)$. Post-infusion symptoms were transient and usually resolved within 4 days. There were no differences in overall incidence of adverse events between groups after 3 days $(79.7 \%$ vs $78.0 \%)$.

Conclusion(s): A single infusion of ZOL $5 \mathrm{mg}$ achieved more rapid and greater reductions in biochemical markers of bone resorption with similar effects on bone formation versus weekly oral ALN.

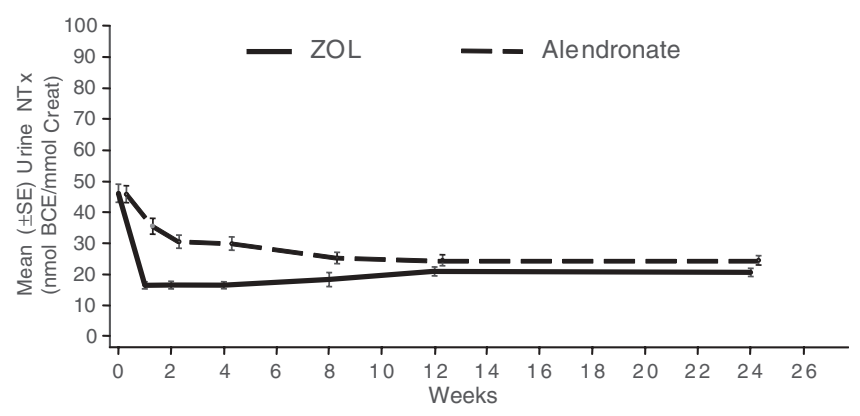

P185. THE EFFECTIVENESS OF ALENDRONATE (70 mg WEEKLY) IN WOMEN WITH POSTMENOPAUSAL OSTEOPOROSIS

L.A. Martchenkova, A.V. Dreval, I.V. Kryukova, R.S. Tishenina, N.M. Milov; Moscow Regional Research Clinical Institute, Moscow, Russia

The aim of this study was to assess effectiveness and tolerability of alendronate in treatment of postmenopausal osteoporosis.
Methods: 15 postmenopausal women with primary osteoporosis (age $61.0 \pm 4.8(\mathrm{M} \pm \mathrm{s})$ years) received alendronate in dosage $70 \mathrm{mg}$ weekly for 12 months. Control group consisted of 19 women suffered from postmenopausal osteoporosis (age 61.7 \pm 5.2 years) did not receive any treatment for study period. Effectiveness of alendronate was evaluated by measurement of BMD, biochemical markers of bone turnover and calcium homeostasis and back pain index.

Results: BMD (T-score) increased in lumbar spine from $-3.2 \pm 0.9$ to $-3.0 \pm 0.7$ in 6 months $(p<0.05$ vs baseline) and to $-2.9 \pm 0.7$ in 12 months ( $\mathrm{p}<0.05$ vs baseline), in femoral neck from $-2.3 \pm 0.9$ to $-2.1 \pm 0.8$ in 6 months $(\mathrm{p}<0.05$ vs baseline) and in trochanter from $-1.8 \pm 0.8$ to $-1.7 \pm 1.0$ in 12 months $(\mathrm{p}<0.05$ vs 6 months $)$ in treated patients. Total proximal femur and distal forearm BMD did not change significantly in both groups. We found a marked decrease in serum calcium from $1.24 \pm 0.03$ to $1.21 \pm 0.03 \mathrm{mmol} / 1$ in 6 months ( $\mathrm{p}<0.05$ vs baseline) and to $1,2 \pm 0.04 \mathrm{mmol} / 1$ in 12 months ( $<<0.05$ vs baseline), in alkaline phosphatase from $98.7 \pm 44.8$ to $63.5 \pm 15.6 \mathrm{u} / 1$ in 6 months $(\mathrm{p}<0.05 \mathrm{vs}$ baseline $)$ and to $60.7 \pm 13.8 \mathrm{u} / 1$ in 12 months $(\mathrm{p}<0.05$ vs baseline) in alendronate group and there was no significant change in serum osteocalcin and blood parathyroid hormone. Back pain improved in 9 and 12 months $(\mathrm{p}<0.05$ vs baseline) in alendronate group. Tolerability of alendronate was satisfactory but 3 patients stopped the treatment due to gastrointestinal side effects.

Conclusion: Those results demonstrate that alendronate in dosage $70 \mathrm{mg}$ weekly increases BMD in axial and peripheral skeleton and decreases serum calcium, bone turnover markers and back pain in women with postmenopausal osteoporosis.

\section{P186. OSTEOPOROSIS AND OSTEOPENIA IN WOMEN WITH BREAST} CANCER

A. Kistauri ${ }^{1}$, G. Tsikhiseli $^{2}$, S. Nijaradze ${ }^{2}$, M. Zodelava ${ }^{1}$, N. Tskhovrebashvili ${ }^{3}$, T.Mamaladze ${ }^{3}$, M. Tskhovrebashvili ${ }^{4}$, D Gabunia ${ }^{1}$; ${ }^{1}$ Department of Internal Medicine, Tbilisi State Medical University, Tbilisi, Georgia, ${ }^{2}$ Clinic "Caraps Medline", Department of Oncology, Reconstructive and Plastic Surgery, Tbilisi, Georgia, ${ }^{3}$ Clinic "Caraps Medline", Department of Osteoporosis and Diabetic foot, Tbilisi, Georgia, ${ }^{4}$ Clinic "Endmedici", Endocrinology, Metabology and Dietology Center, Tbilisi, Georgia

Objective: Breast cancer (BC) patients should be particularly alert to the decrease of bone mineral density (BMD), as many BC patients receive treatments that may increase their risk of developing osteoporosis. The aim of the present study was to determine $\mathrm{BMD}$ in women with $\mathrm{BC}$, to consider for them adjuvant therapy after successful treatment for BC with the following BMD monitoring.

Material and Methods: We have studied 80 women with new case histories of BC aged 35-76 years. All patients were divided in four age-matched groups. BMD was measured at three sites (distal radius, midshaft tibia and proximal phalanx) using the ultrasound bone sonometer (Sunlight, Omnisense). Results were interpreted according to the criteria adopted by the WHO by T score.

Results: The mean data for $\mathrm{T}$ score in the I group of patients with $\mathrm{BC}(\mathrm{n}=14$ age before 45 years $)$ was: distal radius $-0,2 \pm 0,02$; midshaft of the tibia $-0,2 \pm 0,05$; proximal phalanx $-0,8 \pm 0,11$; in the II group ( $\mathrm{n}=24,46-56$ years) T score: $-0,8 \pm 0,09 ;-1,8 \pm 0,15$; $-1,6 \pm 0,11$; in the III group $(n=20,57-65$ years $)$ T score: $-1,8 \pm 0,18 ;-1,7 \pm 0,13 ;-2,5 \pm 0,04$; and in the IV group $(\mathrm{n}=22$, 66 years and up) $\mathrm{T}$ score $-2,2 \pm 0,14 ;-2,4 \pm 0,08 ;-3,4 \pm 0,11$ at the same measurement sites respectively.

Conclusions: In patients with $\mathrm{BC}$ was shown a high rate of decrease of BMD from osteopenia to osteoporotic changes, especially in the older postmenopausal groups, where BC and osteoporosis are common, and although both are dependent on estrogens, this leads to conflicting implications for the treatment: estrogen reduce the risk of fractures but increase the risk of $\mathrm{BC}$. 
In this aspect selective estrogen modifiers (SERM) hold great promise, as they decrease both the fracture risk and the BC risk. So, it is important that BMD must be determined in all women with BC, appropriately monitored and, when necessary, be prophylactically protected so, that the full benefit of adjuvant therapy with SERM (e.g. Raloxifene) can be enjoyed by BC patients. This study is the first step in this field in Georgia. In the future we are planning follow up monitoring of all these patients considering their postoperative therapy.

\section{P187. ROUTINE CARE OF OSTEOPOROSIS BY OFFICE-BASED ORTHO- PEDISTS IN GERMANY - HOW MANY DRUGS ARE NEEDED AND WHAT DO THEY COST?}

H. Gothe, A. Freytag, B. Häussler; IGES - Institute for Health and Social Research GmbH, Berlin, Germany

Objectives: While significant improvements in prevention and therapy of osteoporosis can be gained by adequate drug treatment, it is supposed that ambulatory care physicians, especially orthopedists, are restrained from an evidence-based prescription behavior by budget caps and individual practice prescription limits. To find out how many patients routinely seen by officebased orthopedists suffer from osteoporosis ('practice prevalence'); to assign severity levels to these patients and to determine their need of anti-osteoporosis treatment; to quantify the amount of money required for evidence-based pharmaceutical care of osteoporosis.

Material and Methods: In order to guarantee for an unambiguous assignment of osteoporosis patients to delimited treatment groups, the DVO (orthopedists' professional umbrella association in Germany) recommendations were transformed into algorithms. A questionnaire was developed which served as a documentation tool for the chart review the participating orthopedists had to perform. The total number of patients in the individual practice setting had to be stated and every osteoporosis patient was classified according to the defined severity levels. This procedure allowed to assign every patient to a specific therapy requirement based on his/her individual osteoporosis level.

Results: 123 randomly selected orthopedists participated in the survey, 90 of them were eligible for the study. A total of $n=17,717$ osteoporosis patients (ref. to the $2^{\text {nd }}$ quarter of 2004) were enrolled. This corresponds to an adjusted medium number of 189 osteoporosis patients per orthopedist. 160 of them $(85 \%)$ were in need of anti-osteoporosis treatment (baseline or comprehensive therapy), which accounted for 13.00 EUR to be spent on anti-osteoporosis drug treatment per claims form per quarter. For bisphosphonate therapy only, the corresponding figure was 7.96 EUR. The amount allowed by the practice prescription limit ranges between 6.28 EUR (general health insurance, KV Nordrhein) and 23.02 EUR (pensioners' health insurance, KV Berlin).

Conclusion: To our knowledge, this is the first study investigating routine care requirements of ambulatory anti-osteoporosis drug treatment provided by orthopedists. The study quantifies the amount of drugs actually needed in a realistic bottom-up calculation. Results suggest that guideline-based sole bisphosphonate therapy drains the orthopedists' budget dramatically, while full anti-osteoporosis drug treatment might completely exceed the budget limits.

P188. MORPHOMETRIC X-RAY ABSORPTIOMETRY (MXA) REFERENCE VALUES: RELATIONSHIP BETWEEN VERTEBRAL HEIGHTS, AGE AND YEARS SINCE MENOPAUSE

D. Diacinti ${ }^{1}$, C.M. Francucci ${ }^{2}$, C.E. Fiore $^{3}$, P. Pennisi ${ }^{3}$, M. Rossini $^{4}$, A. Barone ${ }^{5}$ A. Giusti ${ }^{5}$, G.B. Rini ${ }^{6}$, D. Bruno ${ }^{6}$, B. Frediani $^{7}$, T. Bartalena ${ }^{8}$, R. Del Fiacco ${ }^{1}$, S. Minisola ${ }^{1}$, E. D'Erasmo $^{1}$, G.F. Mazzuoli1; ${ }^{1}$ Department of Clinical Sciences, University "La Sapienza", Rome, Italy, ${ }^{2}$ Department of Endocrinology, University of Ancona, Italy, ${ }^{3}$ Department of Internal
Medicine of Catania, Italy, ${ }^{4}$ Department of Internal Medicine, University of Verona, Italy, ${ }^{5}$ E.O. Hospital Galliera of Genova, Italy, ${ }^{6}$ Department of Internal Medicine, University of Palermo, Italy, ${ }^{7}$ Department of Rheumatology, University of Siena, Italy, ${ }^{8}$ General Hospital of Imola, Italy

Aim: to evaluate relationship between vertebral body heights, age and years since menopause to define reference values by morphometric X-ray absorptiometry (MXA).

Design: the 8 centres participating in the study (Ancona, Catania, Genova, Imola, Palermo, Roma, Siena Verona) enrolled 1500 women, according to Black method, including a sample of fertile women $(<44 \mathrm{yrs})$ and postmenopausal women from 45 to $>74$ yrs. All women enrolled had MXA, three centres using the Hologic QDR4500A, five centres using the GE Lunar Prodigy. Both systems acquired spine images with dual-energy (high-definition). MXA images were centrally analyzed by an experienced radiologist. The age and years since menopause stratificated mean $( \pm$ SD) of the three vertebral heights and their ratios for all vertebral levels were calculated for both systems.

Results: vertebral heights and their ratios decreased with aging, but in younger women from 25 to 53 years only a small decrement of vertebral heights was observed $(r=-0.06$, n.s.). Whereas, in older women, all postmenopausal, was observed a significant inverse relationship with age $(r=0.6, p<0.001)$ between age and mean vertebral heights and their ratios, that showed the major decrement for anterior height at T6-T8 level. Vertebral heights ratios significantly correlated with years since menopause $(\mathrm{r}=0.5, \mathrm{p}<0.001)$ decreasing much more in the first 5 years after the menopause. The modal distributions of vertebral heights in pre- and postmenopausal women are clearly distinguished, with a statistically significant difference between the means $(\mathrm{p}<0.01)$.

Conclusions: these results demonstrate that vertebral heights are smaller with aging and years since menopause. Vertebral heights decrement is sustained by menopause as well age-related factors. Therefore, it is necessary also for MXA to use reference values stratified for age and for years since menopause.

\section{P189. OSTEOPOROSIS IN WOMEN AND FAMILY HISTORY FOR FRA- GILITY FRACTURES IN MOTHER}

D. Ruci ${ }^{1}$, V. Ruci ${ }^{1}$, A. Tafaj ${ }^{1}$, A. Priftanji ${ }^{1}$, F. Toti ${ }^{2} ;{ }^{1}$ Department of Rheumatology, Tirana, Albania, ${ }^{2}$ Department of Endocrinology, "Mother Teresa" University Hospital, Tirana, Albania

Purpose: The relationship of osteoporosis in women and heredity is the aim of the study. The evaluation of risk for osteoporosis and documented osteoporosis in mothers in these subjects.

Material and method: We made a quantitative study based on the measurements of bone mineral density using an heel ultrasound bone densitometer (Pegasus). For each patients a questionnaire was filled, recording history for osteoporosis in mother (fragility fractures), age, height, weight. We studied 670 women from $20-75$ years old. 356 of them were more than 50 years old. We made the evaluation for this group also.

Results: The first group was composed of 245 women with a positive history for fragility fractures in their mothers. 44 or 17,6 $\%$ resulted with osteoporosis (T-score less then $-2,5 \mathrm{SD})$. In the second group with no history for fragility fractures we studied 425 women. Only 20 of them or $4,7 \%$ resulted with osteoporosis. $(p<0,01)$, odds ratio(95\% CI) $4,4(2,5--7,7)$. We make the evaluation for the women over 50 years old. We found $25,9 \%$ osteoporosis (38 out of 147) in subjects with maternal history for fragility fractures and 7,6\% osteoporosis (16 out of 209) in subjects without positive history for fragility fractures in their mothers. $(p=0,001)$.

Conclusions: History for documented osteoporosis in mother (fragility fractures ever happened) is a major risk factor for osteoporosis in women. Age also is an important and independent risk factor for these women . 
P190. REGULATION OF BONE BALANCE - SEQUENTIAL MICRONUTRITION

M. Lugand Bourgeois, A. Cornely; Bioresearch and Partners, Monthey, Switzerland

Osteoporosis is becoming a real public health issue. Bioresearch \& Partners' objective is to exploit a new nutritional approach: micronutrition, which can be used in prevention as well as to help and strengthen classic therapeutics. Micronutrisequences are a combination of microgramme-dosed dietary substances organized in sequence. The micronutritional approach developed by Bioresearch \& Partners is a new mode of utilizing nutrients which targets an action on the global regulation of bone balance, the nutrients have been selected to participate in a nutritional strategy aiming to help the restoration of bone metabolism homeostasis.

The aim of the nutritional intervention was the measurement in monotherapy in open study of bone balance regulation by the dosage of biological markers of bone formation (osteocalcin) and resorption (24-hour urine desoxypiridonoline at the creatinine dosage), as well as the increase of bone density observed with bone density test in menopaused women presenting a disturbance of bone balance markers and osteopenia or osteoporosis (BMD).

The inclusion in the trial was excluding any treatment. The nutritional intervention lasted two years. Were excluded of the trial the patients who were immobilized or taking corticoids, having osteomalacia or unstable diseases: diabetes, thyroid, crohn disease, kidney or active hepatic diseases. Nutrisequence (capsules containing micronutrition granules) was taken three times a day during 3 months. Population studied: 24 women aged 64 in average. All 24 patients are followed on bone markers balance, 10 are complementarily followed in absorptiometry.

Results: Of the 17 cases out of 24 cases which were presenting at the beginning of the trial a bone balance with an excessive increase of dpyr, $94 \%$ re-stabilized their bone balance, $82 \%$ totally normalized their balance (in 17,3 months in average). Results on bone density: Of the 10 cases followed in BMD: 7 patients out of 9 had osteopenia or osteoporosis and showed an increase of the trabecular density by $7 \%$.

Conclusion: the regulation of bone markers $(82 \%)$ and the increase of bone density $(+7 \%)$ offer a satisfactory response to the required therapeutic aims in a population presenting an excessively negative bone balance. Micronutrisequence is efficient and perfectly adapted to a systematic preventive action and can be associated with any other drug therapeutics.

\section{P191. MALE OSTEOPOROSIS AMONG SAUDI ARABIAN POPULATION: WILL WE MISS THE BUS AGAIN?}

M. Sadat-Ali, A. Mohsin Elk; College of Medicine, King Faisal University Dammam and King Fahd University Hospital, AlKhobar, Saudi Arabia

Background: The prevalence of postmenopausal osteoporosis among Saudi Arabian females was underestimated and it took the arrival of the new millennium to face the reality. The situation appears very similar when it comes to accept the occurrence of osteoporosis among the male population of Saudi Arabia. This prospective study assessed the prevalence of male osteoporosis among the Saudi Arabs attending King Fahd University Hospital, Al-Khobar, Saudi Arabia.

Patients and Methods: Saudi Arabian males over the age of 50 years, attending the hospital with unrelated complaints were the subjects. Patients had their weight and height measured to calculate their body mass index (BMI) and investigation to rule out secondary osteoporosis. All had bone mineral density (BMD) measurement of hip area and the spine using DEXA scan, Hologic Inc. Patients with a BMD of $<-2.6$ was taken as osteoporotic and those between $<-1$ to -2.5 was taken as osteopenic for analysis.

Results: We were able to analyse the data of 127 patients with an average age of $61.51(50-76) \pm 7.16$ with mean BMI of 24.59
$(18.42-35.5) \pm 3.72$. Analysis of the scans of the hip revealed that $27.55 \%$ of the patients were osteoporotic with BMD of $0.837 \mathrm{gm} /$ $\mathrm{cm}^{2}(0.697-0.936) \pm 0.04$ and T-Score of $-2.8(-2.6--3.4)$ \pm 0.269 . Seventy-three $(57.48 \%)$ were osteopenic with BMD of $0.936 \mathrm{gm} / \mathrm{cm}^{2}(0.863-1.065) \pm 0.05$ and mean $\mathrm{T}$ Score of -1.6 $(-1.1--2.5) \pm 0.361$. Analysis of BMD of the spine showed that the prevalence of osteoporosis was $54(42.5 \%)$ with T-Score of $-3.45(-2.7--5.1) \pm 0.74$ and $37 \%$ were osteopenic with a $\mathrm{T}$ Score of $-1.9(-1.3--2.5) \pm 0.332$.

Conclusions: Our study indicates that the prevalence of osteoporosis among the Saudi Arabian males is higher than reported in the western literature. More studies are needed throughout the Kingdom to get a national prevalence of male osteoporosis. It is recommended that serious measures to be undertaken to prevent male osteoporosis and stop any future epidemic of catastrophic osteoporosis related fractures.

\section{P192. IS THERE AN ALTERNATIVE TO NSAIDS AND COX-2 INHIBITORS IN THE MANAGEMENT OF OSTEOARTHRITIS OF KNEE?}

M. Sadat-Ali, I. Al-Habdan, M. Al-Bluwi, A. Al-Omran, D.A AlDhakeel, A.Y. El-Hassan; University Department of Orthopaedic Surgery And Radiology, King Fahd Hospital of the University, Al-Khobar, Saudi Arabia

Background: Osteoarthritis of knee (OAK) is common among ethnic Saudi Arabian population, which has been blamed due to the cultural postures of this part of the world. Primary treatment of mild and moderate OAK is by NSAIDs (Non-Steroidal antiinflammatory drugs) and recently by Cox-2 Inhibitors (C2I). The complications of these drugs are well known. The use of Glucosamine-Chondroitin Complex (GCC) was reported to be effective in the management of OAK.

Objective: The aim of this study was to test the efficacy of GCC in comparison with the NSAIDs and C2I.

Methodology: Two hundred and fifty Saudi patients with mild to moderate OAK were included in the study. They were randomly divided into two groups. Group A received GCC of $(1000 \mathrm{mg}$ of Glucosamine and $500 \mathrm{mg}$ of Chondroitin Sulphate) Joint Renew2 ${ }^{\circledR}$ per day in divided doses. Group B formed the control group who continued to receive NSAIDs or C2I as they were taking before the start of the study. Before starting the trail the patients had a complete examination and assessment of the activity by the modified Lequense grading (MLG), Visual Analogue Scale (VAS) by the patient and the physician $(0=$ no pair and $9=$ unbearable pain $)$ and the weight-bearing radiographs were graded as per the Kellgren and Lawrence Grading (KLG). Patients were seen every three months for detailed examination as done at the starting of the trial. On the follow-up radiographs, the radiologist was blinded in the reading of the $\mathrm{x}$-rays.

Results: All patients were Saudi Arabian nationals and were suffering from OAK between 10.2 years (Groups $A$ ) and 9.6 years (Group B). The data of 116 Group A patients and 119 of Group B patients was available for analysis. The average age of Group A was 59.9 (45-64) years ( \pm SD4.9) and the Group B was 57.6 (45$62)$ years $( \pm$ SD 5.2$)$. The improvement in MLG was significant from 6 month onwards $(\mathrm{p}<0.05)$ and by 24 months it was highly significant of $\mathrm{p}<0.0001$ in Group A and KLG started to become significant from 18 months onwards. The VAS for pain and range of movements at the time of study was 5 and at 6 months it was 2.6 at 12 months 1.7 and after 18 months it was 1.1 at 24 months. In Group B the patients continued to suffer from pain, VAS between (4-5) during the follow up. In Group A 5 patients $(4.3 \%)$ initially complained of dyspeptic symptoms but continued the treatment.

Conclusion: This prospective randomized study indicates that Glucosamine Chondroitin Complex (Joint Renew2) not only relieved the symptoms of OAK but also modified the progression of the disease. The results of our two-year study allow us to recommend GCC as an alternative to NSAIDs in the management of mild to moderate osteoarthritis of knee. 
P193. OSTABOLIN-CTM: A PHASE I TRIAL TO ASSESS ITS SAFETY, TOLERABILITY AND PHARMACOKINETICS IN HEALTHY OLDER ADULTS

C.L. Barclay ${ }^{1}$, R. Anderson ${ }^{1}$, L. Champoux-Knott ${ }^{2}$, G. Morelli ${ }^{2}$, P. Morley ${ }^{1}$ ' ${ }^{1}$ Zelos Therapeutics Inc., Ottawa, ON, Canada, ${ }^{2}$ MDS Pharma Services, Montreal, QC, Canada

Aims: The biological activity, safety, tolerability and pharmacokinetic profile of subcutaneously (SC) administered Ostabolin-CTM (cyclized hPTH - (1-31) $\mathrm{NH}_{2} ; 10-160 \mu \mathrm{g}$ ) was assessed in a randomized, double blind, placebo-controlled single escalating dose study in healthy older volunteers.

Methods: Forty subjects (20 males and 20 females; 45-96 kg; 4573 years of age) in 5 cohorts received a single subcutaneous injection of Ostabolin-C ${ }^{\mathrm{TM}} 10,20,40,80$ or $160 \mu \mathrm{g}$ or placebo. Each cohort contained 6 active ( 3 male and 3 female) and 2 placebo ( 1 male and 1 female) treated subjects. Pre- and post-dose urinary and plasma cyclic AMP levels, serial serum calcium (0.25-24 hours post-dose), ECG, vital signs, plasma Ostabolin-C ${ }^{\mathrm{TM}}$ levels using a validated ELISA, and Adverse Events (AEs) were recorded.

Results: Following $\mathrm{SC}$ administration $\mathrm{C}_{\max }$ increased proportionally with dose $(26 \mathrm{pg} / \mathrm{ml}$ at $10 \mu \mathrm{g}$ up to $319 \mathrm{pg} / \mathrm{ml}$ at 160 $\mu \mathrm{g})$. The mean $\mathrm{T}_{\max }$ ranged from $0.25-0.75$ hours and the $t_{1 / 2}$ was consistent across dose groups (0.83-1.12 hours). Biological activity, measured by urinary and plasma cyclic AMP, showed significant increases from pre- to post-dose at all doses for urinary cyclic AMP and for 80 and $160 \mu \mathrm{g}$ for plasma cyclic AMP. AEs were rare with 10 and $20 \mu \mathrm{g}$ doses. The most frequently reported AEs were nausea, headache, vomiting, back pain, and dizziness. Tolerability was poor in women receiving $160 \mu \mathrm{g}$. Serial total and ionized serum calcium testing revealed only one subject (a woman treated with $160 \mu \mathrm{g})$ with a single total serum calcium value $(2.65$ $\mathrm{mmol} / \mathrm{L}$ ) marginally above the normal ranges (total $2.11-2.60$ $\mathrm{mmol} / \mathrm{L}$; ionized $0.91-1.13 \mathrm{mmol} / \mathrm{L}$ ). A dose-dependent increase in heart rate was recorded but no clinically significant ECG changes were observed.

Conclusions: Ostabolin-CTM was biologically active at all doses tested. It was safe and well tolerated as a single injection in healthy older adults at doses up to $80 \mu \mathrm{g}$. Elevated serum calcium values were rare (1/30 Ostabolin-CTM treated subjects: $3.3 \%)$, occurring only once and only at the maximum dose tested. The safety profile was otherwise as expected for a drug of this class.

P194. PATIENTS' PREFERENCE FOR OSTEOPOROSIS MEDICATIONS: PREFER-INTERNATIONAL

J. Walliser ${ }^{1}$, S. Bolge ${ }^{2}$, S.S. Sen ${ }^{3}$; ${ }^{1}$ Medical Director of the "Clinical de Metabolismo Oseo y Mineral del Hospital Angeles del Pedregal, Mexico, ${ }^{2}$ Consumer Health Sciences, Princeton, NJ, USA, ${ }^{3}$ Outcomes Research, Merck \& Co., Whitehouse Station, NJ, USA

Objective: To evaluate patients' preferences for two different osteoporosis medication profiles and the reasons for their preferences.

Methods: Physicians were randomly selected in France, Germany, Mexico, Spain and UK, and asked to refer the next 4 osteoporotic women aged 50 years or older seen in their practices. These patients were asked over telephone or face-to-face (Mexico) to indicate their preference between two hypothetical osteoporosis medication profiles, $\mathrm{A}$ and $\mathrm{B}$ which varied on 4 parameters, efficacy, time on market, dosing frequency and dosing procedure. They were also asked to indicate the most importance reason for their medication preference among these 4 parameters.

Results: A total of 3000 patients were interviewed of whom 1500 were on prescription treatment for osteoporosis while the rest were not. The majority of patients $(78 \%)$ preferred drug A over drug B. Effectiveness in reducing risk of fracture was most frequently $(72 \%)$ ranked as the most important reason for their preference followed by time on market $(13 \%)$, dosing frequency $(9 \%)$ and dosing procedure $(6 \%)$.

Conclusion: The drug profile A with proven hip fracture reduction was chosen over drug $\mathrm{B}$ by the majority of the patients

Drug A Drug B

Description

Proven to reduce risk of spine and hip proven to reduce only spine fracture fracture

10 year in regular practice recently introduced

Once a week

30 minutes wait $\quad 60$ minutes wait

Results

Preferred by

$78 \%(0.4)$ of all patients $\quad 22 \%(0.4)$ of all patients

$79 \%(0.4)$ of patients on treatment $\quad 21 \%(0.4)$ of patients on treatment

$76 \%(0.4)$ of patients not on treatment $24 \%(0.4)$ of patients not on treatment

regardless of their treatment status. Effectiveness in reducing risk of fracture was the most important reason for selection of a drug profile.

P195. FRACTURE PREVENTION IN PATIENTS WITH A RECENT CLINICAL FRACTURE. THE APPROACH OF SURGEONS IN THE NETHERLANDS

B. Telgenkamp ${ }^{1}$, S. van Helden ${ }^{1}$, P. Brink ${ }^{1}$, P. Geusens ${ }^{2}$; ${ }^{1}$ Department of General Surgery, Academic Hospital Maastricht, Maastricht, the Netherlands; ${ }^{2}$ Department of Rheumatology, Academic Hospital Maastricht, Maastricht, the Netherlands

Objective: Patients with a history of fragility fracture have an increased risk for new fractures. Patients older than 50 years that present to the hospital with a clinical fracture have a risk of about $11 \%$ to develop a new clinical fracture within 2 years (1). We analysed the current approach of surgeons towards systematic evaluation of patients older than 50 years with a recent clinical fracture for underlying osteoporosis and fall risk, taking into account the availability in the Netherlands of guidelines for osteoporosis $(2,3)$ and fall risk (4).

Methods: Two questionnaires were send to all hospitals in the Netherlands $(\mathrm{n}=100)$, one to a trauma surgeon and one to an orthopaedic surgeon in each clinic, inquiring about the application of the two above mentioned guidelines.

Results: The response rate was $76 \%, 57 \%$ by trauma surgeons and $43 \%$ by orthopaedic surgeons. In $58 \%$ of the hospitals, some evaluation for osteoporosis was performed, based on the guidelines $(24 \%)$ or own criteria $(34 \%)$. In $28 \%$ no evaluation for osteoporosis was performed and in $14 \%$ the answers within the hospital were contradictory. For further evaluation of osteoporosis dual energy absorptiometry was done in $74 \%$ and quantitative computertomography in $5 \%$ of the hospitals. Some evaluation for fall risk was performed by $34 \%$ of the hospitals, based on guidelines $(14 \%)$ or own criteria $(20 \%)$. In $50 \%$ no evaluation for fall risk was performed and in $16 \%$ the answers within the hospital were contradictory.

Conclusion: Some screening for osteoporosis was performed in $58 \%$ of responding hospitals and for fall risk in $34 \%$ of responding hospitals. However systematic application according to guidelines was only $24 \%$ for osteoporosis and $14 \%$ for fall risk.

These data indicate low adherence to local guidelines for osteoporosis and fall risk evaluation in patients of 50 years and older presenting in the hospital for a clinical fracture.

\section{References}

1. van Helden et al. Osteoporosis Int., in press

2. Osteoporosis guidelines in the Netherlands, www. CBO.nl

3. Geusens et al. J Eval Clin Practice, 2005, in press

4. Fall risk guidelines in the Netherlands, www. CBO.nl

P196. STRATEGIES TO DECREASE RISK OF VERTEBRAL REFRACTURE WITH AND WITHOUT VERTEBROPLASTY THROUGH REHABILITATION

C. Schmidt, E. Huntoon, M. Sinaki; Department of Physical Medicine and Rehabilitation, Mayo Clinic, Rochester, MN, US

Aims of this study were to compare refracture rates in patients who had at least one prior osteoporotic compression fracture and 
then were treated using either vertebroplasty (VP), Rehabilitation-Osteoporosis Program of Exercise (ROPE) or both.

Materials and Methods: Subjects were retrospectively obtained from a large database of an academic medical center by computer search. Inclusion criteria were; age greater than 60 at least one radiologically confirmed compression fracture and participation in either or both interventions. Exclusion criteria were: secondary causes of osteoporosis or death less than 24 months after treatment initiation unless a refracture had occurred prior to death. Patients receiving VP had a pre-intervention MRI confirming an acute compression fracture and a physician examination demonstrating severe pain localizing to the same level. Patients receiving ROPE were evaluated by a physical medicine physician, counseled in osteoporosis management, and instructed in a posture training program if appropriate. They participated in a physical therapy instruction session for back extensor strengthening and posture correction. Patients receiving both interventions had ROPE at variable points in the course of their management.

Results: Of 342 records reviewed, 55 met the inclusion criteria for the study and 40 received VP or ROPE. Refracture rates in the 20 patients treated with VP were $60 \%$, which occurred an average of 7.7 months after VP. Refracture rates in 20 patients treated with ROPE were $20 \%$ and occurred an average of 37.8 months after initial diagnosis. Patients $(\mathrm{n}=15)$ receiving both interventions had a refracture rate of $40 \%$ with variable onset of refracture. These fractures frequently occurred prior to the ROPE component of the treatment.

Conclusions: In this preliminary study, the ROPE intervention groups had lower refracture rates when compared to the group not receiving ROPE intervention. If VP is combined with ROPE we can expect decreased refracture rates.

\section{P197. INVESTIGATION OF BONE MARKER ALTERATIONS AFTER TREADMILL GAIT TRAINING USING NEUROMUSCULAR ELECTRICAL STIMULATION AND PARTIAL BODY WEIGHT SUPPORT IN QUADRI- PLEGIC SUBJECTS}

D.C. Leite de Carvalho ${ }^{1}$, C.R. Garlipp ${ }^{2}$, P.V. Bottini ${ }^{3}$, S.H. Afaz $^{3}$, M.A. Moda ${ }^{3}$, A. Cliquet ${ }^{1,4}$; ${ }^{1}$ Orthopaedics Department, Faculty of Medical Sciences, State University of Campinas (UNICAMP), Campinas, Brazil, ${ }^{2}$ Department of Clinical Pathology, Faculty of Medical Sciences, State University of Campinas (UNICAMP), Campinas, Brazil, ${ }^{3}$ Division of Clinical Pathology, University Hospital - UNICAMP, ${ }^{4}$ Department of Electrical Engineering, University of São Paulo (USP at São Carlos), Brazil

Osteoporosis is a known consequence after spinal cord injury (SCI), due to the lack of muscle contraction and mechanical load below the lesion level, which increase bone fragility and risk of fractures. Many studies have been performed towards finding efficient treatments for bone loss and in many cases bone markers have been used in order to evaluate the effect of short period of treatment.

To evaluate the effect of treadmill gait training using neuromuscular electrical stimulation (NMES), with 30-50\% weight relief, on response of biochemical markers of bone metabolism, 21 quadriplegics with incomplete motor loss (ASIA B) were divided into gait group (11), which performed the training for six months, twice a week, during 20 minutes each session (Figure 1) and control group (10), which did not perform gait. Biochemical markers (osteocalcin, bone alkaline phosphatase, pyridinoline and deoxipyridinoline) were measured, at the beginning and after six months. Data from bone markers were analyzed individually due to the large individual variations. In the gait group $81.8 \%$ presented a significant increase of bone formation; $66.7 \%$ also presented a significant decrease of bone resorption markers. In the control group $30 \%$ did not present any change of markers and $20 \%$ presented an increase of bone formation. Individuals from control group, who presented an increase of formation markers increased their independence during daily activities (they managed to get their driver licenses and became less dependent on accompanying persons), during the six months period.
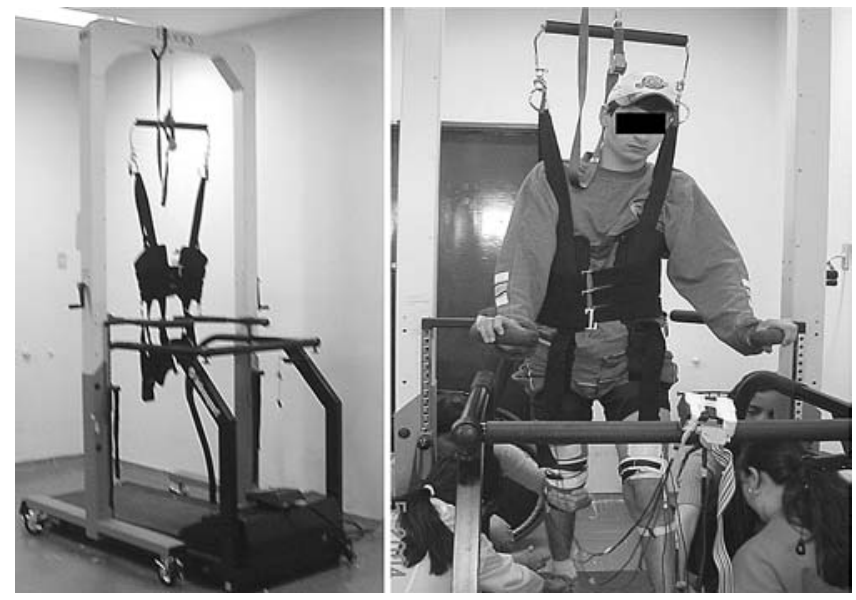

Fig. 1 Treadmill gait training associated to NMES and body weight support

Results have suggested that treadmill gait training, even with $30-50 \%$ of body relief, was efficient to promote biomechanical stress in order to stimulate bone formation process.

\section{P198. PAPANICOLAOU (PAP) SMEAR: A SIMPLE TOOL TO IDENTIFY WOMEN AT INCREASED RISK OF OSTEOPOROSIS}

A. Repše-Fokter ${ }^{1}$, S.K. Fokter ${ }^{2}$, R. Komadina ${ }^{3}$; ${ }^{1}$ Dept. of Pathology and Cytology, Celje General Hospital, Celje, Slovenia, ${ }^{2}$ Dept. of Orthopaedic Surgery and Sports Trauma, Celje General Hospital, Celje, Slovenia, ${ }^{3}$ Dept. of Traumatology, Celje General Hospital, Celje, Slovenia

Objective: The Papanicolaou (Pap) test is an important screening method of detecting precancerous and cancerous changes of the uterine cervix. It is routinely used in organised and non-organised screening programmes in most developed countries. The present study attempted to find out possible coherence between morphologic characteristics in Pap smears on one side, and BMD as measured by DXA on the other.

Methods: DXA measurement was performed in 136 women aged 33-76 years (mean 55 years) in whom Pap smears for routine cervical cancer screening were obtained. The smears were grouped into atrophic and mature cell pattern. Then we compared T-values measured by DXA on the femoral neck and lumbar spine with both groups.

Results: T-scores were significantly lower in atrophic smear pattern group $(\mathrm{p}<0.001)$. Among 73 women with atrophic smears, there were 70 with osteopenia or osteoporosis. In mature cell pattern group (63 women), 44 patients had osteopenia or osteoporosis and 19 had normal bone mineral density. Sensitivty of $61,4 \%$, specificity of $86,4 \%$, positive predictive value of $95,9 \%$, negative predictive value of $30,2 \%$, and accuracy of $65,4 \%$ were achived.

Conclusion: Women with atrophic smear pattern are at greater risk for osteopenia or osteoporosis and a lot of them could be detected with the routine Papanicolaou screening test without additional costs.

\section{P199. BONE DENSITY IN PATIENTS WITH PREVIOUS OSTEOPOROTIC FRACTURE, THE ANALYSIS OF CONSERVATIVE AND SURGICAL TREATMENT AND FURTHER OBSERVATION}

V. Vyskočil ${ }^{1,2}$, T. Pavelka ${ }^{2} ;{ }^{1}$ Dpt. Of Orthopaedic Surgery, Bone Disease Center, Plzen, Czech Republic, ${ }^{2}$ Charles University Hospital Plzen, Bone Disease Center Plzen, Czech Republic

The authors observed the group of patients with 231 lowtraumatic osteoporotic fractures $(87$ fractures in proximal femur, 84 vertebral fractures, 60 ones of distal forearm and 
proximal humerus). All the patients were treated at the Dpt. Of Surgery and Traumatology of University Hospital in Plzen within a year 2004. After the surgical treatment, bone densitometry was assessed in all the patients. According to densitometry results, antiosteoporotic medication was started. Bone mineral density (BMD) was measured in vertebral column and hip. Patients with backspine injurey had significantly lower vertebral BMD, T-score -2.14 in L1-L4 region and -1.6 in hip. The patients with hip fractures showed different situation, their T score of vertebral BMD was -1.6 and hip BMD (L1-L4) had $\mathrm{T}$ score -2.02 . The fractures of proximal humerus and distal foremarm demonstrated lower standard deviation of $\mathrm{T}$ score BMD in both localizations only - 1.3. The patients with distal forearm fracture had mean age 62 years, those ones with vertebral fractures were 67years old and subjects suffering hip fracture were 70 years old. Relatively lower mean age of patients with hip fracture was modified by their ability to come for BMD measurement. With regard to the patientśmobility, all the subjects with hip fracture were treated surgically; the other fractures were treated conservatively in the majority of cases. Patients with low-traumatic fractures and having BMD lower than $-2.5 \mathrm{~T}$ score were treated with antiresorption therapy at the specialized center. Considering high risk of fractures in the remainder, vitamin $\mathrm{D}$ and calcium medication was the other alternative of conservative antiosteoporotic treatment. In conclusion, the authors discussed the applied surgical techniques and their emerging complications.

\section{P200. SCREENING FOR RISK FACTORS FOR OSTEOPOROSIS}

K. Hristozov, H. Bohchelian, M. Chavdarova, D.Najdenova; Department of Endocrinology, Medical University- Varna, Varna, Bulgaria

Osteoporosis is a medical and social problem. Its prevention is in connection with early identification of risk factors for diminished bone density.

Aim of the study: was to investigate the frequency of osteoporotic risk factors in a cross - sectional study.

Patients and methods: A screening for osteoporosis has been carried out. Object of investigation were 200 men and women $(\mathrm{M} / \mathrm{F}=1 / 7)$, mean age $-48+-9,7$ years. An osteodensitometry was performed by Osteodensitometer DTX 200 at the distal radius and ulna. An interview was carried out after a standard list of questions concerning basic risk factors for osteoporosis.

Results: Osteopenia was found in $7 \%$ of all premenopausal women and in $23 \%$ of postmenopausal women. Osteoporosis was found in $2 \%$ of premenopausal women and in $4 \%$ in postmenopausal women. The most frequent risk factors found in subjects with osteopenia and osteoporosis were as follows postmenopause, diminished physical activity, inadequate diet, smoking, family history for osteoporosis.

Conclusions: Osteopenia and osteoporosis are frequent in postmenopausal women. Their early diagnosis is necessary for adequate treatment. Screening is essential for early identification of risk factors and initial stages of disease.

\section{P201. KNOWLEDGE, HEALTH BELIEFS, AND HEALTH-RELATED BEHAVIORS IN FIRST-DEGREE RELATIVES OF WOMEN SUFFERING OSTEOPOROSIS: A COMPARATIVE STUDY}

S.-F. Chang; Department of Nursing, NTCN Doctoral Program Student, Department of Industrial Education, NTNU, Taipei, Taiwan

Background: No previous study has examined knowledge, health beliefs, and health-related behaviors in first-degree relatives (FDRs) of osteoporosis sufferers, especially focusing on Asian women.

Aim: This study explored osteoporosis knowledge, beliefs, and behaviors of women with a family history of osteoporosis, and drew a comparison with women with no such history.
Design: This study recruited women at a large public health center in northern Taiwan. A questionnaire was applied on FDRs and non-FDRs women with a focus on osteoporosis knowledge, health beliefs, and behaviors. Descriptive analysis was initially conducted. Differences between FDRs and non- FDRs were rated via Student's t-tests for continuous variables and the X2 test for categorical variables.

Results: Overall, most of the participants were aware of some osteoporosis-related information but the proportions of correct responses to the questions that tested knowledge between FDRs and non-FDRs were only $44.0 \%$ and $42 \%$, respectively. Meanwhile, participants in the FDRs group not only reported higher concern in developing the disease but also perceived higher barriers compared to the non-FDRs group. As the study demonstrates, for health-related behaviors, the FDRs group did not undertake actual preventive behaviors, and only bone mineral density screening behavior differed significantly from the nonFDRs group.

Conclusions: This study highlights the inadequate information on osteoporosis and constraining beliefs of FDRs women. Additionally, since preventive behaviors of osteoporosis are not noticed in FDRs group, community health nurses and researches should make participants' concerns and assist women to reduce the difficulties of taking practically preventive behaviors not merely by the bone density results. Relevance to clinical practice. This investigation reviews the knowledge, beliefs, and behaviors of the FDRs group for Taiwanese women with osteoporosis. The results of this work can be used to provide effective implementation guidelines for preventing osteoporosis especially for women with a family history of the disease.

\section{P202. RECOVERY AND DECLINE: ONE-YEAR QUALITY OF LIFE RE-} SULTS FROM THE OSSO STUDY

D. Torgerson ${ }^{1}$, C. Cooper ${ }^{2}$, F. Jakob ${ }^{3}$, F. Marín ${ }^{4}$, E. MartínMola $^{5}$, P. Fardellone ${ }^{6}$, S. Adami ${ }^{7}$, N.C. Thalassinos ${ }^{8}$, J. MeloGomes ${ }^{9}$, T. Nicholson ${ }^{4}$, C. Chinn ${ }^{4}$; ${ }^{1}$ Department Health Sciences, University of York, York, UK, ${ }^{2}$ MRC Environmental Epidemiology Unit, Southampton General Hospital, Southampton, UK, ${ }^{3}$ Dept. Orthopaedics, Julius-Maximilians-Universität, Wurzburg, Germany, ${ }^{4}$ Lilly Research Center, Windlesham, UK, ${ }^{5}$ S. Reumatología, Hospital La Paz, Madrid, Spain, ${ }^{6} \mathrm{~S}$. Rhumatologie, Hopital Nord, Amiens, France, ${ }^{7}$ Centro Ospedaliero Clinicizzatodi Valeggio sul Mincio, Verona, Italy, ${ }^{8}$ Dept. Endocrinology, Evangelismos General Hospital, Athens, Greece, ${ }^{9}$ Instituto Portugues de Reumatologia, Lisbon, Portugal

Objective: We describe here how quality of life (QOL) changed over one year in patients included in OSSO (Observational Study on Severe Osteoporosis).

Patients and Methods: OSSO is a prospective, observational study in six European countries that included 2,322 postmenopausal women with established osteoporosis and an inadequate clinical response to osteoporosis drug treatment. Patients had either experienced a fragility fracture (index fracture) despite osteoporosis drug therapy for at least 12 months; (IF: $n=1,313$ ) or had discontinued osteoporosis drug therapy due to compliance problems and/or side effects; $(\mathrm{C} / \mathrm{SE}: \mathrm{n}=1,009)$. QOL was assessed using EuroQoL (EQ-5D) and the Quality of Life questionnaire of the European Foundation for Osteoporosis (QUALEFFO). QOL was calculated for each cohort and also for all study patients with $(8.8 \%)$ or without $(91.2 \%)$ at least one incident fracture during the follow up period.

Results: EQ-5D health state values (HSV) increased in both cohorts mean (SD): IF $0.47(0.34)$ to $0.56(0.31)$; C/SE $0.53(0.32)$ to $0.60(0.30)$ - Total QUALEFFO scores decreased in both cohorts mean (SD): IF 49.1 (18.4) to 44.9 (18.4); C/SE 44.1 (18.6) to 40.3 (18.6)). The table shows changes in QOL in patients with or without an incident fracture.

Conclusion: In OSSO, women with severe osteoporosis have poor quality of life. This improves when there is a period with no further fracture. However, the incident fracture rate was high at $8.8 \%$ and associated with a further deterioration in quality of life. 


\begin{tabular}{|c|c|c|c|c|c|c|c|c|}
\hline & & \multicolumn{7}{|c|}{ Extreme problems in: } \\
\hline & & $\begin{array}{l}\text { Total } \\
\text { Qualeffo } \\
\text { score }\end{array}$ & HSV & Mobility & $\begin{array}{l}\text { Self- } \\
\text { Care }\end{array}$ & $\begin{array}{l}\text { Usual } \\
\text { Activities }\end{array}$ & $\begin{array}{l}\text { Pain/ } \\
\text { s Discomfort }\end{array}$ & $\begin{array}{l}\text { Anxiety/ } \\
\text { Depression }\end{array}$ \\
\hline \multirow{2}{*}{$\begin{array}{l}\text { With an } \\
\text { incident } \\
\text { fracture } \\
n=166\end{array}$} & baseline & $\begin{array}{l}50.2 \\
(19.4)\end{array}$ & $\begin{array}{l}0.45 \\
(0.36)\end{array}$ & $3.0 \%$ & $3.7 \%$ & $15.2 \%$ & $25.2 \%$ & $11.0 \%$ \\
\hline & 1-year & $\begin{array}{l}53.4 \\
(20.4)\end{array}$ & $\begin{array}{l}0.39 \\
(0.37)\end{array}$ & $3.4 \%$ & $9.7 \%$ & $25.0 \%$ & $25.5 \%$ & $11.7 \%$ \\
\hline \multirow{2}{*}{$\begin{array}{l}\text { Without } \\
\text { incident } \\
\text { fracture } \\
\mathrm{n}=1719\end{array}$} & baseline & $\begin{array}{l}47.3 \\
(18.9)\end{array}$ & $\begin{array}{l}0.49 \\
(0.31)\end{array}$ & $1.1 \%$ & $3.0 \%$ & $8.7 \%$ & $22.8 \%$ & $11.0 \%$ \\
\hline & 1-year & $\begin{array}{l}41.6 \\
(18.1)\end{array}$ & $\begin{array}{l}0.60 \\
(0.29)\end{array}$ & $0.5 \%$ & $1.6 \%$ & $6.2 \%$ & $10.3 \%$ & $6.2 \%$ \\
\hline
\end{tabular}

Physicians should consider the potential impact of fractures on QOL when making treatment decisions.

\section{P203. STRONTIUM RANELATE REDUCES THE RISK OF VERTEBRAL FRACTURE IN PATIENTS WITH OSTEOPENIA}

E. Seeman ${ }^{1}$, A. Sawicki ${ }^{2}$, J.-Y. Reginster ${ }^{3}$, C. Roux ${ }^{4}$, A. Rubinacci $^{5}$, M. Diaz-Curiel ${ }^{6}$, J.-M. Kaufman ${ }^{7}$, M.C. de Vernejoul ${ }^{8}$, J.P. Aquino $^{9}$, P.J. Meunier ${ }^{10}$, ${ }^{1}$ Endocrine Unit, Augustine Hospital, Melbourne, Australia, ${ }^{2}$ Warszawskie Centrum Osteoporozy, Warsaw, Poland, ${ }^{3}$ Department of Epidemiology and Public Health, University of Liège, Liège, Belgium, ${ }^{4}$ Hôpital Cochin, Paris, France, ${ }^{5}$ Bone Metabolic Unit, Scientific Inst. H. San Raffael, Milano, Italy, ${ }^{6}$ Servicio de Medicina Interna, Fundación Jimenez Diaz, Madrid, Spain, ${ }^{7}$ Polyclinique d'endocrinologie, Gent University Hospital, Gent, Belgium, ${ }^{8}$ Hôpital Lariboisière, Paris, France, ${ }^{9}$ Clinique Médicale de la Porte Verte, Versailles, France, ${ }^{10}$ Department of Rheumatology and Bone Diseases, Edouard Herriot Hospital, Lyon, France

Objective: There is little evidence to demonstrate that any of the available anti-resorptive or anabolic drugs reduce the risk of vertebral fractures in patients with osteopenia. In part, this may reflect the low incidence of fractures in this population rather than lack of anti-fracture efficacy of the drugs.

Materials and methods: To address this question, we analysed the pooled data from SOTI $(\mathrm{n}=1649)$ and TROPOS $(\mathrm{n}=5091)$ in which postmenopausal women received strontium ranelate $2 \mathrm{~g} /$ $\mathrm{d}$ orally or placebo for 3 years in a randomized double blind fashion. Each patient received calcium and vitamin D supplementation according to baseline nutritional status. In this sample, 409 patients with lumbar and/or femoral neck T-score between -1 and -2.5 and both $\mathrm{T}$-scores $>-2.5$, with or without prevalent fractures were included. Vertebral X-rays were performed yearly.

Results: No relevant differences between groups were detected for the main baseline characteristics: mean (SD) age: 73(6) years; time since menopause: 25(8) years; mean (SD) Lumbar T-score: 1.20(1.15); mean (SD) Femoral Neck T-score: $-2.06(0.44)$. In the intent-to-treat population, strontium ranelate was associated with a $62 \%$ reduction in the relative risk of vertebral fracture over 3 years (as assessed through semi-quantitative method by a central reading centre $)(\mathrm{RR}=0.38,95 \% \mathrm{CI}[0.21 ; 0.70], \mathrm{p}=0.001)$. Amongst the 409 described patients $57 \%$ of patients had a prevalent fracture. In this subgroup, strontium ranelate reduced the risk of vertebral fracture by $56 \%$ over 3 years $(\mathrm{RR}=0,44 ; 95 \%$ CI $[0,22-0,87](p=0.015)$. The other $43 \%$ of patients presented an osteopenia according to their BMD values (described above) and had no prevalent fracture. In this subgroup, strontium ranelate reduced the risk of vertebral fracture by $72 \%$ over 3 years $(\mathrm{RR}=0.28 ; 95 \%$ CI $[0.07 ; 0.99])(\mathrm{p}=0.045)$.

Conclusion: We infer that strontium ranelate, a new antiosteoporotic agent, reduces the risk of vertebral fractures in women with osteopenic range of BMD with and without any prevalent fracture.
P204. VERTEBRAL FRACTURE RISK REDUCTION WITH STRONTIUM RANELATE IN WOMEN WITH POST-MENOPAUSAL OSTEOPOROSIS IS INDEPENDENT OF BASELINE RISK FACTORS

C. Roux ${ }^{1}$, J.-Y. Reginster ${ }^{2}$, J. Fechtenbaum ${ }^{1}$, S. Kolta ${ }^{1}$, A Sawicki $^{3}$, Z. Tulassay ${ }^{4}$, G. Luisetto ${ }^{5}$, J.M. Padrino ${ }^{6}$, D. Doyle $^{7}$, R. Prince ${ }^{8}$, P. Fardellone ${ }^{9}$, O. H. Sorensen ${ }^{10}$, P.J. Meunier ${ }^{11}$; ${ }^{1}$ Department of Rheumatology, Cochin Hospital, René Descartes University Paris, France, ${ }^{2}$ Department of Epidemiology, Public Health and Health Economics, University of Liège, Liège, Belgium, ${ }^{3}$ Warsawian Center of Osteoporosis and Calcium Metabolism, Warsaw, Poland, ${ }^{4} 2^{\text {nd }} \mathrm{Dpt}$ of Internal Medicine, Semmelweis Medical University, Budapest, Hungary, ${ }^{5}$ Dipartimento Scienze Mediche e Chirurgiche, Universita Degli Studi Padova, Padova, Italy, ${ }^{6}$ Servicio de Reumatologia, Hospital 12 de Octubre, Madrid, Spain, ${ }^{7}$ Consultant and Hon Reader in Rheumatology, Whipps Cross University Hospital, United Kingdom, ${ }^{8}$ Department of Medicine, Sir Charles Gairdner Hospital, Perth, Australia, ${ }^{9}$ Department of Rheumatology, Nord Hospital, Amiens, France, ${ }^{10}$ The Osteoporosis Research Center, Department 545, Hvidovre University Hospital, Hvidovre, Denmark, ${ }^{11}$ Department of Rheumatology and Bone Diseases, Edouard Herriot Hospital, Lyon, France

Objective: Strontium ranelate is an orally active treatment able to decrease the risk of vertebral and hip fractures in osteoporotic post menopausal women. The aim of this study was to assess the efficacy of strontium ranelate according to the main determinants of vertebral fracture risk: age, baseline BMD, prevalent fractures, familial history of osteoporosis, baseline BMI and addiction to smoking.

Materials and methods: A population of 5082 women from the pooled analysis of SOTI and TROPOS made of patients with a baseline and at least one post baseline assessment for vertebral fracture risk (2536 receiving strontium ranelate $2 \mathrm{~g} / \mathrm{d}$ and 2546 receiving a placebo), 74 years on average, was followed for 3 years.

Results: Over this period the treatment decreased the risk of both vertebral $(\mathrm{RR}=0.60[0.53-0.69], \mathrm{p}<0.001)$ and non-vertebral $(\mathrm{RR}=0.85$ [0.74-0.99], $\mathrm{p}=0.03)$ fractures. The decrease in risk of vertebral fractures was significant whatever the class of age considered: $37 \% \quad(R R=0.63[0.46-0.85], p=0.003)$ in women $<70$ years, $42 \%(R R=0.58$ [0.48-0.68], $\mathrm{p}<0.001)$ in women aged [70-80[ years, and $32 \%(\mathrm{RR}=0.58 \quad$ [0.50-0.92], $\mathrm{p}=0.013)$ in women $\geq 80$ years. The relative risk of vertebral fracture was 0.28 [0.07-0.99] in osteopenic and 0.61 [0.53-0.70] in osteoporotic women, and baseline BMD was not a determinant of efficacy. The incidence of vertebral fractures in the placebo group increased with the number of prevalent vertebral fractures, but this was not a determinant of the effect of strontium ranelate. In 2605 patients, the risk of experiencing a first vertebral fracture was reduced by $48 \%(R R=0.52$ [0.40-0.67], $\mathrm{p}<0.001)$. The risk of experiencing a second vertebral fracture was reduced by $45 \%$ $(\mathrm{RR}=0.55$ [0.41-0.74], $\mathrm{p}<0.001)(1100$ patients $)$. Moreover, the risk of experiencing more than two vertebral fractures was reduced by $33 \%(\mathrm{RR}=0.67$ [0.55-0.81], $\mathrm{p}<0.001)(1365$ patients $)$. Familial history of osteoporosis, baseline BMI and addiction to smoking were not a determinant of efficacy as well.

Conclusion: This study demonstrates that a 3-year treatment with strontium ranelate leads to anti-vertebral fracture efficacy in post menopausal women independently of age, initial BMD, prevalent vertebral fractures, familial history of osteoporosis, initial BMI and addiction to smoking.

P205. GLUCOSAMINE SULFATE INHIBITS IL-1-STIMULATED GENE EXPRESSION AT CONCENTRATIONS FOUND IN HUMANS AFTER ORAL INTAKE

T. Piepoli ${ }^{1}$, T. Zanelli ${ }^{1}$, O. Letari ${ }^{1}$, S. Persiani ${ }^{2}$, L.C. Rovati ${ }^{2}$, G. Caselli ${ }^{1}$, ${ }^{1}$ Pharmacology Dept., Rottapharm, Monza (MI), Italy, ${ }^{2}$ Clinical Pharmacology Dept., Rottapharm, Monza (MI), Italy

Objective: Studies in man have shown that after administration of oral therapeutic doses of glucosamine sulfate for osteoarthritis, 
plasma concentrations of glucosamine are in the $10 \mu \mathrm{M}$ range (Persiani et al. Osteoarthritis Cartilage 2005; 13: 1041-9). Our aim was therefore to investigate in a human chondrocyte-like cell model, the effects of glucosamine sulfate at these concentrations on IL-1-induced gene expression of different inflammation or matrix degradation markers, and of NF-kB family members.

Materials and Methods: Human chondrosarcoma cells SW 1353 were grown to confluence and stimulated with $2 \mathrm{ng} / \mathrm{ml} \mathrm{IL-}$ $1 \beta$, i.e. the optimal stimulatory concentration in pilot experiments. Cells were incubated in drug-free medium or containing glucosamine sulfate 0.001 to $100 \mu \mathrm{M}$. RealTime PCR was used to quantify gene expression levels after optimal exposure: 6 hours for Cox-2, iNOS, IL-1 $\beta$, IL-6 and MMP-3, 1 hour for TNF $\alpha$ and 24 hours for ADAMTS5 (aggrecanase-2). In addition, gene expression levels of the following NF-kB protein subunits were studied after 2-hour incubation: p50 (NF-kB1), p52 (NF-kB2), p65 (RelA) and RelB. All experiments were run in triplicate. Results were expressed as glucosamine concentrations able to decrease by $50 \%$ (IC50) the stimulation by IL- $1 \beta$.

Results: Glucosamine sulfate decreased IL-1 $\beta$-stimulated gene expression of all markers considered, with glucosamine IC50 close to $10 \mu \mathrm{M}$ or lower (Table 1).

Table 1 Glucosamine IC $50(\mu \mathrm{M} \pm \mathrm{SE})$ on IL-1 $\beta$-stimulated gene expression of different markers

\begin{tabular}{|c|c|c|c|c|c|c|}
\hline Cox-2 & iNOS & IL-1 $\beta$ & IL-6 & MMP-3 & TNF- $\alpha$ & $\begin{array}{l}\text { ADAMT- } \\
\text { S5 }\end{array}$ \\
\hline
\end{tabular}

$11.2 \pm 1.2 \quad 13.8 \pm 5.6 \quad 6.2 \pm 3.0 \quad 4.4 \pm 1.1 \quad 10.2 \pm 2.3 \quad 12.8 \pm 2.0 \quad 2.8 \pm 0.7$

The compound also inhibited the cytokine-induced gene expression of NF-kB subunits at even lower concentrations (Table 2).

Table 2 Glucosamine IC50 ( $\mu \mathrm{M} \pm \mathrm{SE})$ on IL-1 $\beta$-stimulated gene expression of NFkB subunits

\begin{tabular}{llll}
\hline P50 & p52 & p65 & RelB \\
\hline $0.4 \pm 0.2$ & $0.6 \pm 0.5$ & $1.0 \pm 1.8$ & $0.3 \pm 0.4$ \\
\hline
\end{tabular}

Conclusions. Glucosamine sulfate inhibits IL-1-stimulated gene expression of different inflammation or matrix degradation markers in a human chondrocyte-like cell model, at glucosamine concentrations found in human plasma after oral therapeutic doses. While these events are probably initiated by a decrease in NFkB nuclear translocation (Largo et al, Osteoarthritis Cartilage $2003 ; 11: 290-8)$, they might be sustained thereafter by the inhibition of the expression of its subunits.

\section{P206. GLUCOSAMINE PLASMA AND SYNOVIAL FLUID CONCENTRA- TIONS BEFORE AND AFTER ORAL ADMINISTRATION OF CRYSTALLINE GLUCOSAMINE SULFATE IN KNEE OSTEOARTHRITIS PATIENTS}

S. Persiani ${ }^{1}$, R. Rotini ${ }^{2}$, G. Trisolino ${ }^{2}$, D. Paganini ${ }^{1}$, L.C. Rovati $^{1}$, M. Locatelli ${ }^{3}$, A. Roda ${ }^{3}$; ${ }^{1}$ Clinical Pharmacology Dept., Rottapharm, Monza (MI), Italy, ${ }^{2}$ Codivilla Dept., Rizzoli Orthopaedic Institute, Bologna, Italy, ${ }^{3}$ Dept. of Pharmaceutical Sciences, University of Bologna, Bologna, Italy

Objective: Our group has recently described the pharmacokinetics of glucosamine in plasma (Persiani et al, Osteoarthritis Cartilage: 2005; 11, 1041-1049) after oral administration to healthy volunteers of crystalline glucosamine sulfate, i.e. the only glucosamine formulation shown to be a symptom- and structuremodifying drug in osteoarthritis (Towheed et al, The Cochrane Library 2005, Issue 2). The aim of the present study was to assess glucosamine levels in plasma and synovial fluid before and after administration of crystalline glucosamine sulfate at therapeutic doses to patients with knee osteoarthritis.

Materials and Methods: Five patients ( 3 men, 2 women) with knee osteoarthritis received oral crystalline glucosamine sulfate soluble powder $1500 \mathrm{mg}$ once-a-day (Rottapharm, Monza, Italy) for 14 consecutive days. Blood and synovial fluid samples were collected before the first drug administration and 3 hours after the last administration, when steady state plasma concentrations were at maximum in the previous healthy volunteer study. Glucosamine concentrations were determined by a Liquid Chromatography with Mass Spectrometry detection (LC-MS/MS) method, with a quantitation limit of $2.1 \mathrm{ng} / \mathrm{ml}$.

Results: The mean (SE) age, height, weight and BMI of the patients were $53 \pm 5$ years, $171 \pm 6 \mathrm{~cm}, 80 \pm 7 \mathrm{~kg}$ and $26.8 \pm 1.3 \mathrm{~kg} /$ $\mathrm{cm}^{2}$, respectively. Endogenous glucosamine was detected in baseline plasma and synovial fluid, with mean (SE) concentrations of $39.5 \pm 5.1 \mathrm{ng} / \mathrm{ml}(0.2 \pm 0.03 \mu \mathrm{M})$ and $15.1 \pm 6.8 \mathrm{ng} / \mathrm{ml}$ $(0.08 \pm 0.04 \mu \mathrm{M})$, respectively. Three hours after the last glucosamine sulfate administration, baseline-subtracted concentrations were $1419 \pm 711 \mathrm{ng} / \mathrm{ml}(7.9 \pm 3.9 \mu \mathrm{M})$ in plasma and $1291 \pm 576$ $\mathrm{ng} / \mathrm{ml}(7.2 \pm 3.2 \mu \mathrm{M})$ in the synovial fluid.

Conclusions: As in healthy volunteers, endogenous glucosamine is present in plasma of knee osteoarthritis patients and, in the latter is detectable also in the synovial fluid. After repeated oral doses of glucosamine sulfate, the plasma and synovial fluid concentrations are similar and in the $10 \mu \mathrm{M}$ range. This concentration is effective in selected human chondrocyte models (Piepoli et al, Arthritis Rheumatism 2005; 9 Suppl: 1326). Further studies are needed to investigate glucosamine mechanism of action at these concentrations, as well as the pathophysiological relevance of different endogenous levels in osteoarthritis patients and the correlation between the levels achieved after exogenous administration and the treatment response.

\section{P207. TESTOSTERONE AND BONE MASS INDICES IN ADOLESCENT BOYS}

L.A. Scheplyagina, T.Y. Moisseyeva, E.N. Tsygina; Research Center of Child Health, Russian Academy of Medical Sciences, Moscow, Russia

Objective(s): To assess relationship of serum testosterone level with bone mineral density indices in adolescent boys.

Materials and Methods: In all, 43 healthy boys at the age from 10 to 16 years and selected using random sampling were examined. All children were measured body height and body mass, total sexual development score were calculated. Moreover, level of biologic development was established using both X-ray of hand bones or accordance of bone age to passport age. Sex hormones supply was studied according serum testosterone level. Mineral bone density was evaluated by dual-energy absorbciometry $\left(\mathrm{L}_{2}-\right.$ $\mathrm{L}_{4}$ ) using densitometer "DPX-MD +" Lunar.

Results: Mean age of examined children was 12,4 years. Physical development in observed boys mainly was harmonious $(81,4 \%)$. Obesity of I-II stage was established in $13.9 \%$ and lower body mass - in $4.7 \%$ of children. In boys, total score of sexual development corresponded to age $(81.4 \%)$ and in $18.6 \%$ pubertas retardation was revealed.

Results of densitometric study demonstrated that every forth child had BMC and BMD values lower than norm. This permitted to identify development of osteopenia. Osteopenia prevalence $(25 \%)$ did not differ significantly from corresponding values in children population of same age and gender. In every forth boy lower values of serum testosterone (less than $10 \%$ percentile) were determined. In examined adolescents, testosterone level correlated with age $(\mathrm{r}=0.6 ; \mathrm{p}<0,000)$, body height $(\mathrm{r}=0.8 ; \mathrm{p}<0,000)$, total score of sexual development $(\mathrm{r}=0.8 ; \mathrm{p}<0,000)$, BMC $(\mathrm{r}=0.7$; $\mathrm{p}<0,000)$, BMD $(\mathrm{r}=0.7 ; \mathrm{p}<0,000)$ and cortical bone thickness $(r=0,4 ; p<0,05)$. As a rule, lower testosterone values were accompanied by lower values of BMD and BMC. In case of pubertas retardation decrease of serum testosterone were established much more often $(90 \% ; \mathrm{p}<0,05)$.

Conclusions: Study findings permit to refer adolescent boys with pubertas retardation to risk group of osteopenia development. 
P208. ESTRADIOL AND BONE MINERALIZATION IN ADOLESCENT GIRLS

L.A. Scheplyagina, T.Y. Moisseyeva, I.V. Kruglova; Research Center of Child Health, Russian Academy of Medical Sciences, Moscow, Russia

Objective(s): To analyze relationship between bone mass and main indicators of pubertas in adolescent girls.

Materials and methods: Random sampling was applied to select 95 healthy girls at the age of $13-17$ to be examined. In all children physical development (body height, body mass, body mass index), total score of sexual development and Tanner's puberty stages. Bone mineral density $\left(\mathrm{L}_{2}-\mathrm{L}_{4}\right)$ was determined using osteodensitometer DPX-MD + Lunar. Osteocalcine (bone formation marker) and estradiol were analyzed in blood serum using immune enzymatic method.

Results and Discussion: Mean age of menarche in examined girls was 12,7 years. It is established that as a rule body height and body mass in examined girls did not differ norm and was positioned within the limits of $25-75 \%$ percentiles. In observed adolescents, stages of pubertas corresponded established norm.

According densitometry results, in $22 \%$ of girls osteopenia was established. This did not differ substantially from corresponding index in children population of the same age and gender.

It is established that values of BMC and BMD, independently of Z-score, correlated with body height $(\mathrm{r}=0.6, \mathrm{p}<0.01)$, body mass $(r=0.7, p<0.05)$, body mass index $(r=0.4, p<0.05)$, total score of sexual development $(\mathrm{r}=0.5, \mathrm{p}<0.01)$, Tanner's puberty's stages $(\mathrm{r}=0.6, \mathrm{p}<0.01)$.

Relationship between osteocalcin level and body height $(\mathrm{r}=0$, $5, \mathrm{p}<0.05)$ and age of menarche $(\mathrm{r}=-0,5, \mathrm{p}<0.05)$ was established.

It is demonstrated that in girls, before age of 14, unidirectional increase of body height, osteocalcin level, BMS, BMD and estradiol takes place. It is noticed that after age of 16 and with establishment of menstrual function blood estradiol increased significantly (up to $49,9 \mathrm{pg} / \mathrm{ml}$ ). At the same time decreased longitudinal growth rate, osteocalcin level and bone mineralization.

Conclusions: Study findings permit to suggest that estradiol impact on bone mineral density in girls during pubertas is caused by relationship of estradiol with longitudinal growth rate and bone mineralization.

\section{P209. AGE-SPECIFIC CHARACTERISTICS OF AXIAL SKELETON MIN- ERALIZATION IN CHILDREN}

L.A. Scheplyagina, T.Y. Moisseyeva, I.V. Kruglova; Research Center of Child Health, Russian Academy of Medical Sciences, Moscow, Russia

Objective: To analyze formation of bone mass in children.

Materials and Methods: In cross-sectional study, 357 healthy children (194 boys and 163 girls) aged from 5 to 17 years were examined. Mineral bone density was evaluated by dual energy absorptiometry $\left(\mathrm{L}_{2}-\mathrm{L}_{4}\right)$ using osteodensitometry "DPX-MD+", LUNAR, USA. Absolute values of BMC $(\mathrm{g})$ and BMD $\left(\mathrm{g} / \mathrm{cm}^{2}\right)$ were assessed.

Results: Significant variability of age-specific values of BMC and BMD is established. It is demonstrated that on average BMC increases from $13.5 \mathrm{~g}$ to $46.4 \mathrm{~g}$ in boys and from 13.72 to $46.23 \mathrm{~g}$ in girls. It is established that with age BMD increase repeats BMC increase but its rate is slower. So, BMD increased from 0.64 to $1.09 \mathrm{~g} / \mathrm{cm}^{2}$ in boys and from 0.65 to $1.09 \mathrm{~g} / \mathrm{cm}^{2}$ in girls. It is revealed that intensive mineralization $\mathrm{L}_{2}-\mathrm{L}_{4}$ occurs from I to III Tanner's stage of pubertas. In all, during pubertas (from 10 to 16 years) BMC increases on $90.7 \%$ in boys and on $97.6 \%$ in girls. BMD increase comes to $34.6 \%$ and $39.7 \%$ accordingly. During pubertas bone mineral density in lumbar part of spine increases in 1.5 times more than in neutral age.
It is established that mean values of $\mathrm{BMC}$ and $\mathrm{BMB}$ in girls younger than 14 years in general were higher than in boys. After the age of 15 BMC and BMD levels in boys began to exceed corresponding parameters in girls. It is demonstrated that BMC and BMD independently of gender have close correlation with body height $(\mathrm{r}=0,8 ; \mathrm{p}=0,000)$ and body mass $(\mathrm{r}=0,7$ $\mathrm{p}=0,000)$.

Conclusions: Age-specific patterns of bone growth in children are characterized by mutual conditionality of body height increase mineral accumulation and bone mineral density increase which are most vital during pubertas.

\section{P210. QUANTITATIVE ULTRASONOMETRIA OF RADIUS AND TIBIA BONES IN HEALTHY CHILDREN}

E.O. Samokhina, L.A. Tcheplyagina, T.Y. Moisseyeva, T.K. Martchenko; Research Center of Children Health, Russian Academy of Medical Sciences, Moscow, Russia

Objective: To assess values of bone strength in healthy children depending of age and gender.

Material and methods: In all, 634 healthy children (298 boys and 336 girls) aged from 3 to 16 years and residing in Moscow were examined. Skeleton bone strength was evaluated by rate of bone speed of ultrasound (SOS, $\mathrm{m} / \mathrm{s}$ ) at distal part of radius and at the mid-shaft tibia. All measurements were made using device "Omnisense 7000S" (Sunlight Medical Ltd, Israel). Data statistic was processed with integrated software package "STATISTICA 6 for MS Windows".

Results: It is established that in examined children SOS values in both sites were noted for variability and, as a rule, they were the higher the older was child independently of gender. In overall, in children at the age from 3 to 16 years SOS values increased from $3588,2 \mathrm{~m} / \mathrm{s}$ to $3829,08 \mathrm{~m} / \mathrm{s}(+6,7 \%)$ in boys and from 3551,4 $\mathrm{m} / \mathrm{s}$ to $3978,09 \mathrm{~m} / \mathrm{s}(+12 \%)$ in girls. During pubertas, SOS values of radius increased in boys and girls on $2.2 \%$ and $5.6 \%$ respectively. Relationship between SOS values of radius and age $(r=0,4$ $\mathrm{p}=0,000)$, body height $(\mathrm{r}=0,3 ; \mathrm{p}=0,000)$ and body mass $(\mathrm{r}=0,3$ $\mathrm{p}=0,000)$ is established. Analysis of data of tibia quantitative ultrasonometria in children at the age from 3 to 16 years revealed that level of SOS value of mid-shaft tibia increased from 3527,1 $\mathrm{m} / \mathrm{s}$ to $3812,55 \mathrm{~m} / \mathrm{s}(+8 \%)$ in boys and from $3489,83 \mathrm{~m} / \mathrm{s}$ to $3795,4 \mathrm{~m} / \mathrm{s}(+8,7 \%)$ in girls. During pubertas, SOS value of tibia increases in boys and girls on $6.4 \%$ in and $5.3 \%$ respectively. Relationship between SOS value of tibia and age $(r=0,4$ $\mathrm{p}=0,000)$, body height $(\mathrm{r}=0,3 ; \mathrm{p}=0,000)$ and body mass $(\mathrm{r}=0,3$ $\mathrm{p}=0,000)$ is revealed. Relationship between SOS value of radius and SOS value of tibia $(r=0,4 ; p=0,000)$ is also established.

Conclusions: Age-specific values of quantitative ultrasonometria of radius and tibia in examined children can be applied as age-specific standards in assessment of bone strength in healthy and ill children.

\section{P211. RELEVANCE OF THE PRODUCTION CYTOKINES IN RHEUMATOID ARTHRITIS}

L. Petrovic-Rackov, Clinic of Rheumatology and Clinical Immunology, Beograd, Serbia, Crnotravska 17, Beograd, Serbia and Montenegro

Citokines TH1 lymphocyte with products of sinoviocytes disrupts natural balance in citokine net inside sinovial tissue, which leads to immunoinflammatory reaction and joint damage. The latest experimental researches in vitro and in vivo on the mice model approved that the interleukins (IL)-12, IL-15 and IL-18 participate in the rheumatoid arthritis (RA) geneses. According to the available literature, researches in vivo have not been conducted on human population so far, thus production and the level of these cytokines in synovial fluid (SF) and blood remains unknown as well as the correlation of the production these three cytokines and the production of the TNF-alpha. The aim of the research is to determine whether 
there is a correlation of the concentrations of the TNF-alpha, IL-18, IL-15 and IL-12 in blood and synovial fluid samples of the patients having active RA. Concentrations of cytokines in serum and SF of 64 patients, whose disease activity was valuated according to the DAS 28 index, and 25 patients with osteoarthrosis (OA), was measured using ELISA whales for human interleukines. The comparison of the cytokine concentrations in serum and synovial fluid showed that patients with RA had considerable higher $(p<0.05)$ concentrations of the TNF-alpha, IL-18 and IL-15 in synovial fluid than in serum, which affirms presumption that these cytokines are produced in a joint and can be good indicators of the local inflammation. We affirmed the existence of the correlation of the concentrations and the production of the TNF-alpha with the production of the IL-18, IL-15 and IL-12 in blood and ST $(p<0,01)$. These cytokines relate to the production of the TNF-alpha, which is one of the key mechanisms that maintain inflammation process in RA, both locally and in the systematically. Only the correlation of the concentrations of the IL-18 and IL-12 in ST could not proven.

\section{P212. AN EVALUATION OF RELATIVE IMPORTANCE OF DIFFERENT ATTRIBUTES OF OSTEOPOROSIS MEDICATION: RESULTS FROM THE PREFER-INTERNATIONAL STUDY}

J. Walliser ${ }^{1}$, S. Bolge ${ }^{2}$, S.S. Sen ${ }^{3}$; ${ }^{1}$ Medical Director of the "Clinical de Metabolismo Oseo y Mineral del Hospital Angeles del Pedregal, Mexico, ${ }^{2}$ Consumer Health Sciences, Princeton, NJ, USA, ${ }^{3}$ Outcomes Research, Merck \& Co., Whitehouse Station, NJ, USA

Background: As per Anderson's health belief model, a patient's decision to take action is dependent upon the patient's perception of advantages and disadvantages of the treatment itself. A better understanding of patients' preference for different attributes of osteoporosis medication may indicate what would be the driving factor for enhancing their adherence to therapy.

Objective: To evaluate patients' attitudes towards different attributes of osteoporosis medication.

Methods: Physicians were randomly selected in France, Germany, Mexico, Spain and UK and asked to recruit consecutive 4 osteoporotic women aged 50 years or older seen in their practices. These patients were contacted and those who consented to an interview were asked to rank and rate importance of efficacy in reducing risk of fracture, side effects, out-of-pocket costs, dosing frequency, formulation (oral, vs. IV), time on market and dosing procedure on a scale of 1 to $5 \quad(1=$ not important at all and $5=$ extremely important). The correlation between patient's ranking for each attribute and importance rating for that attribute was evaluated to assess the consistency in patients' response.

Results: A total of 859 physicians were contacted. Based on their referrals 3000 patients were interviewed of whom 1500 were on prescription treatment for osteoporosis while rest were not. A majority $(57 \%)$ of the patients ranked effectiveness in reducing risk of fracture as the most important reason for their preference for osteoporosis prescription medication, while side-effects $(20 \%)$, and out-of-pocket costs $(7 \%)$ were the second and third most frequently cited most important reason among all the 7 attributes of osteoporosis medication. Effectiveness in reducing risk of fracture also had significantly $(P<0.05)$ higher mean importance rating (4.6) than side-effects, out-of-pocket costs, dosing frequency, formulation, time on market or dosing procedure. There were significant correlations $(\mathrm{p}<0.05)$ between patients' ranking of each attribute and their importance rating.

Conclusion: A majority of patients ranked and rated effectiveness in reducing risk of fracture as the most important reason and of highest importance of all attributes in preferring an osteoporosis medication. Patients ranking of attributes as per their importance were consistent with their importance rating of each of these attributes indicating internal consistency of the questionnaire used.
P213. INTERLEUKIN-6 MEDIATES SUBCHONDRAL OSTEOBLASTS-INDUCED CARTILAGE DEGRADATION

C. Sanchez ${ }^{1}$, E. Kaut ${ }^{1}$, M. Deberg ${ }^{1}$, J.P. Delcour ${ }^{2}$, Y. Henrotin ${ }^{1}$; ${ }^{1}$ Bone and Cartilage Research Unit, University of Liège, Liège, Belgium, ${ }^{2}$ Bois de l'Abbaye Hospital, Seraing, Belgium

Objective: Previously, we have demonstrated that osteoblasts from the sclerotic zones of OA subchondral bone express a particular phenotype characterized by an overproduction of interleukin (IL)-6, transforming growth factor (TGF)-beta1, alkaline phosphatase and osteocalcin but similar amount of IL-1 beta than non-sclerotic osteoblasts. Further, we have observed in a co-culture model that osteoblasts from the sclerotic zones affected chondrocytes metabolism. This work was designed to identify the mediators involved in these osteoblasts-induced effects.

Material and methods: Human chondrocytes were isolated from OA cartilage and cultured in alginate beads for 4 days in the absence or in the presence of non sclerotic or sclerotic OA subchondral osteoblasts in monolayer (co-culture system). During co-culture, monoclonal antibody (Mab) neutralizing IL-6 was added. Aggrecan, sox 9 and matrix metalloproteases (MMP) -3 and -13 mRNA levels in chondrocytes were quantified by real time polymerase chain reaction. Aggrecan and MMP-3 production was assayed by a specific ELISA.

Results: In co-culture, sclerotic, but not non-sclerotic, osteoblasts significantly decreased $(-27 \%, p<0.001)$ aggrecan production and aggrecan gene expression by human chondrocytes. In parallel, sox9 expression was decreased $(-52 \%, \mathrm{p}<0.001)$ whereas MMP-3 and MMP-13 gene expression were increased (+ 44 and $+76 \%$, respectively, $\mathrm{p}<0.001)$. Anti-IL-6 Mab, prevents all these osteoblasts-induced effects.

Conclusions: OA subchondral osteoblasts could contribute to cartilage degradation by stimulating chondrocytes to produce more matrix metalloproteases and by inhibiting aggrecan synthesis. Herein, we have identified IL-6 as a key mediator of the osteochondral pathophysiological axis. To neutralize IL-6 biological activity prevents the negative effects of sclerotic osteoblasts on cartilage metabolism.

P214. COLL 2-1, COLL 2-1 NO ${ }_{2}$ AND MYELOPEROXYDASE IN OA PATIENTS BEFORE AND AFTER HIP OR KNEE REPLACEMENT

M Deberg ${ }^{1}$, A Labasse ${ }^{1}$, E Quettier ${ }^{2}$, JE Dubuc ${ }^{2}$, A Bosseloir ${ }^{3}, Y$ Henrotin'; ${ }^{1}$ Bone and Cartilage Research Unit, CHU SartTilman, 4000 Liège, Belgium ${ }^{2}$ Hospital Princess Paola, 6900 Marche-en-Famenne, Belgium, ${ }^{3}$ Zentech, Liège Science Park, 4031 Angleur, Belgium

Objectives: To determine Coll 2-1 level, a peptide of type II collagen triple helix, its nitrated form $\left(\right.$ Coll 2-1 $\left.\mathrm{NO}_{2}\right)$ and myeloperoxydase (MPO) in serum of patients with knee or hip osteoarthritis (OA) before and three months after joint replacement.

Material and methods: Coll 2-1, Coll 2-1 $\mathrm{NO}_{2}$ and MPO were measured by specific immunoassays in 56 asymptomatic aged donors and in 103 patients with knee or hip OA candidates for joint replacement. Sera were taken the day before the surgery and three months after the hip or knee replacement.

Results: Before surgery, Coll 2-1, Coll 2-1 $\mathrm{NO}_{2}$ and MPO levels were higher in serum of $\mathrm{OA}$ patients than in asymptomatic aged donors [Coll 2-1: median: 146.90 (min: 74.53max: 368.01) $\mathrm{nM}$ vs 119.50 (min: 38.03- $\max : 242.90) \mathrm{nM}$ $(\mathrm{p}<0.05)$, Coll 2-1 $\mathrm{NO}_{2}$ : median : 0.54 (min: 0.10- $\left.\max : 4.21\right)$ $\mathrm{nM}$ vs 0.30 (min: 0.06- $\max : 0.89) \mathrm{nM}(\mathrm{p}<0.001)$ and MPO: median : 147 (min: 0.00- max: 1213.00) ng/ml vs 67.00 (min: 0.00-max: 212.00$) \mathrm{ng} / \mathrm{ml}(\mathrm{p}<0.001)]$. Three months after joint replacement, Coll 2-1 and MPO concentrations in serum of OA patients decreased to the level of the asymptomatic aged donors, whilst Coll 2-1 $\mathrm{NO}_{2}$ levels tended towards a decrease after surgery [median: 0.50 (min: 0.14- max: 1.05) nM]. Moreover, a significant correlation between serum levels of Coll 2-1 and MPO before and after joint replacement was observed (Coll 2-1: $\mathrm{r}=0.23, \mathrm{p}=0.02$; MPO: $\mathrm{r}=0.33, \mathrm{p}=0.003$ ). 
Conclusions: Coll 2-1 is elevated in serum of OA patients and decreases after surgery suggesting that Coll 2-1 is indicative of structural changes in one single joint. Further, MPO levels are increased in OA patients indicating that an excessive activation of neutrophils in late $\mathrm{OA}$ is present.

\section{P215. BONE MINERAL STATUS IN COPD PATIENTS: A CROSS-SEC- TIONAL STUDY}

H. Zmierczak ${ }^{1}$, E. Derom ${ }^{2}$, M. Behaegel ${ }^{2}$, R. Demuynck ${ }^{1}, \mathrm{H}$. Myny $^{1}$, H. Vlieghe ${ }^{1}$, J.M. Kaufman ${ }^{1}$, S. Goemaere ${ }^{1}{ }^{1}$ Unit for Osteoporosis and Metabolic Bone Diseases, ${ }^{2}$ Department of Pneumology Ghent University Hospital, Ghent, Belgium

Objective: Patiënts with moderate to severe chronic obstructive pulmonary disease (COPD) have an increased risk for fractures, specifically for vertebral and rib fractures. The contributing factors to this increased fracture risks are constituted by metabolic factors (relative acidosis, sex hormone and vitamine D deficiency, calcium losses by loop diuretics), mechanical factors (low physical activity level, muscle atrophia) and (intermittent) glucocorticoid treatment. The present study decribes the frequency of osteoporosis in severely affected COPD patients requiring systemic corticotherapy.

Methods: COPD patients $(\mathrm{n}=81)$ fulfilling the following criteria were referred for bone evaluation: (1) chronic corticotherapy $>5 \mathrm{mg}$ prednisolone equivalent/day (2) hospitalization due to COPD exacerbation during the last year, (3) COPD exacerbation with intermittent corticotherapy in the last 3 years. BMD at L1L4 and femoral neck and vertebral deformity assessment (IVA) were performed by DXA. In females osteoporosis was defined by a $\mathrm{T}$-score of -2.5 . Low bone mass in men was defined by a Zscore of -2.0 . Vertebral deformities were graded semi-quantitatively.

Results: Men represented $89 \%$ of the referrals. Mean age was $67 \mathrm{y}$ for men and $58 \mathrm{y}$ for women. The COPD severity was scored by the GOLD criteria: I (8\%), II (16\%), III (52\%) and IV (24\%). Chronic corticotherapy, hospitalization and intermittent corticotherapy for COPD exacerbation in the last 3 years were reported in respectively $13 \%, 54 \%$ and $32 \%$. Osteoporosis defined by low bone density criteria was found in $61 \%$. Prevalent vertebal deformities on IVA were documented in $33 \%$. Only $6 \%$ of these patients medical treatment for osteoporosis. The following advices were formulated for the referring and treating pneumologist: calcium and vitamin D supplementation only in $41 \%$ and further diagnostic evaluation possibly followed with anti-resorptive treatment in $48 \%$.

Conclusion: The present study confirms that this COPD subpopulation is at high risk for osteoporosis and (vertebral) fracture. Low rates of bone health treatment are observed. Without a systematic approach a substantial proportion of this high-risk patients are missed for diagnosis and treatment with a high probability of cost-effective intervention towards fracture prevention. The study was sponsored by an unrestricted grant of MSD Belgium.

\section{P216. DXA-BASED ASSESSMENT OF MALE PATIENTS USING STAN- DARDIZED BONE DENSITY VALUES AND A NATIONAL REFERENCE DATABASE}

S. Goemaere ${ }^{1}$, D. Vanderschueren ${ }^{2}$, J.M. Kaufman ${ }^{1}$, J.-Y. Reginster $^{3}$, Y. Boutsen ${ }^{4}$, S. Poriau ${ }^{5}$, J. Callens ${ }^{6}$, F. Raeman ${ }^{7}$, G. Depresseux ${ }^{8}$, J.P. Devogelaer ${ }^{8}$, H. Borghs ${ }^{2}$, S. Boonen ${ }^{2}$; ${ }^{1}$ Unit for Osteoporosis and Metabolic Bone Diseases, Ghent University Hospital, Ghent, ${ }^{2}$ Leuven University Center for Metabolic Bone Diseases, Katholieke Universiteit Leuven, ${ }^{3}$ Centre Universitaire d'Investigation du Métabolisme Osseux et du Cartilage Articulaire, State University of Liège, ${ }^{4}$ Department of Rheumatology, Université catholique de Louvain Mont Godinne, ${ }^{5}$ Rheumatology, St Elisabeth Ziekenhuis, Sijsele, ${ }^{6}$ Physiotherapy, OLV Ter Linden, Knokke, ${ }^{7}$ Rheumatology, Jan Palfijn Ziekenhuis, Merksem, ${ }^{8}$ Arthritis Unit, Université Catholique de Louvain, Belgium
Objective: Dual energy X-ray absorptiometry (DXA) measurements from different manufacturers provide different bone mineral density (BMD) values and derived T-scores and Z-scores. The differences result partly from technical differences in the algorithms for the determination of bone mineral content (BMC) and bone area and partly from the use of different manufacturerderived reference databases. The purpose of the present study was to implement an uniform expression of BMD in male patients in Belgium, by using standardized BMD (sBMD) values and referring to a newly established national male reference population.

Methods: In 8 bone densitometry centers throughout Belgium, 229 young healthy men were measured on Hologic or GE-Lunar bone densitometers. Quality control procedures were implemented and site cross-calibration performed using the European Spine Phantom. Absolute BMD values were converted to standardized values by previously validated formulae (sBMD).

Results: Clinically acceptable between-center differences were noted. No discrepancy was observed in terms of mean sBMD and standard deviations at the lumbar spine and proximal femur between the Belgian and the NHANES US reference populations. Region-specific sBMD thresholds for the diagnosis of male osteoporosis were calculated.

Conclusions: The current data provide a basis to implement a nation-wide, uniform expression of BMD in male patients and allow harmonization of the BMD-based diagnosis of osteoporosis and the reimbursement of drug therapy in Belgian men with osteoporosis.

\section{P217. DETECTION OF VERTEBRAL FRACTURES ON ROUTINE CHEST X-RAYS: THE STOF STUDY}

S. Goemaere ${ }^{1}$, Y. Boutsen ${ }^{2}$, P. Durez ${ }^{3}$, J. Vanslembrouck ${ }^{4}$ J.F Nisolle $^{5}$, K. Verstraete ${ }^{4}$, J.M Kaufman ${ }^{1}$, J.P. Devogelaer ${ }^{3}$; ${ }^{1}$ Unit for Osteoporosis and Metabolic Bone Diseases, Ghent University Hospital, Ghent, Belgium, ${ }^{2}$ Department of Rheumatology, University Hospital Mont-Godinne, Yvoir, ${ }^{3}$ Department of Rheumatology, St-Luc University Hospital, Brussels, ${ }_{5}^{4}$ Department of Radiology, Ghent University Hospital, Gent, ${ }^{5}$ Department of Radiology, University Hospital Mont-Godinne, Yvoir

Objective: Osteoporosis related vertebral fractures (VFs) have important health care impact and are also strong predictors of future vertebral and other fractures, leading to impaired quality of life and associated mortality. About two thirds of the VFs occur unobserved and are undetected by simple clinical observation. They may be found on chest X-ray performed for unrelated reasons. The purpose of the present study was to describe the prevalence of unrecognized VF, as a missed opportunity for the detection of osteoporosis based on routine X-rays.

Methods: Chest X-rays of female patients older than 50 years were systematically reviewed by a dedicated radiologist for the prevalence of VFs after the routine X-ray protocol. A BMD assessment was proposed directly towards the patients or the referring physician.

Results: From the 900 chest X-rays were reviewed, a total of 115 cases with VFs were documented; mean age was 72 year. By semi-quantitative evaluation most of the cases had low grade severity: grade I (55\%), grade II $(35 \%)$ and grade III $(10 \%)$. The number of VFs per case was 1 in $52 \%, 2$ in $24 \%$ and more than 2 in $24 \%$ of the cases. Review of the routine protocol revealed a manifest underreporting of these fractures. Only in $10 \%$ of the reports "vertebral fracture" was notified either as an unambiguous term or clear diagnosis.

DXA data were obtained for 29 patients $(25 \%)$. Mean Tscores were at the lumbar spine -1.2 , at the total hip -1.9 and the femoral neck -2.5 . A T-score below -2.5 at the lumbar spine was observed in 8 patients $(28 \%)$. Including DXA at the proximal femur 13 additional osteoporosis patients $(48 \%)$ were identified. Systematic data collection about the treatment advices was not possible due to the lack of organized care.

Conclusion: The report of chest X-rays should make a clear statement about the diagnosis and prevalence of vertebral fracture ideally. The clinical experience in the present study demonstrates 
the need for the elaboration of clinical pathways by which health care-givers could take further steps for the evaluation and treatment of osteoporosis in patients with VF on routine chest X-ray. Disclosure: This study was supported by an unrestricted grant of Eli Lilly Belgium.

\section{P218. THE ECONOMICS OF TARGETING FRAGILITY FRACTURES IN AN} ORTHOPAEDIC TREATMENT UNIT

B. Sander ${ }^{1,2}$, V. Elliot-Gibson ${ }^{3}$, D.E. Beaton ${ }^{3}$, E.R. Bogoch ${ }^{3}$, A. Maetzel ${ }^{1,4}$; ${ }^{1}$ University of Toronto, Department of Health Policy, Management and Evaluation, Toronto, ON, Canada, ${ }^{2}$ University Health Network, Division of Clinical Decision Making, Toronto, ON, Canada, ${ }^{3}$ St. Michael's Hospital, Mobility Program Clinical Research Unit, Toronto, ON, Canada, ${ }^{4}$ Amgen Europe $\mathrm{GmbH}$, Lucerne, Switzerland

Objective: The orthopedic unit at a university teaching hospital hired an osteoporosis (OP) coordinator to manage a collaborative program to identify fragility fracture patients and arrange for investigation and treatment of OP and patient education. This study evaluates the cost-effectiveness for an academic hospital to hire a coordinator and identifies areas for future research.

Materials and Methods: Using patient-level data from the first year of the program and data from the literature, a 1-year decision analytic model was developed. The model calculates the annual incidence of a further hip fracture dependent on type of index fracture (hip, wrist, humerus, other), attribution to OP, age and gender. Referral uptake, initiation of OP treatment and compliance modified the incidence of further hip fractures in the presence of a coordinator. Deterministic and Bayesian modeling techniques were used to evaluate the cost-effectiveness and its consequences for decision making. An expected value of perfect information (EVPI) analysis was performed to identify areas where more research would be most valuable.

Results: A coordinator who manages 500 patients yearly would reduce further hip fractures from 35 to 25 , a net cost saving to the hospital of $\mathrm{C} \$ 138,450$. A coordinator was cost-saving: 1) if the costs of treating a further hip fracture are greater than $\mathrm{C} \$ 8,000,2)$ if only half of patients initiated treatment and only half of these complied, 3 ) if treatment efficacy reduced fractures by as low as $20 \%$ and 4 ) if only 190 patients were seen annually. The probability of the coordinator being a cost-effective intervention exceeds $80 \%$ if willingness to pay (WTP) is C $\$ 16,000$ per hip fracture avoided or greater. More research is most valuable for the treatment costs of further hip fractures (C\$90,000 at a WTP threshold of C\$15,000).

Conclusion: Employment of a coordinator to manage fragility fracture patients may reduce further hip fractures and was a dominant strategy in the deterministic analysis. The Bayesian analysis showed a high probability of cost-effectiveness for this intervention from the hospital's perspective if WTP exceeds C\$16,000 per hip fracture avoided. However, if WTP is low, more research on treatment costs of further hip fractures is warranted.

\section{P219. DIFFERING EFFECTS ON HIP AND NONSPINE FRACTURE RISK AMONG N-CONTAINING BISPHOSPHONATES: REVIEW AND META- ANALYSIS}

U.A. Liberman ${ }^{1}$, M.C. Hochberg ${ }^{2}$, P. Geusens ${ }^{3}$, P.D. Ross ${ }^{4}$; ${ }^{1}$ Felsenstein Medical Research Center, Department of Physiology \& Pharmacology, Sackler School of Medicine, Tel-Aviv University, Tel-Aviv, Israel, ${ }^{2}$ Division of Rheumatology and Clinical Immunology, Department of Medicine, University of Maryland School of Medicine, Baltimore, Maryland, USA, ${ }^{3}$ Biomedical Research Institute - LUC, Universitaire Campus, Diepenbeek, Belgium and Maastricht, The Netherlands, ${ }^{4}$ Merck Research Laboratories, Merck \& Co., Inc., Rahway, NJ, USA

Objectives: N-containing bisphosphonates reduce the risk of vertebral fractures (VFx); however, results have not shown con- sistent effects on hip and other nonspine fractures. Systematic reviews are useful to summarize effects that often vary among individual trials, and meta-analysis provides a more precise estimate when results are consistent across pooled trials.

Methods: We surveyed earlier systematic reviews and metaanalyses ${ }^{1}$ together with subsequent reports ${ }^{2-4}$ of randomized, placebo-controlled trials with data on nonspine and/or hip fractures, and used meta-analysis where appropriate to test for heterogeneity and derive pooled estimates.

Results: For risedronate (RIS) ( $\mathrm{n}=12958$ subjects in trials), the relative risk (RR) reduction was $27 \%$ for nonspine fracture $(\mathrm{RR}=0.73 ; 95 \% \mathrm{CI}=0.61,0.87)^{1}$ and $26 \%$ for hip fracture $(\mathrm{RR}=0.74 ; 0.58,0.94)$; there was no significant interaction of treatment and age $>80$ and there was no heterogeneity by dose ( 2.5 or $5 \mathrm{mg} /$ day). For alendronate (ALN), heterogeneity existed; doses $\geq 10 \mathrm{mg} /$ day were significantly more effective for reduction of nonspine and hip fracture. The RR reduction with ALN $\geq 10$ $\mathrm{mg}(\mathrm{n}=3723)$ was $49 \%$ for nonspine fractures $(\mathrm{RR}=0.51 ; 0.38$, $0.69)^{1}$ The RR reduction for hip fracture was $55 \%(\mathrm{RR}=0.45$ $0.28,0.71$ ) when osteoporotic women in FIT (ALN $5 \mathrm{mg}$ /day in years $1-2$ then $10 \mathrm{mg}$ /day for remainder of trial) were included $(\mathrm{n}=6804)$, and $55 \%(\mathrm{RR}=0.45 ; 0.18,1.13)$ for doses $\geq 10 \mathrm{mg} / \mathrm{day}$ $(\mathrm{n}=3723) .{ }^{1}$ One post-hoc analysis suggested an effect of ibandronate (IBN) on non-spine fractures in patients with severe osteoporosis, but the limited fracture data and differences in dosing precluded pooling studies for meta-analysis. No data are available on the effect of IBN on hip fracture. Consequently, there is no consistent evidence of hip or nonspine fracture risk reduction available for meta-analysis with IBN at a dose of $2.5 \mathrm{mg} / \mathrm{day}^{4}$.

Conclusions: The magnitude of effect on hip fracture appears to differ among drugs. Based on current data, ALN reduces the risk of hip and nonspine fracture by $49-55 \%$ and RIS by $26-27 \%$. There is insufficient and/or inconsistent evidence of an effect on these fractures for ibandronate.

1. Cranney et al. Endocr Rev 2002;23:570-8.

2. Papapoulos Osteoporos Int 2005;16:468-74.

3. McClung et al. NEJM 2001;344:333-40.

4. Chesnut et al. JBMR 2004;19:1241-9.

\section{P220. REPRODUCIBILITY OF BONE TEXTURE ANALYSIS ON RADIO- GRAPHIC IMAGES OBTAINED WITH DIRECT DIGITIZATION}

E. Lespessailles ${ }^{1}$, C. Gadois ${ }^{1}$, G. Lemineur ${ }^{2}$, JP Do-Huu ${ }^{2}$ C.L. Benhamou ${ }^{1}$; ${ }^{1}$ Ipros, Service de Rhumatologie CHR d'Orléans, France, ${ }^{2}$ D3A Medical Systems Orleans, France

Objective: Bone texture analysis on radiographs may account for trabecular bone microarchitecture. Assessment of bone microarchitecture may help to predict osteoporotic fractures as bone fragility is both due to a decrease of bone mineral density and trabecular microstructure alterations. We have previously validated a texture analysis on radiographs, and determined its value to discriminate osteoporotic patients with bone fractures from controls. These data were developed after evaluating the reproducibility of this technique. Direct digitization of radiographic images is able to improve convenience and reproducibility of the process. The present work describes the reproducibility of a texture analysis obtained with a home made radiologic prototype.

Material and Methods: The images were obtained on calcaneus by direct digitization, then the ROI was determined semiautomatically, and the analysis was achieved by the maximum likelihood estimator giving the result as Hmean parameter $(\mathrm{H}=$ 2 - D) for one image (1). The radiological parameters were standardized $(20 \mathrm{mAs}, 55 \mathrm{kV}$, focal distance $115 \mathrm{~cm})$. Short-term (S) and mid-term (M) precision errors were estimated by the CV. Inter observer and intra observer reproducibility were assessed by the Root Mean Square CV.

In vitro: reproducibility has been studied on $21 \times 2$ images performed in 48 hours on the same phantom. For the in vivo reproducibility: thirty women aged $58.2 \pm 6.8$ years had two measurements of the left heel, on the same day, with repositioning 
in order to assess the $\mathrm{S}-\mathrm{CV}$. For $\mathrm{M}-\mathrm{CV}$, fourteen subjects (three men and eleven women) aged $44.2 \pm$ years were evaluated at three time points (one week intervals). For inter and intra observer reproducibility ten healthy women aged $61.9 \pm 11.3$ years were evaluated. Each image from the ten women was analysed once by three observers. Each observer was unaware of previous results. Intra observer reproducibility was assessed by analysing ten images three times by the same observer. For Interprototype comparison: thirty patients were radiographed successively on two similar devices.

Results: In vitro CV was evaluated at $0.24 \%$. Results of in vivo intra-observer $\mathrm{CV}$, inter-observer $\mathrm{CV}, \mathrm{S}-\mathrm{CV}, \mathrm{M}-\mathrm{CV}$ were respectively: $0.10 \%, 0.30 \%, 1.06 \%$, and $1.37 \%$. The inter-prototype comparison $\mathrm{CV}$ was evaluated at $0.80 \%$.

Conclusion: These data suggest that the reproducibility of results obtained with this prototype is better than those previously obtained with radiological films. (M - CV $1.37 \%$ versus $2.07 \%$ ) (1). This reproducibility is comparable to that of BMD and ultrasound evaluation. The new prototype studied in this work provides good reproducibility data allowing to undertake inter group comparisons as well as multicenter cross-sectional and prospective studies.

${ }^{(1)}$ Benhamou C.L. et al (1994) Fractal organization of trabecular bone images on calcaneus radiographs. J. Bone Miner. Res. 9, 1909-1918.

\section{P221. CALCITIONIN DIRECTLY MODULATE CHONDROCYTE ACTIVITY THROUGH THE CALCITONIN RECEPTOR, AND INDUCTION OF CAMP: EX VIVO AND IN VIVO EVIDENCE}

M.A. Karsdal ${ }^{1}$, B.C. Sondergaard ${ }^{1}$, H. Wulf ${ }^{1}$, S. Oestergaard ${ }^{2}$, P. Qvist $^{2}$, K. Henriksen ${ }^{1}$, C. Christiansen ${ }^{2}$; ${ }^{1}$ Nordic Bioscience Diagnostics, Herlev, Denmark, ${ }^{2}$ Center for Clinical and Basic Research (CCBR), Ballerup, Denmark

Purpose: To investigate the expression of the calcitonin resorption in articular chondrocytes and evaluate possible direct effects of calcitonin ex vivo. Secondly, to investigate a novel formulation of oral calcitonin on cartilage turnover, in a nontraumatic model of osteoarthritis (OA).

Methods: Localisation and expression of the calcitonin receptor on articular chondrocytes were investigated by immunhistochemistry, RT-PCR and stimulation of cAMP levels by salmon calcitonin [0.0001-1M]. Osteoclasts were used as positive control. Anti-catabolic effects of calcitonin were evaluated in articular cartilage explants. Cartilage degradation was induced by cytokine stimulation of TNF- $\alpha$ [20ng/ml] + oncostatin M (OSM) [10ng/ $\mathrm{ml}$, in the presence or absence of calcitonin. Chondroanabolic actions of calcitonin were evaluated in articular cartilage explants in the presence of $[0.0001-1 \mu \mathrm{M}] \mathrm{sCT}$, followed by investigation of collagen synthesis by $\left[{ }^{3} \mathrm{H}\right]$-proline and proteoglycan synthesis by ${ }^{35} \mathrm{~S}$-sulfate incorporation. In vivo, the effects of calcitonin were evaluated in 7 months rats that underwent ovariectomy (OVX) for 9 weeks. Sham, OVX + estrogen, OVX $+2 \mathrm{mg} / \mathrm{kg}$ calcitonin and $150 \mathrm{mg} / \mathrm{kg} 5-\mathrm{CNAC}$. Fasting blood samples for biochemical marker analysis were taken at baseline, week 1, 2, 4, 6, 9.

Cartilage degradation was both assessed by measurement of collagen type II degradation resulting in the release of C-terminal telopeptides of collagen type II (CTX II ELISA) and histological scoring of articular cartilage erosion in rat knees.

Results: The calcitonin receptor was identified by RT-PCR and immunohistochemistry. Calcitoinn dose-dependently induced cAMP level in chondrocytes. Ex vivo, calcitonin dose dependently inhibited cartilage degradation induced by OSM and TNF- $\alpha$, and stimulated collagen and proteoglycan syntheis. In vivo, as measured by CTX-II, calcitonin completely abrogated cartilage degradation. Similar chondroprotective effects were obtained from histological scoring of articular cartilage in the rat OVX experiment.

Conclusion: Calcitonin might both exert direct chondroprotective and chondroanabolic effects on chondrocytes, thereby be an important mediator of chondrocyte metabolism. These results may promise chondroprotective effects of calcitonin in a clinical setting, albeit this still remains to be investigated.

\section{P222. QUALITY OF LIFE AFTER KYPHOPLASTY AND VERTEBROPLASTY FOR THE TREATMENT OF OSTEOPOROTIC VERTEBRAL COMPRESSION FRACTURES}

L. Massari, G. Caruso, M. Paganelli, E. Kiokia; University of Ferrara, Department of Orthopaedics, Ferrara Clinic, Italy

Objective: This study investigates the clinical outcomes in relation to the quality of life after kyphoplasty and vertebroplasty for the treatment of osteoporotic vertebral compression fracture (VCFs).

Materials and methods: A painful osteoporotic VCFs can be a significant burden for petients and their families, impairing physical function and quality of life ${ }^{1}$.

Kyphoplasty and vertebroplasty are new minimally invasive techniques that have been developed to help stabilize the fractured vertebra and decrease the pain and improve the function of individuals debilitated by painful osteoporotic $\mathrm{VCFs}^{2,3,4,5}$.

Eighty-five osteoporotic VCFs were treated during forty-two kyphoplasty procedures and forty-three vertebroplasty procedures in fifty-nine patients who did not respond to medical therapy. Symptomatic levels were identified by correlating the clinical data with radiographs and, in presence of two adjacent collapses vertebral body, with MRI findings demonstrating edema in the symptomatic levels. Fractures presenting for $>3$ months were included in the vertebroplasty group, whereas fractures presenting for $<3$ months were included in the kyphoplasty group.

Outcome data were obtained by comparing preoperative and latest postoperative SF-36 and visual analogic scale (VAS) data and the statistical analyses wer done using Wilcoxon signed-rank tests and Mann-Whithney test. A P value of $<0.01$ was deemed significant.

Results: Patient surveys revealed significant pain reduction within the first week after surgery and improved activity levels for a majority of patients. The SF-36 scores showed a significant improvement and the VAS score showed a significant reduction for both procedures. Cement extravasation was seen at $7.6 \%$ of patients treated with kyphoplasty procedures and at $14 \%$ of patients treated with vertebroplasty procedures, both without clinical complications.

Conclusions: Kyphoplasty and vertebroplasty, performed in appropriately selected osteoporotic patients with painful vertebral fractures, are promising addition to current medical treatment. These procedures improves physical function and reduces pain. Vertebroplasty has the advantage of being a relatively fast and inexpensive procedure, kyphoplasty is associated with increased cost and surgical time but offers the potential to improve spinal alignment if it is carried out within six-eight weeks from the collapse.

1. Oleksik A, et al: "Health related quality of life in postmenopausal women with low BMD with or without preexisting fractures”. J. Bone Miner. Res. 2000; 15: 1384-1392.

2. Wong W, Reiley MA, Garfin S: "Vertebroplasty/Kyphoplasty". J W I 2000;2:117-24.

3. Lieberman IH, Dudeney S, et al.: "Initial outcome and efficacy of Kyphoplasty in tht treatment of painful osteoporotic vertebral compression fractures". Spine 2001;26:1631-38.

4. Garfin SR, Yuan HA, et al.: "New technologies in Spine: kiphoplasty and vertebroplasty for the treatment of painful oteoporotic compression fractures". Spine 2001;26:1511-15.

5. Phillips FM, Ho E, et al: "Early radiographic and clinical results of balloon kyphoplasty for the treatment of osteoporotic vertebral compression fractures". Spine 2003;28: 2260-2267.

P223. ACCURACY AND PRECISION OF THE LUNAR IDXA, A NEW FANBEAM DENSITOMETER

K.G. Faulkner, C. Verboven, W.K. Wacker, K.P. Riewe, J.R. Franz, H.S. Barden; GE Healthcare, Madison, WI, USA 


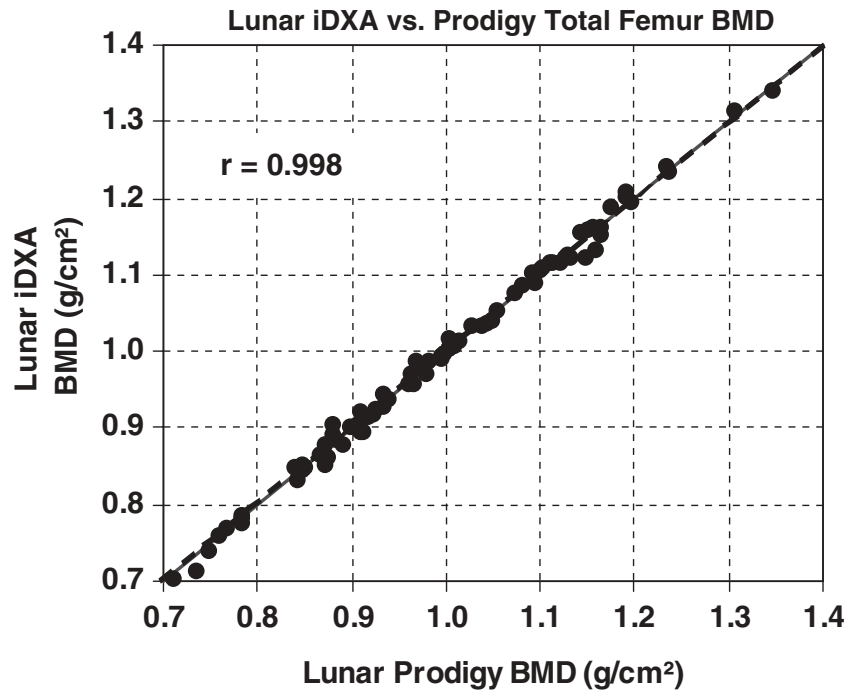

Expanding interest in osteoporosis worldwide has furthered demand for densitometry systems that accurately and precisely measure bone mineral density (BMD) and provide improved visual assessment for analyzing scans and detecting vertebral fracture. Recently, GE Healthcare introduced the Lunar iDXA, a highdefinition imaging densitometer featuring a new direct-to-digital $\mathrm{CZT} \mathrm{HD}^{\mathrm{TM}}$ detector with a staggered-element array to deliver precise and accurate results with near-radiographic image quality.

Materials and Methods: We compared the precision and accuracy of the Lunar iDXA to Lunar Prodigy. Forty subjects, 30 females and 10 males (mean age 56.7 yrs, SD 13.7), were measured 3 times on both densitometers at the spine, femur and total body with repositioning between scans.

Results: BMD measurements for Prodigy and iDXA were highly correlated at the L1-L4 spine $\left(\mathrm{r}^{2}>0.98\right)$, femur neck, trochanter, and total femur $\left(r^{2}=0.99\right)$, and total body $\left(r^{2}>0.96\right)$. Linear regression analysis comparing iDXA with Prodigy showed a unity slope at the L1-L4 spine, the trochanter and total femur, and a small, clinically insignificant, bias at the femur neck $(-0.003$ $\left.\mathrm{g} / \mathrm{cm}^{2}\right)$. There were no significant differences in precision $(\mathrm{CV})$ at the L1-L4 spine ( $1.1 \%$ vs $1.2 \%)$, femur neck ( $1.3 \%$ vs $1.4 \%)$, trochanter $(1.4 \%$ vs $1.2 \%)$, and total femur $(0.8 \%$ vs $0.7 \%)$ for iDXA and Prodigy, respectively. Total body precision was significantly better with iDXA $(\mathrm{CV}=0.6 \%$ vs $0.9 \%)$.

Conclusions: In conclusion, BMD measurements of the spine and hip on the Lunar iDXA and Prodigy are equivalent. Precision at the spine and femur was nearly identical between instruments and total body precision was significantly better with Lunar iDXA.

\section{P224. A COMPARISON OF MANUAL AND COMPUTER-GENERATED MEASUREMENTS OF MANDIBULAR CORTICAL WIDTH AS PREDICTORS OF OSTEOPOROSIS: THE OSTEODENT PROJECT}

C. Lindh ${ }^{1}$, K. Karyanni ${ }^{2}$, J. Graham ${ }^{3}$, K. Horner ${ }^{3}$, H. Devlin ${ }^{3}$, R. Jacobs ${ }^{4}$, P. van der Stelt ${ }^{5}$; ${ }^{1}$ Department of Oral Radiology, Malmö University, Malmö, Sweden, ${ }^{2}$ Department of Oral Diagnosis and Radiology, University of Athens, Athens, Greece, ${ }^{3}$ University Dental Hospital of Manchester, Manchester, UK, ${ }^{4}$ Oral Imaging Center, School of Dentistry, Oral Pathology and Oral Surgery, Leuven, Belgium, ${ }^{5}$ Department of Oral Radiology, Academic Center for Dentistry, Amsterdam, Netherlands

Objective: Manual measurement of mandibular cortical width has been shown to be significantly correlated with bone mineral density (BMD) at the hip, spine and forearm. These latter sites are fracture-prone in post-menopausal osteoporosis. We have developed computer software technology, based on active shape modelling, that will automatically detect the mandibular cortex on panoramic radiographs and then measure its width. We wanted to compare manual measurement of mandibular cortical width with semi-automated constrained fit and automated unconstrained fit methods.

Material and Methods: The diagnostic ability of these different methods at detecting osteoporosis of the femoral neck was compared using dual energy X-ray absorptiometry as the "gold standard "at these sites. 671 subjects were recruited and their BMD measured at the femoral neck.143 (21.4\%) subjects were osteoporotic, classified as a standardized t-score of $<-2.5$ at any site. The ability of one observer's manual measurement of mandibular cortical width to act as a diagnostic test for detecting osteoporosis at the femoral neck was assessed using ROC analysis. The AUC under the ROC curve was $=0.748,(\mathrm{se}=0.027$ $95 \% \mathrm{CI}=0.713$ to 0.780 ). Using a previous training set of 132 DPR images, a point distribution model (PDM) was created of the inferior mandibular cortex. Two experts performed this task independently using a graphical user interface, outlining the inferior mandibular cortex by placing equally spaced points on the computer images between the mental foramen and antegonial region. In the constrained fit, four manually defined reference points were placed on the inferior mandibular surface of the images at the left and right antegonial and mental foramen regions. These points served as starting points for the active shape model and the point distribution model was stretched and positioned to match these points.

Results: As a diagnostic test in detecting osteoporosis at the hip, the semi- automated constrained fit produced an AUC of 0.755 ( $\mathrm{se}=0.026 ; 95 \% \mathrm{CI}=0.721-0.788)$. In detecting osteoporosis at the hip, the automated unconstrained fit produced an AUC of 0.739 ( $\mathrm{se}=0.028 ; 95 \% \mathrm{CI}=0.704$ to 0.772 ). There were no significant differences in AUC between the manual, and either the constrained or unconstrained fits $(\mathrm{p}>0.05)$.

\section{P225. WHAT IS THE RISK OF INSTITUTIONALIZATION AFTER HIP FRACTURE?}

Niklas Zethraeus ${ }^{1,2}$ Oskar Ström ${ }^{2}$, Fredrik Borgström ${ }^{2,3} ;{ }^{1}$ Centre for Health Economics, Stockholm School of Economics, ${ }^{2}$ Stockholm Health Economics, Stockholm Sweden, ${ }^{3}$ Medical Management Centre, Karolinska Institutet, Stockholm, Sweden

Objective: In health economic evaluations of osteoporosis the long-term costs of hip fractures are usually based on the proportion of patients going into long term care (LTC). The aim of this study was to quantify these age-specific proportions and also to consider whether increased fracture related costs are incurred.

Material and Methods: Data was collected for 2747 patients that were admitted for hip fracture surgery during the year 1990 in the Stockholm county council in Sweden. The patient sample included both male and female patients. The patient's accommodation status was registered before and 1 year after the hip fracture. About $25 \%$ of the patient's died within the first and were thus excluded from the estimates. Also, the risk of being institutionalized due to hip fracture had to be adjusted down by the proportion of patients having special accommodation before the fracture. The calculations are based on the assumption that already institutionalized patients not will incur additional accommodation costs when going to LTC.

Results: results are presented in the table below.

Table 1 Accommodation before and after hip fracture in Sweden

\begin{tabular}{llll}
\hline Age & $\begin{array}{l}\text { Proportion going fromProportion initially } \\
\text { own home to LTC } \\
\text { after 1 year }\end{array}$ & $\begin{array}{l}\text { Adjusted proportion } \\
\text { living at home }\end{array}$ & \\
\hline $50-59$ & $7.2 \%$ & $92.6 \%$ & $6.7 \%$ \\
$60-69$ & $7.1 \%$ & $92.4 \%$ & $6.5 \%$ \\
$70-79$ & $12.8 \%$ & $79.7 \%$ & $10.2 \%$ \\
$80-89$ & $19.0 \%$ & $77.5 \%$ & $14.7 \%$ \\
$90-$ & $30.8 \%$ & $73.3 \%$ & $22.6 \%$ \\
All & $15.6 \%$ & $79.7 \%$ & $12.4 \%$ \\
\hline
\end{tabular}

* proportion of all surviving hip fracture patients going to LTC and also incurring additional accomodation costs due to fracture 
Conclusions: The results from this study indicate the risk of being institutionalized after a hip fracture to be constant in patients between 50 and 70 years of age. In older patients the risk seems to increase exponentially with age.

\section{P226. SERUM CALCIUM CONCENTRATION IN ELDERLY PATIENTS}

E. Franek ${ }^{1}$, L. Napiórkowska ${ }^{1}$, K. Safranow ${ }^{2}$, K. Stępie ${ }^{3}$; ${ }^{1}$ Dept of Internal Dis, Endocrinology and Diabetology, ${ }^{2}$ Dept of Clin Biochemistry, Pomeranian Medical Academy, Szczecin, Poland, ${ }^{3}$ Central Laboratory Unit, CSK MSWiA, Warszawa, Poland

Objectives: During the process of aging several processes occur, that may influence serum calcium concentration. Aging causes in most subjects diminished kidney function and impaired vitamin $\mathrm{D}$ hydroxylation, which may decrease serum $\mathrm{Ca}$. From the other side, frequency of neoplastic disease (with possible hypercalcaemia) is increased. In most sources, however, given normal serum $\mathrm{Ca}$ values are not age-dependent. From the other side, Roche Diagnostic manual for serum $\mathrm{Ca}$ assessment shows decreasing with age range of serum $\mathrm{Ca}$, giving the normal value for subjects aged more than $50 \mathrm{y}$ from 2,10 to $2,42 \mathrm{mmol} / 1$. The purpose of the study was to assess serum Ca concentration in the population of patients older than $50 \mathrm{y}$.

Materials and methods: 7278 patients (6645 hospitalized and 633 ambulatory) in whom serum Ca concentration was assessed in the years 2001-2004 in Central Clinical Hospital MSWiA in Warszawa, were included to the study. Mean serum Ca concentration was assessed for particular years and decades of life.

Results: Weighted means (for the $+/-5$ years span) of serum Ca concentration for particular age are shown in Fig 1. As can be seen, mean $\mathrm{Ca}$ level is decreasing slowly from 70 year of age, but after 95 yaer of age the prevalence of hypercalcaemia increases, increasing also the mean value. The ranges $(+/-2 \mathrm{SD})$ in particular decades of life are as follows: 50-59: 2.16-2.50; 60-69: 2.18 2.50 ; 70-79: 2.15-2.49; > 80: 2.08-2.48 mmol/1 (values given for 6645 patients with serum creatinine concentration $<1.5 \mathrm{mg} / \mathrm{dl}$ ).

Conclusion: Serum calcium concentration in the examined population decreases slowly only after 70 year of age. Therefore, one should be cautious using the normal Ca value established by Roche Diagnostics for the population aged over 50 .

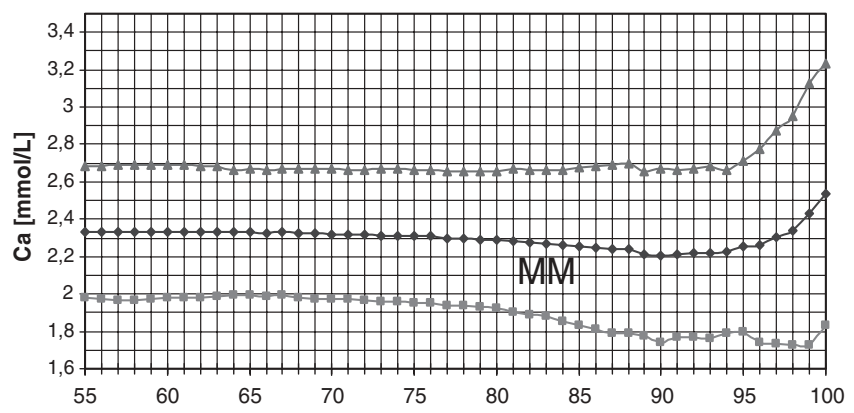

P227. THE ASSESSMENT OF ECONOMIC ASPECTS OF HIP AND DISTAL FOREARM FRACTURES IN MOSCOW REGION

A.V. Dreval, L.A. Martchenkova, I.V. Kryukova; Moscow Regional Research Clinical Institute, Moscow, Russia

The aim of our study was to assess incidence of hip and distal forearm fractures and direct financial expenses connected with those fractures in Moscow Region in the period of 1998-2002. For performing study among population aged more then 50 years Kolomensky area was chosen which is located at South-East of Moscow Region and had total population about 190000 persons at 1 Jan 2002. Source documents about patients with fractures were obtained from traumatologic hospitals, traumatologic stations and municipal polyclinics of Kolomensky area. Cost of medical aid was calculated on the basis of the prices of the State Obligatory Medical Insurance of Russian Federation. 527 hip fractures and 2420 distal forearm fractures were registered during
1998-2002 years. Hip fractures incidence varied from 77.1/100 000 in 1998 to $156.6 / 100000$ in 2001 among males $(\mathrm{p}<0.05)$ and from 166.1/100 000 in 1998 to $216.0 / 100000$ in 2001 among females $(\mathrm{p}>0.05)$. Distal forearm fractures incidence changed from $247.1 / 100000$ in 1999 to $309.3 / 100000$ in 2002 among males $(\mathrm{p}>0.05)$ and from $1213.0 / 100000$ in 1999 to $1025.0 / 100000$ in 2000 among females $(\mathrm{p}>0.05)$. Incidence of both fractures was significantly higher $(\mathrm{p}<0.01)$ in women than in men at all years of the study. Moscow Region population was about 6618000 persons at 1 Jan 2002. We consider provisional amount of hip fractures may be about 23500 and amount of distal forearm fractures may be about 105300 during 1998-2002 in Moscow Region. Direct Financial expenses for treatment of patients with fractures have made in average $582 \$$ for a hip fracture and $10 \$$ for a distal forearm fracture during first 2 months after fracture. Early medical care cost for was about $13677000 \$$ for all hip fractures and $1053000 \$$ for all distal forearm fractures in the period of 1998-2002 in Moscow Region. The investigation among Moscow Region population aged more than 50 years revealed high incidence of osteoporotic fractures both in males and females and very high financial assets which were spent for early medical care for patients with those fractures.

\section{P228. GLUCOCORTICOID-INDUCED OSTEOPOROSIS IN WOMEN USING ORAL (OGC) OR INHALED GLUCOCORTICOIDS (IGC) IN POSTMENO- PAUSE}

A.V. Dreval ${ }^{1}$, L.A. Martchenkova ${ }^{1}$, E.U. Polakova ${ }^{1}$, N.K. Chereiskaya $^{1}$, I.P. Ermakova ${ }^{2}$, V.P. Buzulina ${ }^{2}$, N.M. Milov ${ }^{1}$ Moscow Regional Research Clinical Institute, Russia, ${ }^{2}$ Research Institute of Transplantation and Artificial Organs, Moscopw, Russia

The aim of the study was to assess bone mineral density (BMD), fracture risk, biochemical parameters of bone metabolism and risk factors for corticosteroid-induced osteoporosis in menopausal women chronically treated with OGC or IGC. The study comprised 81 menopausal women with asthma aged 41-75 yrs: 55 ones received OGC and 26 ones treated with IGC. Control group consisted of 123 menopausal women at the same age hadn't ever received any GC therapy. Examination of study participants showed that OGC-group had significantly lower BMD in lumbar spine and proximal femur vs. control and IGC-group. There was revealed a higher frequency of osteoporosis in spine, femoral neck and forearm and frequency of vertebral deformities in OGCgroup vs. control. We didn't found an influence of IGC intake on $\mathrm{BMD}$ and fracture risk. Biochemical examination revealed in OGC-group a marked decrease in serum phosphorus and Crosslaps vs. IGC-group, a decrease in serum osteocalcin vs. control and a PTH hypersecretion. There was an increase in bone resorption biochemical markers and decrease in serum ionized calcium in IGC-group vs. control. The main risk factors for osteoporosis are consider to be age, duration of menopause, low body weight and duration of OGC-therapy in menopausal women treated with OGC and age, duration of menopause and daily dose of IGC in menopausal women received IGC. These results demonstrate that prolong OGC therapy increases risk of osteoporosis and vertebral deformities in menopausal women. IGC do not effect on BMD and fracture risk but high dosage of IGC intake may be an additional risk factor for osteoporosis in postmenopause.

P229. BONE MINERAL DENSITY (BMD) REDUCTION AND INCREASED CHOLESTEROL LEVELS ARE CO-MORBID CONDITIONS AMONG WOMEN AGED 65 YEARS AND OLDER: RESULTS FROM NHANES III

J.P. Bilezikian ${ }^{1}$, M. Davidson ${ }^{2}$, S. Hendrix ${ }^{3}$, L. Liu ${ }^{4}$, M. Louie ${ }^{5}$; ${ }^{1}$ Columbia University Medical Center, New York, NY, USA, 2 Rush University Medical Center, Chicago, IL, USA, ${ }^{3}$ Wayne State University Health Center, Detroit, MI, USA, ${ }^{4}$ Pfizer Inc., New York, NY, USA, ${ }^{5}$ Weill Medical College of Cornell University, New York, NY, USA 
Objective: Low bone mineral density (BMD) is known to be prevalent among women aged 65 years and older, but the coprevalence of low BMD and increased total and low density lipoprotein (LDL-C) cholesterol levels in this population has not been previously explored. The purpose of this study was to assess the co-prevalence of low BMD and high total and LDL-C cholesterol levels among women aged 65 years and older in the United States.

Method: We analyzed BMD and cholesterol data from the third National Health and Nutrition Examination Survey (NHANES III), conducted by the Centers for Disease Control (CDC) from 1988-1994. NHANES data are weighted to be representative nationally. All women aged 65 years and older who participated in the survey were identified for this study. Based on central BMD measurements, these women were classified into three groups as defined by World Health Organization (WHO) criteria for BMD T-scores: normal $(>-1.0)$, osteopenic $(-1.0$ to $-2.5)$, and osteoporotic $(<-2.5)$. We also examined total and LDL-C cholesterol levels among the study cohort.

Results: Among the 19.0 million women aged 65 and older estimated from this national database, 9.6 million $(50 \%)$ were found to be osteopenic, 3.9 million $(21 \%)$ were found to be osteoporotic, and 5.5 million (29\%) had normal BMD. Seventy-three percent of the osteopenic individuals had total cholesterol (TC) levels of $200 \mathrm{mg} / \mathrm{dl}$ or greater and $63 \%$ had LDL-C levels of $130 \mathrm{mg} /$ $\mathrm{dl}$ or greater. Among women with osteoporosis, $63 \%$ had high TC and $53 \%$ had increased LDL-C levels. Among women with normal BMD, $78 \%$ had elevated TC and $61 \%$ had increased LDL-C.

Conclusion: The NHANES III analysis indicates that greater than $70 \%$ of all American women aged 65 and older have either osteopenia or osteoporosis, and the majority of these women also have high total and LDL-C cholesterol levels. The fact that the percentage of normal subjects and osteopenic/osteoporotic subjects who have elevated TC and LDL-C is similar raises questions about the relationship between coronary artery disease and bone loss in postmenopausal women. Nevertheless, the presence of high TC and LDL-C amongst patients with osteopenia/osteoporosis presents a significant comorbidity profile that may have important clinical implications.

\section{P230. STUDY REGARDING THE MODIFICATION OF BONE REMODELING MARKERS UNDER THE INFLUENCE OF PHYSICAL EXERCISE}

G. Mologhianu, A.S. Nica, A. Murgu; The Third Rehabilitation University Clinic in Bucharest, Romania

Osteocalcin is a non-colagen protein contained in the structure of the bone matrix, produced by the osteoblasts during the mineralization phase and thus reflecting their activity. It is a good marker for bone syntesis, quantified using radioimmunological techniques.

Our object of study was the modification of bone markers, especially osteocalcin, under the influence of physical exercise. The study was performed on a number of 32 patients with osteoporosis diagnosed using DXA, over a period of 6 months between June 012004 and November 302004 at the third Rehabilitation University Clinic in Bucharest. The patients were divided into two groups: the first group was given physical exercise and no anti-resorptive medication, and the second group was given physical exercise and also anti-resorptive medication (Fosamax). Seric osteocalcin was determined for every patient before starting the exercise program. The exercise program, consisting of exercises usually prescribed for osteoporosis, was conducted at the clinic, three times a week for 50 minutes and with the recommendation for exercising at home. Several factors were monitored such as middle age and risk factors such as life style and associated disease; the evolution of osteocalcin values from the initial level to before entering the physical program and at the end of it.

Results: Osteocalcin levels dropped in patients of both groups after 6 months of controlled physical exercise. the patients in the Fosamax group has slighltly lower osteocalcin values, but the difference lacked statistical value.
Conclusions: Osteocalcin is influenced by physical effort (the study was conducted over a big enough time to register the variations); osteocalcin, an important bone formation marker, can be influenced by the administration of antiresorptive drugs such as Fosamax that reduce bone turnover; physical exercise, if performed constantly, may influence the bone's metabolic activity by improving the resorbtion - formation balance and may be an important aid for the drug therapy in osteoporosis; Further studies regarding the influence of controlled physical exercise on bone remodellig are mandatory, in order to prove the effects of this therapeutic method on osteoporosis patients.

\section{P231. USE OF CALCITONIN THERAPY IN PREVENTION OF BONE LOSS IN PRE-DIALYSIS CHRONIC RENAL FAILURE PATIENTS}

L.P. Martynyuk, S.I. Smiyan; O.I. Dyachan, Ternopol State Medical University, Department of inner medicine, Ternopil, Ukraine

Objective: The aim of this study was to study the safety and efficacy of of a combined low-dose therapy of alphacalcidol, calcium carbonate and calcitonin in the prevention of bone loss in chronic renal disease (CRD) pre-dialysis patients with mild to moderate renal failure and osteoporosis.

Material and methods: The study included 18 patients in the calcitonin group and 11 in the control group. All 29 patients were diagnosed II -III stages of CRD (glomelular filtration rate $58,6+5,4 \mathrm{ml} / \mathrm{min}$ ). Serum concentration of calcium, phosphorus and alkaline phosphatase were studied before starting treatment and after 12 months. Bone mineral density (BMD) was measured at 2 sites- lumbar spine (L1-L4) and femoral neck using dual energy X ray absorptiometry at baseline and after 12 months of treatment. Patients were randomized into two groups: in first group 18 patients were administered calcium carbonate at daily dose 1,0 gram of elemental calcium, alfacalcidol at a daily dose of 0,25 microg and calcitonin 100 IU x 3 times weekly intramuscular. In second group (11 patients) calcium carbonate at daily dose 1,0 gram of elemental calcium was administered.

Result: In calcitonine group the mean iPTH level declined significantly, serum calcium increased and both the phosphorus level and alkaline phosphatase activity declined significantly after 12 months of treatment. In the control group the levels of iPTH and phosphorus increased. Calcium level and alkaline phosphatase activity were not significantly different at baseline and after 12 months. In the control group, the mean BMD) at lumbar spine decreased in $-5,57 \pm 0,56 \%$ from at baseline. In calcitonin group it increased in 2,12 $\pm 0,68 \%$ from baseline $(p<0,01)$

Conclusions: The study supports the idea for beneficial effect of calcitonin and low dose of alphacalcidol treatment in prevention bone loss in mild to moderate CRF patients with osteoporosis.

\section{P232. ACTION “ARE YOU IN THE RISK GROUP FOR OSTOPOROSIS”}

\section{Iriski; Health Center Indjija, Serbia}

Goal of the research: detection of a problem of low bone density in work active women on the theritory of community of Indjija, located at the north of Serbia.

Material and methodology: study of a section of the bone density state with 202 patients and analisys of potential risk-factors. From July 18-25, 2005 there has been an campaign «A week of fight against osteoporosis» at Health Center Indjija. This is unique action for prevention of ostoporosis that has been carried out at some health center of primary health care in Serbia. Public has been informed through posters, local television and newspapers about campaign as well as about this illness. Visiting different administrative institutions, through immidiate interwiev and filling out one minute test, IOF for ostoporosis has regruted 202 work-active women at the age between $25-55$ for this action.

To all of them bone density has been mesured by QUISS densitometry, and before measuring, they all filled out the test for risk-factors for this illness. Women with low bone density and at 

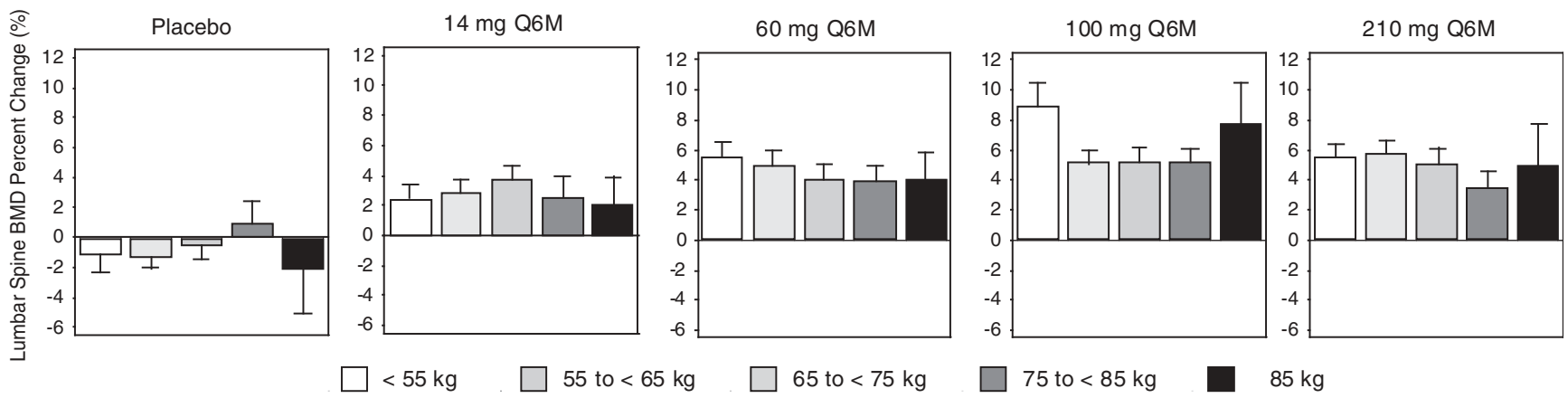

least 2 ostoporosis risk-factors were sent to DEXA measuring of bone density, after which they had plan for prevention or osteoporosis treatments made.

Results: Total of $31 \%$ of women had osteoporosis, and even $49.8 \%$ had osteopenia according to QUISS measurement. After DEXA measuring, result changed but they havent cross the limits of statistical importance $(p>0.05)$. Osteoporosis had $24.2 \%$ of women and osteopenia $55.5 \%$ of women. Presence of 2 risk factors was detected with $82,2 \%$ of women with low bone density, 3 risk factors had $53 \%$, and more than 3 factors had $47 \%$ of women. Interesting is data that even $4.95 \%$ of women with ostoporosis haven't had any risk factor.

Conclusion: with this action, first and only of this type in our area, has been touched the top of a low bone density problem in women population of this territory. Proportions of this problem were detected, which nobody paid any atention before and knowledge about this illness is very small among population and health workers too. Action should be widen to total women population that is work-active to prevent ostoporosis which is much cheaper and more efective than ostoporosis therapy.

\section{P233. SUBANALYSES AND SUPPLEMENTAL SAFETY OF DENOSUMAB (AMG 162) IN A PHASE 2 STUDY IN POSTMENOPAUSAL WOMEN WITH LOW BONE MINERAL DENSITY (BMD)}

P.D. Miller ${ }^{1}$, E.M. Lewiecki ${ }^{2}$, Y. Liu ${ }^{3}$, D.J. Zack ${ }^{3}$, L.A. Fitzpatrick $^{3}$, S.B. Cohen ${ }^{4}{ }^{1}$ Colorado Center for Bone Research, Lakewood, CO, USA, ${ }^{2}$ New Mexico Clinical Res and Osteoporosis Cent, Albuquerque, NM, USA, ${ }^{3}$ Amgen Inc., Thousand Oaks, CA, USA, ${ }^{4}$ Radiant Research, Dallas, TX, USA

Objective: Denosumab (AMG 162) is a fully human monoclonal $\left(\mathrm{IgG}_{2}\right)$ antibody that inhibits osteoclast differentiation, function, and survival by binding to RANKL. Previous results of a randomized, dose-ranging, phase 2 study in postmenopausal women with low BMD showed that 1 year of denosumab treatment rapidly decreased bone turnover markers and increased BMD compared with placebo. Here we report further subgroup analyses and safety results.

Materials and Methods: BMD data were analyzed according to subgroups defined by three baseline characteristics: weight, BMI, and lumbar spine BMD T-score. Safety, including parameters of immune function, was assessed for all treatment groups.

Results: After 1 year of treatment, denosumab (6-monthly) significantly increased lumbar spine BMD compared with placebo across all baseline weight (Figure) and BMI subgroups. When analyzed by baseline lumbar spine BMD T-score, 6-monthly denosumab increased lumbar spine BMD by (LS Mean \pm SE) $4.8 \%$ $\pm 0.6 \%$ in patients with T-score $<-2.5$ compared with $-1.3 \% \pm$ $1.1 \%$ for placebo. The corresponding changes in patients with baseline T-score $\geq-2.5$ were $4.4 \% \pm 0.3 \%$ for denosumab and $-0.5 \% \pm 0.6 \%$ for placebo. Incidence of adverse events was similar among denosumab-, placebo-, and alendronate-treated patients. Upper respiratory tract infection was the most common infection

(13\% placebo, $19 \%$ denosumab, $17 \%$ alendronate). No clinically meaningful differences in white blood cell or lymphocyte counts or T, B, or NK cell numbers occurred across treatment groups.
Conclusion: In this analysis denosumab treatment increased lumbar spine BMD regardless of patient baseline weight or $\mathrm{T}$ score. Further studies are ongoing to evaluate denosumab for use in the treatment of osteoporosis.

\section{P234. VALIDATION AND COMPARATIVE EVALUATION OF FOUR OSTE-} OPOROSIS RISK INDEXES IN MOROCCAN MENOPAUSAL WOMEN

A. El Maghraoui, A. Habbassi, M. Ghazi, L. Achemlal, A. Mounach, A. Nouijai, A. Bezza; Rheumatology and Physical Rehabilitation Centre, Military Hospital Mohammed V, Rabat, Morocco

Background: measuring bone mineral density (BMD) is a widely accepted strategy for identifying subjects with an increased risk of fracture. However, because of limited availability of BMD technology in some communities and cost considerations, it has been proposed that BMD measurements be targeted to subjects with risk factors for osteoporosis. Recently, many risk assessment indices have been developed to identify women who are more likely to have low BMD and thus undergo BMD testing.

Objective: To compare the performance of four risk indices for osteoporosis in White women in Morocco.

Methods: we analysed in an epidemiological cross-sectional study the records for 986 postmenopausal White Moroccan women seen at an out-patient rheumatology centre. Four osteoporosis risk index scores were compared to bone density T-scores. The ability of each risk index to identify women with low BMD (T-score $<-2.0)$ or osteoporosis $(\mathrm{T}<-2.5)$ was evaluated.

Results: Using an Osteoporosis Self-Assessment Tool (OST) score $<2$ to recommend DXA referral, sensitivity ranged from $61 \%$ at the lumbar spine to $85 \%$ at the total hip to detect BMD T-scores of -2.5 , and specificity ranged from $62 \%$ at the lumbar spine to $67 \%$ at the total hip. The negative predictive value was high at all skeletal sites $(79-98 \%)$, demonstrating the usefulness of the OST to identify patients who have normal BMD and should not receive DXA testing. All risk indices performed similarly and showed better results in identifying women with osteoporosis or low BMD based on hip measurement.

Discussion: This is the first study that validated several risk osteoporosis indexes in Moroccan women. The performance of these risk indices among women in Morocco was similar to that reported earlier for other samples in Asian countries, the US, and Belgium. The OST and other risk indices are effective and efficient tools to help target high-risk women for DXA measurement.

P235. DAILY RISEDRONATE PREVENTS BONE LOSS IN OSTEOPENIC POSTMENOPAUSAL WOMEN: A 2-YEAR MULTICENTER TRIAL IN EUROPE

M. Välimäki ${ }^{1}$, J. Heikkinen ${ }^{2}$, M. Muñoz-Torres ${ }^{3}$, H. Mulder ${ }^{4}$, E.S. Øfjord ${ }^{5}$, G. Toss $^{6}$; ${ }^{1}$ Division of Endocrinology, Helsinki University Central Hospital, Helsinki, Finland, ${ }^{2}$ Osteoporosis Clinic, Deaconess Institute of Oulu, Oulu, Finland, ${ }^{3}$ Hospital 
Clínico San Cecilio, Grenada, Spain, ${ }^{4}$ Osteosupport, Rotterdam, The Netherlands, ${ }^{5}$ Centre for Clinical Studies AS, Paradis, Norway, ${ }^{6}$ Department of Endocrinology, University Hospital, Linköping, Sweden

Objectives: Risedronate is well established for treatment of postmenopausal osteoporosis. Our objective was to confirm the superiority of risedronate compared with placebo for preventing bone loss in late postmenopausal women with osteopenia.

Materials and Methods: We conducted a two-year, multicenter, double blind, randomized (2:1 design), placebo-controlled trial, to evaluate the effect of risedronate $5 \mathrm{mg} / \mathrm{d}$ (RIS) on bone mass and bone turnover in 170 women $(65.9 \pm 6.8 \mathrm{yr})>5 \mathrm{yrs}$ post menopause with lumbar spine bone mineral density (LS BMD) T-scores between -2.5 and -1.0 , and at least 1 risk factor for osteoporosis. All women treated with RIS $(\mathrm{n}=114)$ or placebo (PLAC, $\mathrm{n}=56$ ) received daily supplementation of $1000 \mathrm{mg}$ elemental calcium and 400 IU vitamin D. The primary efficacy variable was percent change from baseline to endpoint (Month 24 ) in LS BMD. We also assessed percent changes from baseline in LS BMD at 12 months and urinary type I collagen crosslinked N-telopeptide (uNTX) at 12 and 24 months. Safety was evaluated by adverse event reports and changes in routine clinical laboratory tests and vital signs.

Results: For the RIS group, LS BMD significantly increased from baseline to endpoint by $4.49 \pm 0.38 \%(\mathrm{p}<0.0001)$, whereas PLAC was unchanged $(0.05 \pm 0.54 \%)$. The mean difference between groups $(4.44 \% ; 95 \% \mathrm{CI}, 3.14 ; 5.74)$ was significant $(p<0.0001)$. Similar results were seen after Month 12 for the change in LS BMD from baseline (RIS, $3.07 \pm 0.33 \%$; PLAC, $0.44 \pm 0.50 \% ; p<0.0001)$. Significant decreases in uNTX from baseline for RIS and no change from baseline for PLAC resulted in significant mean differences $(\mathrm{p}<0.0001)$ between the groups of $-37.9 \%(95 \% \mathrm{CI},-49.0 \% ;-26.8 \%)$ and $-31.3 \%(95 \% \mathrm{CI}$, $-42.3 \% ;-20.4 \%])$ at Month 12 and endpoint, respectively. Upper gastrointestinal (UGI) treatment emergent adverse events (TEAEs) and/or other clinically-relevant GI events were reported by $25.5 \%(n=14)$ of women in PLAC and $18.3 \%(n=21)$ of women in RIS.

Conclusions: Risedronate is well tolerated and prevents bone loss in late postmenopausal women with osteopenia who are at increased risk for developing future fractures.

\section{P236. PERFORMANCE OF THE LUNAR IDXA IMAGING DENSITOMETER}

M K O'Connor, D.J. Enright; Department of Radiology, Mayo Clinic, Rochester, MN, USA

Objective: The purpose of this study was to evaluate a new dual-energy x-ray absorptiometry (DXA), the iDXA system (GE Lunar, Madison, WI). This system incorporates a fan-beam DXA device which utilizes a more powerful $x$-ray tube and a high resolution CZT detector.

Materials and Methods: We compared the precision and accuracy of the Lunar iDXA to the Lunar Prodigy at the spine and femur regions in 30 subjects $(27$ females, 3 males, mean age 40.2 years). All subjects were scanned twice on each system, at the spine and bilateral femur.

Results: There was very high correlation between BMD values obtained on the iDXA and Prodigy systems at the L1L4 spine $(\mathrm{r} 2=0.988)$, femoral neck $(\mathrm{r} 2=0.995)$, trochanter $(\mathrm{r} 2=0.992)$ and total femur $(\mathrm{r} 2=0.994)$. Regression analysis showed a small bias $\left(0.028 \mathrm{~g} / \mathrm{cm}^{2}\right)$ for spine measurements. At all other regions, bias was less than $0.005 \mathrm{~g} / \mathrm{cm}^{2}$. Slopes of the regression lines were $1.007,0.991,1.045$ and 1.010 for the spine, femoral neck, trochanter and total femur respectively. There was a small improvement in precision at all measured sites (see table), possibly attributable to the improved spatial resolution of the iDXA unit. Qualitatively, image quality was significantly improved over that obtained on the Prodigy system.

Conclusions: We conclude that BMD correlation between iDXA and Prodigy densitometers was excellent at all measure-

\begin{tabular}{lllll}
\hline Precision $(\% \mathrm{CV})$ & & & \\
\hline System & Spine & $\begin{array}{l}\text { Neck } \\
(\mathrm{Lt} / \mathrm{Rt})\end{array}$ & $\begin{array}{l}\text { Trochanter } \\
(\mathrm{Lt} / \mathrm{Rt})\end{array}$ & $\begin{array}{l}\text { Total Femur } \\
(\mathrm{Lt} / \mathrm{Rt})\end{array}$ \\
\hline iDXA & 0.79 & $1.10 / 0.64$ & $1.13 / 1.00$ & $0.59 / 0.49$ \\
Prodigy & 0.94 & $1.21 / 1.27$ & $1.16 / 1.19$ & $0.83 / 0.68$ \\
\hline
\end{tabular}

ment sites. The iDXA system has a higher table weight limit and wider patient portal. With the increasing incidence of obesity, this system will be important for the measurement of bone density in larger patients.

\section{P237. EFFECT OF ABDOMINAL THICKNESS ON THE ACCURACY OF BONE DENSITOMETRY MEASUREMENTS USING THE LUNAR IDXA SCANNER}

\section{M.K. O'Connor; Mayo Clinic, Rochester, MN, USA}

Objective: To determine the effect of increased abdominal thickness on the accuracy of bone mineral density (BMD) measurements on the iDXA (GE Lunar, Madison, WI) dual energy xray bone densitometer.

Methods: We evaluated a new dual-energy x-ray densitometer, the iDXA system (GE Lunar, Madison, WI). This system incorporates a fan-beam DXA device, which utilizes a more powerful x-ray tube and a high resolution CZT detector. The iDXA unit was evaluated using 2 spine phantoms. The Hologic spine phantom consisted of 5 vertebrae embedded in Lucite and had a BMD of $\sim 1 \mathrm{gm} / \mathrm{cm}^{3}$. The RSD phantom consisted of a spine phantom in a water bath. The spine was sliced into 2 sections to provide spines with BMD values of $\sim 0.6 \mathrm{gm} / \mathrm{cm}^{3}$ and $1.3 \mathrm{gm} / \mathrm{cm}^{3}$. Increased abdominal thickness was achieved both by increasing water depth and thickness of Lucite sheets on top of the phantoms. Simulated abdominal thicknesses ranged from $\sim 10 \mathrm{~cm}$ up to $\sim 37 \mathrm{~cm}$. Scans of the spine were acquired at 3 different x-ray fluxes (corresponding to thin, normal and thick mode for patient studies). For each phantom, 5 scans were acquired in each mode at each depth. Mean and standard deviation of BMD and effective abdominal thickness were recorded.

Results: At high and medium BMD values, results for all 3 scan modes were identical for abdominal thicknesses of $25 \mathrm{~cm}$ or less. At the low BMD value, thin mode was required for thicknesses below $\sim 17 \mathrm{~cm}$ to prevent detector saturation. Thin mode yielded precise results up to $\sim 25 \mathrm{~cm}$ thickness. Normal mode gave consistent results up to $\sim 30 \mathrm{~cm}$, while thick mode could image $\sim 35 \mathrm{~cm}$ of tissue. At thickness beyond the limit of each mode, apparent BMD values increased dramatically.

Conclusions: The iDXA bone densitometer should be capable of image patients with a supine abdominal thickness of up to 35 $\mathrm{cm}$. Coupled with an imaging table capable of supporting up to $200 \mathrm{~kg}$ weight, this should enable accurate measurement of bone mineral density in patients with severe ascites and patients who are morbidly obese.

\section{P238. APPROACH TO OSTEOPOROSIS IN LEBANON}

Y. Yaghi ${ }^{1}$, F. Yaghi ${ }^{2}$, R. Halabi ${ }^{1}$,A. Elbaba ${ }^{1}$, D. Makki ${ }^{1}$; ${ }^{1}$ Hammoud Hospital, University Medical Center, Saida, Lebanon. ${ }^{2}$ American University of Beirut, Beirut, Lebanon

Objectives: To assess management of osteoporosis and its economic costs among Lebanese subjects.

Subjects and Methods: Retrospective analysis was performed on questionaires filled by 1000 patients who had previously undergone their first BMD study. The questionaire asked about age, risk factors for osteoporosis types of specialist who ordered the test and the indication of testing. The height and weight were measured on the day of BMD testing and added to the questionaire sheet.

Results: BMD has been performed unnecessarily in 330 patients $(33 \%)$, as this group of women had no risk factors for 
osteoporosis and the study was ordered as a part of routine health care. Orthopedic surgeons and Gynecologists ordered more than $70 \%$ of these tests. (table 1). The calculated cost for these unnecessary BMD tests was 24 million Lebanese Lira(16500 \$).

Conclusions: Lebanese physicians must base their decisions strictly on indications and accepted guidelines and should order BMD test in presence of risk factors. Knowledge of the risk factors will lead to cost containment strategies in osteoporosis management.

\section{P239. OSTEOPOROSIS IS LARGELY UNDERDIAGNOSED AND UNDER- TREATED: THE LAUSANNE SURVEY OF PATIENTS ADMITTED FOR A POTENTIALLY OSTEOPOROTIC FRACTURE}

O. Lamy ${ }^{1}$, N. Simard ${ }^{1}$, MA. Krieg ${ }^{1}$, S. Cuénot ${ }^{1}$, O. Borens ${ }^{2}$, P.F. Leyvraz; ' 'Service of internal medicine and Osteoporosis Unit, ${ }^{2}$ Orthopaedic Hospital, University Hospital, Lausanne, Switzerland

Objectives: Osteoporosis is a major health problem. Osteoporosis may be suspected on the basis of several risk factors (age, previous fracture,...) and may be diagnosed by DEXA (T-score $<-2.5 \mathrm{DS})$. A large proportion of fractures in subjects $>50$ years are attributable to osteoporosis: hip (80-95\%), forearm $(70-84 \%)$, spine $(82-89 \%)$. Treatments of osteoporosis are effective (reduction of fracture: $30-60 \%$ ) and particularly costeffective in patients at high risk for fracture. The aim of this study was to evaluate the medical management of patient at high risk for osteoporosis before the admission for a fracture.

Material and Methods: All consecutive urban patients $>50$ years admitted for a fracture at the emergency room of the university hospital of Lausanne were interviewed by a study nurse (October 2004 - October 2005). Patients with a fracture of the face / skull, or related to traffic accident were excluded. A simple questionnaire included age, sexe, previous clinical fracture, previous DEXA, previous treatment for osteoporosis, was administered. The patient 'GP was asked about the DEXA performed.

Results: 643 fractures occurred in 600 patients (men 27\%; women $73 \%$; mean age: $74 \pm 13) .474(79 \%)$ patients were hospitalised (length of hospital stay: $12 \pm 8$ days) and 292/474 (62\%) were transferred in a rehabilitation unit. $59 \%$ of patients have had a previous fracture in adulthood. $84 \%$ of fractures were due to low energy traumatism. Sites of fractures: hip 34\%; forearm $15 \%$; humerus $14 \%$; ankle $10 \%$; vertebral $7 \%$; shin/fibula $5 \%$; pelvis $4 \%$; costal $3 \%$. The medical management before this episode of fracture was as follow: DEXA performed $8 \%$; calcium and/or vitamin D 22\%; bisphophonates 4\%; hormonal therapies $1 \%$.

Conclusions: The medical cost of fracture is high, particularly when we consider the length of hospital and rehabilitation stay. Very few patients at high risk for fracture (age, previous fracture) have had a clinical investigation and/or medical management for osteoporosis. Patients with fractures not habitually recognised as typical for osteoporosis must probably be investigated for osteoporosis. Implementation of strategies to increase the identification and treatment of patients at high risk is needed.

P240. NERIDRONATE IN THE TREATMENT OF POSTMENOPAUSAL OSTEOPOROSIS

A. Delle Sedie, M. Mazzantini, M. Cazzato, O. Di Munno; Rheumatology Unit, Department of Internal Medicine, University of Pisa, Italy

Oral bisphosphonates (BPs), such as alendronate and risedronate, are now considered the first choice drugs in the treatment of postmenopausal, male and glucocorticoid-induced osteoporosis (OP). Other BPs that are administered parenterally mar represent an alternative option for those patients who show upper gastrointestinal adverse events or contraindication to oral BPs. Among parenteral BPs, the aminoBP neridronate has been recently approved for the treatment of Osteogenesis Imperfecta and has been shown to be effective in the Paget's disease of bone, in hypercalcemia of malignancy, in the prevention of bone loss in postmenopausal osteoporotic women and in men with androgenic deprivation for prostate cancer. Aim of the present study is to assess by DXA (QDR 4500, Hologic) the 1-year changes of bone mineral density (BMD) of lumbar spine, femoral neck and total hip in women with postmenopausal OP, treated with neridronate $25 \mathrm{mg} /$ monthly plus daily 1.2 calcium and 800 IU vitamin D. Twentythree patients have been evaluated, with a mean age of $66 \pm 7$ years (age range 52-81). Basal and 1-year BMD (T-score, mean \pm SD) values were $-3.12 \pm 0.9$ and $-2.96 \pm 1.08$, respectively, at the lumbar site; $-2.00 \pm 0.82$ and $-1.91 \pm 0.89$, respectively, at the total femur; and $-2.37 \pm 0.68$ and $-2.23 \pm 0.61$, respectively, at the femoral neck. The 1-year variation was found to be statistically significant at lumbar site $(\mathrm{p}=0.0066)$ while a trend towards improvement was registered at the femur. During the study, one patient had a post-traumatic peripheral fracture (humerus). No other adverse effects were noted. The preliminary result of this study suggests that neridronate shows a favourable effect on lumbar and femur BMD, with a good safety profile.

\section{P241. THE ROLE OF CASPASE PATHWAY ON EXPERIMENTAL OSTE- OPOROSIS MODEL AFTER TREATMENT WITH ALENDRONAT}

H.S. Vatansever ${ }^{1}$, C. Köse ${ }^{1}$, F. Taneli ${ }^{2}$, M. Vatansever ${ }^{3}$, S. Seven ${ }^{2}$, C.1 Ulman ${ }^{2}$; ${ }^{1}$ Department of Histology-Embryology, Faculty of Medicine, Celal Bayar University, Manisa, Turkey, ${ }^{2}$ Department of Clinical Biochemistry, Faculty of Medicine, Celal Bayar University, Manisa, Turkey, ${ }^{3}$ Year of 82 Government Hospital, Turgutlu, Manisa, Turkey

Objectives: Bone remodeling and regeneration take place permanently and are the result of a perfect balance between osteoclastic bone resorption and osteoblastic bone synthesis performed in the bone multicellular unit. The some growth factors and cytokines that promote bone cell differentiation have an important role in the prevention of cell death that is usually produced by apoptosis. In the treatment of osteoporosis, Alendronate an aminobisphosphonate, is a potent inhibitor of osteoclast-mediated bone resorption and is used for the treatment of bone disorders, osteoporosis and Pagets disease of bone. The purpose of this study was to investigate the effects of alendronate on caspase pathway during osteoporosis treatment.

Material and Methods: Sixty Swiss albino rats were divided into four groups $(n=15)$ : control, sham, Alendronat treated and Salmon calcitonin treated groups. Experimental osteoporosis was induced to all groups except control group by ovarectomy operations. All rats were sacrificed after 6 weeks of treatments, tibiae were fixed with $10 \%$ formalin, decalcificated and embedded in paraffin. Distributions of caspase- 6 , caspase- 8 and caspase- 9 were analyzed on sections using indirect immunoperoxidase technique.

Results: Caspase-6 immunoreactivity was detected in both Alendronat and Salmon calcitonin treated groups. However, intensity of caspase- 6 was stronger in Alendronat treated group than Salmon calcitonin treated group. Weak or negative immunoreactivity of caspase- 8 and -9 was observed in treated groups. All immunoreactivities were negative in both control and sham groups.

Conclusion: During normal bone remodeling, the rate of supply of new osteoblasts and osteoclasts and the timing of the death of osteoclasts, osteoblasts, and osteocytes by apoptosis are critical determinants of the initiation of new BMUs and the extension or reduction of the lifetime of existing ones. Our results suggest that, alendronat therapy was provided to increase expression of caspase pathway for control of apoptosis on osteoporosis. 
P242. STRONTIUM RANELATE NORMALIZES BONE MINERAL DENSITY IN OSTEOPENIC PATIENTS

O. Malaise, O. Bruyère, R. Deroisy, I. Jupsin, J.-Y. Reginster; WHO Collaborating Center for Public Health Aspects of Osteoarticular Disorders University of Liège, Belgium

Objective: Recent epidemiological studies have shown that a substantial fraction of fractures occurs in patients with osteopenia. Strontium ranelate was shown to reduce the incidence of vertebral and nonvertebral fractures in postmenopausal osteoporosis. The objective of this study is to assess the capacity of Strontium ranelate to restore normal BMD (OMS definition: T-score $\geq-1$ ) in postmenopausal osteopenic women (T-score between -1 and -2.5 ).

Material and methods: In SOTI study (Spinal Osteoporosis Therapeutic Intervention) focused on vertebral fracture efficacy of strontium ranelate, the patients included were osteoporotic at vertebral level and in TROPOS study (Treatment of Peripheral Osteoporosis) focused on non vertebral efficacy the patients included were osteoporotic at femoral neck level. Among these osteoporotic patients, 4240 were also osteopenic at the nonosteoporotic site. A post-hoc analysis from SOTI and TROPOS studies including these 4240 patients was performed. The patients received either a $2 \mathrm{~g}$ a day of Strontium ranelate or placebo for three years. BMD was measured at baseline and each year during three years. The results were analyzed on an intention-to-treat basis.

Results: At lumbar spine, after one, two and three years of treatment with Strontium ranelate, 28.4, 45.4 and $58.7 \%$ respectively of the osteopenic patients normalized their BMD compared to 4.9, 6.7 and $11.2 \%$ in the placebo group (all $\mathrm{p}$ $<0.001)$. At the total hip, the percentage of patients normalizing their BMD was 7.3, 12.6 and $21.1 \%$ in the Strontium ranelate group and 1.8, 1.5 and $2.0 \%$ in the placebo one (all $\mathrm{p}$ $<0.001$ )

Conclusion: Strontium ranelate is able to normalize BMD in a significant proportion of osteopenic patients.

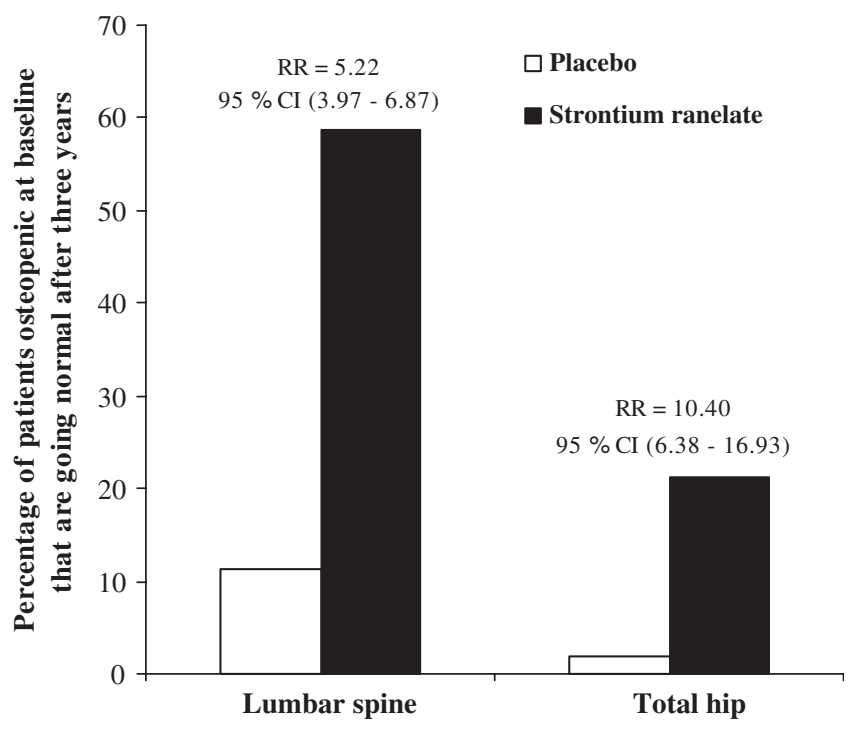

P243. ESTIMATION OF THE DIRECT COSTS BASED ON MEDICAL RESOURCE UTILIZATION RELATED TO INCIDENT FRACTURES IN GREEK POSTMENOPAUSAL WOMEN WITH OSTEOPOROSIS AND AN INADEQUATE RESPONSE TO ANTI-RESORPTIVE MEDICATION

A. Ginis ${ }^{1}$, N. Thalassinos ${ }^{2}$, E. Charalampidou ${ }^{1}$, the HELLENIC OSSO Study Group; ${ }^{1}$ Department of Medical Research, Pharmaserve - Lilly, Greece, 'Director of Endocrinology, Diabetes and Metabolism Department, General Hospital "Evangelismos", Greece
Introduction: The rising number of osteoporotic fractures and their associated morbidity will place a heavy burden on future health care resources. The majority of this direct cost is spent on the acute surgical and medical management following fracture, and the subsequent rehabilitation.

Objectives: OSSO is a 12-month, European, prospective, observational study aiming to assess the changes in Health Related Quality of Life (HRQoL) in females with osteoporosis and an inadequate clinical response to antiresorptive (AR) medications and to determine the direct costs of treatment for those patients.

Methods: The study population consist of postmenopausal women with osteoporosis with an inadequate response to AR medications according to one or more of the following two criteria: a) Patient (pts) who sustains a new clinical or radiographic fragility fracture (vertebral or non-vertebral), despite prescription of any approved a AR treatment at least 12 months prior to this fracture (Group A) b) Pts who discontinue AR therapy due to compliance problems and/or side effects (Group B). In Greece, data were obtained from 271 pts with severe osteoporosis. 263 $(97 \%)$ met the inclusion criteria.

Results: The most common sites of index fracture, which made them eligible for the study, were vertebra $(60 \%)$, forearm/wrist $(9.0 \%)$ and hip (7\%). From 93 pts who had visited the hospital, $26.9 \%$ needed to be hospitalized in the general ward for a mean [SD] duration of 2.6 [5.5] days while $2.1 \%$ needed to be admitted in the intensive care unit (ICU) for a mean [SD] duration of 0.2 [1.6] days. $91.2 \%$ had visited a consultant as health care provider with a mean [SD] number of 3.5 [3.1] visits while $45.3 \%$ used the primary care physician with a mean [SD] number of 1.9 [3.1] visits. 39.6\% required rehabilitation and /or physiotherapy with a mean [SD] number of 7.1 [11.7] visits.

Conclusion: The estimated direct costs, based on medical resource utilisation related to incident fractures, of treating women with osteoporosis and an inadequate response to AR medications in this study in Greece, is approximately $58.143 €$ year. The latter include fracture-related hospitalisation days $(4.643,5 €)$, physician visits $(24.432 €)$, rehabilitation services $(1.618,8 €)$ and hip fracture-related surgical procedures $(27.500$ $€)$

P244. PUTATIVE ANTIOSTEOARTHRITIC EFFECTS OF RALOXIFENE IN WOMEN WITH KNEE OSTEOARTHRITIS

J. Badurski ${ }^{1}$, S. Daniluk ${ }^{1}$, E. Jeziernicka ${ }^{1}$, A. Dobreńko ${ }^{1}$, N. Nowak ${ }^{1}$, J. Busłowska ${ }^{2}$, W.Holiczer ${ }^{1} ;{ }^{1}$ Center of Osteoporosis \& Osteoarticular Diseases, Bialystok, Poland, ${ }^{2}$ Technical University, Bialystok, Poland

The aim of this study was to evaluate the effect of the 12 month-therapy with raloxifene (RLX) in women with osteoarthritis of knees (OAK) on WOMAC and Lequesne indexes and on cartilage degradation and bone remodeling markers.

The study was conducted in 65 women aged 52-79, mean 65.5 years, with OAK diagnosed according to ACR criteria and rated on a 3-point Kellgren-Lawrence scale, with low bone mass (BMD of Hip Neck and/or L1-L4 LS T-score between -1.0 and 2.5) without contraindication to be treated with RLX. Patients were randomly assigned in ratio $1: 2$ to the control (C), or to group (E) being treated for 12 months with $60 \mathrm{mg}$ of RLX p.o./ day. All the participants took calcium up to $1200 \mathrm{mg}$ /day plus 800 IU Vit. D, and in the case of pain, Tramadol $1-2 \times 50 \mathrm{mg} /$ day.

Evaluation of RLX effect was based on WOMAC and Lequesne indices, and on cartilage-specific C-terminal tetrapeptide of type II collagen CTX-II/Cr, and oligometric matrix protein COMP with ELISA method. The bone metabolism was monitored by bone-specific alkaline phosphatase b-ALP, osteoprotegerin OP, bone specific C-terminal tetrapeptide of type I collagen CTX-I, and soluble NF-kappa-B receptor activator sRANKL with ELISA method. The patients and markers were examined before and after 3,6 and 12 months of treatment with RLX. 
The results pointed to a significant improvement of WOMAC index $(p=0.002)$, Lequesne's test results were on the border of significance $(\mathrm{p}=0.05)$ and testing level of probability for CTX-II/ $\mathrm{Cr}$ and WOMAC marker correlation was $\mathrm{p}=0.058$. Regressive analysis of time changes showed statistically significant decrease in OP, CTX-II, CTX-II/Cr in the group E and lack of any marker changes in the $\mathrm{C}$ one. The drop of CTX-II and CTX-II/Cr after 12 months in the group E was $30 \%$ and $40 \%$ respectively, with regard to the values before treatment. There were neither differences in time and between the groups $\mathrm{E}$ and $\mathrm{C}$ as for markers of bone metabolism nor their correlation with markers of cartilage degeneration nor COMP changes.

Conclusion: It seems that RLX may display a curative effect on cartilage degradation with improvement of clinical outcomes of OAK.

\section{P245. OLD BONES SUPPORT OSTEOCLASTOGENESIS SUPERIOR TO THAT OF YOUNG BONE, RESULTING IN PREFERENTIAL RESORPTION OF OLD BONE}

M.A. Karsdal ${ }^{1}$, I. Byrjalsen ${ }^{1}$, D.J. Leeming ${ }^{1}$, M.G. Sorensen ${ }^{1}$, R.H. Nielsen ${ }^{1}$, C. Christiansen ${ }^{2}$, K. Henriksen ${ }^{1}$, P. Qvist ${ }^{1}$; ${ }^{1}$ Nordic Bioscience, Herlev, Denmark, ${ }^{2}$ Center for Clinical and Basic Research, Ballerup, Denmark

Purpose: How osteoclasts target old bone in order to sustain the quality of the skeleton by constant remodelling is currently not known. We investigated whether young bone and old bone differ in their capacity to control osteoclast function. For this purpose, human osteoclast precursors were assessed for their ability to survive, adhere, differentiate and resorb old versus young bone.

Methods: The quantitative differences between young and old bones were assessed by measuring the amount of native ( $\alpha$-CTX) and age-modified ( $\beta$-CTX) collagen type I. Human CD14+ monocytes were isolated by magnetic bead sorting. Differentiation of osteoclasts on young (9-months) and old (8-years) bone slices was tested, by culturing the monocytes in the presence of RANKL and M-CSF for 28 days. Differentiation and resorption were investigated by measuring the TRAcP activity, CTX release in the conditioned medium and by scoring the pit area. Osteoclast numbers were assessed by counting calcitonin receptor positive cells and by measuring TRAcP activity in the conditioned medium. Young and old bones were incubated for 180 days at $37^{\circ}$ to increase age modified $\beta$-collagen.

Results: Old bones contained 3 times lower $\alpha$-CTX $/ \beta$-CTX ratios than those from young bones $(p<0.01)$. On young bone slices the formation of osteoclasts from osteoclast precursors and bone resorption was reduced by more than $90 \%$ compared to those formed on old bone $(\mathrm{p}<0.001)$. By counting of the number of osteoclasts, old bones were shown to have 10 times as many osteoclasts as young bone $(\mathrm{p}<0.001)$. Incubation of young bone slides for 180 days followed by culture of osteoclasts precursors, decreased $\alpha / \beta$ ratios to the old bone level and increased osteoclastogenesis albeit not to the levels of that of old bones.

Conclusion: These data show that old bones support osteoclastogenesis better than young bones and indicate that old bone in vivo accumulate signals that are promoting osteoclastogenesis. Hence, osteoclastic bone resorption on old bone is highly increased compared to that on young bone. Thus, the age of the bones might be an important factor in regulating the amount of resorption and thereby lead to targeted remodeling of old bones during the remodeling process.

\section{P246. LONG-TERM COST-EFFECTIVENESS OF ZOLEDRONIC ACID VERSUS RISEDRONATE IN PATIENTS WITH PAGET'S DISEASE OF THE BONE IN GERMANY}

A.A. Kurth ${ }^{1}$, W. Kotowa ${ }^{2}$, D. Goel ${ }^{3}$, J.A. Maiwenn ${ }^{4}$; ${ }^{1}$ Orthopädische Universitätsklinik Stiftung Friedrichsheim, Frankfurt am Main, Germany, ${ }^{2}$ Fricke \& Pirk GmbH - Member of the IMS Health Group, Nuremberg, Germany, ${ }^{3}$ Novartis Pharma GmbH, Nuremberg, Germany, ${ }^{4}$ Institute for Medical Technology Assessment, Erasmus Medical Center, Rotterdam, Netherlands
Objective: To assess the cost-effectiveness of a single intravenous dose of zoledronic acid $5 \mathrm{mg}$ (ZOL) compared to a daily regimen of oral risedronate $30 \mathrm{mg}$ (RIS) in the long-term (2-year) management of Paget's disease of the bone (PDB) in Germany.

Materials and methods: A model-based incremental costeffectiveness analysis was conducted over four half-year cycles. Clinical efficacy was reflected in four health states: response, nonresponse, relapse, no-relapse. Response was defined as normalization of the serum alkaline phosphatase (SAP) value after 6 months. Relapse was defined as an increase in SAP of at least $50 \%$ from the value at 6 months and at least 1.25 times the upper normal limit. Retreatment was assumed in case of non-response or relapse. Efficacy data were obtained from two 6-month randomized clinical trials which compared ZOL with RIS in patients with PDB. Relapse data were obtained from the extended observation phase of the trial, in which SAP was measured at 6monthly intervals to determine whether patients were still in response. The analysis was conducted from the German payers' perspective. Only direct healthcare costs such as those for physician visits, SAP measurement and drug administration were considered. Cost data were derived from published sources for the year 2005. Costs and effects in the second year were discounted by $5 \%$. A probabilistic sensitivity analysis (PSA) was performed to investigate the robustness of the results.

Results: Over 2 years, treatment with ZOL resulted in an incremental of 6.2 months in response and a cost saving of 243 $€$ compared to RIS. The higher percentage of responders and the longer time in response in the ZOL versus RIS group resulted in a lower average number of treatment courses needed per year -0.59 and 0.94 , respectively. Consequently the average annual treatment costs were lower with ZOL. The results of the PSA indicated that ZOL was more effective and cost-saving with $100 \%$ certainty.

Conclusion: Due to its superior efficacy and lower treatment requirements, ZOL proved to be more cost-effective than RIS in patients with PDB. Hence, ZOL presents a dominant treatment option in the management of PDB indicating superior effectiveness at a lower cost.

\section{P247. ONCE-MONTHLY DOSING OF ORAL IBANDRONATE IS HIGHLY EFFICACIOUS: 2-YEAR RESULTS FROM MOBILE}

J.-Y. Reginster ${ }^{1}$, J.A. Stakkestad ${ }^{2}$, R. Lorenc ${ }^{3}$, C. Hughes ${ }^{4}$, N. Mairon ${ }^{4}, \mathrm{~K}$. Coutant ${ }^{4}, \mathrm{~S}$. Adami ${ }^{5}$; ${ }^{1}$ University of Liège, Liège, Belgium, ${ }^{2}$ CECOR AS, Haugesund, Norway, ${ }^{3}$ The Children's Memorial Institute, Warsaw, Poland, ${ }^{4}$ F. Hoffmann-La Roche Ltd, Basel, Switzerland, ${ }^{5}$ University of Verona, Verona, Italy.

Objectives: Given the strong patient preference for monthly dosing, ${ }^{1}$ once-monthly oral ibandronate (Bonviva) has been developed to further improve the currently suboptimal level of therapeutic adherence with oral bisphosphonates. The MOBILE study is comparing the efficacy and safety of once-monthly ibandronate with daily ibandronate (3-year vertebral fracture risk reduction: $62 \%^{2}$ ). At 1 year, monthly dosing was proven at least as effective as daily dosing for bone mineral density (BMD) endpoints. Here we present the 2-year efficacy results.

Materials and methods: MOBILE was a randomised, doubleblind, non-inferiority study of monthly $(50+50 \mathrm{mg}, 100 \mathrm{mg}$ or $150 \mathrm{mg})$ and daily $(2.5 \mathrm{mg})$ oral ibandronate. All participants received daily calcium $(500 \mathrm{mg})$ and vitamin D (400IU). Women, aged 55-80 years and $\geq 5$ years postmenopausal, with osteoporosis (lumbar spine BMD T-score $<-2.5$ and $\geq-5$ ) were recruited.

Results: A total of 1,609 women were randomised. Substantial lumbar spine BMD gains were obtained in all treatment groups $(5.0 \%, 5.3 \%, 5.6 \%$ and $6.6 \%$ in the daily, $50+50 \mathrm{mg}, 100 \mathrm{mg}$ and $150 \mathrm{mg}$ groups, respectively; Figure). As in the 1-year analysis, all monthly regimens were proven non-inferior to the daily regimen. The $150 \mathrm{mg}$ dose was also shown to be superior to daily $(\mathrm{p}<0.001)$ for the lumbar spine BMD endpoint. Sizeable increases in total hip, femoral neck and hip trochanter BMD were observed in all treatment arms. Greatest increases were consistently obtained 


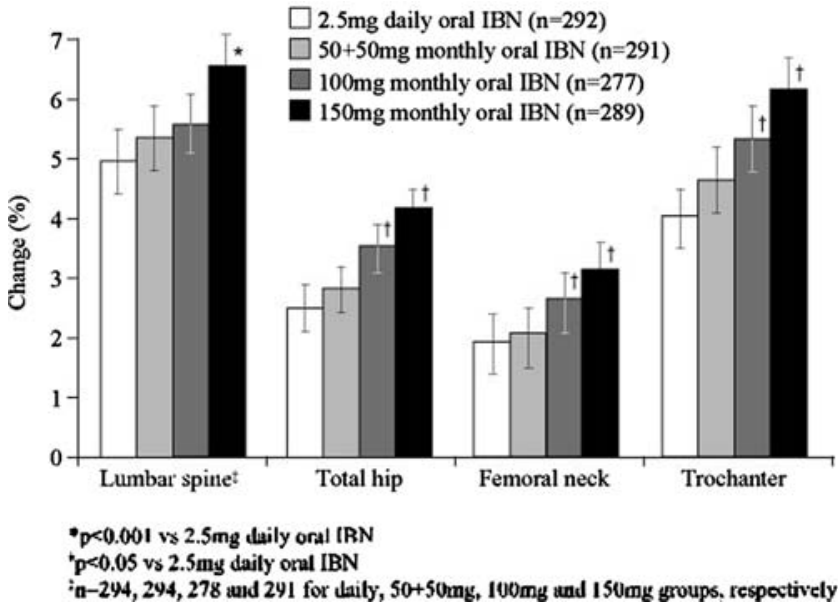

Fig. 1 Mean change $(\%)$ from baseline in BMD at 2 years

with the 150mg dose (Figure). With all regimens, the clinically meaningful reductions in serum CTX concentrations observed at 3 months (50.0-66.4\%) were maintained for the 2-year treatment period $(56.1-67.7 \%)$.

Conclusions: Once-monthly oral ibandronate is an effective treatment option for postmenopausal osteoporosis. Compared with the daily schedule, the superior efficacy provided by the $150 \mathrm{mg}$ regimen may provide additional clinical benefits.

1. Emkey R, et al. J Bone Miner Res 2005;20(Suppl. 1):S416(Abstract M435).

2. Chesnut CH, et al. J Bone Miner Res 2004:19:1241-9.

P248. CROSS CALIBRATION BETWEEN TWO DENSITOMETERS: HOLOGIC QDR2000 - GE LUNAR PRODIGY ADVANCE

N.G. Chau, J. Glémarec, P. Guillot, J.M. Berthelot, Y. Maugars; Service de Rhumatologie, CHU de Nantes, France

Introduction: The techniques used for measuring bone mineral density by DXA vary from one manufacturer to another, which sometimes yields different results. When a densitometer is changed, certain questions must be asked: is it possible to confidently compare patient results, can published cross-calibration algorithms be used, or should one perform one's own cross-calibration?

Methodology and Patients: Thirty patients (21 women and 9 men) between 29 and 84 years old (average 56,7 years) were measured on both the Hologic QDR 2000 and the Lunar Prodigy Advance system (AP lumbar spine and hip). Linear regression analyses were performed to determine the correlation between the measurements and the significance of slopes and intercepts.

Results: The correlation between the QDR2000 and the Prodigy Advance AP spine BMD values is good $\left(\mathrm{r}^{2}=0,957\right.$, Table 1). The highest correlation for the femur region was observed at the trochanter $(0,910)$.

Discussion: The observed differences could be linked to the different bone edge detection software and the different regions of interest at the femur site. Variation between centers is also influenced by operator experience and training [1]. The femur neck region has lower correlation because of the larger precision error, and because the regions of interest differ (the base area of

Table 1

\begin{tabular}{llll}
\hline & $\mathrm{N}$ & $\mathrm{R}^{2}$ & $\begin{array}{l}\text { Regression Analysis } \\
(\mathrm{y}=\text { Lunar })(\mathrm{x}=\text { Hologic })\end{array}$ \\
\hline AP Spine & 30 & 0,957 & $\mathrm{y}=1,086 \mathrm{x}+0,050$ \\
Femur Total & 59 & 0,891 & $\mathrm{y}=0,884 \mathrm{x}+0,169$ \\
Femur Neck & 59 & 0,820 & $\mathrm{y}=0,907 \mathrm{x}+0,177$ \\
Trochanter & 59 & 0,910 & $\mathrm{y}=1,057 \mathrm{x}+0,078$ \\
Shaft & 59 & 0,899 & $\mathrm{y}=0,936 \mathrm{x}+0,167$ \\
\hline
\end{tabular}

trochanter for Hologic, and thinnest part of the neck for Lunar) AP spine and trochanter results have the highest correlation and are preferred, along with femur total region, for comparative measurements.

Conclusion: Cross calibration between Hologic and Lunar systems give satisfactory results for an individual patient follow-up at the lumbar spine, femur total, and trochanter regions, with weaker agreement at the femur neck. We conclude from this study that a cross calibration adapted to each site is strongly recommended.

\section{References}

[1] Faulkner. DXA precision error: The importance of technology and operator experience; ISCD annual meeting, Feb 2005.

P249. IMPROVING COMPLIANCE IN POST-MENOPAUSAL OSTEOPOROSIS: THE INTEREST OF AN ELECTRONIC BLISTER AND OF A MEDICAL INFORMATION BROCHURE

E. Lespessailles ${ }^{1}$, G. Dubourg ${ }^{2}$, L. Blocquel ${ }^{3}$, B. Coudsy ${ }^{4}$; ${ }^{1}$ Department of rhumatology, Hopital Porte Madeleine, Orlèans, France, ${ }^{2}$ Department of rhumatology, Hôpital La Pitié Salpétrière, Paris, ${ }^{3}$ Medical Department, sanofi aventis France, ${ }^{4}$ Medical Department, Procter\&Gamble Pharmaceuticals, France

Introduction: Among osteoporotic patients, bisphosphonates per os have demonstrated theirs anti-fracture efficacy. But the therapeutic compliance with bisphosphonates is under optimum. Resorting to particular treatment dispositions and to educational programs aiming at maintaining treatment's faithfulness are solutions to better improve compliance.

Objective: The main objective of this study was to evaluate the acceptance of a medical information brochure and of a reminder system (electronic blister) for post-menopausal women with osteoporosis, treated with Risedronate $35 \mathrm{mg}$.

Patients and methods: Longitudinal observational study conducted from April to December 2004 by 3408 GPs who have included the first 5 patients consecutively seen. To be included, the patients were to be menopaused, suffer from a known osteoporosis treated with Risedronate $35 \mathrm{mg}$, were to be seen 3 months later ( \pm 1 month), and be able to fill in a self-questionnaire.

Results: 4622 patients (average age 69.6 years old) have been analysed. $55.1 \%$ thought they had a good health; 35,7\% thought their health poor. $83.5 \%$ of patients used the electronic blister, $74.4 \%$ found it practical to use. $77.7 \%$ considered the electronic blister helped them a little or a lot to take their treatment. $57,8 \%$ of patients wanted to continue to use the electronic blister. The information brochure on osteoporosis has been read at least partly by $92.2 \%$ of patients. The global patients' satisfaction regarding the electronic blister or the brochure is good or very good among $92.1 \%$ of them. The frequency of electronic blister use and of information brochure reading seem to have an impact on the treatment intakes' forgetting or lateness. Among the GPs who gave their opinion on the compliance tools (825 among 1581 participants), $93.6 \%$ thought that at least one of the proposed tools better improved the patients compliance. $92.3 \%$ wish to use them with other patients.

Conclusion: These data show that particular treatment dispositions and educational programs are well accepted among osteoporotic patients. The acceptance of these tools seems to have an impact on compliance (less treatment intakes' forgetting or lateness.).

\section{P250. DXA MORPHOMETRY: THE IMPORTANCE OF DETECTING NEW} VERTEBRAL DEFORMATIONS IN CLINICAL PRACTICE

Y. Maugars, P. Guillot, J. Glémarec, J.M. Berthelot; Service de Rhumatologie, CHU de Nantes, France

Introduction: The detection of a vertebral deformation (VD) is important for the diagnosis of osteoporosis, to better assess the 
Table 1

\begin{tabular}{|c|c|c|c|c|c|c|c|c|c|}
\hline \multirow[t]{2}{*}{ L1-L4 T- score } & \multirow[t]{2}{*}{$\mathrm{N}$} & \multirow{2}{*}{$\begin{array}{l}\text { Mean } \\
\text { Age }\end{array}$} & \multirow{2}{*}{$\begin{array}{l}\text { VD \% } \\
\text { pts }\end{array}$} & \multicolumn{4}{|c|}{$\mathrm{N}$ with Vertebral Deformations } & \multirow{2}{*}{$\begin{array}{l}\text { N VD } \\
\text { False + }\end{array}$} & \multirow{2}{*}{$\begin{array}{l}\text { N VD } \\
\text { False - }\end{array}$} \\
\hline & & & & New & First & Additional & Known & & \\
\hline$-2,5>\mathrm{T}>-3$ & 45 & 65,6 & $29 \%$ & 3 & 6 & 8 & 1 & 2 & 1 \\
\hline$-3>\mathrm{T}>-3,5$ & 29 & 62,5 & $38 \%$ & 4 & 2 & 4 & 1 & 3 & 0 \\
\hline$-3,5>\mathrm{T}>-4$ & 28 & 70,1 & $39 \%$ & 7 & 3 & 3 & 4 & 2 & 4 \\
\hline $\mathrm{T}<-4$ & 14 & 69,3 & $29 \%$ & 4 & 2 & 3 & 2 & 0 & 4 \\
\hline Total & 116 & 66,4 & $33 \%$ & $18(15,5 \%)$ & $13(11,2 \%)$ & $18(15,5 \%)$ & $8(6,9 \%)$ & $7(5,8 \%)$ & $8(6,6 \%)$ \\
\hline
\end{tabular}

risk of subsequent fractures, and for availability of treatments requiring presence of a vertebral fracture.

The gold standard for detecting vertebral deformations is a radiographic image. However, new DXA machines provide lateral spine images with morphometric measurements that approach the utility of the radiograph. The principle advantages of DXA morphometry are time saved, decreased radiation and improved convenience for the patients. The disadvantages are an inferior image quality, a more difficult analysis above T7 and false positive values (post-Scheuermann's trauma, scoliosis and erosive discarthrosis).

We have evaluated the importance of DXA morphometry for osteoporosis patients in daily practice.

Methodology and patients: In this study, 116 subjects had lumbar spine and proximal femur BMD, as well as lateral spine morphometry assessed with a Lunar Prodigy device.

Results: A total of 121 vertebral deformations were detected, with at least one deformation detected in $33 \%$ of the subjects (Table 1). 52 of the deformations in 28 subjects were severe. The frequency and the severity of the vertebral deformation were correlated to the spine T-score values.

Previously undetected vertebral deformations were discovered in $31 \%$ of subjects. This represented the first known fracture for $11 \%$ of subjects. 38 subjects $(33 \%)$ had already experienced peripheral fractures. The false positives $(6 \%)$ and false negatives $(7 \%)$ detected by standard X-ray were clinically reasonable. Six subjects incorrectly reported previous vertebral fractures.

Conclusion: DXA morphometry contributes vital information in the management of osteoporotic patients. Lateral spine images can be performed quickly, and technical limitations are minor and can be overcome with careful positioning and rigorous analysis.

P251. THE EFFECTS OF ALENDRONATE ON MALE PATIENTS ON CHRONIC TREATMENT WITH ANTICONVULSIVANT DRUGS

M. Guicciardi ${ }^{1}$, M. Fasani ${ }^{1}$, D. Fiore ${ }^{1}$, M. R. Romagnuolo ${ }^{1}$, E. Bartelucci $^{2}$, Magari ${ }^{2}$, M. Celestini ${ }^{1}$; ${ }^{1}$ Department of Physical Medicine and Rehabilitation, ASL Roma E, Italy, ${ }^{2}$ Opera Don Guanella Maschile, Rome, Italy

Chronic treatment with anticonvulsivant drugs is one of the risk factors in developing osteoporosis, even in males. In Italy there are about 500.000 patients affected by epilepsia. The association of secondary osteoporosis with the predisposition to fall explains the high rate of fractures in these patients.

International literature reports a few papers on secondary osteoporosis, particularly in a so homogeneous group of men.

Objectives: The first aim of our study was to confirm the decrease of BMD after one year of observation in male patients on chronic treatment with antiepileptic drugs. The second aim was to show the improvement of BMD after one year of treatment with alendronate (Fosamax $70 \circledR$ ).

Materials and Methods: We studied 34 institutionalized male patients (mental disabled patients, average age 45 years) on treatment with anticonvulsivant therapy for more than 25 years. All the subjects had similar diet, sunlight exposure, daily physiotherapy and conditions of life. We evaluated them by ultrasound MOC (Hologic-Sahara). We considered BMD at basal time (Tb) and after one year without antiresorptive therapy (T0). For the following year we gave patients alendronate $(70 \mathrm{mg}$ once

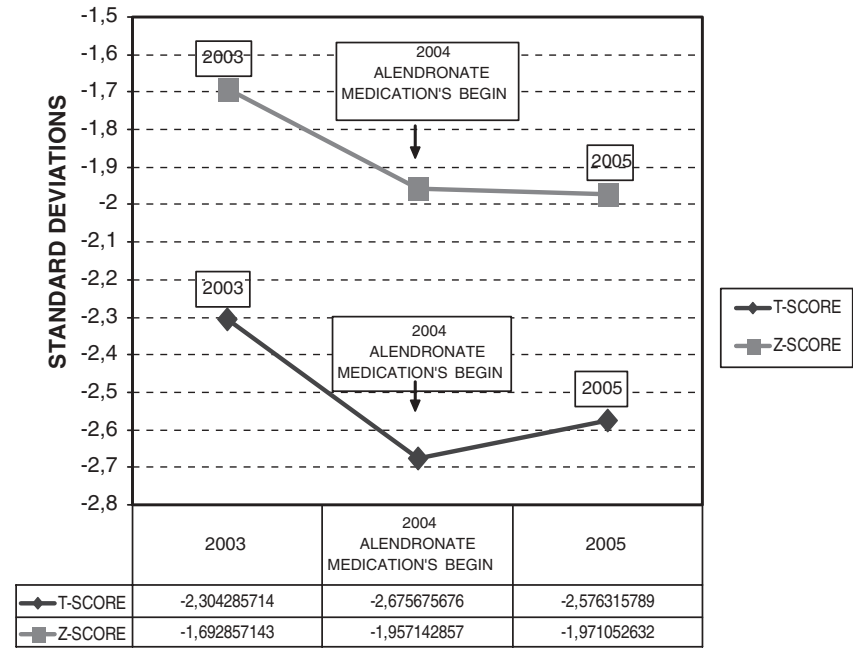

a week) plus calcium and vitamin $\mathrm{D}$; then we again evaluated BMD (T1).

Results: At Tb: medium T-score was $-2,3$ (medium Z-score $-1,7) ; 30$ patients $(88,2 \%)$ had BMD below normal values and 13 $(38,2 \%)$ were osteoporotic (T-score $<-2,5)$. At T0: medium T-score was $-2,7$ (medium Z-score -2.0 ); osteoporotic patients increased from $13(38,2 \%)$ to $21(61,8 \%)$. At T1: medium T-score $-2,5$ (medium Z-score -1,9); osteoporotic patients decreased from $21(61,8 \%)$ to $15(44,1 \%)$.

Conclusions: Our results show that alendronate is effective treatment to stop the decrease of BMD in patients on chronic treatment with anticonvulsivant therapy.

These patients are on high risk of fractures, so dangerous for their life and autonomy, due to the treatment received and the predisposition to fall as consequence of their neurological disease. It's necessary to monitor BMD and treat this patients by antiresorptive therapy (bisphosphonates), calcium, vit. D and regular physical exercise.

\section{P252 -FRACTURE OCCURANCE IN PATIENTS WITH OSTEOPENIA -THE} SEARCH FOR A NEW TREATMENT THRESHOLD

Ch. Muschitz, J. Patsch, Th. Woegerbauer, N. Pascher, E. Buchinger, E. Edlmayr, H.Resch; St. Vincent Hospital, Medical Department II, Vienna, Austria

Objectives: According to current recommendations pharmacological intervention to prevent fractures starts at a T-Score below -2.5 standard deviation.

Our retrospective analysis focussed on the prevalence of fractures in patients with osteopenic T-scores.

Material and Methods: The analysis included 188 Caucasian pre- and postmenopausal women and men aged 20 to 92 years. BMD was assessed by DXA-scan (lumbar spine \& hip). Vertebral fractures were identified by lateral $x$-ray examinations of the lumbar spine. Previous peripheral fractures were patient selfreported.

Results: Gender distribution was $85 \%$ female and $15 \%$ male. Mean age was $64.9 \pm 17.05$ years. The mean T-score showed 
moderate osteopenic values at lumbar spine $(-1.26 \pm 0.95)$ and total hip $(-1.66 \pm 0.36)$. Fracture analysis revealed $27 \%$ vertebral and $8.5 \%$ peripheral fractures. $6.4 \%$ had fractures at both sites.

Fractures most significantly occurred in the sixth and seventh decade of life $(p<0.0005)$. Primary diseases such as chronic inflammatory bowel disease, eating disorder (e.g. anorexia or bulimia) or chronic back pain $(p=0.0008)$ and increased BMI (26.37 vs 23.06; $p=0.000022)$ had influence on the occurrence of vertebral fractures.

Conclusion: Co-existing risk factors such as age, sex and prevalent adult fractures must be assessed to estimate the fracture risk of individual osteopenic patients. The influence of BMI on fractures still remains controversial.

Taking into account the above mentioned results the redefinition of the intervention threshold will be a major target for the future.

P253. A HEALTH-ECONOMIC MODEL FOR THE USE OF RHBMP-2 IN SPINE SURGERY - GERMAN EXAMPLE AND EUROPEAN OUTLOOK

V. Alt ${ }^{1}$, H. Haas ${ }^{2}$, M.A. Rauschmann ${ }^{3}$, C. Carstens ${ }^{4}$, A. Eicher ${ }^{1}$, A. Bitschnau ${ }^{1}$, R. Schnettler ${ }^{1}$; ${ }^{1}$ Department of Trauma Surgery, University Hospital Giessen-Marburg, Giessen, Germany, ${ }^{2}$ Department of Orthopaedic Surgery, University Hospital Giessen-Marburg, Giessen, Germany, ${ }^{3}$ Department of Orthopaedic Surgery, University Hospital Frankfurt/Main, Frankfurt/Main, Germany, ${ }^{4}$ Department of Orthopaedic Surgery, University Hospital Heidelberg, Heidelberg, Germany

Background and purpose: Low back pain related to osteoarthritic changes of the lumbar spine has a significant economic impact on health care budgets worldwide. Surgical treatment by spinal fusion is an option after unsuccessful non-operative therapy. Recombinant human BMP-2 (rhBMP-2) replaces autogenous bone grafting in lumbar one-level anterior lumbar interbody fusions (ALIF). The current German-DRG system and the most other European health care systems do not reimburse the upfront price of $2970 €$ per BMP-2 application. The purpose of the current study is to create a health economic model to evaluate financial savings for health care providers (hospitals) and health care payors (health care insurance) that can be achieved by the use of BMP-2 in spine surgery for Germany and other European countries.

Methods: A previously published meta-analysis was used in which rhBMP-2 showed significant improvements in the treatment after ALIF surgery compared to autogenous bone grafting, including return to work time. These medical findings were transformed into economic data based on current regulations of the German health system leading to a theoretical health economic model for Germany.

Results: The significant faster return to work time and reduced secondary interventions by rhBMP-2 treatment generate important financial savings for health care insurances which are $5831 €$ and $3552 €$ for open and laparoscopic ALIF procedures, respectively. These savings offset the upfront prize of $2970 €$ for rhBMP-2. Savings for hospitals are mainly related to shorter surgery time by the absence of the bone grafting procedure and faster discharge and contribute to savings per case of $233 €$ for open and $892 €$ for laparoscopic interventions. For other European countries savings mainly depend on specific regulations of sickness payments.

Conclusions: The current study showed that innovative technologies with a certain upfront price may achieve net savings for health care providers in the osteoarthritic field due to the fact that improved medical outcome for the patient is related to significant financial savings. The standard use of rhBMP-2 in ALIF surgery would help to lower costs for surgically treated patients with degenerative changes of the lumbar spine in Germany combined with a better medical outcome for the patient. This combination renders this rhBMP-2 in ALIF surgery into a "dominant strategy" from a health economic point of view.
P254. ONCE-MONTHLY ORAL IBANDRONATE IN POSTMENOPAUSAL OSTEOPOROSIS: MOBILE 2-YEAR SAFETY AND TOLERABILITY ANALYSIS

D. Felsenberg ${ }^{1}$, M. Stone ${ }^{2}$, V. Zikan ${ }^{3}$, C. Hughes ${ }^{4}$, A. Burdeska ${ }^{4}$, B. Minic ${ }^{4}$, C. Cooper'; ${ }^{1}$ Charité-University Medicine Berlin, Berlin, Germany, ${ }^{2}$ Llandough Hospital, Cardiff, UK, ${ }^{3}$ Charles University, Prague, Czech Republic, ${ }^{4}$ F. Hoffmann-La Roche Ltd, Basel, Switzerland, ${ }^{5}$ University of Southampton, Southampton, UK

Objectives: Many women with postmenopausal osteoporosis do not receive the full benefit of bisphosphonate treatment due to poor adherence. Tolerability issues are frequently cited as the main reason for discontinuing treatment. ${ }^{1}$ Combining a favourable safety and tolerability profile with a reduced potential for post-dose adverse events by introducing a less-frequently dosed bisphosphonate could improve adherence. In the MOBILE study, the safety and tolerability profile of once-monthly oral ibandronate (Bonviva), a potent, nitrogen-containing bisphosphonate, was studied over a 2-year period.

Materials and methods: MOBILE was a randomised, doubleblind study, involving 1,609 women (aged 55-80 years; $\geq 5$ years since menopause) with osteoporosis (lumbar spine BMD T-score $<-2.5$ and $\geq-5)$. Patients received either monthly $(50+50 \mathrm{mg}$, $100 \mathrm{mg}$ or $150 \mathrm{mg})$ or daily $(2.5 \mathrm{mg})$ oral ibandronate therapy, plus calcium $(500 \mathrm{mg})$ and vitamin D (400IU) supplements. Adverse events, including clinical osteoporotic fractures, were continuously monitored throughout the study period.

Results: The overall incidence of adverse events, including those considered related to treatment or leading to withdrawal, was similar across all treatment groups (Table). Of the serious adverse events reported, very few were considered treatment related ( $<1 \%$ per group; Table). Upper gastrointestinal adverse events were reported with a similar incidence in the oncemonthly and daily treatment groups (Table). Also, no imbalance in the clinical fracture rate was observed between the treatment groups.

Table Overall summary of safety at 2 years $(\mathrm{n}, \%)$

\begin{tabular}{|c|c|c|c|c|}
\hline & $\begin{array}{l}2.5 \mathrm{mg} \\
(\mathrm{n}=395)\end{array}$ & $\begin{array}{l}50+50 \mathrm{mg} \\
(\mathrm{n}=396)\end{array}$ & $\begin{array}{l}100 \mathrm{mg} \\
(\mathrm{n}=396)\end{array}$ & $\begin{array}{l}150 \mathrm{mg} \\
(\mathrm{n}=396)\end{array}$ \\
\hline \multicolumn{5}{|l|}{ Overall } \\
\hline Any adverse event & $302(76.5)$ & $313(79.0)$ & $318(80.3)$ & $317(80.1)$ \\
\hline $\begin{array}{l}\text { Any drug-related } \\
\text { adverse event }\end{array}$ & $128(32.4)$ & $119(30.1)$ & $143(36.1)$ & $146(36.9)$ \\
\hline $\begin{array}{l}\text { Any drug-related } \\
\text { adverse event leading } \\
\text { to withdrawal }\end{array}$ & $30(7.6)$ & $20(5.1)$ & $26(6.6)$ & $27(6.8)$ \\
\hline $\begin{array}{l}\text { Any serious adverse } \\
\text { event }\end{array}$ & $38(9.6)$ & $54(13.6)$ & $55(13.9)$ & $45(11.4)$ \\
\hline $\begin{array}{l}\text { Any drug-related } \\
\text { serious adverse event }\end{array}$ & $2(0.5)$ & $2(0.5)$ & $3(0.8)$ & $1(0.3)$ \\
\hline $\begin{array}{l}\text { Any drug-related } \\
\text { serious adverse event } \\
\text { leading to withdrawal }\end{array}$ & $1(0.3)$ & $1(0.3)$ & 0 & $1(0.3)$ \\
\hline $\begin{array}{l}\text { Upper gastrointestinal } \\
\text { adverse events }\end{array}$ & $90(22.8)$ & $79(19.9)$ & $102(25.8)$ & $89(22.5)$ \\
\hline $\begin{array}{l}\text { Clinical osteoporotic } \\
\text { fractures }\end{array}$ & $24(6.1)$ & $29(7.3)$ & $24(6.1)$ & $27(6.8)$ \\
\hline
\end{tabular}

Conclusions: Over 2 years, once-monthly oral ibandronate was as well tolerated as a daily regimen that has previously shown tolerability similar to placebo. ${ }^{2}$ Compared with the other investigational regimens, no safety disadvantage was observed with the highest ibandronate dose $(150 \mathrm{mg})$. The tolerability and convenience of the once-monthly ibandronate regimen may support greater therapeutic adherence in postmenopausal osteoporosis.

\section{References}

1. International Osteoporosis Foundation 2005.

2. Chesnut CH, et al. J Bone Miner Res 2004;19:1241-9. 
P255. THE MONTHLY ORAL THERAPY WITH IBANDRONATE FOR OSTEOPOROSIS INTERVENTION (MOTION) STUDY: RATIONALE AND DESIGN

S. Boonen ${ }^{1}$, C. Cooper $^{2}$, P. Hadji ${ }^{3}$, P. Mahoney ${ }^{4}$, B. El Azzouzi, ${ }^{4}$ D. Masanauskaite ${ }^{4}$, P.D. Delmas ${ }^{5} ;{ }^{1}$ Leuven University Center for Metabolic Bone Diseases and Division of Geriatric Medicine, Katholieke Universiteit Leuven, Leuven, Belgium, ${ }^{2}$ MRC Epidemiology Resource Centre, University of Southampton, Southampton, UK, ${ }^{3}$ University of Marburg, Marburg, Germany, ${ }^{4}$ F. Hoffmann-La Roche Ltd, Basel, Switzerland, ${ }^{5}$ Claude Bernard University and INSERM Research Unit 403, Lyon, France

Objectives: In osteoporosis, the relative efficacy and safety of alternative bisphosphonate treatment options, schedules and formulations can be assessed by comparative study. However, as antifracture efficacy is unfeasible to assay in this context, validated surrogate markers must be used. Recently, change (\%) from baseline in lumbar spine bone mineral density (BMD), one of the parameters of bone strength, has been used in a comparative study of weekly oral alendronate and risedronate. The MOTION study is a similar comparative study that will establish the effect on bone density, bone turnover and safety of oncemonthly oral ibandronate and weekly oral alendronate.

Materials and methods: MOTION is a randomised, doubleblind, double-dummy, non-inferiority study. For 1 year, approximately 1,800 women (aged 55-84 years and at least 5 years since menopause) with postmenopausal osteoporosis (lumbar spine [L2-L4] BMD T-score $<-2.5$, but $\geq-5.0$ ) will receive either $150 \mathrm{mg}$ once-monthly oral ibandronate plus weekly placebo, or $70 \mathrm{mg}$ weekly oral alendronate plus monthly placebo. Participants will also receive daily calcium $(500-1500 \mathrm{mg})$ and vitamin D (400IU) supplements. At 1 year, changes $(\%)$ from baseline in lumbar spine and total hip BMD will be measured as co-primary study endpoints. The relative effect on bone density of the two regimens will then be assessed by non-inferiority test (margin: $1.14 \%$ and $0.87 \%$ for lumbar spine and total hip BMD, respectively). Changes (\%) in hip trochanter BMD at 1 year will also be assessed, as will changes in the biochemical markers of bone turnover, serum CTX and P1NP, in $30 \%$ of patients at several time points. Adverse events, including clinical vertebral and non-vertebral fractures, will be monitored throughout the study.

Conclusions: MOTION is a head-to-head study that will compare the effect on bone density, bone turnover and safety of once-monthly ibandronate and weekly oral alendronate.

\section{Rosen C, et al. J Bone Miner Res 2005;20:141-51.}

\section{P256. PREVENTION OF AROMATASE INHIBITOR-INDUCED BONE LOSS IN BREAST CANCER WITH BISPHOSPHONATES: UNEXPECTED IN VI- TRO RESULTS}

F. Journé ${ }^{1}$, C. Chaboteaux ${ }^{1}$, G. Leclerq ${ }^{2}$, G. Laurent ${ }^{3}$, J.-J. Body ${ }^{1} ;{ }^{1}$ Laboratory of Endocrinology and Bone Diseases, ${ }^{2}$ Laboratory of Breast Cancer Research, Institut J Bordet, Université Libre de Bruxelles, Brussels, ${ }^{3}$ Laboratory of Histology, Faculty of Medicine and Pharmacy, Université de Mons-Hainaut, Mons, Belgium

Recent clinical data demonstrate the usefulness of aromatase inhibitors (AIs) as a first line endocrine therapy and as adjuvant treatment for estrogen receptor-positive $(\mathrm{ER}+)$ breast cancer. They induce complete estrogen deprivation that, however, leads to clinically significant bone loss. Several trials are ongoing to combine AIs with bisphosphonates (BPs) in order to reduce the risk of treatment-induced osteoporosis. BPs are powerful inhibitors of osteoclast activity and they have demonstrated their efficacy in the treatment of bone metastases. In addition, they can inhibit proliferation and induce apoptosis in tumor cell lines. Nevertheless, we recently reported that the first generation BP clodronate can stimulate the growth of ER + breast cancer cells cultured in steroid-free medium (SFM), an environment that can be viewed as a model for the use of AIs in breast cancer (Journé et al. Br J Cancer 2004).

We hypothesized that inhibitory effects of the third generation nitrogen-containing BPs could be masked by other potent stimulatory effects and we tested ibandronate and zoledronic acid on MCF-7 cells in SFM.

We observed that $100 \mu \mathrm{M}$ BPs stimulated the transcription of an estrogen-responsive reporter gene. The effects of BPs on transactivation were: (i) synergistically increased by 17b-estradiol, suggesting that they may potentiate genomic actions of estrogens, and (ii) suppressed by antiestrogens (tamoxifen and Faslodex), indicating that they are mediated by ER. Moreover, we showed that BPs transiently activated ERK1/2 and phosphorylated ER on serine residues 118 and 167 . The activation of ER through crosstalk with the MAPK pathway was previously reported to potentiate genomic actions of ER. In addition, we showed that the specific MEK inhibitor PD98059, which inactivates ERK1/2, (i) suppressed ER phosphorylations and ERE-dependent reporter gene transactivation induced by BPs, indicating that ERK1/2 mediated BPs effects, and (ii) synergistically increased the effects of $100 \mu \mathrm{M}$ BPs on cell growth inhibition. Furthermore, we also disclosed in MCF-7 cells exposed to low doses of ibandronate and zoledronic acid $(0.01-1 \mu \mathrm{M})$ a stimulation of growth that correlated with estrogen sensitivity. This unexpected mitogenic effect of BPs may be mediated by ER and appears to implicate ERK1/2 activation since it was suppressed by antiestrogens and MEK inhibitors.

In conclusion, nitrogen-containing BPs may activate ERK $1 / 2$, potentiate ER, and consequently stimulate ERE-mediated gene transcription in MCF-7 cells cultured in SFM. The positive effects of BPs in ER + breast cancer cells could antagonize their cytotoxicity and even stimulate proliferation depending on BPs concentrations and cell sensitivity to estrogens. The clinical implications of these in vitro findings remain to be demonstrated.

\section{P257. INHIBITION OF FPP-SYNTHASE IN OSTEOBLASTS MAY EXPLAIN THE BLUNTING OF THE BONE ANABOLIC RESPONSE TO PTH OBSERVED AFTER CHRONIC EXPOSURE OF RATS TO BISPHOSPHONATES}

J.A. Gasser, P. Ingold, A. Rebmann, M. Susa, J.R. Green; Musculoskeletal Diseases, Novartis Institutes for BioMedical Research, Basel, Switzerland

Chronic exposure to the bisphosphonate (BP) alendronate (ALN) was shown to blunt the bone anabolic response to parathyroid hormone (PTH) in rats (Gasser J, J Musculoskel Neuron Interact 2000;1:53) and humans (Finkelstein JA, NEJM 2003;349:1216). We investigated the potential mechanism behind the BP-PTH interaction. Skeletally mature Wistar rats were treated with ALN $(28 \mu \mathrm{g} / \mathrm{kg}$ s.c. twice per week) or vehicle for 16 weeks before starting daily s.c. injections of $100 \mu \mathrm{g} / \mathrm{kg} \mathrm{hPTH}(1-$ 38) 5 times per week. The anabolic response was monitored by serial quantitative computed tomography (pQCT) and mechanical testing. The response to PTH was significantly blunted in ALN-pretreated rats. pQCT measurements indicated a 2-week delay in the anabolic action of PTH. Also, ALN-pretreated rats did not develop the full anabolic response observed in vehiclepretreated rats over time. A second study investigated equipotent therapeutic doses of the BPs ALN $(200 \mu \mathrm{g} / \mathrm{kg})$ and zoledronic acid (ZOL; $32 \mu \mathrm{g} / \mathrm{kg}$ or $322 \mu \mathrm{g} / \mathrm{kg}$ ) administered as single i.v. injections. Daily s.c. PTH $(1-34)$ treatment $(40 \mu \mathrm{g} / \mathrm{kg}, 5 \mathrm{x} /$ week) was initiated $24 \mathrm{~h}$ later and continued for 6 weeks. Serial measurements of BMD, cortical and trabecular architecture in the proximal tibia metaphysis by pQCT and in vivo microCT indicated a normal bone anabolic response in all structural parameters in ALN and ZOL treated animals, including those given the supra-pharmacological dose of ZOL. Although osteoblasts (Obs) are not generally considered to be a target for BP inhibition, in vitro experiments provided direct evidence that BPs reduce protein prenylation in MC3T3-1b cells as measured by Rap1A immunoblotting. Since small $G$ proteins, such as 
Rap1 participate in cytoskeletal reorganization, BPs may thus affect the cytoskeleton in osteoblasts. PTH is able to activate flat bone lining cells into cuboid, collagen-synthesizing osteoblasts within $6 \mathrm{~h}$ after administration. Our data suggest that chronic BP exposure may reduce the ability of PTH to activate bone lining cells into matrix-secreting Obs, a crucial step in the early bone anabolic response to PTH. Due to the inefficient uptake of BPs into Obs, this mechanism is only observed after chronic treatment in vivo and may explain the blunting of the anabolic response to PTH observed after chronic exposure of patients and rats to BPs.

\section{P258. TWO-YEAR SAFETY AND TOLERABILITY PROFILE OF INTRAVE-} NOUS IBANDRONATE INJECTIONS: THE DIVA STUDY

S. Adami ${ }^{1}$, R. Prince ${ }^{2}$, J. Halse $^{3}$, C. Leigh ${ }^{4}$, D. Masanauskaite ${ }^{4}$, I. Jonkanski ${ }^{4}$, D.M. Reid ${ }^{5}$; ${ }^{1}$ University of Verona, Verona, Italy, ${ }^{2}$ University of Western Australia, Perth, Australia, ${ }^{3}$ Osteoporoseklinikken, Oslo, Norway, ${ }^{4} \mathrm{~F}$. Hoffmann-La Roche Ltd, Basel, Switzerland, ${ }^{5}$ University of Aberdeen, Aberdeen, UK

Objectives: Oral bisphosphonates may be unsuitable or contraindicated for some patients. Intravenous (i.v.) dosing could be beneficial for these patient groups. The potent, nitrogen-containing bisphosphonate, ibandronate (Bonviva), can be administered as a rapid (15-30 seconds) i.v. injection with extended intervals. The clinical utility of two novel intermittent i.v. regimens was assessed in the DIVA study. Here, we present data from the 2-year safety and tolerability analysis.

Materials and methods: DIVA, a randomised and doubleblind study, included postmenopausal women with osteoporosis (aged 55-80 years, $\geq 5$ years postmenopause, lumbar spine [L2L4] BMD T-score $<-2.5$ and $\geq-5.0$ ). Patients received either i.v. ( $2 \mathrm{mg}$ every 2 months [q $2 \mathrm{mo}$ ] or $3 \mathrm{mg}$ every 3 months [q3mo]) or oral $(2.5 \mathrm{mg}$ daily) ibandronate treatment. Calcium $(500 \mathrm{mg}$ daily) and vitamin D (400IU daily) were also provided. Adverse events (AEs), including clinical fractures, were continuously monitored.

Results: In total, 1,395 women were randomised. A similar overall incidence of AEs (85-89\%) was reported across the treatment groups during the 2 -year study period. Although no imbalance in associated withdrawals was detected (10-12\%), a higher overall incidence of drug-related AEs was reported in the i.v. treatment arms $(46 \%$ in $\mathrm{q} 2 \mathrm{mo}$ and $42 \%$ in q $3 \mathrm{mo})$ than the oral arm $(37 \%)$. In general, this imbalance was caused by a higher incidence of symptoms commonly associated with i.v. bisphosphonate administration, namely flu-like illness and transient musculoskeletal symptoms (e.g. myalgia and arthralgia). For flulike illness, rates were higher in the i.v. arms $(4.5-4.7 \%)$ than oral arm $(0.9 \%)$ at 2 years, although only a small number of events were reported during the second year of study. In general, such events were associated with the initial administration only, mild to moderate in intensity, transient in nature and resolved without treatment. The number of drug-related serious AEs was low and similar $(0.4-1.1 \%, \mathrm{n}=11)$, with most events considered unrelated to treatment. The incidence of clinical fractures was low in all treatment arms $(6-7 \%)$.

Conclusions: In women with postmenopausal osteoporosis, i.v. ibandronate injections are well tolerated, with a generally comparable safety profile to daily oral dosing, apart from symptoms characteristically associated with i.v. bisphosphonate administration.

P259. WOMEN WITH POSTMENOPAUSAL OSTEOPOROSIS PREFER ONCE-MONTHLY ORAL IBANDRONATE TO WEEKLY ORAL ALENDRONATE: RESULTS OF BALTO II

P. Hadji ${ }^{1}$, C.-L. Benhamou ${ }^{2}$, V. Devas ${ }^{3}$, D. Masanauskaite ${ }^{4}$, E. Barrett-Connor ${ }^{5}$; ${ }^{1}$ University of Marburg, Marburg, Germany, ${ }^{2}$ Porte Madeleine Hospital, Orléans, France, ${ }^{3}$ GlaxoSmithKline, Collegeville, PA, USA, ${ }^{4}$ F. Hoffmann-La Roche Ltd, Basel, Switzerland, ${ }^{5}$ University of California, San Diego, CA, USA
Objectives: Women with postmenopausal osteoporosis (PMO) prefer ${ }^{1}$ and adhere better ${ }^{2}$ to weekly than daily bisphosphonates, but adherence overall remains suboptimal. ${ }^{2}$ Less frequent than weekly bisphosphonate regimens may further improve adherence. ${ }^{3}$ The BALTO I study, conducted in the US, demonstrated a strong patient preference for monthly ibandronate over weekly alendronate. ${ }^{4}$ BALTO II had an identical design, but was conducted in centres in both the US and Europe to further evaluate patient preference and convenience.

Materials and methods: A total of 350 women with PMO enrolled in a 6-month, randomised, two-sequence, two-period, crossover, open-label study. Participants received either oncemonthly oral ibandronate $150 \mathrm{mg}$ for 3 calendar months immediately followed by weekly oral alendronate $70 \mathrm{mg}$ for 12 weeks (sequence $\mathrm{A} ; \mathrm{n}=176$ ) or the same treatments in reverse order (sequence $\mathrm{B} ; \mathrm{n}=174$ ). The women completed a preference questionnaire at the final study visit in which they assessed monthly ibandronate and weekly alendronate for preference and convenience. The modified intent-to-treat population $(n=321)$ comprised women who received at least one dose of each trial medication and completed the preference questionnaire.

Results: Most women preferred monthly ibandronate to weekly alendronate and found it more convenient. Of those women expressing a preference $(\mathrm{n}=299 ; 93.1 \%), 211(70.6 \%)$ preferred monthly ibandronate and $88(29.4 \%)$ weekly alendronate. The preference rate for the once-monthly regimen was statistically significant $(p<0.0001)$. The most common reasons for preferring the monthly regimen were ease of long-term adherence $(81.5 \%)$ and better lifestyle fit $(75.4 \%)$. For women expressing an opinion on convenience $(\mathrm{n}=273), 209(76.6 \%)$ found monthly ibandronate more convenient, while $64(23.4 \%)$ favoured weekly alendronate. The result for monthly ibandronate was statistically significant $(p<0.0001)$. Safety profiles of the two regimens were similar.

Conclusions: As in BALTO I, most women preferred oncemonthly oral ibandronate to weekly oral alendronate and considered it more convenient. Once-monthly bisphosphonate dosing has a strong potential to enhance patient adherence in osteoporosis.

1. Simon JA, et al. Clin Ther 2002;24:1871-86.

2. Cramer JA, et al. Curr Med Res Opin 2005;21:1453-60.

3. Simon JA, et al. The Female Patient 2005;30:31-6.

4. Emkey R, et al. Curr Med Res Opin 2005;21:1895-1903.

\section{P260. BONE MINERAL DENSITY OF THE SPINE AND FEMUR IN HEALTHY MOROCCAN WOMEN}

A. El Maghraoui ${ }^{1}$, A. Guerboub ${ }^{1}$ L. Achemlal $^{1}$, A. Mounach ${ }^{1}$, A. Nouijai ${ }^{1}$, M. Ghazi ${ }^{1}$, A. Bezza ${ }^{1}$, M.A. Tazi ${ }^{2}$; ${ }^{1}$ Rheumatology and physical Rehabilitation Department, Military Hospital Mohammed V, ${ }^{2}$ Epidemiology Department, Ministry of Health, Rabat, Morocco

Background: Bone mineral density (BMD) measurements are widely used to diagnose osteoporosis and to assess its severity. Commercial dual-energy X-rays absorptiometry (DXA) scanners used to determine BMD contains reference data for different populations. No data exists for the Moroccan population.

Aim: The aim of this study was to determine reference values for Moroccan female population and to compare them with values from western and other Arab countries.

Methods: A cross-sectional study of 569 Moroccan women, randomly selected in the area of Rabat, the capital of Morocco, aged between 20 and 79 years was carried out using DXA scan to establish reference values of bone mineral density. Measurements were taken at the lumbar spine and proximal femur using DXA (Lunar Prodigy Vision, GE). The data were compared with normative taken by Caucasians, Kuwaiti, Lebanese, Qatari and Saudi women over six decades of age. 
Results: Our results showed that the Moroccan women showed the expected decline in BMD at both sites with age after peaking at 20-29 years age group. The BMD values of the spine of Moroccan women were lower than Caucasian and Kuwaiti women but higher than the Lebanese, Qatari and Saudi women. The BMD values of the total femur were similar to western (European and American), and Kuwaitis, but higher in Moroccan females than Lebanese and Saudis. Using our reference database, $19.8 \%$ of our population had spine osteoporosis vs $25.4 \% ; 23.7$ and 14.0 using American, European and Middle-eastern values respectively. At the femurs, $4.2 \%$ had osteoporosis vs $3.5 \%$; $3.3 \%$ and $1.5 \%$ using American, European and Middle-eastern values respectively.

Conclusion: Osteoporosis is common among menopausal Moroccan women and should be considered a matter of public concern. Our study emphasises the importance of using population-specific reference values for BMD measurements to avoid overdiagnosis and/or underdiagnosis of osteoporosis.

\section{P261. BONE STRONTIUM DISTRIBUTION AND DEGREE OF MINERALI- ZATION OF BONE IN POSTMENOPAUSAL OSTEOPOROTIC WOMEN TREATED WITH STRONTIUM RANELATE FOR 2 AND 3 YEARS}

G. Boivin ${ }^{1,2}$, D. Farlay ${ }^{1,2}$, C. Simi ${ }^{1}$, P.J. Meunier ${ }^{1}$; ${ }^{1}$ INSERM Unité 403, Faculté de Médecine R. Laennec, Université Claude Bernard Lyon1, Lyon, France, ${ }^{2}$ Centre Technologique des Microstructures, Université Claude Bernard-Lyon1, Villeurbanne, France

Strontium ranelate (Protelos ${ }^{\circledR}$ ) is a new effective treatment for postmenopausal osteoporosis which provides early and sustained antifracture efficacy and increases the bone mineral density $(\mathrm{BMD})^{1,2}$. Here, strontium $(\mathrm{Sr})$ interactions with bone mineral were investigated in iliac bone biopsies from postmenopausal osteoporotic women. Samples were obtained at the end of 2 sets of studies: a 2-year placebo-controlled (PLA) phase II study $\left(\mathrm{STRATOS}^{3}\right)$ with 3 different doses of Protelos $(0.5 \mathrm{~g} /$ day $\mathrm{n}=6 ; 1 \mathrm{~g} /$ day $\mathrm{n}=6 ; 2 \mathrm{~g} /$ day $\mathrm{n}=8)$ or PLA $(\mathrm{n}=7)$; and the 3 -year phase III studies (SOTI and TROPOS $\left.{ }^{1,2}\right)$ with PLA $(n=15)$ or Protelos $(2 \mathrm{~g} /$ day $n=15)$. All patients received daily calcium and vitamin $\mathrm{D}$ supplements according to their needs. Bone samples were quantified by X-ray microanalysis for $\mathrm{Sr}$ uptake and distribution and by microradiography to measure the degree of mineralization of bone (DMB $)^{4}$. In Protelos-treated women, $\mathrm{Sr}$ is deposited dependently of dose in bone ( $\mathrm{p} \leq 0.0002$ vs PLA), with significantly $(p=0.0001)$ higher content in newly formed bone structure units (BSUs) than in old BSUs which are constantly devoid of $\mathrm{Sr}$ even after 3 years of treatment. At $2 \mathrm{~g}$ /day, bone $\mathrm{Sr}$ content (BSC) is only slightly higher after 3 years of treatment than after 2 (NS) confirming the chemical analysis showing that a plateau in BSC is reached after 3 years. In new BSUs containing $\mathrm{Sr}$, the maximal $\mathrm{Sr}$ content is similar after a 2 or 3-year treatment, suggesting that $\mathrm{Sr}$ is adsorbed onto the mineral surface rather than substituting calcium ions. Such a situation is in favor of rapid Sr clearance from bone. DMB is not different in Protelos and PLA groups regardless of the dose used in STRATOS or the treatment duration. Heterogeneity index of mineralization does not change after a 2- or 3-year treatment. To conclude, these data suggest that the increased BMD observed during Protelos treatment could be due to increased bone mass, as BSC reaches a plateau between 2 and 3 years of treatment and DMB is not modified.

1. Meunier et al. 2004 NEJM 350:459-68

2. Reginster et al. 2005 JCEM 90:2816-22

3. Meunier et al. 2002 JCEM 87:2060-6

4. Farlay et al. 2005 JBMR 20:1569-78

P262. TERIPARATIDE RELIEF OF PAIN IN THE POSTMENOPAUSAL WOMEN WITH OSTEOPOROTIC VERTEBRAL FRACTURES

J. S. Hwang J. F. Chen; Division of Endocrinology and Metabolism, Department of Internal Medicine, Chang Gung
Memorial Hospital, Linkou, Chang Gung University, Taiwan, Republic of China

Objective: Osteoporotic vertebral fractures occasionally lead to collapse of vertebral body, kyphosis, persistent back pain, and disability. The aim of this study was to obtain pain relief of postmenopausal women with established osteoporosis with teriparatide treatment.

Material and Methods: We performed a prospective study of patients with a painful osteoporotic vertebral compression fractures. At the time of enrollment, back pain were evaluated using a visual analog scale (VAS) (ranging from 1 to 10 ), and at 1 month and 3 months after teriparatide $20 £ g g$ daily injection. The minimal, clinically important change of the VAS is 2 points.

Results: Twelve patients with vertebral compression fractures were evaluated. The mean age of the patients was 74.3 years. Based on VAS scores, pain was decreased in $83 \%$ patients after teriparatide treatment. The mean pain rating score decreased from 6.7 to 4.2 , and 6.7 to 3.7 at one, and three month after the teriparatide treatment $(\mathrm{p}<0.001)$.

Conclusion: We conclude that teriparatide provides positive effects on relief of pain in patients with osteoporotic vertebral fractures.

\section{P263. BONE RESORPTION IS EFFECTIVELY NORMALISED BY INTER-} MITTENT INTRAVENOUS IBANDRONATE INJECTIONS: THE DIVA STUDY

D.M. Reid ${ }^{1}$, P.N. Sambrook ${ }^{2}$, P. Garnero ${ }^{3}$, C. Hughes ${ }^{4}$, D. Masanauskaite ${ }^{4}$, J.A. Stakkestad ${ }^{5}$; ${ }^{1}$ University of Aberdeen, Aberdeen, UK, ${ }^{2}$ University of Sydney, Sydney, Australia, ${ }^{3}$ INSERM Research Unit 403 and Synarc, Lyon, France, ${ }^{4}$ F. Hoffmann-La Roche Ltd, Basel, Switzerland, ${ }^{5}$ CECOR AS, Haugesund, Norway

Objectives: When taken correctly, daily and weekly oral bisphosphonates effectively normalise the elevated rate of bone resorption caused by postmenopausal osteoporosis, as shown by biochemical markers. With less frequent dosing schedules, the response is less well established, especially with intravenous administration. In the DIVA study, the impact on bone resorption of ibandronate (Bonviva) when given as continuous oral therapy or by intermittent intravenous injection was compared in women with osteoporosis. Observations for the 2-year study period are presented.

Materials and methods: DIVA was a randomised, doubleblind study in 1,395 women $(55-80$ years old; $\geq 5$ years postmenopause) with osteoporosis (lumbar spine [L2-L4] BMD Tscore $<-2.5$ and $\geq-5$ ). Participants received either $2 \mathrm{mg}$ ibandronate every 2 months [q $2 \mathrm{mo}$ ] or $3 \mathrm{mg}$ ibandronate every 3 months [q3mo] by rapid (15-30 seconds) i.v. injection or $2.5 \mathrm{mg}$ ibandronate as daily oral therapy. Calcium $(500 \mathrm{mg})$ and vitamin D (400IU) supplements were provided. Concentrations of the biochemical marker of bone resorption, serum CTX (sCTX), were assayed in blood samples collected at 2, 4, 6, 12 and 24 months in the $\mathrm{q} 2 \mathrm{mo}$ arm and 3, 6, 12 and 24 months in the $\mathrm{q} 3 \mathrm{mo}$ arm, just prior to the next scheduled dose.

Results: At the first assessment, pronounced decreases in sCTX were observed in both i.v. treatment arms (Table). At 6 months (first parallel assessment for the q2mo and q3mo arms), a similar and substantial magnitude of biomarker suppression

Table Median change $(\%, n)$ from baseline in sCTX

\begin{tabular}{llll}
\hline Month & $\begin{array}{l}2.5 \mathrm{mg} \text { daily } \\
\text { oral IBN }\end{array}$ & $\begin{array}{l}\text { 2mg q2mo } \\
\text { i.v. IBN }\end{array}$ & $\begin{array}{l}\text { 3mg q3mo } \\
\text { i.v. IBN }\end{array}$ \\
\hline 2 & $-45.0(173)$ & $-47.9(340)$ & - \\
3 & $-54.1(189)$ & - & $-43.3(349)$ \\
4 & $-58.7(170)$ & $-61.4(343)$ & - \\
6 & $-63.4(358)$ & $-65.3(342)$ & $-58.1(345)$ \\
12 & $-63.5(360)$ & $-64.7(342)$ & $-59.0(347)$ \\
24 & $-59.9(310)$ & $-55.6(301)$ & $-53.4(298)$ \\
\hline
\end{tabular}


was observed in all treatment groups (Table). In both i.v. arms, similar, pronounced and clinically meaningful reductions in sCTX were observed at all subsequent assessment points, which were generally comparable with those in the oral arm (Table).

Conclusions: I.v. ibandronate injections effectively normalise bone resorption in women with postmenopausal osteoporosis over a 2-year treatment period.

P264 - DETERMINANTS OF NON-COMPLIANCE WITH BISPHOSPHONATE THERAPY IN WOMEN WITH POST-MENOPAUSAL OSTEOPOROSIS

CH.A. van den Boogaard $^{1}$, F.J.A.Penning-van Beast $^{1}$, J.A. Erkens $^{1}$, A.M.T. Engbersen ${ }^{2}$, R.M.C. Herings ${ }^{1}$; ${ }^{1}$ PHARMO Institute, Utrecht, The Netherlands, ${ }^{2}$ Novartis Pharma AG, Basel, Switzerland

Objective: To identify factors associated with non-compliance with bisphosphonate therapy in women with post-menopausal osteoporosis.

Material and methods: Data were obtained from the PHARMO database, which includes linked drug-dispensing records and hospital records of more than two million individuals in defined areas in the Netherlands. New female users of daily or weekly alendronate or risedronate therapies, aged 45 years and older or with diagnosed post-menopausal osteoporosis in the period of 1 January 1999 - 30 June 2004, were included in the study cohort. One-year compliance was measured using the Medication Possession Ratio (MPR) and defined as the sum of days' supply for all prescriptions of the initial drug (daily or weekly bisphosphonate) in the first year after starting treatment. To identify determinants of non-compliance, non-compliant patients (MPR $<50 \%$ ) were compared to compliant patients (MPR $\geq 80 \%$ ). The effect of type of initial drug, patient age, prescriber, co-medication and fractures on non-compliance with bisphosphonates was investigated. For those independent factors increasing the risk of non-compliance, the impact on the patient population was determined by the Population Attributable Risk percentage (PAR\%), which takes into account the proportion of non-compliant patients and the odds ratio.

Results: The study cohort included 8,822 new users of bisphosphonates, of whom 4,699 (53\%) were compliant and 3,089 $(35 \%)$ were non-compliant with their initial therapy after one year. $1,034(12 \%)$ of patients had a MPR $\geq 50 \%$ and $<80 \%$. Use of daily bisphosphonates, number of different co-medications used in the year preceding bisphosphonate therapy, and use of gastrointestinal medication during the first year of bisphosphonate therapy were associated with an increased risk of non-compliance (OR 3.1, 95\% CI 2.7-3.5; OR 1.8, 95\% CI 1.3-2.3 for e.g. $>10$ different co-medications; and OR 1.2; 95\% CI 1.1-1.4, respectively). The corresponding PAR $\%$ values were $42 \%, 14 \%$ and $6 \%$, respectively. In contrast, higher age, first prescription from a specialist, hospitalization for osteoporosis or osteoporotic fracture in the year preceding bisphosphonate therapy, and use of NSAIDs in the year prior to bisphosphonate therapy independently decreased the risk of non-compliance with bisphosphonates.

Conclusion: These results indicate that a daily, rather than a weekly, dosing regimen is the most important independent determinant of non-compliance with bisphosphonates. However, compliance for both regimens can be considered to be suboptimal and leaves room for improvement.

\section{P265. TREATMENT PATTERNS WITH DAILY AND WEEKLY BIS- PHOSPHONATE REGIMENS IN WOMEN WITH POST-MENOPAUSAL OSTEOPOROSIS}

CH.A. van den Boogaard ${ }^{1}$, F.J.A. Penning-van Beest ${ }^{1}$, J.A. Erkens $^{1}$, A.M.T. Engbersen ${ }^{2}$, R.M.C. Herings ${ }^{1}$; ${ }^{1}$ PHARMO Institute, Utrecht, The Netherlands, ${ }^{2}$ Novartis Pharma AG, Basel, Switzerland
Objective: To determine treatment patterns with daily and weekly bisphosphonate regimens in women with post-menopausal osteoporosis.

Material and methods: Data were obtained from the PHARMO database, which includes linked drug-dispensing records and hospital records of more than two million individuals in defined areas in the Netherlands. New female users of daily or weekly alendronate or risedronate therapies, aged 45 years and older or with diagnosed post-menopausal osteoporosis in the period 1 January 1999 - 30 June 2004, were included in the study cohort. One-year persistence of treatment was determined by using episodes of bisphosphonate treatment, using the method of Catalan. For non-persistent patients, the first change in osteoporosis treatment pattern (switch, discontinuation or stop) in the one-year period after starting treatment was determined. Treatment patterns were analyzed separately for new users of daily and weekly bisphosphonate regimens.

Results: The study cohort included 4,222 new users of daily bisphosphonates and 4,600 new users of weekly bisphosphonates. One-year continuation rates with bisphosphonates were low: only $1,257(30 \%)$ of daily bisphosphonate users and $2,099(46 \%)$ of weekly bisphosphonate users remained on their initial therapy after one year. Non-persistent users of daily bisphosphonates mostly discontinued $(27 \%)$ or definitely stopped $(27 \%)$ their initial treatment during the first year. Likewise, most non-persistent users of weekly bisphosphonates discontinued (29\%) or definitely stopped $(20 \%)$ treatment during this period. For patients discontinuing treatment, the median length of time to restart treatment was 22 days. Treatment was resumed with the original drug regimen in $86 \%$ and $96 \%$ of daily and weekly bisphosphonate users, respectively.

Conclusion: The results of this study indicate that bisphosphonates are not used as recommended. Fewer than half of daily as well as weekly bisphosphonate users remain on their treatment after one year. However, about $30 \%$ of non-persistent bisphosphonate users resume treatment in the first year. It is likely that this pattern of bisphosphonate use has a detrimental impact on treatment efficacy.

P266. PRESCRIPTION PATTERN OF HUMAN RECOMBINANT 1-34 PARATHYROID HORMONE (TERIPARATIDE, FORSTEO ${ }^{\circ}$ ) IN SWEDEN

Ö. Ljunggren ${ }^{1}$, A. Toll ${ }^{2}$, K.-J. Myren ${ }^{2}$, T. Mullarney ${ }^{3}$; ${ }^{1}$ Department of Medical Sciences, University of Uppsala, Uppsala, Sweden, ${ }^{2}$ Eli Lilly Sweden AB, Stockholm, Sweden, ${ }^{3}$ Eli Lilly UK Ltd, Erl Wood, England

Objectives: To determine the severity of osteoporosis in Swedish patients prescribed teriparatide and to assess the alignment with the criteria for reimbursement of the Swedish Pharmaceutical Benefits Board (PBB).

Material and methods: Patients were recruited at 22 Swedish osteoporosis centers. The study design was non-interventional. All consenting patients prescribed teriparatide in normal clinical practice were eligible for enrollment, Data from the first 188 patients, estimated to represent approximately half of all Swedish patients prescribed teriparatide during the recruitment period, were included in this analysis.

The PBB had determined that patients who fulfill the criteria for at least one of the following categories should be reimbursed for teriparatide:

1. Bone mineral density (BMD) $<-3,0$ and at least one prevalent fracture,

2. Established osteoporosis and intolerance to bisphosphonate treatment due to contraindications or adverse events,

3. BMD $<-2,5$ and at least two new fractures during bisphosphonate treatment.

Number of previous fractures and BMD were recorded at baseline. Back pain during the last month was measured by a Visual Analog Scale from 0-100. 
Results: The majority of patients $(90,4 \%)$ met at least one of the reimbursement criteria and $46,8 \%$ of the patients met two or more of the criteria.

Overall, the patients had a mean lumbar spine (LS) BMD Tscore of $-3,5 \pm 1.1$ (mean $\pm \mathrm{SD}$ ). The mean number of prevalent fractures was $3,4 \pm 2,0$. The mean VAS score for back pain the last month was $49 \pm 25$ and $56,1 \%$ of patients stated that they had back pain every day or almost every day. For the 150 patients fulfilling the criteria for category 1, the mean LS BMD was $-3,8 \pm 0,9$ and the mean number of fractures was $3,3 \pm 1,9$. The 18 patients that did not meet any of the criteria had either several prevalent fractures (4 or more) or very low $\operatorname{BMD}(<-4,1)$.

Conclusions: Swedish physicians have prescribed teriparatide in alignment with the PBB criteria and treated very severe osteoporotic patients.

P267. VERTEBRAL ASSESSMENT BY PLAIN FILM X-RAY: ACCURACY AND PRECISION OF MORPHOXPRESS $₫$ - A MORPHOMETRIC, SEMIAUTOMATIC SOFTWARE

G. Guglielmi ${ }^{1}$, A. Brett ${ }^{2}$, D. de $\mathrm{Feo}^{3} ;{ }^{1}$ Radiology, Scientific Institute Hospital "CSS", San Giovanni Rotondo, Foggia, Italy, ${ }^{2}$ Image Metrics PLC, Regent House, Heaton Lane, Stockport SK4 1BS, UK, ${ }^{3}$ Procter \& Gamble, Rome, Italy

Objective: Osteoporosis is a progressive skeletal disease characterised by reduction in bone mass, resulting in an increased risk for fractures. Vertebral fractures are a risk factor for further vertebral and nonvertebral fractures with 1 in 5 women experiencing a new vertebral fracture in the year following a vertebral fracture. Vertebral fractures often remain undiagnosed. In clinical practice, vertebral fractures are qualitatively detected on lateral spinal radiographs. Two methods are used for quantitative assessment of vertebral fractures. Using a "semiquantitative" approach, each vertebrae is visually examined and classified according to standardised definitions of degree and type of fractures. Using the second method, six (or more) points are placed manually on the vertebral margins and the anterior, middle and posterior heights are calculated. A number of semi-automatic morphometric software solutions have been described to assist the physician. A novel software (MorphoXpress ${ }^{\circledR}$ ) designed to semi-automatically assess digitised lateral spine x-rays using 6-point morphometry was tested for accuracy and reproducibility.

Methods: The software uses a previously trained statistical model-based template of the vertebrae to analyse unseen images by fitting the template in an optimal way. 92 previously unseen digitised $\mathrm{x}$-rays were evaluated. X-rays showing normal and fractured vertebrae were randomly selected over several months from female patients at an Italian hospital-based osteoporosis center. Accuracy was calculated by vertebral label (T5 to L4) and normalised by vertebral width. Vertebrae exhibiting errors greater than 3 SD from the mean error were treated as failures of automatic annotation and excluded from calculation of the overall mean error.

Results: Mean percentage errors ranged from 1.39 to 3.90 for the anterior-posterior reference (mean of $2.50+/-0.72$ ) and from 1.50 to 3.22 for the superior-inferior reference (mean of $2.16+/-0.5)$, with higher accuracy on the lumbar spine. The mean of the signed point errors was approximately $0.4 \%$ vertebral width indicating little or no systematic error in automatic annotation. In the precision test, no vertebrae had mean errors greater than $3 \mathrm{SD}$ from the mean error of all tests. Values ranged from a minimum of $0.8 \%$ for lower lumbar vertebrae to a maximum of $3.1 \%$ for $\mathrm{T} 5$ in the AP reference. The mean result over all vertebrae in the test set was $2.6+-1.3 \%$ for vertebral widths.

Conclusion: MorphoXpress ${ }^{\circledR}$ can support physicians in their clinical practice for detecting vertebral fractures with a good reproducibility.
P268. WHOLE BODY COMPOSITION ASSESSMENT IN ELDERLY OSTEOPENIC AND OSTEOPOROTIC PATIENTS: COMPARISON BETWEEN DUAL X-RAY ABSORPTIOMETRY (DXA) AND BIOELECTRICAL IMPEDANCE ANALYSIS (BIA)

J. Swanenburg ${ }^{1,2}$, E.D. de Bruin ${ }^{1,2,3}$, M. Stauffacher ${ }^{1,2}$, D.

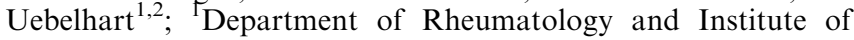
Physical Medicine, University Hospital Zurich, Zurich, Switzerland, ${ }^{2}$ Centre For Prevention and Treatment of Osteoporosis, University Hospital Zurich, Zurich, Switzerland, ${ }^{3}$ Institute for Human Movement Sciences, Swiss Federal Institute of Technology, Zurich, Switzerland

Objectives: A cross-sectional study aimed at comparing wholebody composition (WBC) with dual-energy X-ray absorptiometry (DXA) and hand-to-foot bioelectrical impedance analyses (BIA) in a population of elderly people diagnosed with severe osteopenia or osteoporosis was conducted at the Centre for Prevention and Treatment of Osteoporosis of the University of Zurich.

Materials and Methods: WBC measurements where performed in a total of 24 female patients, aged (mean \pm SD) $76 \pm 7$ yrs, BMI: $26.2 \pm 4.7 \mathrm{~kg} / \mathrm{m} 2$ presenting with severe osteopenia or osteoporosis. The whole body composition (WBC) including fat body mass (FBM), lean body mass (LBM) and the percentage of body fat $(\% \mathrm{BF})$ of each patient were measured both with DXA (Hologic QDR 4500 Elite) and BIA (101 Body Impedance Analyser, Fresenius Kabi Inc.).

Results: Scatter-plots showed comparability. The Bland and Altman (differences against the mean) approach showed for the $75 \%$ FBM within $\pm 1 \mathrm{SD}(3.1 \mathrm{~kg})$ standard deviation boundaries; $75 \%$ of the LBM within $\pm 1 \mathrm{SD}(2.9 \mathrm{~kg})$ and $70 \%$ of $\% \mathrm{BF}$ data are within $\pm 1 \mathrm{SD}(5.8 \%)$. The means of the differences are at $-1.0 \mathrm{~kg}$ of FBM, $1.6 \mathrm{~kg}$ of LBM) and $5.9 \%$ of $\% \mathrm{BF}$.

The intra class coefficients (ICC) showed significant differences between DXA and BIA WBC in all patients.

Conclusion: Scatter-plots and differences against the mean plots (Bland and Altman) showed good comparability between both DXA and BIA methods. Nevertheless, the ICC showed significant differences between the measurement methods. This contradictory result could be due to a calibration problem. Further research should identify both measurement errors to be able to make a better calibration of the devices and allow better clinical applicability for the assessment of WBC.

P269. PERSISTENT BISPHOSPHONATE USE REDUCES THE RISK OF OSTEOPOROTIC FRACTURES IN DAILY PRACTICE

W.G. Goettsch ${ }^{1}$, F.J.A. Penning-van Beest ${ }^{1}$, J.E. Erkens ${ }^{1}$, N.O. Lynch $^{3}$, A. Novak ${ }^{4}$, R.M.C. Herings ${ }^{1,2}$; ${ }^{1}$ PHARMO Institute, Utrecht, The Netherlands, ${ }^{2}$ Department of Pharmacoepidemiology and Pharmacotherapy, Utrecht Institute for Pharmaceutical Science, Utrecht University, The Netherlands, ${ }^{3}$ GSK, Greenford, United Kingdom, ${ }^{4}$ Roche Nederland BV, Woerden, The Netherlands

Objective: This study aimed to investigate the effect of persistent bisphosphonate usage on the risk for hospitalization due to osteoporotic fractures.

Methods: The PHARMO database, which includes linked drug-dispensing records and hospital discharge records of more than one million subjects in defined areas in the Netherlands, was used to identify new female users of alendronate, etidronate or risedronate $>50$ years in the period Jan ' 96 - Jan '03. Persistence with bisphosphonate treatment was determined using the method of Catalan. Within the cohort a matched case control study was performed. Cases were selected on the basis of a first hospitalization for an osteoporotic fracture (index date). Controls were matched 10:1 to cases on month of inclusion in the cohort and were assigned a random index date. The association with risk for fractures was assessed for bisphosphonate use at the index date and for persistent bisphosphonate use before the index date. 
Results: The study cohort included 8,845 new female bisphosphonate users; 334 women $(3.8 \%)$ were hospitalized for a fracture after inclusion in the cohort. 3,280 controls were matched to the 334 cases. Bisphosphonate use at the index date significantly reduced the risk for osteoporotic fractures (RR 0.78; 95\% CI 0.61-0.99, adjusted for age, previous fractures and comedication). At least one year of persistent bisphosphonate usage reduced the risk for osteoporotic fractures even more substantially (adjusted RR 0.70; 95\% CI 0.50-0.99).

Conclusions: These results emphasize the importance of persistent bisphosphonate usage to obtain the maximal protective effect. Previous studies have demonstrated that persistence with bisphosphonates is higher with less frequent dosing regimens but is still suboptimal. This study has demonstrated that improving persistence results in reduced hospitalization for osteoporotic fractures.

\section{P270. BONE MINERAL DENSITY AND FREQUENCE OF RISK FACTORS IN THE INDUSTRIAL AREA IN SERBIA}

N. Prodanovic, B. Bozic, I Zgradic; Military Medical Academy, Belgrade, SCG, Serbia and Monetnegro

Objectives: The central problem with osteoporosis (OP) is fracture. According to these facts, it is necessary to prevent them on time. The risk factors (RF) for osteoporotic fractures are age, gender, rase, BMD, family history of OP and fracture, life style, etc. The aim of this study was to determine the percentage of RF for OP, fracture frequency and bone mineral density (BMD) in Serbia.

Material and methods: In the period from January 2005 to June 2005 the Society for osteoporosis in Serbia and Montenegro carried out a study which included 232 persons from a factory in an industrial zone (city of Pancevo), average age was 57 . During the examination, all persons filled a questionnaire which included questions related to RF (early surgery menopause, consuming more than 3 cups of coffee a day, smoking more than 5 cigarettes a day, physical inactivity, history of previous fractures, etc.). BMD was measured by ultrasound "Sahara"-Hologic device, on the heel. All the persons diagnosed with osteopenia with one or more RF, or OP after the ultrasound examination, had their BMD measured by Lunar DEXA DPX 2000 device on lumbar spine (L1-4) and/or femur.

Results: Out of 232 (92 men and 140 women) examinated persons, 22 of them $(9,05 \%)$ had osteopenia and $14(6 \%)$ of them had OP. The most common RF in Pancevo was consuming coffee, early surgery menopause, diseases influencing BMD and intake of drugs with an influence on BMD. $127(54,74 \%)$ persons had BMD measured by DXA device. $44(34,65 \%)$ of them had OP, 49 $(38,58 \%)$ persons had osteopenia and $34(26,77 \%)$ persons had normal BMD. $53(41,73 \%)$ out of 127 who had their BMD measured by DXA device had previous osteoporotic fractures.

Conclusions: Based on the results shown, we can conclude that a high number of osteoporotic fractures and a number of risk factors was noted in the industrial area. Very important risk factors for bone loss in that industrial zone are early surgery menopause, diseases and drug intake. We noted an alarming number of osteoporosis patients and previous osteoporotic fractures in this small industrial area. Our results indicate that more attention should be paid in this industrial area to prevention of osteoporosis problems.

\section{P271. TERIPARATIDE TREATMENT INCREASES BONE MINERAL DENSITY IN PATIENT WITH SPINAL CORD INJURY INDUCED OSTEO- POROSIS: A CASE REPORT}

B. Uebelhart, R. Rizzoli; Service of Bone Disease, Department of Rehabilitation and Geriatrics, Cantonal hospital, Geneva, Switzerland

Patients with spinal cord injury are known to develop severe osteoporosis with high prevalence of non-traumatic fractures leading to a marked increase of morbidity. In the acute injury phase, bone resorption is increased and the use of bisphosphonate treatment has been shown to be effective in reducing bone loss. In the chronic phase, bone remodelling as assessed by histomorphometry analysis or biochemical markers is rather low. Teriparatide, a potent bone anabolic agent, could be of interest to stimulate bone formation and to decrease bone fragility and hence to reduce fracture risk. We report the case of a 48-year-old-man suffering from a tetraplegia with traumatic fracture of the 5th cervical vertebra since the age 23 . He presented a right hip fracture at the age of 47 during a transfer from his chair to a car and a spontaneous right diaphysal femoral fracture, 6 months later. At the time of the first fracture, bone mineral density measurements showed a severe osteoporosis at the hip and radius levels. Before treatment, total alkaline phosphatase value was normal (77IU/ $\mathrm{L}, \mathrm{N}: 30-125)$ and d-pyridinoline was slightly increased (19.7nmol/ mmol-creatinine, N:8-18)reflection of bone resorption. Teriparatide treatment was clinically well tolerated with a slight increased in albumine-corrected calcemia from 2.15 to $2.31 \mathrm{mmol} / \mathrm{L}(\mathrm{N}: 2.2-$ 2.6) after one month of treatment. After 7 months of teriparatide and despite no significant differences in markers of bone remodelling, bone mineral density values increased at all sites: Pretreatment Tériparatide Delta BMD T-score BMD T-score g/ $\mathrm{cm}^{2} \mathrm{SD} \mathrm{g} / \mathrm{cm}^{2} \mathrm{SD}$ Lumbar spine $0.871-1.50 .906-1.2+4 \%$ Femoral hip $0.306-4.80 .367-4.4+20 \%$ Total hip $0.330-5.0$ $0.422-4.4+28 \%$ Total radius $0.523-3.20 .557-2.5+6.5 \%$ This patient with spinal cord injury of long term duration presents a severe osteoporosis with two recent major long bone fractures. Bone response to teriparatide treatment was rapid and of high magnitude. No new fracture occurred during the 7-month followup. Thus teriparatide appears to be efficacious in a patient with severe osteoporosis consecutive to spinal cord injury.

\section{P272. ALENDRONATE IMPROVES THE STRUCTURAL GEOMETRY OF THE PROXIMAL FEMUR IN POSTMENOPAUSAL OSTEOPOROTIC WOMEN}

T.J. Beck ${ }^{1}$, J.A. Cauley ${ }^{2}$, A.E. de Papp ${ }^{3}$, L.E. Wehren ${ }^{3}$, D.T. Baran $^{3}$; ${ }^{1}$ Johns Hopkins Medical Institutions, Baltimore, MD, USA, ${ }^{2}$ University of Pittsburgh, Pittsburgh, PA, USA, ${ }^{3}$ Merck Research Laboratories, Horsham and Upper Gwynned, PA and Rahway, NJ, USA

Objectives: We sought to determine if alendronate treatment produced positive changes in the structural geometry of the proximal femur in postmenopausal women with either prevalent vertebral fractures (VF) or femoral neck BMD T-Scores less than -2.5 .

Material and Methods: A subset of 319 women from the University of Pittsburgh clinic enrolled in the Fracture Intervention Trial (FIT) were evaluated after 3 years (VF) or 4 years (BMD T-score $\leq 2.5$; no VF) of treatment with placebo ( $\mathrm{PBO}$, $\mathrm{N}=155$ ) or alendronate (ALN (5 mg/2 yrs; $10 \mathrm{mg} / 3-4 \mathrm{y}) \mathrm{N}=164$ ). Hips were scanned using Hologic QDR2000 DXA scanners and scan images were compared to baseline using the Hip Structure Analysis (HSA) software. Conventional BMD and cross-sectional geometries were evaluated at the narrowest point on the femur neck (NN), across the intertrochanteric (IT) region along the bisector of the neck and shaft axes and across the shaft at $1.5 \mathrm{x}$ minimum neck width distal to the axes intersection. Follow-up parameters were adjusted for prevalent VF and the baseline value by ANCOVA.

Results: Compared to PBO, at all three cross-sectional regions, ALN-treated women showed significantly $(\mathrm{p}<0.05)$ increased BMD (4-6\%), cortical thickness (4-7\%), bone crosssectional areas (4 to $7 \%$ ), and section moduli (4 to $8 \%$ ) and reduced buckling ratios $(-4$ to $-7 \%)$. Changes in the latter 3 parameters indicate improvement in resistance to axial and bending loads, as well as increased cortical stability. Treatment increased periosteal apposition at the IT region, but not at the $\mathrm{NN}$ or shaft regions.

Conclusion: We conclude that alendronate has favorable effects on hip structural geometry. 
P273. TREATMENT OF POSTMENOPAUSAL OSTEOPOROTIC WOMEN WITH PARATHYROID HORMONE 1-84 FOR 18 MONTHS IMPROVES TRABECULAR BONE ARCHITECTURE: A STUDY OF ILIAC CREST BIOPSIES USING MICRO-COMPUTED TOMOGRAPHY

D.W. Dempster ${ }^{1}$, J. Fox ${ }^{2}$, I. Moreau ${ }^{3}$, A. Varela ${ }^{3}$, L.G. SteMarie $^{4}$, M.K. Newman ${ }^{2}$, S.Y. Smith ${ }^{3}$, R.R. Recker ${ }^{5}$; ${ }^{1}$ Helen Hayes Hospital, West Haverstraw, NY, USA, ${ }^{2}$ CTBR, Senneville, PQ, Canada, ${ }^{3}$ Charles River Laboratories, Preclinical Services Montreal Inc., Canada, ${ }^{4}$ NPS Pharmaceuticals, Salt Lake City, UT, USA and Nycomed, Roskilde, Denmark, ${ }^{5}$ Creighton University, Omaha, NE, USA.

Iliac crest biopsies were obtained from postmenopausal osteoporotic women enrolled in the TOP Study after 18 months of daily sc injections of placebo or $100 \mu \mathrm{mg}$ parathyroid hormone 184 (PTH). All subjects received daily treatment with calcium (700 $\mathrm{mg}$ ) and vitamin D (400 IU). There were no significant differences between groups in age, weight, bone turnover markers, or spine and hip bone mineral density at baseline.

Prior to sectioning for histomorphometry, the biopsies $(n=8$ /group) were subjected to micro-computed tomography (MicroCT 20, Scanco Medical, AG).

The values for trabecular structure obtained by $\mu \mathrm{CT}$ were very similar to those obtained by histomorphometry (presented at ASBMR2004). Compared to placebo-treated subjects, mean cancellous bone volume (Cn.BV/TV) was $45 \%$ higher in PTHtreated subjects $(\mathrm{PTH}=23.3 \% \pm 2.0$ vs placebo $=16.1 \% \pm$ $1.4 ; P=0.036)$. The higher $\mathrm{Cn} . \mathrm{BV} / \mathrm{TV}$ was the result of $12 \%$ and $17 \%$ increases, in mean trabecular number (Tb.N) $(\mathrm{PTH}=1.40 /$ $\mathrm{mm} \pm 0.06$, placebo $=1.25 / \mathrm{mm} \pm 0.04 ; P=0.093)$ and mean trabecular thickness $(\mathrm{Tb} . \mathrm{Th})(\mathrm{PTH}=186 \mu \mathrm{m} \pm 12$, placebo $=$ $159 \mu \mathrm{m} \pm 11 ; P=0.128)$, respectively, with a $10 \%$ lower mean trabecular separation (Tb.Sp) $(\mathrm{PTH}=696 \mu \mathrm{m} \pm 27$, placebo $=$ $771 \mu \mathrm{m} \pm 23 ; P=0.036)$. Importantly, mean connectivity density (Conn.D) was $22 \%$ higher $\left(\mathrm{PTH}=4.81 / \mathrm{mm}^{3} \pm 0.34\right.$, placebo $\left.=3.93 / \mathrm{mm}^{3} \pm 0.52 ; P=0.074\right)$. The mean structure model index (SMI) was also significantly lower $(55 \%)$ in PTH-treated subjects relative to placebo-treated subjects $(0.49 \pm 0.25$ vs $1.09 \pm 0.16$, respectively; $P=0.046$ ), indicating a better connected trabecular architecture with a more plate-like structure, both indicative of a stronger bone.

In conclusion, relative to placebo, treatment of osteoporotic women for 18 months with PTH resulted in marked increases in Cn.BV/TV, improved trabecular connectivity and a more platelike structure. Structural variables obtained by $\mu \mathrm{CT}$ and bone histomorphometry were remarkably similar. All of these results are consistent with an increase in bone strength and the marked reduction in vertebral fracture incidence observed in the TOP Study.

\section{P274. ENDOSCOPICALLY CHECKED TRANSPEDICULAR SCREW INSERTION IN OSTEOPOROTIC PATIENTS}

T. Yazar, B. Guclu; Ankara University School of Medicine, Ibn-i Sina Hospital, Department of Orthopedics and Traumatology, Ankara, Turkey

Background: Transpedicular screws are one of the most important components of the posterior segmental fixation systems. The most feared complication of transpedicular screw insertion is the possibility of neural damage due to contact during insertion process or continuous irritation of the malpositioned screw on the neural structures. There are many reports in the literature about complications related directly to malpositioning of the transpedicular screws. Currently the position of the screws is checked by palpation of the pedicle walls by the help of a guide wire and fluoroscopic control. Our objective was to inspect the screw hole endoscopically would prevent malpositioning of the transpedicular screws and decrease complications related to them.

Methods: In 20 cases who underwent segmental spinal fixation by transpedicular screws for a variety of reasons, mostly osteo- porotic brittle bone structure. We endoscopically examined 76 screw holes prior to insertion of the screws.

Results: In three screws anterior penetration of the hole through the corpuscular wall was detected and a shorter screw was inserted. Lateral penetration of the vertebra was observed in one case and screw hole was rerouted. In most of the cases osteopenic bone was visualized and the pedicle was grafted before insertion of the screw. Above all, during the direct visualization process of the lamellar structure we could not see any structural change after one year medication for osteoporosis.

Conclusions: Complications due to malpositioned screws can be prevented by endoscopic visualization of the screw hole. Direct visualization of the hole aids in prevention of complications related to insertion of the screw through defective area. We believe, although it adds to the total operative time, avoiding the unpredictable complications worth the time spent.

\section{P275. GROWTH AND MORPHOGENESIS OF MANDIBLE IN GROWING RATS UNDER EFFECT OF CYCLOFERONTM}

V. Luzin, O. Andrieieva, D. Astrakhantsev; Department of Normal Anatomy, State Medical Univercity, Luhansk, Ukraine

Objectives: Immunomodulating drugs like Cycloferon ${ }^{\mathrm{TM}}$ are widely used in clinical practice. It is known that bone morphogenesis depends on immune status to a great extent though the effect of immunomodulators on development of the skeleton of face has not been studied closely. This study is aimed at investigation of the growth and morphogenesis of the mandible in growing rats under long-term effect of Cycloferon ${ }^{\mathrm{TM}}$

Materials and methods: In the experiment we used 60 male rats aged 3 weeks with the initial mass of $30-40$ grams. The experimental animals received Cycloferon ${ }^{\mathrm{TM}}$ daily once a day IM in dosage of 6 $\mathrm{mg} / \mathrm{kg}$ according to a common scheme. The control group comprised the animals that received $0.9 \%$ sodium chloride solution by the same scheme. The observation terms were $7,15,30$ and 90 days The mandibles were measured as follows: mass, maximum length and ramus height. Basing on the data collected we calculated Simon's index (a ratio of maximum length to a cubic root of a bone mass) and height-to-length ratio (height-to-length percentage).

Results: In the controls in all observation terms maximum length of mandible increased from $22.73 \pm 0.26 \mathrm{~mm}$ to $30.98 \pm 0.24 \mathrm{~mm}$, ramus height increased from $9.52 \pm 0.10$ to $13.47 \pm 0.14 \mathrm{~mm}$ and height-to-length increased from $41.87 \pm 0.14$ to $43.48 \pm 0.67$. This testifies to active growth processes in the facial skeleton.

In the experimental group growth rate decreased reaching maximum in the $30^{\text {th }}$ day of the experiment. By this term maximum length, ramus height and height-to-length ratio decreased by $15.37 \%, 18.64 \%$ and $3.85 \%$ respectively $(\mathrm{p}<0.05)$. The Simon's index was significantly lower than in controls. By the 90th day however the growth inhibiting effect of Cycloferon ${ }^{\mathrm{TM}}$ had reduced significantly.

Conclusions: Daily IM $0.6 \mathrm{mg} / \mathrm{kg}$ Cycloferon ${ }^{\mathrm{TM}}$ reduces bone growth rate, decreases height-to-length ratio and robusticity index. The changes reach maximum at the 30th day of the experiment.

P276. WEEK-BY-WEEK EFFECTS OF MONTHLY IBANDRONATE ON BIOCHEMICAL MARKERS OF BONE RESORPTION: RESULTS OF A 3-MONTH RANDOMIZED TRIAL

J. Walliser ${ }^{1}$, R. Royavo ${ }^{2}$, P. Geusens ${ }^{3}$, A.W. Kung ${ }^{4}$, J.N Verbruggen ${ }^{5}$, L.E. Wehren ${ }^{5}$, M.E. Melton ${ }^{5}$; ${ }^{1}$ Hospial Angeles del Pedregal, Padierna, Mexico, ${ }^{2}$ Central University of Ecuador, Eloy Alfaro, Ecuador, ${ }^{3}$ Universitaire Campus Gebouw C, Diepenbeek, Belgium, ${ }^{4}$ Queen Mary Hospital, Hong Kong, China, ${ }^{5}$ Merck Research Laboratories, Brussels, Belgium, Rahway, NJ, USA, and Whitehouse Station, NJ, USA

Objectives: The number and depth of active bone resorption sites are considered important determinants of bone strength and 
fracture risk. Bisphosphonates (BPs) bind preferentially to active bone remodeling sites where they decrease bone resorption through direct effects on osteoclasts. The resorption phase of a remodeling site is typically completed within 2-3 weeks. Thus, some resorption sites may proceed to completion if BPs are given at intervals greater than 1-2 weeks. No data are available on the effect of monthly dosing of ibandronate on bone markers in the interval between doses. This study examined the pattern of urinary and serum resorption markers in a monthly dosing regimen of ibandronate.

Material and Methods: After a 4-week run-in of calcium and vitamin D, 203 postmenopausal osteoporotic women were randomized to receive 3 once-monthly doses of ibandronate $100 \mathrm{mg}$ or $150 \mathrm{mg}$, or matching placebo. Patients and all study staff remained blinded to treatment allocation throughout the study. Serum CTx and urine NTx were measured at baseline (Week 0, just prior to first dose), one and 4 weeks after each dose and weekly after the third dose (Weeks 9-12). Geometric mean percent change from baseline was determined.

Results: Dose-dependent decreases in resorption were observed. In each cycle, sCTx and uNTx decreases were less 4 weeks post-dose than at one week post-dose. Marker levels increased progressively throughout the 4 weeks following the third dose of ibandronate, but remained below placebo at all time points.

Conclusions: Reduction of both resorption markers with monthly ibandronate was cyclic. The clinical implications of this effect merit further investigation.
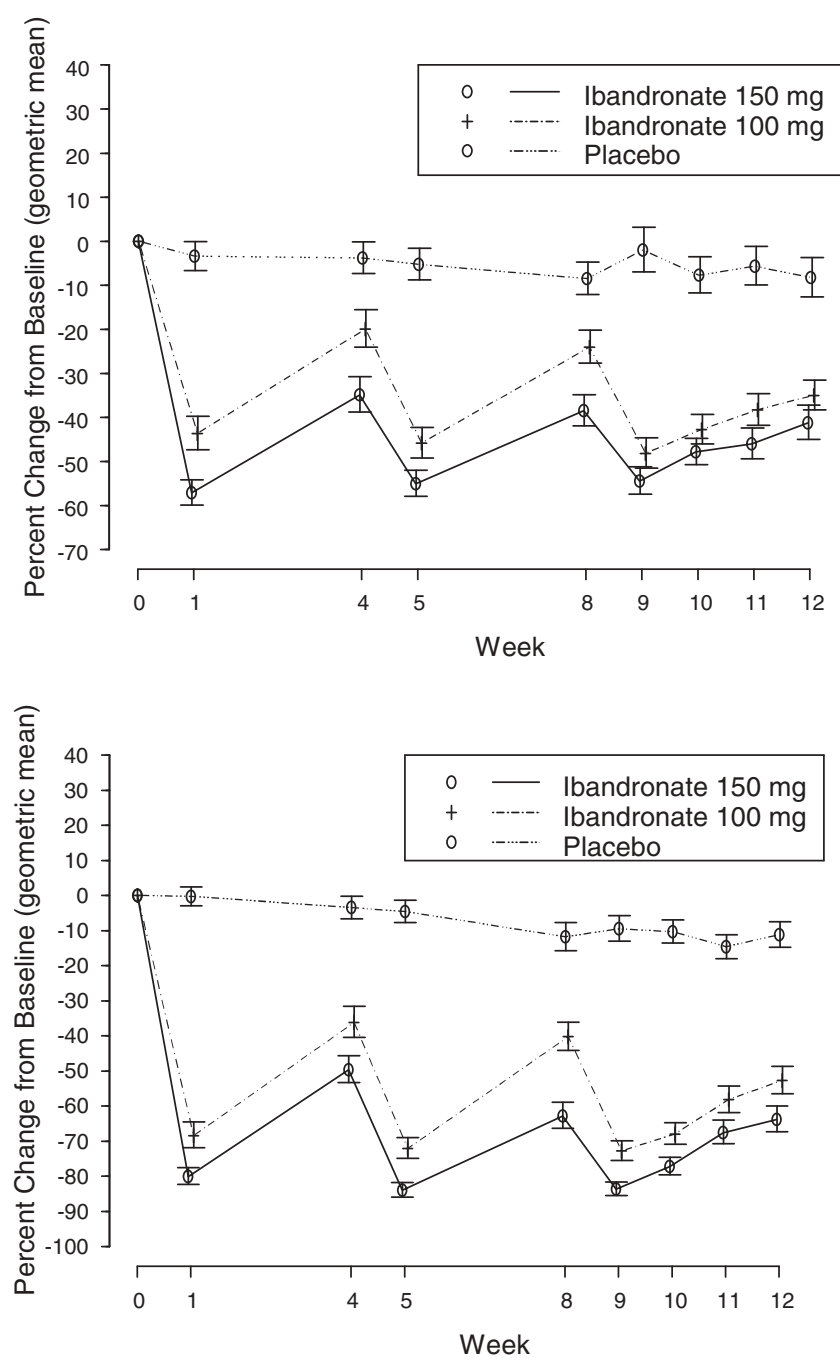

P277. THRESHOLD RESPONSE OF SERUM CTX TO MONTHLY ORAL IBANDRONATE THROUGHOUT DOSING INTERVAL: RESULTS OF A 3-MONTH RANDOMIZED TRIAL

J. Walliser ${ }^{1}$, R. Royavo ${ }^{2}$, P. Geusens ${ }^{3}$, A.W. Kung ${ }^{4}$, J.N. Verbruggen ${ }^{5}$, L.E. Wehren ${ }^{5}$, M.E. Melton ${ }^{5}$; ${ }^{1}$ Hospial Angeles del Pedregal, Padierna, Mexico, ${ }^{2}$ Central University of Ecuador, Eloy Alfaro, Ecuador, ${ }^{3}$ Universitaire Campus Gebouw C, Diepenbeek, Belgium, ${ }^{4}$ Queen Mary Hospital, Hong Kong, China, ${ }^{5}$ Merck Research Laboratories, Brussels, Belgium, Rahway, NJ, USA, and Whitehouse Station, NJ, USA

Objectives: Intermittent oral ibandronate, given once per month, does not maintain stable reduction in bone resorption. This suggests that some persons taking the drug may have little reduction in turnover for at least part of the interval. Since clinical efficacy of bisphosphonates is correlated with the extent of reduction in turnover, this may have important clinical consequences. In this study we examined the proportion of participants who reached specified threshold levels of resorption marker reduction.

Material and Methods: Postmenopausal osteoporotic women participated in a 3-month, randomized, double-blind, placebocontrolled clinical trial. Enrolled participants were randomized to receive ibandronate $150 \mathrm{mg}$ or $100 \mathrm{mg}$ or matching placebo every 4 weeks for 3 doses. Serum CTx was measured just prior to each dose, one week after each dose, and weekly for 4 weeks after the final dose. Geometric mean percent change from baseline was calculated, as was the proportion that had marker reductions of at least $40 \%$, $50 \%$, and $60 \%$ at each time point after the third dose (Weeks 9-12).

Results: At each week after dosing, for both ibandronate doses, a progressively smaller proportion of women met the defined SCTx reduction thresholds. Fewer than half of women taking $150 \mathrm{mg}$ of ibandronate had sCTx reductions of at least $60 \%$ at all 4 weeks after the third dose (Weeks 9-12) and fewer than three-quarters $(71.9 \%)$ of women had reductions of at least $40 \%$ at all four weeks.

Conclusions: The proportion of women who attain specified reduction in SCTx declines progressively with time between doses of once-monthly ibandronate. To what degree the observed cyclic changes in bone resorption between doses might affect efficacy deserves further study to evaluate the clinical implications.

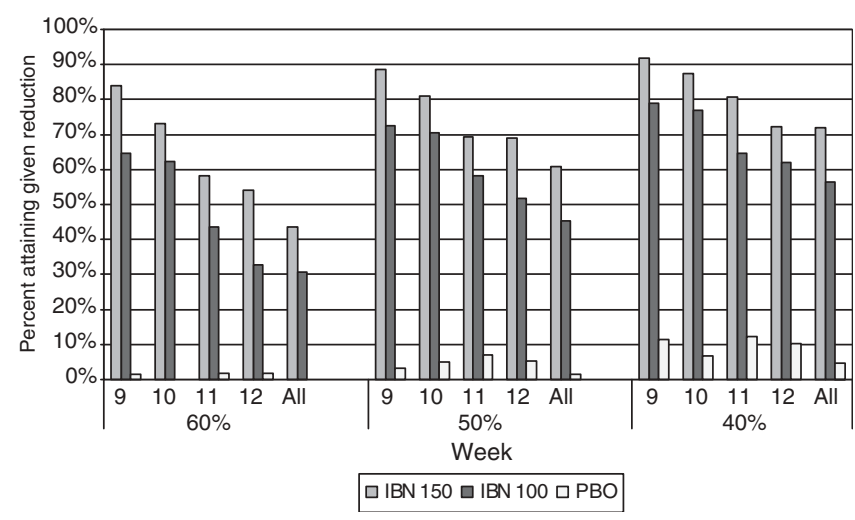

P278. RELATIONSHIP BETWEEN TERIPARATIDE-MEDIATED CHANGES IN BMD AND MICROARCHITECTURE IN POSTMENOPAUSAL WOMEN WITH OSTEOPOROSIS

R. Recker ${ }^{1}$, H. Resch ${ }^{2}$, P. Chen ${ }^{3}$, D. Misurski ${ }^{3}$, I. Pavo ${ }^{3}$, A. Sipos ${ }^{3}$; ${ }^{1}$ Creighton University Medical Center, Osteoporosis Research Center, Omaha, NE, USA, ${ }^{2} 2^{\text {nd }}$ Department of Internal Medicine, St. Vincent Hospital, Vienna, Austria, ${ }^{3}$ Eli Lilly and Company, Indianapolis, IN, USA

Objectives: Bone strength is determined by bone mineral density (BMD) and other elements of bone quality, including bone microarchitecture. Teriparatide (TPTD) treatment increases 


\begin{tabular}{lll}
\hline $\begin{array}{l}\text { Change in Micro } \\
\text { architectural }\end{array}$ & $\begin{array}{l}\text { Change in } \\
\text { Parameters } \\
(22 \text { months })\end{array}$ & \\
& Lumbar Spine & Femoral Neck \\
\cline { 2 - 3 } & & \\
\hline 2-D Histomorphometry & & \\
Trabecular volume & $0.45(\mathrm{n}=26, \mathrm{p}=0.02)$ & $0.35(\mathrm{n}=25, \mathrm{p}=0.09)$ \\
Cortical thickness & $0.02(\mathrm{n}=26, \mathrm{p}=0.92)$ & $-0.07(\mathrm{n}=25, \mathrm{p}=0.75)$ \\
Wall thickness & $0.41(\mathrm{n}=29, \mathrm{p}=0.03)$ & $0.56(\mathrm{n}=28, \mathrm{p}=0.002)$ \\
Marrow star volume & $-0.09(\mathrm{n}=24, \mathrm{p}=0.67)$ & $-0.14(\mathrm{n}=23, \mathrm{p}=0.52)$ \\
\hline $\begin{array}{l}\text { 3-D } \mu \text { CT } \\
\text { Connectivity density }\end{array}$ & $0.41(\mathrm{n}=32, \mathrm{p}=0.02)$ & $0.32(\mathrm{n}=31, \mathrm{p}=0.08)$ \\
$\begin{array}{l}\text { Bone volume/ } \\
\text { total volume }\end{array}$ & $0.48(\mathrm{n}=32, \mathrm{p}=0.006)$ & $0.51(\mathrm{n}=31, \mathrm{p}=0.004)$ \\
Trabecular number & $0.44(\mathrm{n}=32, \mathrm{p}=0.01)$ & $0.44(\mathrm{n}=31, \mathrm{p}=0.01)$ \\
Trabecular separation & $-0.37(\mathrm{n}=32, \mathrm{p}=0.04)$ & $-0.46(\mathrm{n}=31, \mathrm{p}=0.01)$ \\
$\begin{array}{l}\text { Trabecular thickness } \\
\text { Structure model index }\end{array}$ & $0.22(\mathrm{n}=32, \mathrm{p}=0.22)$ & $0.34(\mathrm{n}=0.06, \mathrm{p}=0.02)$ \\
$\begin{array}{l}\text { Degree of anisotropy } \\
\text { Cortical porosity }\end{array}$ & $-0.54(\mathrm{n}=32, \mathrm{p}=0.001)$ & $-0.55(\mathrm{n}=31, \mathrm{p}=0.001)$ \\
Cortical thickness & $-0.01(\mathrm{n}=32, \mathrm{p}=0.94)$ & $-0.02(\mathrm{n}=31, \mathrm{p}=0.92)$ \\
\hline & $0.20(\mathrm{n}=28, \mathrm{p}=28, \mathrm{p}=0.88)$ & $0.10(\mathrm{n}=27, \mathrm{p}=0.63)$ \\
\hline
\end{tabular}

BMD and improves both cortical and trabecular bone microarchitecture. Increases in lumbar spine (LS) BMD account for approximately 30 to $41 \%$ of the vertebral fracture risk reduction with TPTD treatment. We sought to determine whether increases in BMD are associated with improvements in cortical and trabecular microarchitecture.

Methods: Paired iliac crest biopsies were obtained at study baseline and after a mean treatment duration of $22 \pm 2$ months (range, $19-24$ months) in a subset $(\mathrm{n}=36)$ of postmenopausal women with osteoporosis who participated in the Fracture Prevention Trial (placebo [ $\mathrm{n}=13$ ], TPTD $20 \mu \mathrm{g} /$ day [ $\mathrm{n}=13]$, TPTD $40 \mu \mathrm{g} /$ day $[\mathrm{n}=10])$. The biopsy samples were analyzed by $2-\mathrm{D}$ histomorphometry and 3-D microcomputed tomography $(\mu \mathrm{CT})$. LS and femoral neck (FN) BMD were measured at baseline and 12 months. Pearson correlation was performed to assess the relationship between actual changes in BMD and actual changes in microarchitectural parameters.

Results: Changes in LS and FN BMD correlated with changes in trabecular, but not cortical microarchitecture, suggesting that BMD increases are associated with improvements in trabecular bone microarchitecture.

Conclusion: Improvements in trabecular bone microarchitecture may contribute to BMD-associated increases in bone strength during TPTD treatment.

\section{P279. LINKAGE TO A REGION ON CHROMOSOME 11 IN TWO MALTESE FAMILIES WITH SEVERE OSTEOPOROSIS}

C. Vidal ${ }^{1}$, R. Galea ${ }^{2}$, M. Brincat ${ }^{2}$, A. Xuereb Anastasi ${ }^{1,3}$; ${ }^{1}$ Department of Pathology, University of Malta Medical School, Malta, ${ }^{2}$ Department of Obstetrics and Gynaecology, University of Malta Medical School, Malta, ${ }^{3}$ Institute of Health Care, University of Malta, Malta

Osteoporosis is a metabolic bone disease with a strong genetic component. Family based linkage studies were performed by a number of investigators to try to identify loci that might contain genes responsible for an increased susceptibility for osteoporosis.

A whole genome linkage scan using 400 microsatellite markers was performed in 27 members from two Maltese families with a highly penetrant form of osteoporosis. The phenotype was defined by lumbar and femoral z-scores calculated after measurement of bone mineral density (BMD) by DEXA. Both males and females were among the affected individuals. Multipoint parametric and non-parametric linkage analyses were performed by EasyLinkage v4.0 using GENEHUNTER v2.1, assuming dominant and recessive modes of inheritance with variable penetrance.

Evidence of linkage was observed to a marker at $11 \mathrm{p} 13$ where a non-parametric LOD score (NPL) of 4.99 was obtained. A maximum heterogeneity LOD (HLOD) score of $2.74(p=0.0091)$ for this region was obtained for the dominant mode of inheritance with $90 \%$ penetrance and a phenocopy rate of $1 \%$. Suggestive linkage was observed at a number of other chromosomal loci including 5q34, 6q22, 9q21, 13q33 and 17q21.

These results suggest that a major gene responsible for osteoporosis might be present in region $11 \mathrm{p} 13$ while other contributing genes might be present on other chromosomes. Identification of these genes is important for the early identification of individuals at risk and for the development of effective treatments.

\section{P280. DISABILITY WEIGHTS FOR OSTEOPOROSIS AND OSTEOPOROTIC FRACTURES}

M.F. Janssen, E. Birnie, G.J. Bonsel; AMC, Public Health Epidemiology, Amsterdam, The Netherlands

Objectives: To compare a newly developed method to derive disability weights for risk factor diseases, like osteoporosis, with the standard Burden of Disease approach (SBoD). A risk factor disease, like osteoporosis, is an asymptomatic condition with a risk of a symptomatic event. SBoD estimates burden of risk by multiplying the probability of an event by the burden of that event, ignoring the impact of risk awareness. In osteoporosis, fracturerisk awareness and associated mortality may affect the disease burden.

Methods: Disability weights were derived by a panel of the general public in the Netherlands $(n=142)$ as part of a larger valuation study. All health states were presented to participants on a standardized, preformatted sheet ('vignette') containing disease specific information and a generic description (EQ-6D5L). All vignettes were valued by time-trade-off (TTO). Vignettes for osteoporosis showed an asymptomatic state with varying fracture-risks $(1,2,5$, or $10 \%)$, which were valued directly. To derive osteoporotic weights by SBoD osteoporotic fracture vignettes were included. SBoD weights were obtained indirectly, calculated as hip fracture weight multiplied by fracture risks. In total, 14 vignettes were valued, of which three included a mortality risk.

Results: Taking fracture-risk awareness into account, mean TTO disability weights for osteoporosis ranged from $0,035(1 \%)$ to $0,151(10 \%)$. Calculated SBoD weights are $0,001(1 \%)$ to 0,011 $(10 \%)$, a factor 9 to 34 lower.

Conclusions: The burden of osteoporosis can be quantified via disability weights using direct methods. Ignoring fracture-risk awareness and associated mortality leads to gross underestimation of the burden of osteoporosis. The inclusion of burden of risk would significantly influence current QALY/DALY disease rankings and would accommodate the empirical gap between SBoD and the clinical practice. It would better explain the sizeable resources spent on risk 'management' (eg diagnostic tests, disease surveillance) and prevention in terms of cost per QALY.

\section{P281. PATIENTS PREFER PROVEN EFFICACY OVER DOSING REGIMEN IN OSTEOPOROSIS THERAPY WITH BISPHOSPHONATES}

R. Keen ${ }^{1}$, S. van der Geest ${ }^{2}$, B. Wick ${ }^{3} ;{ }^{1}$ University College London, London, UK, ${ }^{2}$ Procter\&Gamble Pharmaceuticals SARL, Petit Lancy, Geneva, Switzerland, ${ }^{3}$ Procter\&Gamble Pharmaceuticals Germany GmbH, Weiterstadt, Germany

There is a move towards less frequent dosing with bisphosphonates in the management of postmenopausal osteoporosis. Recent studies have suggested that patients may prefer a monthly over a weekly oral treatment, with this being attributable primarily to the longer interval between doses. Patient preference is however, complex and will be influenced by many factors. In this study we have assessed whether patients' preference would be altered by knowledge about drug efficacy in the prevention of osteoporotic fractures.

Postmenopausal women (age $\geq 55$ yrs) with osteoporosis participated in face-to-face interviews across the UK. Participants were presented with a hypothetical scenario in which they had the choice between a weekly or a monthly bisphosphonate with similar dosing instructions and similar types of side effects. The 
weekly bisphosphonate was demonstrated to have a proven efficacy to reduce the fracture risk at the spine and hip, whereas the monthly had a proven efficacy to reduce the fracture risk at the spine but not at the hip. Participants were asked to express a preference for the weekly or monthly product, and the main reason for their preference was also recorded.

Data was available for 250 women (mean age $71 \mathrm{yrs}$ ). Of these, $50 \%$ were already taking bisphosphonates. A statistically significance difference was found in their preference for the weekly dosing regimen with proven efficacy at spine and hip $(74 \%$; $\mathrm{p}<0.001)$. This was independent of bisphosphonate use $(p=0.355)$. The highest preference was found in the age groups $55-59$ yrs and $>70$ yrs with $76 \%$ and $82 \%$, respectively. The majority of those preferring the weekly product, expressed efficacy ('reduced risk of broken bones') as the main reason followed by the weekly dosage regimen.

This study demonstrates that patients prefer a weekly bisphosphonate with a proven fracture efficacy at spine and hip over a monthly with proven fracture efficacy only at the spine. These findings suggest that demonstrated efficacy of a bisphosphonate in reducing fracture risk at the hip is important for patients when deciding on treatment, and patients should be fully informed about the differences in proven efficacy between drugs.

\section{P282. IS PERSISTENCE TO BISPHOSPHONATE THERAPY RELATED} ONLY TO DOSING REGIMEN?

T.P. Van Staa ${ }^{1}$, H.G.M. Leufkens ${ }^{2}$, C. Cooper ${ }^{3}$; ${ }^{1}$ Epidemiology, Procter\&Gamble Pharma, Egham, UK, ${ }^{2}$ Department of Pharmacoepidemiology, Utrecht Institute for Pharmaceutical Sciences, Utrecht, The Netherlands, ${ }^{3} \mathrm{MRC}$ Resource Center in Epidemiology, Southampton Hospital, Southampton, UK

Persistence to osteoporosis treatment is important given the chronic nature of the underlying condition. It has been suggested that persistence can be substantially improved by just changing the dosage regimen. It was evaluated whether persistence is only related to dosing regimen.

Patients who started alendronate or risedronate treatment were identified from two UK general practitioner medical records databases (data collection 1987-2003): General Practice Research Database and The Health Improvement Network research database. Patients with Paget's disease were excluded. The persistence of bisphosphonate treatment (i.e., the proportion of patients who continued treatment over time) was determined by measuring repeat prescribing of bisphosphonates. Survival and time-dependent Cox analyses were used.

11,998 patients initiated alendronate or risedronate treatment (average age 69.0 years, $83 \%$ women, $52.5 \%$ on weekly). Using a time-period of 3 month, the 1-year persistence was $68.7 \%$ with weekly and $57.5 \%$ daily bisphosphonate. Persistence also increased during the observation period. The 1-year persistence in daily users was $52.2 \%$ in 1996 and $66.2 \%$ in 2002 $(\mathrm{P}$-value of trend $<0.05)$. For weekly users, this was $62.9 \%$ in 2001 and $70.4 \%$ in 2002 ( $\mathrm{P}$-value of trend $<0.05$ ). The degree of persistence was strongly related to the time-period chosen to evaluate repeat prescribing. Persistence was considerably higher if larger time gaps between two repeat prescriptions were allowed. Gaps in prescribing occurred more frequently in patients with a recent major illness $(\mathrm{RR}=2.00 ; 95 \%$ CI $1.57-2.53)$ or hospitalisation $(\mathrm{RR}=1.80 ; 95 \%$ CI $1.44-2.25)$. Weekly dosing did not change the likelihood of having a gap in prescribing $(\mathrm{RR}=1.05 ; 95 \%$ CI $0.99-1.12)$. Treatment discontinuation was most likely to occur after the first prescription. Overall, $70.2 \%$ of patients with a repeat after the first prescription were still taking bisphosphonates after 1 year (weekly $75.3 \%$ and daily $65.8 \%$ ).

Patients often have gaps in prescribing, e.g. due to intercurrent illness. If these gaps are ignored, persistence will be substantially underestimated. Also, persistence has increased during the observation period in both daily and weekly users which also narrowed the gap between daily and weekly bisphosphonate persistence. This suggests that dosage regimen is only one of the many factors determining persistence.

\section{P283. CROSS CALIBRATION OF GE-LUNAR IDXA AND PRODIGY BONE DENSITOMETERS}

L. Cole, E.A. Mossman, M.R. McClung; Oregon Osteoporosis Center, Portland, OR, USA

Bone mineral density (BMD) measurement at the lumbar spine and proximal femur with dual-energy X-ray absorptiometry (DXA) is the acknowledged gold standard for diagnosing and monitoring patients with osteoporosis and assessing fracture risk. The recently introduced Lunar iDXA (GE Healthcare, Madison, WI) fan-beam densitometer uses a new CZT detector that provides markedly improved image quality. We evaluated the BMD performance of the iDXA compared to the Lunar Prodigy at the spine and proximal femur.

Thirty postmenopausal women (mean age 61.3 years) were measured once at the spine on both devices, and 29 postmenopausal women (mean age 61.8 years) were measured once at the femur on each of the 2 DXA systems.

\section{Lunar iDXA vs Prodigy Total Femur BMD}

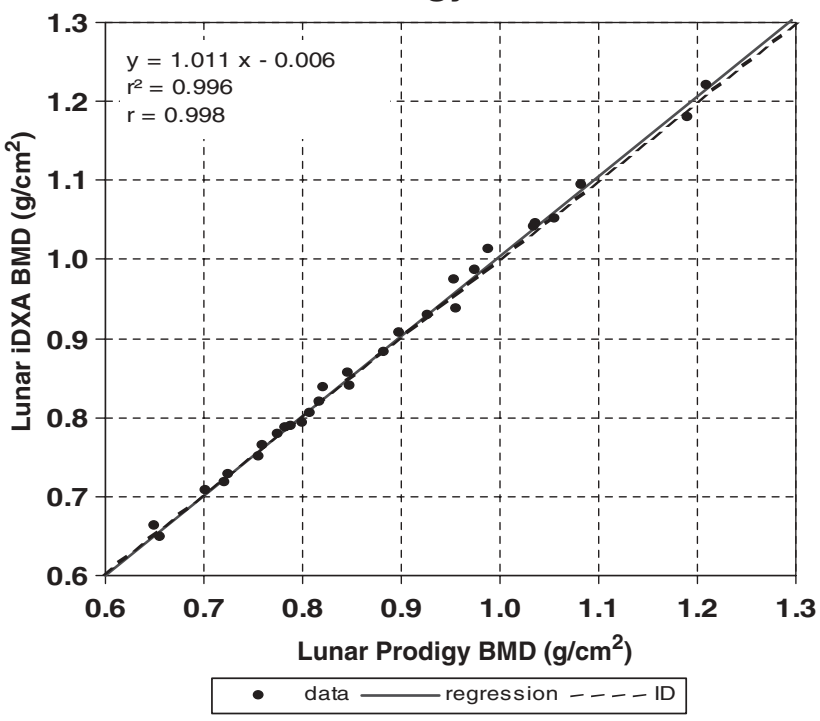

\section{Lunar iDXA vs Prodigy L1-L4 Spine BMD}

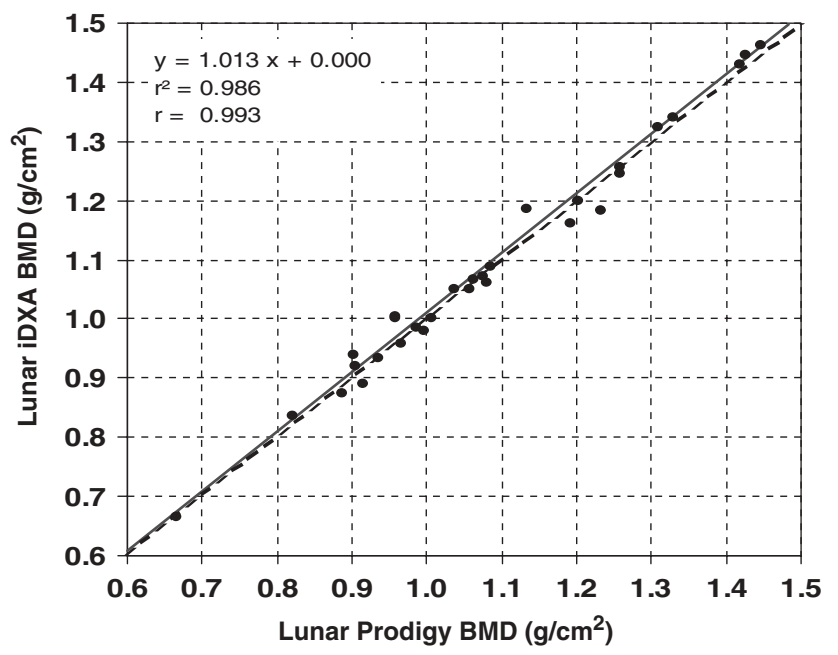

- data regression _- - ID 
BMD measurements for Prodigy and iDXA were highly correlated $\left(r^{2}=0.99\right)$ at the L1-L4 spine, femur neck, trochanter, and total femur. Neither slope nor intercept differed significantly from the identity values of 1 and 0 respectively (alpha $=0.05)$. There was a small bias of $+0.004 \mathrm{~g} / \mathrm{cm}^{2}$ for total femur by paired t-test.

We conclude that BMD agreement between iDXA and Prodigy densitometers was excellent at the spine, femur neck, trochanter, and total femur measurement sites.

This study was funded by a grant from GE Healthcare.

P284. POSTMENOPAUSAL OSTEOPOROTIC PATIENTS PROFILE AND TREATMENT COMPLIANCE: OSTEPI STUDY

F. Blotman ${ }^{1}$, B. Avouac ${ }^{1}$, B. Cortet $^{2}$, P. Hilliquin ${ }^{3}$, D. Pouchain ${ }^{4}$, F.A. Allaert ${ }^{5}$, A.F. Gaudin ${ }^{6}$, A. El Hasnaoui ${ }^{6}$; ${ }^{1}$ OFOS association, Paris, France, ${ }^{2} \mathrm{CHU}$ Lille, France, 3 Sud Francilien Hospital, Créteil, France, ${ }^{4} \mathrm{GP}$, Vincennes, France, ${ }^{5} \mathrm{CHRU}$ Dijon, France, ${ }^{6}$ GlaxoSmithKline, Marly-le-Roi, France

Objective: To describe postmenopausal women with osteoporosis in terms of risk factors and evaluate their compliance to osteoporosis treatment.

Material and method: An observational, cross-sectional study in general practice (GP). General Practitioners (GPs) filled out a register of women patients with osteoporosis, treated for osteoporosis or not. They completed a more detailed questionnaire on the first three women patients listed in the register, currently receiving or who received treatment of osteoporosis, during the past two years. Osteoporosis risk factors and compliance to actual treatments were evaluated during the inclusion visit.

Results: GPs included 2522 patients in the registers. Mean menopausal age of these patients was $49.7 \pm 4.1$ years and $10.8 \%$ of menopauses occurred at $<45$ years old. One third of women $(18.6 \%)$ presented familial history of osteoporosis, $13.7 \%$ had BMI $<19 \mathrm{Kg} / \mathrm{m} 2,13.2 \%$ had alcohol and/or tobacco consumption, $12.1 \%$ had long duration treatment (corticoids, heparin, ...) and $7.1 \%$ had bed rest $>3$ months. Then 1011 patients were interviewed. $39.8 \%$ of these patients met the criteria for osteoporosis based on low bone mineral density (Tscore ?-2.5), 36.9\% based on previous fracture and $23.4 \%$ based on both. $97.3 \%$ were treated for osteoporosis, among them $80.6 \%$ by bisphosphonates and $18.1 \%$ by SERM. Compliance to these treatments was quoted with a specific questionnaire, which indicated $60.9 \%$ of good compliance only. Further analysis showed that compliance is strongly affected by the intake frequency of the treatment: $66.7 \%$ of good compliance for bisphosphonate weekly vs $45.3 \%$ for bisphosphonate daily $(\mathrm{p}<0.0001)$.

Conclusion: These results indicated that a lower frequency of drug intake improved compliance to osteoporosis treatment but the compliance was steel suboptimal.

\section{P285. A RANDOMIZED, PROSPECTIVE STUDY OF THE EFFECTS OF EXERCISE ON QUALITY OF LIFE IN POSTMENOPAUSAL WOMEN}

L. Evstigneyeva, O. Lesnyak; Sverdlovsk Regional Clinical Hospital, Ekaterinburg, Russia

Objective: To evaluate the effectiveness of exercise on quality of life in postmenopausal women with vertebral fractures.

Material and methods: Forty-five healthy postmenopausal women were randomized into the exercise group $(n=23)$ and control group $(\mathrm{n}=22)$. The mean age was $67.2 \pm 7.49$ SD years in the exercisers and 68.6 $\pm 6.7 \mathrm{SD}$ years in the control $(\mathrm{p}>0.05)$. All women had at least one osteoporotic vertebral fracture and suffered from chronic back pain. Baseline variables in QUALEFFO score were not significantly different between groups. Exercise program included stretching, strength training and weight-bearing exercises. The program was conducted three times per week 60 minute a day for 6 months. Participants in the control group were instructed to continue with their usual daily activities. Participants were assessed at baseline, 3 months, 6 months using the Quality of Life Questionnaire of the European Foundation for Osteoporosis (QUALEFFO-41) and the "Timed Up and Go" test.

Results: There was improvement in total QUALEFFO score over 3 months (4.19) and over 6 months (4.2)in the exercise group compared to baseline $(\mathrm{p}<0.05)$. Quality of life significant improved in the domains of pain, physical function (mobility) and social function. In the control group quality of life did not change. A small decrease of the time of performance «Timed Up and Go» test observed in the exercise group $(\mathrm{p}<0.05)$ and there were no change in the control group.

Conclusion: exercise improves quality of life in elderly women with vertebral fractures.

\section{P286. COMPARISON OF GE HEALTHCARE LUNAR PRODIGY AND LUNAR IDXA DENSITOMETERS}

D. Krueger, M. Checovich, N. Vallarta-Ast, D. Gemar, R. Clodfelter, N. Binkley; University of Wisconsin Osteoporosis Research Program, Madison, WI, USA

Objective: GE Healthcare has recently developed a new fanbeam densitometer, the Lunar iDXA. This study assessed comparability of BMD measurements and precision obtained on a Lunar iDXA and Lunar Prodigy densitometer.

Materials and methods: In 245 subjects, 153 women and 91 men, age (mean [range]) 52.8 years, [20.0 - 91.5] lumbar spine and dual proximal femur measurements were obtained in the routine clinical manner. Their mean lowest BMD T-score from either the L1-4 spine, total femur or femur neck was -0.8 [3.4 to -4.3$]$. Additionally, in 30 postmenopausal women age 69.8 years, [61.778.8] from the above group, a precision assessment of the lumbar spine and proximal femur was conducted on each instrument in accordance with ISCD recommendations.

Results: BMD at the L1-4 spine, mean total femur and mean femur neck was very highly correlated $\left(\mathrm{r}^{2} \geq .98\right)$ between these two instruments. Bland-Altman analyses confirmed the absence of clinically significant bias. Specifically, biases at the L1-4 spine, mean total femur and mean femoral neck regions were $0.011,-0.002$ and -0.008 grams $/ \mathrm{cm}^{2}$ respectively. Moreover, the BMD least significant change was similar when comparing iDXA to Prodigy $\left(0.041\right.$ and $0.044 \mathrm{grams} / \mathrm{cm}^{2}$ respectively at the L1-4 spine and 0.013 and 0.010 grams $/ \mathrm{cm}^{2}$ at the mean total femur).

Conclusion: There is excellent BMD correlation between iDXA and Prodigy densitometers. Similarly, BMD precision with these two instruments at the L1-4 spine and femur is virtually identical.

P287. THE IMPACT OF DOSING FREQUENCY ON USE OF BIS PHOSPHONATE THERAPY AMONG POST-MENOPAUSAL WOMEN: A COMPARISON OF COMPLIANCE AND PERSISTENCE BETWEEN UK AND US COHORTS

N.O. Lynch ${ }^{1}$, M. Walker ${ }^{2}$, W. Cowell ${ }^{2}$, U. Rigney ${ }^{3}$, N. Suppapanya ${ }^{3}$; ${ }^{1}$ GlaxoSmithKline, Greenford, UK, ${ }^{2}$ Roche Products Ltd, Welwyn, UK ${ }^{3}$ GlaxoSmithKline, Collegeville, PA, USA

Objectives: Bisphosphonates are among the most effective therapies for the treatment of osteoporosis. Patient usage (compliance and persistence) with daily and weekly bisphosphonate treatment has been shown to be sub-optimal. This study compares the results from the US with those from a UK study.

Methods and materials: The UK Mediplus database, containing data on 2.5 Materials patients from approximately 600 GPs, was used to identify post-menopausal women ( $>50$ years) newly prescribed once weekly (OW) or once daily (OD) bisphosphonates (2001-2005). US data on bisphosphonate na patients was derived from an administrative claims database (19972003) from 30 health plans. In both studies women were grouped into two cohorts; once daily (alendronate $5 \mathrm{mg}$ or $10 \mathrm{mg}$ and risedronate $5 \mathrm{mg}$ ) and once weekly (alendronate $70 \mathrm{mg}$ and risedronate $35 \mathrm{mg}$ ) and followed for at least 12 months from their 
initial prescription. Compliance was calculated as days on therapy (Medication Possession Ratio - MPR) and persistence as the number of days from the initial prescription to a lapse of $>30$ days after the end of a previous prescription.

Results: The MPR for the combined study cohorts was $63.3 \%$ in the UK and $60.6 \%$ in the US, but in both countries OW users had a significantly higher MPR than OD users (Table 1). Overall persistence rates at 12 months were similar in both countries, averaging 207 days in UK and 196 days in US. In both the UK and US, persistence with treatment was significantly better for OW users when compared to OD users, with a higher proportion of OW users persisting with treatment for 12 months (Table 1).

Table 1 Comparison of compliance and persistence

\begin{tabular}{lllllllll}
\hline & $\begin{array}{l}\text { OW N } \\
\text { cohort }\end{array}$ & $\begin{array}{l}\text { OD N } \\
\text { Cohort }\end{array}$ & $\begin{array}{l}\text { OW } \\
\text { MPR }\end{array}$ & $\begin{array}{l}\text { OD } \\
\text { MPR }\end{array}$ & $\begin{array}{l}\text { OW Days } \\
\text { Persist }\end{array}$ & $\begin{array}{l}\text { OD Days } \\
\text { Persist }\end{array}$ & $\begin{array}{l}\text { OW \% } \\
\text { Persist }\end{array}$ & $\begin{array}{l}\text { OD \% } \\
\text { Persist }\end{array}$ \\
\hline UK & 5,102 & 860 & $70.3^{*}$ & 51.7 & $228^{*}$ & 186 & 43.6 & 33.3 \\
US & 731 & 2,010 & $56.3^{*}$ & 37.7 & $227^{*}$ & 185 & 44.2 & 31.7 \\
\hline
\end{tabular}

* OW vs OD All p <0.0001

Conclusions: Findings were similar in the US and UK. Both studies highlighted that compliance and persistence with bisphosphonates can be improved substantially with a less frequent dosing regimen. A further decrease in dosing frequency may improve compliance and persistence.

\section{P288. BONE STRENGTH AND BONE MINERAL CONTENT IN RATS WITH EXPERIMENTALLY INDUCED LIVER CIRRHOSIS}

V. Ferencz ${ }^{1}$, B. Kari ${ }^{2}$, Sz. Meszaros ${ }^{1}$, J. Gaal ${ }^{3}$, F. Szalay ${ }^{1}$, A. Mester ${ }^{4}$, Cs. Horváth ${ }^{1}$; ${ }^{1}$ Semmelweis Univ., I.st. Dept. of Medicine, ${ }^{2}$ Dept. of Diagnostic Radiology and Oncotherapy, ${ }^{3}$ Technical Univ. Budapest, Polymer Engineering and Textile Technology

Our aim was to investigate the relationship between bone strength and bone mineral content at different skeletal sites in experimentally induced osteoporosis in rats. Bone mineral content (BMC) was determined by single photon absorptiometry (SPA, Gamma NK-364) at femoral metaphysis and a value well proportioned to BMC was measured by microSEGAMS at trochanter on soft radiographs (Siemens Mammomat) digitalised by Osiris in cirrhotic rats. Maximal loading capacity (Fmax) was determined by biomechanical tests (Zwick-020) at femoral metaphysis. The results were compared to healthy controls. In healthy controls strong correaltion was found both between cortical BMC and metaphyseal Fmax $(r=0,901, p<0,001)$, and between cancellous (trochanteric) bone mass and metaphyseal Fmax $(r=0,906, p=0,002)$. Srong correlation was detected between cortical and cancellous bone mass $(r=0,809, p=0,015)$ at femora of healthy rats. However, in cirrhotic animals only cortical BMC showed relationship to metaphyseal Fmax $(r=0,769$, $\mathrm{p}=0,003)$ and no correlation was found between cortical and trochanteric bone mass. Our results showed, that maximal loading capacity at femoral metaphysis could be described by both cortical and cancellous bone mass in healthy controls. In cirrhotic animals, however, cortical and trabecular bone loss is not parallel. Therefore, metaphyseal loading capacity can not be determined by trabecular bone density. Moreover, our results confirm that digitised radiographs could be used to determine a value correlating to bone mineral content.

\section{P289. EFFECT OF CIGARETTE SMOKING ON BONE QUALITY PARAM-} ETERS

Sz. Mészáros ${ }^{1}$, E. Csupor $^{2}$, E. Tóth ${ }^{3}$, V. Ferencz ${ }^{1}$, M. Deli ${ }^{1}$, É. Hosszú $^{4}$, K. Bors ${ }^{5}$, Cs. Horváth ${ }^{1}$; ${ }^{1} 1$ st Department of Internal Medicine, Faculty of Medicine, Semmelweis University, Budapest, Hungary ${ }^{2}$ The Health Service, Budavar Local Authorities,
Budapest, Hungary ${ }^{3}$ Department of Reumatology, Ferenc Flor County Hospital, Kerepestarcsa, Hungary ${ }^{4}$ 2nd Department of Pediatrics, Semmelweis University, Budapest, Hungary ${ }^{5}$ Regional Osteoporosis Centre Ferencvaros, Budapest, Hungary

Smoking is a risk factor for osteoporosis. Our previous study showed lower bone density among smokers in a group of postmenopausal women. After this finding we would like to investigate how smoking could influence bone quality. Forty-five (range: 25-72 ys) smoker women were compared with 45 non-smoker women adjusted for age and antropometric parameters. Quantitative ultrasound (QUS) method was used to determine the speed of ultrasound (SOS) and the ultrasound attenuation (BUA) transmitting the left heel (Achilles In Sight). Dual photon absorptiometry method was applied to investigate the bone mineral density of lumbar spine and left femoral neck (Prodigy, GE Lunar) and single photon absorptiometry was used to determine the bone mineral content of radius at the non dominant side (NK-364, Gamma). No difference was found between smokers and non-smokers among the premenopausal group, however, postmenopausal smoker women had slightly lower SOS and BUA values than their non-smoker mates. Postmenopausal smoker women suffering from bone fracture had significantly lower SOS than postmenopausal non-smoker women (1508.9 vs $1525.3 \mathrm{~m} / \mathrm{s}$, respectively), despite their bone density did not differ from each other. Similar differences was not find in the premenopausal group. Our data augmet the knowledge about the injurious effect of smoking. The increasing risk for bone fracture among smokers could be explained by the decreasing of bone mass, which was previously described, and the decreasing of bone elasticity.

P290. COMPARISON OF OSTEODENSITOMETRIC AND QUANTITATIVE ULTRASOUND PARAMETERS AMONG PATIENTS SUFFERING FROM PSEUDO-PSEUDOHYPOPARATHYROIDISM, SEUDOHYPOPARATHYROIDISM TYPE I/A AND PRIMARY HYPERPARATHYROIDISM

E. Csupor ${ }^{1,2}$, I. Szabolcs ${ }^{3}$, V. Ferencz ${ }^{2}$, G. Iván ${ }^{3}$, M. Goth ${ }^{3}$, L. Kovács $^{3}$, G. Győri ${ }^{2}$, E. Tóth ${ }^{4}$, Sz. Mészáros ${ }^{2}$, É. Hosszú ${ }^{5}$, Cs. Horváth ${ }^{2}$; ${ }^{1}$ The Health Service of Budavári Local Authorites, Budapest, Hungary, ${ }^{2} 1$ st Department of Internal Medicine, Semmelweis University Medical School, Budapest, Hungary, ${ }^{3}$ National Medical Center, Department of Internal Medicine, Budapest, Hungary, ${ }^{4}$ Department of Rheumatology, Ferenc Flor County Hospital, Kerepestarcsa, Hungary, ${ }^{5}$ 2nd Department of Paediatrics, Semmelweis University Medical School, Budapest, Hungary

Parathyroid hormone (PTH) excretion is increased both in primary hyperparathyroidism (pHPT) and in pseudohypoparathyroidism type Ia (PHP Ia). Pseudo-pseudohypoparathyroidism (P-PHP) is considered to be the normocalcemic form of pseudohypoparathyroidism type Ia. Our aim was to measure bone mineral content and to estimate bone quality, furthermore, to investigate whether PTH influence these parameters in the above mentioned disorders. Ten patients who were suffering from pseudo-pseudohypoparathyroidism (P-PHP, age: 41.6 \pm 5.4 ys) were compared to 10 patients with primary hyperparathyroidism (pHPT) and to 10 healthy subjects. Moreover, 9 pseudohypoparathyroidism type I/a patients (PHP, age: $34.2 \pm 5.43$ ys) were compared to 9 primary hyperparathyroidism and 9 healthy controls adjusted by age and gender. We assessed the incidence of bone fractures and we measured the bone mass (on axial bones by dual photon absorptiometry, on forearm by single photon absorptiometry), and quantitative ultrasound (QUS) measurement was performed both on the calcaneus (BUA, SOS) and on the proximal phalanxes (AdSOS). Moreover, we measured some laboratory parameters of calcium metabolism. No difference was found between PHP I/a and pHPT in bone mass. The lowest value was revealed on the radius. QUS parameters: pathologically low AdSOS on the phalanxes was found in PHP I/a and it was lower than in pHPT patients, BUA showed similar tendency at the calcaneus. Bone mass did not significantly differ between P-PHP and 
healthy controls, however, it was decreased at the forearm. Pathological AdSOS was measured in P-PHP, which was lower than in pHPT. SOS at the calcaneus was lower in P-PHP than in $\mathrm{pHPT}$, however it was not considered pathological. Disease specific laboratory results were found and the radiological examinations confirmed the diagnosis. Bone fractures developed in three cases among PHP I/a, in two cases among P-PHP, while no fractures occurred in pHPT and healthy group. The bone loss among PHP I/a patients is considered to be the result of PTH excess on bone tissue. The decline in bone quality and architecture may play a role in developing bone fractures.

\section{P291. SUSTAINED REDUCTION OF BONE TURNOVER MARKERS THROUGHOUT THE TIME-INTERVAL BETWEEN WEEKLY DOSES OF ALENDRONATE OR RISEDRONATE}

H. Bone ${ }^{1}$, P. Miller ${ }^{2}$, E. Rosenberg ${ }^{3}$, E. Chen ${ }^{3}$, A. de Papp ${ }^{3}$; ${ }^{1}$ Michigan Bone \& Mineral Clinic, PC, Detroit, Michigan, ${ }^{2}$ Colorado Center for Bone Research, Lakewood, CO, ${ }^{3}$ Merck \& Co., Inc., West Point, PA, USA

Objectives: For bisphosphonates, the duration of the timeinterval between doses has become longer. This post-hoc analysis of the randomized, double-blind FACT trial determined whether reductions in bone turnover markers (BTMs) that occur in response to treatment with once-weekly $(\mathrm{OW})$ alendronate or risedronate are consistently maintained between doses.

Methods: OW alendronate $70 \mathrm{mg}$ or OW risedronate $35 \mathrm{mg}$ was taken on any chosen day of the week by postmenopausal women with low bone density. BTMs (urine N-telopeptide of type I collagen [NTX] and serum C-telopeptide of type I collagen [CTX], bone-specific alkaline phosphatase [BSAP], and N-terminal propeptide of type 1 procollagen [P1NP]) were measured at baseline and 3 months. Mean percent reduction from baseline in BTMs was compared among 878 per protocol patients, who were grouped according to how many days prior to their 3-month BTM measurement they had taken their last bisphosphonate dose.

Results: Increasing time (measured in days) since last alendronate dose did not significantly influence the reduction from baseline in NTX $(P=0.122)$. Increasing time since last risedronate dose resulted in a trend toward decreased NTX response $(\mathrm{P}=0.041)$. However, no significant changes in response over time since the last dose of either drug were detected for CTX, BSAP, or P1NP.

Conclusions: Both OW bisphosphonates (OW alendronate 70 $\mathrm{mg}$ and $\mathrm{OW}$ risedronate $35 \mathrm{mg}$ ) provided a sustained reduction of bone resorption and bone formation markers throughout the dosing interval, similar to the effect achieved with daily dosing.

\section{P292. BASELINE CHARACTERISTICS OF NON-RESPONDERS TO BIS- PHOSPHONATE THERAPY IN THE FOSAMAX ACTONEL COMPARISON TRIAL (FACT)}

K. Saag ${ }^{1}$, A. Sebba ${ }^{2}$, S. Bonnick ${ }^{3}$, E. Rosenberg ${ }^{4}$, E. Chen ${ }^{4}$, A.E. de Papp ${ }^{4}$; ${ }^{1}$ University of Alabama, Birmingham, AL, UK, ${ }^{2}$ Arthritis Associates, Palm Harbor, FL, USA, ${ }^{3}$ Clinical Research Center of North Texas, Denton, TX, USA, ${ }^{4}$ Merck \& Co., Inc., West Point, PA, USA

Objective: Bone mineral density (BMD) non-response to therapy is of potential concern to clinicians. The objective of this analysis was to compare apparent BMD non-responders between treatment groups, in a 12-month extension of the randomized, double-blind FACT study, and to identify the baseline characteristics of BMD non-responders compared to responders.

Methods: 833 postmenopausal women with low BMD entered the FACT extension and continued their same doubleblind treatment (once-weekly [OW] alendronate [ALN] $70 \mathrm{mg}$ or OW risedronate [RIS] $35 \mathrm{mg}$ ). For purposes of this analysis, "non-responders" were defined as those individuals with measured BMD losses of $>0 \%$ in at least 2 of the 4 BMD sites (lumbar spine, femoral neck, hip trochanter, or total hip) after 24 months.

Results: Non-responders were more frequent among RIS patients $(37.6 \%)$ than ALN patients $(14.5 \%), \quad \mathrm{P}<0.001$. Non-responders were younger at the onset of menopause (nonresponders 45.3 years, responders 47 years, $\mathrm{P}=0.013$ ) and more likely to have a family history of osteoporosis (non-responders $50 \%$, responders $38.4 \%, \quad \mathrm{P}=0.002)$. Non-responders had numerically higher baseline BMD at all sites and numerically lower baseline levels of all bone turnover markers examined (NTX, CTX, BSAP, and P1NP). Non-responders and responders did not differ in baseline fracture history or baseline use of tobacco, alcohol, or caffeine.

Conclusions: More patients receiving OW RIS than OW ALN for 24 months were apparent BMD non-responders to therapy. Although small differences were seen in baseline characteristics between non-responders and responders, identifying nonresponders prior to initiation of bisphosphonate therapy is likely to be difficult in the clinic.

\section{P293. METHODOLOGY FOR DETERMINATION OF FRACTIONAL CALCIUM ABSORPTION}

R. Sherrell ${ }^{1}$, P. Field ${ }^{1}$, S. Shapses ${ }^{1}$, R. Robson ${ }^{2}$, M. Liu ${ }^{3}$, W Bayne $^{3}$, E. Woolf ${ }^{3}$, L. Wehren ${ }^{3}$, A. Santora ${ }^{3}{ }^{1}{ }^{1}$ Rutgers University, New Brunswick, NJ, USA, ${ }^{2}$ Christchurch Clinical Trials, Christchurch, New Zealand, ${ }^{3}$ Merck Research Laboratories, Rahway, NJ, USA

Background: Vitamin D is important for skeletal metabolism because of its essential role in optimizing calcium absorption from the gut. The effectiveness of orally-administered vitamin D can, therefore, be determined by measuring fractional calcium absorption (FCA).

Material and Methods: FCA was measured by dual tracer method at baseline and after 4 weeks of treatment in 56 participants in a randomized, controlled clinical trial comparing a once-weekly tablet containing alendronate $70 \mathrm{mg}$ and vitamin $\mathrm{D}_{3} 2800$ IU to matching placebo. Dietary calcium intake was stabilized prior to and during treatment to a target of 1100 to $1300 \mathrm{mg} / \mathrm{day}$, by use of 3-day food diaries, dietary counseling, and calcium supplements. The IV and oral calcium formulations were enriched with stable isotopic forms of calcium, ${ }^{42} \mathrm{Ca}$ and ${ }^{43} \mathrm{Ca}$, respectively, and were administered simultaneously. Isotope doses were chosen to achieve approximately $10 \%$ enrichment of urine over the natural abundance. Ten hour urine samples were collected; calcium was precipitated as the oxalate salt and ashed to remove organics. Isotopic composition of calcium was determined by mass spectrometry. ${ }^{44} \mathrm{Ca}$ was used as the internal standard, and baseline (pre-dose) ${ }^{42} \mathrm{Ca}$ and ${ }^{43} \mathrm{Ca}$ used in determinations of FCA were the actual natural abundances of these isotopes in urines collected at baseline. Power/sample size calculations were based on $7.7 \%$ variability in FCA estimates previously reported by Ensrud et al. (Ann Intern Med 2000), and on an estimated correlation between baseline and on-treatment values of 0.50 . A sample size of 25 patients per group had $90 \%$ power to detect a $7.2 \%$ between-group difference and $80 \%$ power to detect a $6.3 \%$ change from baseline.

Results: Blinded interim variability estimates confirmed these assumptions, with an observed variance of $6.3 \%$, based on the first 24 patients enrolled. Observed variability at study end was $6.5 \%$.

Conclusions: The dual tracer method of estimating FCA using mass spectrometry was shown to be an excellent means for assessing treatment effects. This method avoids the use of radioisotopes, sparing patient exposure and eliminating the need to handle radioactive substances. The observed variability of FCA supports the power/sample size calculations for the study. 
P294. 24 MONTHS OF OW ALENDRONATE 70 MG PRODUCES GREATER GAINS IN BMD AND GREATER REDUCTION IN MARKERS OF BONE TURNOVER THAN OW RISEDRONATE 35 MG WITH SIMILAR TOLERABILITY

S.L. Bonnick ${ }^{1}$, K. Saag ${ }^{2}$, D.P. Kiel ${ }^{3}$, M. McClung ${ }^{4}$, D. Thompson $^{5}$, E. Rosenberg ${ }^{5}$, E. Chen ${ }^{5}$, A.E. de Papp ${ }^{5}$; ${ }^{1}$ CRC of North Texas, Denton TX, USA, ${ }^{2}$ University of Alabama, Birmingham, AL, USA, ${ }^{3}$ Beth Israel Deaconess Medical Center, Boston, MA, USA, ${ }^{4}$ Oregon Osteoporosis Research Center, Portland, OR, USA, ${ }^{5}$ Merck \& Co., Inc., West Point, PA, USA

Objectives: We performed a 12-month extension to a one year, randomized, double-blind trial of once weekly $(\mathrm{OW})$ alendronate (ALN) $70 \mathrm{mg}$ and OW risedronate (RIS) $35 \mathrm{mg}$ in postmenopausal women with low bone mineral density (BMD) (T-score $\leq-2.0$ at hip trochanter, total hip, femoral neck, or spine).

Methods: 833 subjects were consented into the 12-month extension and maintained their original blinded treatment allo-
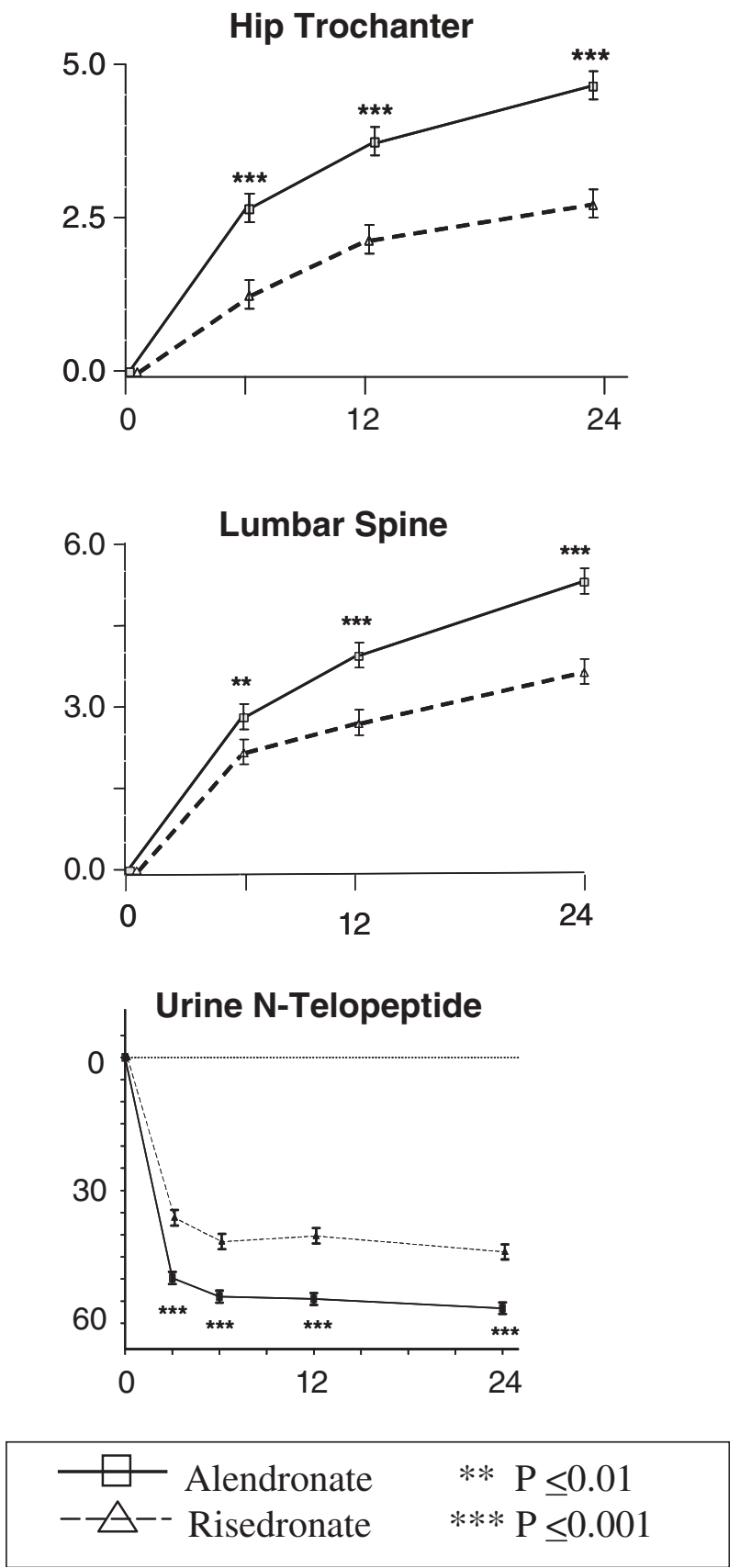

cations. The primary endpoint of the extension was the percent change from baseline in the hip trochanter BMD at 24 months. Secondary endpoints included total hip, femoral neck and lumbar spine BMD, percentage of patients achieving prespecified gains or losses in BMD, markers of bone turnover (U-NTX/Cr, serum CTX, BSAP, and P1NP), and overall and upper GI tolerability.

Results: ALN produced significantly greater increases in BMD at all sites at 24 months than did RIS $(\mathrm{p}<0.001)$. The mean percent change from baseline in BMD at 24 months at the hip trochanter (primary endpoint) was $4.6 \%$ with ALN and $2.5 \%$ with RIS (treatment difference of $2.1 \%, \mathrm{p}<0.001$ ). More ALN than RIS patients had measured BMD increases of $\geq 3 \%$ $(\mathrm{P}<0.001)$, and fewer ALN than RIS patients had BMD declines of $\geq 3 \%$ ( $P \leq 0.01)$. Significantly greater reduction in all markers of bone turnover was seen with ALN compared to RIS at 24 months $(p<0.001)$. There was no significant difference in the percent of patients reporting overall or any upper GI AE $(24.8 \%$ ALN, $22.9 \%$ RIS, $p=0.567$ ) or discontinuation due to an upper GI AE $(1.7 \%$ ALN, $1.2 \%$ RIS, $p=0.577)$.

Conclusions: After two years of follow-up, OW ALN $70 \mathrm{mg}$ produced significantly greater gains in BMD and greater reductions in markers of bone turnover than OW RIS $35 \mathrm{mg}$, with no differences in overall or upper GI tolerability.

P295. HAS THE PREVENTION FOR GLUCOCORTICOID INDUCED OSTEOPOROSIS (GIOP) BEEN ACCOMPLISHED IN PATIENTS WITH RHEUMATOID ARTHRITIS (RA)?

S. Falcão, R. Barros, J.M.B. Pimentão, J.C. Branco; Rheumatic Unity, Hospital Egas Moniz, SA, Lisbon, Portugal

Glucocorticoid therapy is the most common secondary cause of bone loss; the severity of the bone loss in glucocorticoid-treated patients may vary from an approximately 3 to 20 percent decrease in bone density over 1 to 2 years. American College of Rheumatology (ACR) recommendations for the prevention and treatment of GIOP suggest that patients initiating glucocorticoid therapy at a dose equivalent to $5 \mathrm{mg}$ or more of prednisone/day for 3 months or more should: modify risk factors for osteoporosis; start weight-bearing physical exercise; initiate intake of calcium and vitamin $\mathrm{D}$; and bisphosphonate therapy. Objective: To evaluate in a population receiving long-term glucocorticoid therapy which are followed-up according to international recommendations for prevention of GIOP.

Method: In a group of 61 consecutive patients with RA, followed-up in our rheumatic unity, we gathered data referring to sex, age, glucocorticoid therapy, intake of calcium and vitamin D, bisphosphonate therapy, bone mineral density (BMD) and the existence of osteoporotic fractures.

Results: In our data, $78.6 \%$ were medicated with prednisone $5 \mathrm{mg} /$ day for more than 3 months; $81.3 \%$ were doing calcium and vitamin D; $51.2 \%$ were medicated with bisphosphonate therapy. Regarding BMD values: $62.9 \%$ not normal (T-score below -1 ) $5.7 \%$ normal; for $31.4 \%$ of our cases we didn't get any data.

Conclusion: Based in the fact that we had a high percentage of cases with BMD abnormalities we consider exceptionally low the number of patients medicated with bisphosphonate therapy and therefore the prevention and treatment for GIOP has been compromised

P296. PROGLUMETACIN (PROTAXON/- FORTE): PORTRAIT OF AN EFFECTIVE NSAID WITH EXCELLENT SAFETY

K.K. Förster; Medical Dept.-Clin. Research, Opfermann Arzneimittel GmbH, Wiehl, Germany

Introduction: Classical non-steroidal anti-inflammatory drugs (NSAIDs) are used in osteoarthritis (OA) to reduce pain and flares as well as to ameliorate joint function. But NSAIDs are often accompanied by (gastrointestinal) adverse events (AEs) because of their mechanism of action: cyclooxygenase (COX) inhibition. Even potentially severe complications like bleeding and perforation may occur. 
Problem solution: This led to different strategies to prevent such AEs. One strategy was the combination of NSAID therapy with antacids, histamine-2-antagonists or proton pump inhibitors. Another strategy of topical interest led to preferential/ selective COX-2-inhibitors [Vane et al. 1996].

Amelioration of safety: The development of proglumetacin (Protaxon forte, Protaxil), a molecular combination of indomethacin with proglumide, a gastrin and cholecystokinin antagonist [Rovati et al. 1983], was also aimed to reduce gastrointestinal AEs. A molecular variation of the effective indomethacin led to a NSAID with a good safety profile: Proglumetacin (Münzenberg et al. 1980). Several NSAID controlled gastroscopical studies show proglumetacińs excellent safety as well as a Meloxicam controlled study (Bonn et al. 1999).

Broad experience: Under daily practice experiences, efficacy and safety were proven in a multi-centre study with more than 4600 rheumatic patients. $96 \%$ of patients assessed the therapy as ,good" or ,,successfull“". Proglumetacin was tolerated well also by patients of high risk (higher age, patients with gastral, liver, or renal diseases). Rate of primarily non severe AEs was $11,5 \%$ (Reichelt et al. 1986).

Aspects of use in sports medicine: In a randomized, placebocontrolled, double-blind multi-centre study 114 patients with acute epicondylitis of the elbow were treated with taping and proglumetacin or placebo. After one/two weeks the success rate with proglumetacin (plus taping) was $74 / 86 \%$, while the placebo (plus taping) success was 49/72,5\%. This success was shown again in those patients with acute epicondylitis, induced by sports activities like tennis and golf (Förster et al. 1997).

Conclusion: Concerning treatment of OA, other rheumatic diseases, sports induced injuries like tennis elbow, and prophylaxis of ectopic ossification after total hip replacement, clinical studies show a potent efficacy of proglumetacin, combined with an excellent (gastrointestinal) safety. Therefore, proglumetacin is an effective and very safe alternative NSAIDs.

\section{P297. EFFECT OF ONCE-WEEKLY ALENDRONATE 70 MG AND VITAMIN D3 2800 IU ON FRACTIONAL CALCIUM ABSORPTION IN POSTMENO- PAUSAL OSTEOPOROTIC WOMEN: A 4-WEEK, DOUBLE-BLIND, RAN- DOMIZED, CONTROLLED MULTI-CENTER CLINICAL TRIAL}

R. Robson ${ }_{5}^{1}$, D. Kendler ${ }^{2}$, M. Handel ${ }^{3}$, S. Shapses ${ }^{4}$, Z. Yang ${ }_{5}^{5}$, T. Wilson ${ }^{5}$, M. Liu ${ }^{5}$ A. Mantz, L. Wehren ${ }^{5}$, A. Santora'; ${ }^{1}$ Christchurch Clinical Trials, Christchurch, New Zealand, ${ }^{2}$ Osteoporosis Research Centre, Vancouver, British Columbia, Canada, ${ }^{3}$ Prince of Wales Hospital, Randwick, New South Wales, Australia, ${ }^{4}$ Rutgers University, New Brunswick, New Jersey, USA, ${ }^{5}$ Merck Research Laboratories, Rahway, New Jersey, USA

Objectives: Although vitamin D is essential for efficient calcium absorption and is important for management of osteoporosis, recent studies have shown that a large minority of patients do not regularly take supplements; similarly, the majority of patients treated for osteoporosis have serum vitamin $\mathrm{D}$ levels $<30$ $\mathrm{ng} / \mathrm{mL}(75 \mathrm{nmol} / \mathrm{L})$. This study was designed to examine the effect of a once-weekly tablet containing $70 \mathrm{mg}$ of alendronate and vitamin D3 2800 IU (ALN D2800) on fractional calcium absorption (FCA).

Material and Methods: After a 4-week placebo run-in and dietary stabilization period, 56 postmenopausal women with osteoporosis (T-score $\leq-2$ at lumbar spine, total hip, femoral neck, or trochanter) were randomized to receive drug or matching placebo for 4 weeks. FCA was measured at baseline and at Week 4 from urines collected for 10 hours following IV and oral administration of stable isotopes of calcium (dual tracer method), using mass spectrometry. Change in FCA was analyzed using an ANCOVA model with treatment as a model factor and baseline FCA and 25-hydroxyvitamin D as covariates.

Results: FCA absorption was significantly increased from baseline after 4 weeks of treatment with once-weekly ALN D2800 compared to placebo ( $p<0.001$ ). The absolute LS mean increase with ALN D2800 was 7.0\% from the baseline of

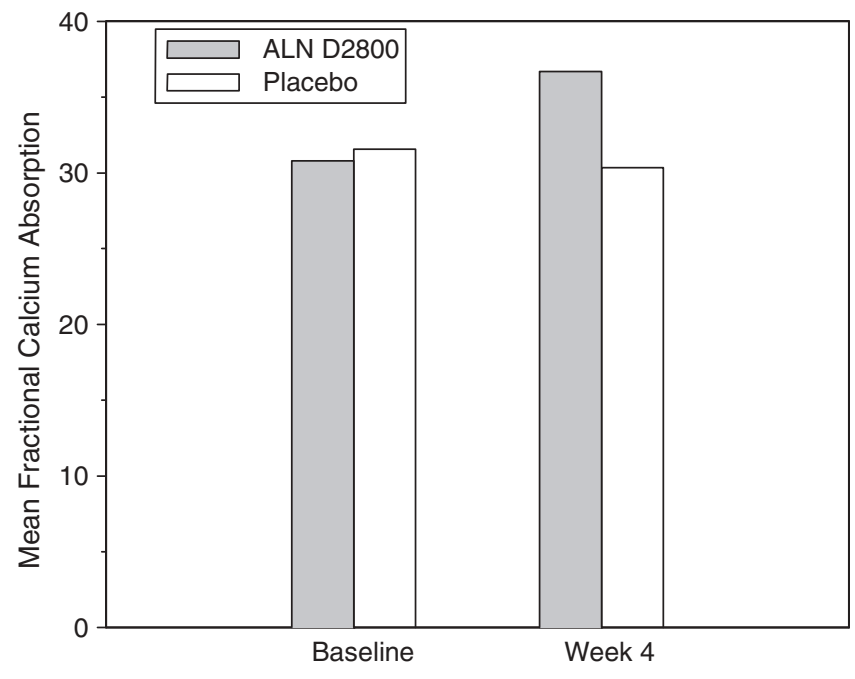

$30.8 \%$ (22.7\% relative increase); FCA decreased $1.6 \%$ in the placebo group from a baseline value of $31.6 \%$ (5.1\% relative decrease).

Conclusions: Four weeks of treatment with once-weekly alendronate $70 \mathrm{mg}$ vitamin D3 2800 IU was associated with approximately a $23 \%$ relative increase in FCA. The absolute increase of $7.0 \%$ is likely to be clinically meaningful and would support the increase in BMD observed during the first year of treatment with alendronate. This medication containing alendronate and vitamin D3 improves FCA, thereby helping patients absorb more calcium.

\section{P298. FACTORS THAT WERE ASSOCIATED WITH NEED FOR CARERS AMONGST PATIENTS WITH FRAGILITY FRACTURES}

T.C. Lau ${ }^{1}$ on behalf of the NHG Osteoporosis Workgroup ${ }^{2}$; ${ }^{1}$ Department of Rheumatology Allergy and Immunology, Tan Tock Seng Hospital, Singapore, ${ }^{2}$ National Healthcare Group, Singapore

Aim: This paper examined the factors which were associated with need for carers amongst patients with fragility hip or spine fractures

Method: Patients with previous vertebra or hip fragility fracture were invited to join the program unless they were bed ridden or have a history of oesophagitis or renal impairment. Baseline data pertaining to demographics, mobility status, comorbidities, and need for carers were collected. Statistical analysis was done using multivariate logistic regression modeling.

Results: Out of a total of 984 participants recruited between Aug 2002 to Jan 2005, $262(26.6 \%)$ were patients with only hip fractures, $640(65.0 \%)$ with only vertebra fractures and 82 $(8.3 \%)$ with both hip and vertebra fractures. Their mean age was 73 years (45-92, SD 10.2), with a female preponderance (877 vs 106). There were $898(91.5 \%)$ Chinese, $44(4.5 \%) \mathrm{Ma}-$ lays, $32(3.3 \%)$ Indians. Majority has no formal education $(68 \%)$ or only primary education $(15.9 \%) .96 \%$ of the participants were staying at home. Only $61.6 \%$ of participants with vertebra fracture and $29.0 \%$ with hip fracture were ambulant without aid. $58 \%$ of the participants were independent with self-care, the remainder required care by maid $(20 \%)$, relative $(19 \%)$ or others $(3 \%)$. Factors associated with need for carers were increasing age, falls, reduced mobility, stroke, diabetes mellitus, renal disease and hip fracture.

Conclusion: Amongst patients with fragility fracture, only $58 \%$ were independent with self-care. Knowing the factors associated with need for carers might help to predict the health care needs of the patients. 
P299. DO A DEDICATED COORDINATOR AND AWARENESS PROGRAM IMPROVE THE DOCUMENTATION OF OSTEOPOROSIS IDENTIFICATION AND CARE IN AN ACUTE CARE FRACTURE CLINIC?

S.E. Ward ${ }^{1}$, J.J. Laughren ${ }^{1}$, E.R. Bogoch ${ }^{1}$, V. Elliot-Gibson ${ }^{1}$, D.E. Beaton ${ }^{1}$; ${ }^{1}$ Mobility Program, St. Michael's Hospital, University of Toronto, Toronto, Ontario, Canada

Objective: To evaluate osteoporosis (OP) documentation and follow-up in fragility fracture patients following implementation of an Osteoporosis Exemplary Care Program (OECP).

Material and Methods: A case control study using chart audit was performed on 166 patients with fragility fractures. Patients in the post-OECP group (cases, $\mathrm{n}=83$ ) were selected from a registry of patients identified as fragility fractures of the hip or wrist during the first four months of the program. Age, sex and fracture type matched controls $(n=83)$ were obtained through medical records and had their fracture prior to program implementation. Group characteristics were compared using $\chi^{2}$ tests.

Results: Following OECP implementation, chart documentation of OP risk/diagnosis improved from $50.6 \%(42 / 83)$ to $72.3 \%(60 / 83)$ (unadjusted OR $2.55 ; 95 \%$ CI $1.3-4.9)$. Documentation of referral for OP follow-up increased from $19.3 \%$ (16/83) to $42.2 \%(35 / 83)$ (unadjusted OR 3.05 ; $95 \%$ CI $1.5-6.1$ ) and documentation of appropriate OP treatment increased from $25.3 \%(21 / 83)$ to $67.5 \%(56 / 83)$ (unadjusted OR $6.12 ; 95 \%$ CI 3.1-12.0). However, because all patients in the database were contacted by the coordinator and $97 \%$ received appropriate osteoporosis education and referral or treatment, we are confident that the documented treatment rate was lower than the actual care.

Conclusions: Being in the OECP program significantly improved the odds of having a diagnosis of OP in the chart, or having care for the probable OP. Documentation of OP treatment or referral is less common on charts then in our registry. However the chart remains the avenue of communication and critical for continuity of care, therefore we have changed our protocol to include the coordinator's notes in the medical chart.

\section{P300. BONE MINERAL DENSITY IN PATIENTS WITH DIFFERENT} ETIOLOGY OF SUBCLINICAL HYPERTHYROIDISM

L. Rozhinskaya ${ }^{1}$, G.A. Melnichenco ${ }^{1}$, Z.E. Belaya ${ }^{1}$, T.M. Ale$\mathrm{kseeva}^{2}$, O.K. Dorofeeva ${ }^{2}{ }^{1}$ National Research Center for Endocrinology, Moscow, Russia, ${ }^{2}$ Endocrinology Unit, Moscow, Russia

Aims: To compare the effect of subclinical hyperthyroidism with different causes on bone mineral density (BMD) in postmenopausal women.

Methods: 89 postmenopausal women (age 64 (59-68) years (Median: lower-upper quartiles), were divided into four groups: group (1) $(\mathrm{n}=21$; TSH $0.198(0.026-0.241))$ - women with toxic multinodular goiter (TMG) without history of clinical hyperthyroidism; group (2) $(\mathrm{n}=25$; SH $0.060(0.015-0.192))$ - patients with levothyroxine suppressive therapy $(125(125-150) \mu \mathrm{g}$ /day during 3 (1.5-6) years); group (3) $(\mathrm{n}=21$; TSH $0.083(0.014-0.287))-$ patients with Graves' disease (GD) with antithyroid treatment (3 (2-7) years), group (4) - a control group $(n=22$; TSH 1.862 $(0.941-2.22)$ ). All patients had free thyroxine (fT4) and tri-iodothyronine (fT3) levels within the reference range. There were no differences between groups in age $(p=0.11)$, body mass index (BMI) $(\mathrm{p}=0.69)$. BMD was measured by dual energy $x$-ray absorptiometry (DXA) (Lunar) in the lumbar spine (L1-L4), femoral neck, total hip and radius. TSH-receptor antibodies were detected with the h-TBII assay (TRAK Dynotest BRAMS) in order to differentiate GD from TMG.

Results: ANOVA and the multiple comparison test (The Fisher LSD test) were used to compare the difference in BMD between the four groups. BMD was $10.6 \%$ lower $(p=0.023)$ in L1-L4 and $9.5 \%$ lower $(p=0.017)$ in femoral neck in group (1);
BMD was $11.6 \%$ lower $(\mathrm{p}=0.006)$ in group (1) and $10.3 \%$ lower $(\mathrm{p}=0.013)$ in group (3) in total hip; and also BMD was $14.5 \%$ lower $(p=0,003)$ in group $(1)$ and $17.3 \%$ lower $(p=0.0004)$ in group (3) in total radius as compared to the control group. There were no differences in BMD between groups (2) and (4).

Conclusions: The etiology of subclinical hyperthyroidism exerts influence on BMD in postmenopausal women. Postmenopausal women with endogenous subclinical hyperthyroidism have significantly lower BMD in all regions of the skeleton versus postmenopausal women without thyroid disease. On the other hand, exogenous subclinical thyrotoxicosis has no effect on BMD. The lowest BMD has been found in the distal and proximal cortical bone.

P301. EFFICACY OF ALFACALCIDOL TREATMENT FOR SECONDARY HYPERPARATHYROIDISM IN PATIENTS WITH CHRONIC RENAL FAILURE BEFORE DIALYSIS

L. Rozhinskaya ${ }^{1}$, A.N. Il'ina ${ }^{1}$, A.V. Borisov ${ }^{2} ;{ }^{1}$ National Research Center for Endocrinology, Moscow, Russia, ${ }^{2}$ Center for Extracorporal therapy "Fesfarm", Moscow, Russia

Aims: Secondary hyperparathyroidism (SHP) is observed in some patients with end-stage renal disease (ESRD) as the glomerular filtration rate (GFR) falls below 60 \{mili $1 /$ minute and is a severe complication of uraemia. To determine the effective doses of Alfacalcidol for SHP in patients with ESRD before dialysis.

Methods: 57 patients (42 women and 15 men) with creatinine level from 150 to 450 unit/1 and GFR from 58 to 13 \{mili\}1/minute were examined. $55 \%$ from these patients were with diabetes mellitus. The basic level of parathyroid hormone (PTH) was from 43 to 1032 \{pico $\}$ g/ $\{$ mili $\} 1.38$ patients $(66 \%)$ suffered from SHP with mean PTH level 417 \{pico $\} \mathrm{g} /\{$ mili $\} 1$. Alfacalcidol was prescribed individually in doses from 0,5 to 3,75 micro g per week in patients with PTH level more than $200\{$ pico $\} \mathrm{g} /\{$ mili\}1. Patients were treated during the one year and it was possible to correct dose during the treatment. PTH, calcium, phosphorous, creatinine, bone resorption marker CTx levels were measured in serum several times during the treatment. Bone mineral density (BMD) was measured by dual energy X-ray absorptiometry (DXA) (Lunar) in the lumbar spine, femoral neck, total hip and radius.

Results: PTH was $50 \%$ lower from basic level in patients who received more than 1,75 micro\}g Alfacalcidol per week. When Alfacalcidol was prescribed in dose less than 1,75 \{micro $\}$ g PTH level decreased insignificantly or even increased. The treatment was ineffective in $9(24 \%)$ patients. The creatinine level increases in average at $21 \%$ among patients with Alfacalcidol treatment however it was independent of Alfacalcidole dose. There were not found any significant differences in calcium, phosphorous levels and BMD in all regions of the skeleton. The CTx level did not significantly change during the treatment and was 1,766 \{plusmin 0,877 (mean \{plusmin\}standart deviation) before and 1,724 plusmin 0,735 after treatment.

Conclusions: Thus Alfacalcidol is effective for SHP treatment in patients with ESRD before dialysis if it is received in dose more than 1,75 micro\}g per week. Treatment with Alfacalcidol leads to BMD stabilization. There were not found any differences in CTx level apparently due to progress of main disease and relatively low doses of Alfacalcidol.

P302. SUB-CLINICAL, UNDETECTED HYPERPARATHYROIDISM AND HYPOTHYROIDISM IN INDIVIDUALS CONSUMING HIGH FLUORIDE INTAKE IN JEDDAH-SAUDI ARABIA-IMPLICATIONS TO HEALTH AND ECONOMY

S. Bahijri ${ }^{1}$, A. Al-Fares ${ }^{1}$, T. Al-Khateeb ${ }^{2}$, A. Mufti ${ }^{3} ;{ }^{1}$ Faculty of Medicine, Jeddah, Saudi Arabia, ${ }^{2}$ Faculty of Dentistry, Jeddah, Saudi Arabia, ${ }^{3}$ Faculty of Earth Sciences, King Abdul Aziz University, Jeddah, Saudi Arabia

Objectives: To investigate the prevalence of fluoride excessive consumption in children and adults living in the city of 
Jeddah, and its effects on thyroid and parathyroid function reflected on plasma levels of FT3, FT4, TSH, PTH, calcium and phosphate.

Methods: 145 healthy subjects of different ages (7-50years) were recruited. Dietary intake of fluoride was recorded using food frequency questionaire. Subjects were examined dentally for caries and fluorosis, and clinically for any symptoms of disease. They were also questioned on their dental hygiene practice. Fasting blood samples were obtained for the estimation of FT3, FT4, TSH, PTH, calcium, phosphate and albumin. Samples of drinking water and fasting urine were also obtained for the estimation of fluoride. Fluoride was estimated in all types of drinking water and beverages consumed by subjects. Fluoride intake from all sources was calculated for all subjects, and they were divided into age groups and subgroups according to their total fluoride intake.

Results: Fluorosis was detected in 15 adults. High intake of fluoride was detected in a large percentage of subjects, some of which exhibited depressed thyroid activity and hyperparathyroidism accompanied by decreased mean plasma phosphate and calcium in some groups. The effects were reversed when fluoride intake was decreased.

Conclusion: Excessive fluoride intake is common in our population, associated with undetected hyperparathyroidism and hypothyroidism. Long-term effects could include increased incidence of metabolic bone diseases including osteoporosis, which would increase the economic burden on individuals and on the state. Use of fluoride supplements and highly fluoridated dental products on regular bases and excessive tea drinking must be discouraged.

\section{P303. ASSOCIATION BETWEEN OSTEOPOROSIS AND CARDIOVASCU- LAR RISK FACTORS IN POSTMENOPAUSAL WOMEN}

A. Tancredi ${ }^{1}$, A.-S. Polet ${ }^{1}$, B. Bosio-Le Goux ${ }^{2}$, B. AugendreFerrante ${ }^{2}$, J.-Y. Reginster ${ }^{1}$; ${ }^{1}$ World Health Organisation Collaborating Centre for Public Health Aspects of Rheumatic Diseases and Department of Public Health, Epidemiology and Health Economics, University of Liège, Liège, Belgium, ${ }^{2}$ Lilly France, Suresnes, France

Objective: Osteoporosis and cardiovascular diseases may occur in women after menopause. Previous investigations support the existence of a link between these two chronic morbidities. To identify whether french postmenopausal women requiring an anti-osteoporotic treatment, in real life setting, are at increased risk for cardiovascular disorders.

Material and method: This observational survey was conducted in France by 8000 General practitioners, 900 Gynecologists and 500 Rheumatologists. They were asked to recruit four consecutive postmenopausal women in whom they intended to initiate, continue or modify an anti-osteoporotic treatment. A questionnaire, including the most frequent risk factors for osteoporosis and cardiovascular disease, was designed to standardize the procedure of data collection. A global score of osteoporosis risk was developed by summing all reported risk factors. Univariate and multivariate statistical analyses were performed to investigate the link between the two chronic conditions.

Results: The mean age of 19,337 French postmenopausal women who participated in the survey was $66.0( \pm 8.2)$ years, ranging from 42 to 97 years. Previous family history of osteoporosis $(47.8 \%)$, poor physical activity $(48.5 \%)$, hypertension $(39.7 \%)$ and hypercholesterolemia $(37.8 \%)$ accounted for the most prevalent risk factors in the French postmenopausal women. The number of cardiovascular risk factors increased as a function of the number of osteoporosis risk factors $(p<0.001)$. Canonical correlation analysis indicated that postmenopausal women who reported a low physical activity and a fracture after the age of 45 years are also inclined to present with hypertension, hypercholesterolemia and obesity even after correction for age. The prevalence of fracture after the age of 45 years increases with the number of cardiovascular risk factors $(\mathrm{p}<0.001)$.
Conclusion: Our results suggest a modest association between osteoporosis and cardiovascular disease in postmenopausal women. They highlight the importance of concomitant investigation and, if possible, management, of both diseases.

\section{P304. POSTMENOPAUSAL OSTEOPOROSIS PREVALENCE IN A SOUTH} BULGARIAN CITY

P. Solakov, S. Kuzmanova; University Clinic of Rheumatology, Plovdiv, Bulgaria

Objective: Ideally, BMD would screened by dual energy x-ray absorptiometry (DXA) of lumbar spine, proximal femur, total body, forearm and tibia. The presence of severe osteophytes of the spine and the hips are strongly related to increase bone mass. Spinal degenerative disease often elevates DXA measured bone mass in postmenopausal women. Peripheral bone mass measurement by quantitative ultrasound densitometry (QUD) is inaffected by osteoarthritis and is largely used in everyday practice. To find a cheaper alternative for diagnosis of osteoporosis, we determined the combination of central BMD by DXA with QUD of forearm and tibia.

The objective of this study is to evaluate the prevalence of osteoporosis at lumbar spine (LS) by DXA and simultaneously at radius and tibia by QUD in postmenopausal women 50 years of age or more in South Bulgarian city.

Material and method: In our epidemiological study the bone density was measured in women dwelling in a Plovdiv health area comprising more than 300000 people. The measurement was performed by DXA Hologic QDR1000 of the LS and by ultrasonic Sunlight Omnisence for radius and tibia respectively. Total 445 subjects have been recruited, 417 have enrolled. 28 patients refused complete participation from various reasons. All investigated women completed a simple questionnaire "Risk factors for osteoporosis".

Results: The results from the questionnaire for group of women with T-score $>-2.5$ are shown on the table 1 .

\begin{tabular}{|c|c|c|c|}
\hline $\begin{array}{l}\text { Risk factors / } \\
\text { Age groups (n) }\end{array}$ & $\begin{array}{l}50-59 y \\
153\end{array}$ & $\begin{array}{l}60-69 y \\
151\end{array}$ & $\begin{array}{l}\text { age }>70 y \\
113\end{array}$ \\
\hline Decrease physical activity & $41(26.8)$ & $62(41.0)$ & $79(69.9 \%)$ \\
\hline Cigarette smoking & $14(9.1)$ & $11(7.3)$ & $7(6.2)$ \\
\hline Alcohol abuse & $25(16.3)$ & $22(14.6)$ & $14(12.4 \%)$ \\
\hline Cofein abuse & $51(33.8)$ & $49(32.4)$ & $27(23.9)$ \\
\hline Diseases relevant for OP & $21(13.7)$ & $28(18.5)$ & $29(25.7)$ \\
\hline $\begin{array}{l}\text { Family history of OP } \\
\text { and fracture }\end{array}$ & $19(12.4)$ & $21(13.9)$ & $17(15.0)$ \\
\hline Early menopause & $10(6.53)$ & $13(8.6)$ & $11(9.7)$ \\
\hline Low BMI & $16(10.4)$ & $20(13.4)$ & $19(16.8)$ \\
\hline Loss of height & $22(14.3)$ & $38(25.2)$ & $66(58.4)$ \\
\hline
\end{tabular}

The simple linear regression showed that central DXA correlate significantly with "decrease physical activity" and in women after 60 years old correlate with "loss of height", and "low BMI". No significant relationships were found between total questionnaire score and any BMD measurement.

The frequency of women with central DXA and peripheral QUD with T-score $>-2.5$ are shown on the table 2:

\begin{tabular}{llll}
\hline \multirow{2}{*}{ BMD } & \multicolumn{2}{l}{ T-score $>-2.5$} & \\
\cline { 2 - 4 } & L spine DXA & QUD Radius* & QUD Tibia* \\
\hline Age groups (n) & $(\mathrm{n} / \%)$ & $(\mathrm{n} / \%)$ & $(\mathrm{n} / \%)$ \\
$50-59$ years $(153)$ & $19(12.4)$ & $15(9.8)$ & $14(9.1)$ \\
$60-69$ years $(151)$ & $38(25.2)$ & $44(29.1)$ & $23(15.2)$ \\
Age $>70(113)$ & $24(21.2)$ & $32(28.3)$ & $23(20.3)$ \\
Total $(417)$ & $81(19.4)$ & $91(21.8)$ & $60(14.4)$ \\
\hline
\end{tabular}

When comparing the ultrasound with DXA, the determined ultrasound indexes of radius were in $94.2 \%$ in good agreement with central DXA results. According the tibia ultrasound measurements only $85.1 \%$ of patients with DXA T-score $>-2.5$ were 
with osteoporosis. The lumbar spine DXA identified 81 women as osteoporotic, QUD of radius - 91 and QUD of tibia - 60 .

QUD of radius and tibia predict central BMD in postmenopausal women. Total questionnaire score did not improve predictability of osteoporosis.

Percentage of postmenopausal women with low BMD is nearly the same like other countries in South Europe $(1,2,3)$.

Conclusion: Approximately $20 \%$ of postmenopausal women aged 55-86 years from a South Bulgarian city have central DXA and peripheral QUD T-score $>-2.5$. The measurement of lumbar spine DXA, QUD of radius and tibia simultaneously increases the number of women identified as osteoporotic $(p<0.05)$. Results at the radius and tibia ultrasound measurements in elderly women show good agreement with central DXA values. The differences reflect some limited utility of central DXA in older population due to the high prevalence of spinal degenerative disease.

\section{References}

1. Pilipovic N.Z. et al. Bone mineral density and fracture in urban women in Belgrade. ARD European congress of Rheumatology 2003; Abstract SAT0090:312.

2. Manisali $\mathrm{M}$ et al. Bone mineral density reference values in the normal female and male population of Izmir, Turkey. Eur Radiol 2003;13:157--62.

3. Paker $\mathrm{N}$ et al. Bone mineral density of the spine, proximal femur, total body and forearm in healthy turkish women. ARD European congress of Rheumatology 2004; Abs SAT0213:448

P305. EARLY MENOPAUSE AS A RISK FACTOR FOR OSTEOPOROSIS FIRST MACEDONIAN OFFICIAL DENSITOMETRY DATA

N. Nasteska, M. Panajotovik, D. Grujoska-Veta; PRAMA MEDICA Skopje and Orthopedic clinic - Skopje, Skopje, Republic of Macedonia

Osteoporosis is one of the most common metabolic diseases of the bone tissue. The aim of this research is to demonstrate the influence of early menopause as a risk factor for osteoporosis.

Methods and materials: A group of 84 patients was observed, varying from 45 to 75 years of age. They all had early menopause occurrence (before the age of 45). Simultaneously, a group of 84 women between the age of 45 and 75 was observed, where menopause occurred after their $48^{\text {th }}$ year of age. All patients were scanned on the DEXA (Lunar DPX) apparatus, during a 6month period. They were all scanned on at least 3 bodily regions (lumbal part of the backbone L1-L4 and dual femur - both hips, while 35 of them were scanned on the forearm).

Results: Out of 84 scanned patients with early menopause, 48 were diagnosed with osteoporosis with a T-score higher than $-2,5$, 30 were diagnosed with osteopenia with a T-score of $-1,0$ to $-2,5$, while 6 patients did not have osteoporosis. Out of the control group, 23 women were diagnosed with osteoporosis, 25 with osteopenia, while 36 patients had normal densitometry results.

Discussion: Considering the consequences of osteoporosis which result in a high percentage of handicap and mortality, the necessity of a timely diagnosis with a DEXA scan occurs as a standard.

P306. SMALL HEIGHT AND SMALL BONES AS A PREDETERMINING FACTOR FOR OSTEOPOROSIS - FIRST MACEDONIAN OFFICIAL DENSITOMETRY DATA

M. Panajotovik, N. Nasteska, D. Grujoska-Veta; PRAMA MEDICA Skopje and Orthopedic clinic- Skopje, Skopje, Republic of Macedonia

The aim of this research is to demonstrate the incidence of osteoporosis at women with small height (smaller than $158 \mathrm{~cm}$ ) as a relevant predetermined factor for osteoporosis.

Methods and materials: A group of 155 women was observed, whose symptoms included back, joint and bone pains. The patients' age varied from 45 to 75 years with height smaller than $158 \mathrm{~cm}$. The control group consisted of 155 women who had the same symptoms (back, joint and bone pains) at the age of 45 to 75 , but taller than $165 \mathrm{~cm}$. They all went under the DEXA (Lunar DPX) scan on at least 3 bodily regions (L1-L4, dual femur).

Results: Out of the group of women with small body height, 60 of them were diagnosed osteopenia with a T-score varying from $-0,1$ to $-2,5 ; 81$ were diagnosed with osteoporosis with a $\mathrm{T}$ score higher than $-2,5$, while 14 patients had normal densitometry results. Out of the control group of women with a bodily height higher than $165 \mathrm{~cm}, 57$ were diagnosed with osteopenia, 35 were diagnosed with osteoporosis, while 63 had normal densitometry results. All patients were tested for the level of ionized Calcium in their blood.

Discussion: The analysis demonstrated that small height is an important predetermined factor for osteoporosis, not depending on the patient's age.

\section{P307. LONGITUDINAL STUDY OF MAGNETIC RESONANCE IMAGING AND FIXED-FLEXION RADIOGRAPHY TO ASSESS PROGRESSION OF OSTEOARTHRITIS OF THE KNEE}

O. Bruyer̀ ${ }^{1}$, M. Kothari², C. Peterfy ${ }^{2}$, S. Zaim ${ }^{2}$, D. White ${ }^{2}$, N. Burlet ${ }^{1}$, F. Richy ${ }^{1}$, D. Ethgen ${ }^{3}$, T. Montague ${ }^{3}$, C. Dabrowski ${ }^{3}$, H. Genant ${ }^{2}$, J.-Y. Reginster ${ }^{1}$; ${ }^{1}$ WHO Collaborating Center For Public Health Aspects Of Osteoarticular Disorders, University Of Liège, Liège, Belgium, ${ }^{2}$ Synarc Inc., San Francisco, California, USA, ${ }^{3}$ Glaxosmithkline, Collegeville, Pa, USA

Objective: To investigate, over one-year, the relationship between radiography and magnetic resonance imaging (MRI) findings in patients with knee osteoarthritis (OA).

Material and methods: $62 \mathrm{OA}$ patients (46 women), with mean age (SD) of 64.9 (10.3) years and mean body mass index of 28.1 (4.7) $\mathrm{kg} / \mathrm{m}^{2}$, were followed for one year. Both knees were radiographed at baseline and after one year using the fixed-flexion technique with a positioning frame. Radiographs were digitized and minimum medial and lateral femorotibial joint-space widths were measured using a validated semi-automated software. $1.5 \mathrm{~T}$ MRI of the knees was also acquired at baseline and one year. Volume and thickness of cartilage of the medial tibia, the lateral tibia and the femur were measured using a validated semiautomated software. Knees were also assessed with a multi-feature whole-organ MRI scoring system (WORMS) (Peterfy et al. Osteoarthritis and Cartilage 2004;12:177-90) at each time point. This score combined individual scores for articular cartilage, osteophytes, bone marrow abnormality, subchondral cysts and bone attrition in 14 locations. It also incorporated scores for the medial and lateral menisci, anterior and posterior cruciate ligaments, medial and lateral collateral ligaments and synovial distension.

Results: Medial femorotibial joint-space narrowing (JSN) after 1 year correlated significantly with loss of medial tibial cartilage volume $(\mathrm{r}=0.25, \mathrm{p}=0.046)$ and thickness $(\mathrm{r}=0.28$, $\mathrm{p}=0.025$ ), over the same period. The area under ROC curve for medial tibial cartilage volume change as a predictor of radiographic JSN $>0.3 \mathrm{~mm}$ was $0.71(95 \%$ CI 0.53 to 0.88$) ; \mathrm{p}=0.023$. We found also a significant correlation between progression of WORMS and radiographic medial JSN over one year $(\mathrm{r}=-0.35 ; \mathrm{p}=0.006)$. These results remained statistically significant after adjusting for age, sex and body mass index.

Conclusion: This study showed a significant association between MRI and radiographic assessment of progression of knee OA.

\section{P308. EARLY POSTMENOPAUSAL WOMEN HAVE HIGH PREVALENCE OF INADEQUATE SERUM VITAMIN D LEVELS. RESULTS OF A EURO- PEAN STUDY}

O. Bruyer̀e, O. Malaise, A. Neuprez, J.-Y. Reginster; WHO Collaborating Center for Public Health Aspect of Osteoarticular Disorders and Department of Public Health, Epidemiology and Health Economics, University of Liège, Liège, Belgium 
Objective: A high prevalence of vitamin D Inadequacy has been widely documented in elderly women. Low vitamin D level is associated with secondary hyperparathyroidism, increased bone turnover and bone loss, which increase fracture risk. The objective of this study is to assess the prevalence of inadequate serum vitamin D levels in women aged less than 70 years old.

Material and methods: Postmenopausal osteoporotic women were recruited from 9 European countries (France, Belgium, Denmark, Italy, Poland, Hungary, United Kingdom, Spain and Germany). Assessment of 25-Hydroxyvitamin D [25(OH)D] was performed with a commercial radioimmunoassay (DiaSorin) in all patients. There are no clear international agreements on what constitute a level of vitamin D inadequacy. However, recent publications suggest that the circulating level of vitamin D should be over $80 \mathrm{nmol} / \mathrm{L}$ or at least between $50 \mathrm{nmol} / \mathrm{L}$ and $80 \mathrm{nmol} / \mathrm{L}$. We used, in the present study, two cut-offs of $25(\mathrm{OH}) \mathrm{D}$ fixed according to these publications $(<80 \mathrm{nmol} / \mathrm{L}$ and $<50 \mathrm{nmol} / \mathrm{L})$.

Results: A total of 8532 women with a mean (range) age of $74.2(45-100)$ years were included in this study. The mean (SD) level of $25(\mathrm{OH}) \mathrm{D}$ was $61.0(27.2) \mathrm{nmol} / \mathrm{L}$. There was a slight, not clinically significant, correlation between the age of the patients and the level of $25(\mathrm{OH}) \mathrm{D}(\mathrm{r}=-0.10 ; \mathrm{p}<0.0001)$. In the whole study population $(\mathrm{n}=8532)$ the prevalence of vitamin $\mathrm{D}$ inadequacy was $79.6 \%$ and $32.1 \%$ when considering cut-off of 80 and $50 \mathrm{nmol} / \mathrm{L}$, respectively. In women aged less than 70 years old $(n=1631)$, the prevalence was $80.3 \%$ and $37.6 \%$, respectively. The prevalence of vitamin D inadequacy reaches $78.9 \%$ (cut-off of $80 \mathrm{nmol} / \mathrm{L}$ ) and $38.2 \%$ (cut-off of $50 \mathrm{nmol} / \mathrm{L}$ ) in patients aged less than 60 years old $(n=285)$.

Conclusion: This study confirms the high prevalence of vitamin D inadequacy in osteoporotic European postmenopausal women. However, age does not seem to substantially influence this prevalence.

\section{P309. HIGH PREVALENCE OF INADEQUATE SERUM VITAMIN D LEVELS IN OSTEOPOROTIC EUROPEAN POSTMENOPAUSAL WOMEN}

O. Bruyere, O. Malaise, A. Neuprez, J.-Y. Reginster; WHO Collaborating Center for Public Health Aspect of Osteoarticular Disorders and Department of Public Health, Epidemiology and Health Economics, University of Liège, Liège, Belgium

Objective: Inadequate vitamin D level is associated with secondary hyperparathyroidism, increased bone turnover and bone loss, which increase fracture risk. There are no clear international agreements on what constitute a level of vitamin D inadequacy. Recent publications suggest that the circulating level of vitamin D should be over $80 \mathrm{nmol} / \mathrm{L}$ or at least between $50 \mathrm{nmol} / \mathrm{L}$ and 80 $\mathrm{nmol} / \mathrm{L}$. The objective of this study is to assess the prevalence of inadequate serum vitamin D levels in postmenopausal European women.

Material and methods: Assessment of 25-Hydroxyvitamin D $[25(\mathrm{OH}) \mathrm{D}]$ was performed with a commercial radioimmunoassay (DiaSorin) in 8532 osteoporotic European postmenopausal. Europenan countries included France, Belgium, Denmark, Italy, Poland, Hungary, United Kingdom, Spain and Germany. Two cut-offs of $25(\mathrm{OH}) \mathrm{D}$ inadequacy were fixed : $<80 \mathrm{nmol} / \mathrm{L}$ and $<50 \mathrm{nmol} / \mathrm{L}$.

Results: Mean (SD) age of the patients was 74.2 (7.1) years, body mass index was $25.7(4.1) \mathrm{kg} / \mathrm{m}^{2}$. Level of $25(\mathrm{OH}) \mathrm{D}$ was $61.0(27.2) \mathrm{nmol} / \mathrm{L}$. There was a highly significant difference of vitamin D level across European countries $(p<0.0001)$. The lowest level of vitamin D was found in France [51.5 (26.1 nmol/ $\mathrm{L})]$ and the highest in Spain [85.2 (33.3) nmol/L). In the whole study population, the prevalence of $25(\mathrm{OH}) \mathrm{D}$ inadequacy was $79.6 \%$ and $32.1 \%$ when considering cut-offs of 80 and $50 \mathrm{nmol} / \mathrm{L}$, respectively. The prevalence of low $25(\mathrm{OH}) \mathrm{D}(<80 \mathrm{nmol} / \mathrm{L})$ reaches $90.4 \%$ in France and $45.8 \%$ in Spain.

Conclusion: This study confirms the high prevalence of vitamin $\mathrm{D}$ inadequacy in osteoporotic European postmenopausal women. The prevalence could be even higher in some particular countries. We believe that a greater awareness of the importance of vitamin D inadequacy is needed to address this public health problem.
P310. QUANTITATIVE CALCANEAL ULTRASONOMETRY; NORMATIVE DATA FOR THE TURKISH POPULATION AND AGE RELATED VARIATION IN STIFFNESS INDEX

B. Durmaz, S. Oncel, Y. Kirazli, S. Alper, O. Peker, M. Saridogan, Y.G. Kutsal, G. Dincer, F.Atalay, N. Eskiyurt; Turkish Osteoporosis Society, Turkey

Objectives: The aim of the present study was to establish a normative data for calcaneal stiffness index (SI) as a quantitative ultrasound measurement in healty Turkish population and to evaluate the age related changes in this value.

Material and methods: This was a cross-sectional, epidemiological study. The subjects with medical conditions affecting bone metabolism (chronic diseases or medications) were excluded. A total number of 10435 subjects were measured with Achilles devices; 8156 subjects (1389 male and 6767 female) in the age range 18-89 years from both rural and urban area were included in the study. Normative data was expressed using two statistical models mean and 95\% percentile, and regression analysis.

Results: The peak value for SI in female subjects was achieved in the 30-39 years age group. The total age related decrease was $24.26 \%$ for SI in female subjects. For SI measurements in male subjects, the peak value was obsered in the age range 18-29 years. The total age related reduction in SI values of male subjects was $17.8 \%$. There was a statistically significant reduction in male SI data between 40-49 and 50-59 years. The female SI data showed significantly decrease between the age groups $40-49$ and 50-59; 50-59 and 60-69; and 60-69 and 70-79.

In 4401 women from 40 years to 59 years of age, the mean SI was significantly higher in pre-menopausal women $(\mathrm{N}=2002)$ than in post-menopausal women $(\mathrm{N}=2399)$.

Conclusion: The curve representing the SI change according to age is best fitted by the regression analyses of cubic model.

The results of this study could be useful as a guide for comparing the data of individual studies.

P311. OPG, RANKL, RANKL/OPG RATIO, OPT IN POSTMENOPAUSAL WOMEN POPULATION

M. D'Amore ${ }^{1}$, S. D'Amore ${ }^{2}$, A. Fontana ${ }^{3}$, M. Fanelli ${ }^{4}$, G. Minenna ${ }^{1}$; DIMIMP-Sezione di Reumatologia, Università degli Studi di Bari, Bari, Italia, ${ }^{2}$ DIMIMP-Sezione di Medicina Interna, Università degli Studi di Bari, Bari, Italia, ${ }^{3}$ Unità Operativa Patologia Clinica I A.O. Policlinico Bari, Bari, Italia, 4DIMIMP-Sezione Diagnostica per Immagini, Statistica Medica Università degli Studi di Bari, Bari, Italia

The aim of this study is to evaluate the serum concentration of OPG, RANKL, RANKL/OPG ratio, Osteopontin, bone-type alcaline phosphatase (BAP), serum-N-Telopeptide of type I collagen (serum-NTX), bone mineral density (BMD) in postmenopausal women population. A cohort of 163 women in postmenopausal, not in therapy for osteoporosis, has been followed up at the osteoporosis centre of Policlinico in Bari. The population with main age of 55,5 years (range 43-64), in menopausal disorder since 2 years, has been divided into three separate groups, depending from their T-score: osteoporosis nr. 28/163; osteopenic nr. 100/163; normal nr. 35/163. Comparison of the variables in the three groups has been executed through kruskalWallis test. Correlations have been calculated using Spearman's correlation coefficient. All values have been presented using mediana with range. The comparison among the three groups is significant for RANKL $(\mathrm{Kw}=14,86, \mathrm{p}=0,0006)$, RANKL $/ \mathrm{OPG}$ ratio $(\mathrm{Kw}=13.24, \mathrm{p}=0.0013)$, NTX $(\mathrm{Kw}=11.92, \mathrm{p}=0.0026)$ and OPN $(\mathrm{Kw}=7,58, \mathrm{p}=0,022)$, but non for OPG and BAP. The correlation among considered variables has shown significant statistical variation in the group of osteopenic women: a negative correlation between OPG and RANKL ( $\mathrm{rs}=-0,242, \mathrm{p}=0,0153$ ), positive one between RANKL and OPN ( $r s=0,304, p=0,0021)$, RANKL and serum-NTX ( $\mathrm{rs}=0,213, \mathrm{p}=0,033)$, OPN and serum-NTX ( $r s=0,465, p<0,0001)$, OPN and RANKL/OPG ratio $(\mathrm{rs}=0,261, \mathrm{p}=0,0086), \mathrm{BAP}$ and serum-NTX $(\mathrm{rs}=0,510$, 
$\mathrm{p}<0,0001$ ), serum-NTX and RANKL/OPG ratio ( $\mathrm{rs}=0,202$, $\mathrm{p}=0,044)$. Our study shows an important variation of the bone metabolism markers including RANKL, OPG and OPN in postmenopausal women with a seriuos engagement of the bone turnover above all in the pre-osteoporotic phase.

\section{P312. A PROSPECTIVE STUDY OF ALGINATE SEEDED WITH MATURE ALLOGENIC HUMAN CHONDROCYTES IN THE TREATMENT OF ARTIC- ULAR CARTILAGE DEFECTS}

K.F. Almqvist ${ }^{1}$, P. Verdonk ${ }^{1}$, R. Verdonk ${ }^{1}$, K. Verstraete $^{2}$, E.M. Veys $^{3}$, G. Verbruggen ${ }^{3}$; ${ }^{1}$ Dept. of Physical Medicine and Orthopaedic Surgery, Ghent, Belgium, ${ }^{2}$ Dept. of Radiology, Ghent, Belgium, ${ }^{3}$ Dept. of Rheumatology, Ghent University Hospital, Ghent University, Ghent, Belgium

Aim of the Study: To produce tissue-engineered cartilage by human articular chondrocytes cultured in a biocompatible, biodegradable matrix for the treatment of (osteo)chondral symptomatic lesions to avoid osteoarthritis in the younger patient.

Materials and Methods: Earlier studies have shown that chondrocytes proliferate in alginate, and synthesize a cartilagelike matrix for up to 8 weeks.

Allogenic human chondrocytes were cultured in 1.0\% alginate beads for 2 weeks before implantation into cartilage defects of the knee joint. Prior to the surgical intervention a small part of the alginate beads was dissolved and the chondrocytes were tested for their phenotypical stability. The (osteo)chondral lesion of the femoral condyle in 22 patients ( 3 lateral - 19 medial) was debrided by an open technique, and covered by an autologous periosteal flap. Subsequently the defect was filled with the alginate beads. The cartilage lesion was finally injected with $0.5 \%$ fibrin gel. Parameters that were followed pre- and postoperatively, were the Lequesne index, the WOMAC, the KOOS and VAS-score, and Gd-DTPA MRI. A follow-up arthroscopy to assess the macroscopic aspect and the integration of repair tissue into surrounding native cartilage was performed at 12 months postoperatively.

Results: Clinically, major adverse reactions to the scaffold seeded with the cartilage cells, were not observed. The results (mean follow-up: 19months) of clinical examination of the involved joint as well as the functional scores improved with time. Follow-up arthroscopy at 12 months showed an intact articular surface, with good integration into the surrounding cartilage, and a practically normal indentation was felt on palpation.

Conclusion: This surgical procedure is performed in one step. Biodegradable, biocompatible scaffolds could be used in the treatment of cartilage defects, with the advantage that the artificial matrix provides an initial support to the chondrocytes, making the implant theoretically initially biomechanically superior to the model in which cells are injected as a suspension under a periosteal flap. This method will have an important place in the prevention of posttraumatic osteoarthritis.

\section{P313. LEPTIN SERUM LEVELS IN PATIENTS AFFECTED BY SERO- NEGATIVE ARTHRITIS}

G. Minenna ${ }^{1}$, S. D'Amore ${ }^{2}$, P. Maggiolini ${ }^{3}$, M. Fanelli ${ }^{4}$, M. D'Amore ${ }^{1}$; ${ }^{1}$ DIMIP-Sezione di Reumatologia, Università degli Studi di Bari, Bari, Italia, ${ }^{2}$ DIMIMP-Sezione di Medicina Interna, Università degli Studi di Bari, Bari, Italia, ${ }^{3}$ Unità Operativa patologia Clinica I A.O. Policlinico Bari, Bari, Italia, ${ }^{4}$ DIMIMP-Sezione Diagnostica per Immagini, Statistica Medica Università degli Studi di Bari, Bari, Italia

Leptin has an important role in the regulation of body weight and modulates immunity and inflammation. A positive feedback mechanism has been described between tumor necrosis factor and leptin, and it has been suggested that leptin potentiates inflammation in patients with rheumatoid arthritis. Objectives.
Aims of this study are to evaluate leptin serum concentration in patients affected by seronegative arthritis and to compare the results with the ones obtained by healthy population. Methods. 40 patients have been recruited affected by seronegative arthritis $(\mathrm{M}: \mathrm{F}=14: 26)$ with an average age of 54,4 years (range 18-80), average disease duration of 3,5 years (range $1-6$ ). Leptin concentration has been measured by ELISA method both in patients then in healthy population of 80 subjects equally divided between male and female and with same average age. The difference between the two considered groups has been analyzed using Student's test.

Results. Leptin medium concentration has been $10,701 \mathrm{ng} / \mathrm{ml}$ with SD 9,718; while 5,358 ng/ml with SD 2,286 in healthy population. The difference between the two considered groups is significant $\mathrm{t}=4,661, \mathrm{p}<0,0001$.

Conclusion. The increase in leptin production during inflammation suggests that leptin is part of the cytokine cascade which governs the inflammatory-immune response and the host defense mechanism. However, both pro and anti-inflammatory effects have been described for leptin according to the experimental model investigated. New studies with other variables will be indispensable for explaining the obtained result.

\section{P314. VALIDITY AND RELIABILITY OF DXA FOR ASSESSING FAT MASS REGIONAL DISTRIBUTION}

S. Di Gregorio ${ }^{1}$, L. Del Rio ${ }^{1}$, L. Ibañez ${ }^{2}$, C. Sole ${ }^{1}$, A. Ferrer ${ }^{2}$, E. Bonel $^{1}$, J.Rosales ${ }^{1}$; ${ }^{1}$ CETIR, Centre Mèdic, Barcelona, Spain, ${ }^{2}$ Hospital San Juan de Dios, Barcelona, Spain

Dual-energy X-ray absorptiometry (DXA) is mainly used to measure bone mineral density and body composition. Recent improvements in software have made it possible to automatically determine regional fat mass distribution. Hyperandrogenism is associated with an excess of fat mass, especially in the abdominal region. We evaluated the validity and reliability of DXA for the assessment of regional fat mass distribution in teenagers with hyperandrogenism, and the ability to detect changes in follow-up measurements.

Patients/Methods: 36 teenagers with hyperandrogenism (mean age: $15 \pm 2.5$ years) had DXA total body assessments at treatment baseline and at least six months later at time of final treatment. They were classified according to different treatments: without (G0); with oral contraceptive and/or metformine, flutamide (G1) and with metformine and/or flutamide only (G2) Anthropometric parameters were measured (weight; height, waist circumference-WC-; hip circumference-HC-). The total and regional body composition was measured on a GE-Lunar Prodigy, using 8.1 software version. This version measures regional fat mass in waist and hips regions, to determine the fat distribution in the abdomen (android-AF-) and around the hips (gynoid-GF-), expressed by percentage. In 11 subjects visceral fat was also measured by MRI.

Results: The DXA AF showed a significant correlation with visceral fat measured by MRI $(\mathrm{r}=: 0.989 ; \mathrm{p}<0.0001)$. We also found a correlation between the WC and AF $(r=0.667$; $\mathrm{p}<0.0001)$ and between the HC and GF $(r=0.56 ; \mathrm{p}<0.001)$.

The G0 group showed a positive correlation between the length of treatment and both weight change $(r=0.650 ; p=0.03)$ and total mass $(\mathrm{kg})(\mathrm{r}=0.882 ; \mathrm{p}=0.02)$, but there was no correlation between length of treatment and fat and lean mass distribution.

In the G1 group we didn't find any significant correlations.

The G2 group showed a positive correlation between treatment period and both total fat $-\%-(r=0.554 ; p=0.04)$ and $G F$ $(r=0.589 ; p=0.03)$, but there was no correlation between treatment period and WC or waist/hip ratio.

Conclusion: This study shows that the Prodigy DXA is a valid, economical, and rapid method to evaluate the regional fat distribution with better sensitivity than anthropometric parameters to evaluate the changes in the follow up. 
P315. EFFECT OF TWO-YEAR DISCONTINUATION AFTER FIVE YEARS ALENDRONATE TREATMENT IN PATIENTS WITH POSTMENOPAUSAL OSTEOPOROSIS AND IMPAIRED FASTING GLUCOSE

M. Cokolic ${ }^{1}$, R. Hren ${ }^{2}$; ${ }^{1}$ Department of Endocrinology and Diabetology, Internal Clinic, Teaching Hospital, Maribor, Slovenia, ${ }^{2}$ Institute of Mathematics, Physics, and Mechanics, University of Ljubljana, Ljubljana, Slovenia

Background and Aims: In our previous reports, we have shown that postmenopausal osteoporosis can be effectively and safely treated in patients with impaired fasting glucose (IFG; serum levels of fasting glucose $>6.1$ and $<6.9 \mathrm{mmol} / \mathrm{l}$ ) for up to five years. In this study, we evaluated the remaining effects of a 5year treatment with alendronate in 24-month follow-up after withdrawal of alendronate treatment.

Material and Methods: Eleven women with postmenopausal osteoporosis (T-score below $-2.5 \mathrm{SD}$ ) and IFG were enrolled in a seven-year prospective study. At the enrollment, patients were 55 to 73 years old (mean: 65 years) and 5 to 19 years (mean: 12,6 years) after the menopause. They were treated with alendronate $(10 \mathrm{mg} / \mathrm{d}$ in Years $1-4$ and $70 \mathrm{mg} / \mathrm{w}$ during Year 5) in combination with $500-\mathrm{mg} / \mathrm{d}$ elemental calcium; in Years 6-7 alendronate treatment was discontinued. During the seven-year follow-up, the BMD in the lumbar spine (L1-L4) and left hip was measured in all patients using dual energy X-ray densitometry (Hologic QDR $2000+)$. The serum levels of glucose, HbAlc, Ca, alkaline phosphatase (ALP) and creatinine were measured every 6 months. All patients were treated for IFG with diabetic diet only.

Results: In Years 1-5, BMD increased on average by $11.1 \%$ in the lumbar spine (L1-L4) and by $6.4 \%$ in the left hip. In Years 67 , BMD increased in the lumbar spine by additional $1.1 \%$ (range $0.714-0.864$ ), but decreased on hip by $4 \%$ (range $0.592-0.781$ ). Levels of Ca, ALP and creatinine were within normal limits, while serum levels of fasting glucose and the average level of HbAlc showed no statistically significant changes during the entire period of 7-year follow-up; also, no clinical side effects were observed.

Conclusions: Results of our study indicate that residual effects of alendronate treatment are seen in the lumbar spine region, but not in the hip region. Moreover, more than $60 \%$ of improvement observed during the 5-year treatment with alendronate was lost in just 2 years after cessation of the treatment. As the risk for hip fractures markedly increases with the decline in BMD of the hip, alendronate treatment should be continued to maintain its benefits.

\section{P316. PULSED SIGNAL THERAPY (PST) FOR THE TREATMENT OF OSTEOPOROSIS - A MULTICENTER INVESTIGATION}

R. Markoll, D. Da Silva Ferreira, H. Martin; Institute for Innovative Medicine, Infinomed, Munich, Germany

Objectives: The concept of bone function regulation by bone's intrinsic electromechanical properties may be traced back to Fukada and Yasuda (1957) who demonstrated that bone has piezoelectric properties. This was subsequently verified by many others and alternatives, including electromagnetic fields, put forth to resolve some bone-related disorders that were incurable by conventional intervention of orthopaedic surgery. It was noted that as a result of bone's biopotentials, repair and remodeling processes that normally occur in response to mechanical loading, could be elicited by an electrical stimulus - an exogenously applied electrical current, including PEMF.

Material and Methods: Pulsed electromagnetic signals, quasirectangular in waveform and varying energy parameters are emitted from a medical device consisting of a magnetic field generator, connected to a ring-shaped coil, or other applicator, by means of an electronic interface producing a pulsed ELF DC magnetic field of $0.28 \mathrm{~W}$, with field intensity $5-15 \mathrm{G}$ and frequency $1-30 \mathrm{~Hz}$. The pulse-modulated signals are non-invasive, but transduced through the affected tissue by direct induction. Biophysically, PST passively induces a pulsating fluid flow and ionic displacement in the targeted area and surrounding connective tissue, generating a type of "fluid shear stress" over cell mem- branes. This piezoelectric effect ("streaming potential") results in interaction of the pulsating signals with cell membranes and activation of various interconnected signaling pathways. PST thus emulates the innate physiological and mechanical stresses evoked and required in bone formation (mechanotransduction).

Results: Over 25 clinical and 5 in vitro studies have confirmed the positive effects of PST in the treatment of musculoskeletal conditions, namely osteoarthritis. In a pilot study, increasing trends in vBMD and an associated decrease in pain was observed in postmenopausal women with osteoporosis (OP), resulting in initiation of a multicenter, post-marketing surveillance investigation. Both DEXA measurements and bone-specific markers for resorption (beta-crosslaps) and formation (alkaline phosphatase) are employed to assess the positive effects of PST on BMD and decreased fracture risk.

Conclusions: Currently, 17 medical centers in central Europe are actively participating in this investigation. A meta-analysis conducted with data from over 50 patients will assess the effects of PST on BMD, in decreasing pain and in reducing fracture risk.

\section{P317. A NEW MUTATION OF PHEX GENE IN A PATIENT WITH HYPERPHOSPHATURIA AND HYPERCALCIURIA}

A. Falchetti, S. Carbonell Sala, A. Gozzini, D. Strigoli, F. Franceschelli, G. Leoncini, E. Croppi, L. Masi, A. Tanini, M.L. Brandi; Department of Internal Medicine, University of Florence, Florence, Italy.

The most common form of familial hyposphatemic rickets (FHR), a group of disorders with similar clinical and biochemical features [hypophosphatemia, hyperphosphaturia, normal levels of $1,25(\mathrm{OH}) 2 \mathrm{D} 3$ and PTH, skeletal deformities, short stature, osteomalacia, dental abscesses bone pain], is represented by the dominant X-linked hyposphatemic rickets (XLH). Individuals with FHR phenotype and a negative familial history in $60-80 \%$ of cases are carriers of mutations in PHEX gene, on chromosome Xp22.2-p22.1. The mice phenotypical analogue of the human XLH is represented by Hyp strand, in which a 3' deletion of Phex removes its $\mathrm{COOH}$-terminal domain. The clinical consequences of PHEX inactivating mutations indicate that its encoded product, an endopeptidase member M13Zn-metallopeptidases family, expressed at the skeletal level by osteoblasts, osteocytes, and odontoblasts, is involved in phosphate regulation and mineral homeostasis. PHEX inactivating mutations widespread along the gene, cause XLH: exons 3-4-11-12-14-15-17-20-22 represent the regions with the higher rate of mutation; such mutations could enable the accumulation of phosphaturic factors and/or mineralization inhibitors. A 26 years old male patient (height $176 \mathrm{~cm}$, weight $65 \mathrm{~kg}$ ) referred to our Centre exhibiting a clear hyperphosphaturia ( $>2000 \mathrm{mg} / 24 \mathrm{~h}$ ), hypophosphatemia, hypercalciuria $(>600 \mathrm{mg} / 24 \mathrm{~h})$, hypocalcemia $(£ 8,1 \mathrm{mg} / \mathrm{die})$, PTH circulating levels at the upper values of the normal range and normal values of $25(\mathrm{OH}) \mathrm{D}$; other symptoms were: deep asthenia, muscle pain and spasms, abundant diuresis $(12,51 \mathrm{t} / \mathrm{die})$. After obtaining the signed informed consent we performed a blood sampling from which genomic DNA has been prepared to analysed PHEX gene. The 22 exons and the intron-exon boundaries of PHEX gene have been investigated by a PCR/Sequencing protocol (ABI-Prism 3100). It has been determined the presence of a hemizygous missense mutation of PHEX gene in codon 401 (CCT/CTT) causing a Pro/Leu substitution in the extracellular domain closely a cysteine residue highly conserved in exon 11 . Nearly future functional studies will be helpful to characterize the molecular mechanisms underlying this mutation.

P318. MUTATIONAL ANALYSIS OF P62/SEQUESTOSOME GENE (p62/ SQSTM1) AND CONSTRUCTION OF A CLINICAL DATABASE IN A MULTICENTRIC ITALIAN SERIES OF PATIENTS AFFECTED BY PAGET'S DISEASE OF BONE (PDB)

A. Falchetti ${ }^{1}$, F. Marini ${ }^{1}$, L. Masi ${ }^{1}$, M. Di Stefano ${ }^{2}$, G. Isaia ${ }^{2}$, G. B. Rini ${ }^{3}$, M. Matucci Cerinic ${ }^{1}$, M. Ulivieri ${ }^{4}$, S. Giannini ${ }^{5}$, L. Sartori ${ }^{5}$, V. Braga ${ }^{6}$, S. Adami ${ }^{6}$, L. Di Matteo ${ }^{7}$, O. Di Munno ${ }^{8}$, 
S. Ortolani ${ }^{9}$, M. L. Brandi ${ }^{1} ;{ }^{1}$ Department of Internal Medicine, University of Florence, Florence, Italy, ${ }^{2}$ Department of Internal Medicine, University of Turin, Turin, Italy, ${ }^{3}$ Department of Internal Medicine, University of Palermo, Palermo, Italy, ${ }^{4}$ Servizio di Radiologia, Ospedale Maggiore di Milano IRCSS, Milan, Italy, ${ }^{5}$ Department of Medical and Surgical Sciences, University of Padova, Padova, Italy, ${ }^{6}$ Rheumatology Unit, University of Verona, Valeggio S/M, Verona, Italy, ${ }^{7}$ Presidio Ospedaliero di Pescara Reparto di Reumatologia, Pescara, Italy, ${ }^{8}$ Rheumatology Unit, Department of Internal Medicine, University of Pisa, Pisa, Italy, ${ }^{9}$ Centre for Metabolic Bone Diseases, Istituto Auxologico Italiano IRCCS, Milan, Italy

We previously reported mutations of exons 7 and 8 of SQSTM1 gene in 62 sporadic Italian PDB patients, including P392L and 2 different mutations at exon 8, consisting of M404V and G425R substitutions, respectively. In this Multicentric Italian study, including PDB cases from Northern, Central and Southern regions of Italy, we have been performing DNA analysis from 298 sporadic (148 M - 150 F) and 11 familial [99 individuals (41 M $58 \mathrm{~F})$ of which 26 clinically affected $(15 \mathrm{M}$ and $11 \mathrm{~F}] \mathrm{PDB}$ cases. Five percent of sporadic PDB resulted to harbor mutations of p62/SQSTM1 gene, while in 10 out 11 Italian PDB kindreds mutations of p62/SQSTM1 gene have been detected $(91 \%$ mutants). Consequently, we identified 34 individuals referred as asymptomatic gene mutant carriers (age range 24-50). We detected the following different mutations at UBA domain of SQSTM1 gene: D335E (7.7\% of mutations of sporadic cases), A381V (10\% of familial cases), Y383Stop ( $7.7 \%$ of mutations of sporadic and $10 \%$ of familial cases), P387L (7.7\% of mutations of sporadic cases), $1205+1 \mathrm{G} / \mathrm{A}$ (IVS7 +1$)(10 \%$ of familial cases), P392L (53\% of mutations of sporadic and $10 \%$ of familial cases), InsT Glu396Stop (10\% of familial cases), S397A (7.7\% of mutations of sporadic cases), M404V (15\% of mutations of sporadic cases and $20 \%$ of familial cases), G425R (20\% of familial cases), and G425E (7.7\% of mutations of sporadic cases). Interestingly, four unrelated families, two originating from confining districts in Central Italy and two originating from nearby Potenza (Southern Italy), shared common mutations, M404V and G425R, respectively, suggesting a possible founder effect. In order to search for a possible genotype/phenotype correlation and to improve our knowledge on PDB in Italy we are building a minimal database for the collection of homogenous clinical data in our Country according to a common clinical protocol. Data on geographical origin, cohort of birth, lifestyle [also including environmental data (rural or urban environment)], age at diagnosis, monostotic/polyostotic involvement, skeletal site of localization, $x$-rays and/or bone scintigraphy assessment, serum levels of Alkaline Phosphatase pre- and post-therapy, type of therapy (including dosage, number of cycles), previous viral diseases, presence of pet or other animal (to be specified), other received therapies (past/ongoing), and other diseases have been collecting. Obviously, such database may be furtherly improved through a direct interaction within the participating Centres. Ongoing studies provide: haplotype analysis of $5 \mathrm{q} 35$ loci in different families sharing the same p62/SQSTM1 mutation and haplotype analysis of $5 \mathrm{q} 35$ loci in different families not sharing the same p62/SQSTM1 mutation but with a common geographical origin. Future perspectives will consist of: a) common clinical follow-up of the identified asymptomatic gene carriers; b) genotype/phenotype evaluation (according to the database under construction); c) increasing of the number of participating centres representing all the Italian regions; and d) adoption of a common diagnostic-clinical protocol for the newly referred patients.

\section{P319. VITAMIN D IN OSTEOPOROSIS: ATTITUDES AND BELIEFS} OF PATIENTS AND PHYSICIANS

H. Resch ${ }^{1}$, L.E. Wehren ${ }^{2}$, S.S. Sen ${ }^{3}$; ${ }^{1}$ Saint Vincent Hospital, Vienna, Austria, ${ }^{2}$ Medical Communications, Merck \& Co., Whitehouse Station, NJ, USA, ${ }^{3}$ Outcomes Research, Merck \& Co., Whitehouse Station, NJ, USA
Objectives: Although osteoporosis treatment guidelines include recommendations for calcium and vitamin D intake, routine use of adequate supplementation of vitamin D is low. This study surveyed physicians and their patients to examine their knowledge and usage of vitamin D and calcium.

Material and Methods: Approximately 50 physicians in the United Kingdom, Mexico, and Austria (as a special situation in which calcium and vitamin D are provided free of charge to patients being treated for osteoporosis) were randomly surveyed, as were the first 10 patients with osteoporosis from each of their practices. Physicians were asked to rate the importance of vitamin $\mathrm{D}$, calcium and exercise in osteoporosis management on a scale of 1 to $10(1=$ not important at all, $10=$ extremely important $)$ and to estimate use of calcium and vitamin D supplements by their patients. Patients were asked about their own intake of vitamin D and calcium.

Results: Altogether 151 physicians completed the telephone survey and 910 of their own patients (350 in Austria, 212 in UK and 348 in Mexico) with osteoporosis completed the telephone interviews. Approximately $86 \%, 28 \%$, and $46 \%$ of physicians rated the importance of vitamin D and calcium as being 9 or 10 in Austria, UK, and Mexico, respectively. Around 34\% of patients in Mexico and UK reported taking vitamin D supplements while $90 \%$ of patients in Austria were doing so. However, only $20 \%$ of patients in Austria reported that they took the supplements daily.

Conclusions: Despite the recognition by both physicians and patients that vitamin D is important for bone health, only a low proportion of osteoporosis patients regularly take supplements containing vitamin D. This is the case even when vitamin D and calcium supplements are provided free with osteoporosis drug prescriptions.

\section{P320. COMPARISON BONE DENSITY OF OPERATIVE AND NON-OPER- ATIVE ARMY PERSONNEL}

I. Mokhtari ${ }^{1}$, M. Kamalifard ${ }^{2}$, K. Azma ${ }^{3}$, F. Rezai Moghadam ${ }^{3}$; ${ }^{1}$ Sport Medicine Research Center, Azad University, Tehran, Iran, ${ }^{2}$ Occupational and Health Research Center, Tehran, Iran, ${ }^{3} \mathrm{PMR}$ Research Center, Army Medical University, Tehran, Iran

Aim: the aim of this study was to assese values of lifestyle factors including physical activity, job, calciuemintake, and smoking in 355 army male personnel.

Methods: from feb-june 2004,355 male (age between 3555 years) in two equal separate group; operative(active field soldiers) and non -operative (army office staff) were evaluated with dual X-ray and laser (DXL) calscan for messurement of calcaneus BMD. life style factors including smoking, calcium intake and physical activity were evaluated by the investigators through a specifically designed questionaire.training programs for operative personel include: 1) marching-at least 30 minute per week 2) walking-at least 30 minutes per day 3) army special field operation-at least 4 times per year. operatives were compared with nonoperative personnel that did not perform the regular active field training maneuvers.

Result: linear regression revealed that BMD is significant associative with job $(\mathrm{p}<.001)$, age $(\mathrm{p}<.001)$, smoking $(\mathrm{p}=.007)$, calcium intake $(\mathrm{p}=.016)$, and years of physical activity $(\mathrm{p}<.001)$ in addition, dual- energy X-ray absorptiometry (DEXA) scans of lumbar spine and femors were performed for 35 participant for sample correlation that the result was same as for DXL ( $p>0.05)$.

Conclusion: this study suggest that years of regular physical activity and mechanical loading were the strongest predictors of calcaneus BMD.

\section{P321. PRESCRIPTION VITAMIN D USE AMONG PATIENTS tAKING ANTI-RESORPTIVE AGENTS IN CANADA}

D.A. Hanley ${ }^{1}$, Q. Zhang ${ }^{2}$, M.C. Meilleur ${ }^{3}$, P. Mavros ${ }^{4}$, S.S. Sen ${ }^{5}$; ${ }^{1}$ University of Calgary, Calgary, Alberta, Canada, ${ }^{2}$ Outcomes Research, Merck \& Co., Whitehouse Station, NJ, USA, ${ }^{3}$ Health Economics, Merck \& C0., Kirkland Quebec, Canada, ${ }^{4}$ Outcomes 
Research, Merck \& Co., Whitehouse Station, NJ, USA, ${ }^{5}$ Outcomes Research, Merck \& Co., Whitehouse Station, NJ, USA

Objective: Although vitamin D is recommended as a basic part of osteoporosis management, recent studies suggest many patients with osteoporosis are not taking vitamin D. This study evaluated the rate of use of prescription vitamin $\mathrm{D}$ among patients who were taking anti-resorptive agents (ARAs) for their osteoporosis in Quebec, Canada.

Methods: We examined reimbursed pharmacy claims in a random patient sample from the public insurance plan Régie d'Assurance Médicament du Québec who were 65 or older with at least one prescription $(\mathrm{Rx})$ for alendronate, risedronate or raloxifene between January 1st1996 and December 31, 2004. Pattern of use of prescription vitamin D and ARAs was assessed by reviewing pharmacy claims during the 1-year observation period following the patient's first (index) Rx for ARA between January 1st, 1996 and December 31, 2003 (index period). A vitamin $\mathrm{D}$ and ARA possession ratio was computed as ratio of total number of days of prescription vitamin D supply to total number of days of ARAs supply during the observation period.

Results: A total of 50,225 patients were reviewed of whom $90.5 \%$ were female. Approximately $28 \%, 28 \%, 22 \%, 14 \%$ and $8 \%$ of patients were $65-69$ years, $70-74$ years, $75-79$ years, $80-84$ years and 85 years or over, respectively. Only $38.25 \%$ of these patients had any use of prescription vitamin D during the observation period. Higher age groups were associated with higher proportion of prescription vitamin D use, with $32 \%, 36 \%$, $40 \%, 45 \%$, and $47 \%$ of patients in the $65-69$ years, $70-74$ years, $75-79$ years, $80-84$ years and 85 years or older age groups respectively. The vitamin $\mathrm{D}$ and ARA possession ratio was 0.55 $( \pm 2.9)$ indicating that patient-days taking prescription vitamin $\mathrm{D}$ were only about half of the days taking ARAs. Similar observations were made for a sub-set of patients who did not change ARAs from their index ARA Rx during the observation period.

Conclusion: Prior studies have shown high prevalence of inadequate vitamin D nutrition in Canada, and a need for vitamin D supplementation. However, even with a diagnosis of osteoporosis requiring the use of ARAs, a majority of patients are not taking vitamin D.

\section{P322. REDUCING HIP FRACTURES IN AN AT-RISK, ELDERLY POPU- LATION}

T. Masud ${ }^{1}$, P. Geusens ${ }^{2}$, I. Barton ${ }^{3}$, M. McClung ${ }^{4}$; ${ }^{1}$ Dept of Musculoskeletal Gerontology University of Derby, UK, ${ }^{2}$ Department of Rheumatology, University Hospital, Maastricht, Netherlands and Limburg University Centre, Diepenbeek, Belgium, ${ }^{3}$ Procter \& Gamble Pharmaceuticals, UK, ${ }^{4}$ Oregon Osteoporosis Center and Providence Medical Center, Portland, USA

Objective: The HIP study (hip intervention program) (1) showed that risedronate statistically reduced the risk of hip fracture compared to placebo in the ITT (intention to treat) population (placebo incidence $=3.9 \%$; risedronate incidence $=2.8 \% ; \mathrm{RR}=0.7 ; 95 \% \mathrm{CI}=0.6-0.9 ; \mathrm{p}=0.020)$. The aim of this study was to determine the hip fracture efficacy of risedronate in an elderly population with established osteoporosis.

\begin{tabular}{lll}
\hline Parameter & $\begin{array}{l}\text { Placebo } \\
\mathrm{N}=566\end{array}$ & $\begin{array}{l}\text { Risedronate } \\
\mathrm{N}=1090\end{array}$ \\
\hline $\begin{array}{l}\text { Age (Years) } \\
\text { Mean (SD) }\end{array}$ & $77(4.8)$ & $77(4.8)$ \\
$\begin{array}{l}\text { Range } \\
\text { Femoral Neck T-Score } \\
\begin{array}{l}\text { NHANES-III) } \\
\text { Mean (SD) }\end{array}\end{array}$ & $68-100$ \\
$\begin{array}{l}\text { Hip Fracture Incidence } \\
\text { Kaplan-Meier }\end{array}$ & $-3.1(0.44)$ & $-3.1(0.43)$ \\
$\begin{array}{l}\text { Hip Fracture Efficacy } \\
\text { RR (95\% CI) }\end{array}$ & $7.4 \%$ & $3.8 \%$ \\
P-Value & - & $0.54(0.32,0.91)$ \\
\hline
\end{tabular}

Method: 9331 women were enrolled in the 3 year, doubleblind, randomised HIP trial. Subjects were enrolled in 2 groups aged between 70 and $79 y$ with low FN BMD, or aged $80+$ with either low FN BMD or a clinical risk factor for hip fracture. All received calcium $1000 \mathrm{mg}$; those with low baseline levels of vitamin D received 500IU. This research focused on a subgroup of the intention to treat population, comprising of women with a baseline femoral neck BMD $<-2.5$ SD (NHANES-III) and at least one prior vertebral fracture. Time to first fracture methodology was used to estimate the 3-year hip fracture incidence of each treatment group and the observed treatment efficacy.

Results: Hip Fracture Reduction in Women aged $70-100$ years

Conclusion: Risedronate has previously been shown to significantly reduce the risk of hip fracture in the ITT population of the HIP trial. In a subgroup of elderly women aged $70-100 \mathrm{y}$ who were at increased risk (i.e. with established osteoporosis), risedronate reduced hip fractures significantly by $46 \%$.

1 McClung MR et al. Effect of risedronate on the risk of hip fracture in elderly women. N Engl J Med 2001;344(5):333-340

P323. ASSESSMENT OF PARAOXONASE 1 ACTIVITY AND MALONDIALDEHYDE LEVELS IN PATIENTS WITH OSTEOPOROSIS

H. Demir ${ }^{1}$, G. Baskol ${ }^{2}$, B. Cavdaroglu ${ }^{1}$, M. Baskol ${ }^{3}$, S. Muhtaroglu ${ }^{1}$; Department of Physical Medicine and Rehabilitation, Erciyes University, Kayseri, Turkey, ${ }^{2}$ Department of Biochemistry and Clinical Biochemistry, Erciyes University, Kayseri, Turkey, ${ }^{3}$ Department of Internal Medicine, Erciyes University, Kayseri, Turkey

Objective: Oxidative stress may regulate cellular function in multiple pathological conditions, including osteoporosis. We aimed to determine serum paraoxonase 1 (PON1) activities as known lipid antioxidant and malondialdehyde (MDA) levels, end products of lipid peroxidation, induced by reactive oxygen species (ROS) for evaluating oxidative stress in osteoporotic patients.

Material and Methods: Twenty-six osteoporotic patients were included in the study and compared with healthy controls $(n=20)$. Serum PON1 activity and MDA levels were measured according to an enzymatic spectrophotometric method.

Results: The serum MDA level was higher in the patient group $(3.8 \pm 1.4 \mathrm{nmol} / \mathrm{ml})$ than controls $(1.4 \pm 0.4 \mathrm{nmol} / \mathrm{ml} ; \mathrm{p}<0.001)$. PON1 activity was found to be lower in the patients group $(141.8 \pm 88.4 \mathrm{U} / \mathrm{l})$ than the control group $(263.4 \pm 99.8 \mathrm{U} / \mathrm{l}$; $\mathrm{p}<0.001)$. There was a negative correlation between MDA levels and PON1 $(\mathrm{r}=-0.495)$ activities with a statistical significance (Pearson's correlation analysis; $\mathrm{p}<0.001$ ).

Conclusion: In conclusion, increased ROS levels in osteoporotic patients may result in a pro-oxidation environment, which in turn could result in decreased antioxidant PON1 activity and increased MDA levels. As a result, lipid peroxidation may have a role in the pathogenesis of the osteoporotic patients. Since PON1 is also an antioxidant agent, effective antioxidant therapy to inhibit lipid peroxidation is necessary and agents to increase PON1 activity may be a therapeutic option in osteoporotic patients.

P324. IS URINARY INCONTINENCE ASSOCIATED WITH FRACTURE

Y. Dionyssiotis, S. Tournis, E. Papakitsou, G. Trovas, A. Katsalira, D. Economopoulos, B. Samdanis, A. Galanos, G.P. Lyritis; Laboratory for Research Musculoskeletal System, University of Athens, KAT Hospital, Kifissia, Greece

Objective: Urinary incontinence is a common problem and a chronic medical condition. A little importance has been attributed to the association of urinary incontinence and fractures, especially in community dwelling women. The aim was to study the influence of urinary incontinence as a risk factor for fracture in a postmenopausal women population. 
Material and methods: During the years 2004 and 2005, 1055 randomly selected community dwelling women, participated in a control program for osteoporosis which was organised by the Hellenic Foundation of Osteoporosis in cooperation with the Hellenic Patient Osteoporosis Society. Incontinence episodes and fractures were assessed by answering a questionnaire about risk factors for low energy fractures by interview. None of the women was taken any antiosteoporotic drug including calcium supplementation. Women were separated in group A: 331 women with incontinence and in group B:724 women without incontinence episodes. The mean age of the participants included in the study was $64,8 \pm 8,8(\mathrm{SD})$ in group $\mathrm{A}$ and $63,8 \pm 8,9(\mathrm{SD})$ in group $\mathrm{B}$. Women were also separated in subgroups according to age decade. Subgroup 1 $(\mathrm{n}=340): 50-59$ years,subgroup $2(\mathrm{n}=363): 60-69$ years and subgroup $3(n=346): 70+$ years.In each subgroup we calculated the prevalence of fractures according to the presence of urinary incontinence. Fisher's test and regression analysis were used to estimate the association between urinary incontinence and fractures (OR, CI).

Results: From group A 97 women $(29,3 \%)$ had fractures vs 193 women $(26,7 \%)$ from group B a non significant (N.S.) result (OR:1,14,CI:0,85-1,52, p=0,373).Subgroups' analysis was given a significant result according to fractures only in subgroup $1: 23,4 \%$ vs $15,6 \% \quad(p=0,05, \mathrm{OR}: 1,74, \mathrm{CI}: 1,1-3,05)$ between women with incontinence and without incontinence respectively.In subgroups 2 and 3 the results were N.S.

Conclusions: Urinary incontinence was associated with increased risk of fracture in early postmenopausal women. The results suggest that these community dwelling women should be considered as a special target group in order to prevent fractures.

\section{P325. EXERCISE AND CALCIUM INTAKE ACT SYNERGISTIC ON BONE IN POSTMENOPAUSAL WOMEN?}

Y. Dionyssiotis, G. Trovas, I. Charopoulos, A. Katsalira, E. Papakitsou, S. Tournis, B. Samdanis, D. Economopoulos, A. Galanosand; G.P.Lyritis Laboratory for Research Musculoskeletal System, University of Athens, KAT Hospital, Kifissia, Greece

Objective: The aim was to study the synergistic action of exercise and calcium intake on bone in postmenopausal women

Material and Methods: During the years 2004 and 2005, 1319 community dwelling postmenopausal women, randomly selected, participated in a control program for osteoporosis which was organised by the Hellenic Foundation of Osteoporosis in cooperation with the Hellenic Patient Osteoporosis Society.Exercise and calcium intake were assessed by answering a questionnaire by interview about weekly exercise activity and duration including walking, dancing, aerobics, muscle strengthening, gardening and about daily calcium intake. In the same session all women performed quantitative ultrasound of the heel with an osteometer ultrasound Achilles Express GE Lunar on the left heel. None of the women was taken bone acting drugs and/or calcium supplementation. Women were separated according to weekly exercise, in 1219 women who never exercised, 67 women who exercised less than two hours and 33 women who exercised more than two hours weekly and according to daily calcium in women with less than $800 \mathrm{mg}$ intake $\{\mathrm{n}=942$, mean age $64,8 \pm 8,8(\mathrm{SD})\}$ and in women with more than $800 \mathrm{mg}$ calcium intake $\{\mathrm{n}=377$, mean age $63,8 \pm 8,9(\mathrm{SD})\}$.

Results: Estimation of mean T-score between women who exercised according to more or less than $800 \mathrm{mg}$ daily calcium intake, revealed an increased significant result $(\mathrm{p}=0,05)$, in women who exercised more than two hours weekly and received more than $800 \mathrm{mg}$ calcium daily. In contrary in women with less than $800 \mathrm{mg}$ daily calcium intake we didn't observe any difference in mean T-score between women, independently of weekly duration of exercise.
Conclusion: These results suggest the importance of adequate calcium consumption as synergistic factor with the proper exercise to induce gains in bone mass.

\section{P326. SHORT-TERM PRECISION OF BONE MINERAL CONTENT AND BONE MINERAL DENSITY MEASURED AT DIFFERENT FOREARM RE- GIONS OF INTEREST USING DUAL-ENERGY X-RAY ABSORPTIOMETRY}

D. Sindel, A. Yaliman, A. Oral; Department of Physical Medicine and Rehabilitation, Istanbul Faculty of Medicine, Istanbul University, Istanbul, Turkey

Forearm bone mineral density (BMD) is recommended to be measured when posteroanterior spine and/or hip cannot be measured or interpreted, in very obese patients, and in those with hyperparathyroidism. However, due to the limitations for its use, the precision of forearm bone mineral content (BMC) and/or BMD measured at different sites within the forearm has not been studied widely. Therefore, it is not clear which region of interest in a radius scan as measured by dual-energy X-ray absorptiometry (DXA) has a better precision. We aimed to compare the precision errors of BMC and BMD measured at the ultradistal, mid-distal, and $33 \%$ radius (also called one-third radius) sites and of the total radius. For evaluating the short-term precision, 30 female subjects aged between 43 and 78 years had duplicate nondominant forearm scans on the same day using DXA (Hologic QDR 1000) with repositioning the forearm after the first scan. The precision errors were calculated as the root-mean-square coefficient of variation (RMSCV\%). The short-term precision errors expressed as RMSCV\% were $2.29 \%$ for BMC, $2.42 \%$ for BMD at the ultradistal site, $1.49 \%$ for $\mathrm{BMC}$ and $1.14 \%$ for BMD at the mid-distal site, $1.64 \%$ for $\mathrm{BMC}$ and $1.35 \%$ for $\mathrm{BMD}$ at the $33 \%$ radius, and $1.16 \%$ for $\mathrm{BMC}$ and $1.13 \%$ for BMD of the total radius. We can conclude that the precision errors of BMC are higher than those of BMD at all regions of interest of the radius except for the ultradistal site. It appears that that the precision errors of the BMD at mid-distal site and BMD of the total radius are lower than those at the other sites, with the ultradistal site having the highest precision error. Since measurements with excellent precision do not always have a high sensitivity, the association between these diferent radius sites and fracture prediction remains to be elucidated.

\section{P327. A COMPARISON OF THE PRECISION OF CALCANEAL QUANTITATIVE ULTRASOUND PARAMETERS AND BONE MINERAL DENSITY AT DIFFERENT SKELETAL SITES AS MEASURED BY DUAL- ENERGY X-RAY ABSORPTIOMETRY}

A. Oral, D. Sindel, A. Yaliman; Department of Physical Medicine and Rehabilitation, Istanbul Faculty of Medicine, Istanbul University, Istanbul, Turkey

Bone mineral density (BMD) as measured by dual-energy Xray absorptiometry (DXA) is considered the gold standard for the management of osteoporosis. Recently, quantitative ultrasound (QUS), which is easy to use, inexpensive, portable, does not use ionizing radiation, and has also been shown to provide information about bone quality and to predict fracture risk, has gained growing interest in this area. However, to be more widely used in the management of osteoporosis, QUS needs to be accurate, reliable, and sensitive to change. Our aim was to compare the precision of QUS parameters [broadband ultrasound attenuation (BUA), speed of sound (SOS), quantitative ultrasound index (QUI), and estimated heel bone mineral density (eBMD)] as measured by a gel-coupled calcaneal QUS device and BMD at the lumbar spine and proximal femur as measured by DXA, in an attempt to evaluate the clinical usefulness of these measurement methods. The short-term precision of the measurements was examined as the root-mean-square coefficient of variation (RMSCV\%) as measured twice in the same female subjects, aged 43 to 78 years, representative of our clinic's patient population, on the same day with repositioning the patient after each measurement. Calculations were based on 30 subjects with duplicate scans for BMD measurements and 28 subjects with duplicate QUS 
measurements, because we failed to make QUS evaluation of the two subjects with lower extremity edema. Least significant change (LSC) was also calculated as $2.77 \times \mathrm{CV} \%$ for the group at $95 \%$ confidence interval. RMSCV $\%$ and LSC $\%$ for QUS indices for the right / left heel were 3.15 / 3.24\% and 8.73 / 8.97\% for QUI, $4.19 / 4.91 \%$ and $11.61 / 13.60 \%$ for BUA, $0.29 / 0.28 \%$ and 0.80 $0.78 \%$ for SOS, and $3.77 / 3.80 \%$ and $10.44 / 10.53 \%$ for eBMD. BMD measurements showed lower precision errors, RMSCV and LSC being $2.11 \%$ and $5.84 \%$ for the lumbar spine (L1-L4), $2.77 \%$ and $7.68 \%$ for the femoral neck, and $1.61 \%$ and $4.47 \%$ for the total hip. In conclusion, the higher short-term precision errors for QUS indices than those of DXA BMD measurements may limit the use of QUS for patient monitoring.

\section{P328. TUMORAL CALCINOSIS: IDENTIFICATION OF A NOVEL RECES- SIVE MUTATION IN FIBROBLAST GROWTH FACTOR-23 (FGF-23)}

L. Masi ${ }^{1}$, A. Gozzini ${ }^{1}$, A. Franchi ${ }^{2}$, R. Capanna ${ }^{3}$, D. Campanacci $^{3}$, V. Martineti ${ }^{1}$, S. Carbonell ${ }^{1}$, A. Amedei ${ }^{1}$, A. Falchetti ${ }^{1}$, D. Strigoli ${ }^{1}$, A. Tanini ${ }^{1}$, M.L. Brandi ${ }^{1}$; ${ }^{1}$ Department of Internal Medicine, University of Florence, Florence, Italy, ${ }^{2}$ Department of Human Pathology and Oncoloy, University of Florence, Florence, Italy, ${ }^{3}$ Orthopedic Unit, University of Florence, Florence, Italy

Tumoral calcinosis (TC) is a rare genetic disorder characterized by periarticular cystic and solid tumorals calcifications. It is characterized by hyperphospatemia and an elevated serum of calcitriol concentration in every patients. The hyperphosphatemia results form an increase in capacity of renal tubular phosphate reabsorption. The identification of phosphotonin family hormones suggest that mutations of these molecules could be involved in the pathogenesis of TC. One of these molecules is represented by FGF-23. The TC phenotype is similar to that described in the FGF-23 knockout mice. In the present study we described a new FGF-23 mutation in a subject affected by TC.

A Caucasian women (years 67) was examined for a history of ectopic calcification. Biochemical exams showed and hyperphosphathemia and hyperphosphaturia with normal value of PTH and inappropriately normal level of $1-25(\mathrm{OH}) 2$ D3. The patient presented a big shoulder calcification and also a calcification of femoral artery. We expanded the family tree through detailed family histories, which importantly revealed that parents were consanguineous. Hystologically the mass was characterized by calcium deposition and granulomatous reaction around the mass. Genomic DNA was extracted from blood collected from the patient, her daughter and her grandchild by standard procedure. DNA was not available from her parents. All three FGF-23 coding exons, as well as conserved splice sites, were amplified by standard PCR procedure. Nucleotide sequences were determined by direct sequencing with a DNA kit and an automated DNA sequencer (ABI PRISM 3100 - Perkiln-Elmer Corp).

We discovered a new homozygous codon 41 , His/Gln (CACCAA) substitution in exon 1 of FGF-23 gene in the affected patient. A heterozygous substitution was present in the daughter. No mutation were found in the two children. FGF-23 gene mutation was not found in the SNP database (www.ncbi.nih.gov/ snp). In summary, a recessive mutation in FGF 23 causes TC. Understanding the functional significance and molecular physiology of this novel mutation will reveal critical information regarding the role of FGF-23 in states of normal and of disorder of phosphate homeostasis.

\section{P329. A GAIN FUNCTION MUTATION IN THE CALCIUM-SENSING RECEPTOR IN AN ITALIAN FAMILY WITH AUTOSOMAL DOMINANT HYPOCALCEMIA}

L. Masi ${ }^{1}$, A. Amedei ${ }^{1}$, G. Leoncini ${ }^{1}$, S. Procopio ${ }^{2}$, A. Gozzini ${ }^{1}$, A. Falchetti ${ }^{1}$, R. Imbriaco ${ }^{1}$, F. Franceschelli ${ }^{1}$, R. Livi ${ }^{1}$, A. Tanini ${ }^{1}$, M.L. Brandi ${ }^{1}$; ${ }^{1}$ Department of Internal Medicine, University of Florence, Florence, Italy, ${ }^{2}$ Department of Pediatric Medicine, University of Florence, Florence, Italy
The calcium sensing receptor $(\mathrm{CaR})$ was first identified in the parathyroid cells and was later found to be expressed in the kidney and other tissues. Inactivating mutations in the CaR gene are responsible of an elevation in extracellular calcium, leading to mild moderate hypercalcemia and relative hypocalciuria called Familial Hypocalciuric Hypocalcemic (FHH). On the other hand, activating mutations in the $\mathrm{CaR}$ gene are associated with reverse phenotype, autosomal dominant hypocalcemic (ADH) and a sporadic hypoparathyroidism. So far, about 20 activating mutations in the CaR gene have been identified and recently a $\mathrm{Ser}^{820} \mathrm{Ph}$ mutation in the CaR has been described in Japanese family.

In the present study we described an Italian family affected by ADH in whom a CaR mutation was identified. The proband was a girl 13 year old affected by a mild hypocalcemia and hypercalciuria with normal range value of PTH. She underwent a computed tomographic examination of the brain because of headache which showed the presence of calcificatin of the basal ganglia. In addition a juvanile hypertension was present in the proband. Baseline biochemical data of the family members showed a mild hypocalcemia and hypertension in the mather and two of four uncles.

Genomic DNA of all subjects was extracted from white blood cells with standard procedure. In the proband, all protein-coding exons (exons 2-7) of the CaR gene were amplified by PCR with standard procedures using primer pairs as described in the literature. Nucleotide sequences of both strands of the PCR products were determined by direct sequencing with a DNA kit and an automated DNA sequencer (ABI PRISM 3100, Perkiln-Elmer Corp). A Ser ${ }^{820}$ Phe activating mutation of the CaR was found.

In summary, we have idenfied the Ser ${ }^{820}$ Phe activating mutation in a Italian family affected by Hypertension and ADH.

\section{P330. STABILIZATION OF THE SKELETAL CYSTIC ANGIOMATOSIS BY BISPHOSPHONATE TREATMENT}

L. Masi ${ }^{1}$, F. Del Monte ${ }^{1}$, A. Franchi ${ }^{2}$, R. Capanna ${ }^{1}$, L. Sinigaglia ${ }^{3}$, M. Muratore ${ }^{4}$, M.L. Brandi ${ }^{1}$; ${ }^{1}$ Department of Internal Medicine, University of Florence, Florence, Italy ${ }^{2}$ Department of Pathology, University of Florence, Florence, Italy, ${ }^{3}$ Orthopedic Institute G. Pini, Department of Rheumatology, University of Milan, Italy, ${ }^{4}$ Rheumatologic Unit, A. Galateo Hospital, San Cesario, Lecce, Italy

Cystic angiomatosis is a rare condition of disseminated multifocal hemangiomatous and/or lymphangiomatous lesions of the skeleton with possible visceral organ involvement. The cause of this condition remain so far unknown. Patients with cystic angiomatosis may be asymptomatic and with absence of pathologic fracture. In the present study we described a 20 -year old men, in otherwise excellent health, had a 2-year history of cervical and dorsal back pain. Radiographs of the column showed the presence of multiple cystic lesions in the cervical, dorsal and lumbar vertebral body. A triple-phase whole-body bone scan demonstrated focal increased up-take within, with no other abnormalities. Destruction of bone was confirmed by CT scans and MRI scans showed angiomatous soft tissue formation. A bone biopsy specimen appeared as a simple cyst or multiple communicating cysts. Serum calcium and phosphorous levels PTH and Vitamin D metabolites at the presentation were within the respective rangers. High levels of serum bone alkaline phosphatase was observed (31.4 U/L ; reference range 10-25) and urine desoxypiridinoline (DPD) levels were in the normal range. For additional studies, we assessed the serum concentrations of the osteoclastic regulators osteoprotegerin (OPG) and osteopontin (OPN) at the baseline and during the follow-up by ELISA (R\&D System, USA; Immundiagnostik, Germany). At the baseline OPN and OPG levels were high (OPN:16 $\pm 0.5 \mathrm{ng} / \mathrm{ml}$ reference value: $5.9 \pm 0.6$ and $\mathrm{OPG} 125 \pm 0.34 \mathrm{pmol} / \mathrm{ml}$ reference value $0-30)$.Patient started an intravenous bisphosphonate infusion $(30 \mathrm{mg}$ pamidronate) every month. A follow-up after 6 months therapy showed an improve of mobility and back pain of the patient. Imaging 
studies including conventional X-ray and CT scans after 3 and 6 months of treatment did not show and progression of bone destruction. After initiation of bisphosphonate tretment serum bone alkaline phosphatase levels normalized. In addition OPN and OPG decreased significantly. In conclusion, an immediate clinical improvement of local pain, an improvement of metabolic resorption and a stable clinical, radiological picture during the first 6 months of follow-up suggests effectiveness of pamidronate treatment alone. Longer-term follow-up and additional studies employing bisphosphonates in skeletal cysitic angiomatosis will help to evaluate this therapeutic approach in further detail.

P331. PRESCRIPTION AND COPRESCRIPTION PATTERNS OF BONEACTIVE SUBSTANCES AMONG POSTMENOPAUSAL WOMEN

F. Richy, O. Bruyère, A. Mawet, J.-Y. Reginster; University of Liège, Faculty of Medicine, Dept of Public Health, Epidemiology and Health Economics, CHU, Sart-Tilman, Belgium, WHO Collaborating Center for the Public Health Aspects of Osteoarticular Disorders, Liège, Belgium

Setting. In a balanced public health and health economics perspective, the accurate prescription and coprescription rates of the numerous substances and drugs affecting bone are a prerequisite for an efficient management of osteoporosis. Indeed very sparse data is available in this field. We designed this study to investigate the rate of prescription and coprescription of vitamin $\mathrm{D}$ and calcium among women aged 60 to 80 years. Methods. Cross-sectional data from 1733 postmenopausal women collected between 1996 and 1999 in an outpatient clinic located in Liège, Belgium. The following classifications were made, according to the presence or absence of osteoporosis at various anatomical sites (hip, femoral neck, lumbar spine, measured by DXA-Hologic QDR 2000): use of calcium, vitamin D, hormone replacement therapy, biphosphonates, calcitonin, SERMS alone or in combination. Results. The mean age (SD) of the subjects was 68.5 (5.2) years. $26 \%$ of the subjects had osteoporosis at the hip, femoral neck, or lumbar level. $69 \%$ of the subjects with osteoporosis received a treatment while $64 \%$ of the subjects free of osteoporosis did. Calcium and vitamin D were the most often prescribed substances for osteoporotic subjects, hence their combination was only seen among $34 \%$ of the patients.

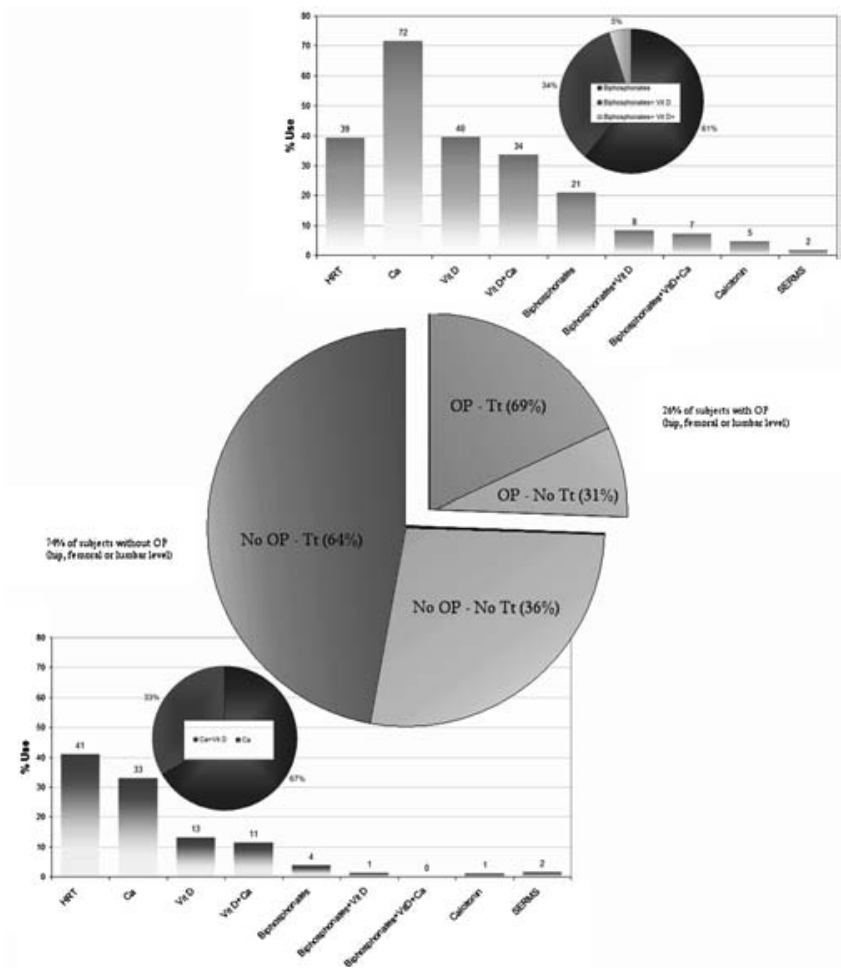

Biphosphonates in conjunction with vitamin $\mathrm{D}$, and in conjunction with calcium, were prescribed among $21 \%$ and $7 \%$ of the subjects with osteoporosis, respectively. Among healthy subjects, hormone replacement therapy was most prevalent $(41 \%)$, followed by calcium $(33 \%)$, vitamin D $(13 \%)$, vitamin D and calcium $(11 \%)$, and biphosphonates $(4 \%)$. Interpretation. This preliminary, pragmatic study has shown that few osteoporotic women receive adequate treatment, and that currently accepted preventive measures, such as calcium and vitamin $\mathrm{D}$, are seen in less than 1 patient at risk out of three. Our preliminary data also provide and insight on the lack of coprescription rates of vitamin $\mathrm{D}$ and calcium, especially regarding biphosphonates. There is a need to evaluate the results on health of the use of these medicines in clinical practice given the discreet efficacy results obtained in clinical trials. Use of calcium and vitamin D should be encouraged because of the deficiencies observed on an international basis. Coprescription of these drugs, together with biphosphonates should be reinforced, or combined therapies should be promoted.

\section{P332. STRUCTURAL-FUNCTIONAL STATE OF BONE LOSS OF THE POSTMENOPAUSAL WOMEN WITH KNEE OSTEOARTHRITIS}

V. Povoroznjuk, N. Grygoryeva; Department of Clinical Physiology and Pathology of Locomotor Apparatus, Institute of Gerontology AMS Ukraine, Kiev, Ukraine.

Background: Osteoarthritis and osteoporosis are the most important diseases of bone and joint system; however, the rates of their development in postmenopausal period have some peculiarities. Many studies show the role of deficient sexual hormones and menopause in the development of osteoarthritis and osteoporosis.

Materials and Methods: In order to study the peculiarities of knee osteoarthritis and structural-functional state of bone mass in elder women we've examined 157 women aged of 50-79 years in postmenopausal period (duration - 2-34 years) with knee osteoarthritis (I-III stages). A diagnosis of osteoarthritis was performed along the criteria of American rheumatology association (1995), its stage - by Kellgren-Lourenz classification. Heel bone examinations were performed by means of ultrasound bone densitometer.

Results: We've revealed peculiarities of structural-functional state of bone loss depending on knee osteoarthritis' stage (Tabl. 1) and age of women. We've also determined the role of increased body weight in development of knee osteoarthritis and onset of structural-functional violations of bone mass in women during postmenopausal period. It was founded that in the women with osteoarthritis which were standardised on body mass index the data of ultrasound densitometry were worse in patients with II-III stage of osteoarthritis. Tab. 1. Body mass index and data of ultrasound densitometry of women in depend of the age and stage of knee osteoartritis. Data / Groups Control group OA (I stage) OA (II-III stage) 60-69 years Body mass index, un. 31,6 $\pm 0,431,4 \pm 0,7$ $32,8 \pm 1,2$ Stiffness index, $\% 80,3 \pm 1,979,5 \pm 2,371,7 \pm 2,5$ a, b Zrange, SD. $0,4 \pm 0,20,3 \pm 0,2-0,3 \pm 0,2 \mathrm{a}, \mathrm{b} 70-74$ years Body mass index, un. $32,5 \pm 0,7 \quad 31,4 \pm 1,0 \quad 32,54 \pm 0,7$ Stiffness index, \% $71,0 \pm 3,8 \quad 72,8 \pm 2,1 \quad 74,9 \pm 1,9 \quad$ Z-range, SD. $-0,03 \pm 0,34$ $0,11 \pm 0,200,27 \pm 0,18 \mathrm{a}-\mathrm{p}<0,05$ compared with control group; $\mathrm{b}$ $\mathrm{p}<0,05$ compared with group with OA (first stage).

P333. SELF-REPORTED VERSUS MEASURED PREVALENCE OF OSTEOPOROSIS. AN AGE-STRATIFIED ANALYSIS OF ITS DIAGNOSTIC COVERAGE

F. Richy ${ }^{1}$, O. Bruyère ${ }^{1}$, P. Maassen ${ }^{2}$, G. Pire ${ }^{3}$, J.-Y. Reginster $^{1}$; ${ }^{1}$ University of Liège, Faculty of Medicine, Public Health, Epidemiology and Health Economics, Liège, Belgium, WHO Collaborating Center for Public Health Aspects of Osteoarticular Disorders, Liège, Belgium, ${ }^{2}$ General Director, Health and Environment, Province of Liège, Liège, Belgium, ${ }^{3}$ Representative in charge of Health, Environment and Quality of life, Province of Liège, Belgium 

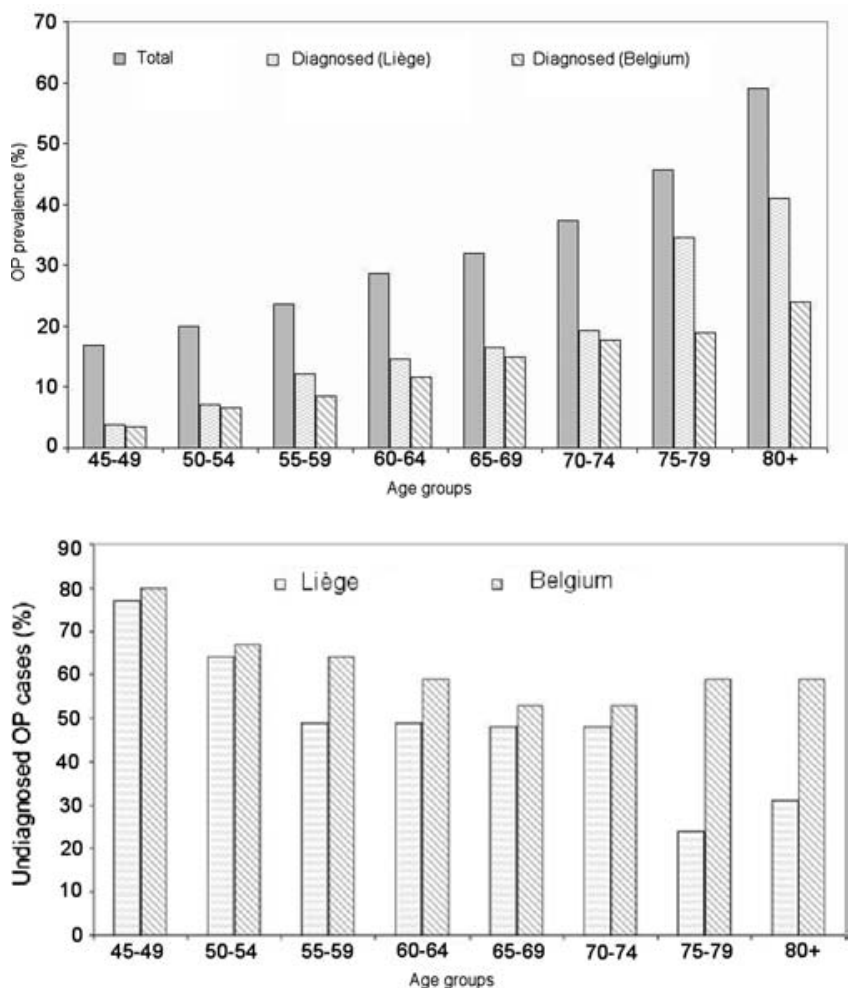

Introduction: The efficacy and efficiency of prophylactic and therapeutic treatments for osteoporosis are limited by the lack of awareness of the patients and the physicians about this condition and by the lack of reimbursement of diagnostic procedures. There is a lack of data regarding what percentage of the osteoporotic population receives diagnosis. Methods: Two databases were crossed to link 1) the age-specific prevalence of the diagnosis of osteoporosis at the total hip, femoral neck and L2-L4 $(n=609)$ assessed during an independent, free osteoporosis-screening campaign and 2) the self-reported prevalence of osteoporosis in a representative sample of postmenopausal women $(n=2726)$. Records were matched on age in a 4 to 1 fashion.

Results: The age-specific prevalence of osteoporosis (considered at any measurement site) presented an exponential increase and ranged from 16.8 to $59.1 \%$. The percentage of awareness of osteoporosis among the osteoporotic subjects ranged from 20 to $69 \%$ and increased steadily with age groups. The positive impact of the free screening campaigns organised for 4 years now in the Province of Liège could be observed on the basis of higher awareness rates compared to global Belgium values.

Implications: Even in a country like Belgium, were the availability of densitometers is one of the highest in Europe, the percentage of diagnosed osteoporosis remains critically poor in the general population. Our data show that up to $59 \%$ to $80 \%$ of the osteoporotic subjects remain undiagnosed, placing them at risk of fractures and weighting down the community with the increasing burden of direct and indirect costs. These data accurately document the need for a better access and reimbursement of densitometry.

1) Richy, F., et al., Development and validation of the ORACLE score to predict risk of osteoporosis. Mayo Clin Proc 2004; 79: 1402-8.

2) The Belgian Health Interview Survey. Web-based interractive analysis.

\section{P334. NUTRITION AND OSTEOARTHRITIS OF KNEE IN POSTMENO- PAUSAL WOMEN}

V. Povoroznjuk, N. Grygoryeva; Department of Clinical Physiology and Pathology of Locomotor Apparatus, Institute of Gerontology, Kiev, Ukraine
Background: There are a literature data about possible influence of some nutrients on the development and progressing of knee osteoarthritis. It was shown, that the patients with low level of vitamin $\mathrm{C}$ in their blood had a triple progressing risk of osteoarthritis. Famous properties of vitamins $\mathrm{E}$ and $\mathrm{A}$ also give a possibility to think about their role in progressing of osteoarthritis. The patients with low concentration of vitamin $\mathrm{D}$ in feeding ration have a raised risk of knee osteoarthritis. The purpose of this study was to determine the relationship of consumption of different nutrients in feeding ration of postmenopausal women and incident and stage of knee osteoarthritis.

Materials and Methods: Influence of diets on development and progressing of knee osteoarthritis was investigated in 133 postmenopausal women.

Results: We did not found the differences in contents of most macro- and micronutrients in depend of incident and stage of osteoarthritis, however it was a tendency in consumption of some microelements and vitamins. The patients with OA compared with control group have a more level of $\beta$-carotin $(F=2,64, p=0,11)$, folacin $(F=2,94, p=0,09)$, less level of vitamin $\mathrm{D}$ (accordingly $0,33 \pm 0,04$ and $0,53 \pm 0,06 \mathrm{mg} / \mathrm{d}$; $\mathrm{F}=6,51, \mathrm{p}=0,01)$. We have not founded the differences in consumption of vitamin $\mathrm{C}(\mathrm{F}=1,96, \mathrm{p}=0,16)$, vitamin $\mathrm{A}$ $(\mathrm{F}=0,05, \quad \mathrm{p}=0,83)$ and $\mathrm{E}(\mathrm{F}=0,85, \quad \mathrm{p}=0,36)$ in patients dependency on presence of arthritis. The tertiles analysis showed that frequency of knee osteoarthritis in the women with low vitamin $\mathrm{D}$ consumption (first tertile) was 48,9\%, high vitamin $\mathrm{D}$ level in daily ration (fourth tertile) $-22,7 \%$. We have not founded the differences in consumption of all macroand micronutrients in depend of stage of osteoarthritis.

Conclusion. Our results shows that in the feeding ration of postmenopausal women with knee arthritis there are significant more level of vitamin D and diminished level of some microelements and vitamins, that can contribute to its progressing.

\section{P335. FALL PREVENTION IS ESSENTIAL TO REDUCE CLINICAL FRACTURE INCIDENCE IN ELDERLY WOMEN - INDEPENDENTLY OF DRUG TREATMENT FOR OSTEOPOROSIS}

H. Boerst, O. Bock, M. Kalbow, D. Felsenberg; Centre for Muscle and Bone Research, Charité - Universitaetsmedizin Berlin, Campus Benjamin Franklin, Berlin, Germany

Objectives: To examine the incidence of falls and clinical fractures and their association with parameters of neuromuscular function, osteodensitometry and drug treatment for osteoporosis in a German cohort of elderly women.

Patients and Methods: 1197 elderly women (60 to 95 yrs) were examined in the "Osteoporosis Risk Factor Survey" in summer 2002 and contacted 24 months later for follow-up (by questionnaire and telephone) to evaluate their actual health and medical supply status with special consideration of falls, clinical fractures and drug treatment for osteoporosis.

Results: Complete follow-up data sets were evaluated for 786 women (mean age 70.9 yrs $+/-7.2 \mathrm{SD}$ ). Among those were 251 women with osteoporosis $(31.9 \%), 341$ with osteopenia $(44.4 \%)$ and 194 with normal BMD $(25.7 \%)$ acc.to WHO criteria. A total of 352 women $(43.8 \%)$ experienced at least one fall during the 24 months follow-up period, 283 of them $(80.4 \%$ of all fallers) had at least one locomotoric fall. Some tests and parameters of neuromuscular function obtained at baseline were associated with the risk to fall, in particular a chair rising test $>7.6 \mathrm{sec}(\mathrm{RR}=1.90$; CI 1.30-2.77). 89 clinical fractures occurred in 83 patients. The overall fracture incidence was $15.6 \%$ and $9.1 \%$ in osteoporotic and osteopenic patients, but $8.2 \%$ in patients with normal BMD as well. 78 fractures $(87.6 \%$ of all fractures) in 72 patients were related to a locomotoric fall. Every third faller in osteoporotic women $(33.7 \%)$ sustained a fracture, every fourth in osteopenic women $(24.8 \%)$ and even every fifth in women with normal BMD $(20.3 \%)$. In the subset of osteoporotic women, the percentage of fallers who sustained a fracture was not different between patients with or without antiresorptive treatment of bone. 
Conclusions: Almost $90 \%$ of all clinical fractures in the age group of 60 years and older were fall related. Whether antiresorptive treatment of bone was given or not, one third of osteoporotic patients who fell sustained also a fracture. These findings emphasise on the importance of fall prevention to reduce clinical fracture incidence in elderly women in general and in osteoporotic women in particular.

\section{P336. CROSS-CALIBRATION OF THE LEXXOS CONE BEAM DENSI- TOMETER WITH THE HOLOGIC QDR-4500}

M. Audran ${ }^{1,2}$, Y. Simon ${ }^{1,2}$, R. Levasseur ${ }^{1,2}$, D. Chappard ${ }^{1}$, E. Legrand ${ }^{1,2} ;{ }^{1}$ Pôle ostéoarticulaire, ${ }^{2}$ Faculté de Médecine and CHU Angers, INSERM EMI-0335, Angers, France

The Lexxos cone beam densitometer (DMS, France) utilizes a 2-D detector and conically collimated X-rays; this allows a short acquisition times of $3 \mathrm{~s}$ using two $\mathrm{X}$-ray exposures $(75 \mathrm{kV}$ and 140 $\mathrm{kV}$ ). The aim of the study was to establish a cross-calibration of the Lexxos system with the Hologic QDR-4500 fan-beam densitometer. In a previous study the root mean square coefficient of variation (RMS CV\%) of the Lexxos densitometer ranged between 1.1 to $1.9 \%$.

Aim: We studied 89 volunteers (74 females, 15 males; mean age: $53 \pm 26$ yrs old); no subject weighted more than $95 \mathrm{~kg}$. The scanned ROIs were the lumbar spine from L1 to L4, the femoral neck and the total hip (using the Hologic foot-positioning system). Bone mineral density (BMD) data obtained with Lexxos and Hologic systems were compared by using linear regression analysis (Passing-Bablock), Bland \& Altman and mountain plots. BMD data from right and left hip measured with each system were also compared

Results: No significant differences were measured between right $(\mathrm{R})$ and left $(\mathrm{L})$ values obtained $\bullet$ either with Hologic : $\mathrm{R} / \mathrm{L}$ FN: $+0.6 \%, \mathrm{p}=0.30 ; \mathrm{R} / \mathrm{L} \mathrm{TH}=+0.7 \%, \mathrm{p}=0.18 \bullet$ or with Lexxos R/L FN: $-0.1 \%, p=0.93$; R/L TH: $.07 \%, p=0.18)$ By using the Passing-Bablock method, we showed, comparing Lexxos to Hologic: - An overestimation by Lexxos at the lumbar spine $(+5.3 \% ; \mathrm{p}<.0001)$, right $\mathrm{FN}(+1.8 \%, \mathrm{p}=.06)$, right $\mathrm{TH}$ $(1.8 \%$, NS) - An underestimation by Lexxos at the left FN $(-1.9 \%)$, left TH $(0.1 \%$, NS).

In conclusion by regression analysis we confirmed a significant linear relationship between the cone-beam Lexxos and the fanbeam Hologic QDR-4500, with correlation coefficients ranging from 0.9 to 0.96 . Nevertheless, there is no strict agreement between the measurements at the lumbar spine, with a significant overestimation of the values by Lexxos.

P337. EVALUATION OF BONE MINERAL DENSITY AND MARKERS OF BONE TURNOVER IN PATIENTS AFTER RENAL TRANSPLANTATION

Arkadiusz Zygmunt ${ }^{1}$, Ilona Kurnatowska ${ }^{2}$, Feliks Kacprzyk ${ }^{2}$, Jolanta Bialkowska ${ }^{3}$, Maciej Jablkowski ${ }^{3}$, Michal Nowicki ${ }^{2}$, Andrzej Lewinski ${ }^{1}$; ${ }^{1}$ Department of Endocrinology and Metabolic Diseases, ${ }^{2}$ Department of Nephrology, Hypertension and Kidney Transplantation, ${ }^{3}$ Department of Infections Diseases, Medical University, Lodz, Poland

It has been shown that attainment of normal metabolic environment after renal transplantation does not always result in resolution of abnormalities of mineral metabolism and the bone loss may persist or even progress in the post-transplant period. Preexisting renal osteodystrophy, hyperparathyroidism, and steroid therapy are all involved in this process.

The aim of this study was to evaluate the relation between bone mineral density (BMD) and specific markers of bone turnover in patients after renal transplantation.

This cross-sectional study included 62 patients $(40$ men aged $43.5 \pm 13.2 \mathrm{yrs}$, and 22 women $43.0 \pm 12.4$ yrs with no history of bone fractures. Mean period after renal transplantation was $61.0 \pm 43.7$ months in men and $37.0 \pm 31.0$ months in men (the difference non significant). All patients had a stable transplant function and were on a standard triple therapy that included steroids in a dose 5-10 $\mathrm{mg}$ prednisone per day.

In all transplanted patients serum levels of beta-CrossLaps, intact parathyroid hormone (iPTH) and alkaline phosphatase (ALP) were measured. BMD was estimated in the lumbar spine and in the hip using dual-energy x-ray absorptiometry. Low BMD (osteoporosis and osteopenia) was found in $77.4 \%(48 / 62)$ patients. Diagnostic criteria of osteoporosis were fulfilled by $27.4 \%$ of subjects, more frequently in men $(30.0 \%)$ than in women $(22.7 \%)$. Osteopenia was observed in $50 \%$ of patients and was more frequent in women $(59.1 \%)$ than in men $(45.0 \%)$. We also observed a positive correlation between serum $\beta$-CrossLaps concentrations and PTH in women $(r=0.74 ; p<0.05)$. Plasma PTH levels correlated positively with a length of haemodialysis therapy before renal transplantation (in women $\mathrm{r}=0.50 ; \mathrm{p}<0.05$ and in men $r=0.58 ; \mathrm{p}<0.05$ ).

Conclusions: The frequency of low bone mineral density in patients after renal transplantation is high. Low bone density is equal in women and men. Longer period of haemodialysis treatment before renal transplantation is associated with higher posttransplant levels of PTH.

P338. IMPACT OF RISEDRONATE ON OSTEOPOROTIC FRACTURES, PATIENT QUALITY OF LIFE AND TREATMENT COSTS IN SPAIN

A. Echevarria ${ }^{1}$, O. Bouin ${ }^{2}$; ${ }^{1}$ Health Economics, Sanofi Aventis, Madrid, Spain, ${ }^{2}$ Global Health Outcomes \& Market Access, Sanofi Aventis, Paris, France

Objective: Osteoporosis, which is characterized by hip, vertebral, wrist and other fragility fractures, is a large and growing disease with significant health care costs and a detrimental impact on quality of life. The objective of this analysis was to assess the cost-effectiveness of Risedronate compared to calcium + vitamin D only or Alendronate in high-risk osteoporotic patients in Spain.

Methods: A published, fracture-incidence based model of osteoporosis was used to estimate the impact of therapy on costs, fractures and quality adjusted life years (QALYs). In addition, the costs per hip fracture averted, and per QALY gained were estimated. The population for the analysis included women aged 65 and older with a bone mineral density $\mathrm{T}$-score of $\leq-2.5$ and a previous history of vertebral fracture, treated over 5 years with either of Risedronate, calcium + vitamin D only or Alendronate. Model inputs included general population mortality, fracture rates, relative risk (RR) of fracture due to risk factors, fracture costs, and prices per day (Actonel ${ }^{\circledR} 1.38 €$; Fosamax ${ }^{\circledR} 1.46 €$ ). RR reduction of fracture of the hip (Risedronate $60.0 \%$; Alendronate $51.0 \%$ ) and vertebral (Risedronate $49.0 \%$; Alendronate $47.0 \%$ ) were based on the results of large randomized controlled trials.

Results: Table 1: Total events, QALYs and costs with 3.0\% cost discounting and $3.0 \%$ outcomes discounting.

\begin{tabular}{llllll}
\hline Therapy & $\begin{array}{l}\text { Total } \\
\text { Fractures }\end{array}$ & QALYs & Costs & $\begin{array}{l}\text { Cost per } \\
\text { Fracture } \\
\text { Averted }\end{array}$ & $\begin{array}{l}\text { Cost per } \\
\text { QALY } \\
\text { Gained }\end{array}$ \\
\hline CALCIUM + & 2,253 & 11,344 & $5.88 \mathrm{M}$ & & \\
$\begin{array}{l}\text { VITAMIN D ONLY } \\
\text { RISEDRONATE }\end{array}$ & 2,082 & 11,437 & $8.08 \mathrm{M}$ & 12,960 & 23,715 \\
ALENDRONATE & 2,091 & 11,431 & $8.40 \mathrm{M}$ & $\begin{array}{l}\text { DOMI- } \\
\text { NATED }\end{array}$ & $\begin{array}{l}\text { DOMI- } \\
\text { NATED }\end{array}$ \\
\hline
\end{tabular}

Conclusions: This analysis extends the results of the clinical trials by demonstrating that treatment with Risedronate can reduce long-term hip and vertebral fracture rates, as well as, associated costs while improving patient quality of life. This clinical and cost-effectiveness results provide strong support for the adoption of Risedronate in the treatment of osteoporosis. 
P339. COST-EFFECTIVENESS OF FOSAVANCE ${ }^{\circledR}$ IN THE TREATMENT AND PREVENTION OF OSTEOPOROSIS IN THE UNITED KINGDOM

J. Jansen ${ }^{1}$, S. Gaugris ${ }^{2}$, G. Bergman ${ }^{3}$, S.S. Sen ${ }^{4}$; ${ }^{1}$ Mapi Values, The Netherlands, ${ }^{2}$ Outcomes Research, Merck \& Co., Whitehouse, NJ, USA, ${ }^{3}$ Mapi Values, The Netherlands, ${ }^{4}$ Outcomes Research, Merck \& Co., Whitehouse Station, NJ, USA

Objective: To evaluate the cost-effectiveness of Fosavance ${ }^{\circledR}$ (alendronate plus vitamin $\mathrm{D}_{3}$ ) versus Fosamax ${ }^{\circledR}$ (alendronate) with dietary supplements of vitamin D (Fosamax-VitD), and versus Ibandronate in the treatment of post-menopausal osteoporosis in the UK

Methods: A lifetime patient simulation model depicting the osteoporosis disease progression was developed adopting a previously published model (Kanis et al. 2000) to estimate the costeffectiveness of Fosavance $\AA$ versus comparators. This discrete state transition model using a 1-year cycle included health states related to hip, vertebral, wrist and proximal humerus fractures, as well as health states for death due to hip fractures and death due to other causes. The model estimated outcomes including number of fractures, quality-adjusted life years (QALYs), direct medical costs, cost per QALY gained and cost per fracture avoided. The impact of 5 years of treatment on the occurrence of fractures and other associated outcomes was modeled with relative risks observed in the Fracture Intervention Trial (FIT) for Fosavance ${ }^{\circledR}$ and Fosamax ${ }^{\circledR}$ and data from Chesnut et al (2004) for ibandronate. It was assumed that vitamin D did not have any effect on risk of fracture (relative risk $=1$ ). Direct medical costs, utilities associated with different health states were derived from existing literature. Analyses were performed for women with a history of vertebral fractures (hVF) and for women with osteoporosis (BMD T-score $\leq-2.5 \mathrm{SD}$ ) and aged 50,60, 70 and 80 years. Probabilistic sensitivity analyses using Monte Carlo simulation were undertaken to estimate the uncertainty of the model outcomes.

Results: In women with osteoporosis and women with a hVF, 50 years and older, Fosavance ${ }^{\circledR}$ is cost-saving over FosamaxVitD. Compared to Ibandronate, Fosavance ${ }^{\circledR}$ is cost-effective for women with a hVF aged 50 years (incremental cost/QALY gained $=£ 19095$; incremental cost per fracture avoided $=£ 1875$; both below the generally accepted threshold for cost-effectiveness: $£ 30000$ ) and dominant for women 60 and over.

Conclusions: In the UK, Fosavance ${ }^{\circledR}$ appears to be a dominant therapy over Fosamax-VitD for women over the age of 50 with osteoporosis or with a hVF. Compared to Ibandronate, Fosavance ${ }^{\circledR}$ is dominant for women with a hVF aged 60 and over.

\section{P340. ONE-YEAR INCREASE IN HIP (HIP OR FEMORAL NECK) BONE MINERAL DENSITY PREDICTS LONG-TERM (3 YEARS) DECREASE IN VERTEBRAL FRACTURE INCIDENCE IN PATIENTS TREATED WITH STRONTIUM RANELATE}

O. Bruyer̀ ${ }^{1}$, P. Fardellone ${ }^{2}$, J.-M. Kaufman ${ }^{3}$, S. Adami ${ }^{4}$, A. Torrijos $^{5}$, J.-Y. Reginster ${ }^{6}$; ${ }^{1,6}$ WHO Collaborating Center for Public Health Aspect of Osteoarticular Disorders, University of Liège, Liège, Belgium, ${ }^{2}$ Hôpital Nord, Service de Rhumatologie, Amiens, France, ${ }^{3}$ U.Z. Gent Department of Internal Medicine, Gent, Belgium, ${ }^{4}$ Rheumatology Unit, University of Verona, Valeggio, Italy, ${ }^{5}$ Hospital La Paz, Madrid, Spain

Objective: Recent studies have shown that strontium ranelate (SR) improves bone mineral density (BMD) and reduces vertebral and non-vertebral fractures incidence in postmenopausal osteoporotic women. However, the relationship between BMD increase and fracture reduction is unknown. The objective of this study is to analyze the associations between one-year changes in BMD and future vertebral fracture risk after 3 years of treatment with SR.

Material and Methods: Analysis from the SOTI (Spinal Osteoporosis Therapeutic Intervention study) and the TROPOS (Treatment of Peripheral Osteoporosis) studies. In these two studies, patients received either $2 \mathrm{~g}$ /day of Strontium ranelate or placebo for three years. BMD of the femoral neck and the total hip was assessed at baseline and after 1 year $(n=2338)$.
Results: The logistic regression analysis, including age, body mass index and baseline BMD as covariates, showed that 1-year increases in neck or hip BMD changes were statistically associated with a reduction in the incidence of new clinical vertebral fractures $(p=0.03)$. For each increase of $1 \%$ in femoral neck or total hip BMD after one year, the risk to experience a new clinical vertebral fracture after 3 years decreased by $3 \%(95 \% \mathrm{CI} 1 \%$ $6 \%)$. The reduction is of similar magnitude $(3 \%[\mathrm{p}=0.04]$ and $2 \%$ $[p=0.06]$ for neck and hip BMD changes, respectively) for all vertebral fractures (clinical and morphometric). Patients experiencing an increase in femoral neck BMD $(>0 \%)$ after 1 year have a $21 \%$ risk reduction $(p=0.04)$ to experience a new vertebral fracture (clinical or morphometric) over 3 years, compared to patients without improvement of BMD.

Conclusion: One-year changes in total hip or femoral neck BMD predicts reduction in vertebral fracture incidence, during treatment with SR.

P341. RELATIONS BETWEEN INCREASE IN FEMORAL NECK BONE MINERAL DENSITY AND DECREASE IN HIP FRACTURE INCIDENCE DURING TREATMENT WITH STRONTIUM RANELATE

O. Bruyer̀ ${ }^{1}$, P.D. Delmas ${ }^{2}$, J.-P. Devogelaer ${ }^{3}$, D.O. Slosman ${ }^{4}$ C. Albanese ${ }^{5}$, J.-Y. Reginster ${ }^{6} ;{ }^{1,6}$ WHO Collaborating Center for Public Health Aspect of Osteoarticular Disorders, University of Liège, Liège, Belgium, ${ }^{2}$ Hôpital Edouard Herriot, Lyon, France, ${ }^{3}$ Université Catholique de Louvain, Bruxelles, Belgium, ${ }^{4}$ Clinique Générale Beaulieu, Genève, Switzerland, ${ }^{5}$ University of Rome La Sapienza, Roma, Italy

Objective: Strontium ranelate (SR) increases bone mineral density (BMD) in postmenopausal osteoporotic women. A recent study has also highlighted the hip fracture reduction observed during a 3-year treatment with SR, in osteoporotic women aged over 74 years with low BMD at the femoral neck (T-score $\leq-2.4$ according to NHANES normative values). The objective of this study is to analyze the associations between BMD changes and hip fractures incidence during treatment with SR.

Material and Methods: Out of two recent studies assessing the anti-fracture efficacy of SR [the Spinal Osteoporosis Therapeutic Intervention study (SOTI) and the Treatment of Peripheral Osteoporosis study (TROPOS)], we have selected a population at high risk of hip fracture. BMD was assessed at baseline and after a follow-up of 3 years at the femoral neck.

Results: 465 patients were included in the present analysis. In patients experiencing a hip fracture over 3 years of treatment with $\mathrm{SR}$, femoral neck BMD increased by (mean [SEM]) 3.41 (1.02) \% compared to $7.23(0.81) \%$ in patients without hip fracture $(p=0.02$ between the two groups). Patients in the highest quartile of neck BMD changes $\left(>11.63 \% \mathrm{~g} / \mathrm{cm}^{2}\right)$ experienced less hip fractures $(0.9 \%)$ than patients in the lowest $\left(<1.65 \% \mathrm{~g} / \mathrm{cm}^{2}\right)$ quartile $(6.0 \%)(\mathrm{p}=0.03$ between the two quartiles). Compared to patients in the lowest quartile of neck BMD changes, the risk to experience a hip fracture is reduced by $76 \%$ in patients within the 3 highest quartiles $(\mathrm{p}=0.01)$.

Conclusion: During 3 years of SR treatment, neck BMD changes is associated with hip fracture incidence reduction.

\section{P342. STRONTIUM RANELATE STIMULATES MURINE OSTEOBLAST REPLICATION INDEPENDENTLY OF CALCIUM SENSING RECEPTOR- MEDIATED ERK1/2 ACTIVATION}

O. Fromigué ${ }^{1}$, A. Barbara ${ }^{1}$, E. Haÿ ${ }^{1}$, C. Petrel $^{2}$, E. Traiffort ${ }^{2}$, M. Ruat $^{2}$, P.J. Marie ${ }^{1}$; ${ }^{1}$ Osteoblast Biology and Pathology Laboratory, Inserm U606 \& University Paris, Hôpital Lariboisière, Paris, France, ${ }^{2}$ Cell and Molecular Neurobiology Laboratory, UPR 9040 CNRS, Gif-sur-Yvette, France

Recent clinical trials showed that strontium ranelate is an effective treatment for osteoporosis. We previously showed that strontium ranelate stimulates cell replication and type I collagen synthesis in rat or mouse osteoblastic cells. However, the cellular mechanisms by which strontium ranelate may act on osteoblasts 
are not fully characterized. We hypothesized that strontium ranelate may promote cell proliferation through activation of the membrane calcium sensing receptor (CaSR) expressed in osteoblasts. To test this hypothesis, we determined the effects of strontium ranelate in osteoblastic cells isolated from wild type $\left(\mathrm{CaSR}^{+/+}\right)$and $\mathrm{CaSR}$ null $\left(\mathrm{CaSR}^{-/}\right)$mice. Primary mouse calvaria pre-osteoblasts were obtained from the first and second collagenase digestions and used for DNA synthesis determination. We found that strontium ranelate and calcium $(1-3 \mathrm{mM})$ increased DNA synthesis at 24-72 $\mathrm{h}$ in pre-osteoblasts from both $\mathrm{CaSR}^{+/+}$and $\mathrm{CaSR}^{-/}$mice, as evaluated by tritiated thymidine incorporation and BrdU ELISA assay. Activation of MAPKs ERK $1 / 2$ is an important signaling pathway acting downstream of $\mathrm{CaSR}$, which is involved in the control of cell replication. We therefore determined whether strontium may activate ERK1/2 and whether this effect is dependent on CaSR expression. In preosteoblasts from $\mathrm{CaSR}^{+/+}$mice, calcium and strontium $(0.5-10 \mathrm{mM})$ rapidly $(10 \mathrm{~min})$ and markedly (3-fold) increased phospho-ERK1/2 levels, as shown by western blot analysis. In contrast, calcium or strontium at the same doses had no effect on phospho-ERK1/2 in pre-osteoblasts from CaSR-null mice, even after prolonged treatment for $30 \mathrm{~min}$. These results show that 1) CaSR expression is not essential for the effect of strontium ranelate on mouse calvaria pre-osteoblast replication, 2) strontium activates ERK1/2 via CaSR in primary pre-osteoblasts, and 3 ) the stimulatory effect of strontium ranelate on pre-osteoblast replication is independent of CaSR-mediated ERK1/2 activation. This work shows that strontium ranelate can stimulate ERK1/2 via the CaSR in osteoblasts. However, the results obtained in the CaSR-null mice allow us to conclude that a receptor distinct from $\mathrm{CaSR}$ could also be involved together with $\mathrm{CaSR}$ in the strontium ranelate effect on the pre-osteoblast replication in mouse primary calvaria cells.

\section{P343. ALTERATIONS OF CORTICAL AND TRABECULAR ARCHITECTURE ARE ASSOCIATED WITH FRACTURES IN POSTMENOPAUSAL WOMEN, INDEPENDENTLY OF DECREASED BMD. THE OFELY STUDY}

E. Sornay-Rendu, S. Boutroy, F. Munoz, P.D. Delmas; Inserm Unit 403 and Université Claude Bernard Lyon1, Lyon, France

Objective: Alterations of bone architecture contribute with low bmd to skeletal fragility, but the independant contribution of bmd, cortical and trabecular architecture to the risk of fracture in postmenopausal women has not been evaluated. We assessed the role of low bmd and impaired architecture in postmenopausal women with fractures.

Methods: We have performed a case-control study in those women from the ofely study who had measurement of density and architecture at the distal radius and tibia with hr-pqct (xtreme ct, scanco medical ag, bassersdorf, switzerland)1. Fifty seven women (mean years $72.0 \pm 5$ ) who sustained a fragility fracture during the 13 years follow-up of the study were age-matched with two controls without fracture. All fractures were radiographically confirmed and vertebral fractures were evaluated on spine radiographs every 4 years. Bmd was measured by dxa at the same visit, at the same site at the radius and at the total hip (qdr 1000+ and 4500, hologic).

Results: There were 50 peripheral fractures in 45 women, 18 vertebral fractures in 14 women and 2 women had both types of fractures.

\begin{tabular}{llllll}
\hline & Dtota & Dtrabb & Cort thc & Tbnd & Tb the \\
\hline Fractures & $222.4 \pm 52.1$ & $105.4 \pm 33.3$ & $0.513 \pm 0.15$ & $1.34 \pm .0 .26$ & $0.065 \pm .0 .01$ \\
Controls & $266.9 \pm 65.7$ & $131.2 \pm 38.4$ & $0.614 \pm 0.18$ & $1.51 \pm 0.32$ & $0.073 \pm 0.02$ \\
$\mathrm{P}$ & $<0.0001$ & $<0.0001$ & 0.0006 & 0.0009 & 0.013 \\
P adjusted & 0.0002 & 0.0004 & 0.01 & 0.015 & 0.015 \\
$\begin{array}{l}\text { for radius } \\
\text { bmd }\end{array}$ & & & & \\
\hline
\end{tabular}

Total a, trabecular b, volumetric bone density, cortical thickness c, trabecular number $\mathrm{d}$ and thickness e
Similar results were found at the distal tibia and $p$ values for dtot, tbn and th remained significant after adjustment for hip bmd.

Conclusion: In postmenopausal women, vertebral and nonvertebral fractures are associated with low volumetric bone density and architectural alterations of trabecular and cortical bone that can be assessed by a non invasive method. These quantitative and qualitative alterations are independent -especially at the radius-of areal bmd assessed by dxa.

Boutroy, S. et al. J clin endocrinol metab 2005; 90:6508-15

\section{P344. MINERAL METABOLISM AND ITS HORMONAL REGULATION IN STRESSED MOTHERS' OFFSPRING}

L.Y. Sergienko, G. Gerashchenko, N. Malova, L. Pivovarevich, O. Kartavceva, T. Bondarenko, G. Cherevko; Department of Histopathology, Institute of Endocrine Pathology Problems, Kharkov, Ukraine

Objective: It has been shown that maternal stress during gestation generates a risk of developing metabolic disorders and endocrine dysfunctions in offspring further life. Our recent investigations confirmed the presence of elevated basal or stressinduced plasma corticosterone and insuline, low level of sexhormones and manifested reduction of reproductive function in these offspring. We also discovered that short immobilization caused formation of osteoporosis in them.

The aim of this study was to investigate the level of calcium (Ca), phosphorus $(\mathrm{P})$, magnesium $(\mathrm{Mg})$ in comparison with calcitonin $(\mathrm{CT})$, parathyroid $(\mathrm{PTH})$ in blood and osteogenic processes in female offspring of stressed mothers.

Material and Methods: 45 female pups were received from pregnant Wistar rats which were daily exposed to social-emotion stress starting from 2 up to 8 day of pregnancy and randomly divided into 3 groups $(\mathrm{G}-1,-2,-3)$. The rats of $\mathrm{G}-1$ were killed at 35 days old, G-2 - at 90 days old, G-3 - at 12 months old. The offspring of intact mothers of the same age and sex were used as control rats $(\mathrm{C})$. The hormone concentrations were determined by the RIA method. $\mathrm{Ca}, \mathrm{P}, \mathrm{Mg}$ were measured spectrophotometrically. The histological examination of the bone was performed by microscope "Olimpus" with a computer analyzer of microstructures.

Results: It has been established that $\mathrm{Ca}, \mathrm{Ca} 2^{+}$in plasma were decreased by $20 \%$ only in G-2 vs same age intact rats $(\mathrm{p}<0,02)$; offspring of G-1 had more eminent $\mathrm{P}$ and $\mathrm{Mg}$ concentration than corresponding control $(p<0,05)$ but in G-2 these informers were decreased (by $30 \%$ and $55 \%$ compared with $\mathrm{C}$ ). P level in G-3 exceeded the control informer $(\mathrm{p}<0,05)$ but $\mathrm{Mg}$ was identical G-1. Offspring had low PTH level $(p<0,02$ vs $C)$ but in animal of G-2 and G-3 PTH was increased $(p<0,02)$. Calcitonin in $\mathrm{G}-2$ also increased but in 12-months old its level decreased (to $16.21 \pm 0.75 \mathrm{nmol} / \mathrm{ml})$. Pathological examination showed that there are many cartilages in bone trabeculas of model groups G-1 and G-2. Rats of G-2 had thined bone cortex and trabeculas with poor integrity, distorted, broken and decreased in size. G-3 was characterized by numerous areas of bone recorption in hip cortex.

Conclusion: Maternal stress in early pregnancy disturbs hormonal regulation of mineral metabolism and causes osteopathology in female offspring.

\section{P345. BONE DENSITY IN RHEUMATOID ARTHRITIS: RELATIONSHIP WITH ISOKINETIC ANKLE MUSCLE STRENGTH}

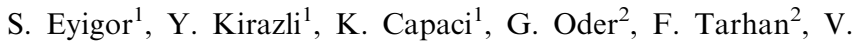
Inal $^{2} ;{ }^{1}$ Ege University Faculty of Medicine Physical Therapy and Rehabilitation Dept, Izmir, Turkey, ${ }^{2}$ Ege University Faculty of Medicine Internal Medicine Dept Rheumatology Division, Izmir, Turkey

Objective: The aim of this study is to examine relationships of bone mineral density (BMD, $\mathrm{g} / \mathrm{cm}^{2}$ ) with ankle isokinetic muscle strength (IMS) in rheumatoid arthritis (RA).

Material and Methods: Twenty-one patients (mean age; $53,90 \pm 12,43$ year, mean disease duration; $12,58 \pm 5,92$ years, 
body mass index; $26,69 \pm 3,60 \mathrm{~kg} / \mathrm{m} 2$ ) diagnosed as RA according to the 1987 criteria of the American Rheumatism Association were selected consecutively from a rheumatology outpatient clinic. Twenty healthy subjects (mean age; 58,90 $\pm 11,68$ year, body mass index; $26,04 \pm 3,90 \mathrm{~kg} / \mathrm{m} 2$ ) matched for gender and age, participated in the study as a control group. Isometric and isokinetic muscle strength were measured in ankle plantar- and dorsiflexion by using an isokinetic dynamometer (Cybex II). BMD of the spine, femoral neck and total femur was measured by dual energy X-ray absorptiometry (DEXA).

Results: There are no significant differences for age, weight, height, body mass index, lumbar and femur BMD values, or any of the isokinetic, or isometric strength measures between the groups $(p>0.05)$. Femoral neck and total femur BMD were correlated with ankle isokinetic measurements both in RA and control groups $(p<0.05)$. The correlation between the ankle dorsiflexor muscle strength and BMD values appeared to be stronger compared with ankle plantar flexor muscle strength. In the RA group, disease duration was correlated with BMD values of all regions $(\mathrm{p}<0.05)$.

Conclusions: Ankle isokinetic muscle strength was correlated with femoral neck and total femur BMD in RA and healthy controls. This finding indicates that muscle strengthening exercises may prevent bone loss not only in RA but also in healthy subjects.

\section{P346. PREFERENCE TO THE THERAPEUTIC SCHEME IN OSTEOPORO- TIC PATIENTS WITH INDICATION TO START BISPHOSPHONATE TREATMENT}

B. Samdanis, S. Tournis, Y. Dionyssiotis, E. Papakitsou, A. Katsalira, D. Economopoulos, A. Galanos, G.P. Lyritis; Laboratory of Research Musculoskeletal System, University of Athens, KAT hospital, Kifissia, Greece

Osteoporosis is a metabolic disease that decreases the mechanical strength of bone tissue, thus increasing the risk of fragility fractures. Therapeutic schemes for osteoporosis are divided in daily, weekly and monthly.

Objectives: To examine the preference to the therapeutic scheme in osteoporotic patients with indication to start bisphosphonate treatment.

Materials and Methods: 349 postmenopausal women, receiving osteoporosis treatment for more than 6 months, were asked to complete a questionnaire regarding:

a) Their intention to begin an alternative therapeutic scheme

b) Their preference to weekly or monthly therapeutic scheme

Results: $284(81,4 \%)$ would rather change their treatment, whilst $45(12,9 \%)$ stated their intention to continue their recurrent treatment. Furthermore $286(81,9 \%)$ answered that they would definitely prefer a monthly applied treatment, $41(11,7 \%)$ would probably take up such a scheme, $1(0,3 \%)$ was uncertain, $4(1,1 \%)$ were probably not keen on changing their weekly therapy and 2 $(0,6 \%)$ answered that they would definitely not change their weekly scheme.

Conclusion: $81,4 \%$ of the population would rather change their current treatment. Moreover the monthly treatment seems to be more attractive to osteoporotic patients, since $93,6 \%$ stated that they would definitely or probably prefer such a scheme, compared to the weekly one.

\section{P347. THE DURATION OF STAY IN TREATMENT IN GREEK POST- MENOPAUSAL OSTEOPOROTIC WOMEN}

D. Economopoulos, S. Tournis, Y. Dionyssiotis, E. Papakitsou, A. Katsalira, B. Samdanis, A. Galanos, G.P. Lyritis; Laboratory of Research Musculoskeletal System, University of Athens, KAT hospital, Kifissia, Greece

Osteoporosis treatment is crucial for the maintenance of a good quality of life by reducing the risk of potential complications, such as fractures and immobilization. Compliance to treatment is an important issue, essential for the preservation of an acceptable therapeutic result.

Objectives: To examine retrospectively the compliance of osteoporotic women, undergoing treatment for a time period of 2 years.

Materials and Methods: From 783 community dwelling women, who expressed an interest in osteoporosis, 460 patients suffered from osteoporosis and were undergoing treatment. They were asked to fill a questionnaire regarding their duration of stay in treatment. Information about the participant's therapy adaptation, over a 6 month, 1 year, 2 years and more than 2 years period, were collected, as well as information about the probable reasons leading to an early therapy discontinuation.

Results: $349(75,9 \%)$ women received treatment for a time period of at least 6 months, $323(70,2 \%)$ for a year, $298(64,7 \%)$ for 2 years, whilst $221(48 \%$ ) complied to treatment for more than 2 years. Regarding the cause of early discontinuation, $26(7,4 \%)$ stopped their treatment following doctor's orders, 95(27,2\%) because they had difficulties receiving their treatment, $15(4,3 \%)$ because they feared of future medication side effects, $40(11,5 \%)$ interrupted on their own decision, $30(8,6 \%)$ due to inconvenience in receiving treatment, $2(0,6 \%)$ ceased treatment for no reason, whereas $141(40,4)$ continued their therapeutic scheme.

Conclusion: The documented compliance to any osteoporosis treatment was better, compared to other studies, but still rather low. A constant decrease of therapy uptake has been documented amongst the time periods. Furthermore, a difficulty in receiving treatment seems to be the primary cause for early discontinuation.

\section{P348. VITAMINE D DEFFICIENCY IN SUBJECTS OVER 60 YEARS OF AGE IN A TROPICAL COUNTRY: VENEZUELA}

G. Riera-Espinoza, J. Ramos, F. Riera, L. Marcano, M. Naressi; UNILIME Hospital Universitario Angel Larralde, Universidad de Carabobo, Valencia, Venezuela

In the human being the vitamin $\mathrm{D}$ increases the absorption of calcium and phosphorus in order to properly mineralize the skeletum. In vitamin D deficit condition, bone is not mineralized suitably and bone diseases take place. Recently a vitamin D deficit has been observed as much in adolescents as in adults and, mainly, in old people. The vitamin D supplements produce a reduction of PTH, diminish the bone remodelation and increase the bone mineral density and are able to diminish the risk of fractures. Vitamin D status is an important factor for understanding mineral metabolism and bone diseases. Corporal reserves of vitamin D are estimated by serum levels of $25(\mathrm{OH}) \mathrm{D} 3$. So called "reference values of 25(OH) D3" are controversial and affected mainly by sunlight exposure, latitude, seasons and diet. The objective is to determine the values of vitamin $\mathrm{D}$ in men of more than 30 years old with low bone mass determined by DXA. The 25-hydroxyvitamin D (25-OH D) levels were measured by enzymeinmmunoassay (OCTEIA 25-Hydroxyvitamin D. IDS.UK). Samples were taken from venous blood in the fasting state, frozen at $-70^{\circ} \mathrm{C}$ and measured in duplicate at the same time. We analyzed 45 male patients with $60.18 \pm 12.45$ years, the Bone Mineral Density (BMD) at total Hip was $0.901 \pm 0.123 \mathrm{~g} / \mathrm{cm}^{2}$, T-score $-1.35 \pm 0.99$; BMD Femoral Neck $0.836 \pm 0.106 \mathrm{~g} / \mathrm{cm}^{3} \mathrm{~T}$-score $-1.7 \pm 0.82$; BMD L1-L4 $1.044 \pm 0.149$ T-score $-1.4 \pm 1.24$; the serum calcium level was $8.88 \pm 0.39 \mathrm{mg} / \mathrm{dl}$; Range $1 \pm$ DS $8.49-$ 9.27 with minimum values and maximum 8.50 and $9.90 \mathrm{mg} / \mathrm{dl}$; vitamin D $52.062 \pm 15.04 \mathrm{ng} / \mathrm{dl}$. Range $1 \pm$ DS $37.02-67.10$ with minimal and maximal values 80 and $250 \mathrm{ng} / \mathrm{dl}$. While co-relation the Vitamin D values with age, BMD and serum calcium levees, there were not significant statistical differences $(\mathrm{E} r=0.134$ $\mathrm{p}=0.381 ;$ TH $\mathrm{r}=0.680 \mathrm{p}=0.678 ; \mathrm{FN} \mathrm{r}=0.015 \mathrm{p}=0.922 ; \mathrm{L} 1-\mathrm{L} 4$ $\mathrm{r}=0.077 \mathrm{p}=0.613 ; \mathrm{CS} \mathrm{r}=-0.109 \mathrm{p}=0.477)$. In conclusion the Vitamin D levels in our sample were near to the Venezuelan premenopausal women average levels $(52.86 \pm 24.28 \mathrm{ng} / \mathrm{ml}$. Range \pm SD 28.58-77.14), regarding the normal values of serum calcium regardless of being patients with low level bone density. 
P349. FALECALCITRIOL IN THE TREATMENT OF HYPERPARATHYROIDISM IN HEMOIDIALYSED PATIENTS

H. Morii, Osaka City University Emeritus Professor, Japan

Falecalcitriol (26,27-hexafluoro-1,25-dihydroxyvitamin D3, F6) has been approved for the treatment of secondary hyperparathyroidism, hypoparathyroidism and osteomalacia since 2001 in Japan. It wss developed as a vitamin D analog for the treatment of bone and caclium abnrmalities in (Tanaka Y et al, Arch Biochem Biophys 229:348-354, 1984). This compound has 6 atoms at 26 and 27 positions in the secosterol structure.

It is converted to 1,23,25-trihydroxy-hexafluoro-vitamin D3 in the cell which has equivalent acivity as the mother compound and thus the total activity is fortified. Another advantage of this compound is that the surppressive effect of PTH is more marked compared with the hypercalcemic effect in relation to activity of calcitriol.

At the end of 2004 there were 248,816 hemodialysed patients in Japan. Among those F6 was prescribed to 4,816 (1.94\%), alfacalcidol $(\alpha \mathrm{D} 3)$ to $52,471(21.14 \%)$ and calcitriol to 17,301 patients $(6.97 \%)$. Most frequently prescribed range of intact PTH level was $200-360 \mathrm{pg} / \mathrm{ml}$ for 3 kinds of vitamin D analogs, but the frequency was $32.1 \%$ for $\mathrm{F} 6,19.3 \%$ for $\alpha \mathrm{D} 3$ and $21.0 \%$ for calcitriol. Most frequently prescribed range of serum calcium for 3 kinds of analogs was $4.5-5.0 \mathrm{mEq} / 1$ and the frequency was $20.7 \%$ for $\mathrm{F} 6,22.6 \%$ for $\alpha \mathrm{D} 3$ and $24.5 \%$ for calcitriol. The incidence of history of myocard infaction was $4.6 \%$ for $\mathrm{F} 6,5.4 \%$ for $\alpha \mathrm{D} 3$ and $5.0 \%$ for calcitriol. The incidence of history of brain infaction was $7.6 \%$ for $\mathrm{F} 6,10.1 \%$ for áD3 and $9.4 \%$ for calcitriol.

In conclusion F6 has been prescribed for patients with relatively definite range of intact PTH compared with other vitamin D analogs. The incidence of history of myocard and brain infaction was the least for F6 compaed with other 2 vitamin D analogs.

\section{P350. FRACTURE FREQUENCY IN PATIENTS WITH ENDOCRINO- PATHIES IN ULTRAVIOLET DEFICIENCY AREA}

M. Nekrasova, L. Suplotova; Tyumen Medical academy, Tyumen, Russia

Objectives: Osteoporosis is the main cause for the low traumatic fractures. It's especially actual for inhabitants of West Siberia, where annual sunny days amount is very small because of its geographical situation in hifh latitudes from 55 to 73 degree. So, West Siberia region takes the first place in osteoporotic fractures frequency in Russia.

Aims: To study osteoporotic fractures frequency in patients with endocrine dysfunction in ultraviolet deficiency area.

Materials and Methods: We studied anamnestically fractures frequency in 577 West Siberian patients with endocrinopaties, icluding 111 persons with thyrotoxicosis (TT), 219 hypothyroid patients (HT), 123 - with diabetes mellitus type 1 (DM) and 124 patients, taking glucocortiicoids (GK) because of bronchial asthma. Comparison group (CG) consisted of 159 practically healthy men and women.

Results: In general fractures were marked in $14,4 \%$ patients with endocrine dysfunction against $5 \%$ in $\mathrm{CG}(\mathrm{p}=0,003)$; in TT $-9,9 \%(p=0,123)$, HT $-11,9 \%(p=0,021)$, DM $-12,2 \%$ $(\mathrm{p}=0,029), \mathrm{GK}-22,6 \%(\mathrm{p}=0,000)$. In TT, HT and GK groups we found positive age correlation $\left(r_{1}=0,28 ; r_{2}=0,3 ; r_{4}=0,3\right.$, $\mathrm{p}<0,05)$ and menopausal status $\left(r_{1}=0,29 ; r_{2}=0,3 ; r_{4}=0,34\right.$, $\mathrm{p}<0,05)$; in TT and GK patients - correlation with main disease severity $\left(r_{1}=0,41 ; r_{4}=0,32, p<0,001\right)$. Icreasing TT severity was assosiated with significant fracture risk growth $(\mathrm{OR}=20,1[2,1$ $189,9])$. In HT patients fracture risk increases twice according to high TSH level $(\mathrm{OR}=2,62 \quad[1,02-6,70])$ : maximal fractures frequency in this group $(17,6 \%)$ was registered in high TSH patients $(p=0,043)$. Fracture risk factors in DM were the age of the diabetes begining $(p=0,034)$ and disease duration $(p=0,014)$. HвA1c level wasn't found significant influence on fractures incidence. In GK group the most fractures frequency was registered in oral GK users $(50 \%)$, that's considerably larger CG level $(5 \%, p=0,001)$. Inhaled and combined (oral + inhaled) GK therapy lead to fracture incidence in $18 \%$ and $36 \%$ accordingly $(\mathrm{p}=0,082)$. In general farcture chance in oral GK users is higher than inhaled $(\mathrm{OR}=4,6[1,3-16,1])$ and combined GK patients $(\mathrm{OR}=9,7[2,0-47,1])$.

Conclusion: Endocrine dysfunction increase osteoporotic fracture risk in West Siberia inhabitants.

P351. THE CORRELATION OF METABOLIC SYNDROME AND OSTEOPOROSIS IN THE ELDERLY

M. Halil ${ }^{1}$, M. Cankurtaran ${ }^{1}$, B.B. Yavuz ${ }^{1}$, Z. Ulger ${ }^{2}$, S. Ariogul ${ }^{1}$; ${ }^{1}$ Division of Geriatrics, Department of Internal Medicine, Faculty of Medicine, Hacettepe University, Ankara, Turkey, ${ }^{2}$ Department of Internal Medicine, Faculty of Medicine, Hacettepe University, Ankara, Turkey

Objectives: Both metabolic syndrome (MS) and osteoporosis (OP) are very common in the elderly. Growing evidence links vascular and bone diseases. These two diseases may share an etiologic factor such as dyslipidemia or inflammation. Although this association is often dismissed as a consequence of aging, elucidation of the relationship between MS and OP may be helpful in understanding the common etiological mechanisms.

Material and Methods: Patients aged 65 years and over without an acute illness that were referred to our geriatric medicine outpatient clinic for comprehensive geriatric assessment were included in this cross-sectional study. MS was defined by using the WHO and the National Cholesterol Education Program (NCEP) definitions. The bone density was measured by dual energy X-ray absorptiometry (DXA) using a Hologic QDR- 4500A bone densitometer. Measurements included BMD of the lumbar spine (L1L4) and femoral neck. T-scores equal to or below -2.5 at both lumbar spine and femoral neck were defined as osteoporosis.

Results: Total 1255 patients, $789(62.9 \%)$ females, 466 $(37.1 \%)$ males with a mean \pm SD age of $71.84 \pm 6.29$ years were included in our study. MS prevalence was $16.2 \%$ with WHO definition and $23.8 \%$ with NCEP definition. Mean levels of triglyceride and body mass index were lower in OP patients ( $p$ values were non-significant) while HDL cholesterol was significantly higher in OP patients. The percentage of OP in patients without MS and with MS was; $59.1 \%$ vs. 50.7 in WHO $(p=0.027)$ and $59.4 \%$ and $52.4 \%$ in NCEP $(p=0.035)$ groups, respectively.

Conclusion: In this cross-sectional study we found that OP is less common in patients with MS than the patients without MS. This result is similar to the previous studies. Although an association between dyslipidemia and OP is shown in previous studies, our results did not support this. The complex relationship of OP with MS and MS components must be evaluated in prospective studies.

\section{P352. CLINICAL AND BIOLOGICAL SAFETY OF LONG TERM USE OF CALCIUM AND VITAMIN D SUPPLEMENTATION IN ELDERLY WOMEN WITH VITAMIN D INSUFFICIENCY}

M. Brazier ${ }^{1}$, S. Kamel ${ }^{1}$, F. Grados ${ }^{1}$, M. Mathieu ${ }^{2}$, P. Fardellone ${ }^{1}$; ${ }^{1}$ Departments of Biochemistry and Rheumatology, University Hospital Center, Amiens, France, ${ }^{2}$ Innothera Laboratories, Arcueil, France

The need for calcium increases with aging, whereas dietary intake and digestive absorption are simultaneously reduced. Moreover, in the Elderly, the digestive malfunction is majored by hypovitaminosis $\mathrm{D}$. This explain the use of calcium and vitamin D as regular treatment in the Elderly. Despite its very common use, few studies have focused on long term calcium and vitamin D supplementation safety. The purpose of the present study was to assess the clinical and biological safety (with special attention to renal function) of a one-year course of treatment with calcium and vitamin $\mathrm{D}$ in a combined formulation in ambulatory women over 65 years with vitamin D insufficiency. 192 women $(74.6 \pm$ 6.9 years; $64.0 \pm 12.5 \mathrm{~kg}$ ) with vitamin D insufficiency [25(OH)D $<12 \mathrm{ng} / \mathrm{ml}$ ] were included in a randomized, double blind, placebocontrolled, multicenter study conducted in France. Women took 
either a combination of $500 \mathrm{mg}$ of calcium carbonate and $400 \mathrm{IU}$ of vitamin D3 in one tablet, or a matching placebo twice daily. Adverse events were recorded over the whole treatment period. Blood and urine samples were collected at baseline and during the course of treatment for laboratory tests, and in particular measurement of creatinine and uric acid levels. Comparison between groups were done using parametric (t test, Chi2) or non-parametric (Wilcoxon) tests. Among the 192 women (97 in the placebo group and 95 in the calcium/vitamin D group), 50 were prematurely withdrawn for various reasons, but without difference between the two groups in this respect. Respectively, $23(24.0 \%)$ and $21(22.1 \%)$ women presented treatment-related adverse events $(\mathrm{p}=0.86)$, mainly metabolic disorders and in particular hypocalcemia, and gastrointestinal disorders. No major complications directly related to the calcium and vitamin $\mathrm{D}$ supplementation occurred. As expected, we observed in women from the calcium + vitamin $\mathrm{D}$ group, an increase in calcium urinary excretion and $25(\mathrm{OH}) \mathrm{D}$ serum level (more than $90 \%$ with $25(\mathrm{OH}) \mathrm{D}>12 \mathrm{ng}$ $\mathrm{ml}$ ), associated with a decrease in intact-parathyroid hormone (about $80 \%$ with normalized values). Although renal function was not altered, the number of women with elevated serum $(\mathrm{p}=0.046)$ and urine $(p=0.08)$ uric acid levels significantly increased in the calcium + vitamin D group. Long term supplementation with calcium and vitamin D appeared to be well tolerated in this population, even though impaired uric acid metabolism was reported in more than half of the subjects in the trial.

P353. MISSION STUDY: PREVALENCE OF OSTEOPOROTIC FRACTURES IN FRANCE, IN 6841 MENOPAUSAL WOMEN WITH OR WITHOUT HORMONE REPLACEMENT THERAPY

P. De Reilhac ${ }^{1}$, M.-C. Micheletti ${ }^{2}$, T. Chevallier ${ }^{2}$, J.-Y. Reginster ${ }^{3}$ and the MISSION Group; ${ }^{1}$ Honorary President of F.N.C.G.M., Nantes, France, ${ }^{2}$ Laboratoire Théramex, Monaco, Monaco, ${ }^{3}$ World Heath Organisation (WHO) Collaborating Centre for Public Health Aspects of Rheumatic Diseases and Osteoporosis, Liège, Belgium

This historico-prospective study is being performed by the F.N.C.G.M., under the guidance of a WHO Collaborating Centre for Public Health Aspects of Rheumatic Diseases and Osteoporosis, with a group of experts and in collaboration with Theramex-Merck Laboratories (1). Its aims are to determine in France : in the first instance (historical approach), the prevalence of breast cancer (2) and of diseases such osteoporotic fractures, etc., in menopausal women under gynaecologists' care and treated or not treated with a "French" type of Hormone Replacement Therapy (HRT) ; then, secondly (prospective phase), the incidence of these pathologies during a follow-up period. Here we present the results of the historical phase concerning osteoporotic fractures.

6841 menopausal women have been recruited: 3509 treated and 3362 untreated. The mean age is 60.7 years old. The average age of menopause onset is 50 years old. The mean BMI is similar in the 2 groups (25). The mean duration of HRT is 8 years. $74 \%$ of the treated women receive cutaneous estradiol (E). Of the women taking a progestative supplement to $\mathrm{E}, 37.4 \%$ receive progesterone and $62.6 \%$ receive retroprogesterone or a norpregnane or pregnane derivative (excluding MPA and nor-testosterone derivatives).

Prevalence of osteoporotic fractures.

\begin{tabular}{llll}
\hline Type & $\begin{array}{l}\text { Treated Group } \\
\mathrm{n}(\%)\end{array}$ & $\begin{array}{l}\text { Untreated } \\
\text { Group n (\%) }\end{array}$ & $\mathrm{p}$ \\
\hline Femoral neck & $7(0.2)$ & $14(0.4)$ & 0.1 \\
Wrist & $48(1.4)$ & $97(2.9)$ & $<0.0001$ \\
Lumbar spine & $17(0.5)$ & $37(1.1)$ & 0.005 \\
\hline
\end{tabular}

There were significantly less osteoporotic fractures (wrist and lumbar spine) in the treated group than in the untreated. The protective effects of HRT against fracture in menopausal women have been reported in WHI study (3). In the MISSION study, the possible protective anti-fracture role of « French » HRT will only be able to be demonstrated in the prospective phase which is currently ongoing.

(1) T Chevallier, et al. J Gynecol Obstet Biol Reprod, 2005

(2) P De Reilhac, et al. Repoduction Humaine et Hormones, 2005

(3) Writing Group for the Women's Health Initiative Investigators, JAMA, 2002.AMA - July 17, 2JAMA002

\section{P354. INTRAVENOUS IBANDRONATE IN ANKYLOSING SPONDILYTI-} S(ASP) OPEN-LABEL

J.J. Scali, J. Salomón, S. Visentini, D. Sevilla, Y. Ju; Rheumatology and Metabolic Bone Diseases Unit, Hospital Durand, Buenos Aires, Argentina

Aim of the Study: To evaluate anti-inflammatory effect and safety profile of Intravenous Ibandronate in pts suffering from ASp with partial response to NSAIDs when given in therapeutic doses.

Inclusion Criteria: Pts with Asp (New York Criteria) with axial or peripheral pain

Exclusion: Hypersensitization to drug, conditions with alterations of Phosphocalcic metabolism.

Treatment: Ibandronic Acid, $2 \mathrm{mg}$ given in a slow IV infusion, dilution in $500 \mathrm{cc}$ of dextrosa $5 \%$ solution, each 2 months during 6 months.

Analysis: At baseline and at the end of the study: comparison of variables by means of Wilcoxon's test.

Material \& Methods: 20 patients (19 men and 1 woman, average age: 55 years old) suffering from ASp (average standing of the disease: 14 years-between: 5 to 41 years), all pts with sacroiliac involvement (MRI confirmed), 19 pts having HLAB27 positive and 6 pts with uveitis, and all pts with refractory response to NSAIDs in therapeutic doses. Pts were on Methotrexate treatment (4 pts), Sulfasalazine: 4 pts, on prednisone: 2 pts, and the rest only with NSAIDs. 10 patients were pensioned because of their handicapped condition and 3 pts over 55 years old were unemployed.

Evaluation: By means of BASDAI y BASFI questionnaries, Schober's Test, Torax Expansion measured in $\mathrm{cm}$, distance fingers-floor, occipucio-wall and laboratory (CRP, ESR).

Results: At the end (6 months) BASDAI fall in $35.5 \%$ $(p=0.002)$, BASFI, ESR and CRP also reduced but no significantly comparing with baseline.

Side effects: Arthralgias/Myalgias in the following days to pulses (almost between 2 to 8 days) in $30.0 \%$ of pts, both symptoms well tolerated for the patients, no GI or renal-urinary symptoms. No changes in Blood pressure or alterations in Cardiovascular System.

Conclusions: This preliminary report indicated that some cases with ASp may benefit with Ibandronate therapy.
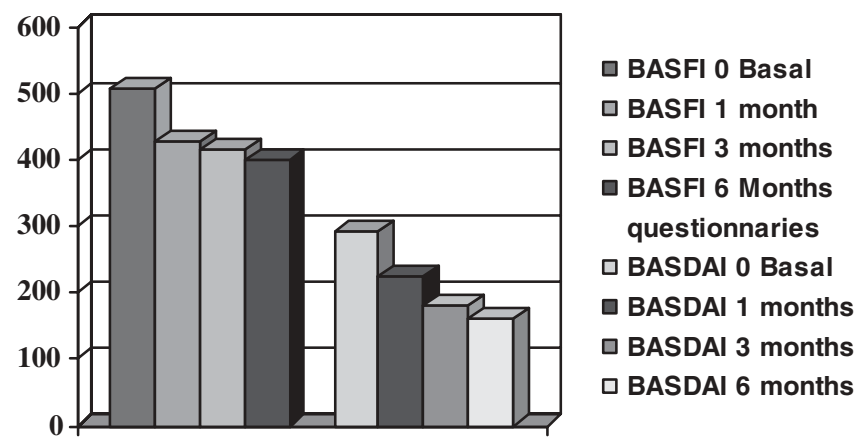

References:

1. Maksymowych W, et al Arthritis Rheum 2002;46:766-773: A six-month randomized, controlled, double blind dose response comparison of intravenous pamidronate $(60 \mathrm{mg}$ versus $10 \mathrm{mg}$ ) 
in the treatment of nonsteroidal antiinflam-matory drug-refractory ankylosing spondylitis"

2. Pfister T, Atzpodien E, Bauss F: Intermittent Intravenous dosing: effects of ibandronate and zoledronate on renal histopathology and function in the rat, Heidelberg Universuty, Mannheim, Germany

\section{P355. PARADOXAL RESULTS IN FRACTURE RISK RATIO ON DIABETES} TYPE I \& II (PRELIMINARY REPORT)

J.J. Scali, J. Salomón, S. Visentini, A. Martinez; Rheumatology \& Metabolic Bone Diseases Unit, Hospital Durand, Buenos Aires, Argentina

We studied diabetic population of our Hospital, and data was collected separately on pts on Diabetes Type I and DB Type II, matched by sex, age (50-78 years old), body weight, lifestyles (use of alcohol or cigarettes, sedentarism, etc) race/ethnic and economic background Pts with another metabolic or potentially osteoporotic disease were excluded from this analysis. None of pts were taking any specific bone active or anti resorptive therapy. Very few of the women on insuline were taking hormone therapy $(<4 \%)$. There was no differences in baseline general characteristics for the two groups of diabetes. Group on insuline has Mean Lumbar Spine (LS) BMD T-score of -2.9 (Men) and -3.4 (Women) and mean femoral neck BMD t-score was -2.1 (men) and -2.9 (women). For the group without insuline LS BMD T score was -2.0 (men) and -2.7 (women) and mean femoral neck BMD T-score was -1.7 (men) and -2.5 (women). Distributions of LS T scores stratified by presence/ absence of prevalent fragility fracture are shown in table II. The relative risk of osteoporosis defined by absolute risk was 1.6 (CI 0,$012 ; 2.86$ ) for pts on insuline prolonged therapy. This was significant for men, women and for the group as a whole during the 5 years follow-up of the study.

Table 1 BONE MINERAL DENSITY, DXA and RX in Diabetic Population

\begin{tabular}{|c|c|c|c|c|c|c|}
\hline \multirow[t]{2}{*}{ N: 114} & \multicolumn{3}{|c|}{ Men: 64} & \multicolumn{3}{|c|}{ Women: 50} \\
\hline & BMI & $\operatorname{OPDXA}(+)$ & $\begin{array}{l}\text { OPRx } \\
(+)\end{array}$ & BMI & OPDXA(+) & $\begin{array}{l}\text { OPRx } \\
(+)\end{array}$ \\
\hline $\begin{array}{l}\text { on insuline } \\
\text { n: } 46 \text { men, } \\
\text { n: } 32 \text { women }\end{array}$ & 26.1 & $11 / 45(23 \%)$ & $\begin{array}{l}0 / 10 \\
(0 \%)\end{array}$ & 25.4 & $\begin{array}{l}8 / 21 \\
(25 \%)\end{array}$ & $\begin{array}{l}0.8 \\
(0 \%)\end{array}$ \\
\hline $\begin{array}{l}\text { no insuline } \\
\text { n: } 18 \text { men, } \\
\text { n:18 women }\end{array}$ & 26.5 & $\begin{array}{l}2 / 17 \\
(12 \%)\end{array}$ & $\begin{array}{l}2 / 2 \\
(100 \%)\end{array}$ & 25.8 & $\begin{array}{l}1 / 17 \\
(6 \%)\end{array}$ & $\begin{array}{l}1 / 1 \\
(100 \%)\end{array}$ \\
\hline
\end{tabular}

The bone density was significantly lower on insuline therapy group for men $(p<0.02)$ and for women, $(p<0.00001)$. The diagnosis of osteoporosis by absolute fracture risk reached significance for non-insuline group for women $\mathrm{p}<0.02$ and a trend for men $\mathrm{p}<0.15$.

In the insuline group there was a significant increase in the prevalence of osteoporosis, and yet for both men and women. The uncontrolled treatment rates in men on subcutaneous insuline may reflect more positive evaluation of osteoporosis.

At 5 years of follow-up, the risk factors assessment, fragility fracture prevalence (vertebral and nonvertebral) in diabetic pts over a range of absolute fracture risk ranged from 20 to $44 \%$ in DB Type I demonstrates a low fragility fractures when compared with DBT II, even thinking that pts's BMD are lower in DBT-I than in DBT-II (Paradoxal results)

Conclusion: Pts on insuline therapy souffering from osteoporosis usually are not on any specific bone treatment independently from treatment effectiveness with concurrent insuline or under diagnosis of osteoporosis in both sexes. In the insuline group there was a significant increase in the prevalence of osteoporosis, for both sexes. The bone mineral density (BMD) was significantly lower on the group of insuline therapy for men $(\mathrm{p}<0.02)$ and for women $(\mathrm{p}<0.00001)$ comparing with no insuline group.
Table 2 Absolute OP Fracture Risk DBT-I and on DBT-II up to 5 years (VERTMN \& NA, BMD-MN \& NA)

\begin{tabular}{lllllll}
\hline $\begin{array}{l}\text { AOFR } \\
\begin{array}{l}\text { Up to } \\
5 \text { years }\end{array}\end{array}$ & $\begin{array}{l}\text { Baseline } \\
\text { T-score }\end{array}$ & $\begin{array}{l}\text { Mean } \\
\text { Age }\end{array}$ & $\begin{array}{l}\text { Percent } \\
\text { PVF }\end{array}$ & $\begin{array}{l}\text { DBT-I* } \\
\text { DBT-II } \\
\text { Incidence }\end{array}$ & $\begin{array}{l}\text { Hazard } \\
\text { Ratio }\end{array}$ & p $<$ value \\
\hline $20 \%$ & $\begin{array}{l}<1.5 \text { to } \\
<-2.0\end{array}$ & 61 & $63.2 \%$ & $14 \% 17 \%$ & 0.58 & $<0.05$ \\
$28 \%$ & $\begin{array}{l}<-2.0 \text { to } 68 \\
>-2.5\end{array}$ & $68.6 \%$ & $19 \% 24 \%$ & 0.63 & $<0.01$ \\
$34 \%$ & $<-2.5$ to 68 & $78.5 \%$ & $26 \% 29 \%$ & 0.61 & $<0.005$ \\
$44 \%$ & $>-3.0$ & 73 & $87.8 \%$ & $27 \% 30 \%$ & 0.44 & $<0.001$ \\
\hline
\end{tabular}

The diagnosis of a new fracture, paradoxally and contrary as we expected, reached more significance for non-insuline group for women $\mathrm{p}<0.02$ and a trend for men $\mathrm{p}<0.13$ but it was less significant for insuline group for both sexes. DB Type I demonstrates less fragility fractures than DBT II remembering that the last one has a high BMD scores but also a higher rate of fracture risk when comparing both group. We need to analyze groups in the future including more population to conclude with those interesting preliminary results.

\section{P356. CALCIUM DAILY FOOD INTAKE IN EUROPEAN POST- MENOPAUSAL WOMEN}

O. Bruyere, O. Malaise, A. Neuprez, J.-Y. Reginster; WHO Collaborating Center for Public Health Aspect of Osteoarticular Disorders and Department of Public Health, Epidemiology and Health Economics, University of Liège, Liège, Belgium

Objective: WHO recommendation for calcium intake is $1300 \mathrm{mg}$ daily for postmenopausal women. The objective of this study is to assess the calcium daily food intake in postmenopausal European women.

Material and Methods: Assessment of calcium food intake was performed with a validated questionnaire in 8532 osteoporotic European postmenopausal women. European countries included France, Belgium, Denmark, Italy, Poland, Hungary, United Kingdom, Spain and Germany.

Results: Mean (SD) age of the patients was 74.2 (7.1) years, body mass index was $25.7(4.1) \mathrm{kg} / \mathrm{m}^{2}$. The mean calcium food intake was 744 (332) $\mathrm{mg} /$ day. There was a highly significant difference of calcium food intake across European countries $(\mathrm{p}<0.0001)$. The lowest level of calcium food intake was found in Hungary [503 (247) $\mathrm{mg}$ ] and the highest in United Kingdom [985 (302) $\mathrm{mg}$. In the whole study population, only $6 \%$ of the women have a daily calcium food intake over $1300 \mathrm{mg}$. There was no clinical variation of calcium food intake with age, with mean level of $778 \mathrm{mg} /$ day and $779 \mathrm{mg} /$ day in women aged less than 70 years old and 60 years old, respectively.

Conclusion: This study confirms the low calcium food intake in European postmenopausal women. This implies that the average need of exogenous calcium supplementation in European postmenopausal women should be above $500 \mathrm{mg}$.

\section{P357. IMPACT OF PRIOR BISPHOSPHONATE THERAPY ON THE ANA- BOLIC EFFECTS OF TERIPARATIDE: RATIONALE AND DESIGN OF THE OPTAMISE STUDY}

J.P. Bilezikian ${ }^{1}$, N.B. Watts ${ }^{2}$, P.D. Delmas ${ }^{3}$, P. Miller ${ }^{4} ;{ }^{1}$ Columbia University College of Physicians and Surgeons, New York, NY, USA, ${ }^{2}$ University of Cincinnati College of Medicine, Cincinnati, OH, USA, ${ }^{3}$ Inserm Research Unit 403 and Claude Bernard University of Lyon, Lyon, France, ${ }^{4}$ Colorado Center for Bone Research, Lakewood, CO, USA

Objectives: Some observations suggest that the anabolic response to TPTD may be delayed in patients previously treated with alendronate (ALN) [Ettinger J Bone Miner Res. 2004;19:745] 
while other studies do not [Cosman N Eng J Med 2005 2005;353:566]. Furthermore, it is not known if the extent to which subsequent responsiveness to teriparatide (TPTD) after bisphosphonate (BP) therapy differs between alendronate (ALN) and risedronate (RIS).

Materials/Methods: The Open-label Study to Determine How Prior Therapy with Alendronate or Risedronate in PostMenopausal Women with Osteoporosis Influences the Clinical EffectivenesS of TeriparatidE (OPTAMISE) is a multinational, parallel-group study designed to compare differences in anabolic response to TPTD in postmenopausal women previously treated with either ALN or RIS. Key entry criteria include lumbar spine (LS) or total hip BMD T-score $\leq-2.0$ with a fragility fracture or T-score $<-2.5$, vitamin D replete $(\geq 16 \mathrm{ng} / \mathrm{mL})$, uninterrupted treatment with ALN or RIS for $\geq 2$ years, and urinary NTX $<50 \mathrm{nmol} / \mathrm{mmol}$ creatinine (to insure compliance with previous BP therapy). Subjects $(n=290)$ are being stratified and paired based on prior BP use and duration of BP treatment. After enrollment, subjects discontinue BP treatment and receive daily TPTD injections $(20 \mathrm{mg})$ for 12 months. Baseline and post treatment measures of bone formation are obtained throughout the study, with particular interest in the early anabolic response to TPTD at 3 and 6 months. The effects of TPTD on BMD and measures of bone strength are also being assessed.

Conclusion: OPTAMISE is the first study to examine prospectively and compare the anabolic effects of TPTD after ALN or RIS therapy, and addresses the question of whether the initial choice of BP therapy influences the anabolic response of TPTD.

\section{P358. POSTMENOPAUSAL OSTEOPOROTIC WOMEN PREFER LESS FREQUENT DOSING FOR BISPHOSPHONATES}

M. Walker ${ }^{1}$, A. Carr ${ }^{2}$, W. Cowell ${ }^{1} ;{ }^{1}$ Roche Products Limited, ${ }^{2}$ Clinimatrix Limited, UK

Objectives: Bisphosphonates are now available at daily, weekly and monthly dosing frequencies. This study aimed to establish patient preference for dosing frequency for bisphosphonate therapy and to elicit the reasons for that notional preference.

Methods: A telephone interview survey in the UK of postmenopausal women aged over 50 years was conducted. Women were recruited via an advertising campaign (radio, press, osteoporosis patient organisations). Eligible women were: bisphosphonate naïve, current bisphosphonate users and lapsed bisphosphonate users. The interview was based on three validated questionnaires and elicited: preferences and valuations for different dosing regimens; reasons for the preference; experience of bisphosphonate therapy; and attitudes towards bisphosphonate treatment.

Results: 1083 women were interviewed: 550 bisphosphonate naïve; 334 current daily or weekly bisphosphonate users; 199 lapsed bisphosphonate users (stopped daily or weekly therapy within 12 months) none of the sample had taken a monthly bisphosphonate. $828(83 \%)$ of women preferred monthly dosing to weekly or daily. $680(82 \%)$ of these would prefer to take a monthly bisphosphonate with a support programme that could offer information, advice, and reminders to take their medication. $92(8 \%)$ women would prefer untreated osteoporosis to taking any bisphosphonate medication. 'Convenience' was the most frequently cited reason for preferring monthly dosing $(n=453$, $55 \%$ ) and women preferring monthly dosing were more likely to perceive their current/past bisphosphonate therapy as 'inconvenient' than those preferring daily/weekly dosing $(p=0.02)$. Women preferring monthly dosing also had more concerns about bisphosphonate medication than those preferring daily or weekly dosing: $44 \%$ of women who preferred monthly dosing and $18 \%$ who preferred daily/weekly dosing were worried about becoming dependent on medication $(\mathrm{p}=0.0001) ; 37 \%$ of women who preferred monthly and $12 \%$ who preferred daily/weekly were concerned about taking long term treatment $(p=0.0001)$.

Conclusions: This study clearly demonstrates a preference for monthly dosing with bisphosphonate therapy in women naïve to bisphosphonates, current and lapsed bisphosphonate users. Preference for monthly dosing is driven by perceptions that it would be more convenient and by concerns about becoming dependent on medication or taking medication over a long time period. Such concerns highlight the potential value of a nurse-led support programme.

P359. GONADAL HORMONES LEVEL, SEXUAL HORMONE BINDING GLOBULIN LEVEL AND GONADOTROPIN LEVEL IN VENEZUELAN MEN WITH LOW BONE MINERAL DENSITY

R. Constantino, J. Cedeño-Taborda, L. Marcano, J. Ramos, G. Riera-Espinoza; UNILIME-UC, Hospital Universitario Dr. Angel Larralde, Valencia, Venezuela

In men there is a decrease in testosterone of $1 \%$ to $2 \%$ year after 30 year of age, other gonadal hormone increase as FSH and in less degree LH. Also the sexual hormone binding globulin (SHBG) increases with age. In contrast there is little change within Estradiol levels; with a direct relationship with the decrease of Bone Mineral Density (BMD) at cortical (2\% per decade) and trabecular bone (12\% per decade). The objective was to determine serum levels of Total Testosterone (TT) Free testosterone (FT) Biodisponible Testosterone (BT) Sexual Hormone Globulin Level (SHBG), Lutein Hormone (LH) and Estradiol (E) in men over 30 years of age with low bone density by DEXA. 64 patients were studied mean age 58.69 \pm 12.91 BMD Total Hip (TH) $0.911 \pm 0.12 \mathrm{~g} / \mathrm{cm}^{2}$ T-Score $-1.2 \pm 0.99 ;$ Femoral Neck (FC) $0.842 \pm 0.10 \mathrm{~g} / \mathrm{cm}^{2} \mathrm{~T}$-score $-1.7 \pm 0.79$; Trocanter $(\mathrm{T}) 0.854 \mathrm{~g} / \mathrm{cm}^{2}$ T-score $\quad-1.66 \pm 0.76 ; \quad$ L1-L4 $\quad 1.057 \pm 0.14 \mathrm{~g} / \mathrm{cm}^{3} \quad$ T-score $-1.34 \pm 1.25$. TT levels $4.81 \pm 1.87 \mathrm{ng} / \mathrm{ml}$; TL $0.282 \pm 0.105 \mathrm{nmol} /$ $\mathrm{L} ;$ BT $7.12 \pm 4.03 \mathrm{nmol} / \mathrm{L} ;$ SHBG $47.89 \pm 24.03 \mathrm{nmol} / \mathrm{L} ; \quad \mathrm{LH}$ $6.44 \pm 4.50 \mathrm{mUI} / \mathrm{ml} ;$ E $30.74 \pm 9.72 \mathrm{pg} / \mathrm{ml}$. Age was co-related with FT $(p=0.01)$, SHBG $(p=0.000)$ and LH $(p=0.001)$. Regarding BMD and Gonadal Hormone we found significant co-relation between BMD TH, T and L1-L4 with TT $(\mathrm{P}<0.007$, $<0.05,<0.004$ respectively); with FT and L1-L4 p=0.04 and with SHBG TH $p=0.002$, FN $p=0.01, T p=0.006$, of these correlations the strongest was between BMD and SHBG; not like with BT, LH and E ( $\mathrm{p}>0.05)$.

We can conclude that the gonadal axis is modified with the process of ageing, producing a direct decrease of serum level of TT increase of SHBG and LH, with a decrease in cortical and trabecular bone mineral density, SHBG seems to be the main hormonal maker in men with low bone density.

\section{P360. BONE MARROW STROMAL CELLS OF YOUNG AND ADULT RATS RESPOND SIMILARLY TO PLATELET-RELEASED SUPERNATANT AND BONE MORPHOGENETIC PROTEIN-6 IN VITRO}

S. Cei ${ }^{1}$, B. Kandler ${ }^{2}$, A. Füg ${ }^{1,2}$, M. Gabriele ${ }^{1}$, J.O. Hollinger ${ }^{3}$, G. Watzek $^{2}$, R. Gruber ${ }^{2}$; ${ }^{1}$ Cattedra di Chirurgia Speciale Odontostomatologica, University of Pisa, Pisa, Italy, ${ }^{2}$ Department of Oral Surgery, Medical University of Vienna, Vienna, Austria, ${ }^{3}$ Bone Tissue Engineering Center, Carnegie Mellon University, Pittsburgh, USA

Objective: Osteoporosis is an age-related disease that is increasingly being recognized to have an impact on periodontal bone regeneration, osseointegration of dental implants and graft consolidation. This in vitro study was intended to show whether aging is associated with a diminished responsiveness of osteoprogenitor cells to growth and differentiation factors.

Material and Methods: We compared the capacity of bone marrow stromal cells harvested from young and adult rats to proliferate, migrate and differentiate into the osteogenic lineage following exposure to platelet-released supernatant or bone morphogenetic protein-6 (BMP-6). Bone marrow stromal cells were isolated from twelve young rats aged 6 weeks and twelve adult rats aged nine months. Proliferation was assessed by $3[\mathrm{H}]$ thymidine incorporation, migration was evaluated with the Boyden chamber assay and osteogenic differentiation was deduced from alkaline phosphatase activity. 
Results: Irrespective of the donor age, bone marrow stromal cells showed increased mitogenic activity and chemotactic motility when exposed to platelet-released supernatant. Adult bone marrow stromal cells had higher alkaline phosphatase activities at baseline and upon incubation with BMP-6 than cells obtained from young animals. There was no difference between the two groups in the slope of the alkaline phosphatase activity curve following stimulation with BMP-6.

Conclusion: The data demonstrate that, irrespective of their age, bone marrow stromal cells respond similarly to platelet-released supernatant and BMP-6 under in vitro conditions. These findings suggest that osteoprogenitor cells within the bone marrow of adult rats retain their juvenile potential to respond to growth and differentiation factors, which are released naturally or are applied therapeutically at sites of bone regeneration.

\section{P361. NORMATIVE VALUES OF SOLUBLE RANKL IN THE SERUM OF HEALTHY ADULTS AND CORRELATION WITH VARIOUS PARAMETERS OF BONE METABOLISM}

S.Kudlacek ${ }^{1,2}$, B. Schneider ${ }^{3}$, W. Woloszczuk ${ }^{2,5}$, P. Pietschmann $^{2,4}$, R. Willvonseder ${ }^{1,2}$ for the Austrian Study Group on Normative Values of Bone Metabolism; ${ }^{1}$ Medizinische Abteilung, Krankenhaus der Barmherzigen Brüder, Wien, ${ }^{2}$ Ludwig Boltzmann Institut für Altersforschung, Wien, ${ }^{3}$ Institut für Medizinische Statistik, Universität Wien, ${ }^{4}$ Institut für Pathophysiologie, Universität Wien, ${ }^{5}$ Ludwig Boltzmann Institut für experimentelle Endokrinologie, Wien Austria

RANKL (receptor activator of nuclear factor-kappa B ligand) is expressed on stromal and osteoblastic cells and in concert with osteoprotegerin (OPG) regulates bone metabolism. OPG functions as a decoy receptor for RANKL. This interaction results in inhibition of osteoclast differentiation and of bone resorption. We determined soluble RANKL levels in 1029 individuals of a normal Austrian population (635 women, 394 men). Serum levels of SRANKL were measured by a sandwich ELISA (Biomedica ${ }^{\circledR}$, detection limit: $0,4 \mathrm{pmol} / 1)$. In women the mean sRANKL level was $0.37 \pm 0.36 \mathrm{pmol} / 1 \quad\left(10^{\text {th }}-90^{\text {th }}\right.$ percentile range: $0.04-$ $0.71 \mathrm{pmol} / \mathrm{l})$; in men the mean sRANKL concentration was 0.46 $\pm 0.46 \mathrm{pmol} / 1\left(10^{\mathrm{th}}-90^{\mathrm{th}}\right.$ percentile range: $\left.0.04-0.85 \mathrm{pmol} / 1\right)$.

Correlation with serum parameters and bone density (lumbar, spine DXA) showed a negative correlation of sRANKL with age in females and males $(p<0.05)$. sRANKL and OPG were negatively correlated in females whereas positively in males. We investigated a certain influence of age on serum levels of bone matrix proteins reflecting bone metabolism.

Kudlacek S., Schneider B., Peterlik M., Leb G., Klaushofer K., Weber K., Woloszczuk W., Willvonseder R. Serum levels of osteoprotegerin increase with age in an healthy adult population Bone 2003; 32, 6, 681-686

\begin{tabular}{lllllllllll}
\hline Gender & $\mathbf{f}$ & $\mathbf{m}$ & $\mathbf{f}$ & $\mathbf{m}$ & $\mathbf{f}$ & $\mathbf{m}$ & $\mathbf{f}$ & $\mathbf{m}$ & $\mathbf{f}$ & $\mathbf{m}$ \\
\hline $\mathrm{N}$ & 229 & 65 & 220 & 117 & 150 & 177 & 31 & 30 & 5 & 5 \\
Mean & 0,68 & 0,40 & 0,53 & 0,39 & 0,53 & 0,39 & 0,38 & 0,34 & 0,43 & 0,39 \\
Median & 0,39 & 0,69 & 0,38 & 0,51 & 0,34 & 0,36 & 0,36 & 0,44 & 0,11 & 0,15 \\
SD & 0,6 & 1,2 & 0,8 & 1,1 & 0,6 & 0.8 & 1,9 & 0,7 & 0,1 & 0,05 \\
Age & $<\mathbf{3 5}$ & & $\mathbf{3 6 - 4 5}$ & & $\mathbf{5 6 - 6 5}$ & & $\mathbf{6 5 - 7 5}$ & & $>\mathbf{7 6}$ & \\
group & & & & & & & & & & \\
\hline
\end{tabular}

\section{P362. OSTEONECROSIS OF THE JAWS AND BISPHOSPHONATES}

A. Wutzl ${ }^{1}$, G. Eisenmenger ${ }^{1}$, E. Biedermann ${ }^{1}$, F. Wanschitz ${ }^{1}, \mathrm{G}$. Millesi-Schobel $^{1}$, A. Baumann ${ }^{1}$, P. Pietschmann ${ }^{2}$, R. Ewers ${ }^{\text {; }}$ ${ }^{1}$ Department of Cranio-Maxillofacial and Oral Surgery, Medical University of Vienna, Austria, ${ }^{2}$ Department of Pathophysiology, Medical University of Vienna, Austria

Objectives: Bisphosphonates are currently used in the treatment of various bone diseases like Paget's disease, osteoporosis, osteogenesis imperfecta and osteolytic bone lesions of multiple myeloma and malignant diseases. A recently described serious intraoral complication of tumour patients is osteonecrosis of the jaws, which is suspected to be related to intravenously administered bisphosphonates.

Methods: We included in our study all patients with oral complications after bisphosphonate therapy who presented at the University Hospital for Maxillofacial and Oral Surgery from July 2004 through June 2005. A total of 17 patients were investigated, all of them suffering from malignant disease. The most common oncologic diagnosis was multiple myeloma in 12 patients. History revealed an i.v. medication with pamidronate in 5 patients, 12 patients had been treated with zolendronic acid. 9 patients presented with lesions in the mandible, whereas 8 patients suffered from maxillary bone involvement. Typical presenting symptoms were exposed bone and pain with signs of local inflammation. In 13 patients a tooth extraction stood in the beginning of the symptoms. In fifteen patients sequestrectomy and decortication with local soft tissue closure was carried out in general anaesthesia.

Results: We report the 6 months post operation results concerning pain score, mode of prosthetic rehabilitation and local complications. Pre- and postsurgical computed tomography was evaluated for prediction of diagnosis and recurrence.

Conclusion: Computed tomography proved to differentiate between metastatic lesions and osteonecrosis. Sequestrectomy and decortication with local soft tissue closure proved to be a possible treatment modality. An evaluation by a dentist before the start of an i.v. bisphosphonate therapy for prophylactic reasons is recommended.

\section{P363. EFFICACY AND TOLERABILITY OF HYALURONIC ACID VISCO- SUPPLEMENTATION WITH SINOVIAL $®$ FOR THE TREATMENT OF KNEE OSTEOARTHRITIS: A RETROSPECTIVE OBSERVATIONAL STUDY}

F. Depont, I. Addra, N. Lechevallier, T. Diatta, A. Grelaud, M.A. Bernard, N. Moore; INSERM U657, Département de Pharmacologie, Université Victor Segalen, F33076 Bordeaux, France

Objective: To assess efficacy and safety of one intra-articular injection of hyaluronic acid (HA, Sinovial $\left.{ }^{\circledR}\right)$ in patients with knee osteoarthritis (OA).

Methods: A retrospective observational study was performed in France. From August 2003 to March 2004, all prescribers of HA were asked to included patients with primary or secondary symptomatic knee osteoarthritis and a first HA injection between January to April 2003. At the time of inclusion, data on OA history, OA treatments, HA injection tolerance, pain and knee function since HA injection were collected. NSAIDs and analgesic use were also collected from a national reimbursement database during the 10 months after the sinovial injection date.

Results: A total of 408 outpatients (146 men and 262 women) aged between 26 and 92 years (mean age - men $63.3 \pm$ 12.2 years / women $67.0 \pm 11.3$ years) were selected by 61 physicians. Mean body mass index indicated that patients were overweight (men: $28.2 \mathrm{~kg} / \mathrm{m}^{2}$ - women: $28.3 \mathrm{~kg} / \mathrm{m}^{2}$ ). $65.4 \%$ of patients had had OA symptoms for more than 2 years $.91 .4 \%$ of patients were treated with analgesics, NSAIDs, and/or corticosteroids during the week preceding the HA injection. For $64.5 \%$ of patients and $68.3 \%$ of physicians, knee function was better or much better since injection. At the time of inclusion ( 8 months after injection on average), $44.3 \%$ of the patients considered their knee pain to be low or null during the day and $76,4 \%$ during the night. Successful injections were associated with recent-onset ( $<5$ years) OA and lower BMI. Among 113 patients treated by NSAIDs 2 months before sinovial injection date, a NSAID prescription was found for only $31 \% 10$ months after the injection. Intra-articular hyaluronic acid administration was well tolerated. Local adverse events (mild burning sensation, erythema or irritation at the injection site) were reported with an overall rate of $4 \%$, and no systemic adverse events were noted in any patient. 
Conclusion: In this observational study, the intra-articular injection of HA (sinovial ${ }^{\circledR}$ ) was associated with improved knee function, less pain and less need for pain medication.

\section{P364. SAFETY AND EFFICACY OF IDEA-033 VERSUS CELECOXIB AND PLACEBO IN OSTEOARTHRITIS (OA) OF THE KNEE}

M. Rother ${ }^{1}$, B.J. Lavins ${ }^{2}$, J. Gu${ }^{2}$, W. Kneer ${ }^{3}$, K. Lehnhard ${ }^{3}$, E. Seidel $^{3}$, S. Mazgareanu ${ }^{1}$, ${ }^{1}$ IDEA AG, ${ }^{2} \mathrm{McNeil}$ CSP, ${ }^{3}$ for the IDEA-033 Clinical Study Group

Objective: Transfersome ${ }^{\circledR}$ carriers are ultradeformable vesicles designed to deliver drugs non-invasively through the skin barrier to target muscles and joints without being cleared by the cutaneous microcirculation. Ketoprofen is an NSAID analgesic with potent additional local anti-inflammatory properties. This study compared the safety and efficacy of $110 \mathrm{mg}$ of ketoprofen in Transfersome ${ }^{\circledR}$ Gel (IDEA-033) applied epicutaneously twice daily (bid) with that of celecoxib (100 mg orally bid) and placebo in treating the signs and symptoms of knee OA.

Method: This six-week, multicenter, randomized, double-blind, double-dummy, parallel-group study was conducted in 397 subjects with OA of the knee. To qualify for the study, subjects had to present at baseline pain with walking of at least $40 \mathrm{~mm}$ on the WOMAC visual analogue scale, with an increase of at least $15 \mathrm{~mm}$ compared to screening, and a physician's global assessment of OA of grade 3 to 5 and at least a one-grade increase from screening.

Results: Three co-primary endpoints were defined a priori. In the intent-to-treat (ITT) analysis, for the WOMAC pain subscale, IDEA-033 $(p=0.0041)$ and celecoxib $(p=0.0004)$ showed a statistically significant improvement from baseline at Week 6 versus placebo. For the WOMAC physical function subscale, celecoxib showed a significant $(\mathrm{p}=0.0100)$ improvement; the improvement for IDEA-033 versus placebo approached statistical significance $(p=0.077)$. For Patient Global Assessment, IDEA$033(\mathrm{p}=0.0015)$ and celecoxib $(\mathrm{p}=0.0145)$ showed a statistically significantly higher response to therapy at Week 6 . Analysis by study week demonstrated that IDEA-033 and celecoxib were associated with progressive improvement over the entire study period. The results of the per-protocol analysis were generally consistent with the ITT analysis; however, for the WOMAC physical function subscale, the improvement after 6 weeks with IDEA-033 was significantly $(\mathrm{p}=0.0118)$ greater than with placebo. IDEA-033 was well tolerated. Overall, $53.6 \%$ of subjects treated with IDEA-033, $50.0 \%$ of subjects treated with celecoxib, and $48.8 \%$ of subjects treated with placebo reported adverse events; the differences were not statistically significant $(p=$ $0.7116)$.

Conclusion: IDEA-033 was more effective than placebo and similar to celecoxib. It may present a promising alternative to systemic anti-inflammatory therapy for OA of the knee.

\section{P365. PREVALENCE OF LOW CALCIUM INTAKE IN POSTMENOPAUSAL OSTEOPOROTIC WOMEN: THE NEED FOR SUPPLEMENTATION}

S. Boonen ${ }^{1}$, S. Magowan ${ }^{2}$, X. Zhou ${ }^{2}$, R.P. Heaney ${ }^{3}$, ${ }^{1}$ Leuven University, Leuven, Belgium, ${ }^{2} \mathrm{P} \& \mathrm{G}$ Pharmaceuticals, Mason, $\mathrm{OH}$, USA ${ }^{3}$ Creighton University, Omaha NE, USA

Guidelines recommend that women over age 50 consume > $1200 \mathrm{mg} /$ day of calcium. Calcium supplementation has been an integral part of all registration trials for osteoporosis since the Study of Osteoporotic Fractures (SOF), 1986-1988. The 19941996 Continuing Survey of Food Intakes by Individuals (CSFII) reports over $90 \%$ of women, 50 years and older, consume less than the recommended level of $1200 \mathrm{mg}$ /day of calcium in their diet. The objective of this analysis is to investigate the mean calcium daily intake and the prevalence of insufficient calcium intake among postmenopausal women based on 6 major osteoporosis Phase III trials conducted in the last 10 years.

The percentage of women with daily baseline intakes below the minimum $1200 \mathrm{mg}$ /day was calculated based on the reported mean and standard deviation of the average daily intakes from each trial. The aggregate baseline mean calcium intake was calculated from mean calcium daily and weighted for the number of patients enrolled in each trial.

This analysis included 11,474 postmenopausal women with mean ages ranging from 53-71 years. The weighted mean calcium intake (all sources) for all 6 trials was $727 \mathrm{mg} / \mathrm{day}$, which was slightly higher than the recently obtained 2004 NHANES data of $660 \mathrm{mg} /$ day. Taken overall, the percent of postmenopausal women with insufficient calcium intake (below $1200 \mathrm{mg} /$ day) was estimated to be $85.0 \%$ (Table 1 ).

\begin{tabular}{|c|c|c|c|c|c|c|}
\hline YEAR & STUDY & GROUP & $\begin{array}{l}\text { NUMBER } \\
\text { (patients) }\end{array}$ & $\begin{array}{l}\text { MEANAGE } \\
\text { (years) }\end{array}$ & $\begin{array}{l}\text { INTAKE } \\
\text { MG (SD) }\end{array}$ & $\begin{array}{l}\text { PERCENT } \\
<1200 \mathrm{mg}\end{array}$ \\
\hline 1995 & $\begin{array}{l}\text { Liberman } \\
\text { et al. }\end{array}$ & All & 994 & 63.0 & 738 (539) & $80.4 \%$ \\
\hline \multirow[t]{2}{*}{1996} & \multirow[t]{2}{*}{ FIT I } & Treatment & 1002 & 70.7 & $652(417)$ & $90.6 \%$ \\
\hline & & Placebo & 1005 & 71 & 619 (397) & $92.8 \%$ \\
\hline \multirow[t]{4}{*}{1998} & \multirow[t]{4}{*}{ EPIC } & Treatment & 452 & 53 & $910(506)$ & $71.7 \%$ \\
\hline & & Treatment & 445 & 54 & $971(565)$ & $65.7 \%$ \\
\hline & & Treatment & 102 & 53 & $935(594)$ & $67.2 \%$ \\
\hline & & Placebo & 461 & 53 & 889 (445) & $75.8 \%$ \\
\hline \multirow[t]{2}{*}{1998} & \multirow{2}{*}{ FIT II } & Treatment & 2214 & 67.6 & $634(405)$ & $91.9^{\circ}$ \\
\hline & & Placebo & 2218 & 67.7 & $638(395)$ & $92.3 \%$ \\
\hline \multirow[t]{4}{*}{2000} & \multirow[t]{4}{*}{ PROOF } & Treatment & 316 & 68.2 & 907 (563) & $69.9 \%$ \\
\hline & & Treatment & 316 & 69 & $911(452)$ & $73.9 \%$ \\
\hline & & Treatment & 312 & 67.9 & $874(480)$ & $75.1 \%$ \\
\hline & & Placebo & 311 & 68.2 & $979(592)$ & $64.6 \%$ \\
\hline \multirow[t]{3}{*}{2001} & \multirow{3}{*}{$\begin{array}{l}\text { Neer } \\
\text { et al. }\end{array}$} & Treatment & 444 & 69 & 786 (443) & $82.5 \%$ \\
\hline & & Treatment & 434 & 70 & 757 (449) & $83.8 \%$ \\
\hline & & Placebo & 448 & 69 & $762(433)$ & $84.4 \%$ \\
\hline REVIEW & $\begin{array}{l}\text { All } \\
\text { STUDIES }\end{array}$ & All & 11,474 & $66.3 \mathrm{yr}$ & $727 \mathrm{mg} / \mathrm{d}$ & $85.0 \%$ \\
\hline
\end{tabular}

* calculation assumed a normal distribution with parameters as reported

These data, derived from the baseline assessments of major osteoporosis trials, suggest that, despite increased public awareness, little has changed in the average daily calcium intake since SOF 1988 (714mg/day). Baseline daily calcium intake of patients is still approximately $500 \mathrm{mg}$ below the recommended intake. Postmenopausal osteoporotic women would benefit from a treatment regimen that includes calcium supplementation with their prescription medicine in order to maximize the fracture risk reduction benefits of osteoporosis therapy.

P366. CALCIUM SUPPLY IN WOMEN WITH POSTMENOPAUSAL OSTEOPOROSIS ON BISPHOSPHONATE THERAPY IN DAILY PRACTICE

J.D. Ringe ${ }^{1}$, G. Möller ${ }^{2}$; B. Wick ${ }^{2}$; ${ }^{1}$ Medizinische Klinik IV, Klinikum Leverkusen, Leverkusen, Germany, ${ }^{2}$ Procter\&Gamble Pharmaceuticals-Germany GmbH, Schwalbach, Germany

The objective of the study was to assess whether women with postmenopausal osteoporosis on bisphosphonate therapy have a sufficient calcium intake.

264 community-based physicians were asked to interview patients with postmenopausal osteoporosis on bisphosphonate therapy regarding their knowledge about the importance of calcium and their daily calcium intake using a questionnaire. The survey was conducted from July to September 2004.

Questionnaires were analyzed from 726 patients. The importance of calcium for their osteoporosis therapy was rated as important or very important by $678(93.4 \%)$ of the patients. Patients estimated their required daily calcium intake to be 1000 mg (median, 25\% 500, 75\% 1000); $75.5 \%$ believed that they take enough calcium per day. Calculation based on the reported intake of calcium-rich foods showed a median of $913.2 \mathrm{mg}$ calcium per day $(25 \% 466.7 ; 75 \% 1514.7) .157(21.6 \%)$ patients reported that they take no additional calcium, and of these patients, $54.8 \%$ believed that they take sufficient calcium with their dairy products. A regular calcium supplement was considered too expensive by $17.8 \%$, too cumbersome by $17.8 \%$, and $16.6 \%$ admitted that they forget to take it.

Although more than $90 \%$ of the women interviewed rated the intake of calcium for their osteoporosis therapy as important 
or very important, they underestimated their required calcium intake. The German Dachverband Osteologie (DVO) recommends $1500 \mathrm{mg}$ daily calcium intake for women with postmenopausal osteoporosis (http://www.lutherhaus.de/osteo/leitliniendvo/index.php). Studies show that the daily calcium intake from food for women aged $\geq 50$ years is below the recommended amount (DGE Report, 2000). Our results confirm these findings, identifying a deficit in daily calcium of $500-600 \mathrm{mg}$ versus the required intake. More than $20 \%$ of patients took no supplemental calcium to reach the daily requirement. When asked for the reason, more than half considered extra calcium supplementation too expensive or too inconvenient. Osteoporosis therapy with bisphosphonates requires a sufficient calcium level. We conclude that a simplified way to supply calcium to patients on bisphosphonate therapy could ensure greater compliance to this evidence-based osteoporosis therapy and, thereby, could be more cost-effective.

\section{P367. IMPACT OF EARLY OSTEOPOROSIS TREATMENT ON SUB- SEQUENT FRACTURE PREVENTION AMONG WOMEN WITH CLINICAL VERTEBRAL FRACTURE}

R. Lindsay ${ }^{1}$, N. Borisov ${ }^{2}$, R. Sheer ${ }^{2}$, M. Steinbuch ${ }^{2,3} ;{ }^{1}$ Helen Hayes Hospital, ${ }^{2}$ Procter\&Gamble Pharmaceuticals, Inc., ${ }^{3}$ Ohio State University, USA

This study evaluated osteoporosis treatment use and its effect on prevention of subsequent fractures among women with a clinical vertebral fracture, utilizing an integrated administrative, medical and pharmacy claims database.

A retrospective cohort study was conducted among women (aged $45+$ ) with a new vertebral fracture verified with a diagnostic code and a record of radiologic exam, between July 1, 2000 and June 30, 2003. The cohort was followed for 12 months after the vertebral fracture to identify a subsequent fracture (vertebral and non-vertebral), osteoporosis treatment and its subsequent fracture prevention effect. The following exclusion criteria were applied to the data: (1) in the 6 months period prior to the vertebral fracture, women with a record of previous (a) vertebral or non-vertebral fractures, (b) treatment with risedronate, alendronate, nasal calcitonin, or raloxifene, or (c) Paget's disease treatment, and (2) at any point of the study, women with a record of malignant neoplasm and/or trauma "E codes".

A total of 7,233 women were identified with a new clinical vertebral fracture. The mean age was 73 years. Majority of the population $(80 \%)$ did not receive any osteoporosis treatment (bisphosphonates or nasal calcitonin); 1,056 women $(15 \%)$ received osteoporosis treatment within 90 days of their vertebral fracture (early-treatment cohort), and 358 women $(5 \%)$ received treatment after 90 days of their vertebral fracture (late-treatment cohort). During the 1-year follow-up, $18 \%$ of untreated women experienced a subsequent fracture (vertebral or nonvertebral, $\mathrm{n}=1,039)$. Among women in the early-treatment cohort only $85(8 \%)$ had a subsequent fracture $(R R=0.51$, $\mathrm{p}<0.01$ compared to no treatment cohort), and in the latetreatment cohort, $76(21 \%)$ had a subsequent fracture $(\mathrm{RR}=1.05, \mathrm{p}=0.73$ compared to no treatment cohort). Osteoporosis treatments were effective in their subsequent fracture prevention compared to no treatment as follows: risedronate $(\mathrm{RR}=0.30, \mathrm{p}<0.01)$, alendronate $(\mathrm{RR}=0.47, \mathrm{p}<0.01)$, and nasal calcitonin $(\mathrm{RR}=0.67, \mathrm{p}=0.03)$.

Among women aged $45+$ years with a clinical vertebral fracture, the majority did not receive any osteoporosis treatment. Among those who received treatment, there was a significantly lower risk of developing a subsequent fracture. The early-treatment cohort trended toward fewer fractures compared to the late-treatment cohort. This suggests that a fragility fracture demands immediate attention and rapid, effective treatment. Risedronate was observed to have a $70 \%$ reduction in the occurrence of subsequent fractures within 1 year of vertebral fracture.
P368. RISEDRONATE DEMONSTRATES ANTIFRACTURE EFFICACY OVER A RANGE OF BASELINE BMD LEVELS

R. Lindsay ${ }^{1}$, S. Magowan ${ }^{2}$, I. Barton ${ }^{3}$, D. Felsenberg ${ }^{4}$, R Adachi ${ }^{5}$; ${ }^{1}$ Helen Hayes Hospital, West Haverstraw, NY, USA, ${ }^{2} \mathrm{P} \& \mathrm{G}$ Pharmaceuticals, Mason, OH, USA, ${ }^{3} \mathrm{P} \& \mathrm{G}$ Pharmaceuticals, Egham, United Kingdom, ${ }^{4}$ Univ Hospital Benjamin Franklin, Berlin, Germany, ${ }^{5}$ McMaster University, Hamilton, $\mathrm{ON}$, Canada

Baseline BMD level and risk factors are important considerations in the decision to initiate osteoporotic therapy. The WHO commentary on the threshold for intervention is not the same as the diagnostic threshold for osteoporosis. Currently, there is little available information concerning the efficacy of osteoporotic therapies over a range of baseline T-scores. This study investigates the fragility fracture efficacy (vertebral and nonvertebral) of risedronate $5 \mathrm{mg}$ /day in postmenopausal women over 4 ranges of femoral neck (FN) T-scores.

This analysis included 2575 postmenopausal women from 4 phase III trials: VERT MN \& NA, BMD MN \& NA. Women were randomized to receive placebo or risedronate and treated for up to 3 years. Subjects were stratified into 4 subgroups based on level of baseline FN T-score. Fractures included in the analysis were vertebral and nonvertebral (composite of 6 sites). The fracture incidence and relative risk reduction for each baseline BMD range are summarized for each subgroup.

In the four BMD strata, fracture incidence in the placebo group increased with decreasing baseline BMD values, potentially confounded by increasing age and an increased percentage of patients with prevalent vertebral fractures (PVF). Risedronate, however, demonstrated significant anti-fracture efficacy in postmenopausal women, regardless of baseline level T-score ranges (Table 1). Treatment by T-score interaction was $\mathrm{p}=0.653$.

Although baseline BMD is an important predictor of fracture risk, Risedronate's demonstrated efficacy against fragility fractures appears to be independent of baseline BMD level.

Table 1 Osteoporotic Fracture Incidence in Placebo and Risedronate Patients with Baseline FN T-score Data < -1.5 (VERT-MN \& NA, BMD-MN \& NA)

\begin{tabular}{|c|c|c|c|c|c|c|c|}
\hline $\begin{array}{l}\text { Baseline } \\
\text { T-score }\end{array}$ & $\mathrm{N}$ & $\begin{array}{l}\text { Mean } \\
\text { Age }\end{array}$ & $\begin{array}{l}\text { Percent } \\
\text { PVF }\end{array}$ & $\begin{array}{l}\text { Placebo } \\
\text { Incidence }\end{array}$ & $\begin{array}{l}\text { Risedronate } \\
\text { Incidence }\end{array}$ & $\begin{array}{l}\text { Hazard } \\
\text { Ratio }\end{array}$ & $\begin{array}{l}\mathrm{P} \\
\text { value }\end{array}$ \\
\hline$\leq-1.5$ to $>-2.0$ & 609 & 67 & $63.1 \%$ & $20 \%$ & $14 \%$ & 0.58 & $<0.05$ \\
\hline$\leq-2.0$ to $>-2.5$ & 822 & 68 & $68.7 \%$ & $27 \%$ & $19 \%$ & 0.63 & $<0.01$ \\
\hline$\leq-2.5$ to $>-3.0$ & 682 & 69 & $78.4 \%$ & $36 \%$ & $26 \%$ & 0.61 & $<0.005$ \\
\hline$\leq-3.0$ & 462 & 71 & $87.7 \%$ & $46 \%$ & $27 \%$ & 0.44 & $<0.001$ \\
\hline
\end{tabular}

P369. TREATMENT-RELATED CHANGES IN BMD ARE NOT ASSOCIATED
WITH THE OCCURRENCE OF A FRAGILITY FRACTURE WHILE ON WITH THE OCCURRENCE

R. Lindsay ${ }^{1}$, S. Magowan ${ }^{2}$, P. Miller ${ }^{3}$, J. Adachi ${ }^{4}$, I.P. Barton ${ }^{5}$, D. Felsenberg ${ }^{6}$, N.B. Watts ${ }^{7}$. Helen Hayes Hospital, West Haverstraw, NY, USA, ${ }^{2} \mathrm{P} \& \mathrm{G}$ Pharmaceuticals, Mason, $\mathrm{OH}$ USA, ${ }^{3}$ Denver Osteoporosis Center, Denver, CO, USA, ${ }^{4} \mathrm{McMas}-$ ter University, Hamilton, ON, Canada, ${ }^{5} \mathrm{P} \& \mathrm{G}$ Pharmaceuticals, Egham, United Kingdom, ${ }^{6}$ Univ Hospital Benjamin Franklin, Berlin, Germany, ${ }^{7}$ University of Cincinnati Bone Health and Osteoporosis Center, Cincinnati, Ohio, USA

Risk factors for osteoporotic fracture in untreated postmenopausal women have been well documented. However, there is a paucity of data addressing the risk determinants for fracture while actually on treatment. This valuable information would be helpful in identifying the higher risk patient. This analysis was designed to determine the effect of different parameters associated with the occurrence of an osteoporotic fracture (vertebral \& nonvertebral) while taking risedronate $5 \mathrm{mg} /$ day. The analysis specifically examined 4,324 postmenopausal women randomized to receive risedronate $5 \mathrm{mg} /$ day in the risedronate phase III placebo-controlled fracture endpoint trials over a 3 year period (VERT-MN, 
VERT-NA and HIP). Women who sustained fragility fractures ( $\mathrm{F}+$ group) were compared to women who remained fracture free (F- group). These two groups of women were analyzed to determine the significance of the following parameters to fracture occurrence while on therapy: baseline LS \& FN BMD, body mass index (BMI), prevalent vertebral fractures (PVF), and LS \& FN BMD percent change from baseline over 3 years. Descriptive statistics were summarized for each fracture group and formally compared using the Wilcoxon Rank Sum test.

Over 3 years 523 postmenopausal women sustained a fragility fracture and 3801 women remained fracture-free while on risedronate $5 \mathrm{mg}$ /day therapy. There was a significant difference in baseline BMD scores, prevalent vertebral fracture status and BMI between women who did or did not sustain a fracture during treatment. (Table 1). Changes in lumbar spine or femoral neck BMD during treatment, however, were not different between patients who did or did not suffer from a fragility fracture during treatment.

Table 1 Patient Characteristics (Median) By Incident Fracture Outcome

\begin{tabular}{llll}
\hline PARAMETER & $\begin{array}{l}\text { Patients with } \\
\text { Fractures }(\mathbf{F}+) \\
(\mathrm{n}=523)\end{array}$ & $\begin{array}{l}\text { Patients without } \\
\text { Fractures }(\mathbf{F}-) \\
(\mathrm{n}=3801)\end{array}$ & $\begin{array}{l}\text { p-value } \\
\mathrm{F}(+) \vee \mathrm{F}(-)\end{array}$ \\
\hline Baseline LS T-score & -3.0 & -2.6 & 0.005 \\
Baseline FN T-score & -2.8 & -2.6 & $<0.001$ \\
Baseline PVFs & 2.0 & 1.0 & $<0.001$ \\
Baseline BMI $\left(\mathrm{kg} / \mathrm{m}^{2}\right)$ & 24.6 & 25.0 & 0.020 \\
LS \% BMD Change & $5.4 \%$ & $5.1 \%$ & 0.359 \\
FN \% BMD Change & $1.6 \%$ & $1.8 \%$ & 0.298 \\
\hline
\end{tabular}

Of the investigated parameters, well-established risk factors for fracture such as low BMD, low BMI and prevalent vertebral fracture status allowed a differentiation between patients who did or did not fracture during treatment. Change in BMD with treatment was no different in patients who sustained a fracture compared with those who remained fracture free.

\section{P370. BIOINFORMATICS AIDED DESIGN OF A HIGHLY SPECIFIC} TNFSF14 ELISA - APPLICATION FOR BONE RESEARCH

N. Brinskelle-Schmal ${ }^{1}$, G. Hawa ${ }^{1}$, A. Lukas ${ }^{2}$, J. Marc ${ }^{3}$; ${ }^{1}$ Biomedica Gruppe, Vienna, Austria, ${ }^{2}$ Emergentec Biodevelopment $\mathrm{GmBH}$, Vienna, Austria, ${ }^{3}$ University of Ljubljana, Faculty of Pharmacy, Ljubljana, Slovenia

Reliability and specificity of modern ELISA systems strongly depend on knowledge about the biochemical role and molecular structure of the molecule which should be analysed. Usage of different antibodies with different, or unknown epitope specificity often makes comparison of published results difficult or even leads to misinterpretation of clinical data, because e.g. different fragments of the target molecule are detected. Thus thorough knowledge of molecular characteristics and the epitope specificity is necessary to develop highly specific ELISA systems. We applied a genuine computer program (discovery BASE) to select proteins with relevance to bone metabolism and the best epitopes for generation of highly specific antibodies. As a model system, TNFSF14, a member of the tumor necrosis factor superfamily, was chosen, because we speculate, that, like RANKL, which belongs to the same class of molecules, TNFSF14 might play a role in diseases with elevated bone turnover.

Discovery BASE was used to calculate suitable epitopes most likely to circulate in serum or plasma (protease fragmentation sites) and showing best surface accessibility as well as antigenicity. Antibodies against those epitopes were raised in sheep, their avidity determined and compared against commercially available antibodies. ELISA prototypes were developed and a small study on samples from patients with osteoporosis, chronical polyarthritis, osteoarthritis, rheumatoid arthritis and with no bone disease performed. The best combination of antisera resulted in an ELISA with significant differentiation between normal $(0.59$
$+/-0.4 \mathrm{pmol} / \mathrm{l})$, osteoporotic $(2.29+/-0.81 \mathrm{pmol} / \mathrm{l})$ and rheumatoid patient samples $(4.9+/-2.4 \mathrm{pmol} / \mathrm{l})$. Clinical and specificity data of the assays used are presented.

\section{P371. THE PROGNOSIS OF OSTEOPOROSIS DEVELOPMENT RISK} AMONG THE INDUSTRIAL WORKERS

A. Ignatyev, T. Yermolenko, L. Batsulya; Odessa State Medical University, Odessa, Ukraine

The considerable spreading (about 70\%) of osteopenia and osteoporosis among the industrial workers and the risk of the fractures in the industrial work can eventually lead to the great material and human loses. But having the same conditions of labor, not all the workers are affected by the professional diseases. Obviously, internal factors cause the increased sensitiveness to the harmful industrial conditions and the resistance to them.

Materials and methods: The 800 workers of the able-bodied age (20-60 years) from the south-Ukrainian factories went under the examination. The examination was made by the ultrasound densitometer "Achilles express", which gives the prognosis of the fractures among the patients during the early changes of the mechanical strength of bone tissue that can't be seen clinically or diagnosed timely. The diagnostic program also included the evaluation of the complaints, the anamnesis, the clinical status, the determination of the functional state of the vegetative nervous system and the conducting of the functional tests.

Results: Among 25\% of workers was found osteoporosis, among $34,5 \%$ was found osteopenia. On the bases of received data the mathematical model was developed, which allows making the prognosis of the osteoporosis development probability by quantitative evaluation of the factors that lead to the MBD decreasing. Using the proposed method, we could distinguish four categories of patients with different risk rate for osteoporosis. It gives us the opportunity to have the differentiated approach in the prevention and treatment of this serious pathology.

Conclusions: The prevention and early diagnosis procedures of osteoporotic fractures can save the material expenses on treatment. The well-timed prophylaxis and treatment will prevent bone-muscular system diseases among the people of able-bodied age and secure the high quality and independence of living among the older age group.

\section{P372. MEASURING FUNCTIONING IN PATIENTS WITH HAND OSTEO- ARTHRITIS: CONTENT COMPARISON OF QUESTIONNAIRES BASED ON THE INTERNATIONAL CLASSIFICATION OF FUNCTIONING, DISABILITY AND HEALTH (ICF)}

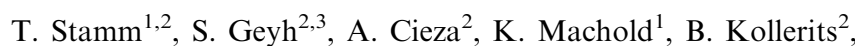
M. Kloppenburg ${ }^{4}$, J. Smolen ${ }^{1}$, G. Stucki ${ }^{2,3}$; ${ }^{1}$ Vienna Medical University, Department of Internal Medicine III, Division of Rheumatology, ${ }^{2}$ ICF Research Branch of the WHO Collaborating Center for the Family of International Classifications at the German Institute of Medical Documentation and Information (DIMDI), Munich, Germany, ${ }^{3}$ Department of Physical Medicine and Rehabilitation, Ludwig-Maximilians-University, Munich, Germany, ${ }^{4}$ Department of Rheumatology, Leiden University Medical Centre, Leiden, The Netherlands

Objective: When selecting a questionnaire, researchers and clinicians need to know whether or not a questionnaire covers the relevant outcomes. The aim of this study was to analyse and compare the content of questionnaires which have been used to assess functioning in patients with hand osteoarthritis based on the International Classification of Functioning, Disability and Health (ICF).

Method: Questionnaires were identified in a structured literature search. All concepts included in the items of the questionnaires were linked to the ICF categories according to 10 established linking rules by two health professionals. The degree of agreement between the two health professionals was determined by means of Kappa statistic. On the basis of the linking, the content of the instruments was compared. For each concept, it 
was examined whether functioning is measured on the level of activity or participation or both activity and participation. Indicators for content density, content diversity and the percentage of linked ICF categories addressing participation were calculated.

Results: HAQ, AUSCAN, Cochin scale, FIHOA, SACRAH and AIMS2-SF were analysed. The result of the kappa statistic for agreement between the two investigators was 0.74 . 163 concepts were identified in the 113 items of all instruments which were then linked to 7 ICF categories of the component body functions, 45 categories of the component activities and participation and 6 categories of the component environmental factors. AUSCAN and SACRAH have the lowest diversity ratio. AIMS2SF shows the highest diversity ratio and the highest percentage of linked ICF categories which address participation.

Conclusion: When selecting an instrument for comprehensive measurement of functioning, researchers and clinicians may wish to include both one instrument with a low diversity ratio (for disease specific aspects) and another instrument with a high diversity ratio (for broader aspects of functioning including some aspects of participation).

P373. CLINICAL WORK-UP AND ANALYSIS OF BONE HISTOLOGY, STRUCTURE AND MATERIAL PROPERTIES IN A YOUNG WOMAN WITH MULTIPLE PERIPHERAL FRACTURES

J. Patsch ${ }^{1}$, C. Muschitz ${ }^{1}$, Th. Wögerbauer ${ }^{1}$, P. Roschger ${ }^{2}$, K. Klaushofer ${ }^{2}$, H. Resch ${ }^{1}$; ${ }^{1}$ St. Vincent Hospital, Medical Department II; Vienna, Austria, ${ }^{2}$ Ludwig-Boltzmann-Institute of Osteology, Vienna, Austria

Objective: There is growing evidence that a reduction in bone mineral density does not solely explain the pathology underlying an osteoporotic fracture. In this context we give a description of the clinical work-up and corresponding tissue processing in a case of idiopathic osteoporosis.

Methods: As the 26-year old woman presented with a history of multiple peripheral fractures profound osteologic workup was initiated.

Beside X-rays of the thoracic and lumbar spine, Bone Mineral Density measurements using DXA and QCT of the hip and lumbar spine were performed as the usual standard procedures.

Furthermore analyses of biochemical bone markers and a transiliac bone biopsy were performed.

Results: Vertebral X-ray did not show any evidence of osteoporosis. An underlying systemic disorder or accelerated bone turn-over could be excluded by blood analysis.

BMD measurements using DXA resulted in osteopenia per definition, both at the hip (BMD total $0,756 \mathrm{~g} / \mathrm{cm}^{2}, \mathrm{~T}=-2,0$, BMD neck $0,882 \mathrm{~g} / \mathrm{cm}^{2}, \mathrm{~T}=-0,8$ ) and lumbar spine (BMD L1$\left.\mathrm{L} 4=0,926 \mathrm{~g} / \mathrm{cm}^{2}, \mathrm{~T}=-2,2\right)$. Quantitative computer tomography showed with a mean of $99,6 \mathrm{mg} / \mathrm{cc}$ calciumphosphate at the lumbar spine and $0,671 \mathrm{~g} / \mathrm{cm}^{2}$ calciumphosphate at the (total) hip values that were clearly below the method-respective fracture thresholds.

Quantitative histomorphometry demonstrated a regular number of both osteoclasts and osteoblasts, normal osteoid thickness (O.Th), osteoid bone volume (OV/BV).

The analysis of bone structure using qBEI (quantitative Backscattered Electron Imaging) resulted in a markedly reduced bone volume $(-34,25 \%)$, reduced trabecular thickness $(-18,3 \%)$ and poor tissue connectivity. At the material level the analysis of bone mineral density distribution (BMDD) finally revealed a shift of the curve to lower mineral concentration values and an increase of heterogeneity.

Conclusion: We conclude from our findings that in a young patient with fragility fractures hypomineralisation at the material level is rather linked to fracture risk than BMD or clinical measures.

\section{P374. THE EFFECTS OF GLYCITEIN ON OSTEOCLAST GENERATION}

M. Winzer ${ }^{1}$, M. Rauner ${ }^{2}$, G. Hofbauer ${ }^{2}$, K. Wahl ${ }^{1}$, P. Pietschmann ${ }^{1}$; ${ }^{1}$ enter of Physiology and Pathophysiology, Medical
University of Vienna, Austria ${ }^{2}$ Ludwig Boltzmann Institute of Aging Research, Vienna, Austria

Objective: The isoflavones glycitein and genistein are plantderived, non-steroidal molecules showing estrogen-like effects in humans. These effects are mainly explained by the interaction with estrogen-receptors and may prevent postmenopausal bone loss. As single isoflavones differ with regard to their biological effects, our aim was to study the effects of the very poorly investigated glycitein.

Material and Methods: Bone marrow cells from four to eight weeks old female mice were cultured in the presence of 10-8 $\mathrm{M}$ of 1,25-dihydroxyvitamin D3 [1,25 $(\mathrm{OH}) 2 \mathrm{D} 3]$, with and without various concentrations of glycitein (10-7 M - 10-12M) for 7 days. The number of osteoclast-like cells was determined by staining for tartrate-resistant acid phosphatase (TRAP), a marker enzyme of osteoclasts. Furthermore, the activity of caspase 3 and caspase 7 was measured by a fluorometric assay.

Results: The number of multinucleated TRAP + cells induced by $1,25(\mathrm{OH}) 2 \mathrm{D} 3(10-8 \mathrm{M})$ was significantly decreased by glycitein $(10-8 \mathrm{M})(\mathrm{p}<0.05, \mathrm{n}=12)$. The apoptosis-assay showed a significant increase of caspase $3 / 7$-activity in the presence of glycitein $(10-8 \mathrm{M})(\mathrm{p}<0.01, \mathrm{n}=4)$.

Conclusion: Data reveal that the stimulating effect of 1,25dihydroxyvitamin D3 on osteoclast differentiation is significantly inhibited by glycitein $(10-8 \mathrm{M})$. This effect is at least in part caused by inducing apoptosis in osteoclast-like cells and/or stromal cells. This study indicates that glycitein may reduce bone loss due to decreased bone resorption.

P375. NUMBERS NEEDED TO TREAT (NNT) FOR VERTEBRAL FRACTURE PREVENTION WITH NITROGEN-CONTAINING BISPHOSPHONATES IN WOMEN WITH POSTMENOPAUSAL OSTEOPOROSIS

J.D. Ringe ${ }^{1}$, M. Kraenzlin ${ }^{2}$, S. Adami ${ }^{3} ;{ }^{1}$ University of Cologne, Leverkusen, Germany, ${ }^{2}$ University of Basel, Basel, Switzerland, ${ }^{3}$ University of Verona, Verona, Italy

Objectives: Postmenopausal osteoporosis (PMO) is predicted to become more prevalent as the population ages, and a corresponding increase in burden in terms of mortality, morbidity and associated healthcare cost is expected. Bisphosphonates have proven antifracture efficacy and are the mainstay of PMO treatment. The number needed to treat (NNT), which conveys both statistical and clinical significance, is a frequently used measure for making treatment decisions in clinical practice. It depends on both the antifracture efficacy of the drug and the baseline fracture risk of the population to be treated. The pivotal studies of bisphosphonates in PMO recruited populations with different background vertebral fracture risk at baseline, despite similar inclusion criteria. Cross-study comparison of NNT requires adjustment for the observed baseline risk. The purpose of this analysis is to compare nitrogen-containing bisphosphonates based on their NNT in relevant patient populations.

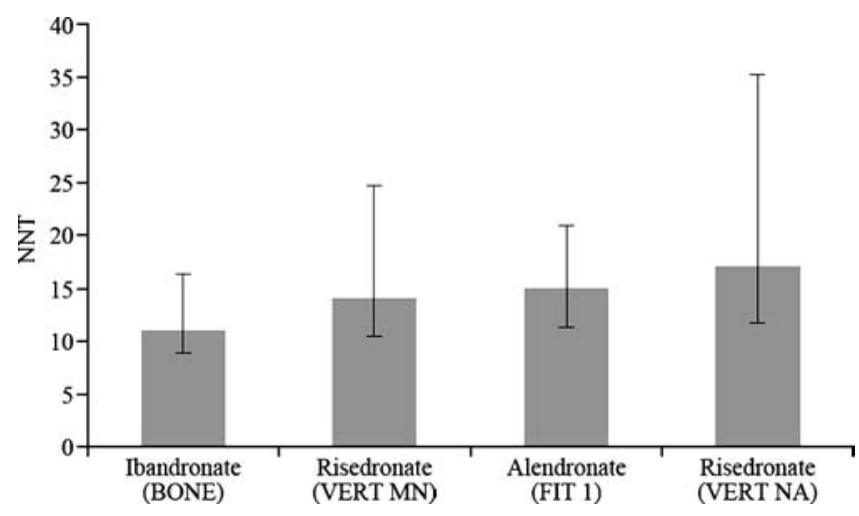

Fig. NNT for 3 years to prevent a vertebral fracture 
Methods: The calculation of NNT for the different drugs assumed a common osteoporotic population with a background (placebo) vertebral fracture incidence of $15 \%$ at 3 years and that the treatment effect (relative risk reduction) observed in the clinical trials would apply to this standard population. The absolute risk reduction (ARR) was calculated as the difference between the estimated fracture rates in the placebo and bisphosphonate treatment groups at a chosen time point. The NNT and its 95\% confidence interval (CI) are the reciprocals of those for ARR. Two slightly different computational methods were used, depending on the availability in the published data of standard errors (SE) or CIs for fracture rates.

Results and conclusion: The NNT for 3 years to prevent one vertebral fracture appears to be similar across the different bisphosphonates studied, with the lowest point estimate for ibandronate in the BONE study and the highest point estimate for risedronate in the VERT NA study (Figure).

P376. BONE TEXTURE ANALYSIS ON CALCANEUS DIGITIZED RADIOGRAPHIC IMAGES: DISCRIMINANT VALUE IN OSTEOPOROSIS

C.L. Benhamou ${ }^{1}$, C. Gadois ${ }^{1}$, G. Lemineur ${ }^{2}$, J.P. Do-Huu ${ }^{2}$, E. Lespessailles ${ }^{1}$; Ipros - Service de Rhumatologie CHR d'Orléans, France, ${ }^{2}$ D3A Medical Systems Orleans, France

Objective: Osteoporosis is characterized by compromised bone strength and increased risk of fracture. BMD remains the standard tool for early assessment of fracture risk. Among the other bone properties, trabecular microarchitecture is considered to play a major role. Bone texture analysis on radiographs may account for trabecular bone microarchitecture. We have previously validated a texture analysis on radiographs, and determined its value to discriminate osteoporotic patients with bone fractures from controls. Direct digitization of radiographic images is able to improve the reproducibility and the convenience of the process. In this study we aim to evaluate the discriminant value of bone texture analysis in post-menopausal osteoporosis.
Material and methods: A new home made radiologic prototype permits in vivo assessment of trabecular bone texture parameters : co-occurrence matrices, run-lengths method, and fractal analysis. The images were obtained on calcaneus by direct digitization. The radiological parameters were standardized $(20 \mathrm{mAs}, 55 \mathrm{kV}$, focal distance $115 \mathrm{~cm})$. The ROI was determined semi-automatically; the fractal analysis was achieved by the likehood estimator using the results as Hmean parameter $(\mathrm{H}=2$ D). On the same ROI, run-lengths and co-occurrence matrices were performed. BMD has been measured at the lumbar spine (LS), femoral neck (FN) and total hip (TH) by DXA (Hologic Delphi W).

We compared for BMD and texture analysis a group of healthy post-menopausal women with no fracture $(\mathrm{C}: \mathrm{n}=116$; age $54-92 ; 73.4 \pm 7.8$ years old) to a group of post-menopausal women with vertebral fracture (VF: $\mathrm{n}=34$; age $54-94 ; 77.1 \pm$ 9.6 years old) and a group of post menopausal women with hip fracture (HF : $n=27 ; 59-97 ; 80.4 \pm 9.2$ years old).

Results: Results were adjusted for age since it was significantly higher both in the HF and the VF groups than in control women. However there were no significant differences concerning the BMI. We found a significant difference for both co-occurrence matrices and run lengths mean values between $\mathrm{C}$ versus $\mathrm{HF}(\mathrm{p}<$ $0.0001 ; p<0.0001)$ and $C$ versus VF $(p=0.001 ; p=0.003)$ Hmean parameter was significantly lower in VF versus $\mathrm{C}: 0.593$ $\pm 0.039 ; 0.609 \pm 0.032(\mathrm{p}=0.024)$. Hmean parameter was also significantly lower in HF versus C : $0.581 \pm 0.034 ; 0.609 \pm 0.032$ $(\mathrm{p}=0.001)$. FN BMD, TH BMD and LSBMD were significantly lower $(\mathrm{p}<0.0001)$ in $\mathrm{HF}$ and VF versus $\mathrm{C}$.

Conclusions: These data show that trabecular bone texture analysis parameters can separate groups of post-menopausal women either with osteoporotic fractures or without fractures. The respective discriminant value of BMD and texture analysis will be examined in these groups. In accordance with previous studies, we confirm the potentially high value of this technique for the diagnosis of osteoporosis. 


\section{Symposia Abstracts}

\section{Osteoporosis Treatment - Patient Preferences, Therapeutic Adherence and Clinical Outcomes Sponsor: ROCHE \& GLAXOSMITHKLINE}

\section{SY400. IMPROVING THERAPEUTIC ADHERENCE THROUGH LESS- FREQUENT DOSING}

R. Rizzoli; Department of Rehabilitation and Geriatrics, University Hospitals, Geneva, Switzerland

Suboptimal therapeutic adherence is a widespread issue in clinical practice. In chronic disease, the rate of patient noncompliance varies from $20 \%$ in asthma to $71 \%$ in arthritis. ${ }^{1}$ In postmenopausal osteoporosis, therapeutic adherence is also suboptimal; the number of patients persisting with daily bisphosphonates is $58 \%$ at 6 months, $49 \%$ at 1 year and only $20 \%$ at 2 years. $^{2}$

Lack of compliance is associated with smaller reductions in the rate of bone turnover. ${ }^{3}$ After 22 weeks of treatment with a daily bisphosphonate, $>60 \%$ of compliant patients had a decrease from baseline in serum type 1 collagen C-telopeptide of $>50 \%$ compared with only $20 \%$ of non-compliant patients. Increases in bone mineral density (BMD) are also significantly reduced; compared with consistent users, inconsistent users had significantly lower gains in BMD at years 1,2 and $3(\mathrm{p}<0.003){ }^{4}$ As a result, non-adherence leads to an increase in fracture risk. Non-persistent users have a $26 \%$ increase in overall fracture risk and a $25 \%$ increase in non-vertebral fracture risk. ${ }^{6}$ Non-persistent users of bisphosphonates also have a $20-30 \%$ greater risk of hospitalisation due to fracture compared with persistent users. ${ }^{7}$

Solutions to improve adherence include offering greater flexibility in dosing regimens and tailoring therapy to individual patients needs. Numerous studies have demonstrated that the number of patients persisting with therapy after 1 year is greater with weekly regimens compared with daily regimens. ${ }^{8-10} \mathrm{Al}-$ though therapeutic adherence is improved with weekly compared with daily dosing, it can still be improved. A less frequent than weekly bisphosphonate regimen may improve patient adherence still further, thus improving the clinical outcomes of treatment.

\section{References}

1. Berg JS, et al. Ann Pharmacother 1993;27:S5-19

2. Lombas C, et al. J Bone Miner Res 2001;16(Suppl. 1):S529 (Abstract M406)

3. Eastell R, et al. Calcif Tissue Int 2003;72:408

4. Sebaldt RJ, et al. Ann Rheum Dis 2004;63(Suppl. 1):S96-97

5. Gold D, et al. J Bone Miner Res 2005;20(Suppl. 1):S399 (Abstract M362)

6. Silverman S, et al. J Bone Miner Res 2005;20(Suppl. 1):S286 (Abstract SU417)

7. Goettsch WG, et al. J Bone Miner Res 2005;20(Suppl. 1): S278 (Abstract SU388)

8. Recker RR, et al. Mayo Clin Proc 2005;80:856-61

9. Bartl R, et al. Osteoporos Int 2005;16(Suppl. 3):S45 (Abstract P195)

10. Cramer JA, et al. J Bone Miner Res 2004;19(Suppl. 1):S446 (Abstract M434).

\section{SY401. BISPHOSPHONATES ARE THE GOLD STANDARD TREATMENT FOR PMO - HOW DO THEY COMPARE?}

J. Cannata-Andia; Universidad de Oviedo, Hospital Universitario Central de Asturias, Oviedo, Spain

The primary aim of osteoporosis treatment is fracture prevention, as fractures severely impact on patient health and quality of life. Daily bisphosphonates reduce vertebral fracture risk by a similar magnitude, although the largest relative risk reduction for vertebral fracture has been reported with ibandronate $(62 \%){ }^{1}$ Clinical data for non-vertebral fracture are variable, as the trial design and fracture risk of the study population influence outcomes. However, the available bisphosphonates are generally considered to share comparable efficacy and tolerability. ${ }^{2,3}$

Bisphosphonates are also associated with increases in bone mineral density (BMD) and reductions in biochemical markers of bone turnover, and comparator studies using BMD as a surrogate endpoint for fracture efficacy have been used to assess less frequent than daily regimens. ${ }^{4-6}$ Preclinical and clinical data obtained for ibandronate administered with an extended dose-free interval have supported the approval of once-monthly oral ibandronate $(150 \mathrm{mg})$ in the US and EU.

The MOBILE study compared the efficacy and safety of oncemonthly oral ibandronate with daily oral ibandronate $(2.5 \mathrm{mg})$ in women with postmenopausal osteoporosis $(n=1,609){ }^{6,7}$ Analysis at 1 year demonstrated the non-inferiority of the $150 \mathrm{mg}$ dose compared with daily ibandronate in terms of producing substantial increases in lumbar spine (L2-L4) BMD. ${ }^{7}$ Data at 2 years were consistent with these findings $(6.6 \%$ vs $5.0 \%$, respectively) and the $150 \mathrm{mg}$ regimen group was proven superior in terms of BMD increase at the lumbar spine $(p \leq 0.002)$, total hip $(p<0.0001)$, femoral neck $(p \leq 0.029)$ and trochanter $(\mathrm{p}<0.0001) .{ }^{6}$ The $150 \mathrm{mg}$ ibandronate regimen was shown to have a similar safety and tolerability profile to the daily regimen.

The MOTION study is comparing once-monthly oral ibandronate $(150 \mathrm{mg})$ with weekly alendronate $(70 \mathrm{mg})$. Until these comparative data become available, an important differentiating factor for consideration when prescribing bisphosphonates will be patient preferences for dosing regimens, e.g. weekly versus oncemonthly.

\section{References}

1. Chesnut CH, et al. J Bone Miner Res 2004;19:1241-9

2. NICE Final appraisal determination. July 2004

3. Cranney A, et al. Endocr Rev 2002;23:570-8

4. Schnitzer T, et al. Aging (Milano) 2000;12:1-12

5. Brown JP, et al. Calcif Tissue Int 2002;71:103-11

6. Reginster J-Y, et al. Ann Rheum Dis 2006. Epub ahead of print

7. Miller PD, et al. J Bone Miner Res 2005;20:1315-22.

\section{SY402. EXPERIENCING ONCE-MONTHLY IBANDRONATE: CONFIRMING THE PATIENT'S PREFERENCE FOR LESS FREQUENT DOSING}

D. Reid; University of Aberdeen, Aberdeen, UK

Patient preference for reducing bisphosphonate dosing frequency from daily to weekly corresponds with an increase in adherence. $^{1,2}$ However, further improvement in therapeutic adherence is required and less-frequent dosing might achieve this objective. With the introduction of once-monthly oral ibandronate (Bonviva), it is important to confirm whether patient preference extends to this new regimen.

A survey questioning 393 women with postmenopausal osteoporosis regarding their preference for either their current weekly bisphosphonate or a proposed new once-monthly bisphosphonate found that, of patients expressing a preference $(93 \%)$, the majority $(67 \%)$ stated they would prefer a once-monthly tablet. ${ }^{3}$ Their main reasons for choosing once-monthly included 'taking medication less often' and 'fitting better with their lifestyle'.

Patient preference for once-monthly ibandronate has been further assessed in a US clinical trial (BALTO I), a 6-month, randomised, open-label, cross-over study. ${ }^{4}$ The 342 participants (bisphosphonate-na or daily bisphosphonate-lapsed women with postmenopausal osteoporosis) received once-monthly ibandronate $(150 \mathrm{mg})$ for 3 months followed by weekly alendronate $(70 \mathrm{mg})$ for 12 weeks, or vice versa. Patient preference and choice for convenience was recorded at study end using a self-completed questionnaire. 
Of the women expressing a preference $(93 \%)$, the majority preferred once-monthly ibandronate $(71 \%$ vs $29 \%$ weekly alendronate, $\mathrm{p}<0.0001$ for monthly preference) and considered ibandronate to be more convenient $(74.6 \%$ vs $25.4 \%$ weekly alendronate, $\mathrm{p}<0.0001$ for monthly preference). Patients stated that once-monthly dosing would be easier to follow over a long period of time and fit better with their lifestyle. The overall incidence of adverse events and upper gastrointestinal adverse events was similar for both treatment regimens; $3.7 \%$ and $5.3 \%$ of patients withdrew due to adverse events with ibandronate and alendronate, respectively. Switching between alendronate and ibandronate did not affect patient preference (Gart-order-effect, $\mathrm{p}=0.1855$ )

These data show that a strong patient preference exists for once-monthly ibandronate over weekly alendronate. A second study conducted in Germany, France and the US, following the same study design, found similar results, ${ }^{5}$ confirming that lessfrequent dosing is an acceptable treatment option that meets patient needs. Such findings may well lead to higher adherence rates with monthly therapy and hence better clinical outcomes.

\section{References}

1. Simon JA, et al. Clin Ther 2002;24:1871-86

2. Recker RR, et al. Mayo Clin Proc 2005;80:856-61

3. Simon JA, et al. The Female Patient 2005;30:31-36

4. Emkey R, et al. Curr Med Res Opin 2005;21:1895-903

5. Hadji P, et al. ECCEO 2006 (Abstract 182).

SY403. THE IMPACT OF SUBOPTIMAL ADHERENCE ON OSTEOPOROSIS TREATMENT OUTCOMES

R. Adachi; McMaster University, Hamilton, Ontario, Canada

Bisphosphonates are highly effective and routinely used to treat postmenopausal osteoporosis. However, less than $50 \%$ of patients continue with treatment for more than 1 year. ${ }^{1}$ A longitudinal, cohort study of medical and pharmaceuticals claims from the Medstat MarketScan ${ }^{\circledR}$ database investigated the link between suboptimal therapeutic adherence and increasing fracture incidence $(n=37,698 ; n=6,825$ diagnosed with postmenopausal osteoporosis). $^{2}$ Over the 24 -month study period, less than half $(48 \%)$ of the women diagnosed with osteoporosis were compliant with daily or weekly bisphosphonate therapy and less than one quarter $(21 \%)$ were persistent. The risk of fracture was reduced by $26 \%$ in refill compliant patients and by $21 \%$ in persistent patients compared with the non-compliant and non-persistent patients $(\mathrm{p}<0.001$ and $\mathrm{p}=0.0069$, respectively). It was calculated that with appropriate treatment and adequate adherence almost 400,000 fractures could be prevented each year in the USA alone.

Therapeutic adherence has been improved by reducing dosing frequency from daily to weekly; $55 \%$ of patients receiving a weekly bisphosphonate persisted with therapy compared with $37 \%$ of daily bisphosphonate users. ${ }^{3}$ However, these levels can still be improved. It is anticipated that further extending the dosefree interval with once-monthly ibandronate (Bonviva) may improve adherence. This is supported by a recently published study that demonstrated that a majority of patients prefer once-monthly ibandronate $(150 \mathrm{mg})$ and find it more convenient than weekly alendronate $(70 \mathrm{mg}){ }^{4}$

Following regulatory approval of once-monthly ibandronate for the treatment of postmenopausal osteoporosis, studies have commenced in the USA and Europe to assess the impact of oncemonthly dosing versus weekly dosing on long-term therapeutic adherence.

\section{References}

1. Lombas C, et al. J Bone Miner Res 2001;16(Suppl. 1):S529 (Abstract M406)

2. Siris E, et al. J Bone Miner Res 2005;20(Suppl. 1):S282 (Abstract SU403)
3. Recker RR, et al. Mayo Clin Proc 2005;80:856-61

4. Emkey R, et al. Curr Med Res Opin 2005;21:1895-903.

Update on Disease Modification and Disease Management in Osteoarthritis Sponsor: ROTTAPHARM GROUP

\section{SY404. PHARMACOLOGICAL CORRELATES OF THE CLINICAL EFFECTS OF GLUCOSAMINE SULFATE IN OSTEOARTHRITIS}

G. Herrero-Beaumont; Department of Rheumatology, Fundacion Jimenez Diaz - Capio, Madrid, Spain

Glucosamine sulfate has been proposed as a symptom- and structure-modifying drug in osteoarthritis (OA). Two independent, 3-year trials have shown that the prescription formulation of crystalline glucosamine sulfate $1500 \mathrm{mg}$ once-a-day was able to delay joint structure changes in knee OA as assessed by standing radiographs. While the data on the symptom-modifying effects are more controversial for glucosamine in general, the same formulation and dose of glucosamine sulfate has been recently shown to relieve pain and improve function over a 6-month treatment course in knee OA patients in the Glucosamine Unum In Die Efficacy (GUIDE) trial.

Therefore it looks like appropriate doses of the correct glucosamine formulation might exert an effect on both symptoms and joint structure changes, i.e. with a unique mechanism among other drugs used in OA

To test this hypothesis, we have assessed the effects of the NSAIDs meloxicam $\left(10^{-6} \mathrm{M}\right)$, diclofenac $\left(10^{-6} \mathrm{M}\right)$, indomethacin $\left(10^{-6} \mathrm{M}\right)$, or of the putative chondroprotectives diacerhein $\left(10^{-5} \mathrm{M}\right)$ and chondroitin sulfate $(1 \mathrm{mg} / \mathrm{ml})$, or of glucosamine sulfate $(1 \mathrm{mg} / \mathrm{ml})$, on the activation of several proinflammatory and structural mediators in cultured human OA chondrocytes and synoviocytes.

Stimulation with $10 \mathrm{U} / \mathrm{ml} \mathrm{IL-1 \beta}$ induced an increase in NFkB activation, $\mathrm{PGE}_{2}$ production, COX-2 expression and metalloproteases (MMP-1/MMP-13) synthesis in both cell types, while NO production was increased only in chondrocytes. Glucosamine sulfate was the only compound able to reverse all these stimulatory activities in both cell types, although the effects on MMPs were limited to chondrocytes, with inhibition rates ranging between $45 \%$ and $75 \%$. Putative chondroprotective agents only partially controlled some of these mediators. NSAIDs were able to significantly inhibit proinflammatory pathways, but NO increased synthesis in chondrocytes was unaltered and metalloproteases production was even super-induced in both cell types.

These pharmacological findings support the effects of glucosamine sulfate both as a symptom- and a structure-modifying drug as shown in recent clinical studies using appropriate formulations and doses, i.e. with a unique profile among other drugs commonly used in OA.

\section{SY405. OSTEOARTHRITIS DISEASE MANAGEMENT WITH GLUCOSA-} MINE SULFATE: A CRITICAL REVIEW

L.C. Rovati; Department of Clinical Pharmacology, Rottapharm, Monza and University of Milano-Bicocca, Italy

Osteoarthritis (OA) disease management requires chronic non-pharmacological and pharmacological approaches. Among the latter, NSAIDs offer short-term benefit but long-term data are lacking or not favourable, with risks of toxicity for chronic use, particularly in elderly patients.

Among putative disease-modifying agents, glucosamine sulfate showed both symptom- and structure-modification in knee $\mathrm{OA}$ in two independent, 3-year, placebo-controlled trials. While the sound data obtained on joint structure with standing knee radiographs may be further confirmed by newer imaging techniques, it was important to clarify whether the symptomatic effects observed were clinically relevant, also given the mild disease characteristics of the patients enrolled. A recent re-analysis 
indeed assessed that out of 259 completers, over $50 \%$ were in Patient Acceptable Symptom State for knee pain at the end of the two studies, but they were $68.4 \%$ for glucosamine sulfate vs. $55.6 \%$ with placebo $(\mathrm{p}=0.033)$ with $38.9 \%$ vs. $27.8 \%$, respectively, achieving at least Minimal Clinically Important Improvement $(\mathrm{p}=0.04)$. When symptom and structure outcomes were considered together, $53 \%$ of patients receiving glucosamine sulfate had joint space narrowing $<0.5 \mathrm{~mm}$ and over $20 \%$ improvement in total WOMAC, vs. $32 \%$ under placebo $(\mathrm{p}=0.0007)$.

If these effects were predictive of disease modification, a decrease in clinically relevant outcomes should occurr after longer observation: indeed, there was a reduction in OA-related surgeries 5 years after glucosamine sulfate withdrawal, with a significant $(\mathrm{p}=0.04) 52 \%$ decrease in risk of knee replacement compared with placebo in the two studies combined.

The symptom- and structure-modifying effects of glucosamine sulfate are probably linked to the inhibition of IL-1 intracellular signalling pathway, resulting in a decreased expression of both pro-inflammatory and pro-degenerative gene products. In facts, these activities are observed in vitro in chondrocytes at glucosamine concentrations in the $10 \mu \mathrm{M}$ range, i.e. those achieved in plasma and synovial fluid of knee $\mathrm{OA}$ patients receiving the $1500 \mathrm{mg}$ once daily prescription formulation of glucosamine sulfate used in the long-term studies. This preparation was also used in the recently reported GUIDE trial, showing symptommodification already over 6 months of disease management, at variance with other short studies using different glucosamine formulations providing lower levels of the active ingredient.

\section{SY406. OSTEOARTHRITIS OF THE KNEE AND GLUCOSAMINE: A COMMENTARY}

J.-Y. Reginster, R.D. Altman; WHO Collaborating Center for Public Health Aspects of Rheumatic Diseases, Liege, Belgium and University of California, Los Angeles, USA

Knee osteoarthritis is a public health concern throughout the world. Therapeutic guidelines have been developed by several groups, including the American College of Rheumatology (ACR) and the European League Against Rheumatism (EULAR), with strong similarities between them. Among the differences, glucosamine sulfate is a prescription drug in continental Europe and the EULAR guidelines assign to it a high level of evidence from clinical trials, while in the US glucosamine in a variety of formulations is considered a dietary supplement. Indeed, randomised controlled clinical trials have produced conflicting results about glucosamine symptom-modifying effects. The 2005 edition of a Cochrane Review suggested that conflicting results might be due to the use of different glucosamine formulations, with the most favourable data being associated with the prescription glucosamine sulfate preparation. Nevertheless, the controversy continues with two recently presented trials.

The NIH-sponsored study labelled GAIT used glucosamine hydrochloride $500 \mathrm{mg}$ three times a day vs. placebo, celecoxib, chondroitin sulfate, or the combination of glucosamine and chondroitin, over 6 months. Only celecoxib achieved a modest but significant difference in symptom improvement compared with placebo in the overall population; nevertheless, a significant difference in symptom improvement was also found in favour of the nutraceutical combination in a subset analysis of patients with higher pain at baseline.

Conversely, the GUIDE trial was performed in Europe with the prescription glucosamine sulfate $1500 \mathrm{mg}$ once daily, that provided significantly higher symptomatic improvement at 6 months compared with placebo, while 3 g daily acetaminophen/ paracetamol failed to reach a significant effect.

Although there has been a public comment that the differences in the trials are due to corporate vs. non-corporate sponsorship, no data were produced to support the allegation. Indeed, one could argue that the differences in results were more from the differences in glucosamine formulations and doses, with different pharmacokinetics and thus pharmacologically effective concen- trations, besides a possible role for sulfates. Differences can also be noted in study design, including use of rescue analgesic medications and instruments adopted to assess the efficacy, and in study populations, namely BMI, genetic/ethnic backgrounds, levels of symptoms upon entry and placebo response rate.

\section{Multidimensional Impact of Postmenopausal Osteoporosis Sponsor: ELI LILLY}

SY407. THERAPEUTIC STRATEGIES FOR, AND ECONOMIC IMPACT OF, SEVERE OSTEOPOROSIS - ROLE OF TERIPARATIDE

C. Cooper; MRC Epidemiology Resource Centre, University of Southampton, Southampton General Hospital, Southampton, UK

The personal and societal burden of osteoporotic fragility fractures can be quantified in broad measures by the quality of life of the patient and the economic cost to the patient and society in general. Estimates for lifetime risk of fracture for women are $40-50 \%$ and for men, up to $22 \%$. Fragility fractures increase exponentially with age, and projections suggest that hip fractures alone will increase up to 3 -fold by 2025 and 4 -fold or more by 2050, at a cost of up to 131 billion in the US (Johnell et al., 1997 Am $\mathbf{J}$ Med). Thus the number of hip fractures alone may affect 7 million to 21 million people in just over 4 decades from now (Johnell \& Kanis OI 2005). Therapeutic agents for the treatment of fractures have demonstrated effectiveness. Recombinant teriparatide, [rhPTH (1-34)] is a bone-formation agent for the treatment of postmenopausal women and men who are at high risk for fracture. Teriparatide markedly reduced the risk of vertebral fractures by $65 \%$ and nonvertebral fragility fractures by $53 \%$ in postmenopausal women with osteoporosis treated with the current marketed dose of $20 \mu \mathrm{g}$ once-daily (Neer et al., 2001, NEJM). Following discontinuation of teriparatide treatment, the reduction in risk of vertebral fractures was maintained for up to 18 months (Lindsay et al., 2004, Archiv Intern Med). The costeffectiveness of teriparatide treatment has been described using a simulation model incorporating data on fracture risk and mortality (Lundkvist et al., 2005 OI). Total accumulated life-time costs, life years and quality-adjusted life years (QALY) were estimated in the model simulation of clinical fractures. The cost per QALY was estimated to be approximately 3 fold lower in patients who were treated immediately after a vertebral fracture (EUR 20,000) compared with those who were treated for a previous vertebral fracture (EUR 64,000). Thus the cost-effectiveness of teriparatide treatment is highly dependent not only upon the risk profile of the treated patient but also the timing of starting treatment relative to the fracture.

\section{SY408. COMPLIANCE WITH LONG-TERM TREATMENT IN PATIENTS WITH OSTEOPOROSIS}

\section{J.D. Adachi; St. Joseph's Healthcare, Hamilton, Canada}

Osteoporosis is a systemic skeletal disease characterized by low bone mineral density and microarchitectural deterioration, resulting in an increased susceptibility to fracture. In recent years, clinical trials have established the efficacy of commonly used treatments including antiresorptive and bone formation agents when taken consistently for the duration of the study trial. The reduction in the risk of vertebral fractures ranges from $30-65 \%$ and nonvertebral fracture risk is reduced $16-53 \%$. Prior to the time of the fracture, however, osteoporosis may be described as a "clinically silent" or asymptomatic disease because the patient is often unaware of the loss in bone density or changes in architecture. Consistent with other chronic, largely asymptomatic conditions, long-term compliance with medication is generally poor (Solomon et al., 2005 Archiv Intern Med). The challenge for the patient is be adherent to the treatment to maximize the potential benefit of these osteoporosis treatments, the prevention of new fractures and chronic disability. 
Adherence consists of 2 components: compliance (taking the drug as prescribed) and persistence (taking the drug for the recommended time). Evidence suggests that $20 \%$ of patients abandon osteoporosis treatments within 6-7 months, and by the end of the first year, approximately $50 \%$ of patients are not adherent to treatment. Suboptimal adherence $(<80 \%)$ is associated with suboptimal outcomes. Poor adherence is reflected in lower gains in bone mineral density, less likelihood of achieving expected changes in markers of bone turnover, and lower reduction in risk of fracture. Barriers to adherence include side-effects, lack of acceptance that the treatment is needed, and complex or inconvenient dosing regimens. Where less frequent prescription options are available, patients were more compliant with weekly regimens than with once-daily bisphosphonate treatments, although the absolute rate of persistence remained below $50 \%$ after 1 year. Of interest is a recent study by Rousculp et al. (ECCEO5, 2005), who found that persistence at 60 days among patients using teriparatide, taken once-daily by self-injection was slightly but significantly higher than that of patients taking a once-daily oral alendronate treatment $(64.6 \%$ vs. $59.9 \%)$. Strategies to improve adherence may include patient education regarding the disease state, providing feedback to the patient on the positive impact of adherence on outcomes such as markers of bone turnover or bone mineral density, and more convenient dosing regimens.

\section{SY409. MULTIRISK ASSESSMENT IN POSTMENOPAUSAL WOMEN}

\section{J.-Y. Reginster; University of Liège, Liège, Belgium}

With an aging population and an increase in life expectancy, women will live an unprecedented average number of years after menopause, with the diminution in estrogen production associated with long-term health implications. The assessment of "total health" in postmenopausal women encompasses 5 areas of care: mental health (depression), cardiovascular health, skeletal health, oncology, and gynecologic health. The 3 most costly challenges to health (annual costs, US dollars) are osteoporosis (13.8 billion); cardiovascular disease (10 billion); and breast cancer ( 6 billion). We present 4 topics for discussion: 1. Association of risks for cardiovascular disease and osteoporosis in postmenopausal women treated for osteoporosis in real life settings. French postmenopausal women $(19,337)$ who were being treated for osteoporosis responded to questions regarding risk factors for osteoporosis, cardiovascular disease, and breast cancer. Women who reported height loss, a marker of vertebral osteoporosis, were more likely to have risk factors for cardiovascular disease. 2. Incidence of fractures, cardiovascular and breast cancer events in the placebo group of the MORE (Multiple Outcomes of Raloxifene Evaluation) Trial. The event rates for a primary endpoint, osteoporotic fractures and secondary endpoints, cardiovascular disease and breast cancer, were compared over 3 years in 2565 postmenopausal women assigned to the active placebo group. Fracture was the most common event. The likelihood of a coronary event, invasive breast cancer, or clinical vertebral fracture was similar. Each of these events was approximately 5 times more likely than hip fracture. 3. Osteoporosis as an independent risk for cardiovascular events in MORE. The incidence of cardiovascular events in the MORE placebo group was 4-fold higher in women with osteoporosis than in women with low bone mass (Tanko et al. 2005, JBMR). Osteoporosis was associated with an increased risk of cardiovascular events independent of age and other cardiovascular risk factors. The risk of cardiovascular events increased incrementally with increasing severity and number of vertebral fractures at baseline, independent of age and other traditional cardiovascular risk factors. 4. Management of multiple risks in postmenopausal women. The challenges for a long-term treatment of osteoporosis include safety, fracture efficacy, and compliance. Raloxifene has estrogenlike effects on bone (increase bone density and decrease fracture incidence) and lipids levels (decrease total and LDL cholesterol) but anti-estrogenic effects at the uterus and breast.

Optimal treatment strategies in postmenopausal women with osteoporosis should include, in addition to antifracture efficacy, extraskeletal effects that preserve health and improve quality of life of these women. Raloxifene is currently approved in most countries only for the prevention and treatment of osteoporosis in postmenopausal women. Ongoing clinical studies are assessing the effects of raloxifene in addressing other health concerns of postmenopausal women, including prevention of breast cancer and coronary heart disease events.

\section{The Future of Osteoporosis Sponsor: PFIZER}

\section{SY410. NEW ADVANCES IN THE UNDERSTANDING OF THE PATHOPHYSIOLOGY OF OSTEOPOROSIS}

C. Christiansen; Center for Clinical and Basic Research, Ballerup, Denmark

Osteoporosis has a multifactorial etiology and pathophysiology. It is a pathological condition characterized by a decline in cancellous and cortical bone mass combined with microarchitectural changes to cancellous bone. This results in an increased risk of fragility fractures and subsequent increases in morbidity and mortality related to falls or accidents. Bone mass and mineral density are determined in later life by peak bone mass in early adulthood and the rate of involutional bone loss thereafter. Histomorphometry has shown that the deterioration in microarchitecture contributes to fracture risk independently of bone mass. Bone fragility is a composite of geometry, low bone mass density, severance of microarchitectural connections in trabecular structures, and altered bone material quality (primarily the result of accumulated fatigue damage). Reduced bone mass is the result of varying combinations of gonadal hormone deficiency, inadequate intakes of calcium and vitamin $\mathrm{D}$, decreased physical activity, comorbidity, and other drugs.

Osteoporosis occurs when there is an imbalance between bone resorption, which is regulated by osteoclasts, and bone formation, regulated by osteoblasts. Hormonal changes are a well-established cause of osteoporosis in postmenopausal women. Estrogen deficiency increases the remodelling rate and the volume of bone resorbed, and decreases the volume of bone formed. These hormone-related changes may also involve an interaction with the immune system. Declining levels of estrogen and androgen may contribute to an increase in local bone levels of cytoclastic cytokines, followed by increases in osteoclastogenesis and bone loss. ${ }^{1}$ In vivo and in vitro models of postmenopausal osteoporosis suggest that the activation and expansion of tumor necrosis factor- $\alpha$-producing T-cells are a key step in estrogen deficiencydriven bone loss and are regulated by multiple interacting cytokines. $^{2}$

Recent advances in our understanding of osteoporosis include the identification of key regulators of bone turnover- receptor activator of nuclear factor-kappaB (RANK) and its ligand (RANKL), which is regulated by cytokines and various hormones including estrogen, glucocorticoids, and vitamin D. ${ }^{3}$ New treatment approaches for osteoporosis could involve the manipulation of these regulators of bone turnover.

As we improve our understanding of the complex pathophysiology of osteoporosis, new and more effective therapeutic approaches will emerge.

\section{References}

1. Joseph C, Kenny AM, Taxel P, et al. Role of endocrine-immune dysregulation in osteoporosis, sarcopenia, frailty and fracture risk. Mol Aspects Med. 2005;26(3):181-201.

2. Weitzmann MN, Pacifici R. Role of immune system in postmenopausal bone loss. Curr Osteoporos Rep. 2005;3:9297.

3. Hofbauer LC, Heufelder AE. Role of receptor activator of nuclear factor-kappaB ligand and osteoprotegerin in bone cell biology. J Mol Med. 2001;79:243-253. 


\section{SY411. DIAGNOSTIC AND THERAPEUTIC BREAKTHROUGHS}

\section{P.D. Delmas; Claude Bernard University, Lyon, France}

Diagnosis of osteoporosis can be approached through the measurement of bone mass and bone quality. Noninvasive tests of bone turnover represent a convenient method to assess bone fragility. This can be achieved by measuring biochemical markers of bone metabolism (eg, collagen), markers of bone formation (eg, osteocalcin and $\mathrm{N}$-telopeptide), markers of bone resorption (eg, hydroxyproline and hydroxylysine), and bone mineral density (BMD).

Measurements of BMD, expressed as a T-score, are used to establish a diagnosis of postmenopausal osteoporosis; determine fracture risk; identify candidates for intervention; and assess changes in bone mass over time. Techniques used to assess BMD include dual-energy $\mathrm{x}$-ray absorptiometry (DXA), quantitative computed tomography (QCT), ultrasonography, single-energy $\mathrm{x}$-ray absorptiometry, and radiographic absorptiometry. DXA of the hip represents the gold standard for the diagnosis of osteoporosis using the World Health Organization (WHO) criteria. ${ }^{1}$

Other important tests that are used to evaluate bone quality include bone biopsies, which have limitations owing to their invasive nature. However, newer noninvasive techniques are under development, such as micro CT, which can assess bone microarchitecture and bone quality.

Because the pathophysiology of fracture is multifactorial, diagnosis and treatment of osteoporosis should be based on a fracture risk assessment. A number of risk assessment tools include the Osteoporosis Risk Assessment Instrument (ORAI), Osteoporosis Pre-screening Risk Assessment (OPERA), Osteoporosis Index of Risk (OSIRIS), Osteoporosis Self-assessment Tool (OST), and Simple Calculated Osteoporosis Estimation (SCORE).

In the future, we can expect clinical guidelines to recommend moving away from specific $\mathrm{T}$-scores toward intervention thresholds that are based on absolute fracture risk.

Of the existing antiresorptive therapies, next-generation selective estrogen receptor modulators are advantageous in that they do not appear to induce deleterious estrogen receptormediated effects. Other therapies include anti-inflammatory drugs such as cathepsin $\mathrm{K}$, which acts by suppressing the proinflammatory nuclear transcription factor-kappaB factor, which is known to be associated with osteoporosis. ${ }^{2}$ Additional therapies that are currently under investigation include the anabolic vitamin $\mathrm{D}$ receptor modulators, vitamin $\mathrm{D}$ analogs, and RANKL antagonists.

\section{References}

1. Assessment of fracture risk and its application to screening for postmenopausal osteoporosis. Report of a WHO Study Group. World Health Organ Tech Rep Ser. 1994;843:1-129.

2. Aubin JE, Bonnelye E. Osteoprotegerin and its ligand: a new paradigm for regulation of osteoclastogenesis and bone resorption. Medscape Womens Health. 2000;5:5.

\section{SY412. INTERVENTION THRESHOLDS IN THE TREATMENT OF OSTEOPOROSIS}

J. Kanis; WHO Collaborating Centre for Metabolic Bone Diseases, University of Sheffield Medical School, Sheffield, UK

Because osteoporosis treatments differ in their tolerability, efficacy in reducing fracture risk, and cost, there is a need to be highly selective in the identification of high-risk patients who would benefit from treatment. Using T-scores alone to estimate fracture risk may lead to inappropriate treatment of low-risk patients or the nontreatment of high-risk patients (eg, elderly with fragility fractures but a slightly low bone mineral density [BMD]).

Current treatment guidelines rely on the use of T-scores and the presence of other risk factors, but differ in the populations they address, T-score cutoffs, and risk factors used. Emerging methodologies are calculating intervention thresholds based on fracture probability and cost-utility analyses. The National Osteoporosis Foundation (NOF) ${ }^{1}$ and World Health Organization $(\mathrm{WHO})^{2}$ developed models for applying cost analysis with a combination of BMD and clinical risk factors. Certain assumptions are required, for instance that fracture outcomes (rather than BMD measurements) are the preferred measure of efficacy, and that fracture costs are proportional to disutility (the loss of utility over time associated with a fracture). Other modelling assumptions include estimates on compliance and cost of therapy.

Intervention thresholds were calculated in a model using fracture data and economic assumptions for Sweden. ${ }^{3}$ It was found to be cost-effective to treat women with a 10 -year risk of hip fracture that ranged from $1.2 \%$ at age 50 to $7.4 \%$ at age 80 .

Applying cost-utility analyses to fracture probability, using a mix of standardized and region-specific assumptions, provides intervention thresholds that allow clinicians to select those patients most likely to benefit from therapy.

The predictive value of BMD can be enhanced by use of other factors such as biochemical indices of bone resorption and clinical risk factors. Because of the many techniques available for fracture risk assessment, the 10-year probability of fracture is a desirable measurement to determine intervention thresholds. Treatment can be cost-effective if hip fracture probability over 10 years ranges from $2 \%$ to $10 \%$ dependent on age. ${ }^{4}$

Intervention thresholds differ substantially from diagnostic thresholds and should be based on absolute fracture probability (T-score and other independent risk factors). Health economic assessment based on probability of fracture is an important area for further research.

\section{References}

1. Osteoporosis: review of the evidence for prevention, diagnosis and treatment and cost-effectiveness analysis. Introduction. Osteoporosis Int. 1998;8(suppl 4):S7-S80.

2. Kanis JA, Oden A, Johnell O, et al. The burden of osteoporotic fractures: a method for setting intervention thresholds. Osteoporosis Int. 2001;12:417-427.

3. Kanis JA, Johnell O, Oden A, et al. Intervention thresholds for osteoporosis in men and women: a study based on data from Sweden. Osteoporosis Int. 2005;16:6-14.

4. Kanis JA. Diagnosis of osteoporosis and assessment of fracture risk. Lancet. 2002;359:1929-1936.

\section{Acting on Both Sides of the Equation in Postmenopausal Osteoporosis Sponsor: SERVIER}

\section{SY413. USING RISK FACTORS FOR THE DIAGNOSIS AND TREATMENT OF OSTEOPOROSIS}

E. McCloskey; Academic Unit of Bone Metabolism, Metabolic Bone Centre, Northern General Hospital, Herries Road, Sheffield S5 7AU, UK

Bone mineral density (BMD) has a well-established role in the assessment of fracture risk, such that the clinical development of osteoporosis therapies has largely focused on the selection of patients on the basis of low BMD. Epidemiological studies demonstrate, however, that over half of all fractures occur in individuals with BMD above the T-score threshold for osteoporosis. Such studies have confirmed that the risk of fracture is multifactorial and that the combination of independent risk factors with BMD can increase the sensitivity of any risk evaluation. Several fracture risk scores have been developed in individual studies but relatively few of these have been validated in independent populations.

A World Health Organization Scientific Group has undertaken a series of meta-analyses, based on individual data from 
prospective population-based studies, to identify clinical risk factors for fracture and determine their dependence upon age, sex, and BMD. The clinical risk factors chosen, including age, body mass index, prior fracture, parental history of fracture, corticosteroid use, and smoking, have been selected for their ease of use in a primary care setting and correlations with low BMD. Case finding on the basis of these clinical risk factors will capture a population with low BMD, a possible prerequisite for ensuring that identified high-risk patients would respond to treatment with effective antiosteoporotic drugs. Other factors that also predict fracture risk but may not be influenced by skeletal therapies (eg, frequent falls) have been excluded from the model. Responsiveness to bone therapies has been shown for patients selected on the basis of low BMD, prior fracture, or the use of oral corticosteroids. Analyses of randomized controlled trials indicate that the other clinical risk factors do not affect the beneficial effects of treatment.

The combined use of clinical risk factors provides an assessment of fracture risk that is at least as good as BMD for the prediction of hip and other osteoporotic fractures and can enhance the performance characteristics of BMD.

\section{SY414. HOW TO REBALANCE BONE TURNOVER IN FAVOR OF FORMATION}

P.J. Meunier, M.E. Arlot, J.P.Roux, N. Portero, B. Burt-Pichat; INSERM Unit 403, Faculty Laennec, Lyon, France

In in vitro studies and in vivo preclinical experiments in animals, strontium ranelate has been found to stimulate bone formation and decrease bone resorption. Strontium ranelate prevented bone loss in ovariectomized rats, increased bone mass in osteopenic animals, and improved bone strength in normal animals. In the SOTI (Spinal Osteoporosis Therapeutic Intervention) and TROPOS (TReatment of Peripheral OSteoporosis) phase 3 clinical studies, strontium ranelate $2 \mathrm{~g}$ /day reduced over 3 years the risk of new vertebral fracture $(-41 \%)$ and of hip fracture in osteoporotic women aged 74 years or more $(-36 \%)$, and induced an augmentation of biochemical markers of formation and a decrease in markers of resorption.

The objectives of our histomorphometric analysis of 136 transiliac bone biopsies from patients recruited in SOTI, TROPOS, and STRATOS (STRontium Administration for Treatment of OSteoporosis, a phase 2 study) were to assess the mechanisms of action of strontium ranelate at both the cell and bone tissue levels and the bone safety of the compound.

Materials and methods: iliac biopsies were obtained, after tetracycline double labeling, at baseline and after 1, 2, 3, 4, and 5 years of treatment with either strontium ranelate or placebo. All patients received calcium and vitamin D supplementation. The statistical analysis compared the pooled data from 1 to 5 years in patients treated with strontium ranelate ("treated" group ; $n=49$ ) with the pooled data from placebo, plus baseline data obtained from patients in the strontium ranelate, group ("untreated" group ; $\mathrm{n}=89$ ). Histomorphometric parameters were measured at cancellous, endosteal, and cortical envelopes.

Results: the stimulating effects of strontium ranelate on bone formation were demonstrated by higher osteoblastic surfaces (Ob.SBS) in treated compared with untreated patients $(+38 \%$; $P=0.047)$ and by a greater mineral apposition rate (MAR) in cancellous and cortical bone $(+8 \% ; P=0.008,+11 \% ; P=0.033$, respectively) At the tissue level, there was no significant change in activation frequency. The effects on resorption consisted of a tendency to lower endosteal eroded surfaces, endosteal and cancellous osteoclast surfaces, and osteoclasts $(-14 \%,-6 \%,-9 \%$, $-9 \%$, respectively, all NS). With the higher Ob. SBS in the treated group, it was expected that higher osteoclast surfaces would also be observed, which was not the case, reflecting the dual mode of action of strontium ranelate. In terms of safety parameters, the cancellous osteoid thickness was found to be significantly lower in treated patients $(P=0.007)$, the MAR was significantly higher in treated patients, with no change in mineralization lag-time and osteoid volume. These results demonstrate that primary mineralization rate is not impaired, but on the contrary is stimulated by strontium ranelate

Conclusion: these findings indicate a stimulating effect of strontium ranelate on the osteoblastic population and MAR and a small decrease in bone resorption. They are in agreement with the changes observed in biochemical markers in clinical studies. They confirm the dual mode of action of strontium ranelate and its ability to rebalance bone turnover in favor of bone formation.

\section{SY415. FIRST-LINE TREATMENT FOR POSTMENOPAUSAL OSTEOPO- ROSIS}

J.E. Compston; Department of Medicine, University of Cambridge School of Clinical Medicine, Cambridge CB2 2QQ, UK

The efficacy of strontium ranelate, $2 \mathrm{~g}$ daily, in postmenopausal women with osteoporosis has been investigated in 2 large randomized, double-blind, placebo-controlled trials, namely SOTI (Spinal Osteoporosis Therapeutic Intervention) and TROPOS (TReatment Of Peripheral OSteoporosis). These studies were preceded by a run-in period, the main aim of which was to start the normalization of calcium and vitamin D status. The primary end point of SOTI was reduction in vertebral fractures and, in TROPOS, reduction in nonvertebral fractures. The number of women recruited was 1649 and 5091, respectively, with a mean age of 69 years in SOTI and 77 years in TROPOS.

In SOTI, strontium ranelate therapy was associated with a significant reduction in vertebral fractures, with a $41 \%$ reduction in relative risk (RR) over 3 years, the fracture incidence being $32.8 \%$ in the placebo group and $20.9 \%$ in the treatment group. This beneficial effect was seen after only 1 year of treatment (RR $0.51 ; 95 \%$ confidence interval $[\mathrm{CI}], 0.36-0.74)$. There was also a significant reduction in clinical vertebral fractures (RR $0.62 ; 95 \%$ CI, 0.47-0.83) which was seen after only 1 year of treatment (RR $0.48 ; 95 \% \mathrm{CI}, 0.29-0.80)$ and in height loss $(P=0.003)$ at 3 years. Pooled subgroup analyses of patients from SOTI and TROPOS demonstrated significant reductions in vertebral fracture risk in women with or without vertebral fracture at baseline, women with osteopenia, and women aged over 80 years.

A significant reduction in nonvertebral fractures was demonstrated in TROPOS, with a relative risk of 0.84 (95\% CI, 0.702 0.995) in treated women. In a post hoc analysis of osteoporotic women aged over 74 years, a significant $36 \%$ reduction was seen in hip fracture risk (RR 0.64; 95\% CI, 0.412-0.997) over 3 years.

These results demonstrate the efficacy of strontium ranelate in reducing fractures at vertebral and nonvertebral sites, including the hip. Its therapeutic spectrum make it an alternative first-line option to bisphosphonates in the prevention of osteoporotic fractures in postmenopausal women.

\section{SY416. PROVEN ANTIFRACTURE EFFICACY OF STRONTIUM RANELATE} ACROSS VARYING RISK PROFILES

C. Roux; Service de Rhumatologie, Centre d'Evaluation des Maladies Osseuses, Hôpital Cochin, Paris, France

Osteoporosis is currently managed with a range of therapies that decrease bone resorption or increase bone formation. The response to the treatment may depend on the patient's characteristics, such as age, bone mineral density (BMD), and prevalent fractures. Strontium ranelate $2 \mathrm{~g}$ per day orally was shown to reduce vertebral and nonvertebral fracture risk in 2 prospective studies, SOTI (Spinal Osteoporosis Therapeutic Intervention) and TROPOS (TReatment Of Peripheral OSteoporosis), and hip fracture in high-risk patients. As the design, centers, and BMD and X-ray central reading centers were common to both studies, it was predetermined to analyze this large database of pooled data for better assessment of the treatment effect estimate.

Thus, a population of 5082 women was prospectively studied and the following potential determinants of vertebral antifracture 
efficacy were assessed: age, BMD, prevalent vertebral and nonvertebral fractures, family history of osteoporosis, body mass index, and addiction to smoking. None of these parameters was a determinant of efficacy. Of particular interest were the data on age: the reduction in risk of vertebral fracture was $37 \%$ $(P=0.003)$ in women aged less than 70 years, $42 \%(P<0.001)$ in women aged 70 to 79 years, and $32 \%(P=0.013)$ in women aged 80 and older. BMD at baseline and prevalent vertebral fracture were not determinants of efficacy. The decrease in relative risk of vertebral fracture was $33 \%$ in women with more than 2 fractures at baseline. In patients without baseline vertebral fracture, the risk of the first fracture was decreased by $48 \% \quad(P<0.001)$. Moreover, in the subgroup of patients without prevalent vertebral fracture, but with a previous peripheral fracture $(\mathrm{N}=910)$, strontium ranelate reduced the risk of the first vertebral fracture by $46 \%$.

These data demonstrate that a 3-year treatment with strontium ranelate leads to antivertebral fracture efficacy in postmenopausal women with a large spectrum of risk for such fractures.

Faces in the Crowd: Emerging Strategies to Find and Treat Osteoporosis Patients at High Risk of Fracture Sponsor: NYCOMED

\section{SY417. BEYOND THE FRACTURE: PROFILING RISK GROUPS}

S.J. Gallacher; Medical Unit, Southern General Hospital, Glasgow, UK Beyond the fracture: profiling risk groups

The presence of vertebral fracture is a proven predictor of both vertebral and non-vertebral subsequent fractures. However, vertebral fractures are often asymptomatic, and it has been estimated that only $35 \%$ of vertebral fractures are clinically diagnosed. The majority of fractures that therefore remain undetected suggest the existence of a hidden population of patients at high risk of fracture who may remain unrecognised and not receive appropriate anti-osteoporosis therapy in the absence of risk factor based case-finding approaches to diagnosis.

To explore further the scale and characteristics of this 'hidden' fracture population, epidemiological data were derived from a database of around 10,000 fracture patients presenting consecutively to the Fracture Liaison Service at the Southern General Hospital, Glasgow, UK. One of the key findings from this study is the surprisingly high incidence of previously undiagnosed vertebral fractures (many of which are multiple). Bone mineral density and clinical fracture risk factors were recorded for these patients, along with the results of morphometric x-ray absorptiometry (MXA). The results obtained define a population of non-vertebral fracture patients who have unrecognised underlying prevalent vertebral fracture(s). This sub-group of patients, at high risk of further fracture, has been further stratified by incident fracture site, age and bone mineral density. Data presented for individual fracture sites are broken down to include those with the lowest bone mineral density, with $\mathrm{T}$-scores of $<-3.0$ and $<-4.0$.

This work represents the first accurate characterisation of the degree of overlap between non-vertebral and vertebral fracture patient populations. Furthermore, these data highlight the small number of patients that need to be scanned (with MXA) in order to identify one patient with vertebral fracture and adjust intervention accordingly, making a robust case for a new standard of diagnostic practice. Improved understanding of the characteristics of groups at high risk of fracture raises the question of how best to manage this sub-set of patients, given their need for rapidly effective fracture prevention treatment.

\section{SY418. CALCIUM AND VITAMIN D SUPPLEMENTATION REVISITED}

S. Boonen; Leuven University Center for Metabolic Bone Diseases and Division of Geriatric Medicine, Katholieke Universiteit Leuven, Belgium
There is general agreement that in patients with documented osteoporosis, calcium and vitamin D supplementation should be an integral component of the management strategy, along with antiresorptive or anabolic therapy. A key objective of osteoporosis management is getting the foundation right for all patients, although there is evidence that this simple, inexpensive therapeutic approach is significantly underutilised.

Current evidence suggests that oral vitamin D supplementation reduces the risk of hip and non-vertebral fractures when calcium supplementation is added and when the vitamin D supplement is appropriately dosed. Interest groups such as the American Medical Women's Association (AMWA) recommend at least 800 to 1000 international units (IU) of vitamin D per day in order to improve bone strength in men and women aged 50 years and above.

The AMWA's recommendation is supported by the results of a recent meta-analysis, carried out to estimate the effectiveness of vitamin $\mathrm{D}$ supplementation in preventing hip and non-vertebral fracture in older patients (60 years and above), as observed in double-blind randomised clinical trials. A key conclusion of this meta-analysis was that whilst doses of supplemental vitamin D between 700 and 800 IU per day appear to reduce the risk of fractures in this at-risk patient group, a dose of $400 \mathrm{IU}$ per day is not sufficient for fracture prevention, thus underlining the importance of sufficient dosing.

Furthermore, vitamin D should be combined with calcium supplementation, with evidence suggesting an optimal dosage at around $1000 \mathrm{mg}$, as patients with vitamin D deficiency also tend to be deficient in calcium. The effects of combined calcium and vitamin $\mathrm{D}$ on parathyroid function and bone mineral density provide a strong rationale for the use of this combination in the prevention and treatment of osteoporosis and osteoporotic fracture.

Just as it is important to ensure accurate identification of patients at risk of fracture in order to ensure appropriate intervention, so too is it important for calcium and vitamin D supplementation to be appropriately dosed and targeted towards those patients most likely to benefit. Target populations for supplemental calcium and vitamin D include calcium and vitamin $\mathrm{D}$ deficient individuals, patients on long-term corticosteroid therapy, and osteoporosis patients taking antiresorptives or anabolic agents. Strong support exists for the use of $1000 \mathrm{mg}$ calcium and $800 \mathrm{IU}$ vitamin D as a first-line prevention strategy for the prevention and treatment of osteoporosis; however, patients with documented osteoporosis will derive further benefit in terms of fracture prevention from the addition of an antiresorptive or anabolic agent.

\section{SY419. MANAGING PATIENTS AT HIGH RISK OF FRACTURE WITH PARATHYROID HORMONE (1-84)}

R. Rizzoli; Division des Maladies Osseuses, Hôpital Cantonal de Genève, Geneva 14, Switzerland

The sub-group of osteoporotic patients at highest risk of fracture has a particular need for effective intervention that provides rapid improvements in both bone quantity and quality. These high risk patients have recently benefited from the arrival of the human recombinant parathyroid hormones (PTHs). These potent anabolic agents represent the natural next step in osteoporosis therapy, offering benefits in terms of fracture prevention when incorporated into patient management strategies.

The introduction of a more thermally stable variant, the fulllength PTH (1-84), will broaden the available options in this important new class. Iliac crest biopsies subjected to microcomputed tomography were obtained from postmenopausal osteoporotic women enrolled in the Treatment of Osteoporosis with PTH (1-84) (TOP) Study, randomised to receive either PTH (1-84) or placebo. Relative to the placebo-treated group, PTH (184) resulted in marked increases in cancellous bone volume, improved trabecular connectivity and a more plate-like structure indicative of stronger bone. 
These results are consistent with the marked reduction in vertebral fracture incidence observed in the TOP Study. Over the course of this 18-month trial, treatment with PTH (1-84) was well tolerated, and associated with a significantly lower incidence of vertebral fractures compared to placebo, a relative risk reduction (RRR) of $61 \%$. In particular, PTH (1-84) significantly decreased the risk of first vertebral fracture, with a RRR of $68 \%$. Treatment with PTH (1-84) was generally well tolerated, with headache, nausea and dizziness occurring with higher frequency in the PTH (1-84)-treated group versus placebo. Although the incidence of hypercalcaemia was higher than in the placebo group, this led to discontinuation of therapy in only $0.5 \%$ of the PTH (1-84)treated group.

The anticipated arrival of this new therapeutic option raises the question of how best to incorporate PTH (1-84) into the osteoporosis treatment armamentarium. To address this, postmenopausal women enrolled in the Parathyroid Hormone and Alendronate (PaTH) Study were randomised to receive PTH (184), alendronate, or both for 1 year. After 12 months, those who had received PTH (1-84) monotherapy were randomly reassigned to 1 additional year with either placebo or alendronate. Results from year two show that foundation therapy with PTH (1-84) was associated with accelerated bisphosphonate benefit in the sequential treatment paradigm in which 1 year of PTH (1-84) is followed by 1 year of alendronate. Trabecular bone mineral density (BMD) at the spine increased $25.5 \%$ over the first year on PTH (1-84); this increase was maintained in the second year when PTH (1-84) was followed with alendronate $(31 \%$ increase from baseline after 2 years). Conversely, the densitometric gains achieved with PTH (1-84) were lost if treatment was not followed with an antiresorptive agent: increases in trabecular BMD at the spine were reduced to $14 \%$ over baseline when PTH (1-84) was followed by placebo in the second year.

Full-length parathyroid hormone, PTH (1-84), is well tolerated and preferentially stimulates natural bone formation, restoring bone geometry and enhancing bone quality to build stronger, more fracture-resistant bone, offering accelerated benefits to patients at high risk of fracture when used within a sequential treatment framework.

\section{Osteoporosis Patients: The Need for Complete Fracture Protection Sponsor: ALLIANCE FOR BETTER BONE HEALTH (PROCTER \& GAMBLE PHARMACEUTICALS AND SA- NOFI-AVENTIS)}

\section{SY420. NON VERTEBRAL FRACTURES: WHO IS MOST AT RISK?}

Ch. Roux; Hôpital Cochin, Université René Descartes, Paris, France

One percent of the UK population per year suffer from non-vertebral fractures. ${ }^{1}$ Non-vertebral fractures account for the greatest amount of morbidity, mortality and cost attributable to osteoporosis. An increase in mortality is associated with hip fractures; the latter accounting for $10 \%$ of all fractures and $40 \%$ of total fractures in individuals greater than 80 years of age. $^{2}$ The incidence of other non-vertebral fractures, such as those of the wrist is also comparable. Post menopausal women have a $16 \%$ risk of such a fracture. ${ }^{3}$ Elderly people have an increased risk of non-vertebral fractures such as those of the proximal humerus, pelvis and ribs. ${ }^{1,4}$ It is therefore impractical to focus on hip fractures as the sole burden of non-vertebral fractures. Doing so underestimates the consequence of osteoporosis and the impact of non-vertebral fractures on society.

The current available treatments for vertebral fractures have demonstrated consistent efficacy in the reduction of vertebral fracture risk. Conversely, the treatment effects on non-vertebral fractures have varied significantly. No head-to-head studies have been conducted comparing the anti-fracture effect of the different treatments; however, these different effects on non-vertebral fracture risk may be a determinant in the choice of treatment for women with post menopausal osteoporosis.

The assessment of non-vertebral fracture risk in post menopausal women is a difficult task as the relationship between risk factors and fractures differ by type of fracture and individuals. 5,6 Previous studies have focused on the Colles' fracture. ${ }^{5,6}$ Risk factors specific to this type include previous wrist fracture, low body weight, low calcium intake and non use of HRT. It also appears that the direction of the fall contributes towards fracture type. Vitamin D deficiency and secondary hyperparathyroidism are common in the elderly and may have a role in the prediction of non-vertebral fractures along with a low bone mineral density (BMD) in osteoporotic women.

The characterisation of these risk factors will greatly aid the clinical management and the selection of the treatment for patients identified at risk for non-vertebral fractures such as the elderly and post menopausal women with osteoporosis.

\section{References}

1. Eastell R, Reid DM, Compston J, Cooper C, Fogelman I, Francis RM et al. Secondary prevention of osteoporosis: when should a non-vertebral fracture be a trigger for action? $Q J$ Med. 2001; 94: 575-597

2. Johnell O, Kanis JA, Odèn A, Sernbo I, Redlund-Johnell I, Petterson C, et al. Mortality after osteoporotic fracture. Osteoporos Int. 2004; 15: 38-42

3. Melton LJ III, Chrischilles EA, Cooper C, lane AW, Riggs BL. How many women have osteoporosis? J Bone Miner Res. 1992; 7: $1005-1010$

4. Kannus P, Palvanen M, Niemi S, Parkkari J, Jarvinen M, Vuori I. Increasing number and incidence of osteoporotic fractures of the proximal humerus in elderly people. $\mathrm{Br}$ Med $\mathrm{J}$. 1993; 313: 1051-1052

5. Graafmans WC, Ooms ME, Bezemer PD, Bouter LM, Lips P. Different risk profiles for hip fractures and distal forearm fractures: a prospective study; Osteoporos Int 1996; 6: 427-431

6. Honkanen R, Tuppurainen M, Kröger H, Alhava E, Saarikoski S. Relationship between risk factors and fractures differ by type of fracture: a population-based study of 12192 perimenopausal women. Osteoporos Int. 1998; 8: 25-31.

SY421. RISK OF NON-VERTEBRAL FRACTURES: DO ALL THERAPIES OFFER THE SAME PROTECTION?

\section{H. Dobnig; Medical University, Graz, Austria}

It has been suggested that there is a high rate of morbidity and mortality associated with non-vertebral fractures in particular with those of the hip. Many of the approved therapies for postmenopausal osteoporosis have documented efficacy in reducing the risk of vertebral fractures. However, data on efficacy in nonvertebral factures are not consistent for these therapies. ${ }^{1}$

The assessment of non-vertebral fracture incidence is an important contributor to our understanding of the effects of osteoporosis. The issue of non-vertebral anti-fracture efficacy has largely been based on post-hoc subgroup analyses and a limited number of antiresorptive agents have demonstrated a significant risk reduction in intention-to-treat analysis (ITT).

Registration of new therapies requires fracture prevention efficacy and analysis of the ITT population. ${ }^{2,3}$ The efficacy and quantification of treatment effects should, therefore, be based primarily on analyses of the ITT population rather than subanalyses. The ITT principle is based on two components: patient population and handling of the data. In practice, this ideal is difficult to achieve and missing data can have an impact on the analysis. Regardless of the fact that ITT data gives the most reliable overall estimate of treatment efficacy it is recommended that sensitivity analyses are also performed and summarised when concluding analyses. ${ }^{1}$ 
A review and meta-analysis of ITT studies has shown that risedronate is the only bisphosphonate to maintain non-vertebral anti-fracture efficacy in more than one study. ${ }^{1}$ However, metaanalyses of trials using risedronate and alendronate showed the two bisphosphonates to be efficacious in preventing non-vertebral fractures. ${ }^{1}$

Efficacy onset is also an important marker in treatment efficacy; risedronate has been shown to significantly reduce clinical vertebral fracture incidence within six months. ${ }^{4}$ This rapidity of risk reduction was also demonstrated in four large Phase III studies in post menopausal women for non-vertebral fractures. An analysis of these studies indicated that risedronate significantly reduces the incidence of osteoporosis-related non-vertebral fractures within six months. ${ }^{4}$

\section{References}

1. S. Boonen, Lann, R.F., Barton, I.P., Watts N.B. Effect of osteoporosis treatments on risk of non-vertebral fractures: review and meta-analysis of intention-to-treat studies. Osteoporosis Int. 2005; 16: 1291-1298

2. Committee for Proprietary Medicinal Products (2001) Note for guidance on postmenopausal osteoporosis in women. Committee for Proprietary Medicinal Products (CPMP), London

3. FDA (1994) Guidelines for preclinical and clinical evaluation of agents used in the prevention or treatment of postmenopausal osteoporosis. US Food and Drug Administration

4. J.T. Harrington, SteMarie, L.G., Brandi, M.L., Civitelli, R., Fardellone, P., Grauer, A., Barton, I., Boonen, S. Risedronate rapidly reduces the risk for non-vertebral fractures in women with postmenopausal osteoporosis. Calcif Tissue Int. 2004; 74(2): 129-35.

\section{SY422. BISPHOSPHONATES: MIGHT WE EXPECT A CLASS EFFECT?}

R. Lindsay; Helen Hayes Hospital \& Columbia University New York, USA

In recent years several studies have indicated that the increase in BMD seen with anti-resorptive agents accounts for only a small proportion of the fracture benefit (8-20\%). Consequently, attention has focused on the other potential mechanisms that might influence fracture protection. The consequence of these studies has been a more thorough understanding of the interactions of agents such as bisphosphonates (BP's) with the skeleton. While there are still uncertainties, it is clear that each bisphosphonate has a unique pattern of effects on both mineral, and enzyme activity. BP's bind to the mineral component of bone with a range of binding affinities; zolendronate has the greatest affinity and clodronate the least. ${ }^{1}$ Perhaps as a consequence of differing affinities and the structure of each agent, binding produces different electrical responses at the surface of bone (the so-called zeta potential). Zolendronate, ibandronate and alendronate increase the zeta potential, while risedronate reduces the potential. ${ }^{1}$ Similarly there are differences in potency when inhibition of farnesyl pyrophosphate synthase is considered, where risedronate appears to be several times more potent than alendronate and about twice as potent as ibandronate., These potencies equate roughly to inhibition of bone resorption observed in rodent models of increased remodeling. ${ }^{4}$ While the data are incomplete, we do know that the combination of risedronate effects on mineral and enzyme activity results in decreased remodeling, increased bone mass, return of mineralization to premenopausal levels, with return of collagen cross linking at sites of bone formation toward normal. The consequence is reduction in the risk of fractures at both vertebral and non-vertebral sites. Because of the complexity of the interactions of BP's with mineral and bone cells, the clinician must place reliance on the available fracture data from randomized controlled clinical trials when choosing a BP for individual pa- tients with osteoporosis. Class effects across the spectrum of these interactions, as well as effects on fracture have not been demonstrated.

\section{References}

1. G.H. Nancollas, Tang, R., Phipps, R.J., Henneman, Z., Gulde, S., Wu, W., Mangood, A., Russell, R.G., Ebetino, F.H. Novel insights into actions of bisphosphonates on bone: Differences in interactions with hydroxyapatite. Bone. 2005

2. J.D. Bergstrom, Bostedor, R.G., Masarachia, P.J., Reszka, A.A., Rodan, G. Alendronate is a specific, nanomolar inhibitor of farnesyl diphosphate synthase. Arch Biochem Biophys. 2000; 373 (1): 231-41

3. J.E. Dunford, Thompson, K., Coxon, F.P., Luckman, S.P., Hahn, F.M., Poulter, C.D., Ebetino, F.H., Rogers, M.J. Structure-activity relationships for inhibition of farnesyl diphosphate synthase in vitro and inhibition of bone resorption in vivo by nitrogen-containing bisphosphonates. $J$ Pharmacol Exp Ther. 2001; 296 (2): 235-42

4. J.R. Green, Muller, K., Jaeggi, K.A. Preclinical pharmacology of CGP 42'446, a new, potent, heterocyclic bisphosphonate compound. J Bone Miner Res. 1994; 9 (5): 745-51.

\section{A New Approach for Improving Bone Health Sponsor: NOVARTIS}

\section{SY423. OSTEOPOROSIS: FROM POLICY TO PATIENT}

\section{P.D. Delmas; Claude Bernard University, Lyon, France}

Osteoporosis is a serious debilitating disease, affecting one in three women and one in eight men over 50 years of age in Europe. Changing demographic patterns and increasing life expectancy in the developing world are predicted to result in osteoporosis assuming global epidemic proportions, with the number of osteoporotic hip fractures projected to rise to 6 million worldwide by 2050, of which 3 million will occur in Asia. As well as having a major impact on patients' physical functioning and quality of life, osteoporosis places a heavy economic burden on healthcare systems because of the prolonged hospitalization and rehabilitation required after osteoporotic vertebral and hip fractures.

To be most effective, awareness-raising campaigns should focus on those individuals at highest risk of osteoporosis postmenopausal women and patients with prior fragility fractures - and those physicians who most commonly see osteoporosis-related fractures -orthopaedic surgeons and radiologists. Postmenopausal women are often unaware of their own risk of osteoporosis and the life-threatening potential of hip fracture and spinal degeneration. Orthopaedic surgeons and radiologists play an important role in the diagnosis of osteoporotic fracture, but this opportunity is often missed. A recent Bone and Joint Decade/International Osteoporosis Foundation survey of more than 3,000 orthopaedic surgeons in France, Germany, Italy, Spain, UK and New Zealand revealed that they often fail to recognize osteoporosis as a cause of fracture and are inconsistent in providing appropriate treatment and referral. Half of those surveyed received little or no training in osteoporosis management. Similarly, although radiologists are well placed to diagnose vertebral fractures, the majority of clinically silent fractures remain undetected. The recent IMPACT study, an international investigation of the diagnostic accuracy of spinal radiography in postmenopausal women with osteoporosis, found that vertebral fracture was under-diagnosed worldwide (false negative rates of $29.5 \%$ in Europe/South Africa/Australia, $45.2 \%$ in North America and $46.5 \%$ in Central and South America).

Key to improving osteoporosis care is improved access to bone mineral density (BMD) testing. Dual energy X-ray absorptiometry, the gold standard for BMD measurement, is a 
cost-effective method for identifying individuals at risk of fracture, but is often unavailable or poorly reimbursed. Therapeutic interventions such as bisphosphonates are cost effective if targeted at individuals at high risk of osteoporotic fracture, but are currently under-prescribed for secondary prevention of osteoporotic fractures. The major disadvantage of the oral bisphosphonates is their poor gastrointestinal tolerability and absorption (typically $<1 \%$ ), and low patient compliance with treatment. The challenge for novel bisphosphonate delivery systems is to achieve improved bioavailability and safety; to this end, various parenteral (intravenous, intranasal, subcutaneous and intramuscular) formulations are currently either under development or have been launched.

\section{SY424. COMPARISON OF ORAL VERSUS INTRAVENOUS BISPHOSPH- ONATES: WHAT IS UNIQUE?}

J.A. Gasser; Novartis Institutes for Biomedical Research, Musculoskeletal Diseases, Basel, Switzerland

Zoledronic acid (ZOL) belongs to the family of nitrogencontaining bisphosphonates, which inhibit a key enzyme in the mevalonate pathway, farnesyl diphosphate synthase (FPPS). Inhibition of this enzyme prevents prenylation of signaling molecules such as Ras, Rho, Rac and Cdc42, which activate several cytoskeletal processes that are essential for osteoclast proliferation and function (bone resorption), including osteoclast migration, membrane ruffling and binding to bone. ZOL is the most potent FPPS inhibitor among the nitrogen-containing bisphosphonates, as indicated by its suppression of recombinant human FPPS activity. The order of potency of these agents in inhibiting FPPS (zoledronic acid $>$ risedronate $>$ ibandronate $>$ alendronate $>$ pamidronate) closely mirrors their order of potency in inhibiting bone resorption in vitro. Thus, inhibition of FPPS appears to be a key mechanism responsible for the antiresorptive action of nitrogen-containing bisphosphonates.

The high potency and binding affinity of ZOL underlie its sustained effect in normalizing bone turnover after a single intravenous infusion; moreover, its high affinity for hydroxyapatite may enhance recycling of the drug from sites of bone resorption into the bone matrix, thereby prolonging its duration of action in vivo. In ovariectomized (OVX) rats, a single intravenous dose of ZOL $20 \mu \mathrm{g} / \mathrm{kg}$ (approximately equivalent to a $5 \mathrm{mg}$ dose in humans) provides full protection against changes in cancellous bone architecture, as determined by micro-computed tomography (CT), for 32 weeks. Importantly, despite its high potency, ZOL maintains adequate bone turnover in adult OVX rats, even at a 4-fold higher dose, and preserves bone strength, as indicated by compression testing of the vertebral core and cortical bone of the femoral diaphysis.

In both rats and humans, chronic exposure to alendronate (ALN) blunts the anabolic response of bone to parathyroid hormone (PTH). Compared with vehicle-treated controls, rats pretreated with subcutaneous ALN twice weekly for 16 weeks showed a 2-week delay in onset of the anabolic response to $\mathrm{PTH}$, and incomplete recovery of this response over time, as indicated by serial quantitative CT. In contrast, a single intravenous injection of ZOL or ALN did not attenuate the anabolic effect of PTH, even when, in the case of ZOL, administered at supra-pharmacological doses. Although the osteoblast is not generally considered to be a target for inhibition by bisphosphonates, the finding that bisphosphonates reduce protein prenylation in $\mathrm{MC} 3 \mathrm{~T} 3-1 \mathrm{~b}$ cells in vitro suggests that these drugs may affect the osteoblast cytoskeleton. Because of the inefficient uptake of bisphosphonates by osteoblasts, chronic bisphosphonate exposure is required to attenuate the PTH-mediated transformation of bone lining cells into matrixsecreting osteoblasts, a crucial step in bone's early anabolic response to $\mathrm{PTH}$

In summary, animal studies confirm that, even at high doses, intravenous ZOL inhibits bone resorption without adversely affecting bone formation or mineralization. This finding adds support to the use of a ZOL $5 \mathrm{mg}$ single-dose intravenous infusion regimen in humans.

\section{SY425. POTENTIAL CLINICAL UTILITY OF A NOVEL, LONG-ACTING} BISPHOSPHONATE IN METABOLIC BONE DISEASE

I.R. Reid; Department of Medicine, University of Auckland, Auckland, New Zealand

Oral bisphosphonates are effective agents for the management of osteoporosis and Paget's disease, but their very limited gastrointestinal absorption and low potency necessitate frequent administration on an empty stomach. In addition, their poor gastrointestinal tolerability restricts the maximum single dose that can be given via this route. Thus there is a need for more convenient bisphosphonate dosing regimens, coupled with more effective agents, to encourage long-term patient compliance and prolong the duration of disease remission.

Zoledronic acid, a potent bisphosphonate administered as a single 15-minute intravenous infusion, has been shown to produce quantitatively similar improvements in markers of bone turnover and bone mineral density (BMD) to those achieved by 12 months' of oral bisphosphonate therapy in women with post-menopausal osteoporosis. Treatment regimens of intravenous zoledronic acid $0.25,0.5$ or $1.0 \mathrm{mg}$ every 3 months, $2 \mathrm{mg}$ every 6 months, or $4 \mathrm{mg}$ as a single dose provided similar increases in BMD of the spine $(4.3-5.1 \%)$ and femoral neck (3.1$3.5 \%$ ) and were equally well tolerated. Mild and transient episodes of musculoskeletal pain, nausea and fever occurred with all dosage regimens, particularly after the initial infusion. Results from a recent randomized, double-blind trial in postmenopausal women with osteoporosis/osteopenia $(n=128)$ indicate that a single intravenous infusion of zoledronic acid $5 \mathrm{mg}$ produces greater and more rapid reductions in biochemical markers of bone resorption than oral alendronate $70 \mathrm{mg}$ once weekly over a 6-month treatment period. Moreover, when offered a choice of bisphosphonate regimen, most patients in this trial preferred to receive a once yearly intravenous infusion rather than a once weekly oral pill. The on-going HORIZONPFT trial (due for completion in late 2006), a Phase III study in more than 7,000 elderly women ( $\geq 65$ years) with postmenopausal osteoporosis, is designed to assess the effects of once yearly intravenous zoledronic acid on the incidence of hip and vertebral fractures.

Information on the efficacy and safety of intravenous zoledronic acid in the treatment of Paget's disease comes from two randomized, double-blind trials that compared a single 15minute intravenous infusion of zoledronic acid $5 \mathrm{mg}(\mathrm{n}=182)$ with a 60-day regimen of oral risedronate $30 \mathrm{mg} /$ day $(n=175)$. Pooled findings from the two studies indicated that a higher proportion of zoledronic acid- than risedronate-treated patients (96\% vs $74 \%, \mathrm{P}<0.001)$ achieved the primary efficacy endpoint ( $\geq 75 \%$ reduction or normalization of total alkaline phosphatase excess) within 6 months. Moreover, the time to onset of therapeutic response was considerably shorter with zoledronic acid than with risedronate (median 64 vs 89 days, $\mathrm{P}<0.001$ ). Zoledronic acid's efficacy advantage applied across all demographic, disease severity, and treatment history subgroups, and to other markers of bone turnover. Improvements in quality of life (physical functioning), as assessed using the SF-36 General Health Survey, occurred at 3 and 6 months after treatment with zoledronic acid, and were significantly greater than those seen in the risedronate group at 3 months. Transient influenza-like symptoms, occurring in the first 3 days after treatment initiation, and hypocalcaemia (occurring in the first 2 weeks) were more frequent with zoledronic acid than with risedronate. Follow-up of treatment responders (median 190 days after completion of the 6-month study) showed a loss of therapeutic response in only $0.9 \%$ of zoledronic acid-treated patients, compared with $26 \%$ of risedronate-treated patients $(\mathrm{P}<0.001)$ Follow-up data now extend to almost 2 years, and confirm the sustained disease remission achieved with zoledronic acid in Paget's disease. 
The RANK/RANKL/OPG Pathway: From Bench to Clinical Development Sponsor: AMGEN

\section{SY426. ROLE OF THE RANK LIGAND PATHWAY IN THE REGULATION OF BONE REMODELING}

\section{Lacey; Amgen Inc., Thousand Oaks, USA.}

Maintenance of skeletal health requires an appropriate balance of bone formation and bone resorption, processes directly governed by osteoblasts and osteoclasts, respectively. Receptor activator of NF-kB ligand (RANKL), RANK, and osteoprotegerin (OPG), comprise a critical pathway in the regulation of bone resorption. Initiated with the discovery of OPG, the RANKL/ RANK/OPG axis was elucidated in large measure by genetic ablation and transgenic expression experiments in mice. The results firmly established the central importance of the RANKL pathway in osteoclast biology and hence its pivotal role in bone mass regulation.

RANKL is a type II membrane protein expressed by osteoblasts that is potentially released in soluble form via proteolytic cleavage. RANK, the cognate receptor of RANKL, is expressed by osteoclasts and osteoclast progenitors. RANKL binds to RANK on the surface of precursors to drive differentiation and on mature osteoclasts it promotes both cellular activation and survival. In the absence of RANKL, osteoclasts cannot form, function or survive.

To modulate the bone resorbing effects of RANKL, the body naturally produces OPG. OPG is essentially a decoy receptor for RANKL that binds to and prevents RANKL from interacting with RANK on the cell surface of osteoclasts and their progenitors. In this way, OPG neutralizes the effects of RANKL on osteoclast differentiation and function thereby inhibiting bone resorption.

The expression of both RANKL and OPG appear to be coordinately regulated by hormones, inflammatory mediators/ cytokines, and likely local factors so that bone resorption can be finely modulated. Skeletal disorders characterized by excessive bone resorption or bone loss likely reflect a disturbance of this regulation. Since RANKL is the primary mediator of bone resorption, therapeutic inhibition of RANKL may be a useful approach for treating a broad spectrum of bone loss disorders including osteoporosis, cancer-related bone destruction, and inflammatory bone diseases.

\section{SY427. RANK LIGAND INHIBITION: NEW INSIGHTS IN THE TREATMENT AND PREVENTION OF BONE LOSS}

\section{A. Diez-Pérez; Hospital del Mar, Barcelona, Spain}

RANKL, along with its two receptors RANK and osteoprotegerin (OPG), play a critical role in regulation of bone remodeling. Denosumab (AMG 162) is a fully human monoclonal antibody $\left(\operatorname{IgG}_{2}\right)$ with high affinity and specificity for RANKL. A Phase 1 study demonstrated that a single subcutaneous dose of denosumab resulted in rapid (within 12 hours), substantial (up to $84 \%$ ), sustained (up to 6 months), and reversible decrease of osteoclast bone resorptive activity in healthy postmenopausal women as demonstrated by a decrease in urinary and serum NTx levels from baseline.

In an ongoing Phase 2 study, the safety and efficacy of denosumab is studied in postmenopausal women with low BMD (BMD T-Score <-1.8). In this randomized, double-blind, placebo-controlled, multi-center, dose ranging study, treatment with denosumab every 3 or 6 months for 1 year significantly decreased bone turnover markers and significantly increased BMD at the lumbar spine, hip and distal $1 / 3$ radius relative to placebo (all sites $\mathrm{p}<0.001$ ). The lumbar spine BMD increased within 1 month of denosumab administration, and at month 12 BMD increased by up to $7 \%$. BMD increases with the denosumab $60 \mathrm{mg}, 6$ monthly dose were similar to, and at some sites greater than, alendronate. Further data analysis showed that denosumab enhanced bone mass at the lumbar spine regardless of patient baseline weight or bone mineral density. Continued denosumab treatment for 24 months resulted in consistent reductions in bone resorption and associated continued, significant increases in BMD from baseline.

No changes in white blood cell counts have been observed including T cells, B cells and NK cells. Denosumab appears to be well tolerated in these studies with no significant increase in the severity and number of adverse events observed in denosumab treatments groups compared to placebo or alendronate; no neutralizing antibodies to denosumab have been observed.

Further studies are ongoing to evaluate the potential of denosumab for use in the treatment for postmenopausal osteoporosis.

\section{SY428. REQUIREMENTS FOR CLINICAL TRIALS IN OSTEOPOROSIS AND OSTEOARTHRITIS: WHAT IS NEW IN 2006?}

J.-Y. Reginster; WHO Collaborating Center for Public Health Aspects of Rheumatic Diseases, University of Liège, Liège, Belgium.

Recent advances in the understanding of the epidemiology of osteoarthritis and osteoporosis suggest that previous guidelines for the registration of drugs in these diseases might be no longer substantiated. Following an extensive review of the literature (1990-2004), it appears that a number of points deserve discussion. They mainly relate to the nature of the indication being granted to new chemical entities (treatment of osteoporosis in women at high risk of fractures instead of prevention or treatment of osteoporosis), the requirements of showing an anti-fracture efficacy on all or major nonvertebral fractures (instead of the hip), the duration of pivotal trials ( 2 years instead of 3 ) and the possibility of considering bridging studies for new routes of administration, new doses or new regimens of previously approved drugs. The indication for the treatment of osteoporosis in males could also be granted on the basis of a placebo controlled study with bone mineral density changes after one year as primary endpoint, for medications approved in the treatment of osteoporosis in women at high risk of fractures. For osteoarthritis, it cannot be recommended to use time to joint surgery as a primary endpoint of failure for structure modifying trials of hip or knee, since the parameter has sensitivity but lacks specificity. In contrast, in existing trials, a lack of progression of joint space narrowing has predictive value of $>90 \%$ for not having surgery. It is suggested utilising joint space narrowing (e.g. $>0.3-0.7 \mathrm{~mm}$ ) combined with a lack of clinically relevant improvement in symptoms (e.g. > 20-25\%) for "failure" as a secondary outcome in structure modifying trials of the hip and knee. The primary endpoints should remain the concomitant evaluation of joint space narrowing, on plain X-rays and a validated scale for the assessment of symptomatic benefits.

Injection of Hyaluronic Acid, Osteoarthritis and Evidence Based Medicine: from Concept to Clinical Application Sponsor: IBSA-GENEVRIER

\section{SY429. MECHANISMS OF ACTION OF HYALURONIC ACID}

X. Chevalier; Department of Rheumatology, Hospital Henri Mondor, Creteil, France.

Intra-articular injections of hyaluronic acid (HA) are increasingly accepted as therapy for knee osteoarthritis. HA is a high molecular weight glycosaminoglycan $\left(2.4 \times 10^{6} \mathrm{Da}\right)$ in normal knee, where it is responsible for viscoelastic properties of the synovial fluid. In osteoarthritic knee its concentration and its molecular weight are reduced, which may impair the viscoelastic protection of articular surfaces. On the other hand, HA is synthesized by the chondrocyte and plays a major role in the extracellular matrix architecture, where it links agrecans to form huge macromolecular complexes. HA binds to numerous cells through specific transmembrane receptors (the CD-44 receptors) and thus participates to the cartilage homeostasis. 
The exact mechanism by which HA produces clinical improvement still remains to be elucidated. Once injected into the joint, HA half-life is very short (12-17 h) though its clinical effect lasts several months. This paradox may be explained by cellular effects of HA on the extra-cellular matrix, immune system and by its anti-inflammatory properties. Therefore the concept of viscosupplementation has evolved in a more complex concept of action named viscoinduction. Thus, it has been demonstrated in in vitro studies that exogenous HA can increase the production of HA by synoviocytes in an autocrine fashion. On chondrocytes HA exerts anabolic effects and can prevent the deleterious effect of IL-1 b on PG depletion and on production of pro-inflammatory mediators such as free radicals and NO. HA also demonstrates an antiapoptotic effect in vitro on Fas-induced apoptosis of chondrocytes and can counteract the deleterious effects of fragments of fibronectin.

Those in vitro studies have been substantiating in in vivo experimental animal models of cartilage injury or osteoarthritis. The majority of the studies show that HA can exert preventive and therapeutic effects on cartilage damage as well as reducing MMPs activity, number of apoptotic cells and in situ production of NO. Few negative studies showing deleterious effects of HA may be related to the time of i.a injection and to an analgesic effect leading to overuse of the joint. HA is also known to reduce pain due to a reduction of nociception and sensorial response. Taken together these experimental studies suggest a chondroprotective action of HA which needs to be confirmed in clinical practice.

In human practice it has been shown that repeated i.a injections of HA lead to an increased concentration of synovial HA, suggesting an autocrine production and/or an effect on cartilage homeostasis.

HA is a promising molecule with numerous cellular effects and potential tissue reparative properties.

\section{SY430. EFFICACY OF INTRA-ARTICULAR HYALURONAN INJECTIONS IN KNEE OSTEOARTHRITIS: WHAT ARE THE EVIDENCE?}

E. Maheu; Hôpital St Antoine, Paris, France

Hyaluronan (HA) intra-articular (IA) injections have become a growing option in the treatment of knee osteoarthritis (OA) in Europe during the 5 past years. The EULAR recommendations clearly state that it might be considered as a second-line therapy. Although recommended by EULAR and ACR, HA efficacy remains discussed, as outlined by recent meta-analyses.

Methods: We performed a review of the published clinical randomized controlled trials conducted in knee osteoarthritis, and meta-analyses. The time period considered ran from 1965 to the end of 2005. Control treatments taken into consideration were placebos (saline solutions), NSAIDs, and IA corticosteroids. All randomized controlled trials which assessed pain and/or function were kept into this review.

Results: More than 60 trials were identified, of which more than 30 met the eligibility criteria. HA used in these trials were various, differing by their origin, extraction, molecular weight, concentration and recommended administration schedule (most of trials were done with 3 to 5 weekly injections). 26 trials compared HA to placebo, most of them conducted with a double-blind design. Ten were negative trials, 16 were positive. The majority of the trials showed a significant superiority of HA over placebo on pain and functional impairment reduction. Efficacy starts after 3 to 6 weeks, and lasts as long as 6 to 12 months. Four trials compared HA to various NSAIDs: NSAIDs were generally more rapid than HA to relieve symptoms, but equally effective after 3 to 6 months of treatment, with less sideeffects in the HA groups. Ten trials compared HA with IA corticosteroids injections: 4 studies were opened-label, while 6 were single or double-blind. All trials were concordant: IA corticosteroids acted faster than HA, but this superiority did not last after the $3 \mathrm{rd}$ week. At months 1,3 or $6 \mathrm{HA}$ was more effective than IA corticosteroids on pain relief and functional improvement. Four meta-analyses have been recently published.
One retained a mild efficacy for HA. Two were positive and concluded that HA was effective on symptoms with a moderate effect-size. One failed to identify any symptomatic effect of HA treatment in knee OA.

Conclusion: Most of the randomised controlled trials have shown that HA was more effective than placebo on OA symptoms. Besides, trials have shown that HA was as effective as NSAIDs and more effective than IA corticosteroids on the long term. Two of the 4 published meta-analyses supported a moderate efficacy of HA injections, as stated by the EULAR group.

\section{SY431. HYALURONIC ACID IN OTHER OSTEOARTHRITIC JOINTS: HIP, THUMB, SHOULDER AND ANKLE}

\section{Legre; Hôpital de la Conception, Marseille, France}

Intra-articular treatment with hyaluronic acid (HA) is part of the treatment of knee OA. Owing to its beneficial benefit/risk profile, it has been proposed in other OA joints such as hip, shoulder, first carpo-metacarpal joint of the thumb and ankle. Most of these studies with an open label design need to be cautiously interpreted. Furthermore the rhythm and the number of intra-articular injections differ from one study to another, making any comparison difficult.

In hip OA, more than 10 studies have been published using different molecular weight HA. In open label studies, including patients with radiological stage II to III in Kellgren-Lawrence classification, the number of responders varies from 50 to $65 \%$ following one to 5 i.a injections. Only 2 randomized placebocontrolled studies have been recently published. The first one showed that 3 i.a. injections of sodium hyaluronate are more efficient at month 3 compared to placebo. The second study compared ultrasound guided i.a. injections of hyaluronate sodium (3 weekly injections), one i.a. injection of depomedrol $40 \mathrm{mg}$, and 2 and 3 placebo i.a. injections. Compared to saline, corticosteroid (CS) was more efficient on "pain on walking" (effect size (ES): $0.6)$ than HA (ES: 0.4). Statistical difference was only reached with CS. However in sub-groups of patients with moderate hip OA ( < IV stage) and without synovial fluid effusion, HA was superior to placebo. Safety profile shows about the same prevalence of inflammatory pain reactions as in knee OA.

Very few studies have been published in the OA of the first carpo-metacarpal joint of the thumb. Two open prospective studies with limited number of patients (respectively 16 and 20) and a number of 2 to 3 and 5 weekly i.a. injections indicated a beneficial effect of HA with a follow-up of 3 and 5 months. A controlled, randomized study comparing 4 weekly i.a injections of HA with a corticosteroid (dexamethasone $4 \mathrm{mg} / \mathrm{mL}$ ) showed a long-term benefit of HA over the CS at month 6. Another 26week controlled randomized study compared 3 i.a. injections of HA to triamcinolone acetonide (TA). TA was significantly better than HA at weeks 2-3, but at week 26, a slight superiority of HA could be observed. Larger placebo-controlled study with fluoroscopy guided i.a. injections are required.

In shoulder pain, only poor quality trials, mixing several diagnoses and different designs of i.a. injections, have been published. Recently a large double-blind, randomized trial of i.a. injection of sodium hyaluronate in chronic shoulder pain has been reported. Five weekly saline i.a. injections were compared to 5 and to 3 HA i.a. injections (150 completers per group). Two thirds of patients were stratified to the OA group (2/3 of them had rotatory cuff tear), others had isolated RCT or adhesive capsulitis. Results showed that both 3 and 5 HA injections demonstrated significant pain reduction from weeks 7 to week 26, with the majority of benefit seen in OA patients. Unfortunately the intra-articular route of injection was not verified and the mixture of diagnoses made interpretation of the data difficult.

OA of the ankle is rare. Two prospective open studies with a limited number of patients (less than 20) showed a benefit of respectively 5 and $3 \mathrm{HA}$ injections up to 6 months. One RPC trial including only 20 patients showed a significant proportion of patients with more than $50 \%$ of benefit at month 6 in the HA group compared to control (55\% vs $12,5 \%$ ). 
Since yet, only trials in hip OA seem to indicate a limited beneficial effect, though the number and the design of injections need to be better defined. For other joints, it is necessary to perform large RPC trials using optimal protocols (fluoroscopy guided i.a. injections, trying to limit the number of i.a. injections of HA) and to confirm some promising results, mainly observed in open trials.

\section{SY432. DATA DRIVEN RECOMMENDATIONS FOR THE PERFORMANCE OF INTRA-ARTICULAR KNEE INJECTIONS}

T. Boyer, Service de rhumatologie Hôpital Bichat Paris

Hyaluronic acid injections must be strictly intra-articular. The frequency of injections which are not correctly carried out was studied by opaque arthrography (Jones BMJ 1993), and by miniair arthrography (Bliddal Ann RheumDis 1999). In a personal study, we performed methylene blue injections immediately before knee arthroscopy. The results are concordant and show that a third of the injections on a dry knee is not intra-articular. The way of injection plays a major part. The lateral juxtapatellar portal makes it possible to obtain the best success rate. The anterior portals are to be proscribed because the product is too much often injected into the fat pad.

Intra articular injections are not so easy and uncontrolled experience does not increase the performance of correct placement. Controlled experience by arthrography, arthroscopy or on cadaver is recommended.

\section{SY433. OVERALL TOLERABILITY AND ANALGESIC ACTIVITY OF SINO-} VIAL IN THE TREATMENT OF KNEE OA

P. Brühlmann; Rheumatology and Physical Medicine Department, University Hospital, Zurich, Switzerland

Viscosupplementation with intra-articular hyaluronic acid (HA) is an alternative to the treatment of symptomatic knee osteoarthritis (OA) with pain relieving drugs. Sinovial, is a sterile, nonpyrogenic $0.8 \%$ solution of highly purified sodium hyaluronate for intra-articular application. The aim of the present study was to investigate the safety and tolerability profile of this preparation in patients with symptomatic knee OA over 24 weeks.

This was a single group, open-label study including outpatients of both sexes, aged between 18 and 85 years, with symptomatic knee OA. All patients underwent weekly intra-articular injections of HA for 5 consecutive weeks and were followed-up for 19 additional weeks. The safety and tolerability profile (primary endpoint) was assessed by adverse event (AE) reporting. The secondary endpoint was efficacy evaluated by changes in WOMAC score vs. baseline. Patient and physician satisfaction were also recorded.

Intra-articular HA was generally well tolerated. The most frequent $\mathrm{AE}$ was pain at the injection site (5.8\% of the injections); no serious treatment-related $\mathrm{AE}$ was reported. The WOMAC score was significantly reduced within the first two weeks of treatment (from $4.02 \pm 1.90$ to $3.55 \pm 2.04, p=0.0011$ ), further decreased by the end of the injection series (week 6: $2.59 \pm 1.90$; $p<0.0001$ ) and maintained during the follow-up (week 24: $2.44 \pm$ $1.88 ; p<0.0001)$. The WOMAC subscores were also significantly reduced from week 4 for "pain" and from week 6 for "stiffness" and "physical function".

In the present study, intra-articular HA was well tolerated and safe in patients with symptomatic knee OA. Based on the sustained improvements in WOMAC score and subscores, a carryover effect lasting for at least 19 weeks after the last injection may be proposed. These results further confirm the evidence of efficacy and safety of intra-articular HA in the management of knee OA.

Reduction of Falls and Osteoporotic Fractures: Plain Vitamin D or D-Hormone Analogs

Sponsor: TEVA
SY434. MULTIFACTORIAL PATHOGENESIS OF FALLS AND FRACTURES AND FALL RISK ASSESSMENT

M. Runge; Aerpah Clinic Esslingen, Esslingen, Germany

Epidemiology and pathogenesis of falls in the elderly and their consequences: Falls are a common event among certain groups of elderly people, accounting for $87 \%$ of all fractures in the age of 65 years and older (NCIPC). A third of all elderly people (age $>65$ years) fall each year. About $5 \%$ of these falls result in a fracture and a fifth of these fractures are hip fractures. From another perspective, more than $90 \%$ of hip fractures are caused by a fall. Furthermore falls also cause injuries of soft tissue and cerebrum, chronic impairment of mood and self-esteem and lead to long-lasting self-restrictions of physical activity. Hip fractures, as the most serious consequences of a fall, have an exponential age-related increase and result in a 15$25 \%$ increase of mortality in the successive year and a $20 \%$ increase in nursing home admissions. The pathogeneses of agerelated neuromuscular decline, falls, bone strength and nonvertebral fractures are inextricably interconnected as a pathogenetic cascade. Rather than having a single cause, falls and fallrelated fractures are the result of a combination of intrinsic, situational, and environmental factors. This process is highly age-related: falls occur among elderly people in a significantly higher rate and with different fall mechanisms, especially as sideway fall with impact to the greater trochanter. The majority of falls occur without disruption of consciousness as nonsyncopal falls during normal daily activities without extraordinary demand to the equilibrium and without overwhelming external force. Non-vertebral osteoporotic fractures of hip, humerus, pelvis and wrist result from a combination of falls and reduced bone strength. This combined pathogenesis demands a combined diagnostic and therapeutic approach comprising both bone strength and fall risk. The key finding is: special characteristics differentiate fallers from non-fallers, and these fall risk factors accumulate in one person as an individual cluster of impairments. Interventions against age-related falls and fractures have to be targeted on these patients with high risk who can be identified by their individual aggregation of specific fall risk factors.

Fall risk assessment in the aged: An evidence-based fall risk assessment addresses parameters which have proven to be independent fall risk factors in multivariate analyses of prospective studies:

\section{Impairments of}

1. muscle power of lower extremities, measured by chair rise test, 2. postural capacity (balance), measured by tandem manoeuvres, 3. vision.

4. Multiple medications as indicator of high comorbidity

5. Drugs with specific impairment of postural capacity

6. Cognitive impairment.

Examining muscle function and gait and balance disorders requires age-, sex- and race-related reference values which are collected (cf. www.mobility-clinic.de). Muscle function and postural competence, which both have been proven independently correlated to fall risk, can be measured respectively with the chair rising test and tandem tests, and with the newly developed Leonardo mechanography.

Multifactorial interventions in prevention and therapy: There is a growing body of evidence that falls can be prevented by multifactorial interventions with muscle and balance training and review of medications (Tinetti et al 1994, Close et al 1999). Until now the traditional pharmacological approach concentrates on increasing bone strength, but neuromuscular functions and postural capacity are apparently open to pharmacological regimens. There is emerging clinical evidence that Alfacalcidol, a pro-drug of D-Hormone, improves muscle power (Sørensen et al. 1979, Scharla et al. 2000, Verhaar et al. 2000). A study has already demonstrated the reduction of falls by D-Hormone in elderly patients, aged 65-77 years with normal Vitamin D status 
(Gallagher et al. 2001). Dukas et al. have shown that $1 \mu \mathrm{g} \mathrm{Al}-$ facalcidol daily reduce significantly the number of falls $(-54 \%)$ and fallers $(-55 \%)$ in community-dwelling elderly women and men with a total calcium intake of more than $500 \mathrm{mg}$ daily and normal vitamin D serum levels (Dukas, Bischoff et al 2004). A reduced creatinine clearance $(\mathrm{CrCl})$ of $<65 \mathrm{ml} / \mathrm{min}$ is significantly associated with low D-hormone serum levels and with a significant four times increased risk of falls. 36 weeks of treatment with Alfacalcidol ( $1 \mu \mathrm{g}$ daily) significantly and safely reduces in community-dwelling elderly women and men with a $\mathrm{CrCl}$ of $<65 \mathrm{ml} / \mathrm{min}$ the low $\mathrm{CrCl}$ associated increased number of fallers $(-74 \%)$ and the high risk of falls $(-71 \%)$ (Dukas, Schacht et al 2004). Based on further confirmation of these findings Alfacalcidol opens a new therapeutic strategy for treating osteoporosis by simultaneously increasing bone strength and decreasing falls. It has a double impact on fall- and osteoporosis-related fractures.

\section{SY435. DECREASED CREATININE CLEARANCE IS A RISK FACTOR FOR FALLS IN UNTREATED ELDERLY WOMEN BUT NOT IN WOMEN TREA- TED WITH CALCITRIOL}

J. C. Gallagher; Bone Metabolism, Creighton University Medical School, Omaha, NE 68005, USA

Falls in the elderly are an important risk factor for fractures. An age related decrease in renal function leads to decreased production of 1,25-dihydroxyvitamin $\mathrm{D}_{3}$ which may be a risk factor for falls in the elderly. In a 3 year double blind placebo controlled study we compared the efficacy of calcitriol,estrogen, a combination of both or placebo in preventing bone loss in 489 elderly women age 65-76 years. We collected also prospective data on Falls over 3 years. In this analysis, we compared the effects of treatment with placebo or calcitriol on Falls in two groups with different renal function, using the KDOQI defininition of stage III chronic renal failure, women with a GFR $<60 \mathrm{ml} / \mathrm{min}$ compared to women with a GFR $>60 \mathrm{ml} /$ min. GFR was calculated from a 24 hour urine creatinine clearance. In an intent to treat analysis using a Poisson regression model, creatinine clearance was found to be a statistically significant predictor of falls in the placebo group $(p=0.05)$ but not in the calcitriol treated group. On placebo women with GFR group $<60 \mathrm{ml} / \mathrm{min}$ had a $33 \%$ increase in falls $(p=0.05)$ compared to women with a GFR $>60 \mathrm{ml} / \mathrm{min}$. On calcitriol the mean cumulative number of falls was 50 percent lower $(\mathrm{p}<$ $0.001)$ compared to the placebo group.A similar reduction in falls was seen in the group treated with calcitriol and estrogen. In the placebo treated group there were significant differences in several parameters between the GFR groups. The group with GFR $<60 \mathrm{ml} / \mathrm{min}$ had significantly lower serum $1,25(\mathrm{OH})_{2} \mathrm{D}_{3}$, lower calcium absorption and lower physical performance measurements; there were no significant differences in serum PTH or serum 25 hydroxyvitamin D.

In conclusion an age related decrease in renal function was associated with an increased incidence of falls, except in women treated with calcitriol. Because there is an age related resistance in calcium absorption to endogenous serum 1,25-dihydroyvitamin D there is a need for higher levels, however the aging kidney is not able to increase production and oral calcitriol is needed to increase serum 1,25-dihydroyvitamin D.Calcitriol may reduce falls by improving physical performance.

\section{SY436. PLAIN VITAMIN D AND FALLS AND FRACTURES: QUO VADIS?}

R.M. Francis; University of Newcastle upon Tyne and Bone Clinic, Freeman Hospital, Newcastle upon Tyne, NE7 7DN, United Kingdom

Vitamin D insufficiency is common in older people, where it is thought to contribute to bone loss, postural instability and an increased risk of falls and fractures. The role of calcium and vitamin D supplementation in the prevention of falls and fractures in older people remains uncertain. Chapuy's study indicates that calcium and vitamin D decreases hip fractures in elderly institutionalised women, whereas the work of Dawson-Hughes and Larsen suggests that it may also reduce non-vertebral fractures in community-dwelling older people. The results of the anti-fracture studies of vitamin $\mathrm{D}$ alone are equivocal, with fracture reduction reported by Heinkinheimo and Trivedi, but no decrease observed by Lips or Meyer.

The results of other large studies of calcium and/or vitamin D supplementation in the UK are now emerging, which challenge existing views on the prevention of falls and fractures in older people. The Wessex Fracture Prevention Trial compared the effect of annual IM vitamin D 300,000 IU or placebo in 9,440 community-dwelling men and women aged over 75 (J Bone Miner Res 2004; 19 Suppl.1: S57). This showed no reduction in either falls or fractures with IM vitamin D over three years.

The MRC RECORD Study is a randomised trial of oral calcium (1,000 mg daily), vitamin D (800 IU daily), both or placebo on the secondary prevention of low trauma fractures in 5,292 men and women aged over 70 (Lancet 2005; 365: 16211628). During the 24 to 62 months' follow-up, there was no difference between the treatment groups in all reported fractures, radiologically confirmed fractures, hip fractures or falls.

The Northern and Yorkshire Region Study is a pragmatic 'open' trial in 3,314 women aged over 70 with a risk factor for hip fracture (any prior fracture, body weight $<58 \mathrm{~kg}$, smoker, family history of hip fracture, fair or poor self-reported health). Participants were randomised to receive oral calcium (1,000 $\mathrm{mg}$ daily) and vitamin D (800 IU daily), together with an information leaflet on diet and falls prevention, whereas the control group received the leaflet alone. After a median follow-up of 25 months, there was no reduction in fracture or falls in women randomised to receive calcium and vitamin D (Br Med J 2005; 330:1003-1006).

The recent studies cast doubt on the role of plain vitamin D, with or without calcium, in the prevention of falls and fractures in older people. New and revised meta-analyses, incorporating the results of these trials, will hopefully clarify the situation.

\section{SY437. REDUCTION OF FALLS AND FALLERS USING D-HORMONE} ANALOGS

L. Dukas ${ }^{1}$, E. Schacht ${ }^{2}$; ${ }^{1}$ Acute Geriatric University Clinic, Kantonsspital, Basel, Switzerland, ${ }^{2}$ Dept. of Rheumatology and Rehabilitation, University Clinic Balgrist, Zurich, Switzerland

Objectives: There is controversial discussion about the use of plain vitamin D or D-hormone analogues in the prevention of falls in the elderly. Below a creatinine clearance $(\mathrm{CrCl})$ of less than $65 \mathrm{ml} / \mathrm{min}$. plain vitamin $\mathrm{D}$ is not metabolized into its active muscle and neuronal sensitizing form Calcitriol, the D-Hormone. Recent results in different populations showed that a $\mathrm{CrCl}$ of $<65 \mathrm{ml} / \mathrm{min}$., as a surrogate for low D-hormone serum levels, is associated with a significantly increased risk for falls and fallers. Since this hypothesis suggest that D-hormone would be the only adequate treatment to prevent falls in elderly people with a $\mathrm{CrCl}$ of $<65 \mathrm{ml} / \mathrm{min}$, we analyzed in such a population the effect of Alfacalcidol, a D-hormone analogue, on number of falls and fallers.

Material and Methods: In a population of 378 Swiss community-dwelling women $(\mathrm{N}=191)$ and men $(\mathrm{N}=187), 70$ years old and older, for 36 weeks randomly 191 received $1 \mu \mathrm{g}$ capsules of Alfacalcidol (AlphaD ${ }_{3}{ }^{\circledR}$ Teva), and 187 received one capsule of placebo daily. With the help of questionnaires we regularly assessed the incidence and frequency of falls. Using multivariatecontrolled logistic regression models we assessed the risk of becoming a faller and the risk of falling according to treatment groups and according to a $\mathrm{CrCl}$ cut off of $65 \mathrm{ml} / \mathrm{min}$. The presented results are from ITT analyses.

Results: The 36 weeks of treatment with Alfacalcidol was, compared to placebo, in participants with a $\mathrm{CrCl}$ of $<65 \mathrm{ml} /$ min., associated with a significant reduction in the number of fallers (14/72 vs. $25 / 70$; OR $0.26,95 \%$ CI $0.08-0.80, p=0.019)$, and a significant reduction of the number of falls (16/72 vs. $28 / 70$; OR $0.29,95 \%$ CI $0.09-0.88, \mathrm{p}=0.028)$. No such association was 
observed in participants with a $\mathrm{CrCl}$ of $\geq 65 \mathrm{ml} / \mathrm{min}$ (for fallers $26 /$ 120 vs. $21 / 116$; OR $0.9295 \%$ CI $0.34-2.52, \mathrm{p}=0.875$; for falls $32 /$ 120 vs. $23 / 116$; OR $0.9395 \%$ CI $0.34-2.54, \mathrm{p}=0.885$ ). No cases of clinically relevant hypercalcemia were observed.

Conclusion: Treatment with Alfacalcidol can significantly and safely reduce the low $\mathrm{CrCl}$ associated increased number of fallers and the high risk of falls in a community-dwelling population of elderly men and women having a $\mathrm{CrCl}$ of $<65 \mathrm{ml} / \mathrm{min}$.

\section{Outcomes in Post-Menopausal Osteoporosis: from Clinical Evi- dence to Regulatory Requirements \\ Symposium organized by the Group for the Respect of Ethics and Excellence in Science (GREES) \\ SY438. PATIENTS POPULATION IN CLINICAL TRIALS: FROM ESTAB- LISHED OSTEOPOROSIS TO HIGH-RISK PATIENTS}

P.D. Delmas; Inserm Research Unit 403 and Université Claude Bernard Lyon I, Lyon, France

Osteoporosis is defined as a generalized skeletal disorder, characterized by compromised bone strength predisposing an individual to an increased risk of fracture. Bone strength primarily reflects the integration of bone density and bone quality. Practically, the WHO operational definition defines an osteoporotic woman on the basis of a BMD measurement (spine or hip) showing a T-score below -2.5 . However over the past years it has become evident that fracture risk was not only driven by the level of BMD but by other parameters including bone size and shape, bone turnover, micro architecture, mineralization, damage accumulation (micro fractures) or collagen structure which play a role in bone strength and, hence, in the risk of osteoporotic fractures. These different parameters are not captured by the assessment of BMD.

This has been confirmed by several epidemiological studies showing that at least half of incident fragility fractures occur in postmenopausal women who have a BMD T-score above -2.5. In these populations, using independent risk factors for fractures allows to identify a substantial proportion of women who will experience fragility fractures.

Recently a WHO working party, re-analyzed extensive European, Australian, Japanese and North American databases to better identify individual risk factors for osteoporotic fractures, independently of BMD measurements. The expected outcome of this working group is the production of fracture risk tables, based on the combination of the BMD values and other independent risk factors, including previous fracture, age, family history of osteoporosis, body mass index, tobacco or alcohol use, and diseases predisposing to osteoporosis. High bone turnover has also been repeatedly shown to be an independent risk factor of future fracture risk. These integrated risk assessments predict that approximately one third of postmenopausal women with T-scores between -1 and -2.5 will also experience a fracture within the next ten years. These findings strongly support the concept that the decision process for initiating a treatment in an individual woman, in order to prevent the occurrence of the first osteoporotic fracture, should be based on the global assessment of specific clinical risk factors rather than relying on a BMD measurement only. Hence the theoretical basis to differentiate the osteoporosis prevention indication from the treatment indication as defined in the present note for guidance does not appear to be substantiated any longer. Decision to treat should be based on a projected 10-year fracture risk rather than on an arbitrary BMD threshold.

Patient populations to be included in pivotal clinical trials could include one of the two main subsets: patients with osteoporosis (BMD T-score below -2.5 with or without prevalent fractures) and patients at high risk of experiencing a first fracture, defined as postmenopausal women with osteopenia (BMD T-score between -1 and -2.5 ) associated with one or more independent risk factors, that result in a 10-year probability of fracture in the same order of magnitude as in osteoporotic women, regardless of the time elapsed since menopause.
If clinical studies performed in one or both populations demonstrate a reduction in fracture risk, this could lead to an indication labelled "treatment of postmenopausal women at high risk of fracture" or "prevention of the first fracture". This wording better reflects the patients included in the trials than the "current" wording "prevention of osteoporosis" and "treatment of osteoporosis".

\section{SY439. BRIDGING STUDIES : WHEN ARE THEY RELEVANT ?}

R. Rizzoli; Service of Bone Diseases, WHO Collaborating Center for Osteoporosis Prevention, Department of Rehabilitation and Geriatrics, University Hospitals, Geneva, Switzerland

The efficacy and safety of a new agent for the treatment of osteoporosis should be tested in well designed and sufficiently powered, of at least 2-year duration, placebo- or comparatorcontrolled studies, with incidence of patients with new fractures as primary endpoint. The population to be studied comprises subjects at high risk of fracture, who will represent therefore the target group for treatment, ie postmenopausal women with osteoporosis. The results of pivotal studies will grant the indication " Treatment of osteoporosis in postmenopausal women at high risk of fracture ». This refers thus to a specific dose, and a given route of administration, in a well defined population. However, an extension of the indication could be given for men with osteoporosis, for a new formulation or a new route of administration. In so-called bridging studies, non inferiority trials are comparing the new formulation or route of administration to the original one having demonstrated significant antifracture efficacy. The endpoints are mean BMD changes, and/or percentage of responders, together with changes in bone remodelling, as assessed by biochemical markers of bone turnover. To avoid fracture studies for the potential new indications, ie studies involving numerous patients and of long duration, a particular attention is paid to the analysis of surrogate endpoints in terms of maximal and pharmacodynamic responses. Indeed, these reponses, which are associated with fracture risk reduction, should be identical to those recorded in the pivotal trials, under the conditions where antifracture efficacy has been demonstrated. Equivalence or non-inferiority margins should be preplanned, clinically meaningfull, and the duration of the trial at least one year. This approach also implies that the new indication, the new formulation or the new schedule do not involve another mechanism of action of the compound tested, for instance when the compound is hormonal, or interacts with sex hormones, if osteoporosis in the male gender is concerned. The correlations between the changes in surrogate markers and bone strength, directly evaluated in preclinical studies, may also be taken into consideration.

\section{SY440. MINIMAL REQUIREMENTS FOR REGISTRATION OF DRUGS TO BE USED IN OSTEOPOROTIC MALES}

J.M. Kaufman; Unit for Osteoporosis and Metabolic Bone Diseases, Ghent University Hospital, Belgium

The age-specific risk of fragility fracture of the spine and the hip in men is about half that in women. Fragility fractures in men nevertheless represent a significant public health issue and a major threat for survival and quality of life of elderly men. Presently, few drugs have been approved for treatment of osteoporosis in men. Therefore, requirements for registration of drugs for treatment of osteoporotic men are of practical public health relevancy.

Epidemiological studies have established that the relationship between fracture risk and BMD (as assessed by DEXA) is similar in men and women with a same fracture risk for a given absolute threshold of BMD, independently of gender. Prevalent osteoporotic fractures also predict the risk of future fracture to the same extent in both genders. Furthermore, clinical trials with both antiresorbing- and bone forming agents have indicated qualitatively and quantitatively similar skeletal responses in osteoporotic postmenopausal women and men in terms of changes in biochemical 
markers of bone turnover and BMD, with similar trends in men and women for relative fracture risk reduction. On this background, albeit demonstration of anti-fracture efficacy remains the gold standard for approval of new chemical entities (NCEs) for treatment of osteoporosis in men, the use of BMD changes can be considered as a primary endpoint in clinical trials designed to demonstrate the efficacy of a NCE. Prerequisites for this approach are that the applicant has unequivocally demonstrated the antifracture efficacy of the NCE in postmenopausal women and that extensive investigation in relevant animal models has not revealed significant gender-specific issues of skeletal toxicity and/or efficacy.

These conditions being fulfilled, once an initial marketing authorisation has been granted to an NCE for the treatment of postmenopausal women at high risk of fracture, a study in male osteoporotic patients showing changes in BMD versus placebo of similar magnitude to those observed in postmenopausal osteoporotic women should be deemed sufficient for granting marketing authorisation in the indication "treatment of osteoporosis in men at high risk of fracture", provided the duration of the study is at least 1 year, the same route of administration is used, the dosage is justified, and the included subjects have a similar absolute fracture risk as in the studies in postmenopausal women. Such a bridging strategy cannot be applied if the mechanism of action of the NCE is gender-specific and/or hormonal, in which case direct demonstration of fracture risk reduction in men is needed.

SY441. UPDATE OF THE EUROPEAN REGULATORY REQUIREMENTS FOR THE EVALUATION OF NEW MEDICINAL PRODUCTS IN THE TREATMENT OF POSTMENOPAUSAL OSTEOPOROSIS

Bruno Flamion; on behalf of the European Medicine Agency (EMEA)
Considering the recent changes in the scientific community's views on postmenopausal osteoporosis, the Committee for Medicinal Products for Human Use (CHMP) of the EMEA has decided, through its Efficacy Working Party, to revise the Note for Guidance on Postmenopausal Osteoporosis in Women, which was initially drafted in 1995 and had been already revised in 2001 . No other guideline of the CHMP has been revised so frequently, which is a sign that the scientific opinion on osteoporosis is constantly shaping up. Compared with the previous draft, the main changes in the new guideline can be summarised as follows. Since the aim of treatment is to decrease the incidence of fractures, the distinction between "prevention" and "treatment" of osteoporosis has been abandoned; the expected indication will be the treatment of osteoporosis in postmenopausal women at high risk of fracture. Predefined levels of risk for fractures in the studied population should be carefully established on the basis of known independent risk factors (BMD, age, prevalent fractures, family history, etc) and not only on BMD T-scores. The minimal duration of randomised trials has been reduced to 2 years, while the importance of collecting post-marketing data on maintenance of prevention of fractures has been stressed. The primary endpoint is the incidence of patients with new spinal and non-spinal fractures. The indication may be restricted, e.g. to the effect on the axial skeleton, depending on the results of the clinical trials Notably, the title of the guideline has been changed to "...treatment of primary osteoporosis" since requirements for a marketing indication for the treatment of osteoporosis in males, including acceptance of bridging studies under some conditions, have been defined (secondary osteoporosis is not discussed in this document). Bridging studies based on BMD and other biomarkers are also acceptable for a new way of administration of an existing product. 